\title{
Predicting the T2K neutrino flux and measuring oscillation parameters
}

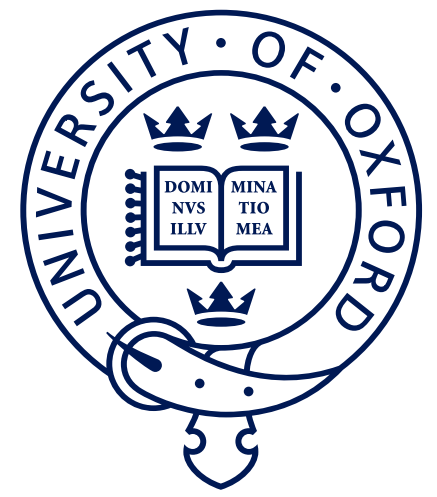 \\ Tomislav Vladisavljevic \\ Balliol College \\ University of Oxford
}

A thesis submitted in fulfilment of the requirements for the degree of Doctor of Philosophy

Trinity 2019 


\section{Abstract}

$\mathrm{T} 2 \mathrm{~K}$ is an experiment designed to study the ways in which neutrinos evolve as they propagate through space. The experiment uses an artificial beam of muon neutrinos, produced at the J-PARC facility in Japan. The spontaneous mixing of these beam neutrinos with other flavours is measured after 295 kilometres, at the location of the Super-Kamiokande detector. The initial beam composition is measured after 280 metres, using a combination of two near detectors. T2K is optimised for measurements of electron neutrino appearance, $\nu_{\mu} \rightarrow \nu_{e}$, and muon neutrino survival. Essential for these measurements is a detailed knowledge of the initial beam composition, before neutrino oscillations had occurred. The production of intense neutrino beams at accelerator facilities is challenging, and requires exceptional understanding of chains of hadronic interactions initiated within thick targets. Most of T2K neutrinos are produced from in-flight decays of focused pions and kaons, emitted from an extended graphite target $(90 \mathrm{~cm})$ bombarded with a $30 \mathrm{GeV}$ proton beam. Besides internal constraints from near detector data, $\mathrm{T} 2 \mathrm{~K}$ relies on hadron production measurements collected by other experiments, most importantly NA61, for accurate modelling of the initial neutrino flux.

This thesis presents a new T2K neutrino flux prediction, using the NA61 replicatarget dataset from 2009. The earlier flux prediction relied on NA61 measurements collected with protons incident on a thin graphite target $(2 \mathrm{~cm})$. The following analysis incorporates NA61 data collected on the full length replica of the T2K target in 2009. In light of this new data, the flux calculation technique has been modified. A dramatic reduction in the unoscillated $\nu_{\mu}$ fractional flux uncertainty has been achieved, from $\sim 10 \%$ to $\sim 5 \%$ around the T2K flux peak. The impact of the improved flux prediction on oscillation measurements has been examined, by performing separate fits to ND280 data, and joint ND280 and Super-K data, in the well-established Bayesian Markov Chain Monte Carlo analysis framework. 


\section{Statement of Originality}

This thesis and the work presented in it are my own and were produced by me as a result of my own original research. Results and figures from published works by others have been clearly attributed, and this work has not been submitted for another qualification at this or any other university.

Chapter 1 is aimed to briefly familiarise the reader with the concept of matterantimatter asymmetry, and how T2K in particular is suited for addressing this paradox. Here, the reader is familiarised with the aims of each chapter.

Chapter 2 is intended to provide a brief background to the field of neutrino physics, presenting some of the main experimental milestones reached over the years. Chapter 3 introduces the T2K experiment, with particular emphasis on the design of the beamline for neutrino production. Chapter 4 provides an introduction the NA61 experiment, which collected dedicated measurements for T2K. In particular, the 2009 replica-target dataset from NA61 described here has been analysed by Alexis Häsler.

Chapter 5 describes the method for flux calculation with thin NA61 thin-target measurements. The framework for this calculation had been refined over the years by many members of the T2K Beam Group, most notably Mark Hartz, Ken Sakashita, Megan Friend, Laura Zambelli, Atsuko Ichikawa and others. I was the analyser responsible for calculating the unoscillated flux prediction for the last three publicly released $\mathrm{T} 2 \mathrm{~K}$ oscillation results, and improved on certain features of the code, but the calculation framework had been put in place over many years by the above-mentioned collaborators.

Chapter 6 describes the flux calculation with NA61 replica-target measurements, newly implemented in this thesis. The results described in Chapters 5 are my own original work, with the exception of one of the beam profile studies in Chapter 5.4, cited from a study by Lukas Berns. The new flux calculation presented in this chapter has been approved as one of the upgrades for the next T2K oscillation result.

Chapter 7 is intended as an introduction to the well-established T2K oscillation analysis in the Bayesian framework with the Markov Chain Monte Carlo (MCMC) technique, progressively developed over the years by $\mathrm{T} 2 \mathrm{~K}$ collaborators, most notably Asher Kaboth, Casey Bojechko, Richard Calland, David Payne, Kirsty Duffy, Clarence Wret and others.

The fit results presented in Chapter 8 are my own, using the existing MCMC framework combined with the new flux inputs prepared for this thesis. Lastly, the conclusions in Chapter 9 reflect my personal interpretation of the presented results. 


\section{Acknowledgements}

Writing this thesis would not have been possible without the generous support and encouragement of many people. Firstly, I would like to thank my supervisors, Mark Hartz and Alfons Weber, for their competent guidance through the different stages of my project, both at Oxford and in Japan. Thank you for always trusting in my abilities, and for all the amazing opportunities that I was given as a member of the T2K collaboration. I would also like to thank the whole neutrino physics community at Oxford, especially Dave, Giles, Xianguo and Phil, for contributing to many engaging discussions that deepened my love for the wonderful and varied field of neutrino physics (and also music thanks to Dave). Special thanks go to all fellow students that I had the pleasure of sharing the office with - Kirsty, Raj, Stephen, David, Charles and Dan - for the camaraderie and upbeat atmosphere which made my time spent at Oxford thoroughly enjoyable.

I am very grateful for the help and support received from fellow T2K collaborators. Especially instrumental for the work presented in this thesis has been the support received from Beam group members, Megan, Arturo, Laura and Lukas. Equally valuable has been the guidance from MaCh3 group members - Asher, Patrick, Clarence, Artur and Will - during the later stages of my project.

The amazing friendships formed during the last four years have enriched my graduate experience in immeasurable ways. Jerome, Ber, Mo, Oli, David and Maria have, each in their own way, contributed to the success of this thesis. My gratitude also goes to Tomma, Yuri, Matet and Tip for being instigators of some truly unforgettable experiences during my two years in Japan. Irrespective of where life takes me from here, I will fondly treasure these memories.

Most importantly, this thesis would never have been possible without the love and support of my family - Goran, Aneta and Vasa. 
To my parents 
List of Figures vii

1 Brief introduction 1

2 Theory 5

2.1 The Neutrino .................... 5

2.2 Neutrino oscillations . . . . . . . . . . . . . . . . . . 14

2.3 Physics of particle beams . . . . . . . . . . . . . . . . 19

3 The T2K experiment $\quad 22$

3.1 Physics goals and results of T2K . . . . . . . . . . . . 22

$3.2 \quad$ J-PARC . . . . . . . . . . . . . . . . . . . . 26

3.3 The $\mathrm{T} 2 \mathrm{~K}$ beamline . . . . . . . . . . . . . . . . 26

3.4 The near detector complex . . . . . . . . . . . . . . 37

3.4.1 The ND280 detector . . . . . . . . . . . . . . . . . . 37

3.4.2 The INGRID detector . . . . . . . . . . . . . . . . . . 41

3.5 The Super-Kamiokande detector . . . . . . . . . . . . . . . . 43

3.6 T2K data-taking summary . . . . . . . . . . . . . . . 52

4 The NA61 Experiment $\quad 54$

4.1 NA61/SHINE Detector . . . . . . . . . . . . . . . . . . . 54

4.2 NA61/SHINE measurements for T2K . . . . . . . . . . . . 58

4.2.1 Thin-target measurements . . . . . . . . . . . . . 58

4.2.2 Replica-target measurements . . . . . . . . . . . . 61

4.2.3 Production cross section measurement . . . . . . . . . 67

5 The T2K flux calculation tuned to thin target data 71

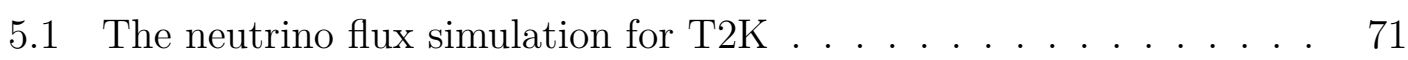

5.2 Introduction to flux tuning . . . . . . . . . . . . . 76

5.3 Multiplicity tuning . . . . . . . . . . . . . . 78

5.3.1 Meson multiplicity weights . . . . . . . . . 78

5.3.2 Baryon multiplicity weights . . . . . . . . . . . . 83

5.3.3 Pion rescattering multiplicity weights . . . . . . . . . . . 87

5.4 Interaction length tuning . . . . . . . . . . . . . . . . . . 88

5.5 Flux uncertainties . . . . . . . . . . . . . . . . . 91

5.5.1 Uncertainties from hadron interaction modelling . . . . . . . 92

5.5.2 Other sources of T2K flux uncertainty . . . . . . . . . 97 
6 The T2K flux calculation tuned to replica-target data 108

6.1 Replica-tuning approach . . . . . . . . . . . . . . . . . . . 108

6.2 Replica-tuned flux prediction . . . . . . . . . . . . . . . . 114

6.3 Replica-tuned flux uncertainty . . . . . . . . . . . . . . . . . . . 119

6.4 Model dependence of NA61 measurement unfolding . . . . . . . . . 126

6.5 Target density effects . . . . . . . . . . . . . . . . . 134

6.6 Proton beam profile effects . . . . . . . . . . . . . . . . 143

6.7 Unconstrained hadronic interactions . . . . . . . . . . . . . . . 152

7 Bayesian statistics and the Markov Chain Monte Carlo technique $\begin{array}{lr}\text { for neutrino oscillation analyses } & 169\end{array}$

7.1 Bayesian Inference . . . . . . . . . . . . . . . . . . . . . . . 169

7.2 Markov Chain Monte Carlo method . . . . . . . . . . . . . . . . . . 172

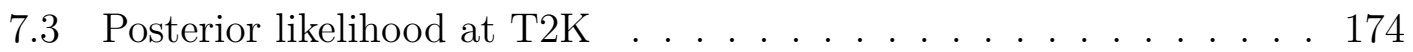

7.4 Systematic parameter inputs . . . . . . . . . . . . . . 176

7.4.1 Flux parameter inputs . . . . . . . . . . . . 176

7.4.2 Cross section parameter inputs . . . . . . . . . . . 178

7.4.3 ND280 and Super-K parameter inputs . . . . . . . . . . . 184

8 Joint $\nu+\bar{\nu} \mathbf{T} 2 \mathrm{~K}$ oscillation analysis with the reactor constraint and $\begin{array}{lr}\text { replica-tuned flux inputs } & 187\end{array}$

8.1 Fits to ND280 data . . . . . . . . . . . . . . . . . . . . 187

8.1.1 ND280 data . . . . . . . . . . . . . . . 187

8.1 .2 Fit results . . . . . . . . . . . . . . . . . . 192

8.2 Joint fits to ND280 and Super-K data . . . . . . . . . . . . . . . 202

8.2.1 Asimov fits . . . . . . . . . . . . . . 202

8.2.2 Fits to combined $\nu+\bar{\nu}$ data . . . . . . . . . . . . 209

9 Conclusions and future prospects $\quad 216$

$\begin{array}{ll}\text { References } & 219\end{array}$ 


\section{List of Figures}

2.1 A schematic representation of the experimental setup devised by Cowan and Reines for detection of neutrinos from the Savannah River Nuclear Reactor. Reprinted with permission from F. Reines and C. L. Cowan, "Free anti-neutrino absorption cross-section", Physical Review, Volume 113, pp. 273-279, 1959[22]. Copyright (1959) by the American Physical Society. . . . . . . . . . . . . . . 7

2.2 Diagram of the constituent particles of the Standard Model. . . . . 8

2.3 Combined measurement of hadronic production cross section around the $\mathrm{Z}^{0}$ resonance, from the ALEPH, DELPHI, OPAL, L3 and SLD experiments. Reprinted from Physics Reports, Volume 427, Issues 56, ALEPH Collaboration et al., "Precision electroweak measurements on the $\mathrm{Z}$ resonance", pp. 257-454, Copyright (2006), with permission from Elsevier[25]. . . . . . . . . . . . . . . . . . .

2.4 Solar neutrino flux measured by the Homestake experiment between 1970 and 1990. The average flux measurement of $(2.32 \pm 0.22)$ SNU, quoted in, is significantly lower from the theoretical prediction of $7.6_{-1.1}^{+1.3} \mathrm{SNU}[36]$. Here, the solar neutrino unit (SNU) is a unit of neutrino flux needed for a target atom capture rate of $10^{-36}$ per second. Reprinted from Progress in Particle and Nuclear Physics, Volume 32, Raymond Davis, "A review of the homestake solar neutrino experiment", pp. 13-32, Copyright (1994), with permission from Elsevier[37].

2.5 Measurement of $\frac{R_{\mathrm{data}}}{R_{\mathrm{MC}}}$ by the Kamiokande detector, as a function of zenith angle subtended by neutrino production and detection points. The dashed lines denote expectations under oscillation hypotheses. Reprinted from Physics Letters B, Volume 335, Issue 2, Y. Fukuda et al., "Atmospheric $\nu_{\mu} / \nu_{e}$ ratio in the multi-GeV energy range", pp. 237-245, Copyright (1994), with permission from Elsevier[38]. . . . .

2.6 Survival probability $\mathrm{P}\left(\nu_{\alpha} \rightarrow \nu_{\alpha}\right)$, as a function of $\frac{\Delta m^{2} L}{4 E}$, showcasing the "sweet" spot $\frac{L}{E} \sim \frac{1}{\Delta m^{2}}$ for probing mass splitting $\Delta m^{2}$. Adapted by permission from Springer Nature Customer Service Centre GmbH: Vieweg+Teubner Verlag, "Neutrinophysik" by Norbert Schmitz, Chapter "Eigenschaften der Neutrinos", Copyright (1997)[55]. Also reproduced in accordance with Copyright (2012) from "Neutrino Physics" by Kai Zuber. Reproduced by permission of Taylor and Francis Group, LLC, a division of Informa plc. 
2.7 The flavour content of each of the mass eigenstates, in both normal and inverted mass hierarchy. The change in flavour content as a function of $\mathrm{CP}$ has been indicated. Here, $\Delta m_{\text {sol }}^{2}=\Delta m_{21}^{2}$ and $\Delta m_{\mathrm{atm}}^{2}=\Delta m_{32}^{2} \approx \Delta m_{31}^{2}$. Reprinted from Progress in Particle and Nuclear Physics, Volume 83, X. Qian and P. Vogel, "Neutrino mass hierarchy", pp. 1-30, Copyright (2015), with permission from Elsevier[56].

2.8 Beam envelope shape, parametrised with emittance and Twiss parameters.

2.9 Evolution of the beam profile subjected to alternating magnets focusing in $x$-direction (QF) and $y$-direction (QD). The solid line shows the beam envelope. The dashed line denotes the track of a single particle. Parts of figure adapted from "Beam optics and lattice design for particle accelerators" by Bernhard J. Holzer[62], under the CC BY copyright licence.

3.1 A pictorial representation of the T2K experiment baseline. Copyright by the T2K Collaboration. . . . . . . . . . . . . .

3.2 The $\nu_{\mu}$ survival and $\nu_{e}$ appearance probabilities as a function of true neutrino energy, at the SK location. The neutrino spectrum after $295 \mathrm{~km}$ is also shown, at different off-axis angles with respect to the beam direction. At the T2K beam energy of $\sim 600 \mathrm{MeV}$, the $\nu_{\mu}$ survival probability is minimised. The $\nu_{e}$ appearance probability is also maximised (note the different scales on the horizontal axis, most $\nu_{\mu}$ have oscillated into $\nu_{\tau}$, but these are too low in energy to detect). Copyright by the T2K Collaboration. . . . . . . . . . . . . .

3.3 The $\left(p_{e}, \theta_{e}\right)$ distribution of the 28 selected $\nu_{e}$ candidate events in the T2K oscillation analysis (Super-Kamiokande exposure of $6.57 \times 10^{20}$ POT, collected from January 2010 to May 2013). Monte Carlo signal and background predictions are based on the T2K best fit value of $\sin ^{2} 2 \theta_{13}=0.140$, under the assumption of normal mass hierarchy. Reprinted figure with permission from K. Abe et al. (T2K Collaboration), Phys. Rev. Lett., vol. 112, p. 061802 (2014)[64]. Copyright (2014) by the American Physical Society. . . . . . . . . . 
3.4 Subfigure a shows the two dimensional $68.27 \%(2 \sigma)$ confidence limit (CL) as a function of $\sin ^{2} \theta_{13}-\delta_{\mathrm{CP}}$ mixing parameters, assuming the preferred normal ordering (NO) of neutrino masses, and considering cases with and without the constraint on $\sin ^{2} \theta_{13}$ from reactor experiments. Subfigure $\mathbf{b}$ shows the two dimensional $1 \sigma$ and $3 \sigma$ confidence limits as a function of $\sin ^{2} \theta_{23}-\delta_{\mathrm{CP}}$ mixing parameters, assuming the preferred normal ordering of neutrino masses and with the reactor constraint. Here, the colour scale corresponds to the $-2 \Delta \ln (\mathrm{L})$ Bayesian likelihood as a function of the value of $\sin ^{2} \theta_{23}-\delta_{\mathrm{CP}}$ parameters. Subfigure c shows the $1 \sigma$ (shaded box) and $3 \sigma$ (error bars) confidence intervals for $\delta_{\mathrm{CP}}$ in normal ordering and inverted ordering. The star, square and vertical line correspond to the best-fit points in subfigures $\mathbf{a}, \mathbf{b}$ and $\mathbf{c}$, respectively. Material from: K. Abe et al. (T2K Collaboration), "Constraint on the matter-antimatter symmetry-violating phase in neutrino oscillations", Nature, published 2020, Springer Nature[8] . . . . . . . . . . . . . . . . . . .

3.5 The orientation of the T2K primary and secondary beamline with respect to the main ring of the J-PARC proton synchrotron. The site of the near detector complex is also shown. Figure reprinted from Progress of Theoretical and Experimental Physics, Volume 2015, K. Suzuki et al., "Measurement of the muon beam direction and muon flux for the T2K neutrino experiment"[65], under the CC BY copyright licence. . . . . . . . . . . . . . . . . . . .

3.6 Rendering of the $\mathrm{T} 2 \mathrm{~K}$ primary beamline, showing the locations of various proton beam profile monitors. Reprinted from Nuclear Instruments and Methods in Physics Research Section A: Accelerators, Spectrometers, Detectors and Associated Equipment, Volume 659, K. Abe et al. (T2K Collaboration), "The T2K experiment", pp. 106-135, Copyright (2011), with permission from Elsevier[63]. . . . .

3.7 Photographs of T2K beam monitors: CT (upper left), SSEM (upper middle), ESM (upper right), BLM (lower left) and OTR (lower right). Four photographs reprinted from Nuclear Instruments and Methods in Physics Research Section A: Accelerators, Spectrometers, Detectors and Associated Equipment, Volume 659, K. Abe et al. (T2K Collaboration), "The T2K experiment", pp. 106-135, Copyright (2011), with permission from Elsevier[63]. One photograph (OTR monitor) reprinted from Nuclear Instruments and Methods in Physics Research Section A: Accelerators, Spectrometers, Detectors and Associated Equipment, Volume 703, S. Bhadra et al., "Optical transition radiation monitor for the T2K experiment", pp. 45-58, Copyright (2013), with permission from Elsevier[66] . . . . . . . . . 
3.8 Rendering of the T2K secondary beamline. Reprinted from Nuclear Instruments and Methods in Physics Research Section A: Accelerators, Spectrometers, Detectors and Associated Equipment, Volume 789, T. Sekiguchi et al., "Development and operational experience of magnetic horn system for T2K experiment", pp. 57-80, Copyright (2015), with permission from Elsevier[67]. . . . . . . . . . .

3.9 Rendering of the T2K target station. Reprinted from Nuclear Instruments and Methods in Physics Research Section A: Accelerators, Spectrometers, Detectors and Associated Equipment, Volume 789, T. Sekiguchi et al., "Development and operational experience of magnetic horn system for T2K experiment", pp. 57-80, Copyright (2015), with permission from Elsevier[67]. . . . . . . . . . .

3.10 Design of the T2K target and the accompanying cooling structure. Reprinted by permission from Springer Nature Customer Service Centre GmbH: Springer Nature, Journal of Radioanalytical and Nuclear Chemistry, "The graphite target for J-PARC neutrino beamline", T. Nakadaira, Copyright (2015)[69].

3.11 Design of the magnetic horns, and the orientation of the target with respect to the first horn, is shown on the left side. The effect of horn current focusing on the neutrino flux observed at Super$\mathrm{K}$ is shown on the right side. Reprinted figure on the left from Nuclear Instruments and Methods in Physics Research Section A: Accelerators, Spectrometers, Detectors and Associated Equipment, Volume 690, A.K. Ichikawa, "Design concept of the magnetic horn system for the T2K neutrino beam", pp. 27-33, Copyright (2012), with permission from Elsevier[71]. Reprinted figure on the right with permission from K. Abe et al. (T2K Collaboration), Phys. Rev. D, vol. 87, p. 012001 (2013)[72]. Copyright (2013) by the American Physical Society.

3.12 A rendering of the muon monitor is shown on the left. The beam direction with respect to the detector has been labelled. An example of the simulated muon profile at the muon monitor is shown on the right. A shift in the muon beam direction by $1 \mathrm{mrad}$ corresponds to a $2 \%$ shift in peak muon energy. Reprinted from Nuclear Instruments and Methods in Physics Research Section A: Accelerators, Spectrometers, Detectors and Associated Equipment, Volume 624, K. Matsuoka et al., "Design and performance of the muon monitor for the T2K neutrinooscillation experiment", pp. 591-600, Copyright (2010), with permission from Elsevier[73] . . . . . . . . . . . . 
3.13 A rendering of the whole near detector complex, placed in a two level underground cavern, at a depth of 30 metres. The off-axis ND280 detector is located on the upper level of the cavern. The horizontal components of the on-axis INGRID detector are on the lower level, and the vertical components span both levels. Copyright by the T2K Collaboration. . . . . . . . . . . . . . .

3.14 An exploded view of the ND280 near detector. The orientation of the detector modules with respect to the beam direction has been indicated. Reprinted from Nuclear Instruments and Methods in Physics Research A, Volume 686, S. Assylbekov et al., "The T2K ND280 off-axis pi-zero detector", pp. 48-63, Copyright (2012), with permission from Elsevier[76]. . . . . . . . . . . . .

3.15 A simplified view of the time projection chamber is shown on the left. The space between the outer and inner wall of the TPC is filled with $\mathrm{CO}_{2}$ for insulation. The micromegas pad segmentation on the anode electron collection plane is $7.0 \mathrm{~mm} \times 9.8 \mathrm{~mm}$. The distribution of energy loss in the TPC as a function of momentum is shown on the right, for positively charged particles. Reprinted from Nuclear Instruments and Methods in Physics Research Section A: Accelerators, Spectrometers, Detectors and Associated Equipment, Volume 637, N. Abgrall et al., "Time projection chambers for the T2K near detectors", pp. 25-46, Copyright (2011), with permission from Elsevier[77]. . . . . . . . . . . . . . . . . .

3.16 A figure showcasing a real ND280 event display. Reprinted from Nuclear Instruments and Methods in Physics Research Section A: Accelerators, Spectrometers, Detectors and Associated Equipment, Volume 659, K. Abe et al. (T2K Collaboration), "The T2K experiment", pp. 106-135, Copyright (2011), with permission from Elsevier[63]. . . . . . . . . . . . . . . . .

3.17 Rendering of the INGRID on-axis near detector. Copyright by the T2K Collaboration. . . . . . . . . . . . . . . .

3.18 Daily event rate measurements at INGRID for T2K physics Runs 1-3[83]. Copyright by the $\mathrm{T} 2 \mathrm{~K}$ Collaboration. . . . . . . . . . .

3.19 Beam profile measurement at INGRID for J-PARC Main Ring Run 32 (14 April - 1 May 2010). The horizontal profile measured by the seven horizontally arranged modules is shown on the left. The vertical profile is shown on the right. The $\mathrm{y}$-axis shows the cumulative number of event candidates detected by each of the INGRID modules over the above-mentioned period[84]. Copyright by the T2K Collaboration. 43 
3.20 Rendering of the SK detector site, and its location with respect to Mt. Ikenoyama. Two horizontal mine shafts lead up to the top of the detector cavern. The locations of the main control room, water purification system and electronics huts are shown. The electron LINAC can be used for testing the SK detector response to electrons of known energy. Reprinted from Nuclear Instruments and Methods in Physics Research Section A: Accelerators, Spectrometers, Detectors and Associated Equipment, Volume 501, S. Fukuda et al. (SuperKamiokande Collaboration), "The Super-Kamiokande detector", pp. 418-462, Copyright (2003), with permission from Elsevier[86]. . . . .

3.21 Schematic diagram showing the developing wavefront of a conical light wave emitted by a Cherenkov radiating particle. The particle of speed $v$ emits Cherenkov radiation if $v>\frac{c}{n}$, where $c$ is the speed of light, $n$ is the dielectric refractive index ( $n=1.33$ for water). From geometrical considerations, it follows that $\cos \theta=\frac{c}{n v}$. The threshold energy for Cherenkov radiation is given by $E_{\min }=\frac{m c^{2} n}{\sqrt{n^{2}-1}} \ldots \ldots$. .

3.22 The total charged-current cross sections for neutrino (left) and antineutrino (right) interactions, per single nucleon and true neutrino energy. The total cross section prediction from the NUANCE simulation[88] is shown, as well as separate contributions from quasi-elastic scattering (QE, dotted), resonance production (RES, dotted-dashed) and deep inelastic scattering (DIS, dashed). At the peak $\mathrm{T} 2 \mathrm{~K}$ beam energy of $\sim 600 \mathrm{MeV}$, quasi-elastic scattering is the dominant charged current interaction mode. Different measurements taken on isoscalar targets are represented by markers of different shapes and colours (for more details refer to [89]). It is worth poting out the different scale on the vertical axis for the two figures $\left(\sigma_{\mathrm{CCQE}}^{\nu} \sim 4 \sigma_{\mathrm{CCQE}}^{\bar{\nu}}\right)$. Reprinted figure with permission from Formaggio, J. A. and Zeller, G. P., Rev. Mod. Phys., vol. 84, pp. 1307-1341 (2012)[89]. Copyright (2012) by the American Physical Society. . . .

3.23 SK event displays for real T2K beam-induced events. Event candidates with single electron-like (top) and muon-like (bottom) Cherenkov rings are shown. The charge deposition on the inner (black unrolled map) and outer (white unrolled map) PMT walls is shown, with the colour of the PMT corresponding to the deposited charge. Event displays taken from [90]. Copyright by the T2K Collaboration. . . . 
3.24 The PID parameter distribution used for distinguishing single-ring $\mu$-like $(\mathrm{PID}>0)$ from $e$-like $(\mathrm{PID}<0)$ events, inferred from Monte Carlo studies with the FiTQun reconstruction algorithm. Observed data with statistical error bars is shown alongside stacked histograms corresponding to Monte Carlo predictions. The fiducial volume cut has already been applied. Figure reprinted from Physical Review D, Volume 96, K. Abe et al. (T2K Collaboration), "Measurement of neutrino and antineutrino oscillations by the T2K experiment including a new additional sample of $\nu_{e}$ interactions at the far detector"[91], under the CC BY copyright licence. . . . . . . . . . .

3.25 The Feynman diagrams for true CCQE neutrino interactions targeted with the $\mathrm{FHC} 1 \mathrm{R}_{\mu}$ and $\mathrm{FHC} 1 \mathrm{R}_{e}$ selection cuts are shown in (a). The $\mathrm{FHC} 1 \mathrm{R}_{e} \mathrm{CC} 1 \pi^{+}$signal selection targets CC RES interactions of $\nu_{e}$ with $1 \pi^{+}$in the final state, detected through its decay electron. These could be Feynman interactions like $\nu_{l}+n \rightarrow l^{-}+n+\pi^{+}$shown in (b), and $\nu_{l}+p \rightarrow l^{-}+p+\pi^{+}$shown in (c). The reconstructed energy formula for the $1 \mathrm{R}_{e} \mathrm{CC} 1 \pi^{+}$sample differs from equation 3.1, to reflect the different final state topology. . . . . . . . . . . .

3.26 The accumulated data for T2K since the first physics run in January 2010 , presented in terms of the delivered number of protons on the target (POT). Note that the beam power is also shown, on the vertical axis on the right side of the diagram. Different colours are used to separate data collected in neutrino and anti-neutrino mode. Copyright by the T2K Collaboration. . . . . . . . . . . . .

4.1 A diagram of the NA61 detector[94]. Figure reprinted from Journal of Instrumentation, Volume 9, p. P06005, N Abgrall et al. (NA61/SHINE Collaboration), "NA61/SHINE facility at the CERN SPS: beams and detector system"[94], under the CC BY copyright licence. . . . . . . . . . . . . . . . . . .

4.2 Specific energy loss $\frac{\mathrm{dE}}{\mathrm{d} x}$ of negatively (left) and positively (right) hadrons propagating through the NA61 TPCs. $\frac{\mathrm{dE}}{\mathrm{d} x}$ is calculated in units of minimum ionizing particle (mip) energy loss, and given as a function of the logarithm of particle momentum. The Bethe-Bloch curves for the average energy loss of different charged hadron species are overlaid on NA61 data. Figure reprinted from The European Physical Journal C, Volume 79, N Abgrall et al. (NA61/SHINE Collaboration), "Measurements of $\pi^{ \pm}, K^{ \pm}$and proton double differential yields from the surface of the T2K replica target for incoming $31 \mathrm{GeV} / \mathrm{c}$ protons with the NA61/SHINE spectrometer at the CERN SPS"[97], under the CC BY copyright licence. . . . . . . . . . . . 
4.3 Invariant mass squared as a function of particle momentum, calculated from readings taken by NA61 time-of-flight detectors, is shown on the left. Distribution of invariant mass squared as a function of specific energy loss, used for reliable particle identification across a wide range of charged hadron momenta, is shown on the right. Figure reprinted from The European Physical Journal C, Volume 79, N Abgrall et al. (NA61/SHINE Collaboration), "Measurements of $\pi^{ \pm}, K^{ \pm}$and proton double differential yields from the surface of the T2K replica target for incoming $31 \mathrm{GeV} / \mathrm{c}$ protons with the NA61/SHINE spectrometer at the CERN SPS"[97], under the CC BY copyright licence. . . . . . . . . . . . . . . .

4.4 The NA61 thin target and replica target configurations used for collecting hadron production measurements for T2K. References to publications accompanying the release of each dataset have been provided. . . . . . . . . . . . . . . .

4.5 The differential $K^{+}$yields as a function of the kaon momentum $p$, and extracted for different ranges of exiting angle $\theta$ with respect to the proton beam direction. The measured particle yield is plotted alongside predictions from GiBUU[102] and the GEANT4 based FTF_BIC[103] generators. The vertical error bars correspond to the total measurement uncertainty. Figure reprinted from The European Physical Journal C, Volume 76, N Abgrall et al. (NA61/SHINE Collaboration), "Measurements of $\pi^{ \pm}, K^{ \pm}, K_{S}^{0}, \Lambda$ and proton production in proton-carbon interactions at $31 \mathrm{GeV} / \mathrm{c}$ with the NA61/SHINE spectrometer at the CERN SPS"[98], under the CC BY copyright licence. . . . . . . . . . . . . . . . . . . .

4.6 Rendering and dimensional drawing of the replica target. The wider section at the upstream end of the target is shown, mounted to the disk-shaped aluminium flange. The replica target on the mechanical drawing has been outlined in red. Figure reprinted from The European Physical Journal C, Volume 79, N Abgrall et al. (NA61/SHINE Collaboration), "Measurements of $\pi^{ \pm}, K^{ \pm}$and proton double differential yields from the surface of the T2K replica target for incoming $31 \mathrm{GeV} / \mathrm{c}$ protons with the NA61/SHINE spectrometer at the CERN SPS"[97], under the CC BY copyright licence. . . . . 
4.7 The NA61 2009 positive pion yields from three representative positions along the graphite target: the most upstream target portion $\left(z_{1}\right)$, one of the middle target portions $\left(z_{3}\right)$ and the most downstream target portion $\left(z_{6}\right)$. The following binning has been adopted by NA61: $0 \mathrm{~cm}<z_{1}<18.0 \mathrm{~cm}<z_{2}<36.0 \mathrm{~cm}<z_{3}<54.0 \mathrm{~cm}<z_{4}<$ $72.0 \mathrm{~cm}<z_{5}<89.99 \mathrm{~cm}$ and $z_{6}$ is used to denote the downstream face of the replica target (selected with $89.99 \mathrm{~cm}<z_{6}<90.01 \mathrm{~cm}$ ). The yields are given as a function of pion momentum, and split into different angular ranges, measured with respect to the incident beam direction. The yield is measured per proton on target, and per pion momentum, in units of $(\mathrm{GeV} / \mathrm{c})^{-1} \ldots \ldots \ldots$

4.8 The systematic uncertainties for the $2009 \pi^{+}$yields from the replica target, split into contributions from different error sources. The uncertainties are given for every longitudinal $z$-bin, for different $\pi^{+}$ momenta and covering the low scattering angle range from 0 to 60 mrad, which is mostly dominated by feed-down and backwards extrapolation systematic uncertainties. Figure reprinted from The European Physical Journal C, Volume 76, N Abgrall et al. (NA61/SHINE Collaboration), "Measurements of $\pi^{ \pm}$differential yields from the surface of the T2K replica target for incoming $31 \mathrm{GeV} / \mathrm{c}$ protons with the NA61/SHINE spectrometer at the CERN SPS"[99], under the CC BY copyright licence. . . . . . . . . . . . . . .

4.9 The systematic uncertainties for the $2009 \pi^{+}$yields from the replica target, split into contributions from different error sources. The uncertainties are given for every longitudinal z-bin, for different $\pi^{+}$momenta and covering the high scattering angle range from 260 to $340 \mathrm{mrad}$, which is mostly dominated by pion loss and backwards extrapolation systematic uncertainties. Figure reprinted from The European Physical Journal C, Volume 76, N Abgrall et al. (NA61/SHINE Collaboration), "Measurements of $\pi^{ \pm}$differential yields from the surface of the T2K replica target for incoming 31 $\mathrm{GeV} / \mathrm{c}$ protons with the NA61/SHINE spectrometer at the CERN SPS"[99], under the CC BY copyright licence. . . . . . . . . . . .

4.10 Portion of the T2K neutrino flux at Super-Kamiokande covered by the 2009 replica target dataset (light grey) and the extrapolated NA61 thin target dataset (dark grey), in both neutrino and anti-neutrino mode. Preference is given to the replica target dataset, which directly constrains $87.2 \%$ of the total $\nu_{\mu}$ flux in $\nu$-mode, and $84.9 \%$ of the total $\bar{\nu}_{\mu}$ flux in $\bar{\nu}$-mode. The part of the T2K flux which cannot be tuned to any NA61 measurements is also shown (black). . . . . . 
4.11 Types of hadron-nucleus scattering in the NA61 adopted formalism.

5.1 A diagram of the T2K beamline geometry handled by the FLUKA simulation. The baffle and target are composed of graphite of the same grade, and helium gas is included in the simulation, filling up the gap inside the baffle, and the path up to the target. . . . . . .

5.2 The combined unoscillated T2K flux in neutrino (top) and antineutrino (bottom) mode of operation, at ND280 (left) and at Super-K (right). The flux has been broken down into contributions from all neutrino species. Only statistical error bars are shown. Copyright by T2K Collaboration. . . . . . . . . . . . . . . . .

5.3 A schematic representation of the fraction of neutrinos at Super-K coming from decays of different parent particles. Here, 2-body and 3-body kaon decays are plotted separately. Each flux component is considered $\left(\mathrm{E}_{\nu}<10 \mathrm{GeV}\right)$ for the neutrino-mode of operation. Copyright by T2K Collaboration. . . . . . . . . . . . . . . . 77

5.4 Two distinct aspects of hadron interactions relevant for flux tuning. $\quad 78$

5.5 The meson multiplicity weights from NA61 data over FLUKA 2011.2b. NA61 measurements have been extrapolated according to the BMPT parametrization. The coarse bins mostly correspond to the NA61 data binning, and the finer bin are from the extrapolation with BMPT fits. Figure reprinted from [85]. Copyright by T2K Collaboration.

5.6 The weights applied to meson multiplicities modelled by GCALOR. From left to right: $\pi^{+}, \pi^{-}$production. From top to bottom: C, Al, $\mathrm{Ti}$ and Fe targets. Figure reprinted from [85]. Copyright by T2K Collaboration. . . . . . . . . . . . . . . .

5.7 Secondary baryon weights for the FLUKA Monte Carlo are shown. These are the weights used to calculate the tuned neutrino flux for T2K analyses. Figure reprinted from [85]. Copyright by T2K Collaboration. . . . . . . . . . . . . .

5.8 Ratio between the pion multiplicities of the HARP data and the FLUKA simulation (on carbon target). Here, weights have been depicted for inclusive in-target interactions with incident $3 \mathrm{GeV} / \mathrm{c}$ $\pi^{+}$, and outgoing $\pi^{+}$. Analogously, weights have been constructed for other combinations of incoming and outgoing pion charge, and for different incident pion momenta. The binning used here is adopted from the release format of the HARP measurement. Figure reprinted from $[85]$. Copyright by T2K Collaboration. . . . . . . . . . . . . 
5.9 Ratio between the pion multiplicities of the HARP data and the GCALOR simulation (on aluminium target). Here, weights have been depicted for inclusive out-of-target interactions with incident $3 \mathrm{GeV} / \mathrm{c} \pi^{+}$, and outgoing $\pi^{+}$. Analogously, weights have been constructed for other combinations of incoming and outgoing pion charge, and for different incident pion momenta. The binning used here is adopted from the release format of the HARP measurement. Figure reprinted from [85]. Copyright by T2K Collaboration. . . . .

5.10 Interaction length weights are applied for both interacting (see Figure 5.10a) and through-going (see Figure 5.10b) particles, refelcting the fect that the interaction and survival probabilities both depend on $\sigma_{\text {prod }} \ldots \ldots \ldots \ldots \ldots \ldots \ldots \ldots \ldots \ldots$

5.11 Comparison of production cross sections for interactions of protons (top left), $\pi^{ \pm}$(top right), $K^{+}$(bottom left) and $K^{-}$(bottom right) on carbon (black) and aluminium (gray), as a function of incident hadron momentum in the lab frame. The markers correspond to data points, and the lines are predictions from different models. The behaviour of the data is best captured by the FLUKA interaction model, whereas predictions from GCALOR deviate more considerably from the measurements. Reprinted figure with permission from K. Abe et al. (T2K Collaboration), Phys. Rev. D, vol. 87, p. 012001 (2013)[72]. Copyright (2013) by the American Physical Society. . . .

5.12 A review of measurements of inelastic (left) and production (right) cross sections of protons at different incident momenta. The following datasets have been considered: Bellettini et al. (full circles) [105], Carroll et al. (empty circles) [121], MIPP (black triangles) [122] and Denisov et al. measurements (grey triangles) [120]. Bellettini et. al, Carroll et. al and MIPP measured the inelastic cross section, while values from Denisov et al. correspond to production cross section as stated in their article, but the quantity is somewhat ambiguous. NA61 measurements are also shown (full and empty squares). To facilitate comparisons between datasets, the quasi-elastic cross section component, estimated with GEANT4, has been added to Carroll et al. and Denisov et al. measurements shown in the left plot, and subtracted from Bellettini et al. measurement shown in the right plot (labelled as MC corrected). Figure reprinted from [85]. Copyright by T2K Collaboration. . . . . . . . . . . . . . . . . 96 
5.13 The quasi-elastic cross section $\sigma_{\text {quasi-el }}(\mathrm{p}+\mathrm{C})$ for protons interacting on carbon, derived from Equation 5.22, plotted as a function of incident proton momentum. The elastic scattering cross sections of nucleons, $\sigma_{\mathrm{el}}(\mathrm{p}+\mathrm{p})$ and $\sigma_{\mathrm{el}}(\mathrm{p}+\mathrm{n})$, are obtained from fits to all existing data, summarised in PDG 2018[54]. Reprinted figure with permission from K. Abe et al. (T2K Collaboration), Phys. Rev. D, vol. 87, p. 012001 (2013)[72]. Copyright (2013) by the American Physical Society. . . . . . . . . . . . . . . .

5.14 Measurements of proton (top left), $\pi^{+}$(top right), $K^{+}$(bottom left) and $K^{-}$(bottom right) production cross sections on carbon, alongside predictions from different simulations. For detailed references to all datasets shown in the Figure, the reader is referred to [72]. Reprinted figure with permission from K. Abe et al. (T2K Collaboration), Phys. Rev. D, vol. 87, p. 012001 (2013)[72]. Copyright (2013) by the American Physical Society. . . . . . . . . . . . . . .

5.15 The asymmetric horn field component modelled in JNUBEAM. On the left is the magnetic scalar potential, and on the right is the associated field. Figure reprinted from [124]. Copyright by T2K Collaboration. . . . . . . . . . . . . . . .

5.16 The hadronic interaction modelling portion of flux uncertainties at Super-K, evaluated when using only 2009 thin target data. The uncertainties have been estimated in neutrino mode (upper four plots) and anti-neutrino mode (lower four plots), for the $\nu_{\mu}, \bar{\nu}_{\mu}, \nu_{e}$ and $\bar{\nu}_{e}$ flux components. Copyright by T2K Collaboration. . . . . . .

5.17 The hadronic interaction modelling portion of flux uncertainties at ND280, evaluated when using only 2009 thin target data. The uncertainties have been estimated in neutrino mode (upper four plots) and anti-neutrino mode (lower four plots), for the $\nu_{\mu}, \bar{\nu}_{\mu}, \nu_{e}$ and $\bar{\nu}_{e}$ flux components. Copyright by T2K Collaboration. . . . . . . . . . 103

5.18 Other sources of flux uncertainties at Super-K. The uncertainties have been estimated in neutrino mode (upper four plots) and anti-neutrino mode (lower four plots), for the $\nu_{\mu}, \bar{\nu}_{\mu}, \nu_{e}$ and $\bar{\nu}_{e}$ flux components. Copyright by T2K Collaboration. . . . . . . . . . . . . . .

5.19 Other sources of flux uncertainties at ND280. The uncertainties have been estimated in neutrino mode (upper four plots) and anti-neutrino mode (lower four plots), for the $\nu_{\mu}, \bar{\nu}_{\mu}, \nu_{e}$ and $\bar{\nu}_{e}$ flux components. Copyright by T2K Collaboration. . . . . . . . . . . . . . .

5.20 Total flux uncertainties at Super-K, evaluated when using only 2009 thin target data. The uncertainties have been estimated in neutrino mode (upper four plots) and anti-neutrino mode (lower four plots), for the $\nu_{\mu}, \bar{\nu}_{\mu}, \nu_{e}$ and $\bar{\nu}_{e}$ flux components. Copyright by T2K Collaboration. 106 
5.21 Total flux uncertainties at ND280, evaluated when using only 2009 thin target data. The uncertainties have been estimated in neutrino mode (upper four plots) and anti-neutrino mode (lower four plots), for the $\nu_{\mu}, \bar{\nu}_{\mu}, \nu_{e}$ and $\bar{\nu}_{e}$ flux components. Copyright by T2K Collaboration.107

6.1 The implementation of pion yields from the replica-target into the flux calculation framework. . . . . . . . . . . . . . . . . . . . . 109

6.2 Contributions to the neutrino flux at Super-K from various hadrons exiting from the $\mathrm{T} 2 \mathrm{~K}$ target. Both the neutrino (upper four figures) and anti-neutrino (lower four figures) modes of operation have been considered, for the $\nu_{\mu}, \bar{\nu}_{\mu}, \nu_{e}$ and $\bar{\nu}_{e}$ flux components. . . . . . .

6.3 Replica-target $\pi^{+}$multiplicity weights used for constraining the unoscillated T2K neutrino flux prediction. Copyright by T2K Collaboration. . . . . . . . . . . . . . . . .

6.4 Replica-target $\pi^{-}$multiplicity weights used for constraining the unoscillated T2K neutrino flux prediction. Copyright by T2K Collaboration. . . . . . . . . . . . . . . . . .

6.5 Ratios of the tuned flux for combined runs 1-9a to the 13a nominal flux, at ND280 (left) and at Super-Kamiokande (right). All species of neutrinos are shown. Errors are not presented. Copyright by T2K

6.6 Ratio of the replica-tuned (13av4.0) flux to the thin-tuned (13av3.0) flux at Super-Kamiokande. Here tuning has been applied on the same set of nominal T2K flux files instead of the combined fluxes for all T2K runs. The total uncertainty on the ratio (vertical error bars) has been calculated by combining both multiplicity and interaction length ( $\sigma_{\text {prod }}$, grey error bands) uncertainties. All other flux uncertainties on the ratio cancel out because of full correlations between the two tuning procedures. Copyright by T2K Collaboration. . . . . . . . . 117

6.7 Ratio of the replica-tuned (13av4.0) flux to the thin-tuned (13av3.0) flux at ND280. Here tuning has been applied on the same set of nominal T2K flux files instead of the combined fluxes for all T2K runs. The total uncertainty on the ratio (vertical error bars) has been calculated by combining both multiplicity and interaction length ( $\sigma_{\text {prod }}$, grey error bands) uncertainties. All other flux uncertainties on the ratio cancel out because of full correlations between the two tuning procedures. Copyright by T2K Collaboration. . . . . . . . . 118 
6.8 A direct comparison of the hadronic interaction modelling portion of flux uncertainties at Super-K with thin-tuning (upper figure) and replica-tuning (lower figure). The solid and dashed black lines in the lower figure correspond to the replica-tuned and the thin-tuned systematic flux uncertainties due to hadron interaction modelling, respectively. The reduction in flux uncertainty at the T2K flux peak (600 MeV), achieved through the implementation of replica-tuning, is clearly visible in the lower figure. Copyright by T2K Collaboration. 120

6.9 The correlation matrix for the replica-tuned flux prediction. Flux is binned in neutrino energy. The starting bins corresponding to flux at both ND280 and Super-K, with different modes of operation (FHC and RHC), and for each neutrino flavour, have been labelled. Copyright by T2K Collaboration. . . . . . . . . . . . . . . .

6.10 The hadronic interaction modelling portion of flux uncertainties at Super-K, evaluated with the addition of 2009 replica-target data. The uncertainties have been estimated in neutrino mode (upper four plots) and anti-neutrino mode (lower four plots), for the $\nu_{\mu}, \bar{\nu}_{\mu}, \nu_{e}$ and $\bar{\nu}_{e}$ flux components. Copyright by T2K Collaboration. . . . . .

6.11 The hadronic interaction modelling portion of flux uncertainties at ND280, evaluated with the addition of 2009 replica-target data. The uncertainties have been estimated in neutrino mode (upper four plots) and anti-neutrino mode (lower four plots), for the $\nu_{\mu}, \bar{\nu}_{\mu}, \nu_{e}$ and $\bar{\nu}_{e}$ flux components. Copyright by T2K Collaboration. . . . . . . . . .

6.12 Total flux uncertainties at Super-K, evaluated with the addition of 2009 replica-target data. The uncertainties have been estimated in neutrino mode (upper four plots) and anti-neutrino mode (lower four plots), for the $\nu_{\mu}, \bar{\nu}_{\mu}, \nu_{e}$ and $\bar{\nu}_{e}$ flux components. Copyright by T2K

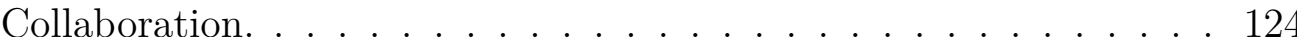

6.13 Total flux uncertainties at ND280, evaluated with the addition of 2009 replica-target data. The uncertainties have been estimated in neutrino mode (upper four plots) and anti-neutrino mode (lower four plots), for the $\nu_{\mu}, \bar{\nu}_{\mu}, \nu_{e}$ and $\bar{\nu}_{e}$ flux components. Copyright by T2K 
6.14 Migration matrices $\mathrm{M}_{\pi^{ \pm}}$describing how to transform from simulated pion multiplicities $\vec{t}$ to what would actually be measured by the NA61 spectrometer $\overrightarrow{\mathrm{r}}$ : $\mathrm{r}_{\mathrm{i}}=\sum_{\mathrm{j}=1}^{762} \mathrm{M}_{\mathrm{ij}} \times \mathrm{t}_{\mathrm{j}}$. The vertical and horizontal axes list correspond to the 762 reconstructed and simulated bins of the NA61 dataset, respectively. The bin numbering scheme iterates across exiting position $z$ (upstream to downstream), angle $\theta$ (lowest to highest) and momentum $p$ (lowest to highest). The matrix element $\mathrm{M}_{\mathrm{ij}}<1$ gives the likelihood for pions exiting from bin $\mathrm{j}$ to be reconstructed as exiting from bin i, given the finite resolution and efficiency of detector components. . . . . . . . . . . . .

6.15 Weights to be applied on measured multiplicities to remove effects of geometric acceptance, detector inefficiencies and bin migrations (resolution), and calculate the "true" pion multiplicities. Efficiencies of various detector components have been absorbed into the unfolding weights, hence weights are generally greater than unity. . . . . . . .

6.16 Ratio of the smeared plus unfolded and the nominal GEANT4 QGSP_BERT generated $\pi^{+}$multiplicities, where the bin by bin unfolding weights were derived from FLUKA. . . . . . . . . .

6.17 Ratio of the smeared plus unfolded and the nominal GEANT4 QGSP_BERT generated $\pi^{-}$multiplicities, where the bin by bin unfolding weights were derived from FLUKA. . . . . . . . . .

6.18 Difference in replica-tuned flux prediction at ND280, depending on the Monte Carlo model used for the unfolding. Different GEANT4 physics lists are compared to the FLUKA model chosen by NA61. The model dependence introduced by the unfolding has a sub-percent effect on the flux. . . . . . . . . . . . . . . . . . .

6.19 Difference in replica-tuned flux prediction at the far detector, depending on the Monte Carlo model used for the unfolding. Different GEANT4 physics lists are compared to the FLUKA model chosen by NA61. The model dependence introduced by the unfolding has a sub-percent effect on the flux. . . . . . . . . . . . . . .

6.20 Variation in predicted positive pion multiplicities exiting from the NA61 target when increasing the measured replica-target density by $1 \sigma \ldots \ldots \ldots \ldots \ldots \ldots \ldots \ldots \ldots$

6.21 Variation in predicted positive pion multiplicities exiting from the NA61 target when decreasing the measured replica-target density by

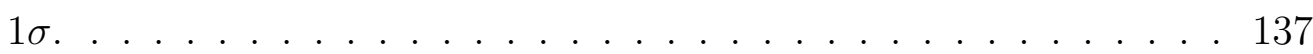


6.22 Comparing the neutrino flux at ND280 constrained with different sets of replica-target weights, where the modelled NA61 target's density was shifted by $\pm 1 \sigma$, to the flux constrained with the standard set of replica-target weights, based on the NA61 target's measured density. 139

6.23 Comparing the neutrino flux at the far detector constrained with different sets of replica-target weights, where the modelled NA61 target's density was shifted by $\pm 1 \sigma$, to the flux constrained with the standard set of replica-target weights, based on the NA61 target's measured density. . . . . . . . . . . . . . .

6.24 Comparison of tuned neutrino fluxes at ND280 modelled with the $\mathrm{T} 2 \mathrm{~K}$ target density set to $\rho_{\text {meas }}^{\mathrm{T} 2 \mathrm{~K}} \pm 1 \sigma$, and the tuned flux modelled with the measured T2K density $\rho_{\text {meas }}^{\mathrm{T} 2 \mathrm{~K}}$. Fluxes had to be calculated from different Monte Carlo samples, accounting for large statistical fluctuations on the ratio at higher neutrino energies with fewer events. The T2K target density was varied for each Monte Carlo sample, and every sample consists of $\sim 1000$ JNUBEAM files, each with 200,000 simulated POT.

6.25 Comparison of tuned neutrino fluxes at the far detector modelled with the $\mathrm{T} 2 \mathrm{~K}$ target density set to $\rho_{\text {meas }}^{\mathrm{T} 2 \mathrm{~K}} \pm 1 \sigma$, and the tuned flux modelled with the measured T2K density $\rho_{\text {meas }}^{\text {T2K }}$. Fluxes had to be calculated from different Monte Carlo samples, accounting for large statistical fluctuations on the ratio at higher neutrino energies with fewer events. The T2K target density was varied for each Monte Carlo sample, and every sample consists of $~ 1000$ JNUBEAM files, each with 200,000 simulated POT.

6.26 The average BPD-3 NA61 proton beam profile used for collecting the 2009 replica-target measurements. . . . . . . . . . . . . . . . . . 144

6.27 Variation in predicted positive pion multiplicities exiting from the NA61 target when increasing the measured NA61 proton beam profile horizontal position by $1 \sigma . \ldots \ldots$. . . . . . . . .

6.28 Variation in predicted positive pion multiplicities exiting from the NA61 target when decreasing the measured NA61 proton beam profile horizontal position by $1 \sigma . \ldots \ldots \ldots$. . . . . . . . . 148

6.29 Comparing the neutrino flux at ND280 constrained with different sets of replica-target weights, where the modelled NA61 proton beam profile centre was shifted by $\pm 1 \sigma$ along either the horizontal or the vertical direction, to the flux constrained with the standard set of replica-target weights, based on the measured NA61 beam profile centre position. 
6.30 Comparing the neutrino flux at the far detector constrained with different sets of replica-target weights, where the modelled NA61 proton beam profile centre was shifted by $\pm 1 \sigma$ along either the horizontal or the vertical direction, to the flux constrained with the standard set of replica-target weights, based on the measured NA61 beam profile centre position. . . . . . . . . . . . . . . . 150

6.31 The vertical axis shows the ratio of the flux tuned with NA61 beam profile weights (kept fixed) with respect to the flux tuned with T2K beam profile weights (varied). The horizontal axis shows the variations in horizontal position (left) and divergence (right) of the T2K beam parameters with respect to the fixed NA61 value. The grey rectangle denotes the scope of variation observed in T2K runs 1-9. Thus, every parameter is varied across a wider range of values than the current maximum difference between the NA61 beam and the T2K beam for any physics run. Extreme displacements of the horizontal beam position of the T2K beam correspond to scenarios where half of the beam misses the target, resulting in a smaller neutrino flux. Reprinted with permission from L. Berns, "Systematic uncertainties for T2K neutrino flux tuning with 2010 NA61/SHINE data", 73rd Annual Meeting of the Japanese Physical Society (2018)[130].

6.32 Incoming and outgoing particle types for unconstrained hadronic interactions of neutrino ancestors, in neutrino mode (left) and antineutrino mode (right). The colour scale in the figures corresponds to the relative number density of the corresponding interactions. . . . 155

6.33 Depiction of the dominant untuned hadronic interactions at the far detector in forward horn current focusing mode. Untuned interactions have been visualised in the phase space of the $\left(x_{F}, p_{T}\right)$ Feynman variables of the daughter hadron, and the colour scale signifies the number density, in arbitrary units, of daughters in each $\left(x_{F}, p_{T}\right)$ bin. The red boundaries specify $6\left(x_{F}, p_{T}\right)$ bins used for estimating the effect of unconstrained interactions on the flux uncertainty. . . . . .

6.34 Depiction of the dominant untuned hadronic interactions at the far detector in reverse horn current focusing mode. Untuned interactions have been visualised in the phase space of the $\left(x_{F}, p_{T}\right)$ Feynman variables of the daughter hadron, and the colour scale signifies the number density, in arbitrary units, of daughters in each $\left(x_{F}, p_{T}\right)$ bin. The red boundaries specify $6\left(x_{F}, p_{T}\right)$ bins used for estimating the effect of unconstrained interactions on the flux uncertainty. . . . . . 160 
6.35 Study of differences in exiting $\pi^{+}$yields predicted by different Monte Carlo models for interactions of $3 \mathrm{GeV}$ positive pions on graphite $\pi^{+}+C \rightarrow \pi^{+}+X$. Plotted are the exiting pion yields in the $\left(x_{F}, p_{T}\right)$ phase space, predicted by FLUKA (top left), FTFP_BERT (top middle) and FTF_BIC (top right). Ratios of pion yields predicted by different models are shown: FTFP_BERT compared to FTF_BIC (middle left), FTFP_BERT compared to FLUKA (middle middle) and FTF_BIC compared to FLUKA (middle right). Finally, the frequency of different values for the yield ratio is examined in the bottom row of figures. . . . . . . . . . . . . . .

6.36 Study of differences in exiting $\pi^{-}$yields predicted by different Monte Carlo models for interactions of $3 \mathrm{GeV}$ positive pions on graphite $\pi^{+}+C \rightarrow \pi^{-}+X$. Plotted are the exiting pion yields in the $\left(x_{F}, p_{T}\right)$ phase space, predicted by FLUKA (top left), FTFP_BERT (top middle) and FTF_BIC (top right). Ratios of pion yields predicted by different models are shown: FTFP_BERT compared to FTF_BIC (middle left), FTFP_BERT compared to FLUKA (middle middle) and FTF_BIC compared to FLUKA (middle right). Finally, the frequency of different values for the yield ratio is examined in the bottom row of figures. . . . . . . . . . . . . .

6.37 Study of differences in exiting $\pi^{+}$yields predicted by different Monte Carlo models for interactions of $3 \mathrm{GeV}$ negative pions on graphite $\pi^{-}+C \rightarrow \pi^{+}+X$. Plotted are the exiting pion yields in the $\left(x_{F}, p_{T}\right)$ phase space, predicted by FLUKA (top left), FTFP_BERT (top middle) and FTF_BIC (top right). Ratios of pion yields predicted by different models are shown: FTFP_BERT compared to FTF_BIC (middle left), FTFP_BERT compared to FLUKA (middle middle) and FTF_BIC compared to FLUKA (middle right). Finally, the frequency of different values for the yield ratio is examined in the bottom row of figures. . . . . . . . . . . . . . .

6.38 Study of differences in exiting $\pi^{-}$yields predicted by different Monte Carlo models for interactions of $3 \mathrm{GeV}$ negative pions on graphite $\pi^{-}+C \rightarrow \pi^{-}+X$. Plotted are the exiting pion yields in the $\left(x_{F}, p_{T}\right)$ phase space, predicted by FLUKA (top left), FTFP_BERT (top middle) and FTF_BIC (top right). Ratios of pion yields predicted by different models are shown: FTFP_BERT compared to FTF_BIC (middle left), FTFP_BERT compared to FLUKA (middle middle) and FTF_BIC compared to FLUKA (middle right). Finally, the frequency of different values for the yield ratio is examined in the bottom row of figures. . . . . . . . . . . . . . . . . 164 
6.39 Study of differences in exiting $K^{+}$yields predicted by different Monte Carlo models for interactions of $3 \mathrm{GeV}$ positive kaons on graphite $K^{+}+C \rightarrow K^{+}+X$. Plotted are the exiting kaon yields in the $\left(x_{F}, p_{T}\right)$ phase space, predicted by FLUKA (top left), FTFP_BERT (top middle) and FTF_BIC (top right). Ratios of pion yields predicted by different models are shown: FTFP_BERT compared to FTF_BIC (middle left), FTFP_BERT compared to FLUKA (middle middle) and FTF_BIC compared to FLUKA (middle right). Finally, the frequency of different values for the yield ratio is examined in the bottom row of figures. . . . . . . . . . . . . . . .

6.40 Study of differences in exiting $K^{-}$yields predicted by different Monte Carlo models for interactions of $3 \mathrm{GeV}$ negative kaons on graphite $K^{-}+C \rightarrow K^{-}+X$. Plotted are the exiting kaon yields in the $\left(x_{F}, p_{T}\right)$ phase space, predicted by FLUKA (top left), FTFP_BERT (top middle) and FTF_BIC (top right). Ratios of pion yields predicted by different models are shown: FTFP_BERT compared to FTF_BIC (middle left), FTFP_BERT compared to FLUKA (middle middle) and FTF_BIC compared to FLUKA (middle right). Finally, the frequency of different values for the yield ratio is examined in the bottom row of figures. . . . . . . . . . . . . . .

6.41 Contributions from unconstrained meson multiplicities to the flux uncertainty at the far detector in forward (top four plots) and reverse (bottom four plots) horn focusing mode. Only uncertainties exceeding $1 \%$ flux error in one of the energy bins have been plotted. Systematic flux uncertainties for neutrinos are shown on the left, and for antineutrinos on the right.

6.42 Contributions from unconstrained meson multiplicities to the flux uncertainty at ND280 in forward (top four plots) and reverse (bottom four plots) horn focusing mode. Only uncertainties exceeding 1\% flux error in one of the energy bins have been plotted. Systematic flux uncertainties for neutrinos are shown on the left, and for antineutrinos on the right. . . . . . . . . . . . . .

7.1 Fractional flux covariance matrix: $\operatorname{sign}\left(V_{i j}\right) \times \sqrt{\left|V_{i j}\right|}$. Here, replicatuning has been used. . . . . . . . . . . . . . . .

7.2 Correlations among cross section parameter inputs for oscillation analyses. Copyright by T2K Collaboration. . . . . . . . . . . . . . . 182

7.3 Fractional ND280 covariance matrix: $\operatorname{sign}\left(\mathrm{V}_{\mathrm{ij}}\right) \times \sqrt{\left|\mathrm{V}_{\mathrm{ij}}\right|}$. The starting bins for different ND280 samples have been labelled. Here, bins correspond to muon momentum and angle. Copyright by T2K Collaboration. . . . . . . . . . . . . . . . . 185 
7.4 Fractional Super-K covariance matrix. The starting bins for different Super-K samples have been labelled. Here, bins correspond to neutrino energy. Copyright by T2K Collaboration. . . . . . . . . . . 186

8.1 Examples of ND280 event displays for $\nu_{\mu} \mathrm{CC} 0 \pi, \nu_{\mu} \mathrm{CC} 1 \pi$ and $\nu_{\mu}$ CCOther FGD1 samples in neutrino mode. Figures from [148]. Copyright by speaker, Kirsty Duffy, on behalf of the T2K Collaboration. The proceedings for this conference talk are available at [149] . . . 188

8.2 Examples of ND280 event displays for CC 1-Track and CC N-Track FGD1 samples in anti-neutrino mode. Figures from [148]. Copyright by speaker, Kirsty Duffy, on behalf of the T2K Collaboration. The proceedings for this conference talk are available at [149] . . . . . . . 188

8.3 MC pre-fit prediction for types of interactions in the ND280 FGD2 CC-0 $\pi$ sample. The observed data from T2K Runs 1-6 is also given. The pre-fit MC prediction shown here assumes the thintuned neutrino flux. Figure reprinted from Physical Review Letters, Volume 121, K. Abe et al. (T2K Collaboration), "Search for $C P$ Violation in Neutrino and Antineutrino Oscillations by the T2K Experiment with $2.2 \times 10^{21}$ Protons on Target"[9], under the CC BY copyright licence. . . . . . . . . . . . . . . . . . . . . . . 190

8.4 ND280 FGD1 data samples in $\nu$-mode (left) and $\bar{\nu}$-mode (right). . . 191

8.5 Comparison of pre-fit (left) and post-fit (right) MC predictions to data, plotted as a function of muon momentum for the $\nu_{\mu} \mathrm{CC}-0 \pi$ sample in FGD1. . . . . . . . . . . . . . . . . .

8.6 Post-fit ratio of ND280 FGD1 MC samples in $\nu$-mode (left) and $\bar{\nu}$ mode (right), in $\left(p_{\mu}, \cos \theta_{\mu}\right)$ phase space. The posterior predictions of the two fits show good agreement despite different nominal neutrino flux inputs.

8.7 Distribution of values for flux parameter $\mathrm{b}_{15}$, for ND280 $\bar{\nu}_{\mu}$ flux with $\mathrm{E}_{\nu} \in[1.5,2.5] \mathrm{GeV} / \mathrm{c}$ in $\nu$-mode, across 750,000 MCMC steps. The parameter posterior is calculated for arithmetic (PDF), Gaussian and highest posterior density (HPD) methods of extraction. For every method, the uncertainty on the mean is also given. The horizontal axis shows the parameter value normalised with respect to the thin-

8.8 Posterior cross section parameters. Parameter values are normalised with respect to the nominal. The prior uncertainties are shown as red error bands. . . . . . . . . . . . . . . . . . . . . . . 196 
8.9 Posterior flux parameters in neutrino mode, evaluated with the arithmetic, Gaussian and highest posterior density methods. All posterior values are normalised with respect to the replica-tuned flux priors. The replica-tuned prior uncertainties are shown as red bands. 197

8.10 Posterior flux parameters in anti-neutrino mode, evaluated with the arithmetic, Gaussian and highest posterior density methods. All posterior values are normalised with respect to the replica-tuned flux priors. The replica-tuned prior uncertainties are shown as red bands. 198

8.11 Fractional covariance matrix and correlation matrix for replica-tuned flux parameters before (left) and after (right) the fit to ND280 data. 199

8.12 Covariance and correlation among cross section parameters before (left) and after (right) the fit to ND280 data. Replica-tuned flux priors are inputs for this fit. . . . . . . . . . . . . . . . . . . 200

8.13 Testing the goodness of MCMC fits to ND280 CC-0 $\pi$ (left) and CC-1Track (right) data samples at FGD1. The red line corresponds

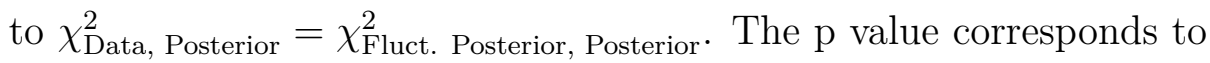
the fraction of points lying below the red line, and has been calculated for each sample. Fits with $\mathrm{p}>0.05$ are considered successful. . . . . 201

8.14 Monte Carlo predictions for different types of events comprising the $1 \mathrm{R}_{e}$ sample at Super-K, in FHC (left) and RHC (right) modes of operation, plotted alongside Run 1-9 data. Copyright by T2K Collaboration.

8.15 Posterior predictive spectra of joint Asimov fits with the reactor constraint. . . . . . . . . . . . . . . . . 205

8.16 Credible intervals in $\sin ^{2} \theta_{13}-\delta_{\mathrm{CP}}$, from joint Asimov $\mathrm{A}$ fits with the $\sin ^{2} \theta_{13}=(2.19 \pm 0.08) \times 10^{-2}$ reactor constraint. The best fit point in the two-dimensional $\sin ^{2} \theta_{13}-\delta_{\mathrm{CP}}$ posterior mode has been labelled. Fits with replica-tuned flux priors are shown in blue. Fits with thin-tuned flux priors are shown in red. . . . . . . . . . . . . 206

8.17 Credible intervals in $\sin ^{2} \theta_{23}-\delta_{\mathrm{CP}}$, from joint Asimov A fits with the $\sin ^{2} \theta_{13}=(2.19 \pm 0.08) \times 10^{-2}$ reactor constraint. The best fit point in the two-dimensional $\sin ^{2} \theta_{23}-\delta_{\mathrm{CP}}$ posterior mode has been labelled. Fits with replica-tuned flux priors are shown in blue. Fits with thin-tuned flux priors are shown in red. . . . . . . . . . . . . . 207

8.18 Credible intervals in $\sin ^{2} \theta_{23}-\Delta m_{32}^{2}$, from joint Asimov A fits with the $\sin ^{2} \theta_{13}=(2.19 \pm 0.08) \times 10^{-2}$ reactor constraint. The best fit point in the two-dimensional $\sin ^{2} \theta_{23}-\Delta m_{32}^{2}$ posterior mode has been labelled. Fits with replica-tuned flux priors are shown in blue. Fits with thin-tuned flux priors are shown in red. 
8.19 Posterior predictive spectra of joint Run 1-9 T2K data fits with the reactor constraint.

8.20 Credible intervals in $\sin ^{2} \theta_{13}-\delta_{\mathrm{CP}}$, from joint data fits with the $\sin ^{2} \theta_{13}=$ $(2.12 \pm 0.08) \times 10^{-2}$ reactor constraint. The best fit point in the two-dimensional $\sin ^{2} \theta_{13}-\delta_{\mathrm{CP}}$ posterior mode has been labelled. Fits with replica-tuned flux priors are shown in blue. Fits with thin-tuned flux priors are shown in red.

8.21 Credible intervals in $\sin ^{2} \theta_{23}-\delta_{\mathrm{CP}}$, from joint data fits with the $\sin ^{2} \theta_{13}=$ $(2.12 \pm 0.08) \times 10^{-2}$ reactor constraint. The best fit point in the two-dimensional $\sin ^{2} \theta_{23}-\delta_{\mathrm{CP}}$ posterior mode has been labelled. Fits with replica-tuned flux priors are shown in blue. Fits with thin-tuned flux priors are shown in red. . . . . . . . . . . . . . . . . . . . . 213

8.22 Credible intervals in $\sin ^{2} \theta_{23}-\Delta m_{32}^{2}$, from joint data fits with the $\sin ^{2} \theta_{13}=(2.12 \pm 0.08) \times 10^{-2}$ reactor constraint. The best fit point in the two-dimensional $\sin ^{2} \theta_{23}-\Delta m_{32}^{2}$ posterior mode has been labelled. Fits with replica-tuned flux priors are shown in blue. Fits with thin-tuned flux priors are shown in red. . . . . . . . . . . . . . . . 214

8.23 Testing the goodness of MCMC joint fits to T2K Run 1-9 data. The $\chi^{2}$ test statistic has been calculated for agreement between observed data and the MC prediction, denoted by $\chi_{\text {Data }}^{2}$, and for agreement between fake data and the MC prediction, $\chi_{\text {Fake Data. For }}^{2} 69 \%$ of parameter sets sampled from the MCMC, the MC prediction is described by data better than fake data $\left(\chi_{\text {Data }}^{2}<\chi_{\text {Fake Data }}^{2}\right) . \ldots$.

9.1 The CCQE inclusive differential cross section, for exiting muon angles $0.94<\cos \theta_{\mu}<1.00$, given as a function of muon momentum $p_{\mu}$. Predictions from NEUT[150] and GENIE[151] interaction generators are shown alongside data. Reprinted figure with permission from K. Abe et al. (T2K Collaboration), Physical Review D, Vol. 87, p. 092003 (2013)[152]. Copyright (2013) by the American Physical Society.

9.2 The potential of the $\mathrm{T} 2 \mathrm{~K}$ experiment to exclude $\sin \delta_{\mathrm{CP}}=0, \pi$ as a function of collected POT, and based on different assumptions regarding improvements in systematic parameters. The following has been calculated for the hypothesis that $\mathrm{CP}$ is maximally violated in the neutrino sector, $\sin \delta_{\mathrm{CP}}=\frac{\pi}{2}$, and assuming normal mass hierarchy and $\sin ^{2} \theta_{23}=0.5$. Figure reprinted from [153], with permission from K. Abe et al. (T2K Collaboration). . . . . . . . . . . . . . . 217 


\section{1 \\ Brief introduction}

What is the Universe composed of? It is remarkable that such a complicated question can be answered in a relatively straightforward, yet scientifically rigorous way. As it turns out, the incredible richness of inanimate objects and living organisms, from stars and planets to cells as fundamental units of life, can be reduced to a handful of constituent particles. The Standard Model of particle physics recognises twelve elementary matter particles, with no internal substructure. The reader will be familiar with notions of particles such as quarks and electrons, which are the constituents of atoms. However, in recent decades, it is arguably neutrinos that have provided the most exciting discoveries for the benefit of the particle physics community, testing and surpassing the validity range of the Standard Model.

Neutrinos are produced in stars during their fusion burning cycles, and trillions of them pass unimpeded through our bodies every second. They are weakly interacting, with no charge and hardly any mass, and are not part of atoms, and yet they are the second most abundant particle in the Universe ${ }^{1}$. The death of massive stars is accompanied with the emission of bursts of neutrinos. In fact, most of the gravitational energy of such dying stars is carried by neutrinos rather than light. Neutrinos could also act as remarkable probes of the early history of the Universe, as many of them decoupled from the rest of matter roughly one second after the Big Bang. The Universe is still filled with these relic neutrinos, cooled down to minuscule kinetic energies, comprising what is referred to as the Cosmic Neutrino Background.

The overwhelming majority of the Universe consists of matter particles. The occasional creation of antimatter, for instance positrons, is rather short lived. They quickly annihilate with electrons, their matter counterparts, emitting a burst of

\footnotetext{
${ }^{1}$ after photons, the carriers of light
} 
light in the process. The widely accepted cosmological theories postulate that the Universe had been created with equal amounts of matter and antimatter. But theories of Big Bang nucleosynthesis, when the Universe was a few seconds old, require a surplus of baryons over antibaryons on the order of one part in billion. So where did antimatter go during those few seconds between the Big Bang and the subsequent start of nuclear formation? Andrei Sakharov formulated the necessary conditions for explaining the abundance of matter in the Universe[1]. There must be processes which violate particle number conservation and crucially CP (chargeparity) conservation, coupled with periods when the Universe departed from thermal equilibrium. Indeed, the mechanism for $\mathrm{CP}$ violation in the quark sector arises naturally through the CKM mixing matrix. However, the measured asymmetry is insufficient [2] to explain the current matter dominated Universe. The Nobel prize-winning discovery of finite neutrino mass[3] reignited hopes that the solution to the problem could lie in leptogenesis[4]. In the PMNS matrix (see Equation 2.17), measurable CP violation could arise for values of $\delta_{\mathrm{CP}} \neq 0, \pm \pi$. Such CP violation has been proposed by certain theoretical models as a requirement for leptogenesis[5-7].

T2K is a neutrino oscillation experiment which measures the mixing of $\nu_{\mu}\left(\bar{\nu}_{\mu}\right)$ with other (anti)neutrino flavours. In T2K, leptonic CP violation would manifest in different oscillation probabilities for neutrinos, $\nu_{\mu} \rightarrow \nu_{e}$, and antineutrinos, $\bar{\nu}_{\mu} \rightarrow \bar{\nu}_{e}$. A robust understanding of the initial beam composition is crucial for making precise oscillation measurements. To achieve this, T2K combines data-driven Monte Carlo flux simulations with measurements from two near detectors, which are sensitive to beam properties prior to any oscillations. The most stringent measurement from the T2K experiment, which recently featured on the cover of Nature magazine[8], places $\delta_{\mathrm{CP}}$ at $-108^{\circ}$, with $-195^{\circ}<\delta_{\mathrm{CP}}<-2^{\circ}$ at the $3 \sigma(99.73 \%)$ confidence interval, for normal mass ordering (preferred by the collected T2K data). The accumulated T2K data generally prefers near maximal $\mathrm{CP}$ violation, and both of the CP-conserving values, $\delta_{\mathrm{CP}}=0$ and $\delta_{\mathrm{CP}}= \pm \pi$, are excluded at the $2 \sigma(95.45 \%)$ confidence interval, irrespective of the mass ordering. If CP is maximally violated $\left(\delta_{\mathrm{CP}}=-\frac{\pi}{2}\right)$, it is expected that the $\mathrm{T} 2 \mathrm{~K}$ experiment could exclude $\mathrm{CP}$-conserving 
values at the $3 \sigma$ confidence interval before the start of data-taking by its successor, the T2HK experiment. In order to make such high precision measurements, besides collecting more data, the reduction of systematic uncertainties is essential. This thesis in particular presents an improved flux calculation technique, achieving a reduction of the $\mathrm{T} 2 \mathrm{~K}$ flux uncertainty from $\sim 10 \%$ to $\sim 5 \%$.

In Chapter 2, major breakthroughs in the field of neutrino physics have been presented, setting up the necessary theoretical background, before proceeding with a detailed description of the $\mathrm{T} 2 \mathrm{~K}$ neutrino oscillation experiment in Chapter 3. Chapter 4 describes the NA61 (SHINE) hadron production experiment, which collected dedicated measurements for T2K. Particular emphasis in Chapter 4 is placed on the NA61 dataset collected using a replica of the T2K target in 2009. Chapter 5 describes the T2K flux calculation largely developed prior to the start of this thesis. As the flux analyser, I have produced predictions of the unoscillated neutrino flux at the near and far T2K detectors used for the last three publicly released T2K oscillation measurements[9-11]. Chapter 6 presents what constitutes a considerable fraction of my original work for this thesis. Certain parts of this work have been reported in [12-15]. Chapter 7 describes the T2K oscillation analysis in the Bayesian mode of inference, utilising Markov Chain Monte Carlo techniques to sample from multi-dimensional posterior probability distributions. This technique is well established in the T2K collaboration, and has been chosen to test the effect of the new flux tuning on the measurement precision. My original work is also described in Chapter 8, which presents the first oscillation measurements at T2K relying on the improved flux calculation from Chapter 6. Finally, in Chapter 9, conclusions are drawn for the impact of this work on both neutrino interaction modelling and future oscillation measurements.

Two aspects of my work during the past four years unfortunately could not be included in this thesis due to length restrictions. These are the timing calibration of the electromagnetic calorimeter of the ND280 near detector, and the analysis I did as a member of the EMPHATIC hadron production experiment. I was responsible for developing the initial Monte Carlo simulation of the EMPHATIC experiment, 
which has later been utilised to estimate systematic uncertainties for the pilot run collected during two weeks of data-taking in January 2018 at the Fermilab Test Beam Facility[16]. In the pilot run, the proton production cross section was measured at a range of incident beam momenta, and on different targets. The proposed physics programme of the EMPHATIC experiment is complementary to that of NA61, and it could ultimately provide valuable data for improving the current understanding of differences between hadron production from thin and thick targets.

Despite every effort being made to always cite publicly available documents, in certain cases it had been necessary to quote internal T2K technical notes, or material presented at internal meetings of T2K working groups. These can be obtained upon request from the T2K Spokesperson and the International Spokesperson, whose contact details can be found at http://t2k-experiment.org/contact-us/. 
I have done a terrible thing:

I have postulated a particle that cannot be detected.

- Wolfgang Pauli ${ }^{a}$

${ }^{a}$ Quoted by Frederick Reines in his forward to [17].

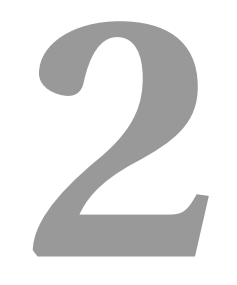

Theory

\subsection{The Neutrino}

Scientific inquiry of the neutrino starts with Wolfgang Pauli, who is credited with postulating the existence of such a particle in an attempt to explain the apparent breaking of energy conservation in $\beta$-decays. Up until then, $\beta$-decays of unstable nuclei were assumed to proceed via the emission of an electron and the accompanying acquirement of an additional +1 unit of charge by the nucleus, which would transform into the nucleus of the adjacent element in the periodic table. For example, tritium would decay into helium via ${ }_{1}^{3} \mathrm{H} \rightarrow{ }_{2}^{3} \mathrm{He}+e^{-}$. In such 2-body decays, the spectrum of emitted electrons had to be monoenergetic. In 1914, James Chadwick's unexpected discovery of a continuous electron spectrum from $\beta$-decays of radium highlighted the need for a better explanation[18]. Energy conservation was seemingly violated. Pauli came to the rescue, proposing the existence of an undetected electrically neutral light particle accompanying the emitted electron, in his now famous letter addressed to "Dear Radioactive Ladies and Gentleman"1 of the Physical Institute of the Federal Institute of Technology in Zurich. In 1932, Chadwick discovered the neutron[20], an electrically neutral building block of the nucleus, with mass remarkably similar to that of the proton. The classical picture of the nucleus was forever changed with the realisation that it consisted of both neutrons and protons. The neutron was far too heavy to explain the electron spectrum from $\beta$-decays. It was Enrico Fermi who then coined the term neutrino, "the little neutral one", to describe Pauli's mysterious particle. Today, it is known

\footnotetext{
${ }^{1}$ Full English translation of letter published in [19]
} 
that $\beta$-decays proceed via the following 3 -body decay:

$$
\mathrm{n} \rightarrow \mathrm{p}+e^{-}+\bar{\nu}_{e}
$$

Fermi also formulated the first rigorous theory of $\beta$-decay, involving the coupling of the neutron, proton, electron and neutrino ${ }^{2}$ at a single interaction point. The force mediating the radioactive $\beta$-decay had to be weak compared to the electromagnetic force, making the detection of the neutrino extremely challenging. It was in 1956 that the neutrino was finally detected, by Frederick Reines and Clyde Cowan at the Savannah River Nuclear Reactor in South Carolina[21]. The nuclear reactor facility provided a large neutrino flux (actually $\bar{\nu}_{e}$, but this had not been known at the time), which reacted with target protons:

$$
\bar{\nu}_{e}+\mathrm{p} \rightarrow \mathrm{n}+e^{+}
$$

producing a neutron and a positron (the antimatter counterpart of the electron). The target protons had been provided by two underground tanks filled with a total of 200 litres of water, placed $\sim 11$ metres from the reactor. Efficient neutron capture had been facilitatied by dissolving $40 \mathrm{~kg}$ of cadmium chloride $\left(\mathrm{CdCl}_{2}\right)$ in the tanks. Interactions induced by $\bar{\nu}_{e}$ could be inferred by detecting simultaneous pairs of 511 $\mathrm{keV}$ photons from positron-electron annihilation $\left(e^{+}+e^{-} \rightarrow 2 \gamma\right)$, followed by a delayed photon signal from the capture of the thermalised neutron

$$
\mathrm{n}+{ }^{108} \mathrm{Cd} \rightarrow\left({ }^{108} \mathrm{Cd}\right){ }^{*} \rightarrow{ }^{108} \mathrm{Cd}+\gamma
$$

where $\left({ }^{108} \mathrm{Cd}\right)^{*}$ denotes the excited state of cadmium. A schematic diagram of the experiment is shown in Figure 2.1. The prompt and delayed (by several $\mu \mathrm{s}$ ) light signals were detected using organic scintillator layers coupled to photomultplier tubes. The detection of the neutrino had been a major breakthrough in this field, and a precursor of more discoveries to follow.

In 1962, Leon Lederman, Melvin Schwartz and Jack Steinberger devised an experiment at the Brookhaven Nuclear Laboratory, with neutrinos from pion decays

\footnotetext{
${ }^{2} \bar{\nu}_{e}$ had not been known at the time
} 


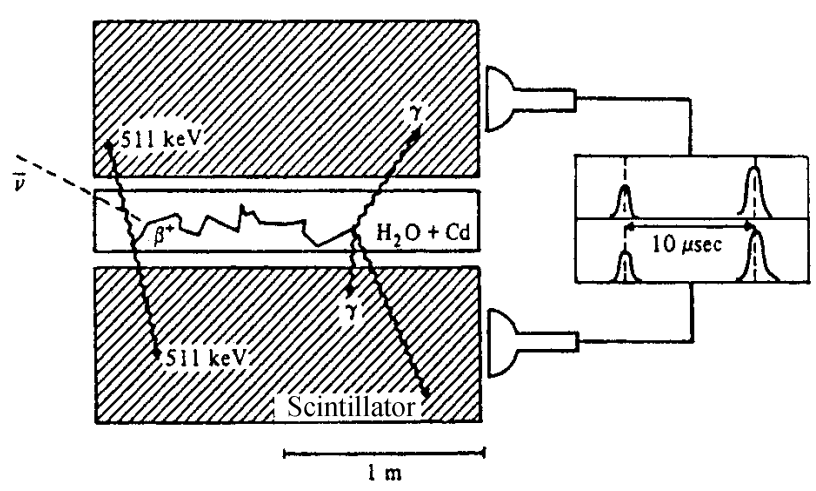

Figure 2.1: A schematic representation of the experimental setup devised by Cowan and Reines for detection of neutrinos from the Savannah River Nuclear Reactor. Reprinted with permission from F. Reines and C. L. Cowan, "Free anti-neutrino absorption crosssection", Physical Review, Volume 113, pp. 273-279, 1959[22]. Copyright (1959) by the American Physical Society.

incident on an aluminium target. The pions were produced through collisions of $15 \mathrm{GeV}$ protons, from BNL's synchrotron, with a beryllium target. These pions were then given sufficient time to decay into muons and neutrinos, before arriving at a 13.5 metre thick steel wall. Today, it is well known that pion decays proceed via $\pi^{+} \rightarrow \mu^{+}+\bar{\nu}_{\mu}$. At the time, the premise of the experiment had been that $\mu^{+}$would be stopped by the steel, and that neutrinos emerging on the other side could interact with an aluminium target placed in a spark chamber. Over several months of data-taking, 51 such neutrino induced interactions were collected. It had been observed that only muons were produced in interactions of these neutrinos with aluminium. Thus, it has been inferred that these neutrinos were somehow "different" from the $e^{+}$producing neutrinos of the Reines-Cowan experiment. This new type of neutrino has been named the muon neutrino $\nu_{\mu}$, in contrast to the previously known electron neutrino $\nu_{e}$.

The first evidence for a third generation of charged leptons, referred to by $\tau^{-}$, came in 1975 at the Stanford Linear Accelerator Center (SLAC). In collisions of electron positron pairs, 64 events with "missing" energy had been observed[23], which were thought to proceed via

$$
e^{+}+e^{-} \rightarrow \tau^{+}+\tau^{-} \rightarrow e^{ \pm}+\mu^{\mp}+4 \nu .
$$



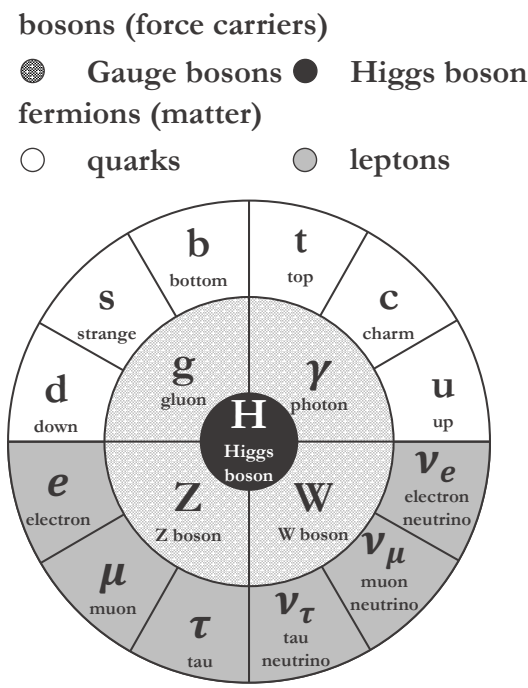

Figure 2.2: Diagram of the constituent particles of the Standard Model.

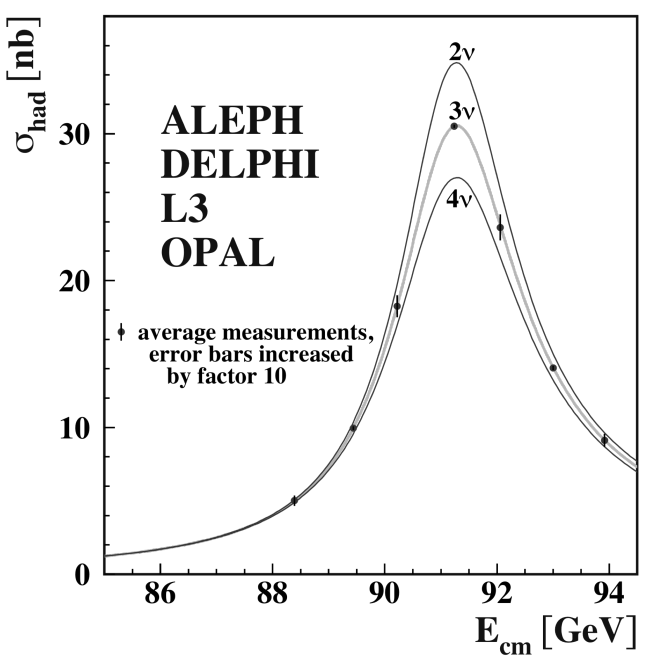

Figure 2.3: Combined measurement of hadronic production cross section around the $\mathrm{Z}^{0}$ resonance, from the ALEPH, DELPHI, OPAL, L3 and SLD experiments. Reprinted from Physics Reports, Volume 427, Issues 5-6, ALEPH Collaboration et al., "Precision electroweak measurements on the $\mathrm{Z}$ resonance", pp. 257454 , Copyright (2006), with permission from Elsevier[25].

The mass of the tau lepton was eventually measured at the DESY experimental facility [24]. With the discovery of $\tau^{-}$, came the implicit expectation for the existence of the accompanying tau neutrino $\nu_{\tau}$. A pattern was starting to emerge, encapsulated in the Standard Model of particle physics, which to this day remains the leading theory for describing particle interactions.

The Standard Model recognises three fundamental forces in the Universe, mediated through carrier particles called gauge bosons. These are the strong force (8 gluon carriers), electromagnetic force (photon) and weak force $\left(\mathrm{W}^{ \pm}\right.$and $\mathrm{Z}^{0}$ bosons). The gravitational force ${ }^{3}$ is notably absent from the Standard Model formalism, since a satisfactory quantum mechanical theory of gravity is yet to be formulated. In any case, the gravitational force is much weaker compared to other forces, and can for all purposes be neglected when considering subatomic interactions. The building blocks of all matter are twelve elementary fermionic

\footnotetext{
${ }^{3}$ Hypothetical Gauge boson carrier called the graviton is yet to be observed.
} 
particles, divided into two subgroups: quarks and leptons. Fermions and bosons differ by an intrinsic property called spin. Bosons have integer spin, and fermions half-integer spin. Quarks come in three pairs (generations), up-down $(u, d)$, charmstrange $(c, s)$ and top-bottom $(t, b)$, where the first particle of each pair has charge $+\frac{2}{3}$ and the second particle charge $-\frac{1}{3}$. Quarks have fractional charge and are never observed isolated, but either in quark antiquark pairs (mesons) or groups of three quarks (baryons), bound together by the strong force. Mesons and baryons always have integer units of charge, and are collectively referred to as hadrons. Leptons also come in three generations (also known as flavours): electron flavour $\left(e^{-}, \nu_{e}\right)$, muon flavour $\left(\mu^{-}, \nu_{\mu}\right)$ and tau flavour $\left(\tau^{-}, \nu_{\tau}\right)$. Unlike quarks, leptons do not have the property of colour, and thus do not engage in strong interactions. In the Standard model, neutrinos are massless and interact exclusively through weak interactions. All massive elementary particles acquire their mass from the Higgs boson, whose existence was postulated in the 1960s[26, 27], but which has been experimentally observed at CERN's Large Hadron Collider in 2012[28, 29]. A schematic diagram of the constituents of the Standard Model is given in Figure 2.2. All elementary fermions apart from the neutrinos occur in both the left-handed and right-handed variety, reflecting the intrinsic particle property called chirality. The weak force is the only force known to be felt by only left-handed particles.

Stringent limits on the number of neutrino generations came from studies of $\mathrm{Z}^{0}$ decays at CERN's Large Electron Positron collider[25]. In the Standard Model, the $\mathrm{Z}^{0}$ boson is predicted to decay into particle antiparticle pairs. Both hadronic decays and decays into charged $\left(l^{-} l^{+}\right)$and neutral $\left(\nu_{l} \bar{\nu}_{l}\right)$ lepton pairs are predicted, and the full width at half maximum of the observed resonance, shown in Figure 2.3, depends on the sum of relative contributions from each of these decay modes:

$$
\Gamma_{\mathrm{Z}}=\Gamma_{\text {had }}+3 \Gamma_{l^{-} l^{+}}+\mathrm{N}_{\nu} \Gamma_{\nu \bar{\nu}}
$$

where $\mathrm{N}_{\nu}$ is the number of light neutrino generations. From measurements of $\Gamma_{\mathrm{Z}}, \Gamma_{\text {had }}$ and $\Gamma_{l^{-} l^{+}}$, and the Standard model prediction for $\Gamma_{\nu \bar{\nu}}$, it has been estimated that there are three neutrino generations, $\mathrm{N}_{\nu}=2.9840 \pm 0.0082[25]$, 
which is consistent with the three experimentally observed charged leptons. If there are any additional neutrino flavours, they would either have to be very massive, $\mathrm{m}_{\nu}>\frac{\mathrm{m}_{\mathrm{z}}}{2}$, or undergo processes very different from those described in the Standard Model (sterile neutrinos).

The tau neutrino was finally measured in 2000 by the DONUT collaboration[30], through the detection of $\tau^{-}$tracks from charged current interactions of $\nu_{\tau}$ in a nuclear emulsion.

Stars are prolific "factories" of neutrinos, produced as by-products of fusion of light into heavier nuclei. For example, in the Sun, release of heat is initiated by fusion of free protons into deuterium nuclei, $\mathrm{p}+\mathrm{p} \rightarrow{ }^{2} \mathrm{H}+e^{+}+\nu_{e}$, which then fuse further into heavier nuclei such as helium, beryllium and so on. Neutrinos from the Sun have first been observed by Raymond Davis in 1969, through neutrino-induced transformation of chlorine nuclei into argon

$$
\nu_{e}+{ }^{37} \mathrm{Cl} \rightarrow{ }^{37} \mathrm{Ar}+e^{-},
$$

at the Homestake mine in South Dakota, and the subsequent detection of unstable ${ }^{37}$ Ar decays. By counting the number of such converted argon atoms, Davis concluded that the $\nu_{e}$ flux from the Sun is roughly one third of the expected value derived from the Solar Model[31], in what became known as the Solar neutrino puzzle[32] (see Figure 2.4). This apparent discrepancy between the expected solar neutrino flux, given the Sun's luminosity, age and chemical composition, and the observed flux at Earth, was ultimately resolved by the SNO$[33,34]$ experiment ${ }^{4}$. Namely, neutrinos change their flavour as they propagate through space. By the time they reach the Earth, the majority of $\nu_{e}$ from the Sun have oscillated ${ }^{5}$ into $\nu_{\mu}$ and $\nu_{\tau}$. The SNO experiment had the capability of measuring both the $\nu_{e}$ flux, $\Phi_{\mathrm{CC}}$, and the total $\left(\nu_{e}+\nu_{\mu}+\nu_{\tau}\right)$ flux, $\Phi_{\mathrm{NC}}$, through charged current (CC) and neutral current $(\mathrm{NC})$ weak interactions on deuteron nuclei,

$$
\begin{aligned}
& \nu_{e}+{ }_{1}^{2} \mathrm{H} \rightarrow e^{-}+\mathrm{p}+\mathrm{p} \quad(\mathrm{CC}) \\
& \nu_{l}+{ }_{1}^{2} \mathrm{H} \rightarrow \nu_{l}+\mathrm{p}+\mathrm{n} \quad(\mathrm{NC}),
\end{aligned}
$$

\footnotetext{
${ }^{4}$ Later confirmed by Super-Kamiokande in [35].

${ }^{5}$ The high electron density in the Sun's core is crucial for a rigorous explanation of oscillations of solar neutrinos. More on matter effects later.
} 
respectively. In addition, measurements of neutrino scattering off from electrons $(\mathrm{ES})$,

$$
\nu_{l}+e^{-} \rightarrow \nu_{l}+e^{-}
$$

probed a linear combination of different neutrino flavours,

$$
\frac{\Phi_{\mathrm{CC}}}{\Phi_{\mathrm{ES}}}=\frac{\Phi_{\nu_{e}}^{\mathrm{sol}}}{\Phi_{\nu_{e}}^{\mathrm{sol}}+0.14\left(\Phi_{\nu_{\mu}}^{\mathrm{sol}}+\Phi_{\nu_{\tau}}^{\mathrm{sol}}\right)},
$$

and had been used for consistency checks. SNO measured only $30 \%$ of the solar neutrinos with flavour $\nu_{e}$, but the total flux now agreed with the Solar model[33, 34].

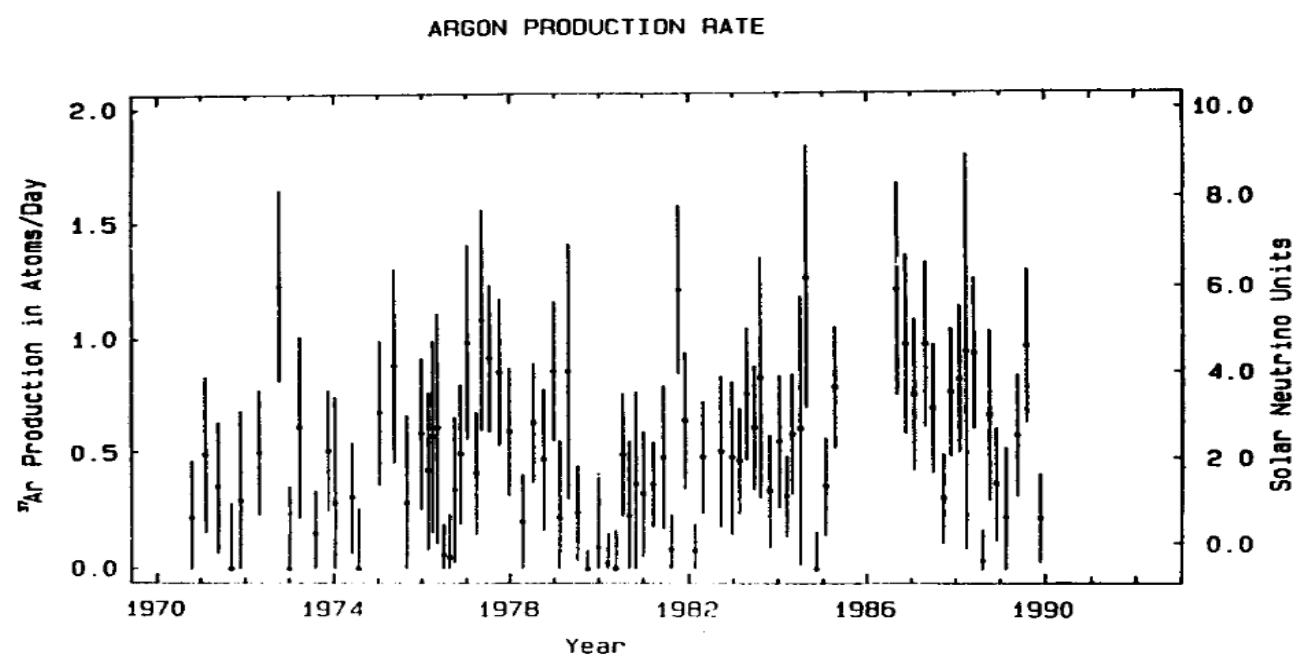

Figure 2.4: Solar neutrino flux measured by the Homestake experiment between 1970 and 1990. The average flux measurement of $(2.32 \pm 0.22) \mathrm{SNU}$, quoted in , is significantly lower from the theoretical prediction of $7.6_{-1.1}^{+1.3} \mathrm{SNU}[36]$. Here, the solar neutrino unit (SNU) is a unit of neutrino flux needed for a target atom capture rate of $10^{-36}$ per second. Reprinted from Progress in Particle and Nuclear Physics, Volume 32, Raymond Davis, "A review of the homestake solar neutrino experiment", pp. 13-32, Copyright (1994), with permission from Elsevier[37].

Besides solar sources, neutrinos are also abundantly produced in upper layers of the Earth's atmosphere, where high energy cosmic rays ${ }^{6}$ interact producing various hadrons. Production of these neutrinos is thus dominated by processes such as $\pi^{+} \rightarrow \mu^{+}+\nu_{\mu}$, and the subsequent $\mu^{+} \rightarrow e^{+}+\nu_{e}+\bar{\nu}_{\mu}$. From these decay modes, the expected ratio of atmospheric neutrino fluxes is given by

$$
R=\frac{\Phi_{\nu_{\mu}}^{\mathrm{atm}}+\Phi_{\bar{\nu}_{\mu}}^{\mathrm{atm}}}{\Phi_{\nu_{e}}^{\mathrm{atm}}+\Phi_{\bar{\nu}_{e}}^{\mathrm{atm}}} \sim 2,
$$

\footnotetext{
${ }^{6}$ comprised mostly of protons and alpha particles
} 
Figure 2.5: Measurement of $\frac{R_{\text {data }}}{R_{\mathrm{MC}}}$ by the Kamiokande detector, as a function of zenith angle subtended by neutrino production and detection points. The dashed lines denote expectations under oscillation hypotheses. Reprinted from Physics Letters B, Volume 335, Issue 2, Y. Fukuda et al., "Atmospheric $\nu_{\mu} / \nu_{e}$ ratio in the multi-GeV energy range", pp. 237-245, Copyright (1994), with permission from Elsevier[38].

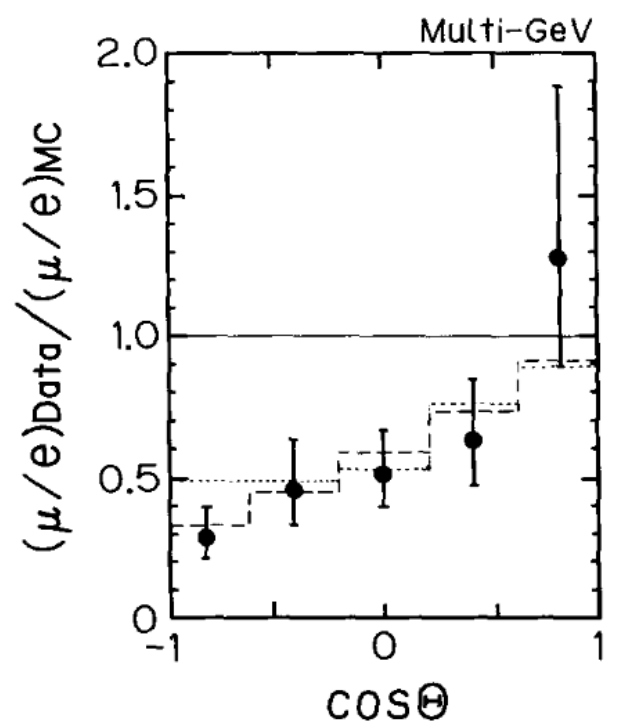

In 1990s, the Kamiokande[38] and IMB[39] experiments observed a deviation of the measured ratio, $R_{\text {data }}$, from the naive theoretical prediction assuming no oscillations, $R_{\mathrm{MC}}$, shown in Figure 2.5 as a function of zenith angle $\theta$. Here, $\cos \theta=1$ corresponds to "downwards" travelling neutrinos, produced in the atmosphere layer above the detector, and $\cos \theta=-1$ corresponds to "upwards" travelling neutrinos, passing through the centre of the Earth before detection. The observed deviation gets progressively more pronounced as the separation between neutrino production and detection points is increased. This became known as the atmospheric neutrino anomaly. The Super-Kamiokande experiment later traced the origin of this anomaly to a deficit in the number of atmospheric $\left(\nu_{\mu}+\bar{\nu}_{\mu}\right)[40]$, which have oscillated into their tau flavoured counterparts by the time of reaching the Super-K tank.

Super-Kamiokande and SNO collaborations shared the 2015 Nobel Prize in Physics for their ground-breaking contributions to the discovery of neutrino oscillations[3]. The major implication of these oscillations is that neutrinos cannot propagate at the speed of light, otherwise in the lab frame of reference the elapsed time between two states of different flavour would be infinite. Following this line of thought, neutrinos have finite mass, providing important evidence of physics beyond the predictions of the Standard Model. The mechanism of mass acquisition in 
neutrino sector exceeds the scope of the Standard model. The main theories require the existence of right-handed neutrinos[41] which have never been observed in nature.

The field of neutrino physics is incredibly rich and offers many exciting prospects for both ongoing and future experiments. The absolute neutrino mass is still to be measured. Cosmological data sets the most stringent upper limit on the sum of neutrino masses, $\sum m_{\nu} \leq 0.2-0.4 \mathrm{eV}[42]$. The KATRIN experiment has been designed with the goal of measuring the absolute mass of $\nu_{e}$ through the detection of $\beta$-decays of tritium[43]. First evidence of neutrinos outside our Solar system came from the observation of a neutrino burst emitted by Supernova 1987A in the Large Magellanic Cloud[44, 45]. Neutrinos from supernovae are powerful probes into stellar evolution, and current experiments such as Super-Kamiokande are being redesigned for their improved detection[46]. Recently, ultra high energy (PeV) neutrinos have been observed by the IceCube detector[47]. The astrophysical objects and processes responsible for the production of these neutrinos are studied by the IceCube collaboration[48]. Crucially, it is still not known whether neutrinos and anti-neutrinos are identical, which is the defining property of Majorana fermions. If proven to be true, this would have interesting theoretical implications, considering that all other Standard model particles are Dirac fermions. This is the aim of several ongoing experiments, which search for hypothetical neutrinoless double-beta decay processes $0 \nu \beta \beta$, proceeding via $(Z, A) \rightarrow(Z+2, A)+2 e^{-}[49]$. Finally, there might be subtle differences between oscillations of neutrinos and anti-neutrinos, the "hints" of which have been measured by active experiments such as T2K[9] and NOvA[50]. It has been expected that physical processes should proceed in identical manner if charge conjugation $(\mathrm{C})$ and parity transformation ${ }^{7}(\mathrm{P})$ are applied, referred to as $\mathrm{CP}$ conservation. Indeed, all measurements point to $\mathrm{CP}$ being a conserved in strong and electromagnetic processes. Evidence of CP violation in weak interactions has been observed in the hadronic sector[51], but on a scale that is insufficient to fully account for the existing matter-antimatter imbalance in the Universe. In leptonic

\footnotetext{
${ }^{7}$ Particles have intrinsic parity +1 , and anti-particles -1 .
} 
sector, $\mathrm{CP}$ violation would manifest in different conversion rates of $\left(\nu_{\mu} \rightarrow \nu_{e}\right)$ and $\left(\bar{\nu}_{\mu} \rightarrow \bar{\nu}_{e}\right)$, and is a major goal of the T2K experiment.

\subsection{Neutrino oscillations}

Crucial for understanding the theory of neutrino oscillations is the distinction between flavour and mass eigenstates. Mass eigenstates $\left|\nu_{i}\right\rangle$, where $i \in\{1,2,3\}$, are eigenstates of the free state Hamiltonian, thus satisfying Schrödinger's equation (in natural units where $c=\hbar=1$ ):

$$
\mathrm{i} \frac{\mathrm{d}\left|\nu_{i}\right\rangle}{\mathrm{d} t}=E_{i}\left|\nu_{i}\right\rangle
$$

and having well-defined momentum:

$$
-\mathrm{i} \frac{\mathrm{d}\left|\nu_{i}\right\rangle}{\mathrm{d} x}=p_{i}\left|\nu_{i}\right\rangle
$$

Consequently, the time evolution of such a mass eigenstate in free space can be expressed via:

$$
\left|\nu_{i}(t)\right\rangle=e^{\mathrm{i}\left(p_{i} x-E_{i} t\right)}\left|\nu_{i}(t=0)\right\rangle
$$

and any neutrino state can be expressed as a linear combination of the three orthogonal mass eigenstates:

$$
|\Psi(t)\rangle=\sum_{i} a_{i} e^{\mathrm{i}\left(p_{i} x-E_{i} t\right)}\left|\nu_{i}(t=0)\right\rangle
$$

Neutrinos engage in interactions with states of well-defined flavour $\left|\nu_{\alpha}\right\rangle$, where $\alpha \in$ $\{e, \mu, \tau\}$, and the probability of a general state $|\Psi(t)\rangle$ having flavour $\left|\nu_{\alpha}\right\rangle$ is obtained by taking the square of the modulus of the corresponding probability amplited $\mathcal{A}$

$$
\operatorname{Prob}=|\mathcal{A}|^{2}=\left|\left\langle\nu_{\alpha} \mid \Psi(t)\right\rangle\right|^{2}
$$

The theory of neutrino oscillations, formalised by Pontecorovo[52] and Maki, Nakagawa and Sakata[53], initially proposed a "mixing" matrix, often referred 
to as the PMNS matrix, relating the mass eigenstates to the flavour eigenstates. The theory had first been developed for two, and later extended to three generations:

$$
\left(\begin{array}{l}
\nu_{e} \\
\nu_{\mu} \\
\nu_{\tau}
\end{array}\right)=\left(\begin{array}{ccc}
\mathrm{U}_{e 1} & \mathrm{U}_{e 2} & \mathrm{U}_{e 3} \\
\mathrm{U}_{\mu 1} & \mathrm{U}_{\mu 2} & \mathrm{U}_{\mu 3} \\
\mathrm{U}_{\tau 1} & \mathrm{U}_{\tau 2} & \mathrm{U}_{\tau 3}
\end{array}\right)\left(\begin{array}{l}
\nu_{1} \\
\nu_{2} \\
\nu_{3}
\end{array}\right)
$$

The PMNS matrix can be separated into different contributions which roughly dominate the oscillations of atmospheric, reactor $^{8}$ and solar neutrinos respectively:

$$
\mathrm{U}=\underbrace{\left(\begin{array}{ccc}
1 & 0 & 0 \\
0 & c_{23} & s_{23} \\
0 & -s_{23} & c_{23}
\end{array}\right)}_{\text {atmospheric }} \underbrace{\left(\begin{array}{ccc}
c_{13} & 0 & s_{13} e^{-i \delta_{\mathrm{CP}}} \\
0 & 1 & 0 \\
-s_{13} e^{i \delta_{\mathrm{CP}}} & 0 & c_{13}
\end{array}\right)}_{\text {short-baseline reactor }} \underbrace{\left(\begin{array}{ccc}
c_{12} & s_{12} & 0 \\
-s_{12} & c_{12} & 0 \\
0 & 0 & 1
\end{array}\right)}_{\text {solar }} \underbrace{\left(\begin{array}{ccc}
1 & 0 & 0 \\
0 & e^{i \lambda_{21}} & 0 \\
0 & 0 & e^{i \lambda_{31}}
\end{array}\right)}_{\text {Majorana }},
$$

where $c_{i j}=\cos \theta_{i j}$ and $s_{i j}=\sin \theta_{i j}$ are the cosine and sine of mixing angle $\theta_{i j}$, respectively, $\delta_{\mathrm{CP}}$ is the $\mathrm{CP}$ phase $\left(\delta_{\mathrm{CP}}=0\right.$ corresponds to conserved $\left.\mathrm{CP}\right)$, and $\lambda_{21}$ and $\lambda_{31}$ are the Majorana phases $\left(\lambda_{21}=\lambda_{31}=0\right.$ for Dirac particles $)$.

For a neutrino produced with flavour $|\Psi(t=0)\rangle=\left|\nu_{\alpha}\right\rangle$, the probability amplitude for the flavour to be in state $\left|\nu_{\beta}\right\rangle$ after time $t$ is given by

$$
\mathcal{A}\left(\nu_{\alpha} \rightarrow \nu_{\beta}\right)=\left\langle\nu_{\beta} \mid \Psi(t)\right\rangle=\left(\sum_{j} \mathrm{U}_{\beta j}^{*}\left\langle\nu_{j}\right|\right)\left(\sum_{i} \mathrm{U}_{\alpha i} e^{\mathrm{i}\left(p_{i} x-E_{i} t\right)}\left|\nu_{i}\right\rangle\right)
$$

which using $\left\langle\nu_{j} \mid \nu_{i}\right\rangle=\delta_{i j}$ simplifies into

$$
\mathcal{A}\left(\nu_{\alpha} \rightarrow \nu_{\beta}\right)=\left\langle\nu_{\beta} \mid \Psi(t)\right\rangle=\sum_{i} \mathrm{U}_{\alpha i} \mathrm{U}_{\beta i}^{*} e^{\mathrm{i}\left(p_{i} x-E_{i} t\right)} .
$$

For neutrinos it is reasonable to assume $E_{i} \gg m_{i}$ and consequently the following set of approximations can be made:

$$
p_{i}=\sqrt{E_{i}^{2}-m_{i}^{2}} \approx E_{i}-\frac{m_{i}^{2}}{2 E_{i}} \text { and } x=t=L
$$

And the oscillation amplitude after travelling a distance of $x=L$ can now be expressed via the following compact notation

$$
\mathcal{A}\left(\nu_{\alpha} \rightarrow \nu_{\beta}\right)(L)=\sum_{i} \mathrm{U}_{\alpha i} \mathrm{U}_{\beta i}^{*} e^{-\mathrm{i} \frac{m_{i}^{2} L}{2 E}}
$$

\footnotetext{
${ }^{8}$ for oscillations of reactor neutrinos over short distances of a few kilometres
} 
where it has been assumed that all mass eigenstates have the same well-defined energy $E$. The oscillation probability, after some manipulation, corresponds to:

$$
\begin{aligned}
\operatorname{Prob}\left(\nu_{\alpha} \rightarrow \nu_{\beta}\right)(L)= & \delta_{\alpha \beta}-4 \sum_{i<j} R e\left[\mathrm{U}_{\alpha i} \mathrm{U}_{\beta i}^{*} \mathrm{U}_{\alpha j} \mathrm{U}_{\beta j}^{*}\right] \sin ^{2} \frac{\Delta m_{j i}^{2} L}{4 E} \\
& +2 \sum_{i<j} \operatorname{Im}\left[\mathrm{U}_{\alpha i} \mathrm{U}_{\beta i}^{*} \mathrm{U}_{\alpha j} \mathrm{U}_{\beta j}^{*}\right] \sin \frac{\Delta m_{j i}^{2} L}{2 E}
\end{aligned}
$$

The T2K experiment, which will be discussed in great detail in this thesis, has been built to measure $\nu_{e}$ appearance and $\nu_{\mu}$ survival probabilities, given the initial high purity $\nu_{\mu}$ beam. The leading order term for $\nu_{\mu}$ survival is given by

$$
\mathrm{P}\left(\nu_{\mu} \rightarrow \nu_{\mu}\right) \approx 1-\sin ^{2} 2 \theta_{23} \sin ^{2}\left(1.27 \frac{\Delta m_{32}^{2}\left[\mathrm{eV}^{2}\right] L[\mathrm{~km}]}{E[\mathrm{GeV}]}\right)
$$

The $\nu_{e}$ appearance probability can be approximated via

$$
\begin{aligned}
\mathrm{P}\left(\nu_{\mu} \rightarrow \nu_{e}\right) & \approx \sin ^{2} \theta_{23} \sin ^{2} 2 \theta_{13} \sin ^{2}\left(1.27 \frac{\Delta m_{32}^{2}\left[\mathrm{eV}^{2}\right] L[\mathrm{~km}]}{E[\mathrm{GeV}]}\right)+\underbrace{\mathrm{P}_{\mathrm{CP}}^{\text {odd }}+\mathrm{P}_{\mathrm{CP}}^{\text {even }}}_{\text {sub-leading terms }} \\
\mathrm{P}_{\mathrm{CP}}^{\text {odd }} & =-\Gamma \sin \delta_{\mathrm{CP}} \sin \left(1.27 \frac{\Delta m_{32}^{2}\left[\mathrm{eV}^{2}\right] L[\mathrm{~km}]}{E[\mathrm{GeV}]}\right) \\
\mathrm{P}_{\mathrm{CP}}^{\mathrm{even}} & =+\Gamma \cos \delta_{\mathrm{CP}} \cos \left(1.27 \frac{\Delta m_{32}^{2}\left[\mathrm{eV}^{2}\right] L[\mathrm{~km}]}{E[\mathrm{GeV}]}\right)
\end{aligned}
$$

where $\Gamma$ is the shorthand notation for

$$
\begin{aligned}
\Gamma= & \cos \theta_{13} \sin 2 \theta_{12} \sin 2 \theta_{13} \sin 2 \theta_{23} \times \\
& \sin \left(1.27 \frac{\Delta m_{32}^{2}\left[\mathrm{eV}^{2}\right] L[\mathrm{~km}]}{E[\mathrm{GeV}]}\right) \sin \left(1.27 \frac{\Delta m_{21}^{2}\left[\mathrm{eV}^{2}\right] L[\mathrm{~km}]}{E[\mathrm{GeV}]}\right) .
\end{aligned}
$$

In these equations, oscillating terms governed by $\Delta m_{21}^{2}$ have been neglected, unless they also depend on $\delta_{\mathrm{CP}}$, such as in the $\mathrm{P}_{\mathrm{CP}}^{\text {odd }}$ and $\mathrm{P}_{\mathrm{CP}}^{\text {even }}$ terms in Equation 2.24. This is a reasonable assumption for the $\mathrm{T} 2 \mathrm{~K}$ experiment, where $\frac{\Delta m_{21}^{2} L_{\mathrm{T} 2 \mathrm{~K}}}{E_{\mathrm{T} 2 \mathrm{~K}}} \ll 1$. Typically, the interplay between neutrino energy and the baseline distance determines the sensitivity of oscillation experiments to different parts of the PMNS matrix. For $\frac{E_{\nu}}{L_{\mathrm{base}}} \gg \Delta m^{2}$, the experiment is too close to the source to observe any significant oscillation effects, as shown in Figure 2.6. For $\frac{E_{\nu}}{L_{\text {base }}} \ll \Delta m^{2}$, neutrinos have 
already gone through many oscillation cycles before reaching the experiment, and the measurement resolution on $\frac{E_{\nu}}{L_{\text {base }}}$ is usually the limiting factor in resolving the fine oscillation pattern. In these cases, oscillation effects are averaged out across this resolving power. Finally, experiments are best suited to probe squared mass splitings satisfying $\frac{1.27 \Delta m^{2} L_{\text {base }}}{E_{\nu}}=\frac{\pi}{2}, \frac{3 \pi}{2}$ etc. The T2K experiment has been designed with baseline $L_{\mathrm{T} 2 \mathrm{~K}}=295 \mathrm{~km}$, and beam energy $E_{\mathrm{T} 2 \mathrm{~K}}=0.6 \mathrm{GeV}$. Thus, the dependence of the $\nu_{e}$ appearance probability (Equation 2.24) on $\Delta m_{21}^{2}=(7.53 \pm$ $0.18) \times 10^{-5} \mathrm{eV}^{2}[54]$, and $\delta_{\mathrm{CP}}$, features only subdominantly, at higher order terms.

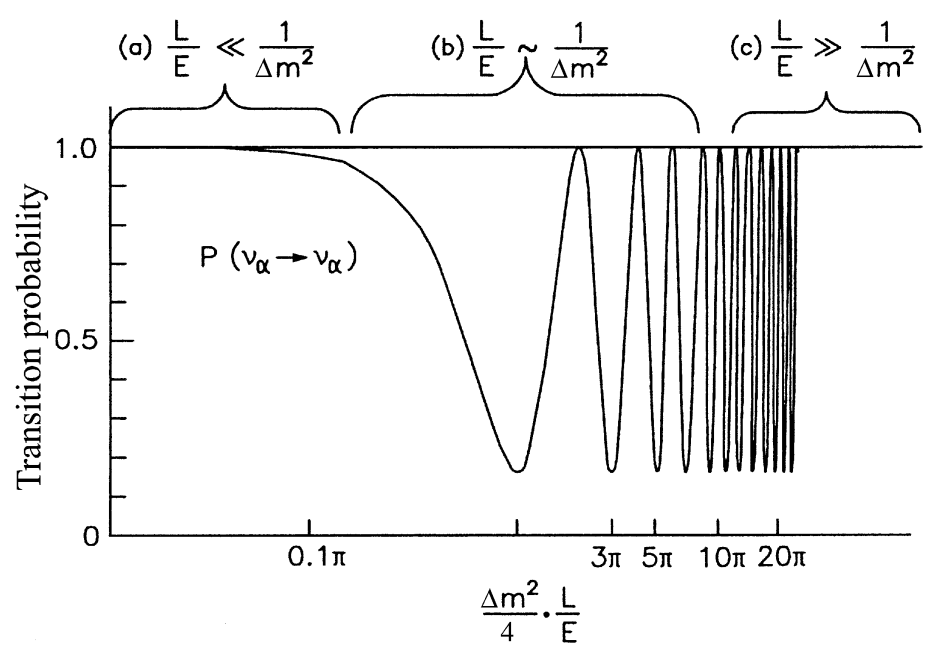

Figure 2.6: Survival probability $\mathrm{P}\left(\nu_{\alpha} \rightarrow \nu_{\alpha}\right)$, as a function of $\frac{\Delta m^{2} L}{4 E}$, showcasing the "sweet" spot $\frac{L}{E} \sim \frac{1}{\Delta m^{2}}$ for probing mass splitting $\Delta m^{2}$. Adapted by permission from Springer Nature Customer Service Centre GmbH: Vieweg+Teubner Verlag, "Neutrinophysik" by Norbert Schmitz, Chapter "Eigenschaften der Neutrinos", Copyright (1997) [55]. Also reproduced in accordance with Copyright (2012) from "Neutrino Physics" by Kai Zuber. Reproduced by permission of Taylor and Francis Group, LLC, a division of Informa plc.

The sign of the $\Delta m_{32}^{2}$ term has not been determined yet from available neutrino data. However, it is known that mass eigenstate $\nu_{3}$ is either significantly heavier, $\Delta m_{32}^{2}>0$, or lighter, $\Delta m_{32}^{2}<0$, than the other two states, $\nu_{1}$ and $\nu_{2}$, known as the normal and inverted mass hierarchy hypotheses, respectively. In fact, from available neutrino data it is known that $\frac{\Delta m_{21}^{2}}{\left|\Delta m_{32}^{2}\right|} \simeq 0.03[54]$. The two possible mass hierarchies, including the average flavour content of each mass eigenstate, are shown in Figure 2.7. The T2K experiment has sensitivity to the sign of $\Delta m_{32}^{2}$, i.e. mass ordering, through the $\mathrm{P}_{\mathrm{CP}}^{\text {odd }}$ term in the electron neutrino appearance probability in Equation 2.24. 


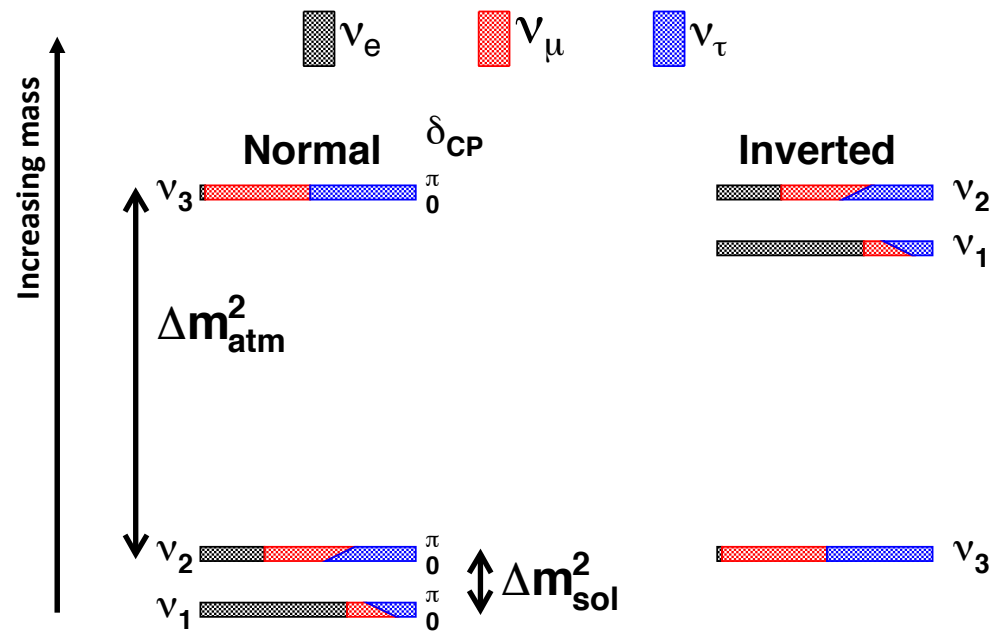

Figure 2.7: The flavour content of each of the mass eigenstates, in both normal and inverted mass hierarchy. The change in flavour content as a function of CP has been indicated. Here, $\Delta m_{\mathrm{sol}}^{2}=\Delta m_{21}^{2}$ and $\Delta m_{\mathrm{atm}}^{2}=\Delta m_{32}^{2} \approx \Delta m_{31}^{2}$. Reprinted from Progress in Particle and Nuclear Physics, Volume 83, X. Qian and P. Vogel, "Neutrino mass hierarchy", pp. 1-30, Copyright (2015), with permission from Elsevier[56].

The oscillation treatment presented here assumes neutrino propagation through vacuum, thus neglecting electrons in the Earth's crust that T2K beam neutrinos could be scattering from. For a more complete consideration including matter effects the reader is referred to [57]. Matter effects arise because the flavour eigenstates are subjected to different interaction modes in presence of high electron density: $\nu_{e}$, unlike $\nu_{\mu}$ and $\nu_{\tau}$, scatter from electrons not only via neutral weak currents, but also through charged weak currents, giving rise to an additional potential energy term in Schrödinger's equation, of form $\sqrt{2} \mathrm{G}_{\mathrm{F}} N_{e}$, where $\mathrm{G}_{\mathrm{F}}$ is the Fermi constant, and $N_{e}$ is the number density of electrons in the medium. The presence of matter effectively induces a flavour dependent index of refraction for neutrinos, analogous to refraction of light. The theoretical framework for describing neutrino oscillations in matter has been introduced first by Wolfenstein[58], and then Mikheyev and Smirnov[59], and is usually referred to as the MSW effect. These matter effects are essential for understanding the propagation of $\nu_{e}$ through the high-density solar medium. Another confirmation comes from the difference in solar neutrino flux during day and night, measured by Super-K[60]. The observed asymmetry 
stems from MSW effects induced by the terrestrial matter density presented to solar neutrinos reaching Super-K at night-time[60].

It has also been assumed that all three mass eigenstates can be expressed as plane waves with the same energy $E$. This assumption considerably simplifies the calculations, arriving nonetheless at the same conclusion as the more rigorous approach, where neutrinos are described with wave packets[61].

\subsection{Physics of particle beams}

$\mathrm{T} 2 \mathrm{~K}$ relies on a well understood proton beam for neutrino production. Thus, the main concepts related to particle beams will be briefly reviewed in this chapter. Conventional particle beams are guided using a combination of dipole magnets for steering and quadrupole magnets for focusing. The evolution of beam particles in the plane transverse to their line of motion obeys the following equation ${ }^{9}$ :

$$
\gamma x^{2}+2 \alpha x x^{\prime}+\beta x^{\prime 2}=\varepsilon
$$

where $\alpha(z), \beta(z)$ and $\gamma(z)$ are Twiss parameters, related via $\gamma=\frac{1+\alpha^{2}}{\beta}, x$ is the transverse beam position and $x^{\prime}=\frac{\mathrm{d} x}{\mathrm{~d} z}$ is the beam divergence, where $z$ is chosen to point along the beam trajectory. The beam emittance, denoted by $\varepsilon$, is usually quoted as an estimate of beam quality. This parametrisation traces out an ellipse with area $\pi \varepsilon$ in the space of $\left(x, x^{\prime}\right)$, as shown in Figure 2.8. Different particles in the beam profile trace out different ellipses, but typically the outermost particle, with highest emittance $\varepsilon_{\max }$, is taken to define the beam envelope at position $z$.

For real beam profiles, such as the ones in Figure ??, it might be difficult to pinpoint the precise edge of the beam. The following is a good estimate of the emittance of a general beam

$$
\varepsilon_{\mathrm{rms}}=\sqrt{\left\langle x^{2}\right\rangle\left\langle x^{\prime 2}\right\rangle-\left\langle x x^{\prime}\right\rangle^{2}}
$$

Emittance can be measured if either the distribution of $\left(x, x^{\prime}\right)$ is known at a certain longitudinal position $z$, or $x$-distributions are sampled at several different

\footnotetext{
${ }^{9}$ Derived by solving Hill's equation of motion.
} 


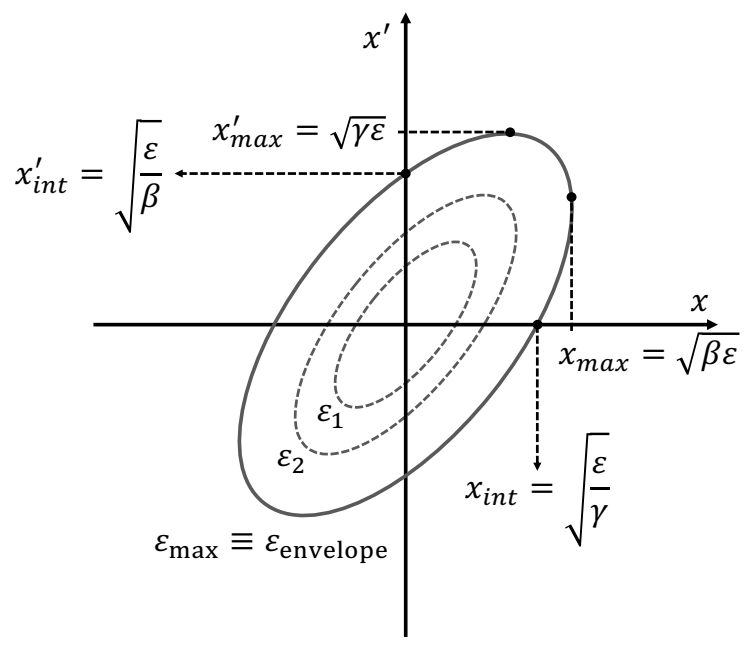

Figure 2.8: Beam envelope shape, parametrised with emittance and Twiss parameters.

$z$ points. The Twiss parameters $\beta$ and $\gamma$ can then be estimated from the spread in position, $x_{\mathrm{rms}}=\sigma_{x}$ (i.e. beam width), and spread in divergence, $x_{\mathrm{rms}}^{\prime}$, of particles in the beam, respectively,

$$
\begin{aligned}
& \beta=\frac{x_{\mathrm{rms}}^{2}}{\varepsilon_{\mathrm{rms}}} \\
& \gamma=\frac{x_{\mathrm{rms}}^{\prime} 2}{\varepsilon_{\mathrm{rms}}} .
\end{aligned}
$$

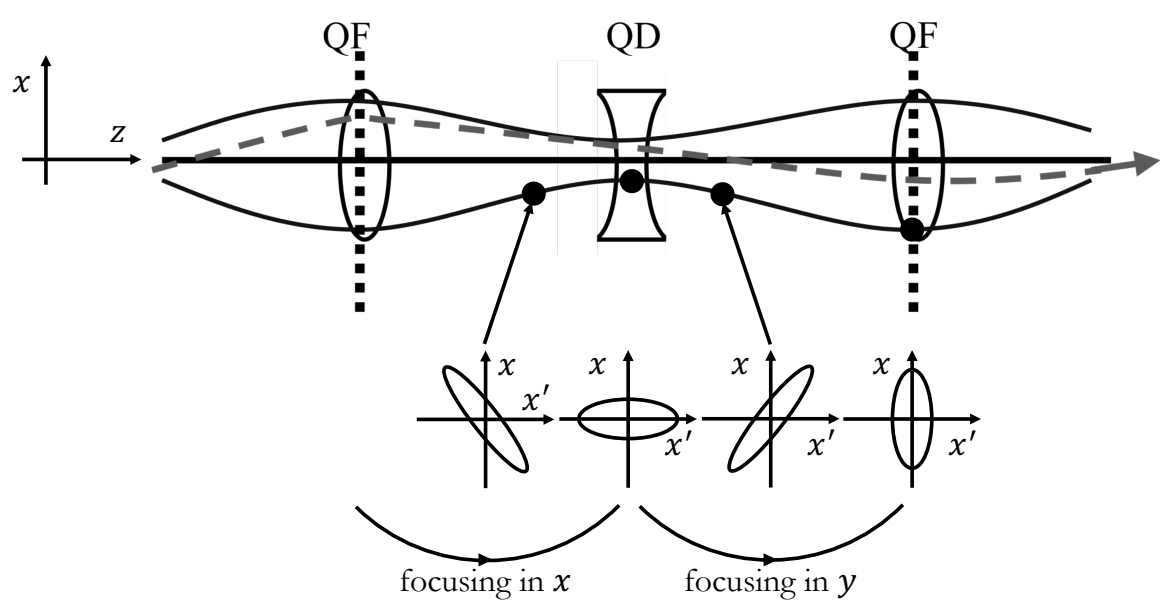

Figure 2.9: Evolution of the beam profile subjected to alternating magnets focusing in $x$-direction $(\mathrm{QF})$ and $y$-direction $(\mathrm{QD})$. The solid line shows the beam envelope. The dashed line denotes the track of a single particle. Parts of figure adapted from "Beam optics and lattice design for particle accelerators" by Bernhard J. Holzer[62], under the CC BY copyright licence. 
For beams subjected to purely conservative forces, such as bending and focusing magnetic fields, the beam emittance $\varepsilon$, and thus the ellipse area, is a conserved quantity. This statement is a result of Liouville's theorem. The beam profile nonetheless changes shape as beam particles propagate along their designated trajectories. This evolution of the beam profile can be explained through Figure 2.9, depicting beam focusing in the transverse plane, using a series of quadrupole magnets focusing alternately in $x$-direction (QF) and $y$-direction (QD). The evolution of the beam envelope is shown, as well as the trajectory of one specific particle, demonstrating that different particles populate the envelope at different $z$ locations along the beamline. The beam emittance remains fixed. 


\section{3 \\ The T2K experiment}

This chapter introduces the T2K experiment, highlighting its physics goals, detector design, and major results published by the collaboration since the commencement of data-taking in January 2010. Particular emphasis is placed on the description of the $\mathrm{T} 2 \mathrm{~K}$ beamline, which is most relevant for the work presented in this thesis.

\subsection{Physics goals and results of T2K}

The T2K (Tokai-to-Kamioka) experiment[63] is a long baseline neutrino oscillation experiment located in Japan. T2K employs the $\nu_{\mu}\left(\bar{\nu}_{\mu}\right)$ enhanced beam, produced at the Japan Proton Accelerator Research Complex (J-PARC). J-PARC is located in the village of Tokai, on the eastern coast of Japan. The intense (anti)neutrino beam is fired across 295 kilometres, to the Super Kamiokande detector, as depicted in Figure 3.1.

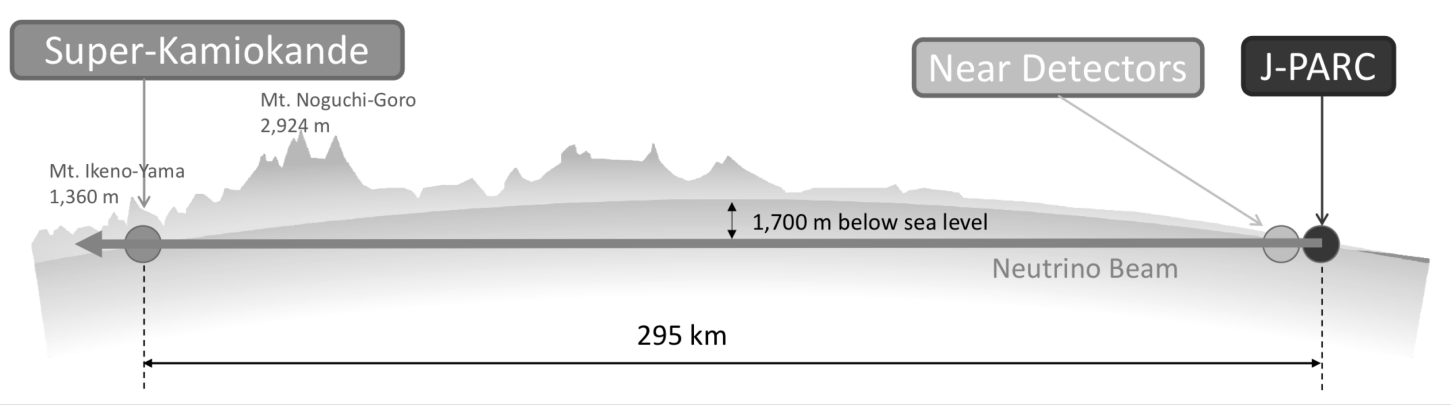

Figure 3.1: A pictorial representation of the T2K experiment baseline. Copyright by the T2K Collaboration.

T2K uses two near detectors, ND280 and INGRID, to precisely measure the neutrino beam 280 metres from the production point. Super Kamiokande (SK) samples the beam composition after 295 kilometres, when the intrinsic beam muon 
(anti)neutrinos have mixed with other neutrino flavours. The neutrino beamline is designed in such a way that the experiment can select for either a neutrino or an anti-neutrino beam to be focused towards the far detector.

T2K was the first ever neutrino experiment to use an off-axis beam. The beam is aimed $2.5^{\circ}$ away from the Super-K (and ND280) detectors. This has been done to produce the neutrino beam with a smaller range of energies. The resulting flux is strongly peaked at the energy at which the probability for observing $\nu_{e}$ appearance at Super-Kamiokande is largest, as shown in Figure 3.2.

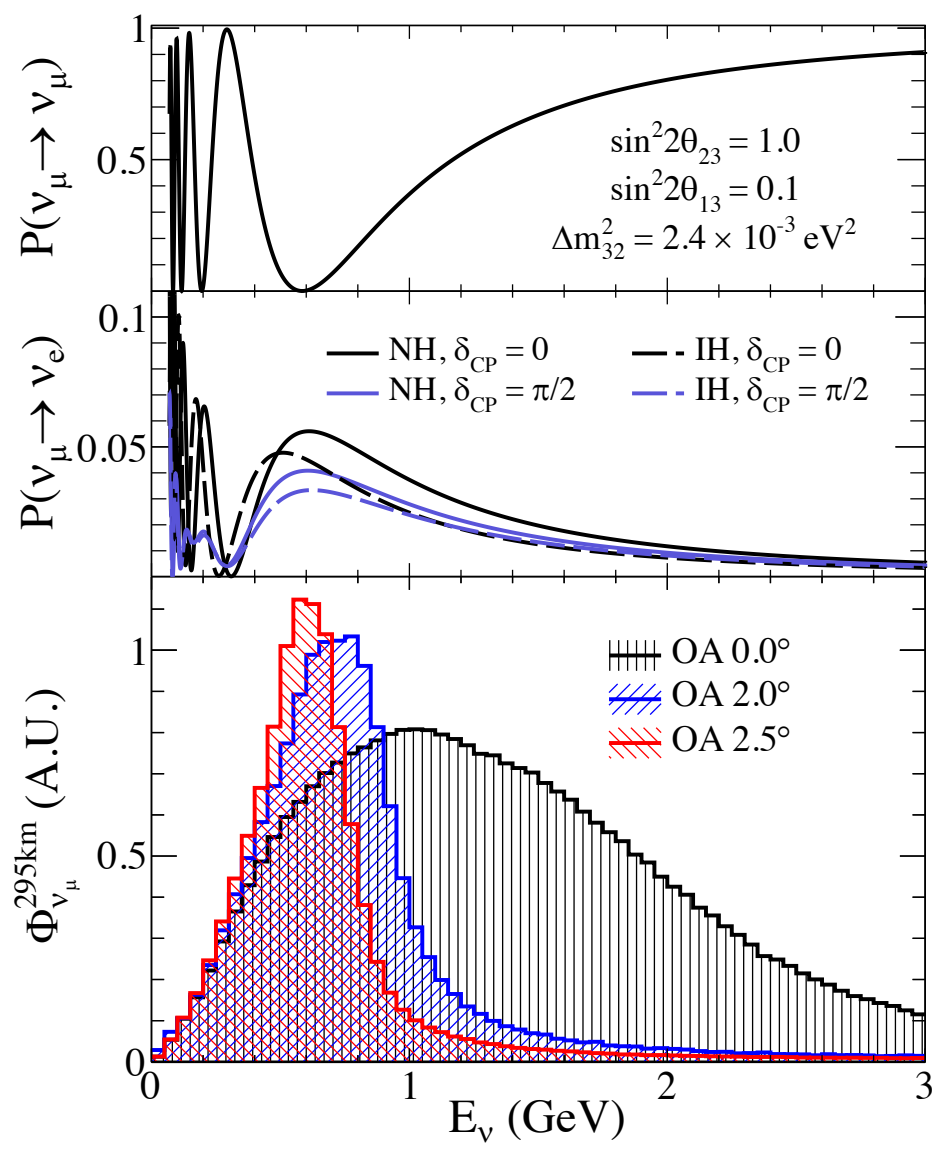

Figure 3.2: $\quad$ The $\nu_{\mu}$ survival and $\nu_{e}$ appearance probabilities as a function of true neutrino energy, at the SK location. The neutrino spectrum after $295 \mathrm{~km}$ is also shown, at different off-axis angles with respect to the beam direction. At the T2K beam energy of $\sim 600 \mathrm{MeV}$, the $\nu_{\mu}$ survival probability is minimised. The $\nu_{e}$ appearance probability is also maximised (note the different scales on the horizontal axis, most $\nu_{\mu}$ have oscillated into $\nu_{\tau}$, but these are too low in energy to detect). Copyright by the T2K Collaboration.

T2K was originally constructed with the goal of observing electron neutrino appearance $\nu_{\mu} \rightarrow \nu_{e}$ in a muon neutrino $\nu_{\mu}$ beam. Complementary to this was 
the precise measurement of $\Delta m_{32}^{2}$ and $\theta_{23}$ oscillation parameters governing the muon neutrino survival. In 2013 the collaboration published a groundbreaking result, conclusively observing the appearance of electron neutrino events in a muon neutrino beam[64]. At the time, T2K observed a total of 28 electron neutrino candidates at the far detector, with a statistical significance of $7.3 \sigma$ compared to the predicted background. The momentum and angle distributions of the electron neutrino events were also consistent with that of an appearance signal, as shown in Figure 3.3. T2K was the first experiment to measure $\sin ^{2} 2 \theta_{13}=0.140_{-0.032}^{+0.038}$ from the mixing in a muon neutrino beam.

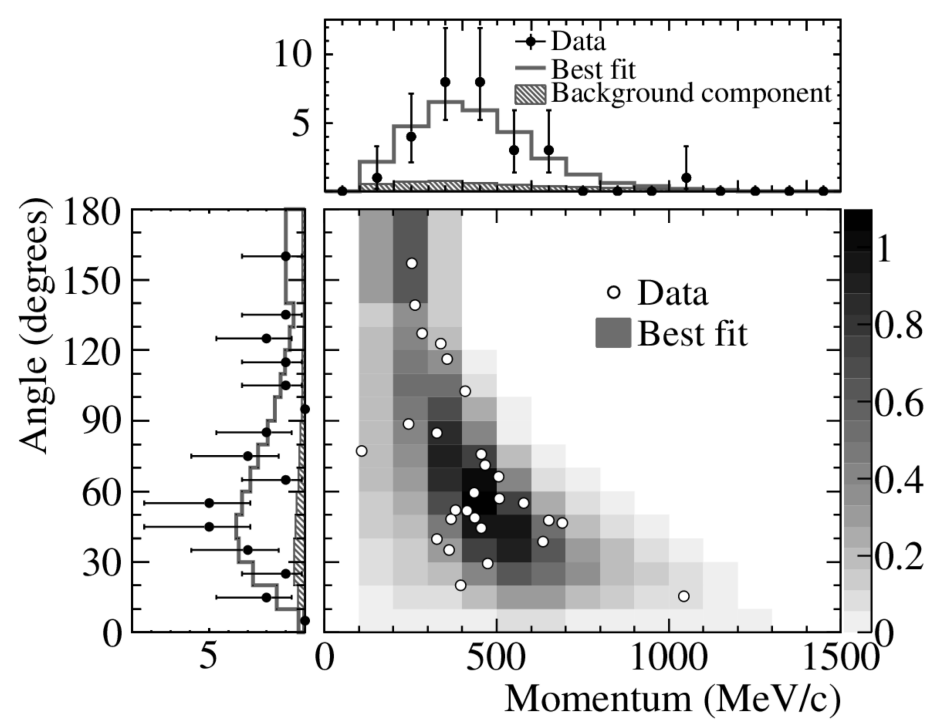

Figure 3.3: The $\left(p_{e}, \theta_{e}\right)$ distribution of the 28 selected $\nu_{e}$ candidate events in the T2K oscillation analysis (Super-Kamiokande exposure of $6.57 \times 10^{20}$ POT, collected from January 2010 to May 2013). Monte Carlo signal and background predictions are based on the T2K best fit value of $\sin ^{2} 2 \theta_{13}=0.140$, under the assumption of normal mass hierarchy. Reprinted figure with permission from K. Abe et al. (T2K Collaboration), Phys. Rev. Lett., vol. 112, p. 061802 (2014)[64]. Copyright (2014) by the American Physical Society.

Since its early days, the goal of the collaboration has shifted towards accumulating comparable amounts of data in both neutrino and anti-neutrino modes of operation, with the aim of searching for $\mathrm{CP}$ violation in the neutrino sector. The latest T2K result, presented in Figure 3.4, reports an exclusion of CP-conserving values of $\delta_{\mathrm{CP}}\left(\delta_{\mathrm{CP}}=0, \pm \pi\right)$ at the $95 \%(2 \sigma)$ confidence level, in addition to the exclusion of $46 \%$ of $\delta_{\mathrm{CP}}$ parameter space, assuming normal mass ordering, 
at the $99 \%(3 \sigma)$ confidence interval[8]. This is the tightest constraint on the value of $\delta_{\mathrm{CP}}$ achieved to date.

In the subsequent text, the reader will be introduced to the different components of the $\mathrm{T} 2 \mathrm{~K}$ experiment.

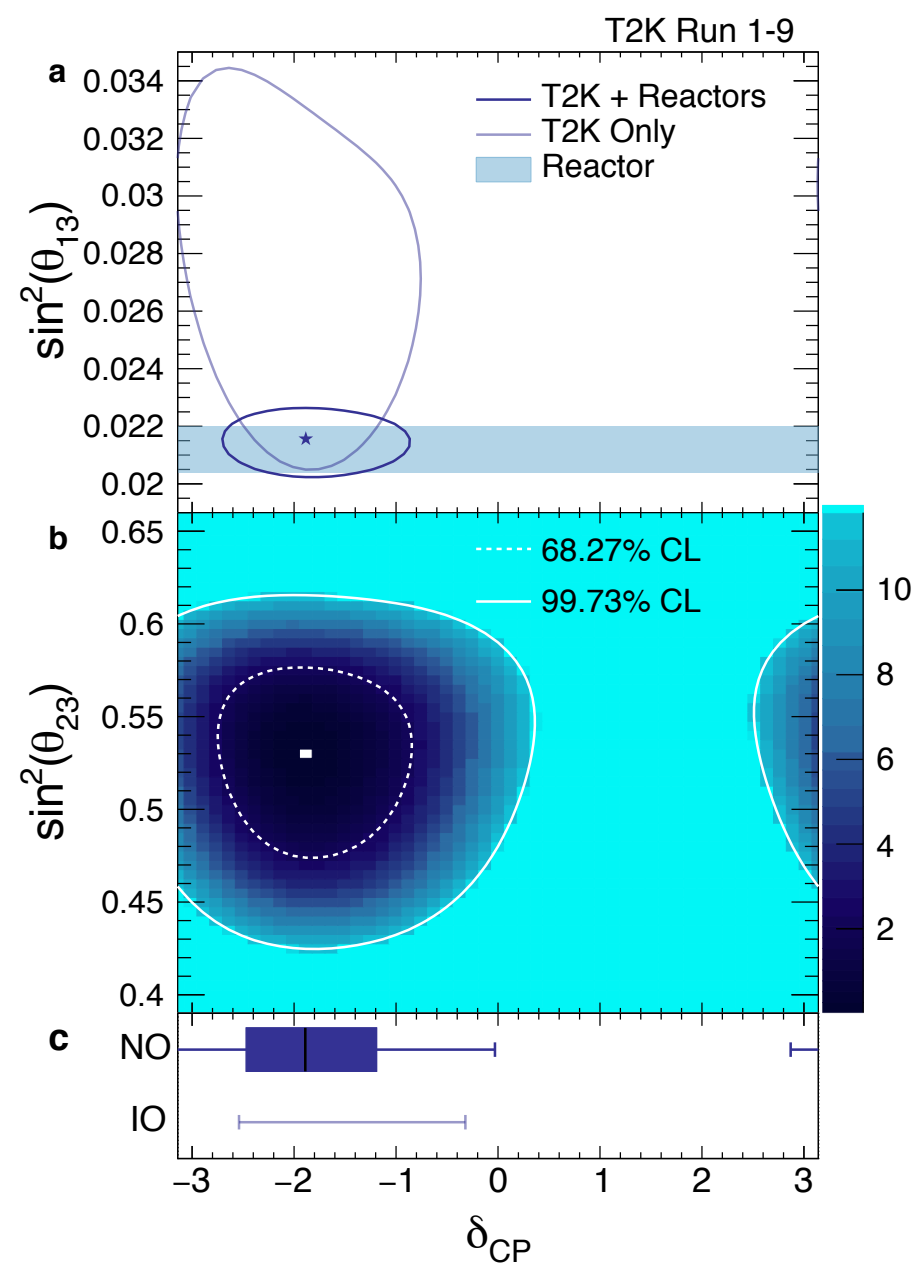

Figure 3.4: Subfigure a shows the two dimensional $68.27 \%(2 \sigma)$ confidence limit (CL) as a function of $\sin ^{2} \theta_{13}-\delta_{\mathrm{CP}}$ mixing parameters, assuming the preferred normal ordering (NO) of neutrino masses, and considering cases with and without the constraint on $\sin ^{2} \theta_{13}$ from reactor experiments. Subfigure $\mathbf{b}$ shows the two dimensional $1 \sigma$ and $3 \sigma$ confidence limits as a function of $\sin ^{2} \theta_{23}-\delta_{\mathrm{CP}}$ mixing parameters, assuming the preferred normal ordering of neutrino masses and with the reactor constraint. Here, the colour scale corresponds to the $-2 \Delta \ln (\mathrm{L})$ Bayesian likelihood as a function of the value of $\sin ^{2} \theta_{23}-\delta_{\mathrm{CP}}$ parameters. Subfigure $\mathbf{c}$ shows the $1 \sigma$ (shaded box) and $3 \sigma$ (error bars) confidence intervals for $\delta_{\mathrm{CP}}$ in normal ordering and inverted ordering. The star, square and vertical line correspond to the best-fit points in subfigures $\mathbf{a}, \mathbf{b}$ and $\mathbf{c}$, respectively. Material from: K. Abe et al. (T2K Collaboration), "Constraint on the matter-antimatter symmetry-violating phase in neutrino oscillations", Nature, published 2020, Springer Nature[8]. 


\section{$3.2 \quad$ J-PARC}

The Japan Proton Accelerator Research Complex (J-PARC) is a multi-purpose proton synchrotron research facility. J-PARC provides different proton beams for experimental facilities dedicated to studying the material and life sciences, hadron and neutrino physics. The production of the beam for T2K starts with negative hydrogen ions $\left(\mathrm{H}^{-}\right)$, which are accelerated to $400 \mathrm{MeV}^{1}$ in the $\sim 250$ metre long linear accelerator (LINAC) section. The ions are then driven through a carbon foil which strips the electrons, leaving bare protons. The protons are then accelerated further to energies of $3 \mathrm{GeV}$ in a rapid-cycling synchrotron, and fed into the Main Ring (MR) proton synchrotron. This is where the protons undergo the final acceleration to kinetic energies of $30 \mathrm{GeV}$. The beam for $\mathrm{T} 2 \mathrm{~K}$ is fast extracted from the main ring with five kicker magnets. The beam spills delivered by J-PARC for T2K consist of 8 narrow proton bunches ( $80 \mathrm{~ns}$ bunch width). The spacing between consecutive bunches within a single spill is $581 \mathrm{~ns}$. With the current operating beam power of $\sim 420 \mathrm{~kW}$, the repetition cycle between beam spills is 2.48 seconds, and the number of protons per bunch is $\sim 2.75 \times 10^{13}$.

\subsection{The T2K beamline}

The T2K $\nu_{\mu}\left(\bar{\nu}_{\mu}\right)$ beam is produced through in-flight decays of mesons, coming from collisions of $30 \mathrm{GeV}$ protons with a cylindrical graphite target. The full beamline is shown in Figure 3.5. It can be split into the upstream and downstream sections.

The high vacuum ${ }^{2}$ portion of the beamline, upstream from the target station, is referred to as the primary beamline. The primary beamline transports proton spills from the main ring (preparation section), bends them to point towards the target (arc section), and focuses the beam profile to the desired shape on the upstream target face (focusing section). The primary beamline employs a plethora of steering, dipole and quadrupole magnets. The arc section achieves a dramatic bend in the

\footnotetext{
${ }^{1}$ Always referring to the kinetic energy.

${ }^{2}$ Pressure in the primary beamline is kept below $3 \times 10^{-6}$ Pa to minimise the beam energy loss through interactions in residual gas.
} 


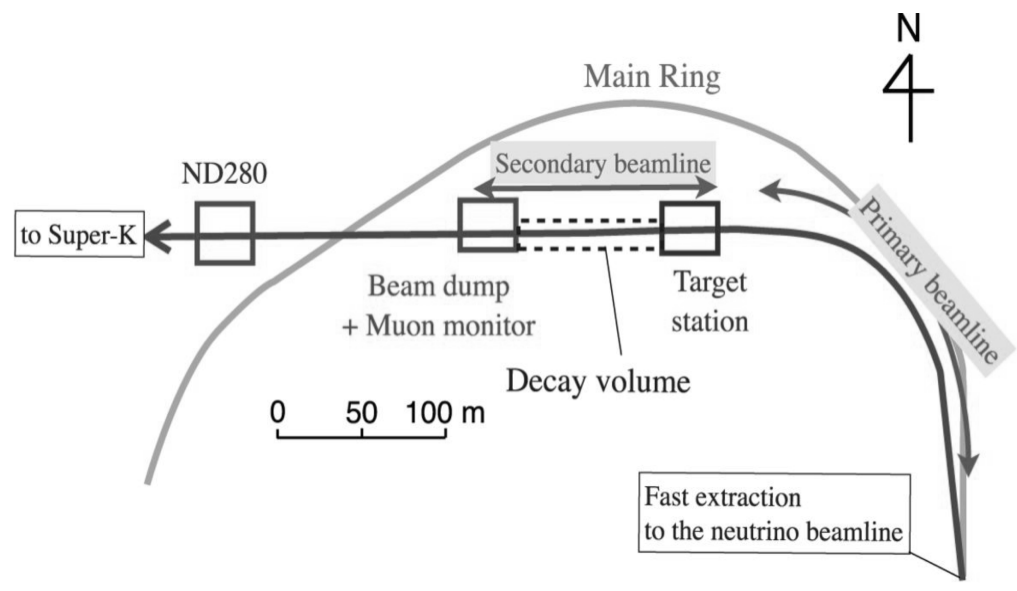

Figure 3.5: The orientation of the T2K primary and secondary beamline with respect to the main ring of the J-PARC proton synchrotron. The site of the near detector complex is also shown. Figure reprinted from Progress of Theoretical and Experimental Physics, Volume 2015, K. Suzuki et al., "Measurement of the muon beam direction and muon flux for the T2K neutrino experiment"[65], under the CC BY copyright licence.

beam direction by $\sim 80.7^{\circ}$, towards the direction of SK. The primary beamline is instrumented with various beam profile monitors: 5 current transformers (CTs), 19 segmented secondary emission monitors (SSEMs), 21 electrostatic monitors (ESMs) and 50 beam loss monitors (BLMs). The locations of various proton beam profile monitors along the primary beamline of the T2K experiment are shown in Figure 3.6.

Current transformers measure the proton beam intensity. They consist of coils wound around toroidal ferromagnetic cores in 50 turns (see Figure 3.7). The centre of the toroidal axis coincides with the beam trajectory. The proton beam generates a magnetic field in the ferromagnet, which in turn induces a current in the coil, proportional to the charge carried by the proton beam. The most downstream current transformer, CT05, is used for taking precise measurements of the protons on target (POT) delivered for every T2K physics run, summarised in Table 3.7. The current normalization uncertainty assigned to CT05 POT measurements is 2\%[63].

Segmented secondary emission monitors measure the beam distribution in the transverse plane, referred to as the beam profile. They consist of $5 \mu \mathrm{m}$ thick titanium foils(see Figure 3.7). The anode collection foil is sandwiched between two striped cathode foils. Secondary electrons emitted from the segmented cathode planes are read out by the single anode plane. Each SSEM placed into the beamline 


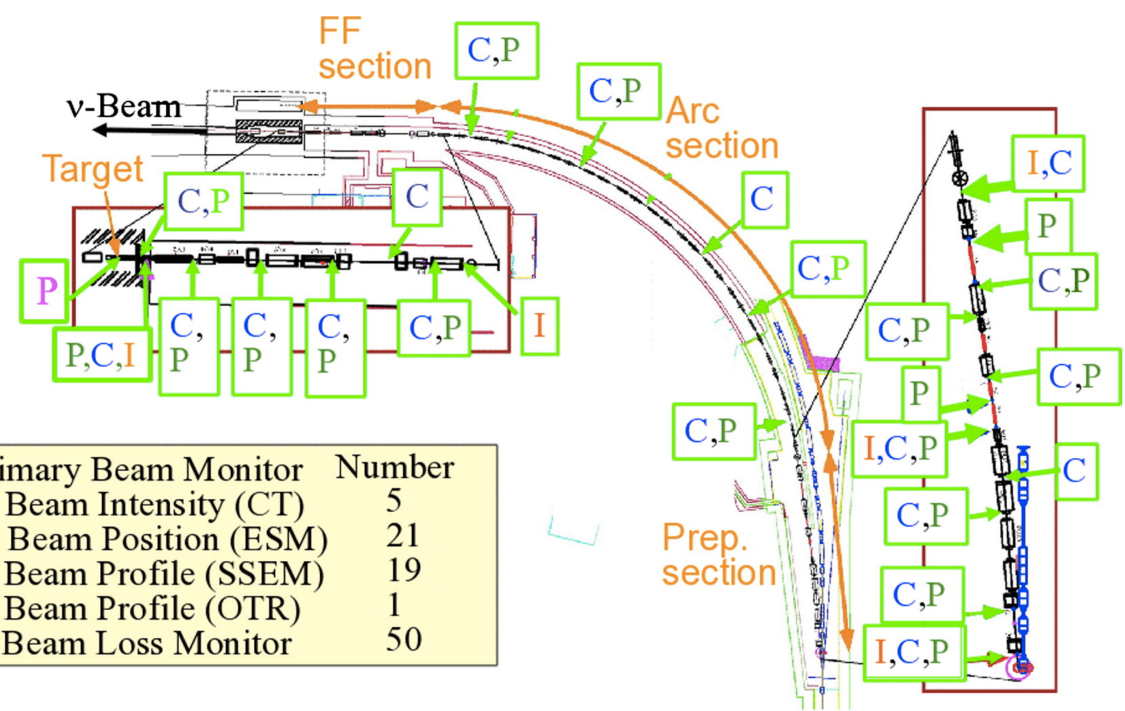

Figure 3.6: Rendering of the T2K primary beamline, showing the locations of various proton beam profile monitors. Reprinted from Nuclear Instruments and Methods in Physics Research Section A: Accelerators, Spectrometers, Detectors and Associated Equipment, Volume 659, K. Abe et al. (T2K Collaboration), "The T2K experiment", pp. 106-135, Copyright (2011), with permission from Elsevier[63].

introduces a $0.005 \%$ beam loss, which is why they are mainly used during single spill beam tuning. Only the most downstream monitor, SSEM19, is kept in the path of the proton beam during continuous operation. If the profile at SSEM19 exceeds the assigned boundaries, the beam is aborted by the interlock system. This system was introduced to protect J-PARC staff and the beamline equipment. SSEMs measure the beam width to precision of $200 \mu \mathrm{m}$.

Electrostatic monitors continuously measure the beam position. Each monitor consists of four segmented cylindrical electrodes, oriented around the nominal beam position (see Figure 3.7). Charge is induced on these electrodes, directly proportional to their distance from the beam. The beam centre is inferred from the observed position of the charge induced on the top-bottom and left-right electrode pairs. This is a non-destructive method, essential for safe beam operation. ESMs are sensitive to the beam position to precision better than $450 \mu \mathrm{m}$, but they have no sensitivity to the beam width.

Wire proportional counters are used as beam loss monitors (see Figure 3.7). 50 BLMs are distributed along the outer walls of the primary beamline, making this a 


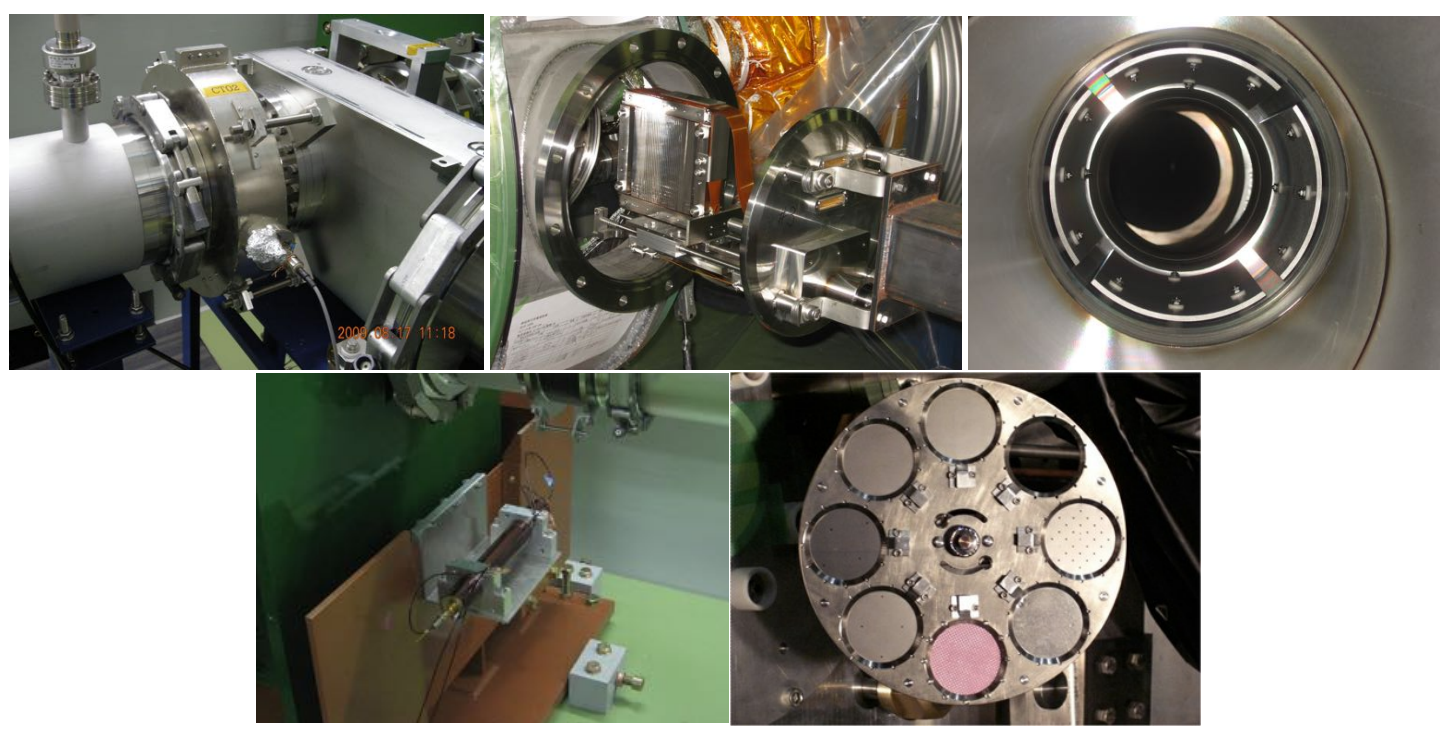

Figure 3.7: Photographs of T2K beam monitors: CT (upper left), SSEM (upper middle), ESM (upper right), BLM (lower left) and OTR (lower right). Four photographs reprinted from Nuclear Instruments and Methods in Physics Research Section A: Accelerators, Spectrometers, Detectors and Associated Equipment, Volume 659, K. Abe et al. (T2K Collaboration), "The T2K experiment", pp. 106-135, Copyright (2011), with permission from Elsevier[63]. One photograph (OTR monitor) reprinted from Nuclear Instruments and Methods in Physics Research Section A: Accelerators, Spectrometers, Detectors and Associated Equipment, Volume 703, S. Bhadra et al., "Optical transition radiation monitor for the T2K experiment", pp. 45-58, Copyright (2013), with permission from Elsevier[66].

non-destructive procedure. Ionizing particles from the beam ionize the $\mathrm{Ar}+\mathrm{CO}_{2}$ gas in the counters. The BLM signal is integrated for every beam spill, and if the beam loss exceeds the assigned maximum value, the beam interlock system generates a signal for aborting the beam. The BLMs are sensitive to beam losses of $16 \mathrm{~mW}[63]$.

The helium filled section of the T2K beamline, housing the T2K target station and all downstream components, is referred to as the secondary beamline (see Figure 3.8). Helium is used to avoid substantial pion losses through interactions with air, as well as to prevent nitrogen oxide production and oxidation of the apparatus. The target station, shown in Figure 3.9, houses various components essential for the generation and real time monitoring of the intense neutrino beam. The station is separated from the high vacuum environment of the primary beamline via a helium cooled titanium-alloy beam window. The graphite baffle is used to protect the equipment by collimating the incoming proton beam. The optical 


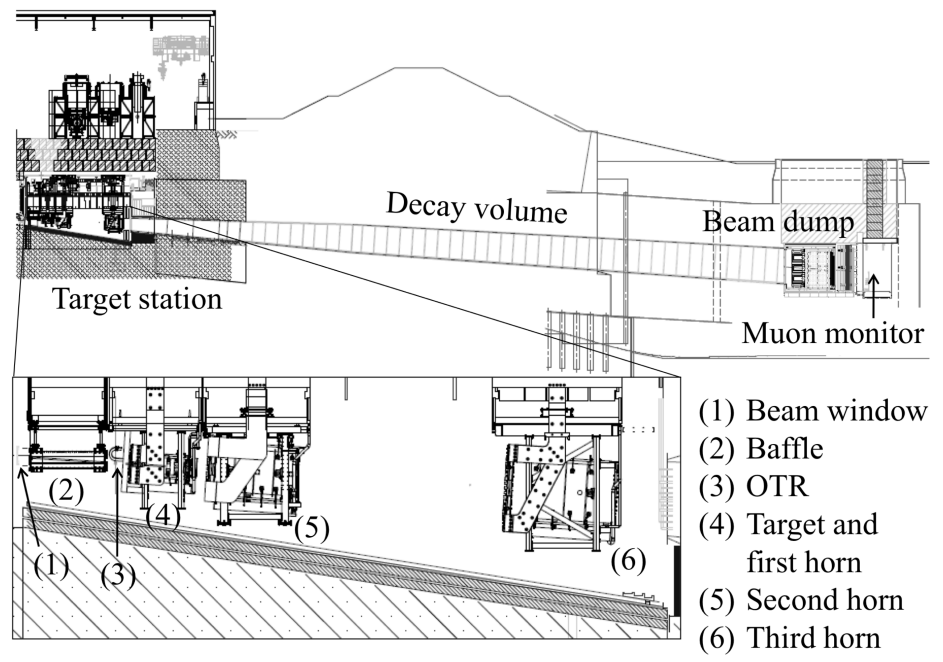

Figure 3.8: Rendering of the $\mathrm{T} 2 \mathrm{~K}$ secondary beamline. Reprinted from Nuclear Instruments and Methods in Physics Research Section A: Accelerators, Spectrometers, Detectors and Associated Equipment, Volume 789, T. Sekiguchi et al., "Development and operational experience of magnetic horn system for T2K experiment", pp. 57-80, Copyright (2015), with permission from Elsevier[67].

transition radiation (OTR) monitor measures the beam profile just upstream from the target. Optical transition radiation is emitted when a charged particle traverses two materials with different dielectric constants. In the case of the OTR monitor, dielectric materials placed in the beam orbit are provided by foils of different material and thickness, installed on a carousel structure (see Figure 3.7). The carousel is oriented at $45^{\circ}$ with respect to the beam, and different foils can be positioned into the beam path by rotating the carousel with a stepping motor. The light emitted from the foil, at $90^{\circ}$ with respect to the beam, is transported to a radiation hard camera via a series of mirrors.

The combined measurements taken by the OTR monitor, ESMs and SSEMs are used to measure the beam profile at the baffle. For every beam spill, the beam centre $(x, y)$ and direction at the upstream baffle face are extrapolated from measurements by ESM19 ${ }^{3}$, ESM20, SSEM19 and the OTR monitor. For the first 100 spills at the start of every data-taking period, all the SSEMs are inserted into the beam orbit. The Twiss parameters $\left(\alpha_{x}, \alpha_{y}\right)$ and beam emittances $\left(\epsilon_{x}, \epsilon_{y}\right)$ are precisely measured from the SSEM1-19 and OTR monitor readings. SSEM1-18

\footnotetext{
${ }^{3}$ ESM19 is used for the vertical beam centre, but not the horizontal
} 


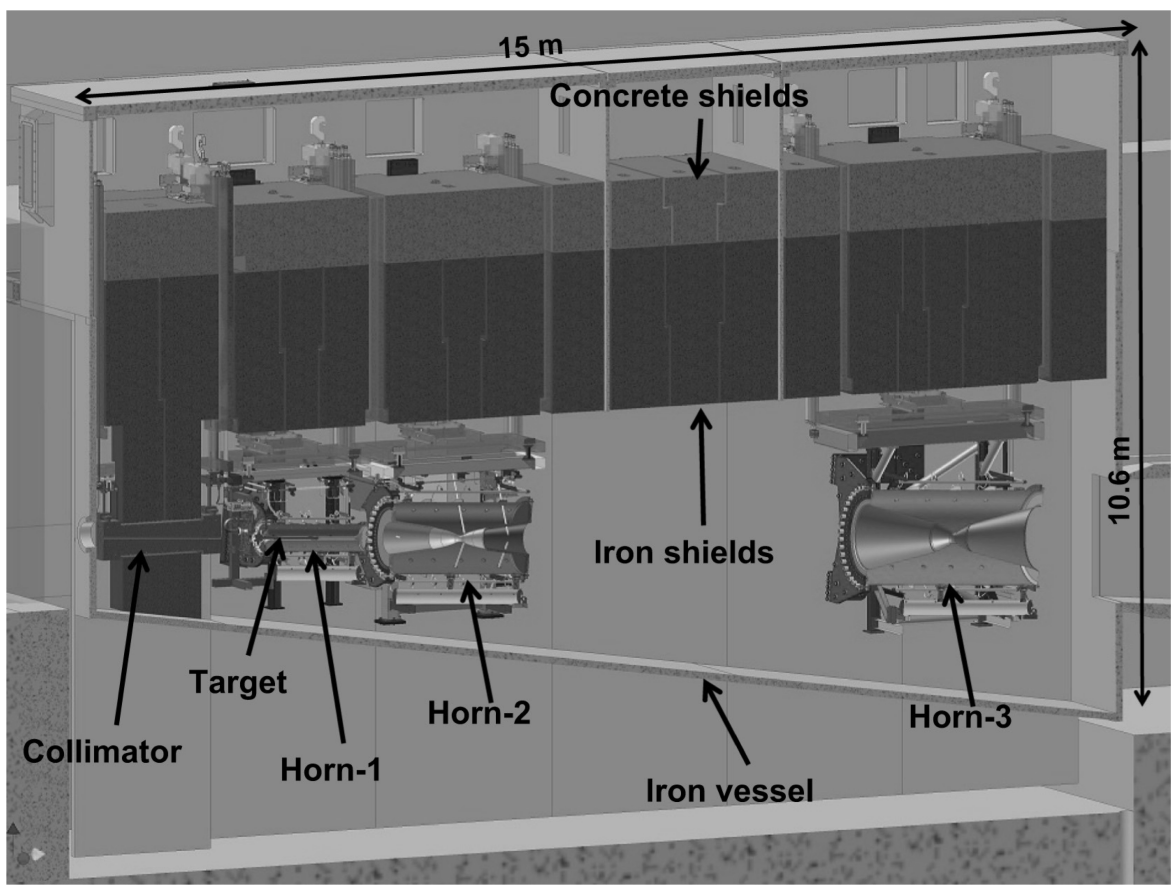

Figure 3.9: Rendering of the T2K target station. Reprinted from Nuclear Instruments and Methods in Physics Research Section A: Accelerators, Spectrometers, Detectors and Associated Equipment, Volume 789, T. Sekiguchi et al., "Development and operational experience of magnetic horn system for T2K experiment", pp. 57-80, Copyright (2015), with permission from Elsevier[67].

are then taken out from the primary beamline to minimise beam losses. For every subsequent spill, the Twiss parameters and emittances are scaled based on SSEM19 and OTR monitor readings. The beam width $\left(\sigma_{x}, \sigma_{y}\right)$ and divergence $\left(\theta_{x}, \theta_{y}\right)$ at the baffle are calculated from the Twiss parameters and emittances. For more details on the calculation of beam parameters from proton beam monitor readings refer to [68]. Beam profile parameters at the baffle are essential inputs for the flux simulation, and are provided in Table 3.1 for runs 1-9. The beam parameters for every T2K run period are obtained by calculating the POT weighted average of beam parameters for individual beam spills.

The uncertainties on measurements of beam position and divergence are shown in Table 3.2. They are dominated by errors in alignment of individual beam monitors and the alignment between the primary and the secondary beamline, as well as errors on individual beam monitor readings. The components of the proton beam position and divergence along the same direction are highly correlated, 


\begin{tabular}{|c|c|c|c|c|c|c|}
\hline Period & & ion $[\mathrm{cm}]$ & $\theta[\mathrm{mrad}]$ & $\sigma[\mathrm{cm}]$ & $\varepsilon[\pi \mathrm{mm} \mathrm{mrad}]$ & Twiss $\alpha$ \\
\hline \multirow{2}{*}{ Run 1} & $x$ & 0.037 & -0.0044 & 0.4237 & 2.13 & 0.60 \\
\hline & $y$ & 0.084 & 0.004 & 0.4167 & 2.29 & -0.09 \\
\hline \multirow{2}{*}{ Run 2} & $x$ & 0.015 & -0.080 & 0.4037 & 5.27 & 0.16 \\
\hline & $y$ & -0.005 & -0.01 & 0.4083 & 5.17 & 0.14 \\
\hline \multirow{2}{*}{ Run 3b } & $x$ & 0.0087 & 0.020 & 0.4134 & 6.50 & 0.16 \\
\hline & $y$ & -0.0024 & 0.043 & 0.3973 & 5.30 & 0.25 \\
\hline \multirow{2}{*}{ Run 3c } & $x$ & -0.0001 & 0.032 & 0.4033 & 4.94 & 0.33 \\
\hline & $y$ & -0.0366 & 0.068 & 0.4220 & 6.02 & 0.34 \\
\hline \multirow{2}{*}{ Run 4} & $x$ & 0.0032 & 0.042 & 0.3755 & 5.00 & 0.15 \\
\hline & $y$ & -0.0865 & 0.182 & 0.4153 & 6.14 & 0.19 \\
\hline \multirow{2}{*}{ Run 5a } & $x$ & -0.0003 & -0.004 & 0.3960 & 3.09 & 0.01 \\
\hline & $y$ & -0.1091 & 1.743 & 0.4214 & 4.08 & 0.05 \\
\hline \multirow{2}{*}{ Run 5b } & $x$ & 0.0273 & 0.070 & 0.3844 & 3.50 & -0.04 \\
\hline & $y$ & -0.0242 & 0.159 & 0.4201 & 4.18 & 0.07 \\
\hline \multirow{2}{*}{ Run 5c } & $x$ & 0.0451 & -0.076 & 0.3891 & 3.98 & -0.05 \\
\hline & $y$ & -0.0435 & 0.130 & 0.4180 & 4.48 & 0.07 \\
\hline \multirow{2}{*}{ Run 6a } & $x$ & 0.113 & -0.013 & 0.4004 & 3.59 & 0.10 \\
\hline & $y$ & -0.0695 & 0.493 & 0.4282 & 4.45 & 0.04 \\
\hline \multirow{2}{*}{ Run 6b } & $x$ & 0.035 & -0.030 & 0.4061 & 2.95 & -0.02 \\
\hline & $y$ & -0.0988 & 0.451 & 0.4367 & 4.22 & 0.29 \\
\hline \multirow{2}{*}{ Run 6c } & $x$ & 0.054 & -0.021 & 0.3849 & 3.42 & 0.09 \\
\hline & $y$ & -0.0753 & 0.453 & 0.4019 & 4.19 & 0.02 \\
\hline \multirow{2}{*}{ Run 6d } & $x$ & 0.0512 & -0.033 & 0.3953 & 3.40 & 0.00 \\
\hline & $y$ & -0.0748 & 0.353 & 0.4027 & 4.26 & 0.03 \\
\hline \multirow{2}{*}{ Run 6e } & $x$ & 0.0324 & -0.017 & 0.4270 & 3.24 & 0.04 \\
\hline & $y$ & -0.0834 & 0.271 & 0.3962 & 3.71 & -0.11 \\
\hline \multirow{2}{*}{ Run 6f } & $x$ & 0.0354 & 0.044 & 0.4434 & 3.07 & -0.01 \\
\hline & $y$ & -0.0769 & 0.316 & 0.4035 & 3.30 & -0.25 \\
\hline \multirow{2}{*}{ Run 7a } & $x$ & 0.0283 & 0.037 & 0.4496 & 3.66 & 1.00 \\
\hline & $y$ & 0.0323 & 0.121 & 0.4176 & 4.38 & -0.18 \\
\hline \multirow{2}{*}{ Run 7b } & $x$ & 0.0089 & -0.020 & 0.4278 & 3.67 & 0.14 \\
\hline & $y$ & -0.0393 & 0.097 & 0.4031 & 4.47 & -0.20 \\
\hline \multirow{2}{*}{ Run 7c } & $x$ & 0.0078 & -0.034 & 0.4240 & 3.50 & 0.19 \\
\hline & $y$ & 0.0146 & 0.038 & 0.4075 & 4.03 & -0.10 \\
\hline \multirow{2}{*}{ Run 8} & $x$ & 0.0157 & -0.066 & 0.4187 & 4.25 & 0.31 \\
\hline & $y$ & -0.0145 & 0.027 & 0.4106 & 3.81 & -0.11 \\
\hline \multirow{2}{*}{ Run 9a } & $x$ & -0.0201 & -0.034 & 0.4198 & 3.95 & 0.16 \\
\hline & $y$ & -0.0421 & 0.158 & 0.4324 & 3.57 & -0.18 \\
\hline \multirow{2}{*}{ Run 9b } & $x$ & -0.0029 & -0.044 & 0.4106 & 4.63 & 0.17 \\
\hline & $y$ & -0.0360 & 0.271 & 0.4267 & 4.24 & -0.21 \\
\hline \multirow{2}{*}{ Run 9c } & $x$ & 0.0138 & -0.008 & 0.4201 & 5.18 & 0.24 \\
\hline & $y$ & -0.0321 & 0.048 & 0.4163 & 3.89 & -0.29 \\
\hline \multirow{2}{*}{ Run 9d } & $x$ & -0.0225 & 0.035 & 0.3820 & 5.16 & -0.11 \\
\hline & $y$ & -0.0350 & -0.046 & 0.4024 & 3.80 & -0.34 \\
\hline
\end{tabular}

Table 3.1: Summary of the beam conditions for runs 1 to 9 , calculated at the baffle. 


\begin{tabular}{l|cc|cc|c}
\hline \hline Period & $\Delta x[\mathrm{~mm}]$ & $\Delta y[\mathrm{~mm}]$ & $\Delta \theta_{x}[\mathrm{mrad}]$ & $\Delta \theta_{y}[\mathrm{mrad}]$ & $\rho\left(y, \theta_{y}\right)$ \\
\hline Run 1 & 0.38 & 0.58 & 0.06 & 0.29 & 0.392 \\
Run 2 & 0.27 & 0.62 & 0.06 & 0.32 & 0.398 \\
Run 3b & 0.28 & 0.58 & 0.06 & 0.29 & 0.427 \\
Run 3c & 0.36 & 0.58 & 0.07 & 0.28 & 0.417 \\
Run 4 & 0.34 & 0.58 & 0.07 & 0.28 & 0.401 \\
Run 5 & 0.34 & 0.57 & 0.07 & 0.28 & 0.409 \\
Run 6a to 6b & 0.34 & 0.58 & 0.07 & 0.28 & 0.433 \\
Run 7 & 0.40 & 0.59 & 0.08 & 0.29 & 0.468 \\
Run 8 & 0.40 & 0.58 & 0.08 & 0.29 & 0.45 \\
Run 9 & 0.40 & 0.58 & 0.08 & 0.29 & 0.45 \\
\hline \hline
\end{tabular}

Table 3.2: Uncertainties on measurements of beam position $(x, y)$ and divergence $\left(\theta_{x}, \theta_{y}\right)$, as well as the correlations between position and divergence in the vertical $(y)$ direction for all run periods.

\begin{tabular}{l|cc|cc|cc}
\hline \hline Systematic Source & $\begin{array}{c}\Delta \epsilon_{x} \\
{[\pi \mathrm{mm} \text { mrad }]}\end{array}$ & $\begin{array}{c}\Delta \epsilon_{y} \\
{[\pi \mathrm{mm} \text { mrad }]}\end{array}$ & $\Delta \alpha_{x}$ & $\Delta \alpha_{y}$ & $\begin{array}{c}\Delta \sigma_{x} \\
{[\mathrm{~mm}]}\end{array}$ & $\begin{array}{c}\Delta \sigma_{y} \\
{[\mathrm{~mm}]}\end{array}$ \\
\hline Beam monitor & 0.10 & 0.12 & 0.11 & 0.11 & 0.10 & 0.10 \\
$p$ dispersion & 0.51 & 0.10 & 0.10 & 0.02 & 0.01 & 0.03 \\
FQ2-4 B field & 0.56 & 0.52 & 0.28 & 1.68 & 0.06 & 0.97 \\
\hline \hline
\end{tabular}

Table 3.3: Uncertainties on measurements of beam emittances $\left(\epsilon_{x}, \epsilon_{y}\right)$, Twiss parameters $\left(\alpha_{x}, \alpha_{y}\right)$ and beam widths $\left(\sigma_{x}, \sigma_{y}\right)$, broken down into contributions from dominant systematic effects [63].

as shown by $\rho\left(y, y^{\prime}\right)$ in Table 3.2. The uncertainties on measurements of beam emittance, Twiss parameter and width are given in Table 3.3, broken down into contributions from different error sources: beam monitor resolution, momentum dispersion $\left(\frac{\Delta p}{p} \sim 0.3 \%\right)$ in the beam, and errors in the modelling of the magnetic field from the focusing quadrupole magnets FQ2, FQ3 and FQ4 (field strength $\frac{\Delta B}{B} \sim 7 \%$ and effective magnet length $\frac{\Delta L}{L} \sim 7 \%$ ).

The T2K target is made of isotropic IG-430 grade graphite, manufactured by the Toyo-Tanso company. The target is cylindrical, with dimensions of $91.4 \mathrm{~cm}$ (length) $\times 2.6 \mathrm{~cm}$ (diameter). The target length is equivalent to 1.9 interaction lengths $\left(\lambda_{\mathrm{I}}\right)$ for $30 \mathrm{GeV}$ protons interacting on carbon. The target is surrounded by a double-sided coaxial cooling tube, with the inner wall made of IG-430 graphite and the outer wall made from a titanium-alloy (Ti-6Al-4V), as shown in Figure 3.10. Helium gas pumped through the cooling tube is used as the refrigerant. The target 
temperature is kept between $400^{\circ} \mathrm{C}$ at the surface and $700^{\circ} \mathrm{C}$ in the centre.

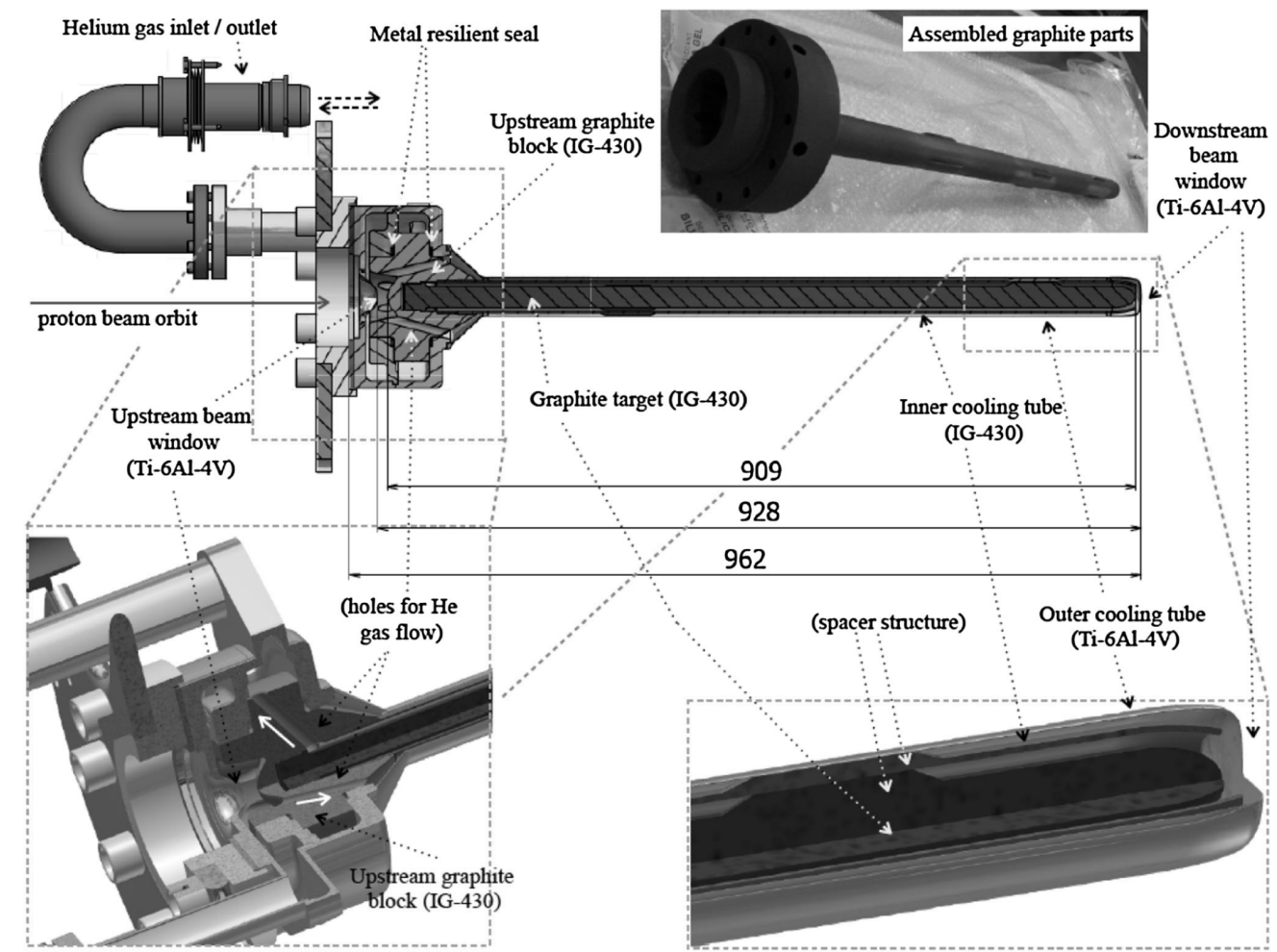

Figure 3.10: Design of the T2K target and the accompanying cooling structure. Reprinted by permission from Springer Nature Customer Service Centre GmbH: Springer Nature, Journal of Radioanalytical and Nuclear Chemistry, "The graphite target for J-PARC neutrino beam-line", T. Nakadaira, Copyright (2015)[69].

The pions and kaons resulting from the collision of beam protons with the T2K target are guided into the 96-metre-long decay volume, using a series of three magnetic horns. The whole target is inserted into the first magnetic horn, as depicted in Figure 3.11. Each horn consists of two coaxial conductors made from an aluminium-alloy (6061-T6). The magnetic field between the two conductors is $\propto \frac{1}{r}$, where $r$ is the distance from the horn axis, and can reach values as high as $1.7 \mathrm{~T}$ when operated with horn currents of $\pm 250 \mathrm{kA}$. The neutrino flux at the far detector is increased by $\sim 17$ times when $+250 \mathrm{kA}$ horn focusing is used compared to the case without focusing, as demonstrated in Figure 3.11. $\pi^{+}$decays are the main channel contributing to neutrino production via $\pi^{+} \rightarrow \mu^{+}+\nu_{\mu}^{4}$. Similarly, anti-neutrinos dominantly come from decays of $\pi^{-} \rightarrow \mu^{-}+\bar{\nu}_{\mu}$. Kaon

\footnotetext{
${ }^{4} 99.9 \%$ branching ratio for $\pi^{+} \rightarrow \mu^{+}+\nu_{\mu}$, rather than $\pi^{+} \rightarrow e^{+}+\nu_{e}$, due to helicity supression.
} 
decays dominate the production of higher-energy neutrinos in the tail of the T2K flux. The wrong flavour contamination in the beam, such as $\nu_{e}$ in a beam composed of predominantly $\nu_{\mu}$, comes from in-flight muon decays within the decay volume $\left(\mu^{+} \rightarrow e^{+}+\bar{\nu}_{\mu}+\nu_{e}\right)$. The first and second horns are used to bend the charged pions and kaons, and the third horn achieves additional focusing. Reversing the horn current polarity from $+250 \mathrm{kA}$ to $-250 \mathrm{kA}$ switches the focusing from positively to negatively charged hadrons, which produces an anti-neutrino enhanced beam via in-flight hadron decays in the decay volume. Thus, the neutrino and anti-neutrino modes of beam operation are also referred to as the forward horn current (FHC, $+250 \mathrm{kA}$ ) and reverse horn current (RHC, -250 kA) modes. Precise measurements of horn current also constitute essential inputs for running the T2K flux simulation. The electrical horn current during data-taking is monitored with Rogowski coils[70], and average horn current readings are provided in Table 3.4.
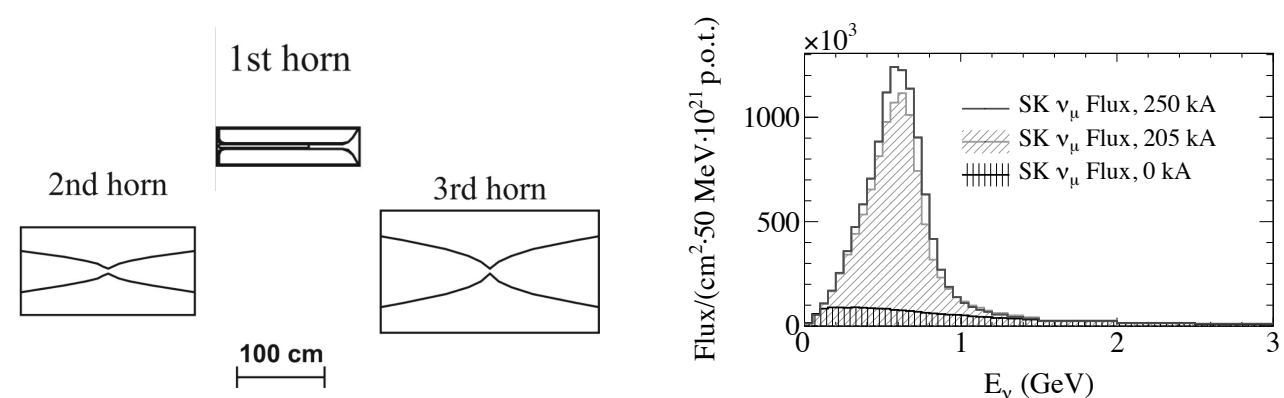

Figure 3.11: Design of the magnetic horns, and the orientation of the target with respect to the first horn, is shown on the left side. The effect of horn current focusing on the neutrino flux observed at Super-K is shown on the right side. Reprinted figure on the left from Nuclear Instruments and Methods in Physics Research Section A: Accelerators, Spectrometers, Detectors and Associated Equipment, Volume 690, A.K. Ichikawa, "Design concept of the magnetic horn system for the T2K neutrino beam", pp. 27-33, Copyright (2012), with permission from Elsevier[71]. Reprinted figure on the right with permission from K. Abe et al. (T2K Collaboration), Phys. Rev. D, vol. 87, p. 012001 (2013)[72]. Copyright (2013) by the American Physical Society.

All the surviving hadrons at the end of the decay volume, and muons of momentum less than $5 \mathrm{GeV} / \mathrm{c}$, are stopped by a beam dump. The dump is made of a graphite core sandwiched on either side with multiple iron plates. It is water cooled and the core temperature is kept stable at $150^{\circ} \mathrm{C}$ for $750 \mathrm{~kW}$ beam operation. The surviving muon flux is correlated with the neutrino flux, 
Table 3.4: Average horn current for each $\mathrm{T} 2 \mathrm{~K}$ run period. The uncertainty on current measurements is $\pm 5 \mathrm{kA}$. Neutrino data is taken in FHC mode with horn currents of roughly $+250 \mathrm{kA}$ (Run $3 \mathrm{~b}$ is taken with weaker $+205 \mathrm{kA}$ focusing). Anti-neutrino data is taken in RHC mode with current values around $-250 \mathrm{kA}$.

\begin{tabular}{lc}
\hline \hline Period & Horn Current [kA] \\
\hline Run 1 +2 & 249.67 \\
Run 3b & 204.7 \\
Run 3c & 248.8 \\
Run 4 & 250.3 \\
Run 5a $+5 \mathrm{~b}$ & 251.6 \\
Run 5c & -251.1 \\
Run 6a & 251.5 \\
Run 6b & -250.0 \\
Run 6c & -251.1 \\
Run 6d & -250.4 \\
Run 6e & -248.8 \\
Run 6f & 249.4 \\
Run 7a & 250.8 \\
Run 7b & -248.1 \\
Run 7c & 249.0 \\
Run 8 & 248.07 \\
Run 9a & 250.47 \\
Run 9b $+9 \mathrm{c}$ & -249.73 \\
Run 9d & -246.96 \\
\hline \hline
\end{tabular}

and can be measured by the muon monitor (MUMON), placed after the beam dump. In T2K, MUMON is used for monitoring the direction and intensity of the neutrino beam, which is then compared to data taken by the INGRID on-axis detector as a consistency check. The precision of the MUMON measurement of neutrino beam direction is within $0.3 \operatorname{mrad}[65]$, on a bunch by bunch basis. A rendering of MUMON is given in Figure 3.12. Two different detection arrays are employed for redundancy: a 7x7 array of silicon-based PIN photodiodes, and an array of 7 ionization chambers (aluminium filled gas tubes) instrumented with 7 sensors each. The material of the ionization chambers is radiation tolerant, whereas the photodiodes are more sensitive and need to be replaced at regular intervals ${ }^{5}$. However, the diodes provide a larger signal response than the ionization chambers

\footnotetext{
${ }^{5}$ The photodiode depletion voltage falls by $50 \%$ after a one month exposure in the T2K muon beam.
} 
at low beam intensities, such as at the start of T2K data-taking.

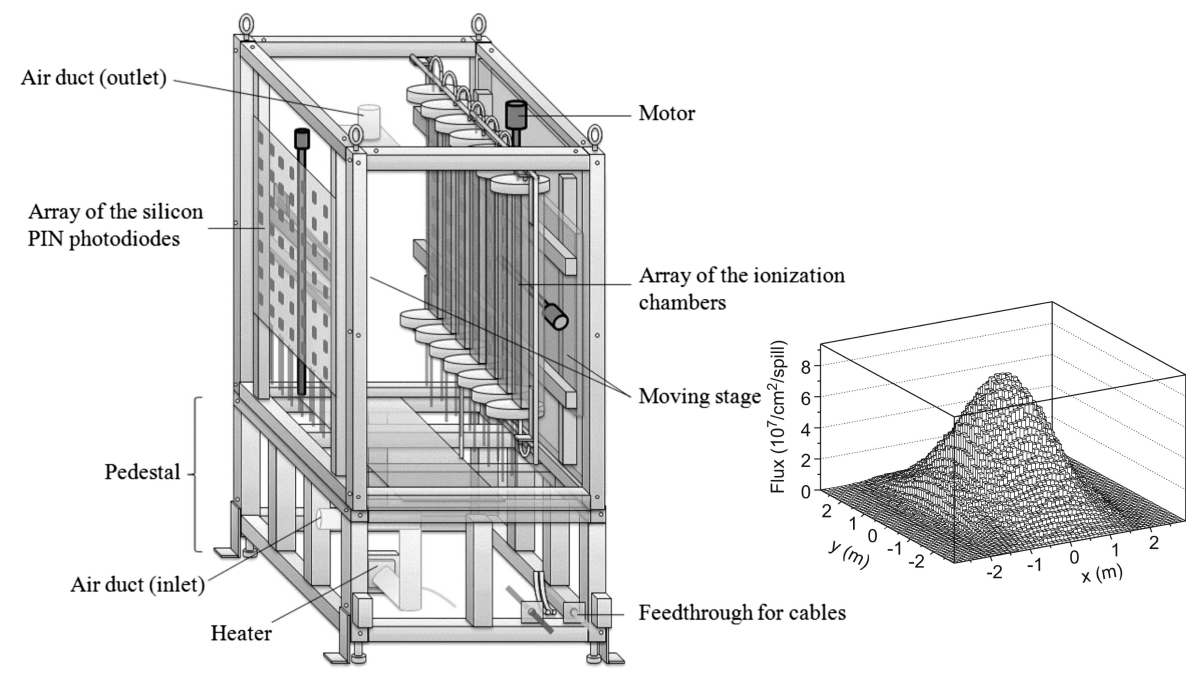

Figure 3.12: A rendering of the muon monitor is shown on the left. The beam direction with respect to the detector has been labelled. An example of the simulated muon profile at the muon monitor is shown on the right. A shift in the muon beam direction by $1 \mathrm{mrad}$ corresponds to a $2 \%$ shift in peak muon energy. Reprinted from Nuclear Instruments and Methods in Physics Research Section A: Accelerators, Spectrometers, Detectors and Associated Equipment, Volume 624, K. Matsuoka et al., "Design and performance of the muon monitor for the T2K neutrinooscillation experiment", pp. 591-600, Copyright (2010), with permission from Elsevier[73].

\subsection{The near detector complex}

T2K uses two near detectors housed in the same complex within the J-PARC site, 280 metres away from the neutrino production point, as depicted in Figure 3.13. ND280 is placed at the same off-axis angle with respect to the neutrino beam as SK. Its components are within a magnetic field, and the detector has good overall particle identification. INGRID is placed on the beam axis. It provides precise measurements of the ND280 off-axis angle for each T2K run. Besides data for oscillation measurements, both ND280 and INGRID also collect data for T2K's rich programme of neutrino cross section measurements.

\subsubsection{The ND280 detector}

The ND280 detector is a fully magnetised tracking detector placed at $2.5^{\circ}$ off-axis with respect to the neutrino beam. The ND280 magnet was refurbished from the 


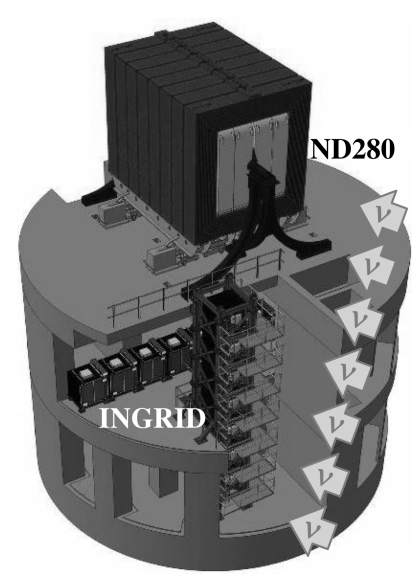

Figure 3.13: A rendering of the whole near detector complex, placed in a two level underground cavern, at a depth of 30 metres. The off-axis ND280 detector is located on the upper level of the cavern. The horizontal components of the on-axis INGRID detector are on the lower level, and the vertical components span both levels. Copyright by the T2K Collaboration.

decommissioned UA1[74] and NOMAD[75] experiments, and provides a dipole field of 0.2 tesla. ND280 has a modular design, with components specifically designed to optimise the detection of different particle types. A rendering of the ND280 detector is shown in Figure 3.14.

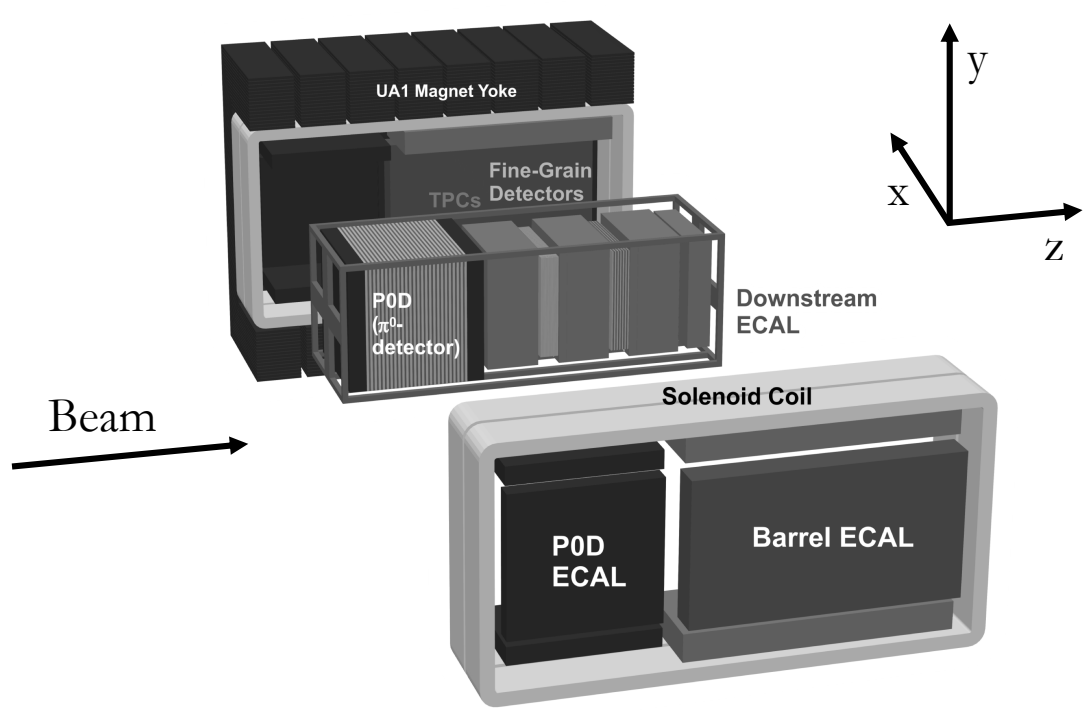

Figure 3.14: An exploded view of the ND280 near detector. The orientation of the detector modules with respect to the beam direction has been indicated. Reprinted from Nuclear Instruments and Methods in Physics Research A, Volume 686, S. Assylbekov et al., "The T2K ND280 off-axis pi-zero detector", pp. 48-63, Copyright (2012), with permission from Elsevier[76]. 
The most upstream component is the $\pi^{0}$ detector (PØD)[76], optimised for the detection of $\pi^{0}$ from neutral-current neutrino interactions ${ }^{6}\left(\nu+\mathrm{N} \rightarrow \nu+\pi^{0}+\mathrm{X}\right)$. $\mathrm{P} \varnothing \mathrm{D}$ is built of scintillator layers interspersed with brass and water layers acting as the target for neutrino interactions. Downstream from PØD is the tracking module, which consists of alternating layers of time projection chambers (TPCs)[77] and fine grained detectors (FGDs)[78].

The TPCs are argon based gas ionization chambers, with excellent 3D track imaging and capable of particle identification through combined measurements of particle momentum and specific energy loss (see Fig. 3.15 on the right). A rendering of the ND280 TPC is shown in Figure 3.15. Charged particles traversing the TPCs ionize the gas. The resulting electrons then drift in a uniform electric field towards readout planes finely instrumented with micromegas detectors. Electrons are first multiplied in an avalanche process before generating a signal on the anode pads of the micromegas[79]. The number of detected electrons is directly proportional to the deposited energy, and the detection time is correlated with the travelled distance. In this way, the trajectory and deposited energy profile of the ionizing particle can be reconstructed. The momentum is measured from the track bending in the magnetic field. For minimum ionizing particles, the resolution on energy loss is $7.8 \%$.

Two FGDs are sandwiched between three TPCs. The target mass for neutrino interactions is provided by the finely segmented bars of polystyrene scintillator, which make up the FGD1 and FGD2 detectors. Alternating layers of scintillator bars are oriented along the mutually orthogonal $x$ and $y$ directions, defined in Figure 3.14 as perpendicular to the beam direction. Because of transverse bar orientations, forward-going particles, i.e. those particles with a significant momentum component along the beam direction, traverse a greater number of scintillator planes than particles emitted at high angles with respect to the beam, resulting in much better track resolution. Every scintillator layer is made up of 192 scintillator bars. The FGD2 detector has the additional feature of water bags placed between scintillator layers, thus providing valuable measurements on the same neutrino target as SK.

\footnotetext{
${ }^{6}$ Main background for $\nu_{e}$ appearance at SK.
} 

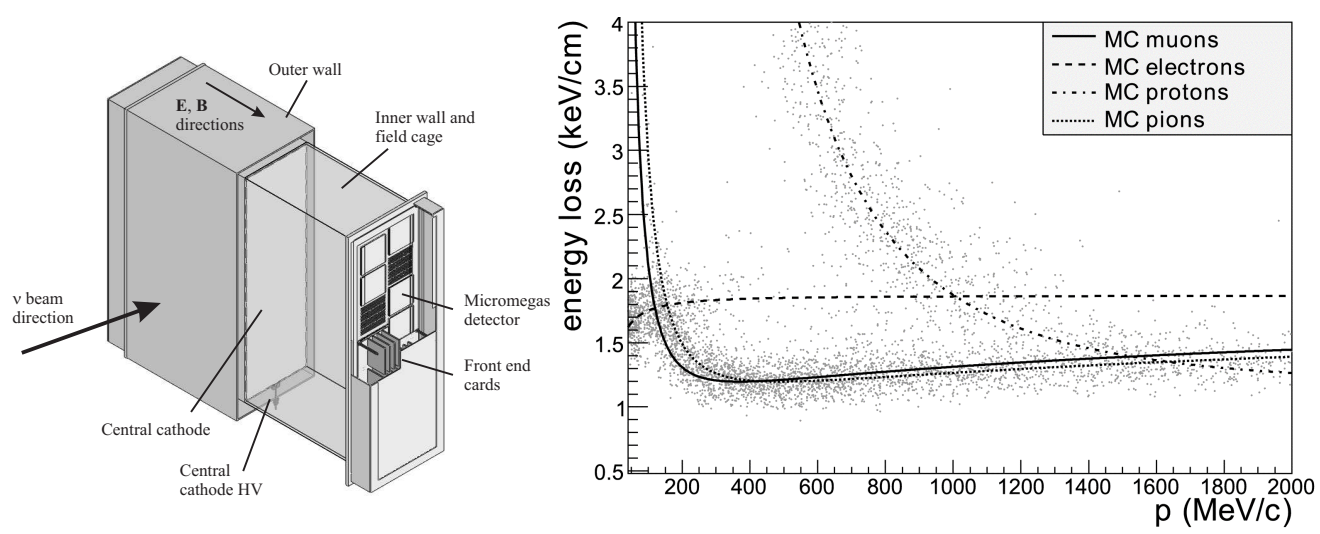

Figure 3.15: A simplified view of the time projection chamber is shown on the left. The space between the outer and inner wall of the TPC is filled with $\mathrm{CO}_{2}$ for insulation. The micromegas pad segmentation on the anode electron collection plane is $7.0 \mathrm{~mm} \times 9.8 \mathrm{~mm}$. The distribution of energy loss in the TPC as a function of momentum is shown on the right, for positively charged particles. Reprinted from Nuclear Instruments and Methods in Physics Research Section A: Accelerators, Spectrometers, Detectors and Associated Equipment, Volume 637, N. Abgrall et al., "Time projection chambers for the T2K near detectors", pp. 25-46, Copyright (2011), with permission from Elsevier[77].

Both the PØD and the TPC and FGD based tracking modules are hermetically enclosed within a barrel-shaped electromagnetic calorimeter (ECal)[80], for the detection of the energy deposited in ND280 in the form of electromagnetic showers. The ECal is comprised of layers of plastic scintillator bars, placed between sheets of lead absorbent, and fully enclosed within the UA1 magnet yoke. The yoke has been instrumented with scintillators for the detection of far ranging muons exiting from the detector. Besides acting as the side muon range detector (SMRD)[81], the yoke provides the magnetic field necessary to determine the particle charge and momentum via the bending of the tracks in the TPCs.

All scintillator based ND280 components, such as the FGD and ECal modules, use the same detection principle. The scintillator bars are typically instrumented with wavelength shifting fibres, going through the centre of each bar. Charged particles passing through the scintillator excite the electrons in the surrounding material, which emit light as they get deexcited back to lower energy levels. This light is then carried via the wavelength shifting optical fibres ${ }^{7}$ to multi-pixel photon

\footnotetext{
${ }^{7}$ The light is first absorbed and then reemitted with a wavelength shifted from the fibre's absorption range.
} 
counters (MPPCs), placed on either both ends or one end of the bar (a reflective coating is used on the other bar end if using only one MPPC). By employing alternating bar layers, oriented perpendicularly to each other, the particle trajectory can be reconstructed by combining measurements from adjacent scintillator layers. The amount of light deposited in each bar can be related to the particle energy.

An example of an ND280 event display is shown in Figure 3.16. Timing information is used to match track segments measured in different ND280 modules. The event display shown here corresponds to an energetic muon entering the ND280 detector, interacting at the boundary between FGD2 and TPC3, and producing various secondary particles which get stopped in the ECal modules.

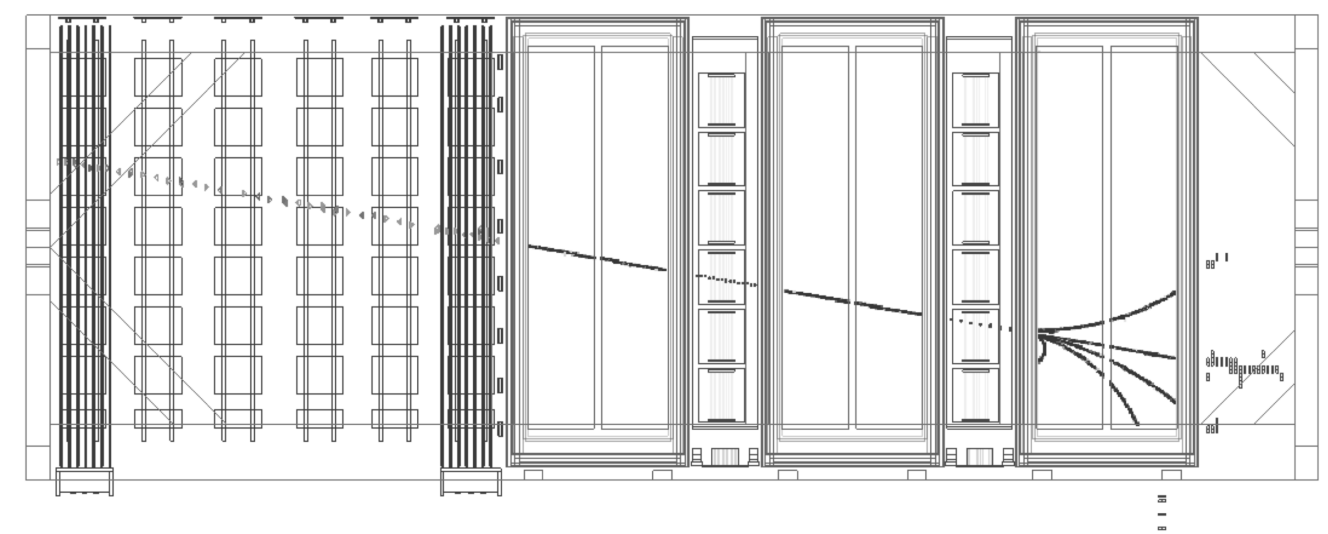

Figure 3.16: A figure showcasing a real ND280 event display. Reprinted from Nuclear Instruments and Methods in Physics Research Section A: Accelerators, Spectrometers, Detectors and Associated Equipment, Volume 659, K. Abe et al. (T2K Collaboration), "The T2K experiment", pp. 106-135, Copyright (2011), with permission from Elsevier[63].

\subsubsection{The INGRID detector}

INGRID (Interactive Neutrino GRID)[82] is the on-axis near detector, responsible for precise measurements of the direction and intensity of the neutrino beam. It consists of 16 identical modules, arranged into a characteristic $10 \mathrm{~m} \times 10 \mathrm{~m}$ cross shaped structure as shown in Figure 3.17. The INGRID modules are made of alternating layers of overlapping $x$ - and $y$-oriented scintillator bars and plates of iron. There are 9 iron plates and 11 scintillator plates in each module (no iron 
plate between the $10^{\text {th }}$ and $11^{\text {th }}$ scintillator modules). The scintillator is used for tracking the charged products of neutrino interactions on iron. The iron target mass for neutrino interactions is 7.1 tonnes per module.

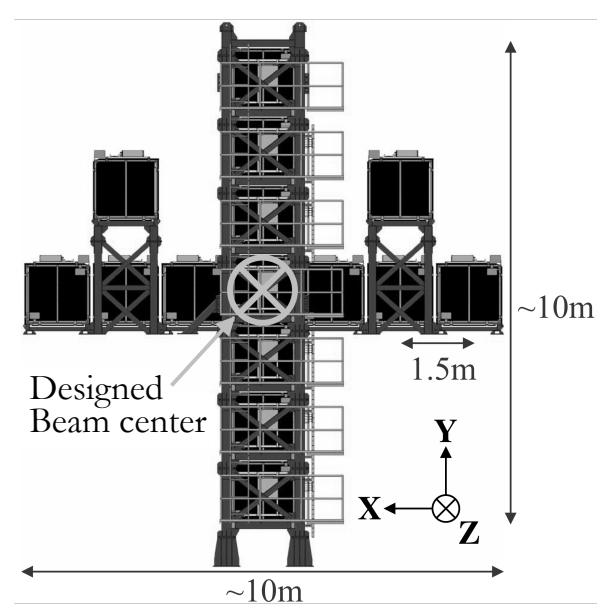

(a) Beam facing view

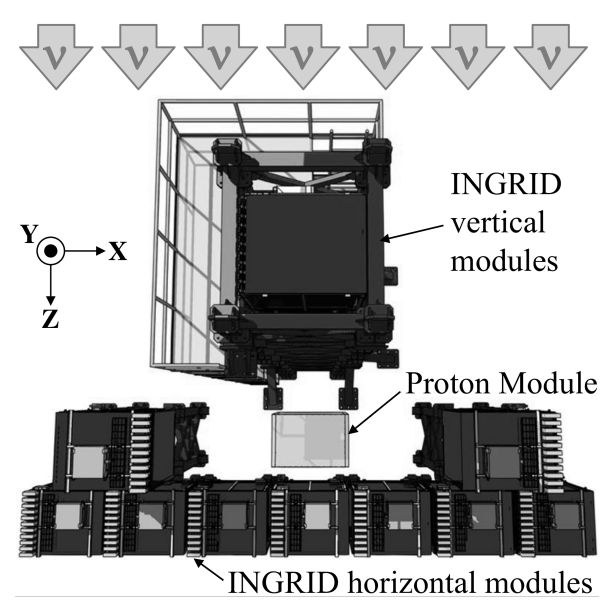

(b) View from the top

Figure 3.17: Rendering of the INGRID on-axis near detector. Copyright by the T2K Collaboration.

The on-axis T2K beam provides sufficient statistics for extracting daily INGRID measurements of beam intensity. The daily event rates for Runs 1-3, for neutrino event candidates with the fourteen cross-forming INGRID modules are shown in Figure 3.18. The beam profile is measured on a monthly basis. One such measurement is shown in Figure 3.19. The beam centre is calculated by fitting a gaussian with the least-squares method to the measured INGRID beam profile. The mean and standard deviation of the fitted gaussian are taken as the beam centre and width, respectively. The beam direction is then taken to be pointing from the T2K graphite target to the measured beam centre at INGRID.

The INGRID measurements of beam direction for T2K Runs 1-9, used in the oscillation analyses presented in this paper, are given in Table 3.5. The beam centre is kept stable within $\pm 0.3 \mathrm{mrad}$ with respect to the designed beam axis during most of $\mathrm{T} 2 \mathrm{~K}$ operation. 


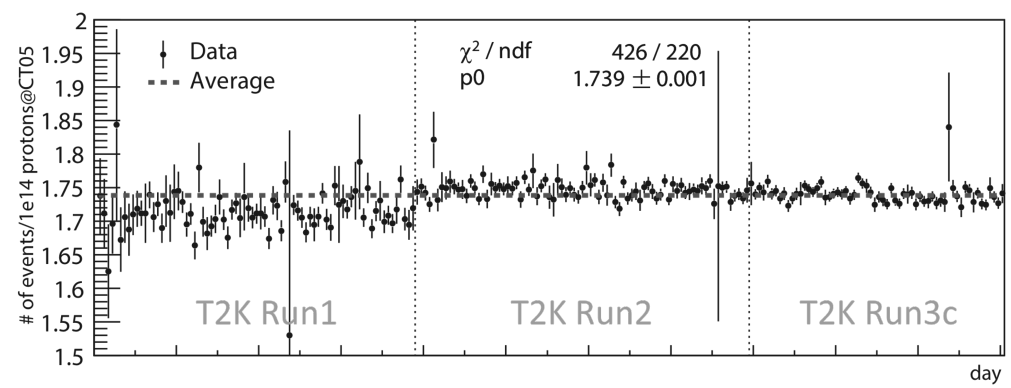

Figure 3.18: Daily event rate measurements at INGRID for T2K physics Runs 1-3[83]. Copyright by the T2K Collaboration.
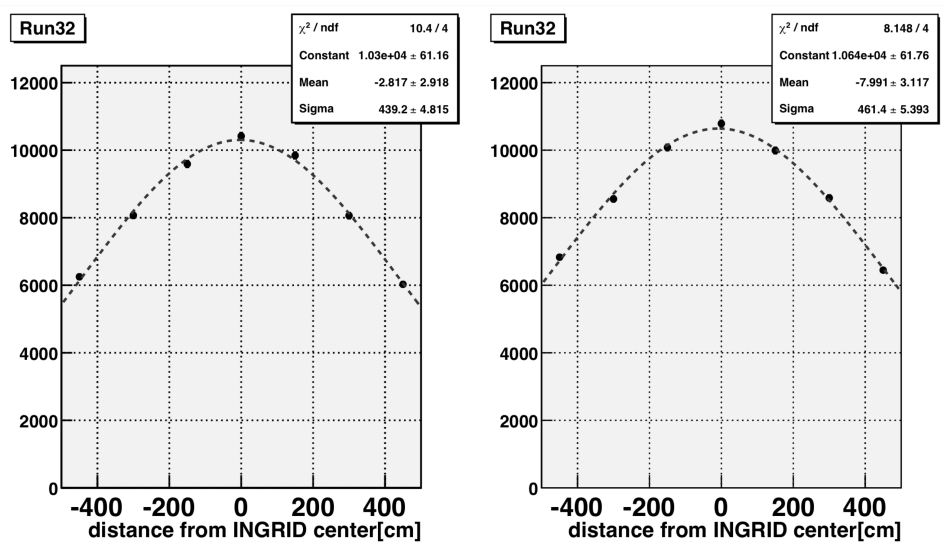

Figure 3.19: Beam profile measurement at INGRID for J-PARC Main Ring Run 32 (14 April - 1 May 2010). The horizontal profile measured by the seven horizontally arranged modules is shown on the left. The vertical profile is shown on the right. The y-axis shows the cumulative number of event candidates detected by each of the INGRID modules over the above-mentioned period[84]. Copyright by the T2K Collaboration.

\subsection{The Super-Kamiokande detector}

Super-Kamiokande (SK)[86], depicted in Figure 3.20, is a large water Cherenkov detector, comprised of a cylindrical tank filled with 50,000 tonnes of ultrapure water. The tank is made of welded stainless steel, 39 metres in diameter and 42 metres in height. It is located roughly 1,000 metres under the peak of Mt. Ikenoyama $(2,700$ metres water equivalent), in Gifu prefecture, Japan. The rock overburden above SK was chosen to reduce the cosmic ray muon flux. For reference, only muons with energy $\sim 1 \mathrm{TeV}$ can penetrate 2700 m.w.e.[87] Inside the cylindrical SK tank is a 55 cm wide stainless steel frame, placed $\sim 2.5$ metres from the tank walls. The steel 
Table 3.5: Summary of beam direction measurements by INGRID. Each measurement is given as (centre value \pm statistical error \pm systematic error)[85].

\begin{tabular}{l|c|c}
\hline \hline Period & Horizontal centre [mrad] & Vertical centre [mrad] \\
\hline \multicolumn{3}{c}{ Neutrino Mode } \\
\hline Run 1 & $0.138 \pm 0.049 \pm 0.094$ & $-0.250 \pm 0.052 \pm 0.105$ \\
Run 2 & $0.094 \pm 0.026 \pm 0.095$ & $-0.041 \pm 0.028 \pm 0.104$ \\
Run 3b & $0.048 \pm 0.097 \pm 0.112$ & $-0.092 \pm 0.110 \pm 0.134$ \\
Run 3c & $0.131 \pm 0.023 \pm 0.094$ & $0.177 \pm 0.025 \pm 0.105$ \\
Run 4 & $0.122 \pm 0.015 \pm 0.094$ & $0.014 \pm 0.016 \pm 0.104$ \\
Run 5 & $0.150 \pm 0.036 \pm 0.096$ & $0.029 \pm 0.058 \pm 0.104$ \\
Run 6 & $-0.018 \pm 0.057 \pm 0.093$ & $0.141 \pm 0.065 \pm 0.141$ \\
Run 7 & $-0.004 \pm 0.038 \pm 0.095$ & $0.162 \pm 0.042 \pm 0.105$ \\
Run 8 & $-0.037 \pm 0.010 \pm 0.095$ & $0.196 \pm 0.011 \pm 0.106$ \\
Run 9 & $0.019 \pm 0.059 \pm 0.093$ & $-0.015 \pm 0.065 \pm 0.107$ \\
\hline \multicolumn{3}{c}{ Anti-neutrino mode } \\
\hline Run 5 & $0.015 \pm 0.066 \pm 0.118$ & $0.185 \pm 0.077 \pm 0.141$ \\
Run 6 & $-0.016 \pm 0.026 \pm 0.121$ & $0.120 \pm 0.029 \pm 0.139$ \\
Run 7 & $-0.055 \pm 0.018 \pm 0.122$ & $0.147 \pm 0.029 \pm 0.140$ \\
Run 9 & $0.005 \pm 0.017 \pm 0.121$ & $0.022 \pm 0.019 \pm 0.140$ \\
\hline \hline
\end{tabular}

framework is used as a support structure for photo-multiplier tubes (PMTs), used for detecting products of neutrino interactions in water. The area between the steel tank and the PMT frame is referred to as the outer detector, and is used to veto cosmic muon events. The area enclosed by the PMT frame is referred to as the inner detector, and is used for collecting data for physics analyses. The inner and outer detector are optically separated by sheets made up of Tyvek, and white and black polyethylene. The inner wall of the frame is instrumented with 11,129 Hamamatsu $50 \mathrm{~cm}$ diameter PMTs, with an inner detector coverage of roughly 40\%. The outer frame wall includes 1,885 Hamamatsu type R1408 $20 \mathrm{~cm}$ diameter PMTs. The side of optically-separating sheet facing the outer detector has a reflective Tyvek coating, to increase the photon detection capabilities of the outer (veto) PMTs. The outer PMT coverage is increased by installing acrylic wavelength shifting plates $(50 \mathrm{~cm} \times$ $50 \mathrm{~cm}$ ) around the photocathode of each tube. Photomultiplier tubes convert light into a measurable electrical signal. Photons incident on the cathode of the tube are first converted into electrons through the photoelectric effect. Before readout, these 
electrons are multiplied in the dynode stack portion of the tube by $\sim 10^{7}$ times. The charge collected by the PMT is proportional to the energy of the incident photons.

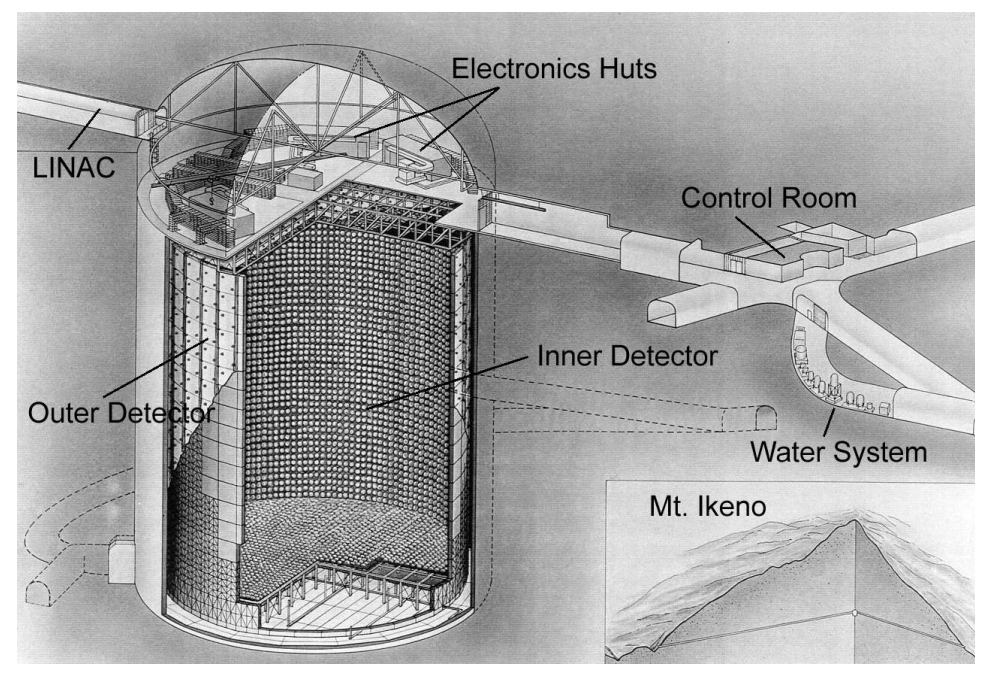

Figure 3.20: Rendering of the SK detector site, and its location with respect to Mt. Ikenoyama. Two horizontal mine shafts lead up to the top of the detector cavern. The locations of the main control room, water purification system and electronics huts are shown. The electron LINAC can be used for testing the SK detector response to electrons of known energy. Reprinted from Nuclear Instruments and Methods in Physics Research Section A: Accelerators, Spectrometers, Detectors and Associated Equipment, Volume 501, S. Fukuda et al. (Super-Kamiokande Collaboration), "The Super-Kamiokande detector", pp. 418-462, Copyright (2003), with permission from Elsevier[86].

The main goal of Super-Kamiokande for the purposes of the T2K oscillation analysis is to measure the flavour and energy of beam neutrinos interacting in the tank. This is done by detecting the Cherenkov radiating charged lepton products of those neutrino interactions. Charged particles emit Cherenkov radiation when moving faster than the phase velocity of light in a dielectric medium, such as water. Cherenkov radiation is emitted in the form of a light cone centred on the particle's direction of propagation. The base angle of the light cone is determined by the particle's speed and the refractive index of the medium, as demonstrated in Figure 3.21. The energy thresholds for the detection of various charged particles produced through neutrino interactions at SK are listed in Table 3.6. Electrons, muons and pions produced in interactions of $\mathrm{T} 2 \mathrm{~K}$ beam neutrinos are typically above the Cherenkov threshold, and thus visible in SK. For the oscillation analysis to work, it is necessary to know the number of Cherenkov rings in every neutrino 
event at Super-K coincident with T2K beam times, in addition to the momentum and direction of charged particles which have generated those rings. Neutrino events from the $\mathrm{T} 2 \mathrm{~K}$ beam are distinguished from atmospheric neutrino induced SK events by the beam's precise timing structure.

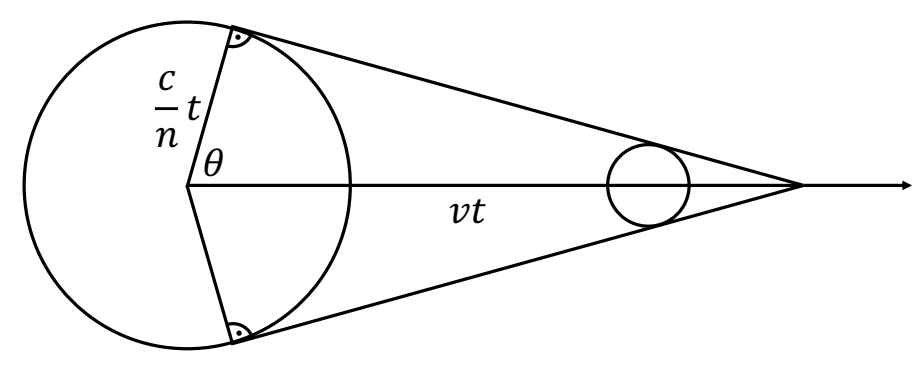

Figure 3.21: Schematic diagram showing the developing wavefront of a conical light wave emitted by a Cherenkov radiating particle. The particle of speed $v$ emits Cherenkov radiation if $v>\frac{c}{n}$, where $c$ is the speed of light, $n$ is the dielectric refractive index ( $n=1.33$ for water). From geometrical considerations, it follows that $\cos \theta=\frac{c}{n v}$. The threshold energy for Cherenkov radiation is given by $E_{\min }=\frac{m c^{2} n}{\sqrt{n^{2}-1}}$.

Table 3.6: Energy thresholds for Cherenkov radiating particles in water.

\begin{tabular}{c|c|c}
\hline \hline Particle & Mass $\left[\mathrm{MeV} / \mathrm{c}^{2}\right]$ & Total energy threshold $[\mathrm{MeV}]$ \\
\hline$e^{ \pm}$ & 0.511 & 0.775 \\
$\mu^{ \pm}$ & 105.6 & 160.7 \\
$\pi^{ \pm}$ & 139.6 & 211.7 \\
$\mathrm{p}$ & 938.3 & 1423.2 \\
$\tau^{ \pm}$ & 1776.8 & 2695 \\
\hline \hline
\end{tabular}

In the Standard Model of particle physics, neutrinos interact with matter via charged and neutral current weak interactions, mediated through the exchange of $W^{ \pm}$and $Z^{0}$ bosons, respectively. Super-Kamiokande, being a Cherenkov detector, is suited for detecting the charged products of those interactions. In charged current interactions, neutrinos convert into the lepton of the same flavour. In neutral current interactions, the incoming neutrino deposits an unknown amount of energy before exiting from the tank. Charged leptons are also produced in neutral current interactions, but with the flavour fully uncorrelated from the through-going beam neutrino. This is why charged current interactions are chosen as the signal for 
the T2K oscillation analysis. The world's combined measurement of the charged current neutrino interaction cross section at a wide range of true neutrino energies is presented in Figure 3.22.
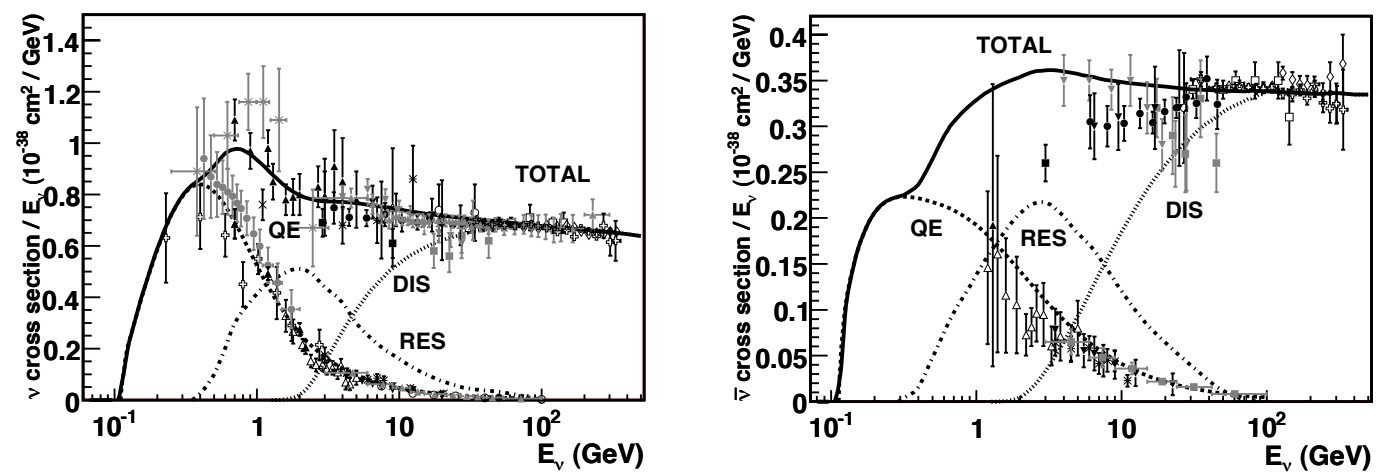

Figure 3.22: The total charged-current cross sections for neutrino (left) and antineutrino (right) interactions, per single nucleon and true neutrino energy. The total cross section prediction from the NUANCE simulation[88] is shown, as well as separate contributions from quasi-elastic scattering (QE, dotted), resonance production (RES, dotted-dashed) and deep inelastic scattering (DIS, dashed). At the peak T2K beam energy of $\sim 600 \mathrm{MeV}$, quasi-elastic scattering is the dominant charged current interaction mode. Different measurements taken on isoscalar targets are represented by markers of different shapes and colours (for more details refer to [89]). It is worth poting out the different scale on the vertical axis for the two figures $\left(\sigma_{\mathrm{CCQE}}^{\nu} \sim 4 \sigma_{\mathrm{CCQE}}^{\bar{\nu}}\right)$. Reprinted figure with permission from Formaggio, J. A. and Zeller, G. P., Rev. Mod. Phys., vol. 84, pp. 1307-1341 (2012)[89]. Copyright (2012) by the American Physical Society.

The dominant $\mathrm{CC} \nu$ interaction mode at the energies of $\mathrm{T} 2 \mathrm{~K}$ beam neutrinos are the charged current quasi-elastic (CCQE) interactions, where neutrinos interact with individual nucleons $n+\nu_{l} \rightarrow p+l^{-}$, where $l \in\{e, \mu, \tau\}$, leaving the nucleus otherwise intact $^{8}$. The proton produced in this way typically escapes the binding potential of the nucleus but is too low in energy to Cherenkov radiate. Other types of $\mathrm{CC} \nu$ interaction modes are through resonance production, most commonly into $\Delta$ baryons, and deep inelastic scattering, where energetic neutrinos probe the quark structure of individual nucleons, producing a multitude of hadrons in the process. On a Feynman diagram level, neutrino interactions might seem deceivingly simple. It is worth pointing out that the direct products of these interactions are often inaccessible to detectors, as they are produced in a nuclear environment. Final state

\footnotetext{
${ }^{8}$ The $\mathrm{T} 2 \mathrm{~K}$ beam energy of $\sim 600 \mathrm{MeV}$ is too low to produce tau leptons in the final state, as demonstrated in table 3.6
} 
interactions (FSI) inside the nucleus can dramatically change the event topology. It is worth pointing out that, for measurements of electron neutrino appearance, intrinsic electron neutrino contamination in the beam constitutes the dominant background.

CCQE interactions are arguably the best suited for probing the physics of neutrino oscillations. Namely, being a class of interactions with only two final state products, the incoming neutrino energy can be reconstructed from the measured energy deposition by the charged lepton. Accurate energy reconstruction is crucial, since it directly affects the neutrino oscillation probability at Super-K. For true CCQE interactions, the incoming neutrino energy can be reconstructed via:

$$
E_{\nu_{l}}^{\mathrm{rec}, \mathrm{CCQE}}=\frac{m_{p}^{2}-\left(m_{n}-V_{b}\right)^{2}+2\left(m_{n}-V_{b}\right) E_{l}+m_{l}^{2}}{2\left(m_{n}-V_{b}-E_{l}+p_{l} \cos \theta_{l}\right)}
$$

where $m_{p}, m_{n}$ and $m_{l}$ are the proton, neutrino and lepton masses, respectively, $\theta_{l}$ is the deflection angle of the lepton's trajectory with respect to the incoming neutrino direction, $p_{l}$ is the lepton momentum and $V_{b}$ is the binding potential that needs to be overcome to free up the neutron. This formula neglects various interactions, and correlations, between nucleons inside the nucleus, which also affect the final state particle kinematics accessible to the detector. Binding energy of $V_{b}=27 \mathrm{MeV}$ is used for interactions on nuclei of ${ }^{16} \mathrm{O}$ at the far detector.

The flavour of beam neutrinos interacting at SK is "measured" by distinguishing between electrons and muons which they produce. The very energetic leptons, and those produced close to the edge of the tank, may leave the detector before their energy drops below the Cherenkov threshold. We refer to these as partially contained events, and they leave filled ring signatures on the PMT instrumented detector walls. Partially contained events do not pass the selection cuts at SK, and are thus excluded from the analysis. Events where the leptons energy drops below the threshold while they are still within the fiducial volume are selected for the analysis and referred to as fully contained events. Muons typically do not scatter much as they propagate the tank, giving rise to sharp Cherenkov rings with clearly defined edges. The lighter electrons scatter more frequently and also produce electromagnetic showers, thus resulting in "fuzzy" Cherenkov rings. For a 
comparison between typical electron and muon Cherenkov ring signatures in SK refer to Figure 3.23. The $\mu$-like or $e$-like property of the Cherenkov ring is inferred with PID cuts with the FiTQun reconstruction algorithm, as demonstrated in Figure 3.24.
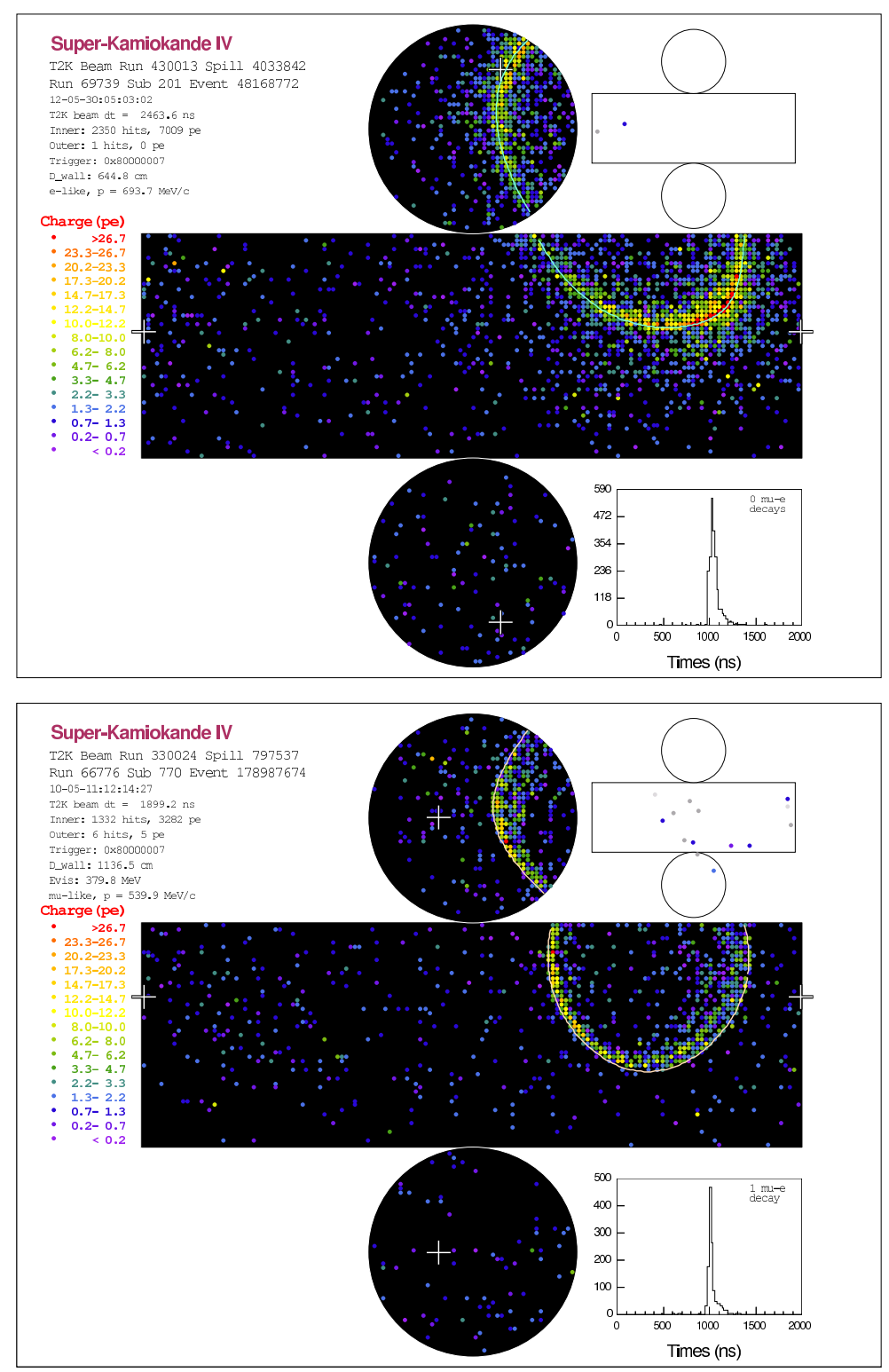

Figure 3.23: $\quad$ SK event displays for real T2K beam-induced events. Event candidates with single electron-like (top) and muon-like (bottom) Cherenkov rings are shown. The charge deposition on the inner (black unrolled map) and outer (white unrolled map) PMT walls is shown, with the colour of the PMT corresponding to the deposited charge. Event displays taken from [90]. Copyright by the T2K Collaboration.

Five signal samples are selected at SK for the oscillation analysis. Selected interactions are specified by the operation mode of the focusing horn current (FHC 


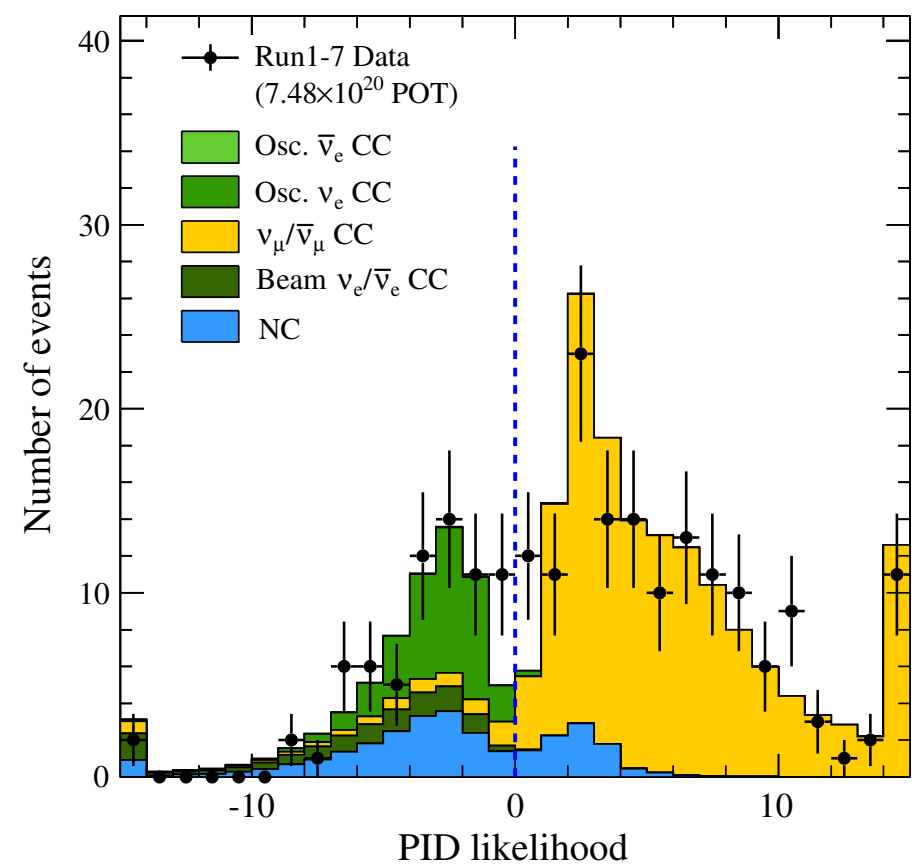

Figure 3.24: The PID parameter distribution used for distinguishing single-ring $\mu$-like $(\mathrm{PID}>0)$ from $e$-like $(\mathrm{PID}<0)$ events, inferred from Monte Carlo studies with the FiTQun reconstruction algorithm. Observed data with statistical error bars is shown alongside stacked histograms corresponding to Monte Carlo predictions. The fiducial volume cut has already been applied. Figure reprinted from Physical Review D, Volume 96, K. Abe et al. (T2K Collaboration), "Measurement of neutrino and antineutrino oscillations by the T2K experiment including a new additional sample of $\nu_{e}$ interactions at the far detector"[91], under the CC BY copyright licence.

or RHC), the particle-like type of the single Cherenkov ring $\left(1 \mathrm{R}_{\mu}\right.$ or $\left.1 \mathrm{R}_{e}\right)$, and if applicable, Cherenkov radiating $e^{+}$from $\pi^{+}$decays. Four of the samples are CCQElike, with a single $\mu$-like or $e$-like Cherenkov ring in FHC and RHC modes: FHC $1 \mathrm{R}_{\mu}, \mathrm{FHC} 1 \mathrm{R}_{e}, \mathrm{RHC} 1 \mathrm{R}_{\mu}$, RHC $1 \mathrm{R}_{e}$. The final sample, FHC $1 \mathrm{R}_{e} \mathrm{CC} 1 \pi^{+}$, selects CC-like $\nu_{e}$ interactions in FHC, with one $\pi^{+}$in the final state detected through the Michel electron. Here, the Michel electron comes from the decay of a muon, which was in turn produced in the decay of the aforementioned $\pi^{+}$. The tree level Feynman diagrams for CCQE and dominant CC RES neutrino interactions targeted by the SK selection cuts are listed in Figure 3.25. There are various backgrounds for each of these samples. For instance, $\mathrm{NC} 1 \pi^{+}$events can be interpreted as $\nu_{\mu} \mathrm{CCQE}$ events if the $\pi^{+}$does not undergo hadronic scattering before dropping below Cherenkov threshold. $\mathrm{NC} 1 \pi^{0}$ events can mimic the $\nu_{e}$ CCQE appearance signal, if one of the 
two photons from the $\pi^{0} \rightarrow 2 \gamma$ decay is backward-going in the $\pi^{0}$ rest frame, and thus boosted to a very low energy in the lab frame ${ }^{9}$. The SK candidate events with one Cherenkov ring in the final state include not just CCQE interactions, but also interactions where additional hadrons (pions) are produced below the Cherenkov threshold. In these cases the reconstructed neutrino energy is underestimated from equation 3.1, which is corrected for in the T2K oscillation analysis based on dedicated Monte Carlo studies. Such CC non-QE events should not be considered as background, since they are sensitive to the neutrino oscillations. They are just signal with a different energy resolution function than the CCQE events.

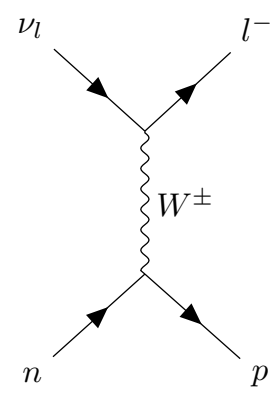

(a) CCQE

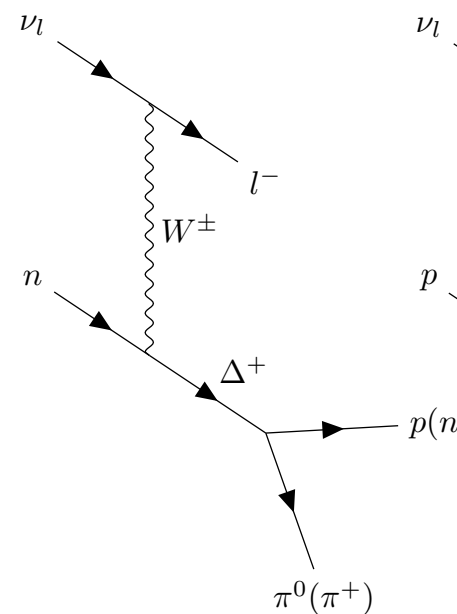

(b) $\Delta^{+}$RES

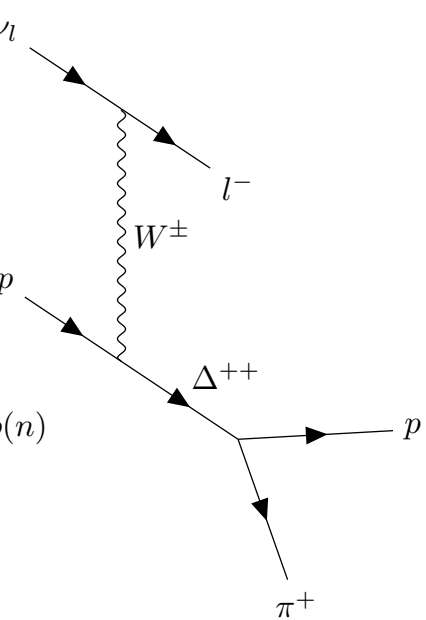

(c) $\Delta^{++}$RES

Figure 3.25: The Feynman diagrams for true CCQE neutrino interactions targeted with the $\mathrm{FHC} 1 \mathrm{R}_{\mu}$ and $\mathrm{FHC} 1 \mathrm{R}_{e}$ selection cuts are shown in (a). The $\mathrm{FHC} 1 \mathrm{R}_{e} \mathrm{CC} 1 \pi^{+}$ signal selection targets CC RES interactions of $\nu_{e}$ with $1 \pi^{+}$in the final state, detected through its decay electron. These could be Feynman interactions like $\nu_{l}+n \rightarrow l^{-}+n+\pi^{+}$ shown in (b), and $\nu_{l}+p \rightarrow l^{-}+p+\pi^{+}$shown in (c). The reconstructed energy formula for the $1 \mathrm{R}_{e} \mathrm{CC} 1 \pi^{+}$sample differs from equation 3.1, to reflect the different final state topology.

Previously, interactions at SK were simply selected if the reconstructed interaction vertex was inside the ID, and more than 2 metres away from the ID walls ( 22,500 tonne fiducial). The latest analysis defines separate fiducial volume cuts for each of the SK candidate samples. The cuts are defined in terms of the closest distance between the ID wall and the interaction vertex (wall), and the distance

${ }^{9}$ Photons at SK can be detected via pair production, if at least one $e^{ \pm}$is Cherenkov radiating. 
from the interaction vertex to the wall along the line of propagation (towall). For more details on the latest fiducial volume cuts refer to [92].

\subsection{T2K data-taking summary}

The oscillation analysis presented in this thesis is based on T2K Run 1-9 data at Super-K, collected from January 2010 to May 2018, with an exposure of $14.938 \times 10^{20}$ protons on target in neutrino mode $(47.7 \%)$, and $16.346 \times 10^{20}$ protons on target in anti-neutrino mode $(52.3 \%)$. The accumulated POT delivered over the years for T2K physics analyses is shown in Figure 3.26. The POT delivered for each physics run, as well as the POT surviving ND280 and SK data quality and selection cuts, and thus used in the analysis presented in this thesis, are shown in Table 3.7. The samples at SK include the full Run 1-9 data, whereas the ND280 samples use only Run 1-6 data. ND280 Run 7-8 samples have not been prepared in time for this result.

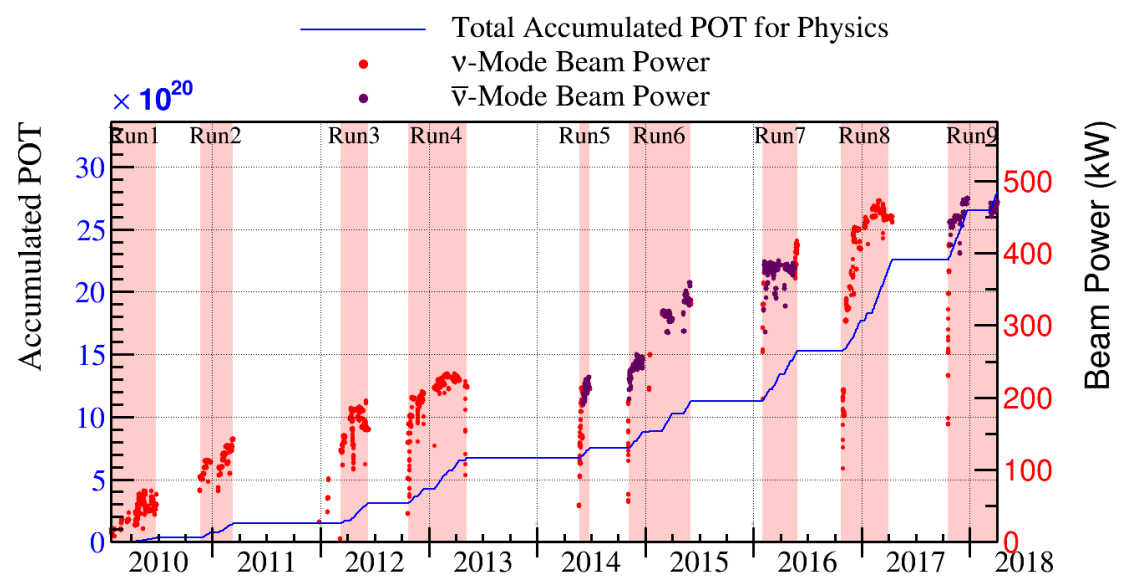

Figure 3.26: The accumulated data for T2K since the first physics run in January 2010, presented in terms of the delivered number of protons on the target (POT). Note that the beam power is also shown, on the vertical axis on the right side of the diagram. Different colours are used to separate data collected in neutrino and anti-neutrino mode. Copyright by the T2K Collaboration. 
Table 3.7: POT delivered to ND280 and SK for the oscillation analysis, as well as the surviving POT after ND280 and SK data quality and selection cuts are applied. All values are listed in terms of $\times 10^{20} \mathrm{POT}$.

\begin{tabular}{l|c|c|c}
\hline Run Period & Delivered & ND280 Cut & SK Cut \\
\hline Run 1 & 0.329 & 0.0 & 0.326 \\
Run 2 & 1.134 & 0.793 & 1.122 \\
Run 3 & 1.608 & 1.581 & 1.599 \\
Run 4 & 3.636 & 3.424 & 3.596 \\
Run 5 & 0.246 & 0.0 & 0.244 \\
Run 5 $\bar{\nu}$ & 0.515 & 0.435 & 0.512 \\
Run 6 & 0.215 & 0.0 & 0.192 \\
Run 6 $\bar{\nu}$ & 3.577 & 3.423 & 3.547 \\
Run 7 & 0.489 & 0.0 & 0.485 \\
Run 7 $\bar{\nu}$ & 3.527 & 2.437 & 3.499 \\
Run 8 & 7.256 & 5.730 & 7.170 \\
Run 9 & 0.205 & - & 0.204 \\
Run 9 $\bar{\nu}$ & 8.887 & - & 8.788 \\
\hline Total $\nu$-mode & 15.118 & 11.528 & 14.938 \\
\hline Total $\bar{\nu}$-mode & 16.505 & 6.295 & 16.346 \\
\hline Total & 31.623 & 17.823 & 31.284 \\
\hline
\end{tabular}




\section{4 \\ The NA61 Experiment}

This chapter introduces the NA61 experiment, focusing on dedicated measurements collected for strengthening the discovery potential of T2K. The work presented in this chapter is not my work, but the product of exceptional work undertaken by the NA61 collaboration. In particular, the NA61 measurement using a replica of the T2K target from 2009 has been analysed by former University of Geneva graduate student Alexis Häsler, and described in more detail in his doctoral thesis[93].

\subsection{NA61/SHINE Detector}

The NA61/SHINE (SPS Heavy Ion and Neutrino physics Experiment)[94] is a fixed target hadron production experiment served by the $\mathrm{H} 2$ beam line of the CERN North Area. The experiment has been proposed in November 2006 and inherited many of its components from NA49[95]. NA61 provides precise hadron production measurements for neutrino experiments with manmade neutrino beams ${ }^{1}$. Dedicated measurements were taken for the T2K and NuMI neutrino beamlines. These "conventional" beamlines produce the neutrino flux by colliding proton beams with radiation-hard targets ${ }^{2}$. The NA61 measurements are essential for better calculations of the neutrino flux in such beamlines.

NA61/SHINE is particularly well suited for measuring the yields of charged particles exiting from any solid target placed into the beam's path, using a combination of time projection chambers (TPCs) and time-of-flight detectors (ToFs). A schematic representation of detector components is shown in Figure 4.1. Two spectrometers are positioned upstream from the NA61 experiment, for triggering

\footnotetext{
${ }^{1}$ The implementation of NA61 measurements in the atmospheric neutrino flux calculation at the location of Super-K is in progress.

${ }^{2} \mathrm{NuMI}$ uses $120 \mathrm{GeV}$ protons incident on a target consisting of $482 \mathrm{~cm}$ thick graphite fins.
} 
on the momentum of the incoming beam. For the measurements presented in this chapter, these spectrometers select particles with momentum of $31 \mathrm{GeV} / \mathrm{c}$, the same as momentum of primary beam protons at T2K. The NA61 beam trigger, $\mathrm{T}_{\text {beam }}$, then selects the desired incoming hadron species, in this case protons, using a series of Cherenkov counters[96], scintillators and beam position detectors (see Figure 4.1). The number of misidentified particles selected in the sample is below $0.8 \%$ [93].

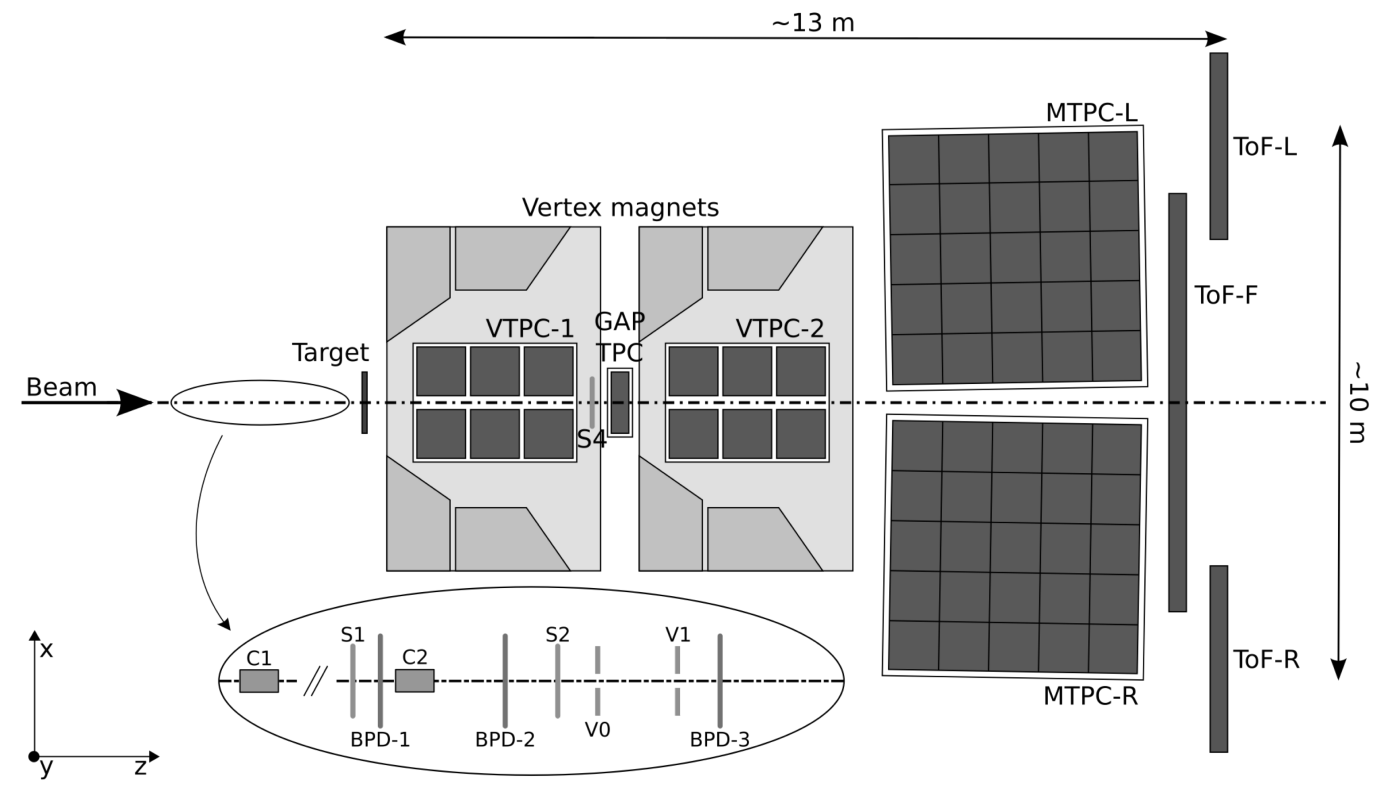

Figure 4.1: A diagram of the NA61 detector[94]. Figure reprinted from Journal of Instrumentation, Volume 9, p. P06005, N Abgrall et al. (NA61/SHINE Collaboration), "NA61/SHINE facility at the CERN SPS: beams and detector system"[94], under the CC BY copyright licence.

The detector comprises of five TPCs. Two vertex TPCs (VTPC-1 and VTPC-2), of dimensions $250 \mathrm{~cm}(\mathrm{~L}) \times 200 \mathrm{~cm}(\mathrm{~W}) \times 98 \mathrm{~cm}(\mathrm{H})$, are placed inside the NA61 superconducting dipole magnets. Between the vertex TPCs is the smaller gap TPC. Finally, the detector also uses the left and right main TPCs (MTPC-L and MTPC-R), placed further downstream from the target, with dimensions of $390 \mathrm{~cm}$ $\times 390 \mathrm{~cm} \times 180 \mathrm{~cm}$. TPCs are filled with an $\mathrm{Ar} / \mathrm{CO}_{2}$ gas mixture and connected to proportional wire chambers for readout. Charged particles traversing the chamber ionize the gas, producing electrons, which, guided by the electric field, drift to the readout plate. The full trajectory of charged particles propagating through the 
detector is reconstructed by combining track segments from all the TPCs. The hadron momentum is measured from the track bending in the vertex TPCs. TPC measurements of specific energy loss $\frac{\mathrm{dE}}{\mathrm{d} x}$ as a function of hadron momentum are shown in Figure 4.2, overlaid with the Bethe-Bloch curves expected for different hadronic species. At low energies, distributions of $\frac{\mathrm{dE}}{\mathrm{d} x}(p)$ can be reliably used for particle identification. However, at higher energies the Bethe-Bloch curves overlap and additional information is needed, provided by time-of-flight detectors.

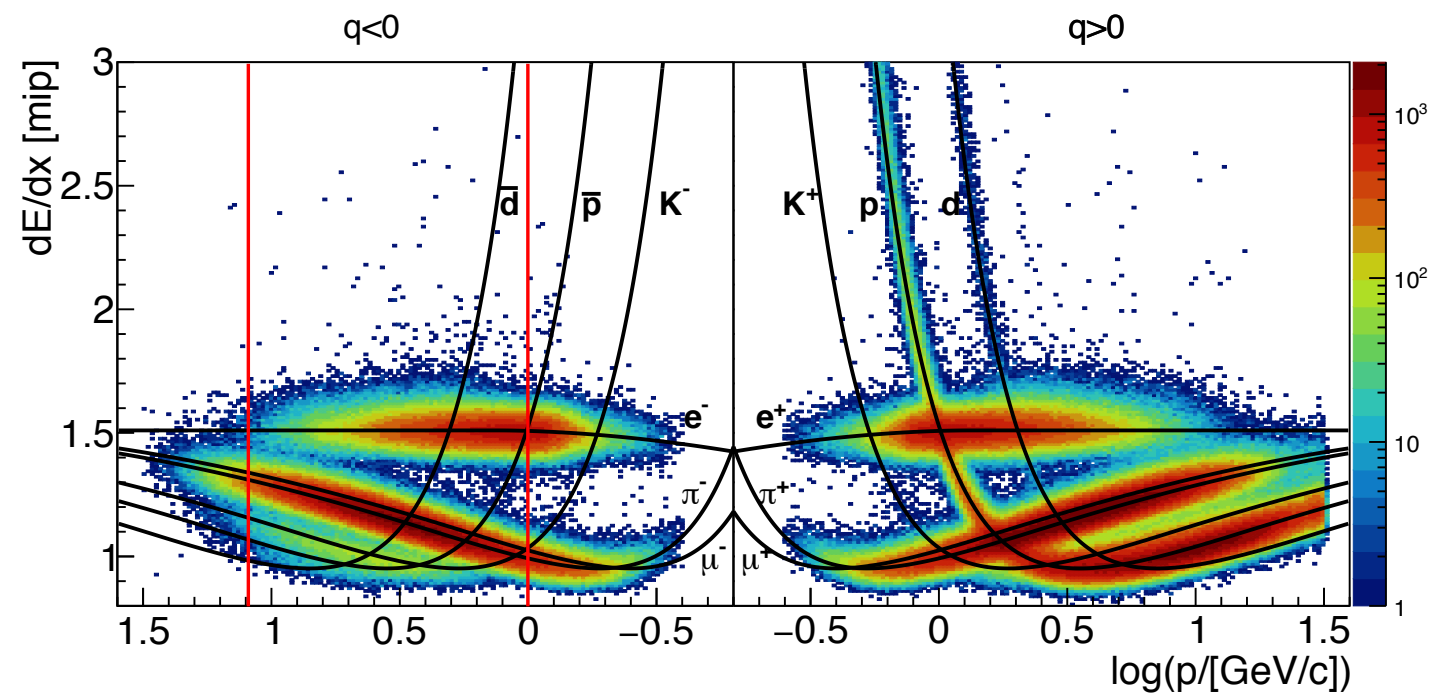

Figure 4.2: Specific energy loss $\frac{\mathrm{dE}}{\mathrm{d} x}$ of negatively (left) and positively (right) hadrons propagating through the NA61 TPCs. $\frac{\mathrm{dE}}{\mathrm{d} x}$ is calculated in units of minimum ionizing particle (mip) energy loss, and given as a function of the logarithm of particle momentum. The Bethe-Bloch curves for the average energy loss of different charged hadron species are overlaid on NA61 data. Figure reprinted from The European Physical Journal C, Volume 79, N Abgrall et al. (NA61/SHINE Collaboration), "Measurements of $\pi^{ \pm}, K^{ \pm}$and proton double differential yields from the surface of the T2K replica target for incoming $31 \mathrm{GeV} / \mathrm{c}$ protons with the NA61/SHINE spectrometer at the CERN SPS"[97], under the CC BY copyright licence.

Three ToF walls are placed downstream from the main TPCs. These are ToF-L, ToF-R and ToF-F, as shown in Figure 4.1. Only ToF-F has sufficient coverage to be used in the NA61 analysis for T2K . ToF-F consists of ten sub-modules, with a total of 80 overlapping scintillator bars. The bars guide the light towards fast Hamamatsu PMTs placed on either end of every bar. The timing resolution of each

\footnotetext{
${ }^{3}$ The geometrical acceptance has a cut-off at low momenta where particles bent by the magnetic field miss the ToF-F
} 
bar is 110 ps [94]. The start timing signal is provided by the scintillator-based S1 counter (see Figure 4.1). By combining the particle time-of-flight measurement $t_{\text {ToF }}$ with track length $l$ and momentum $p$ measurements from the TPCs, the invariant mass squared $m_{\mathrm{ToF}}^{2}$ can be calculated via $m_{\mathrm{ToF}}^{2}=p^{2}\left(\frac{c^{2} t_{\mathrm{ToF}}^{2}}{l^{2}}-1\right)$. The invariant mass squared distribution is shown in Figure 4.3.
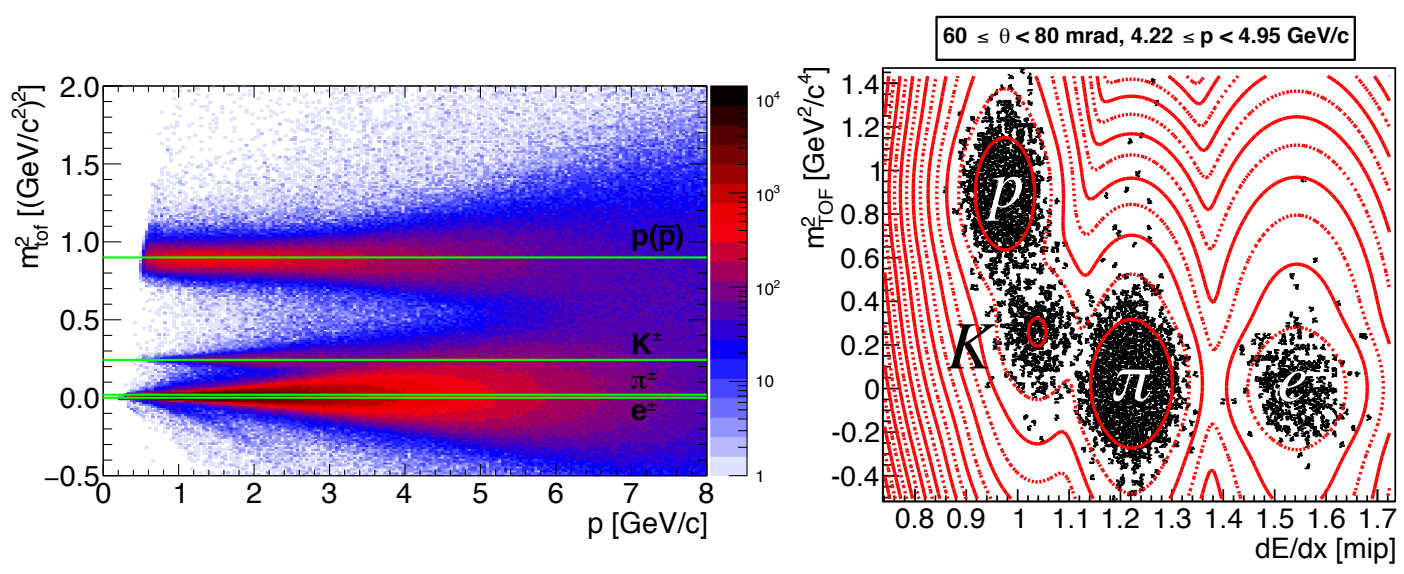

Figure 4.3: Invariant mass squared as a function of particle momentum, calculated from readings taken by NA61 time-of-flight detectors, is shown on the left. Distribution of invariant mass squared as a function of specific energy loss, used for reliable particle identification across a wide range of charged hadron momenta, is shown on the right. Figure reprinted from The European Physical Journal C, Volume 79, N Abgrall et al. (NA61/SHINE Collaboration), "Measurements of $\pi^{ \pm}, K^{ \pm}$and proton double differential yields from the surface of the T2K replica target for incoming $31 \mathrm{GeV} / \mathrm{c}$ protons with the NA61/SHINE spectrometer at the CERN SPS"[97], under the CC BY copyright licence.

Particle identification is achieved by combining measurements of energy loss from the TPCs with the measurements of mass squared reconstructed from ToF-F. Such distributions of $\frac{\mathrm{dE}}{\mathrm{d} x}$ as a function of $m_{\mathrm{ToF}}^{2}$ are shown in Figure 4.3. Complementarity between the two methods for particle identification is vital for covering the wide spread in hadron momentum relevant for $\mathrm{T} 2 \mathrm{~K}$ neutrino production. In the region where Bethe-Bloch energy loss curves overlap (1-3 GeV/c, red lines in Figure 4.2), the ToF-F measurements provide good particle identification: proton separation is good up to $8 \mathrm{GeV} / \mathrm{c}$, whereas kaon from pion separation is reliable up to $3 \mathrm{GeV} / \mathrm{c}$. At higher momenta energy loss measurements become vital for particle identification. 


\subsection{NA61/SHINE measurements for T2K}

A summary of dedicated NA61 measurements for the T2K beamline is given in Table 4.1. NA61 measured hadron yields for T2K with two target configurations, the thin target[98] and the replica target[99] (see Fig. 4.4).

Table 4.1: NA61 measurements for T2K taken with different target configurations.

\begin{tabular}{c|c|c|c|c} 
Beam+Target & $\begin{array}{c}\text { Beam Momentum } \\
\text { GeV } / \mathrm{c}\end{array}$ & Year & $\begin{array}{c}\text { POT } \\
\times 10^{6}\end{array}$ & Hadron Yields \\
\hline p+Thin & 31 & 2007 & 0.7 & $\pi^{ \pm}[100]$ and $K^{+}[101]$ \\
p+Thin & 31 & 2009 & 5.4 & $\pi^{ \pm}, K^{ \pm}, K_{S}^{0}, \Lambda, \mathrm{p}[98]$ \\
p+Replica & 31 & 2007 & 0.2 & $\pi^{ \pm}$ \\
p+Replica & 31 & 2009 & 2.8 & $\pi^{ \pm}[99]$ \\
p+Replica & 31 & 2010 & 10 & $\pi^{ \pm}, K^{ \pm}, \mathrm{p}[97]$
\end{tabular}
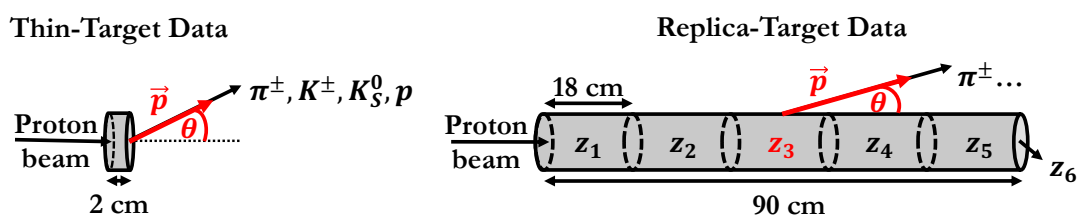

Figure 4.4: The NA61 thin target and replica target configurations used for collecting hadron production measurements for T2K. References to publications accompanying the release of each dataset have been provided.

\subsubsection{Thin-target measurements}

The NA61 thin target configuration uses an isotropic slab of graphite, with dimensions of $2 \mathrm{~cm}(\mathrm{~L}) \times 2.5 \mathrm{~cm}(\mathrm{~W}) \times 2.5 \mathrm{~cm}(\mathrm{H})$. The target is bombarded with a $31 \mathrm{GeV} / \mathrm{c}$ proton beam. At this beam momentum, the target length corresponds to $4 \%$ of the proton interaction length $\left(\lambda_{\mathrm{I}}\right)$. Two separate datasets were collected with this configuration. The pilot run was taken in 2007 , with $0.7 \times 10^{6}$ delivered protons on target. The pilot run had sufficient statistics to measure only the production of charged pions $\pi^{ \pm}$[100], and positively charged kaons $K^{+}$[101]. In 2009, an additional thin target dataset was collected, with $5.4 \times 10^{6}$ protons on target. The production of $\pi^{ \pm}, K^{ \pm}, K_{S}^{0}, \Lambda$ and $p$ was extracted from the 2009 dataset [98]. 
The 2009 NA61 measurements of hadron yields from the thin target have already been implemented in the $\mathrm{T} 2 \mathrm{~K}$ flux calculation framework prior to the commencement of the analysis presented in this thesis.

With the thin target dataset, the released hadronic yields (multiplicities) ${ }^{4}$ $\mathrm{Y}_{\text {thin }}^{\pi^{ \pm}, K^{ \pm}, K_{\mathrm{S}}^{0}, \Lambda, p}(p, \theta)$ are binned by the outgoing hadron momentum $p$ and the angle $\theta$ with respect to the incident beam direction (see Figure 4.4). The measured double differential yield has been calculated with the following formalism in mind:

$$
\mathrm{Y}_{\text {thin }}^{\alpha}(p, \theta)=\frac{d^{2} \mathrm{n}^{\alpha}(p, \theta)}{d p d \theta}=\frac{1}{\sigma_{\text {prod }}} \frac{d^{2} \sigma^{\alpha}(p, \theta)}{d p d \theta}
$$

where $\alpha \in\left\{\pi^{ \pm}, \mathrm{K}^{ \pm}, \mathrm{p}, \mathrm{K}_{\mathrm{S}}^{0}\right\}$ denotes the hadron species, $\sigma_{\text {prod }}$ is the integrated production cross section, which gives rise to production of new hadrons, and $\frac{d^{2} \sigma^{\alpha}}{d p d \theta}$ is the differential inclusive cross section for $\mathrm{p}+\mathrm{C} \rightarrow \alpha+X$ scattering with an $\alpha$ hadron in the final state. For example, measurements of $K^{+}$production from the thin target are given in Figure 4.5.

The production of neutral hadrons $K_{\mathrm{S}}^{0}$ and $\Lambda$ has been measured by selecting their decay channels into pairs of charged hadrons. These are $K_{\mathrm{S}}^{0} \rightarrow \pi^{+}+\pi^{-}$with a branching ratio of $(69.20 \pm 0.05) \%$ and $\Lambda \rightarrow p++\pi^{-}$with a branching ratio of $(63.9 \pm 0.5) \%$. Besides the primary interaction vertex originating at the thin target, topological cuts for selecting these samples required a second reconstructed vertex not more than $0.5 \mathrm{~cm}$ away from the target, with two tracks of opposite charge originating from it. Kinematic cuts required that the invariant mass of the selected pairs of daughter tracks agree with either the $K_{\mathrm{S}}^{0}$ or the $\Lambda$ hypothesis. Cuts on the minimum transverse momentum of daughter tracks ensured that $\gamma \rightarrow e^{+}+e^{-}$were removed. Additional cuts on the decay length, and angle between the incoming neutral hadron and the outgoing charged hadron were applied, for improved $K_{\mathrm{S}}^{0}$ and $\Lambda$ separation.

The likelihood of multiple interactions within the target is negligible, given the $0.04 \lambda_{\mathrm{I}}$ target thickness. Accordingly, this dataset is best suited for understanding the very first interaction of beam protons with graphite nuclei, referred to as the

\footnotetext{
${ }^{4}$ The terms yield and multiplicity will be used interchangeably to describe particle production.
} 

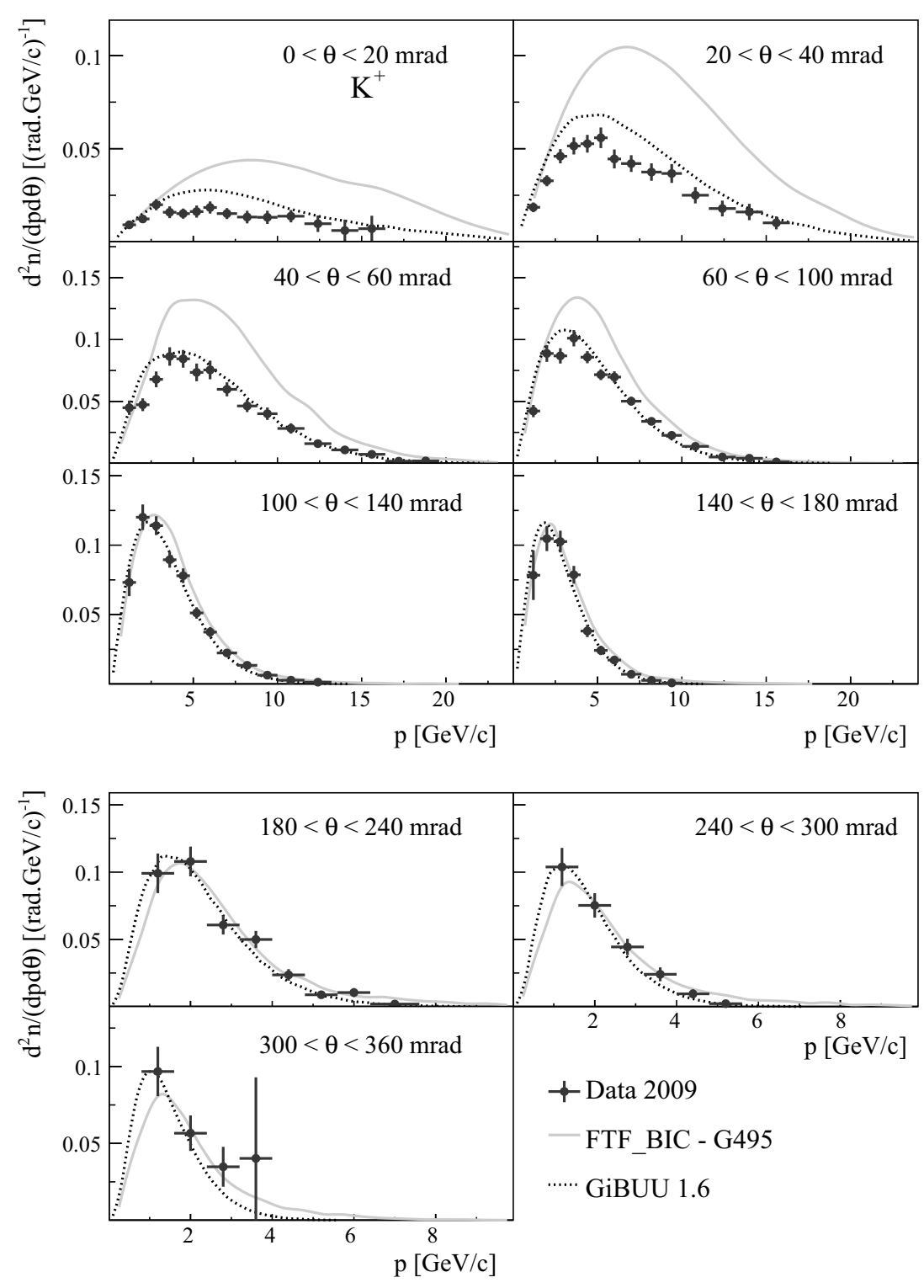

Figure 4.5: The differential $K^{+}$yields as a function of the kaon momentum $p$, and extracted for different ranges of exiting angle $\theta$ with respect to the proton beam direction. The measured particle yield is plotted alongside predictions from GiBUU[102] and the GEANT4 based FTF_BIC[103] generators. The vertical error bars correspond to the total measurement uncertainty. Figure reprinted from The European Physical Journal C, Volume 76, N Abgrall et al. (NA61/SHINE Collaboration), "Measurements of $\pi^{ \pm}$, $K^{ \pm}, K_{S}^{0}, \Lambda$ and proton production in proton-carbon interactions at $31 \mathrm{GeV} / \mathrm{c}$ with the NA61/SHINE spectrometer at the CERN SPS"[98], under the CC BY copyright licence.

primary interaction. Roughly $60 \%$ of $\mathrm{T} 2 \mathrm{~K}$ beam neutrinos come from decays of daughter hadrons from such primary interactions. 


\subsubsection{Replica-target measurements}

Replica-target NA61 measurements have been collected with a replica of the T2K target: a $90 \mathrm{~cm}$ long, rod-shaped piece of graphite, with a circular cross section. The upstream end of the target (length of $2 \mathrm{~cm}$ ) has a $7 \mathrm{~cm}$ diameter, and the rest of the target (length of $88 \mathrm{~cm}$ ) has a diameter of $2.6 \mathrm{~cm}$, as depicted in Figure 4.6. This target widening at the upstream tip was essential for mounting it to the existing NA61 experimental apparatus. The tip is held in place with a disk-shaped aluminium flange, as shown in Figure 4.6. The effect of interactions on the flange has been shown to have a negligible effect for T2K flux considerations ${ }^{5}$.

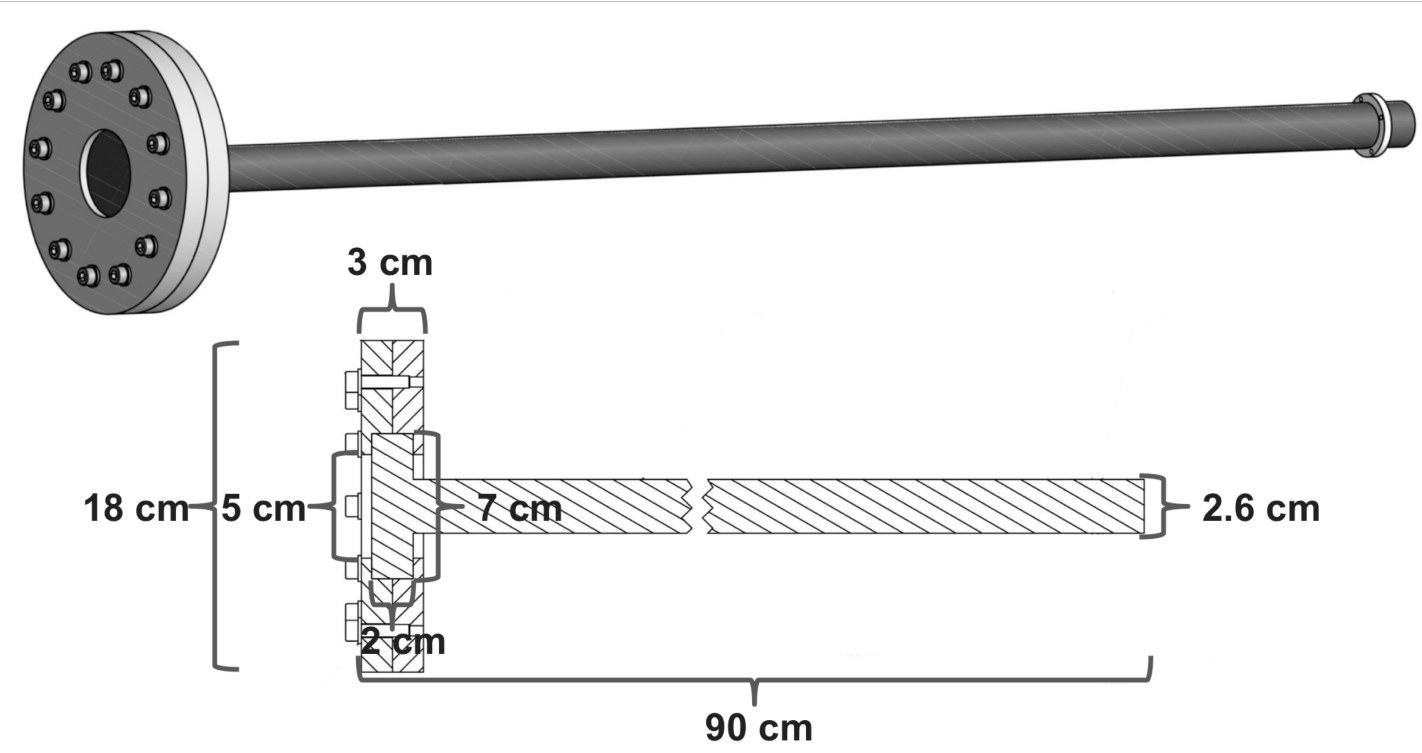

Figure 4.6: Rendering and dimensional drawing of the replica target. The wider section at the upstream end of the target is shown, mounted to the disk-shaped aluminium flange. The replica target on the mechanical drawing has been outlined in red. Figure reprinted from The European Physical Journal C, Volume 79, N Abgrall et al. (NA61/SHINE Collaboration), "Measurements of $\pi^{ \pm}, K^{ \pm}$and proton double differential yields from the surface of the T2K replica target for incoming $31 \mathrm{GeV} / \mathrm{c}$ protons with the NA61/SHINE spectrometer at the CERN SPS"[97], under the CC BY copyright licence.

The run from 2009 was collected with $2.8 \times 10^{6}$ protons on target, providing good statistics for measurements of $\pi^{ \pm}$production[99]. The T2K neutrino flux depends crucially on the longitudinal position of hadron interaction vertices along

\footnotetext{
${ }^{5}$ The real $\mathrm{T} 2 \mathrm{~K}$ target has no flange.
} 
the elongated target, or alternatively, the longitudinal z-position at which hadrons exit from the target. The latter is less model dependent and more readily accessible, since all the tracking components of the NA61 detector are placed downstream with respect to the target. The $\pi^{ \pm}$exiting position is obtained through backwards extrapolation of track parameters measured with the TPCs. Uncertainties on the track parameters are also taken into account. The Runge-Kutta numerical iterative method $[104]$ is used, which incorporates the precise magnetic field map into the backwards track extrapolation. The exiting point is defined as the point where the backwards extrapolated track intersects the volume of the replica target. If there is no intersection between the hadronic track and the target, the distance of closest approach is taken as the exiting position. Out-of-target interactions are efficiently removed by selecting only tracks whose exiting position is either inside the target or within $1 \sigma$ from the target surface ${ }^{6}$. The target surface here is defined as a $90 \mathrm{~cm}$ long cylinder with a uniform radius of $1.3 \mathrm{~cm}$.

The measured yields from the replica target $\mathrm{Y}_{\text {replica }}^{\pi^{ \pm}}(p, \theta, z)$ are binned with respect to the outgoing pion momentum $p$, angle $\theta$, and longitudinal position $z$ at which it exists from the T2K target replica (see Figure 4.4). There are six $z$ bins, five of which divide the $90 \mathrm{~cm}$ long target into $18 \mathrm{~cm}$ thick slabs, and the sixth bin $z_{6}$ corresponds to the downstream face of the replica target. NA61 measures the differential yield, defined via the following equation:

$$
\mathrm{Y}_{\text {replica }}^{\alpha}(p, \theta, z)=\left.\frac{1}{\mathrm{~N}_{\text {P.O.T. }}} \frac{d \mathrm{n}}{d p}\right|_{i, j, k} ^{\alpha}=\frac{1}{\mathrm{~N}_{\text {P.O.T. }}} \frac{\mathrm{N}_{i, j, k}^{\alpha} \mathrm{C}_{i, j, k}}{\Delta p_{i, j, k}}
$$

where $(i, j, k)$ specify the analysis bin numbers containing the $(p, \theta, z)$ point, $\alpha \in$ $\left\{\pi^{+}, \pi^{-}\right\}$denotes the particle species, $\mathrm{N}_{i, j, k}^{\alpha}$ is the reconstructed number of tracks falling into bin $(i, j, k), \mathrm{C}_{i, j, k}$ is the Monte Carlo correction factor and $\Delta p_{i, j, k}$ is the width in momentum space of bin $(i, j, k)$. The yield is normalised to the total number of protons incident on the replica target $\left(\mathrm{N}_{\text {P.о.т. }}\right)$.

An example of NA61 measurements of production from the replica target is shown in Figure 4.7, for the production of $\pi^{+}$from different longitudinal $z$ bins $\left(z_{1}\right.$,

\footnotetext{
${ }^{6} 96.6 \%$ of NA61 tracks pass this cut.
} 
$z_{3}$ and $z_{6}$ overlaid on the same plots) and for different outgoing $\theta$ angle ranges. This pictorial representation of pion yields is intended to communicate certain trends in data. For instance, the very forward going pions are mostly emitted from the downstream face of the replica target, whereas most of the high angle outgoing pions get emitted from the upstream portion of the target. The implementation of these measurements into the $\mathrm{T} 2 \mathrm{~K}$ flux calculation framework was the main goal of the analysis presented in this thesis.
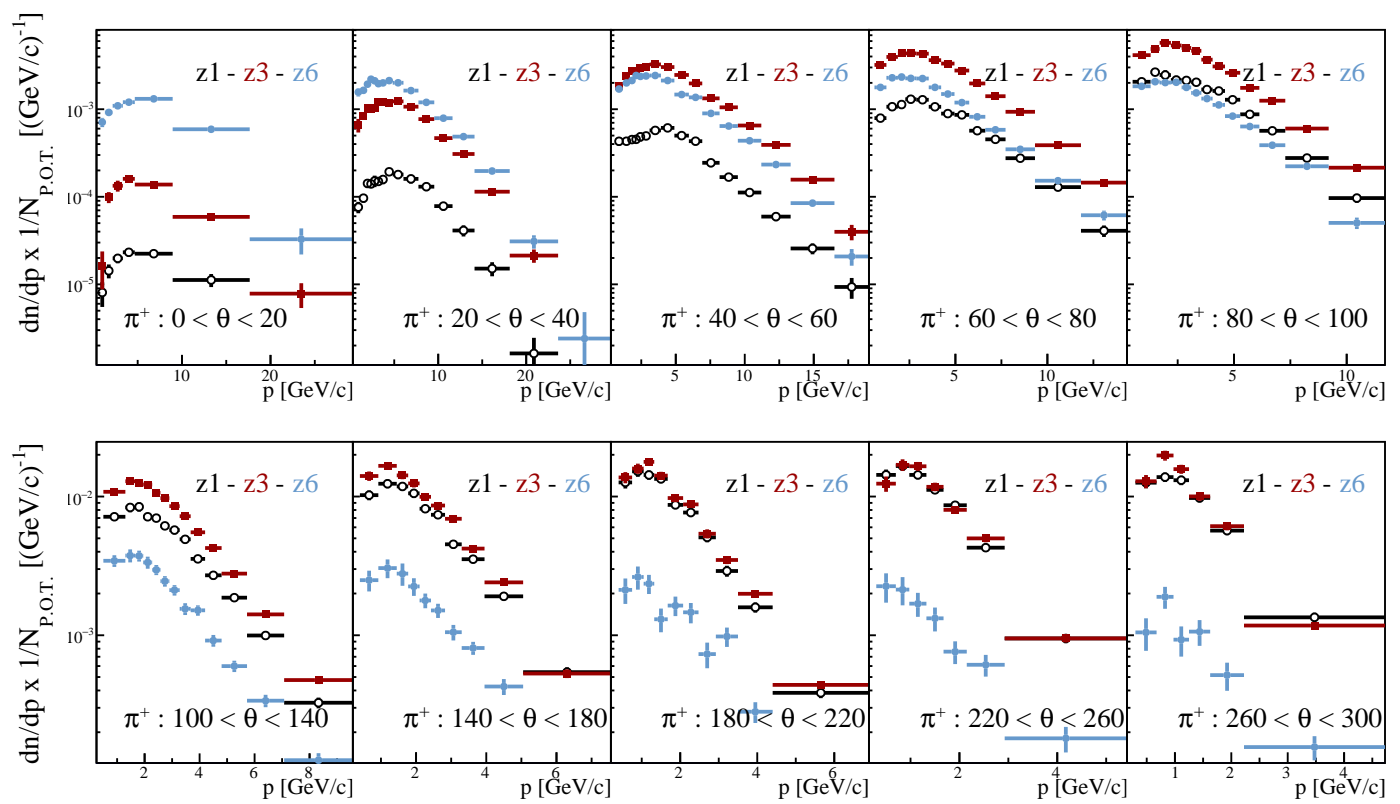

Figure 4.7: The NA61 2009 positive pion yields from three representative positions along the graphite target: the most upstream target portion $\left(z_{1}\right)$, one of the middle target portions $\left(z_{3}\right)$ and the most downstream target portion $\left(z_{6}\right)$. The following binning has been adopted by NA61: $0 \mathrm{~cm}<z_{1}<18.0 \mathrm{~cm}<z_{2}<36.0 \mathrm{~cm}<z_{3}<54.0 \mathrm{~cm}<z_{4}<72.0$ $\mathrm{cm}<z_{5}<89.99 \mathrm{~cm}$ and $z_{6}$ is used to denote the downstream face of the replica target (selected with $89.99 \mathrm{~cm}<z_{6}<90.01 \mathrm{~cm}$ ). The yields are given as a function of pion momentum, and split into different angular ranges, measured with respect to the incident beam direction. The yield is measured per proton on target, and per pion momentum, in units of $(\mathrm{GeV} / \mathrm{c})^{-1}$.

The systematic uncertainties considered for the $2009 \pi^{ \pm}$yields from the replica target are due to particle identification, feed-down corrections, reconstruction efficiency, time-of-flight efficiency, pion loss and backwards extrapolation. An example of NA61 systematic uncertainties for $\pi^{+}$yields, shown as a function of pion momentum, for each $z$-bin and for certain $\theta$ ranges, is given in Figure 4.8 and 
Figure 4.9, for low and high scattering angles, respectively. For a comprehensive summary of NA61 systematic uncertainties for all $(p, \theta, z)$ analysis bins, the reader is referred to the Abgrall et. al publication[99]. Particle identification at higher momenta relies almost exclusively on $\frac{\mathrm{dE}}{\mathrm{d} x}$ measurements from TPCs, described by single gaussians for every particle species and $(p, \theta, z)$ analysis bin. This is not strictly correct, as the width of the energy loss distribution is known to depend on the number of reconstructed track clusters. By fitting a double instead of a single gaussian template to the $\frac{\mathrm{dE}}{\mathrm{d} x}$ distribution, the measured yield changes by as much as $2 \%$ for pion momenta above $10 \mathrm{GeV} / \mathrm{c}$, as reflected in the assigned PID track uncertainty. Feed-down corrections account for pions which are wrongly identified as exiting the target, when in reality they either originate from outof-target interactions, or are the products of hadrons which decayed before they could reach the NA61 tracking modules (e.g. $K^{+} \rightarrow \pi^{+}+\pi^{0}$ and $K_{\mathrm{S}}^{0} \rightarrow \pi^{+}+\pi^{-}$). Such feed-down corrections are inferred from fake data studies with FLUKA, and are highly model dependent. The uncertainty from feed-down corrections can be as high as $5 \%$ for low energy pions but drops substantially at higher momenta. The uncertainty due to track reconstruction efficiency has been estimated by varying the track selection cuts, and by comparing different models for global track reconstruction from individual track segments in different TPCs. A constant 2\% track reconstruction uncertainty has been assigned. The scintillator bars of ToFs are instrumented with two PMTs on either side of the bar. The time-of-flight is taken to be the average time based on the upper and lower PMT readings, with a strict time window cut around the triggered interaction. This cut is applied to remove events with multiple hits in the same scintillator bar, which would be reflected as an apparent broadening of the reconstructed invariant squared mass $m_{\mathrm{ToF}}^{2}$. The ToF efficiency correction has been estimated from data, and a constant $2 \%$ fractional uncertainty was assigned across the complete phase space for this systematic. The pion loss correction accounts for pions which leave tracks in the TPCs but never reach the ToF detector and are thus excluded from the event selection. This could be either due to pion decays or due to out-of-target interactions. The former of the two 
effects dominates this correction. The pion correction is greatest for low momentum pions emitted at high scattering angles, which have to survive for the longest time in order to reach the ToF wall. As already mentioned, the point at which tracks exit from the target is determined by finding the intersection of backwards extrapolated tracks from the TPCs with the target surface. The target position and alignment have to be known to high precision for track extrapolation to be effective. Studies from NA61 data have confirmed that the replica target is aligned with respect to the incident proton beam direction. Data driven studies have measured the centre of the upstream target face to be at position ${ }^{7}\left(x_{\text {target }}, y_{\text {target }}, z_{\text {target }}\right)=(0.16,0.21,-657.62)$ $\mathrm{cm}$, and the associated uncertainty $\left(\Delta x_{\text {target }}, \Delta y_{\text {target }}, \Delta z_{\text {target }}\right)=(0.04,0.04,0.36)$ $\mathrm{cm}$. The change in the number of tracks falling into different $(p, \theta, z)$ analysis bins was investigated as the target position was varied within its measurement uncertainty. The backwards extrapolation uncertainty has been estimated on a bin-by-bin basis. The effect of this uncertainty can be as much as $10 \%$ for the tracks exiting from the most upstream $z_{1}$-bin at low polar angle and the most downstream $z_{6}$-bin at high polar angles. This can be understood by observing that these tracks, emitted from either end of the target, are most likely to no longer intersect the target surface as the target position is varied. For the in-between longitudinal bins, $z_{2}, z_{3}, z_{4}$ and $z_{5}$, this effect largely averages out, as tracks simply migrate between adjacent bins under variations in target position.

An additional dataset was collected in 2010 with $10 \times 10^{6}$ protons on target, providing good statistics for measurements of $\pi^{ \pm}, K^{ \pm}$and proton production [97]. The full implementation of this dataset into the T2K flux calculation framework is planned for the future.

The potential of the replica target dataset lies in its capability to directly constrain both primary interactions, and subsequent reinteractions within the target, thus accounting for $\sim 90 \%$ of the T2K neutrino flux at beam peak energy.

\footnotetext{
${ }^{7}$ The $z$-axis of the NA61 coordinate system is defined by the proton beam direction, and the centre of the coordinate system is chosen so that e.g. scintillator S4 in the beam trigger sequence, shown in Figure 4.1, is located at $z=-211 \mathrm{~cm}$.
} 


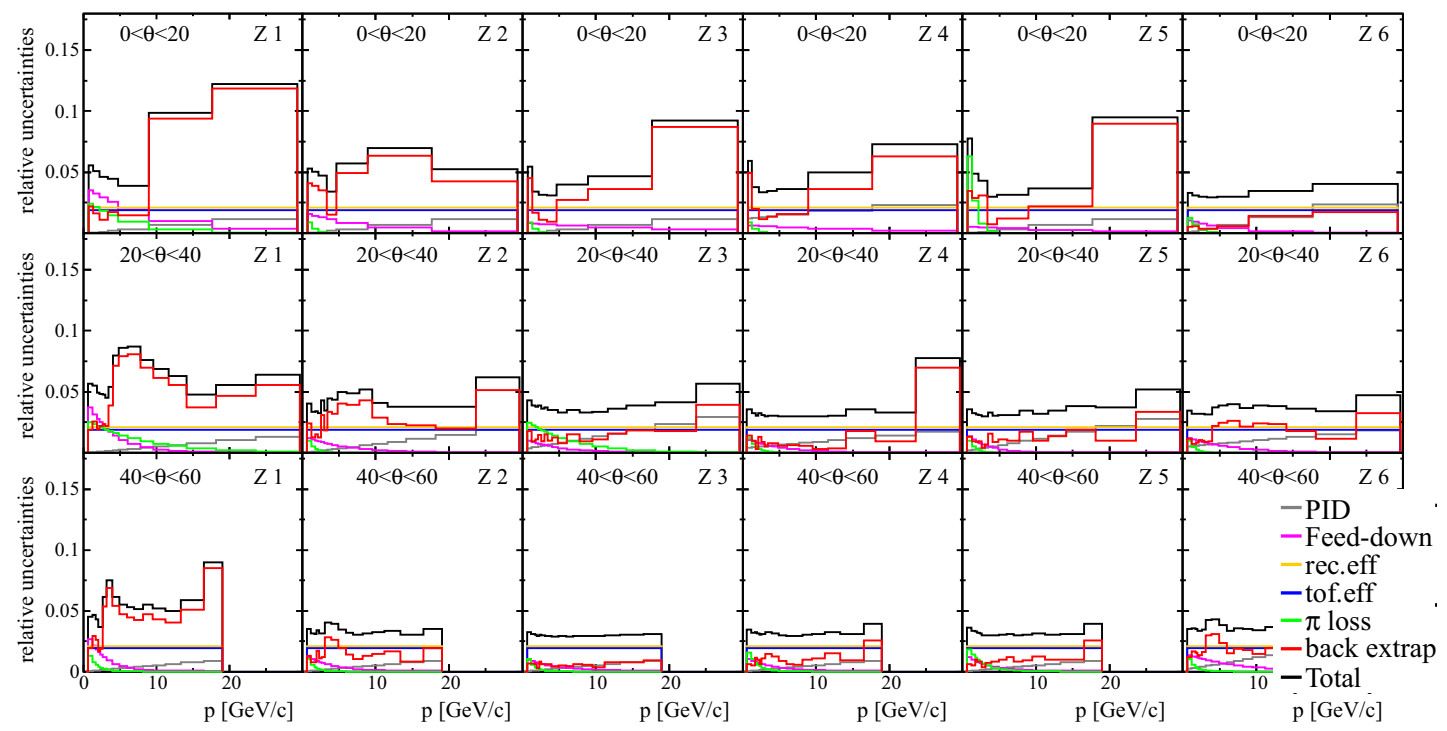

Figure 4.8: The systematic uncertainties for the $2009 \pi^{+}$yields from the replica target, split into contributions from different error sources. The uncertainties are given for every longitudinal $z$-bin, for different $\pi^{+}$momenta and covering the low scattering angle range from 0 to $60 \mathrm{mrad}$, which is mostly dominated by feed-down and backwards extrapolation systematic uncertainties. Figure reprinted from The European Physical Journal C, Volume 76, N Abgrall et al. (NA61/SHINE Collaboration), "Measurements of $\pi^{ \pm}$differential yields from the surface of the T2K replica target for incoming $31 \mathrm{GeV} / \mathrm{c}$ protons with the NA61/SHINE spectrometer at the CERN SPS"[99], under the CC BY copyright licence.

Note the $30 \%$ increase in the fraction of directly constrained interactions compared to the scenario when using only the thin target dataset.

Due to limited statistics, the 2009 replica target dataset consists of charged pion yields only, and thin target data is still used for constraining the neutrino yield originating from other hadron species, as well as for pions outside the coverage of the replica target dataset. The portion of the T2K flux covered with a combination of replica- and thin target measurements, with preference given to replica target data, is given in Fig. 4.10. The regions drawn with the lightest shade of grey are now directly covered with replica target measurements. The darker shaded grey flux portion is tuned to extrapolated thin target measurements (see chapter 5 for more details). The remaining flux fraction that cannot be tuned to NA61 data is also shown, drawn in black. For the signal $\nu_{\mu}\left(\bar{\nu}_{\mu}\right)$ flux in (anti-)neutrino mode, using a combination of replica target and thin target data constrains over $95 \%$ of relevant hadronic interactions, but is less successful in constraining the intrinsic 


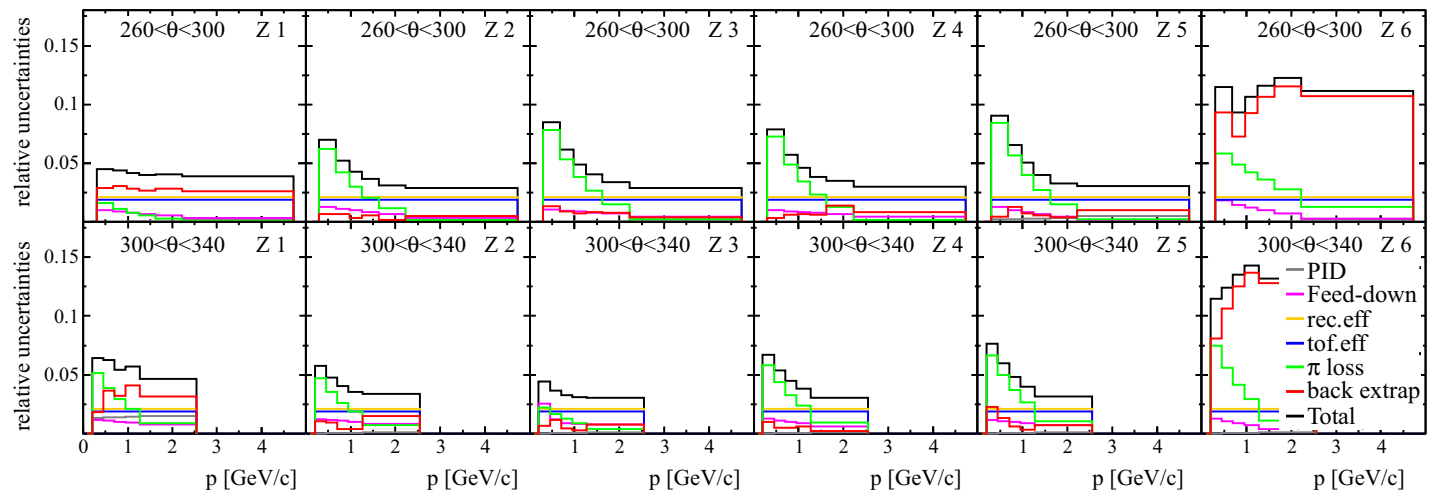

Figure 4.9: The systematic uncertainties for the $2009 \pi^{+}$yields from the replica target, split into contributions from different error sources. The uncertainties are given for every longitudinal $z$-bin, for different $\pi^{+}$momenta and covering the high scattering angle range from 260 to $340 \mathrm{mrad}$, which is mostly dominated by pion loss and backwards extrapolation systematic uncertainties. Figure reprinted from The European Physical Journal C, Volume 76, N Abgrall et al. (NA61/SHINE Collaboration), "Measurements of $\pi^{ \pm}$differential yields from the surface of the T2K replica target for incoming $31 \mathrm{GeV} / \mathrm{c}$ protons with the NA61/SHINE spectrometer at the CERN SPS"[99], under the CC BY copyright licence.

wrong-sign flux background, which is dominated by out-of-target interactions.

\subsubsection{Production cross section measurement}

The phenomenology of hadron-nucleus scattering depends crucially on the incident hadronic momentum. The terminology employed to describe the scattering type, based on the momentum transfer and type of final state products, varies greatly in literature. In this subchapter, the NA61 notation will be adopted. For low momenta, the hadron scatters from the nucleus as a whole. This process is often referred to as (coherent) elastic scattering, and is depicted in Figure 4.11a. The direction of the hadron changes but there is hardly any energy transfer, and no knockout nucleon exits the nucleus. Elastic scattering constitutes roughly $20 \%$ of $\sigma_{\text {tot }}$ for $31 \mathrm{GeV} / \mathrm{c}$ protons. At higher momentum transfer, the incident hadrons start to probe the structure of the nucleus. Scattering from individual nucleons within the nucleus, resulting in a single knockout nucleon, is referred to as quasielastic scattering (see Figure 4.11b). Quasi-elastic scattering constitutes roughly $10 \%$ of $\sigma_{\text {tot }}$ for $31 \mathrm{GeV} / \mathrm{c}$ protons. Finally, at even higher momentum transfer, the hadrons frequently scatter producing other hadrons (e.g. pions, kaons) in the 

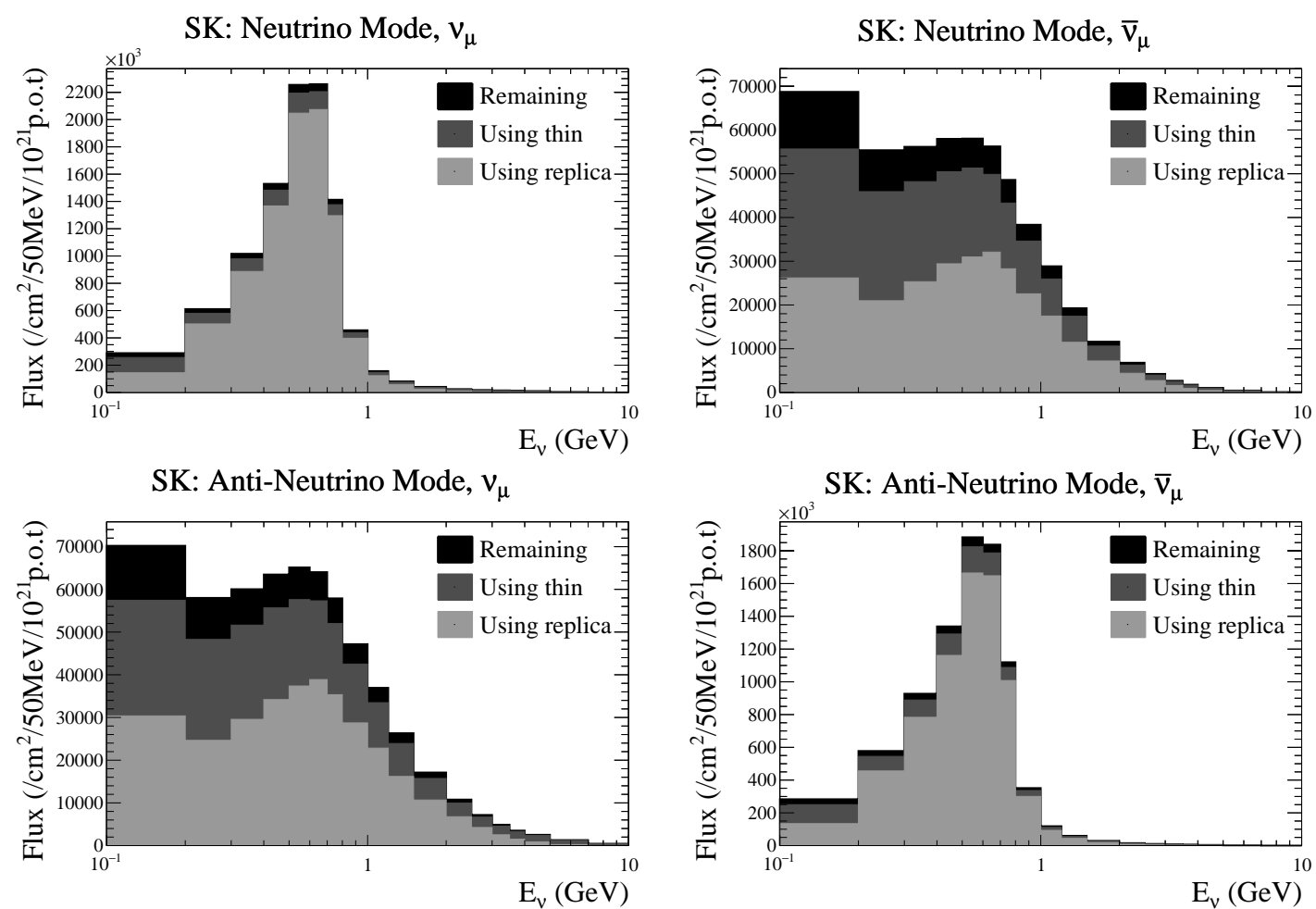

Figure 4.10: Portion of the T2K neutrino flux at Super-Kamiokande covered by the 2009 replica target dataset (light grey) and the extrapolated NA61 thin target dataset (dark grey), in both neutrino and anti-neutrino mode. Preference is given to the replica target dataset, which directly constrains $87.2 \%$ of the total $\nu_{\mu}$ flux in $\nu$-mode, and $84.9 \%$ of the total $\bar{\nu}_{\mu}$ flux in $\bar{\nu}$-mode. The part of the T2K flux which cannot be tuned to any NA61 measurements is also shown (black).

process, typically probing the underlying quark structure of individual nucleons. Hadron production constitutes roughly $70 \%$ of $\sigma_{\text {tot }}$ for $31 \mathrm{GeV} / \mathrm{c}$ protons. One such example of hadron scattering accompanied by pion production is shown in Figure 4.11c. The NA61 inelastic scattering cross section encompasses all scattering processes causing the disintegration of the nucleus (i.e. Figures $4.11 \mathrm{~b}$ and $4.11 \mathrm{c}$ ). The production cross section refers to all processes causing the production of new hadrons (i.e. Figure 4.11c).

Hence, in the terminology employed by NA61, the total inelastic cross section $\sigma_{\text {inel }}$ includes the quasi-elastic $\sigma_{\text {quasi-el }}$ and production $\sigma_{\text {prod }}$ cross sections

$$
\sigma_{\text {inel }}=\sigma_{\text {quasi-el }}+\sigma_{\text {prod }}
$$




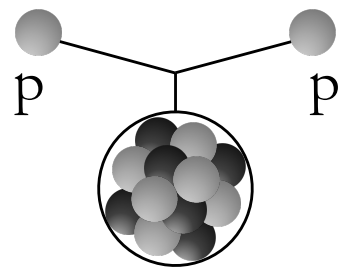

(a) (Coherent) elastic

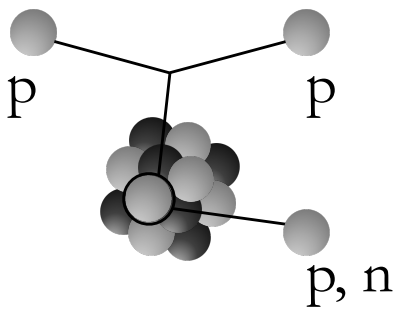

(b) Quasi-elastic

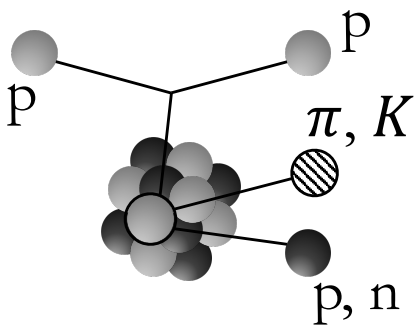

(c) With production

Figure 4.11: Types of hadron-nucleus scattering in the NA61 adopted formalism.

and the total cross section $\sigma_{\text {tot }}$ given by

$$
\sigma_{\text {tot }}=\sigma_{\text {inel }}+\sigma_{\text {el }}
$$

is the sum of the total inelastic and elastic contributions. The NA61 collaboration measured the production and inelastic cross sections for interactions of $31 \mathrm{GeV} / \mathrm{c}$ protons on graphite to be:

$$
\begin{gathered}
\sigma_{\text {prod }}=230.7 \pm 2.8(\text { stat }) \pm 1.2(\text { det })_{-3.5}^{+6.3}(\bmod ) \mathrm{mb} \\
\sigma_{\text {inel }}=258.4 \pm 2.8(\text { stat }) \pm 1.2(\text { det })_{-2.9}^{+5.0}(\bmod ) \mathrm{mb}
\end{gathered}
$$

where stat, det and mod refer to the total statistical, detector systematic and modelling systematic uncertainty. It is worthwhile noticing that these results are dominated by the modelling uncertainty, related to the choice of the physical model used to extract $\sigma_{\text {prod }}$ and $\sigma_{\text {inel }}$ from the directly measured trigger cross section $\sigma_{\text {trig. }}$. The NA61 trigger for cross section measurements, $T_{\text {trig }}=T_{\text {beam }} \wedge \overline{\mathrm{S} 4}$, is defined as the beam trigger $\mathrm{T}_{\text {beam }}$ in anti-coincidence with the $\mathrm{S} 4$ counter, placed between VTPC-1 and the gap TPC (see Figure 4.1). The scintillator based S4 counter has a diameter of $2 \mathrm{~cm}$ and is placed $3.7 \mathrm{~m}$ after the thin target, i.e. the half angle subtended by $\mathrm{S} 4$ counter to the thin target is roughly $2.7 \mathrm{mrad}$. This trigger removes the non-interacting and most of the forward-going elastically scattered protons. However, the precise fraction of elastic $f_{\text {el }}$, quasi-elastic $f_{\text {quasi-el }}$, production $f_{\text {prod }}$ and inelastic $f_{\text {inel }}$ scattering events where all charged products miss the $\mathrm{S} 4$ 
counter, and are thus included in the cross section trigger $T_{\text {trig }}$, can be understood only from model dependent simulations. Thus, by combining equation 4.3 and 4.4, the production and total inelastic cross sections can be expressed in terms of the trigger cross section via

$$
\begin{gathered}
\sigma_{\text {prod }}=\frac{1}{f_{\text {prod }}}\left(\sigma_{\text {trig }}-f_{\text {el }} \sigma_{\text {el }}-f_{\text {quasi-el }} \sigma_{\text {quasi-el }}\right) \\
\sigma_{\text {inel }}=\frac{1}{f_{\text {inel }}}\left(\sigma_{\text {trig }}-f_{\text {el }} \sigma_{\text {el }}\right)
\end{gathered}
$$

where the fractions $f_{\text {el }}, f_{\text {quasi-el }}, f_{\text {prod }}$ and $f_{\text {inel }}$ have been estimated from a GEANT4 simulation[103] of the full NA61 detector with the FTF_BIC physics list. For more details on different physics lists implemented in the GEANT4 simulation framework, the reader is referred forward to Chapter 6.4. The value of the quasi-elastic proton cross section $\sigma_{\text {quasi-el }}$ at $31 \mathrm{GeV} / \mathrm{c}$ has also been estimated from Monte Carlo simulations in the GEANT4 framework. The elastic cross section value $\sigma_{\mathrm{el}}$ from GEANT4 showed significant variation between consecutive GEANT4 software releases and depending on the chosen physics list. It was instead estimated by extrapolating between the existing measurements at $21.5 \mathrm{GeV} / \mathrm{c}[105]$ and at 70 $\mathrm{GeV} / \mathrm{c}[106]^{8}$. Substituting the calculated values of $\sigma_{\text {el }}$ and $\sigma_{\text {quasi-el }}$ back into equations 4.7 and 4.8, gives the final NA61 measurement results given in equations 4.5 and 4.6.

\footnotetext{
${ }^{8}$ Between $21-70 \mathrm{GeV} / \mathrm{c}, \sigma_{\text {el }}$ decreases approximately linearly.
} 


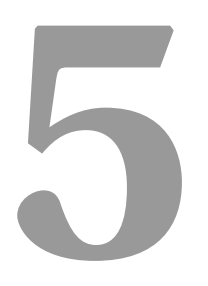

\section{The T2K flux calculation tuned to thin target data}

This chapter introduces the framework for flux calculations, developed over the years by the T2K Beam Working Group. This framework is used to predict the neutrino flux at ND280 and Super-K for every data-taking period, which is essential for making precise measurements of neutrino oscillation parameters. I have been the analyser responsible for producing such flux predictions in the last three oscillation measurements released by $\mathrm{T} 2 \mathrm{~K}[9-11]$, improving upon certain features of the existing code in the process. In addition, the flux tuning technique described here served as a starting point when developing the concept of replica target flux tuning, described in detail in Chapter 6.

\subsection{The neutrino flux simulation for $\mathrm{T} 2 \mathrm{~K}$}

The nominal T2K flux calculation relies on Monte Carlo tools for simulating particle propagation through matter, and various interactions which they might undergo.

The FLUKA2011.2c package[107] is used for modelling the propagation of the beam protons through the beam baffle and the $\mathrm{T} 2 \mathrm{~K}$ target $^{1}$. The $1.9 \lambda_{\mathrm{I}}$ interaction length ensures that most primary protons interact with the graphite target $(\sim 15 \%$ survival probability). In the $\mathrm{T} 2 \mathrm{~K}$ terminology, the first interaction of beam protons is referred to as the primary interaction. FLUKA was chosen for modelling primary interactions because it showed the best agreement with external hadron production measurements. The beamline geometry handled by the FLUKA simulation is depicted in Figure 5.1. It consists of the baffle and T2K target made from graphite,

\footnotetext{
${ }^{1}$ FLUKA2011.2c has since become obsolete, and FLUKA2011.2x will be used for the next flux release.
} 
with helium filling up the space in-between. The FLUKA simulation relies on run-specific in-situ T2K measurements as essential inputs. Primary beam profile measurements specify the trajectories of protons injected into the simulation. Beam profile parameters from Table 3.1 have been used to calculate the neutrino flux for different T2K data-taking periods. Average horn current measurements, listed in Table 3.4, set the horns' magnetic field strengths.

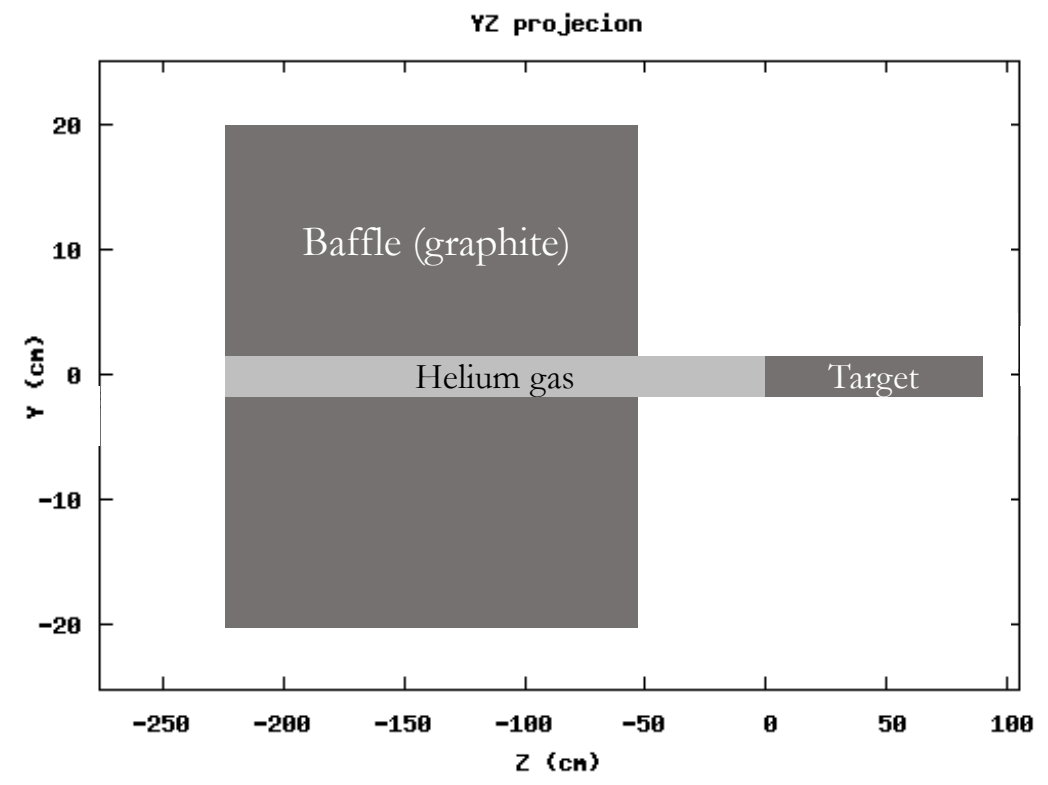

Figure 5.1: A diagram of the T2K beamline geometry handled by the FLUKA simulation. The baffle and target are composed of graphite of the same grade, and helium gas is included in the simulation, filling up the gap inside the baffle, and the path up to the target.

Kinematic information for particles exiting the target is passed on to JNUBEAM. JNUBEAM is a GEANT3[108] based Monte Carlo simulation used for propagating the exiting particles through the horn's magnetic field and the rest of the secondary beamline, allowing for the possibility of further interactions and decays. Out-oftarget interactions are modelled by GCALOR 1.05/04[109]. The final step in the flux calculation consists of tuning the FLUKA and GCALOR models to external data provided by NA61/SHINE and other experiments. Effectively, this means that the likelihood for simulated events gets modified to better reflect existing measurements 
of the modelled interactions. Introducing the flux tuning methodology will be the main focus of this chapter.

The T2K flux simulation inputs differ among the T2K data-taking run periods. For every run, the flux is simulated from $2 \times 10^{8}$ protons incident on the target. The combined measurement of the total T2K flux $\Phi_{\text {combined }}$ is obtained by taking the average flux across the different runs, weighted by the collected protons on target for every run period:

$$
\Phi_{\text {combined }}^{\alpha}=\frac{1}{\mathrm{~N}_{\text {POT total }}^{\alpha}} \times \sum_{\text {run i }} \mathrm{N}_{\text {POT run i }}^{\alpha} \times \Phi_{\text {run i }}^{\alpha}
$$

where $\mathrm{N}_{\text {POT run i }}^{\alpha}$ and $\mathrm{N}_{\text {POT total }}^{\alpha}$ are the total POT from run $\mathrm{i}$ and from all runs, respectively, at detector $\alpha \in\{\mathrm{ND} 280, \mathrm{SK}\}$, which have been summarized in the Table 3.7. The combined flux predictions at ND280 and Super-K obtained using this procedure are presented in Figure 5.2.

Associated with every simulated neutrino event is a series of hadronic interactions leading to its production. This ancestry chain always starts with primary interactions of the original beam protons, and finishes with neutrinos produced as by-products of in-flight particle decays. Decays considered in JNUBEAM as relevant for neutrino production are $\pi^{+}, K^{+}, K_{\mathrm{L}}^{0}$ and $\mu^{+}$decays. The branching ratios for the main decay channels of hadrons and leptons which contribute to neutrino production are summarised in Table 5.1. It is worth adding that since the muon decay amplitude depends on its polarization, the feature has been added to the hadron production generator to save the JNUBEAM true polarization for muons generated through pion and kaon decays. The true kinematic properties of the incident and outgoing particle are also saved for every simulated interaction, in addition to the target nucleon species. As the particles are propagated through the secondary beamline, the distance travelled through different materials is also saved. This neutrino ancestry information is essential for assigning weights in the tuning procedure.

When describing particle interactions, incoming particles are usually referred to as parent particles, and outgoing particles as daughter particles. Daughter particles from the primary interaction might engage in secondary interactions. All subsequent 

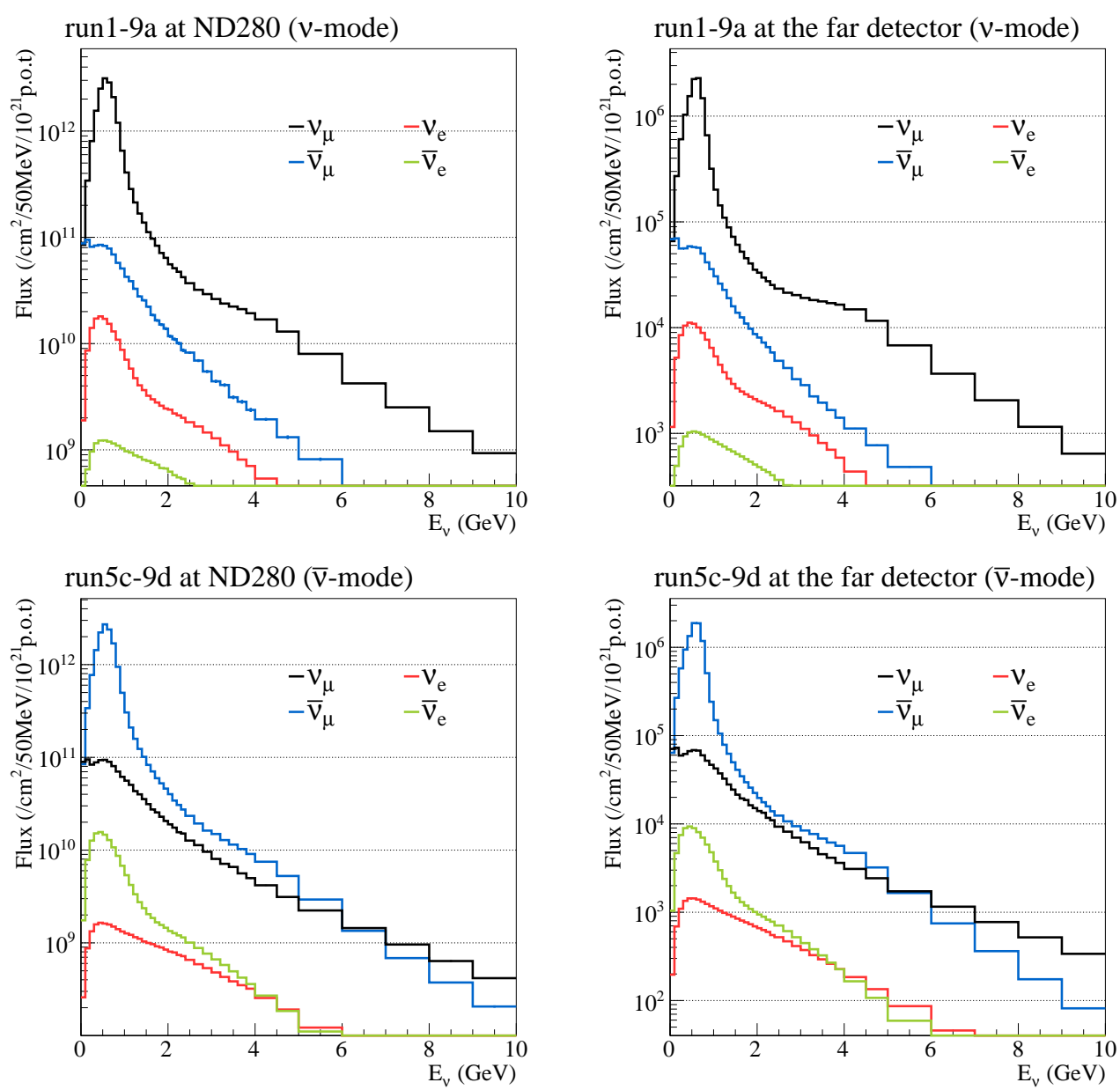

Figure 5.2: The combined unoscillated T2K flux in neutrino (top) and anti-neutrino (bottom) mode of operation, at ND280 (left) and at Super-K (right). The flux has been broken down into contributions from all neutrino species. Only statistical error bars are shown. Copyright by T2K Collaboration.

\begin{tabular}{cc}
\hline \hline Decays for $\nu$ production & Branching Ratio \\
\hline$\pi^{+} \rightarrow \mu^{+} \nu_{\mu}$ & $99.9877 \%$ \\
$\pi^{+} \rightarrow e^{+} \nu_{e}$ & $1.23 \times 10^{-4}$ \\
$K^{+} \rightarrow \mu^{+} \nu_{\mu}$ & $63.55 \%$ \\
$K^{+} \rightarrow \pi^{0} \mu^{+} \nu_{\mu}$ & $3.353 \%$ \\
$K^{+} \rightarrow \pi^{0} e^{+} \nu_{e}$ & $5.07 \%$ \\
$K_{L}^{0} \rightarrow \pi^{ \pm} \mu^{\mp} \bar{\nu}_{\mu}\left(\nu_{\mu}\right)$, called $K_{\mu 3}^{0}$ & $27.04 \%$ \\
$K_{L}^{0} \rightarrow \pi^{ \pm} e^{\mp} \bar{\nu}_{e}\left(\nu_{e}\right)$, called $K_{e 3}^{0}$ & $40.55 \%$ \\
$\mu^{+} \rightarrow e^{+} \bar{\nu}_{\mu} \nu_{e}$ & $100 \%$ \\
\hline \hline
\end{tabular}

Table 5.1: Main T2K neutrino producing decay channels. Branching ratio values taken from Particle Data Group 2018[54]. 
interactions are commonly referred to as tertiary interactions. In T2K, the majority of neutrino parents, $63.2 \%$ of $\nu_{\mu}$ at Super-K, come directly from interactions of the original beam protons (i.e. from primary interactions), as demonstrated in Table 5.2. More than $10 \%$ of $\nu_{\mu}$, and almost $50 \%$ of the wrong-sign $\bar{\nu}_{\mu}$, have parents produced in out-of-target interactions. Out-of-target interactions usually occur on target nuclei other than carbon ${ }^{2}$, and NA61 thin target measurements are thus not immediately useful, before material scaling is applied. Secondary and tertiary interactions, relevant for $36.8 \%$ of $\nu_{\mu}$ in $\nu$-mode, also cannot be directly constrained with NA61 thin target measurements. Namely, the protons which might take part in these interactions have already lost some of their energy, and the NA61 measurements would have to be energy scaled before being used in the tuning procedure.

\begin{tabular}{cccc}
\hline \hline Flux component in $\nu$-mode & 1 interaction & $\geq 2$ interactions & $\geq 1$ out-of-target \\
\hline$\nu_{\mu}$ at ND280 & $63.2 \%$ & $36.8 \%$ & $12.6 \%$ \\
$\bar{\nu}_{\mu}$ at ND280 & $39.5 \%$ & $60.5 \%$ & $49.8 \%$ \\
$\nu_{e}$ at ND280 & $60.1 \%$ & $39.9 \%$ & $13.6 \%$ \\
$\bar{\nu}_{e}$ at ND280 & $50.7 \%$ & $49.3 \%$ & $32.2 \%$ \\
$\nu_{\mu}$ at SK & $63.2 \%$ & $36.8 \%$ & $12.4 \%$ \\
$\bar{\nu}_{\mu}$ at SK & $41.5 \%$ & $58.5 \%$ & $45.1 \%$ \\
$\nu_{e}$ at SK & $61.7 \%$ & $38.3 \%$ & $12.7 \%$ \\
$\bar{\nu}_{e}$ at SK & $54.0 \%$ & $46.0 \%$ & $27.2 \%$ \\
\hline \hline
\end{tabular}

Table 5.2: Number of interactions in the hadronic ancestry for different neutrino flux components $\left(\mathrm{E}_{\nu}<10 \mathrm{GeV}\right)$ at ND280 and Super-K, for operation in neutrino-mode. Cases with one interaction refer to events where the neutrino parents get directly produced through interactions of primary protons (i.e. neutrino parents are daughters of beam protons). In this study, the baffle, magnetic horns, decay volume, beam dump and target cooling structure are all part of the simulated geometry.

The fractional contribution to the neutrino flux at Super-K, coming from decays of various parent particles, is summarised in Table 5.3. The simulated neutrino flux from different parent particles, as a function of neutrino energy, is shown in Figure 5.3. Pion decays dominate the $\nu_{\mu}$ and $\bar{\nu}_{\mu}$ production around the T2K flux peak ( $\sim 600 \mathrm{MeV})$, whereas kaon decays contribute overwhelmingly to neutrino

\footnotetext{
${ }^{2}$ often with incident proton energies lower than the $30 \mathrm{GeV}$ NA61 beam
} 
production in the high energy flux tail. Muon decays at low energies, and kaon decays at high energies, contribute to the intrinsic $\nu_{e}$ and $\bar{\nu}_{e}$ flux contamination.

\begin{tabular}{ccccc}
\hline \hline Neutrino parent & $\nu_{\mu}$ & $\bar{\nu}_{\mu}$ & $\nu_{e}$ & $\bar{\nu}_{e}$ \\
\hline$\pi^{+}$ & $94.91 \%$ & - & $0.97 \%$ & - \\
$\pi^{-}$ & - & $83.22 \%$ & - & $0.36 \%$ \\
2-body $K^{+}$ & $4.73 \%$ & - & - & - \\
3-body $K^{+}$ & $0.25 \%$ & - & $31.71 \%$ & - \\
2-body $K^{-}$ & - & $6.09 \%$ & - & - \\
3-body $K^{-}$ & - & $0.33 \%$ & - & $17.31 \%$ \\
$K_{L}^{0}$ & $0.10 \%$ & $1.49 \%$ & $12.73 \%$ & $76.32 \%$ \\
$\mu^{+}$ & - & $8.87 \%$ & $54.59 \%$ & - \\
$\mu^{-}$ & $0.01 \%$ & - & - & $6.01 \%$ \\
\hline \hline
\end{tabular}

Table 5.3: Fraction of neutrino flux at Super-K coming from decays of different parent particles. All four neutrino flavours contributing to the flux in the neutrino-mode of operation have been considered.

\subsection{Introduction to flux tuning}

Despite best efforts, no single hadronic interaction generator has the capability to successfully describe all existing hadron production datasets, and typically significant variations in Monte Carlo predictions are observed depending on the chosen generator. Thus, nominal T2K Monte Carlo predictions are useful as rough estimates of the expected neutrino flux, but for more stringent predictions it is necessary to tune the nominal MC to a suitable selection of ex-situ measurements. The flux tuning procedure assigns a weight to every simulated neutrino event, based on its hadronic ancestry. The key processes to consider when modelling the hadronic interactions are:

1. How often does a particle, when traversing a given material, interact to produce new particles? The answer to this question can be elucidated from measurements of production cross section (see Figure 5.4a). The simulation is tuned to data by applying what will be referred to as interaction length weights. 

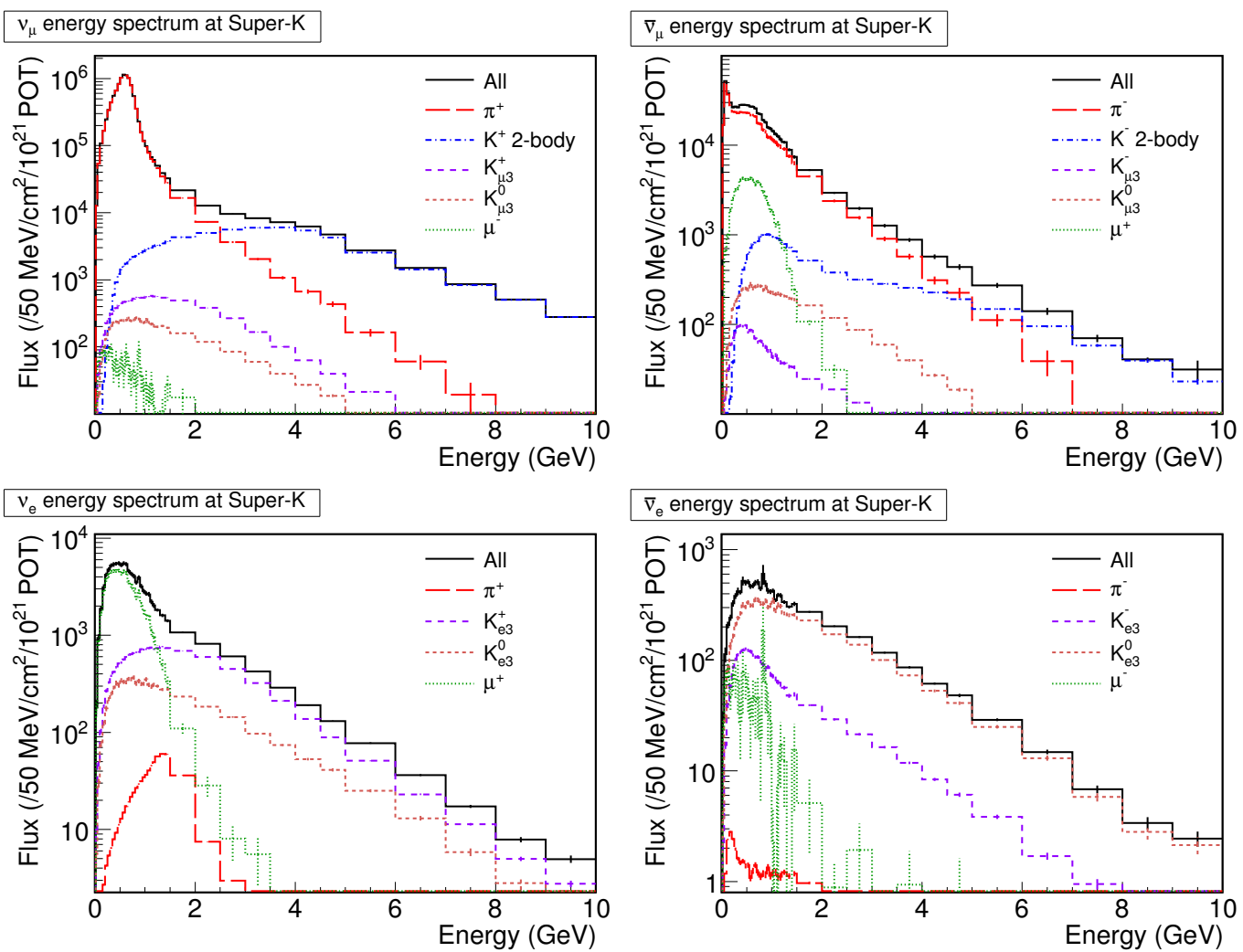

Figure 5.3: A schematic representation of the fraction of neutrinos at Super-K coming from decays of different parent particles. Here, 2-body and 3-body kaon decays are plotted separately. Each flux component is considered $\left(\mathrm{E}_{\nu}<10 \mathrm{GeV}\right)$ for the neutrino-mode of operation. Copyright by T2K Collaboration.

2. How many daughter particles of different species and kinematic properties get produced when the parent particle does interact? Essential for answering this question are measurements of differential production cross section from targets of different thickness (see Figure 5.4b), such as those taken by NA61. Here, the simulated yields are tuned to measurements by applying multiplicity weights.

Thus, the total weight assigned to neutrino events through flux tuning is given by the product of interaction length and multiplicity weights for every simulated interaction in the neutrino's ancestry chain. 


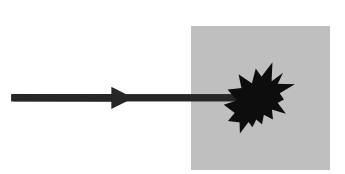

(a) How often does the hadron interact?

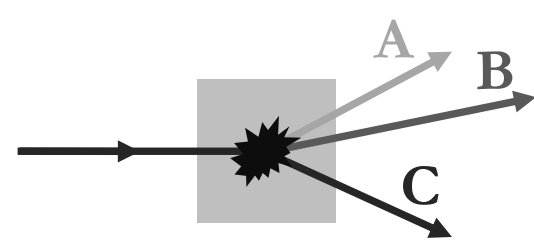

(b) Which particles are most likely to be produced?

Figure 5.4: Two distinct aspects of hadron interactions relevant for flux tuning.

\subsection{Multiplicity tuning}

\subsubsection{Meson multiplicity weights}

The weight applied to each simulated interaction is given by the ratio of measured multiplicity and the multiplicity from the underlying model in the simulation package:

$$
\mathrm{W}_{\text {thin }}^{\alpha}(p, \theta)=\left[\frac{d^{2} \mathrm{n}_{\text {thin }}^{\alpha}(p, \theta)}{d p d \theta}\right]_{\text {NA61 }} /\left[\frac{d^{2} \mathrm{n}_{\text {thin }}^{\alpha}(p, \theta)}{d p d \theta}\right]_{\text {NA61 SIMULATION }}
$$

where $\alpha \in\left\{\pi^{ \pm}, \mathrm{K}^{ \pm}, \mathrm{K}_{\mathrm{S}}^{0}\right\}$ denotes the meson species, and the simulation refers to either FLUKA or GEANT3, depending if the interaction happens in-target or out-of-target, respectively. Here, the NA61 beam profile is incident on the simulated NA61 thin target, and the yields of mesons coming out from the target are stored, to be used for $\left[\frac{d^{2} \mathrm{n}_{\text {thin }}^{\alpha}(p, \theta)}{d p d \theta}\right]_{\text {SIMUlation }}$. The value of $\left[\frac{d^{2} \mathrm{n}_{\text {thin }}^{\alpha}(p, \theta)}{d p d \theta}\right]_{\text {NA61 }}$ can be taken directly from NA61 2009 thin target measurements, introduced in Chapter 4.2.1, for in-target interactions on carbon. For out-of-target interactions, the NA61 data has to be scaled to different target nuclei. It is worth pointing out that Equation 5.2 can be readily used to tune yields of produced mesons. The calculation of weights for baryon tuning is slightly more involved, as the constraint imposed by baryon number conservation must be applied. The $(p, \theta)$ phase-space coverage of the NA61 dataset is extended using the analytical expression for parametrising the secondary particle yields in proton-nucleus interactions. The cross section, according to the 
$\mathrm{BMPT}^{3}$ parametrisation[110], is given by:

$$
\left[E \frac{d^{3} \sigma}{d^{3} \boldsymbol{p}}\right]_{\mathrm{BMPT}}\left(x_{\mathrm{R}}, p_{\mathrm{T}}\right)=\mathbb{A}\left(1-x_{\mathrm{R}}\right)^{\alpha}\left(1+\mathbb{B} x_{\mathrm{R}}\right) x_{\mathrm{R}}^{-\beta}\left(1+\frac{a}{x_{\mathrm{R}}^{\gamma}} p_{\mathrm{T}}+\frac{a^{2}}{2 x_{\mathrm{R}}^{\delta}} p_{\mathrm{T}}^{2}\right) e^{-a p_{\mathrm{T}} / x_{\mathrm{R}}^{\gamma}}
$$

where $p_{\mathrm{T}}$ is the transverse momentum of the produced particle in the centre-of-mass $(\mathrm{CoM})$ frame, and $x_{\mathrm{R}}$ is the ratio of the particle's energy $E^{\mathrm{CoM}}$ and the maximum energy that it could carry $E_{\max }^{\mathrm{CoM}}$ in the CoM frame, i.e. $x_{\mathrm{R}}=\frac{E^{\mathrm{CoM}}}{E_{\max }^{\mathrm{CoM}}}$. The quantities $\mathbb{A}, \mathbb{B}, \alpha, \beta, \gamma, \delta, a$ are fixed constants obtained from fits to data. Additional multiplicative factors of $r_{0}\left(1+x_{\mathrm{R}}\right)^{r_{1}}$ for $\pi^{-}$and $r_{0}\left(1-x_{\mathrm{R}}\right)^{r_{1}}$ for $K^{-}$have been added $^{4}$, reflecting the expected difference in pion and kaon rates depending on charge. Equation 5.3 neglects any forward-backward asymmetry in the CoM frame, which could arise from particle re-interactions in the nuclear environment. This has been corrected for by introducing the modified BMPT differential cross section[72]:

$$
\left[E \frac{d^{3} \sigma}{d^{3} \boldsymbol{p}}\right]_{\text {modified BMPT }}=\left[\frac{\mathrm{A}}{2}\right]^{\left(a+b x_{\mathrm{F}}+c x_{\mathrm{F}}^{2}\right)\left(d+e p_{\mathrm{T}}^{2}\right)}\left[E \frac{d^{3} \sigma}{d^{3} \boldsymbol{p}}\right]_{\mathrm{BMPT}}
$$

where $\mathrm{A}$ is the mass number of the nucleus, $x_{\mathrm{F}}=\frac{p_{\mathrm{L}}^{\mathrm{CoM}}}{p_{\max }^{\mathrm{COM}}}$ is the ratio of the CoM longitudinal momentum $p_{\mathrm{L}}^{\mathrm{CoM}}$ and the maximum momentum the particle could carry $p_{\max }^{\mathrm{CoM}}$, and the factor of 2 appears in the denominator since scattering from deuterium is assumed first, and then target scaling to larger nuclei is applied. The coefficients $a, b, c, d, e$ in the exponent of $\left[\frac{\mathrm{A}}{2}\right]$ are derived from fits to either pion or kaon data, and are summarised in Table 5.4. $x_{\mathrm{F}}$ can be either positive or negative, which introduces the forward-backward asymmetry along the longitudinal direction defined in the CoM frame. Equation 5.4 parametrises the differential cross section. NA61 measures the differential particle yield $\mathrm{Y}(p, \theta)$. These two observables are related via:

$$
\mathrm{Y}_{\mathrm{BMPT}}(p, \theta)=\frac{1}{\sigma_{\mathrm{prod}}} \frac{p^{2}}{E} \times\left[\frac{E}{p^{2}} \frac{d^{2} \sigma(p, \theta)}{d p d \Omega}\right]_{\mathrm{BMPT}} .
$$

In other words, BMPT parametrised differential particle yields can be calculated by normalising the differential cross section by the NA61 production cross section measurement. Thus, for every outgoing meson, the free parameters $\mathbb{A}, \mathbb{B}, \alpha, \beta$,

\footnotetext{
${ }^{3}$ Parametrisation named BMPT after Bonesini-Marchionni-Pietropaolo-Tabarelli de Fatis, the authors in [110].

${ }^{4}$ Here, $r_{0}$ and $r_{1}$ are also constants to be determined from data.
} 
Table 5.4: Estimation of parameters $a, b, c, d, e$ from Equation 5.4, which are used for extrapolating the NA61 measurements. The pion and kaon data in these fits has been taken from Eichten et. al[111] and Allaby et. al[112] (more details on these datasets in Chapter 5.3.1). Best fit values quoted from [72].

\begin{tabular}{l|c|c|c|c|c}
\hline \hline & $a$ & $b$ & $c$ & $d$ & $e$ \\
\hline Fit to $\pi$ data & 0.75 & -0.52 & 0.23 & 1.0 (fixed) & 0.21 \\
Fit to $K$ data & 0.77 & -0.32 & 0.0 & 1.0 (fixed) & 0.25 \\
\hline \hline
\end{tabular}

Table 5.5: The fitted parameters from the modified BMPT fits to NA61 data. The $\chi^{2}$ value for each meson dataset has been normalised by the number of degrees of freedom. Table taken from [85].

\begin{tabular}{l|c|c|c|c|c}
\hline \hline & $\pi^{+}$ & $\pi^{-}$ & $K^{+}$ & $K^{-}$ & $K_{S}^{0}$ \\
\hline$\chi^{2}$ & $465 / 403$ & $370 / 415$ & $75 / 91$ & $124 / 91$ & $1.5 / 12$ \\
\hline $\mathbb{A}$ & $34.3 \pm 13.0$ & $24.1 \pm 14.4$ & $0.899 \pm 0.108$ & $2.83 \pm 0.45$ & $0.67 \pm 2.02$ \\
$\mathbb{B}$ & $1.96 \pm 1.61$ & $0.47 \pm 0.81$ & $5.10 \pm 1.08$ & $-1.25 \pm 0.22$ & $-0.63 \pm 6.11$ \\
$\alpha$ & $3.74 \pm 0.15$ & $3.31 \pm 0.19$ & $3.02 \pm 0.25$ & $4.57 \pm 0.51$ & $2.4 \pm 7.3$ \\
$\beta$ & $0.38 \pm 0.10$ & $0.437 \pm 0.043$ & $0.826 \pm 0.052$ & $0.557 \pm 0.079$ & $1.3 \pm 1.3$ \\
$a$ & $6.058 \pm 0.069$ & $5.659 \pm 0.065$ & $5.884 \pm 0.186$ & $7.089 \pm 0.333$ & $4.5 \pm 1.9$ \\
$\gamma$ & $0.211 \pm 0.008$ & $0.186 \pm 0.009$ & $0.093 \pm 0.026$ & $-0.001 \pm 0.037$ & $0.18 \pm 0.39$ \\
$\delta$ & $0.701 \pm 0.026$ & $0.635 \pm 0.029$ & $0.410 \pm 0.097$ & $0.182 \pm 0.143$ & $0.39 \pm 1.33$ \\
$r_{0}$ & - & $0.853 \pm 0.434$ & - & $1.78 \pm 0.28$ & - \\
$r_{1}$ & - & $1.54 \pm 0.91$ & - & $1.54 \pm 0.52$ & - \\
\hline \hline
\end{tabular}

$\gamma, \delta, a, r_{0}$ and $r_{1}$ (for $\pi^{-}$and $K^{-}$), are fixed from fits to NA61 data, and the best-fit values are given in Table 5.5 .

Now that the BMPT extrapolation has been defined, multiplicity weights can be calculated over a wide range relevant for the T2K flux production in the $(p, \theta)$ phase-space. The weights for tuning meson yields from primary interactions on the $\mathrm{T} 2 \mathrm{~K}$ target are given in Figure 5.5.

\section{Energy and target material scaling}

The multiplicity weights from Figure 5.5 can be used for tuning more than just the primary interactions on graphite, provided that appropriate corrections are applied. When tuning secondary and tertiary interactions, the proton momentum 

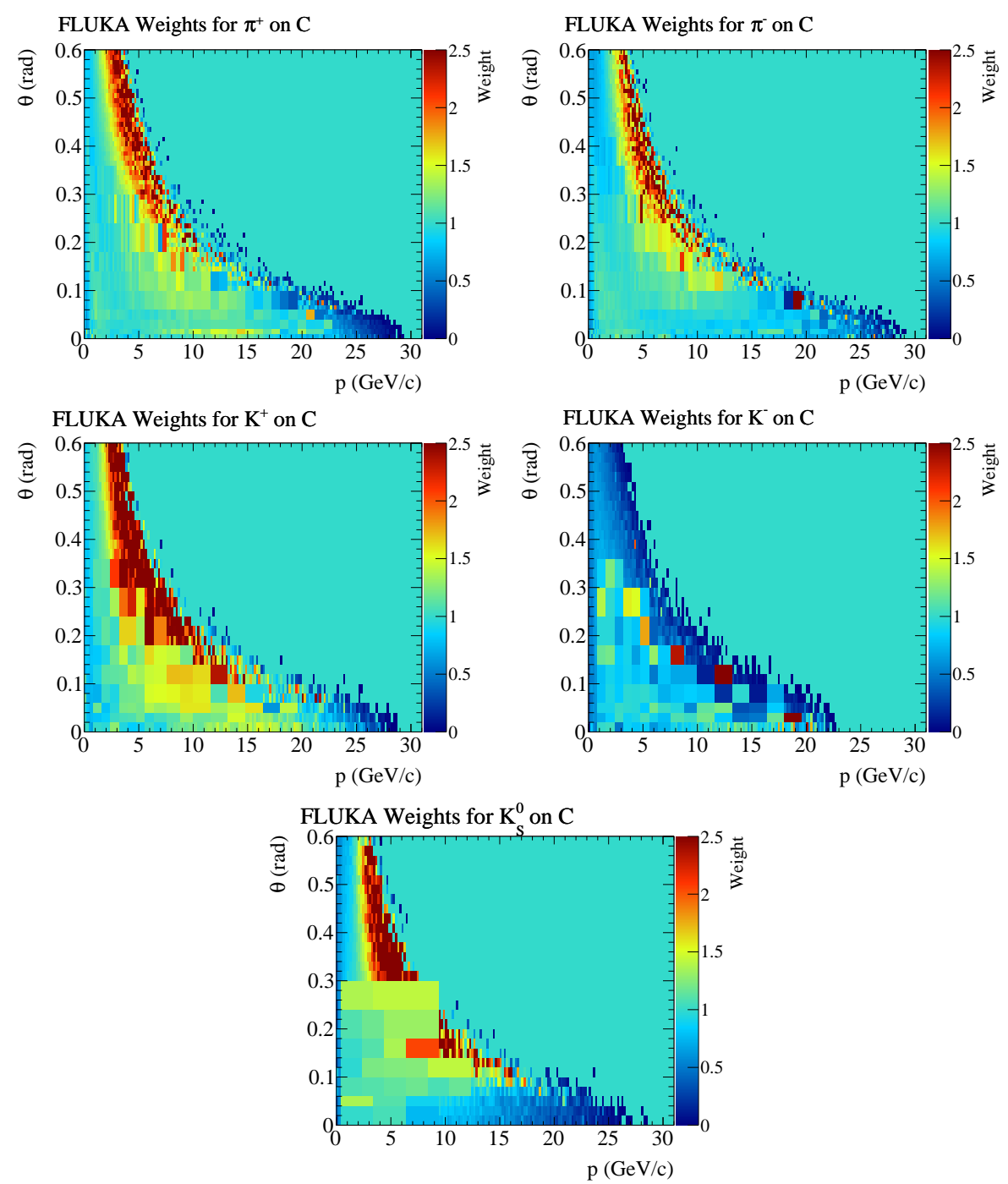

Figure 5.5: The meson multiplicity weights from NA61 data over FLUKA 2011.2b. NA61 measurements have been extrapolated according to the BMPT parametrization. The coarse bins mostly correspond to the NA61 data binning, and the finer bin are from the extrapolation with BMPT fits. Figure reprinted from [85]. Copyright by T2K Collaboration.

is reduced compared to the nominal $31 \mathrm{GeV} / \mathrm{c}$ beam momentum. Richard Feynman argued[113] that in inclusive hadronic collisions of the form $\mathrm{A}+\mathrm{B} \rightarrow \alpha+X$, the inclusive cross section for finding particle $\alpha$, with transverse and longitudinal momenta $p_{\mathrm{T}}$ and $p_{\mathrm{L}}$, respectively, is given by:

$$
d \sigma=f_{\alpha}\left(p_{\mathrm{T}}, x_{\mathrm{F}}\right) \frac{d^{3} \boldsymbol{p}}{E}=f_{\alpha}\left(p_{\mathrm{T}}, x_{\mathrm{F}}\right) \frac{d p_{\mathrm{L}}}{E} d^{2} p_{\mathrm{T}}
$$

where the particle energy $E=\sqrt{m_{\alpha}^{2}+p_{\mathrm{T}}^{2}+p_{\mathrm{L}}^{2}}$, with $m_{\alpha}$ being the particle rest mass. In other words, the probability density for finding the particle $\alpha$ consists 
of the manifestly Lorentz-invariant ${ }^{5}$ part $\frac{d^{3} p}{E}$, and the function $f_{\alpha}\left(p_{\mathrm{T}}, x_{\mathrm{F}}\right)$ which depends purely on the variables $x_{\mathrm{F}}$ and $p_{\mathrm{T}}$, i.e. it contains no explicit dependence on the CoM energy $\sqrt{s}$. This approximation breaks down at lower values of $\sqrt{s}$, when the CoM energy gets close to meson production thresholds. Thus, in order to apply Feynman scaling, the weights in Figure 5.5 are converted from the $(p, \theta)$ to the $\left(p_{\mathrm{T}}, x_{\mathrm{F}}\right)$ phase-space. Multiplicity weights are then assigned to meson yields from secondary and tertiary interactions based on the $\left(p_{\mathrm{T}}, x_{\mathrm{F}}\right)$ value for these interactions. The reader might notice that the BMPT parametrisation introduced in Equation 5.3 offers an alternative differential production cross section expression, which is also without any explicit dependence on the CoM energy of the system. The decision has been made by the T2K Beam Group to use Feynman scaling in the nominal flux calculation to account for different incident hadronic momenta in the lab frame. The BMPT parametrisation is used only when evaluating the flux systematic uncertainty associated with the CoM energy scaling, which will be discussed in more detail in Chapter 5.5.1.

Multiplicity weights can also be assigned for out-of-target interactions, provided that target nucleus scaling has been assigned. The relationship between the cross sections for inclusive scattering $h+A \rightarrow h^{\prime}+X$, of hadrons of species $h$, producing hadron $h^{\prime}$ in the process, is given by:

$$
E \frac{d^{3} \sigma\left(h+A_{1} \rightarrow h^{\prime}+X\right)}{d^{3} \boldsymbol{p}}=\left(\frac{A_{1}}{A_{2}}\right)^{\alpha\left(x_{F}, p_{T}\right)} E \frac{d^{3} \sigma\left(h+A_{2} \rightarrow h^{\prime}+X\right)}{d^{3} \boldsymbol{p}}
$$

where $A_{1}$ and $A_{2}$ are the mass numbers of the two target nuclei. This expression has been adopted from Bonesini et. al[110]. The polynomial parametrization used to describe $\alpha\left(x_{F}, p_{T}\right)$ has been adapted based on studies by Barton et. al[114] and Scubic et. al[115], and is given by:

$$
\alpha\left(x_{F}, p_{T}\right)=P_{0}+P_{1} x_{F}+P_{2} p_{T}+P_{3} x_{F}^{2}+P_{4} p_{T}^{2}+P_{5} x_{F} p_{T}
$$

where the parameters $P_{0}, P_{1}, P_{2}, P_{3}, P_{4}$ and $P_{5}$ are fixed constants to be determined from fits to data. The list of measurements used by T2K to derive the values of these

\footnotetext{
${ }^{5}$ The Lorentz invariant single particle phase space density is proportional to $\frac{d^{3} \boldsymbol{p}}{E}$.
} 
Table 5.6: Data used to derive the target scaling dependence for the double differential production. For the HARP data, only the $12 \mathrm{GeV} / \mathrm{c}$ incident proton data is used in the target scaling fit. Table from [85].

\begin{tabular}{l|c|c|c|c}
\hline \hline Data Set & Target & $p_{\text {in }}, p_{\text {out }}[\mathrm{GeV} / \mathrm{c}]$ & $\theta_{\text {out }}[\mathrm{rad}]$ & $h^{\prime}$ \\
\hline Eichten [11] & $\mathrm{Be}, \mathrm{Al}, \mathrm{Cu}$ & $24.0,4.0-18.0$ & $0.017-0.127$ & $\pi^{ \pm}, K^{ \pm}, p$ \\
Allaby [112] & $\mathrm{Be}, \mathrm{Al}, \mathrm{Cu}$ & $19.2,6.0-16.0$ & $0.013-0.07$ & $\pi^{ \pm}, K^{ \pm}, p$ \\
BNL-E802 [116] & $\mathrm{Be}, \mathrm{Al}, \mathrm{Cu}$ & $14.6,0.5-4.5$ & $0.1-0.9$ & $\pi^{ \pm}, K^{ \pm}, p$ \\
HARP [117] & $\mathrm{Be}, \mathrm{C}, \mathrm{Al}, \mathrm{Cu}$ & $12.0,0.5-8.0$ & $0.025-0.25$ & $\pi^{ \pm}$ \\
\hline \hline
\end{tabular}

parameters have been summarised in Table 5.6. For every outgoing meson species, the ratio of differential production cross section measurements of the heavier to the lightest target $(\mathrm{Be})$ is taken:

$$
\frac{d^{2} \sigma_{h A}}{d p d \theta} / \frac{d^{2} \sigma_{h A_{\min }}}{d p d \theta}=\left(\frac{A}{A_{\min }}\right)^{\alpha\left(x_{F}, p_{T}\right)}
$$

The fitted values of parameters $P_{i}$, where $i \in\{0,1,2,3,4,5\}$, are given in Table 5.7. Separate values have been fitted for each outgoing meson species. Having introduced the target nucleus scaling technique, used in calculating the differential cross section $\frac{d^{2} \sigma_{h A}}{d p d \theta}$, the particle multiplicity can be determined by normalising this differential cross section by the total production cross section $\sigma_{\text {prod }}^{h A}$ (see Equation 4.1). The total production cross section is also obtained from fits to data. Finally, multiplicity weights for tuning out-of-target interactions can now be constructed for the materials building up various components of the T2K secondary beamline. The most important are aluminium (focusing horns), iron (walls of the decay volume), titanium (target cooling support structure) and graphite (beam dump). Examples of some of these weights (focusing on the case of pions) are provided in Figure 5.6.

\subsubsection{Baryon multiplicity weights}

NA61 took measurements of the multiplicities of baryons exiting from the thin target. Thus, baryon multiplicity weights can also be constructed, provided that the constraint of baryon number conservation is imposed. Secondary protons in $\mathrm{T} 2 \mathrm{~K}$ could originate directly from the beam protons scattered off from nuclei. New 

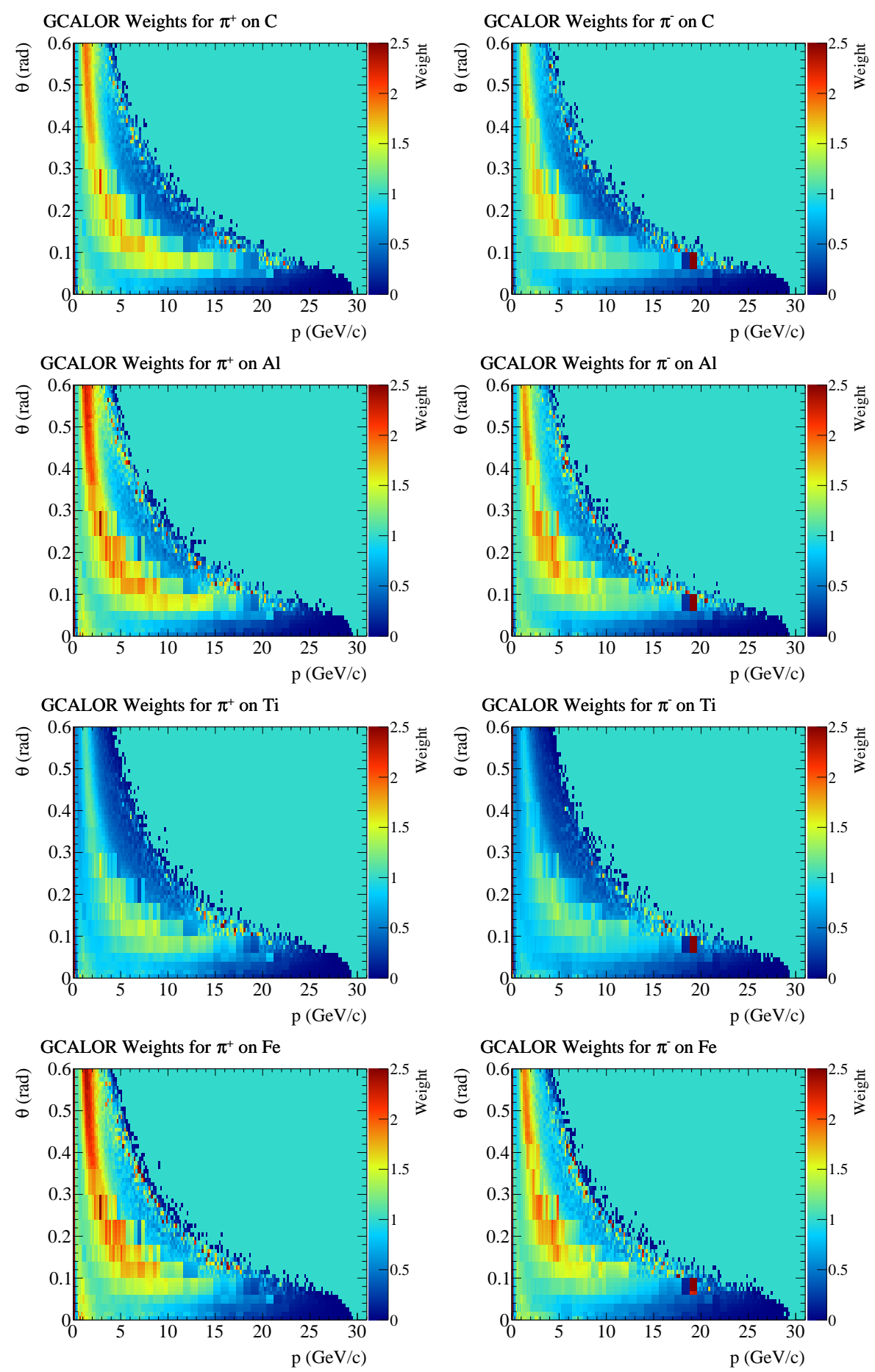

Figure 5.6: The weights applied to meson multiplicities modelled by GCALOR. From left to right: $\pi^{+}, \pi^{-}$production. From top to bottom: C, Al, Ti and Fe targets. Figure reprinted from [85]. Copyright by T2K Collaboration. 
Table 5.7: The fitted target scaling parameters for each particle type. The $\chi^{2} /$ d.o.f listed here include the data point error scaling.

\begin{tabular}{l|c|c|c|c}
\hline \hline & $\pi^{+}$ & $\pi^{-}$ & $K^{+}$ & $K^{-}$ \\
\hline & \multicolumn{4}{|c}{ Fitted $\chi^{2} /$ dof } \\
\hline & $549 / 540$ & $474 / 493$ & $272 / 267$ & $151 / 179$ \\
\hline Parameter & \multicolumn{4}{|c}{ Fitted Value } \\
\hline$P_{0}$ & 0.705 & 0.825 & 0.879 & 0.719 \\
$P_{1}$ & -0.484 & -0.659 & -0.612 & -0.146 \\
$P_{2}$ & 0.199 & -0.142 & -0.157 & -0.333 \\
$P_{3}$ & 0.140 & 0.220 & 0.160 & -0.338 \\
$P_{4}$ & -0.085 & 0.156 & 0.131 & 0.150 \\
$P_{5}$ & 0.045 & 0.252 & 0.323 & 0.725 \\
\hline \hline
\end{tabular}

baryons of all species could also be produced from the break-up of the target nucleus, or through the production of baryon anti-baryon pairs. Typically, the baryon in the final state carrying the vast majority of the total longitudinal momentum, $p_{\mathrm{L}}$, is referred to as the leading baryon. Conservation of the total number of leading baryons has been imposed. In other words, the number of leading baryons before and after tuning should stay unchanged:

$$
N_{\text {lead }}^{\text {before }}=N_{\text {lead }}^{\text {after }} \text {. }
$$

Two distinct sets of weights are applied to secondary baryons produced in the T2K target. For the region of phase space where data is well approximated by the BMPT parametrization, the baryon tuning weight is given by:

$$
\mathrm{W}_{\text {inside }}(p, \theta)=\left[\frac{d^{2} \mathrm{n}_{\text {thin }}}{d p d \theta}\right]_{\text {BMPT }} /\left[\frac{d^{2} \mathrm{n}_{\text {thin }}}{d p d \theta}\right]_{\text {FLUKA }} .
$$

In the region where the BMPT parametrization fails to describe existing data, leading baryons are assigned a constant weight of $\mathrm{W}_{\text {outside }}^{\text {lead }}$, ensuring overall conservation of leading baryon number, whereas non-leading baryons are simply not tuned. Thus, summing over all the $(p, \theta)$ analysis bins, and equating the number of leading baryons before and after tuning, produces the following expression:

$$
1=\frac{N_{\text {lead }}^{\text {before }}}{N_{\text {lead }}^{\text {after }}}=\frac{\sum_{i} N_{\text {lead }, i}^{\text {before }}}{\sum_{i \in \text { inside }} \mathrm{W}_{\text {inside }, i} N_{\text {lead }, i}^{\text {before }}+\sum_{i \in \text { outside }} \mathrm{W}_{\text {outside }}^{\text {lead }} N_{\text {lead }, i}^{\text {before }}},
$$



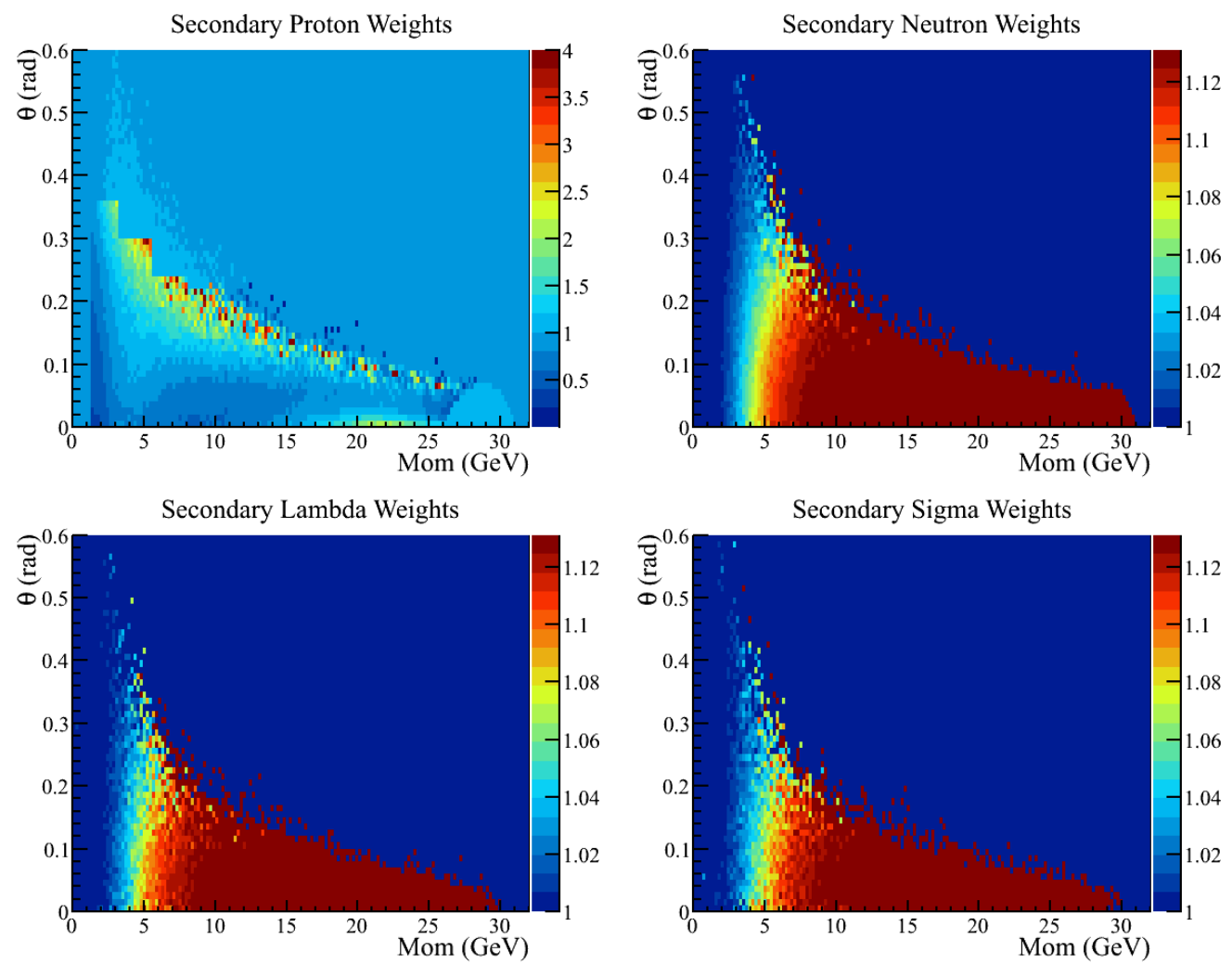

Figure 5.7: Secondary baryon weights for the FLUKA Monte Carlo are shown. These are the weights used to calculate the tuned neutrino flux for T2K analyses. Figure reprinted from [85]. Copyright by T2K Collaboration.

from which the value of $\mathrm{W}_{\text {outside }}^{\text {lead }}$ is to be derived. Thus, for all $(p, \theta)$ bins that cannot be adequately described by the BMPT parametrization, the following baryon multiplicity weight has to be applied to ensure conservation of leading baryon number:

$$
\mathrm{W}_{\text {outside }}(p, \theta)=f_{\text {lead }, i} \times \underbrace{\frac{N_{\text {lead }}^{\text {before }}-N_{\text {lead, inside }}^{\text {after }}}{N_{\text {lead, outside }}^{\text {before }}}}_{\mathrm{W}_{\text {outside }}^{\text {lead }}}+\left(1-f_{\text {lead }, i}\right) \times 1 \text {, }
$$

where $f_{\text {lead }, i}$ denotes the fraction of leading baryons in the $(p, \theta)$ analysis bin before tuning, obtained from the nominal FLUKA simulation, and $N_{\text {lead, inside }}^{\text {after }}$ number of leading baryons after tuning, summed over all the bins where the BMPT parametrization captures the data behaviour. The baryon multiplicity weights constructed in this way for different baryon species are shown in Figure 5.7. 
Table 5.8: Production cross-section interpolated from the T2K flux paper.

\begin{tabular}{l|c|c|c}
\hline \hline Target & $p_{\text {inc }}^{\text {HARP }}[\mathrm{GeV} / \mathrm{c}]$ & $\sigma_{\text {prod }}[\mathrm{mb}]$ & $\Delta \sigma_{\text {prod }}[\mathrm{mb}]$ \\
\hline $\mathrm{C}$ & 3 & 188.4 & 8.0 \\
$\mathrm{C}$ & 5 & 200.0 & 8.8 \\
$\mathrm{C}$ & 8 & 204.0 & 5.1 \\
$\mathrm{C}$ & 12 & 198.5 & 5.1 \\
$\mathrm{Al}$ & 3 & 395.7 & 15.0 \\
$\mathrm{Al}$ & 5 & 389.9 & 15.8 \\
$\mathrm{Al}$ & 8 & 372.0 & 6.4 \\
$\mathrm{Al}$ & 12 & 362.0 & 8.1 \\
\hline \hline
\end{tabular}

\subsubsection{Pion rescattering multiplicity weights}

Conveniently, secondary and tertiary interactions with incident pions can also be tuned, using measurements from HARP[118]. The HARP detector measured the pion production from thin targets (5\% interaction length) and with incident pion beams. HARP took measurements of the inclusive differential production cross sections for interactions of type $\pi^{+}+\mathrm{X} \rightarrow \pi^{ \pm}+\mathrm{Y}$ and $\pi^{-}+\mathrm{X} \rightarrow \pi^{ \pm}+\mathrm{Y}$, with target nuclei $\mathrm{X} \in\{\mathrm{Be}, \mathrm{C}, \mathrm{Al}, \mathrm{Cu}, \mathrm{Sn}, \mathrm{Ta}, \mathrm{Pb}\}$. Measurements were taken at pion incident momenta $p_{\text {inc }}^{\mathrm{HARP}} \in\{3,5,8,12\} \mathrm{GeV} / \mathrm{c}$. The multiplicity is calculated using the usual recipe. First the differential cross section is normalised to the production cross section. For example, a comprehensive summary of production cross section measurements for pions interacting on carbon is shown in Figure 5.11. Using such datasets, the production cross sections can be interpolated to HARP beam energies. Of all the HARP target nuclei, most relevant for T2K are carbon and aluminium. The relevant production cross sections are given in Table 5.8.

Finally, multiplicity weights have been constructed for tuning in-target (see Figure 5.8) and out-of-target (see Figure 5.9) pion scattering. As usual, FLUKA and GEANT3 have been used for modelling in-target and out-of-target interactions, respecively, and the multiplicity weights are constructed as the ratio of the measured and the simulated multiplicity. These multiplicity weights are given at HARP incident momenta. For interactions whose incident pion momentum $p$ lies between 

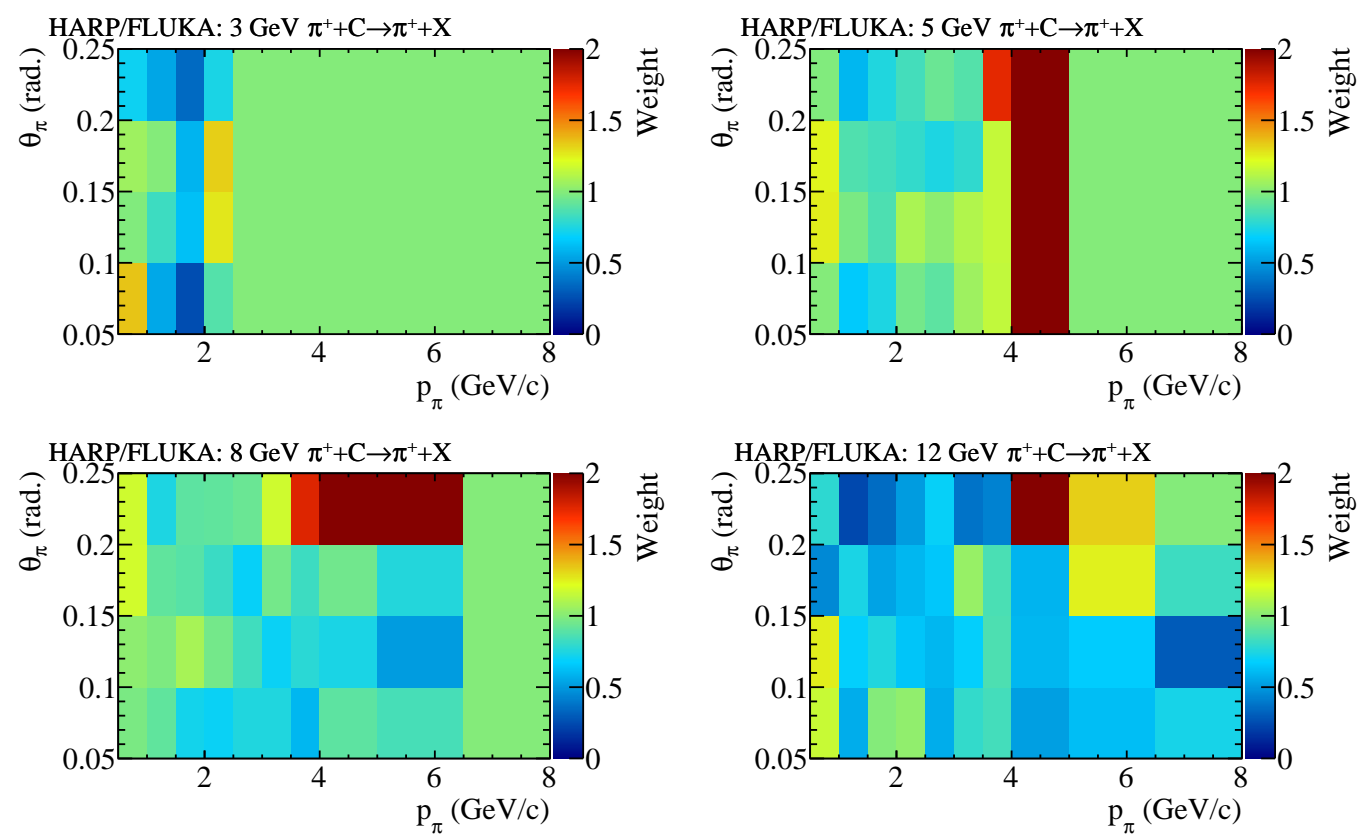

Figure 5.8: Ratio between the pion multiplicities of the HARP data and the FLUKA simulation (on carbon target). Here, weights have been depicted for inclusive in-target interactions with incident $3 \mathrm{GeV} / \mathrm{c} \pi^{+}$, and outgoing $\pi^{+}$. Analogously, weights have been constructed for other combinations of incoming and outgoing pion charge, and for different incident pion momenta. The binning used here is adopted from the release format of the HARP measurement. Figure reprinted from [85]. Copyright by T2K Collaboration.

two HARP values, $p_{\text {inc } 1}^{\text {HARP }}<p<p_{\text {inc } 2}^{\text {HARP }}$, Feynman scaling to both of those HARP momenta is applied. The multiplicity weight at momentum $p$ is taken from a linear extrapolation between the weights at momenta $p_{\text {inc } 1}^{\mathrm{HARP}}$ and $p_{\text {inc } 2}^{\mathrm{HARP}}$. For the case where the momentum of interest lies outside the range of the HARP beam, $p>p_{\text {inc } \operatorname{HAx}}^{\mathrm{HARP}}\left(p<p_{\text {inc min }}^{\mathrm{HARP}}\right)$, the multiplicity weight is taken from Feynman scaling to $p=p_{\text {inc max }}^{\mathrm{HARP}}\left(p=p_{\text {inc min }}^{\mathrm{HARP}}\right)$, respectively.

\section{$5.4 \quad$ Interaction length tuning}

Interaction length weights are applied to tune the simulated production cross section, $\sigma_{\text {prod }}^{\text {sim }}$, to the value $\sigma_{\text {prod }}^{\text {data }}$, measured from data. The relevant process to consider is shown in Fig. 5.10a, and depicts a particle interacting after having travelled a 

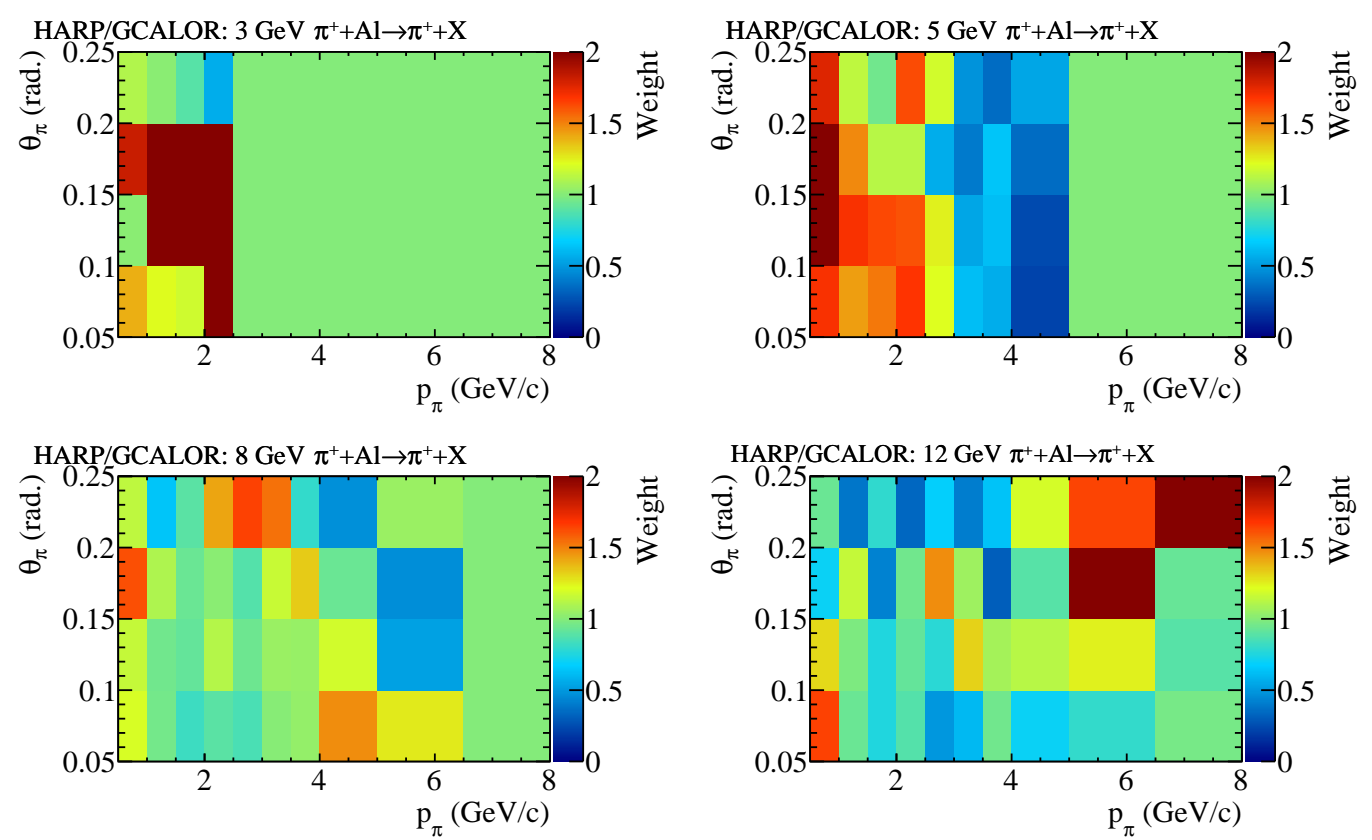

Figure 5.9: Ratio between the pion multiplicities of the HARP data and the GCALOR simulation (on aluminium target). Here, weights have been depicted for inclusive out-oftarget interactions with incident $3 \mathrm{GeV} / \mathrm{c} \pi^{+}$, and outgoing $\pi^{+}$. Analogously, weights have been constructed for other combinations of incoming and outgoing pion charge, and for different incident pion momenta. The binning used here is adopted from the release format of the HARP measurement. Figure reprinted from [85]. Copyright by T2K Collaboration.

distance $x_{1}$ through the material. This probability is given by:

$$
\begin{aligned}
& \mathrm{P}_{\text {int }}\left(x_{1}, \sigma_{\text {prod }}\right)=\int_{x_{1}}^{x_{1}+\Delta x} e^{-\rho \sigma_{\text {prod }} x} \rho \sigma_{\text {prod }} d x= \\
& =e^{-\rho \sigma_{\text {prod }} x_{1}} \rho \sigma_{\text {prod }} \Delta x
\end{aligned}
$$

where $\rho$ is the number density of target nuclei in the material, and $\Delta x$ is the infinitesimal distance over which the interaction occurs. Analogously, the survival probability, for the particle to escape from the target material after travelling across distance $x_{1}$, as shown in Figure $5.10 \mathrm{~b}$, is given by:

$$
\mathrm{P}_{\text {surv }}\left(x_{1}, \sigma_{\text {prod }}\right)=e^{-\rho \sigma_{\text {prod }} x_{1}}
$$

The weight $\mathrm{W}$ that needs to be applied to tune the simulated production cross section, $\sigma_{\text {prod }}^{\text {sim }} \rightarrow \sigma_{\text {prod }}^{\text {data }}$, is given by the ratio of overall probabilities from data and from simulation. For interacting particles the interaction length weight 


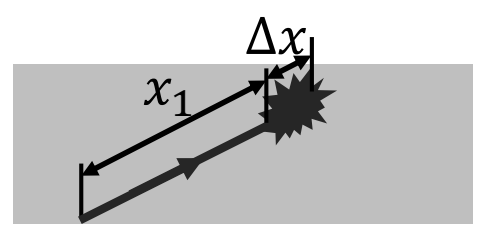

(a) Interacting particle

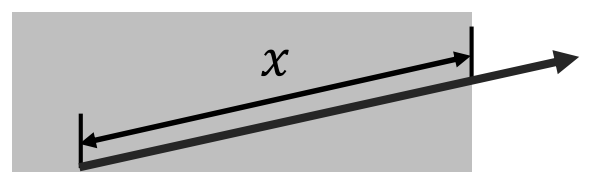

(b) Through-going particle

Figure 5.10: Interaction length weights are applied for both interacting (see Figure 5.10a) and through-going (see Figure 5.10b) particles, refelcting the fect that the interaction and survival probabilities both depend on $\sigma_{\text {prod }}$.

that needs to be applied is:

$$
\mathrm{W}_{\text {int length }}\left(x_{1}, \sigma_{\text {prod }}^{\text {sim }}, \sigma_{\text {prod }}^{\text {data }}\right)=\frac{\mathrm{P}_{\text {int }}^{\text {data }}}{\mathrm{P}_{\text {int }}^{\text {sim }}}=\frac{\sigma_{\text {prod }}^{\text {data }}}{\sigma_{\text {prod }}^{\text {sim }}} e^{-x_{1}\left[\sigma_{\text {prod }}^{\text {data }}-\sigma_{\text {prod }}^{\text {sim }}\right] \rho} .
$$

Conveniently, the infinitesimal distance $\Delta x$ over which the interaction occurs cancels out from the weight expression. For through-going particles, the interaction length does not depend on the production cross section ratio and is given by:

$$
\mathrm{W}_{\text {int length }}\left(x_{1}, \sigma_{\text {prod }}^{\text {sim }}, \sigma_{\text {prod }}^{\text {data }}\right)=\frac{\mathrm{P}_{\text {surv }}^{\text {data }}}{\mathrm{P}_{\text {surv }}^{\text {sim }}}=e^{-x_{1}\left[\sigma_{\text {prod }}^{\text {data }}-\sigma_{\text {prod }}^{\text {sim }}\right] \rho} .
$$

As already mentioned, in-target and out-of-target interactions for T2K neutrinos are handled by FLUKA and GCALOR, respectively. The FLUKA production cross section on carbon shows relatively good agreement with data over a wide range of momenta for different species of incident hadrons, as shown in Fig. 5.11. Thus, the nominal FLUKA in-target production cross sections are not tuned, apart from high energy protons $(p>20 \mathrm{GeV} / \mathrm{c})$ for which the production cross section is tuned to the NA61 measurement of $\sigma_{\text {prod }}=230.7 \pm 2.8($ stat $) \pm 1.2(\text { det })_{-3.5}^{+6.3}(\bmod )[98]$. On the other hand, the agreement between GCALOR cross sections and data for interactions on different secondary beamline materials, such as aluminium, is poor, as demonstarted in Fig. 5.11. Consequently, out-of-target production cross sections modelled by GCALOR are tuned to FLUKA. Using FLUKA as "data" is also convenient since it allows for a smooth production cross section prediction over a range of momenta. In other words, for the purposes of interaction length tuning $\sigma_{\text {prod }}^{\text {data }}=\sigma_{\text {prod }}^{\text {FLUKA }}$ in Equation 5.16 and 5.17 . 

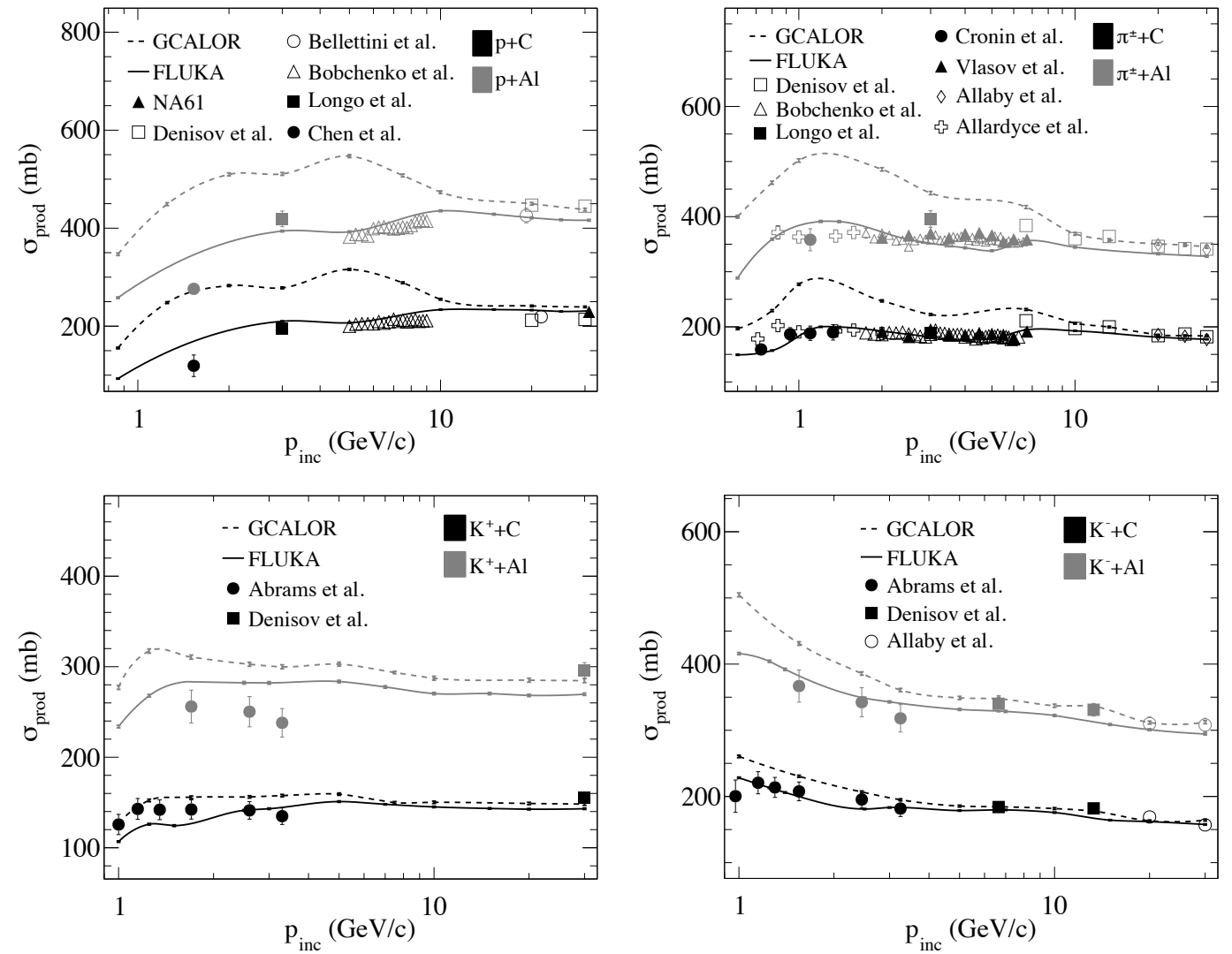

Figure 5.11: Comparison of production cross sections for interactions of protons (top left), $\pi^{ \pm}$(top right), $K^{+}$(bottom left) and $K^{-}$(bottom right) on carbon (black) and aluminium (gray), as a function of incident hadron momentum in the lab frame. The markers correspond to data points, and the lines are predictions from different models. The behaviour of the data is best captured by the FLUKA interaction model, whereas predictions from GCALOR deviate more considerably from the measurements. Reprinted figure with permission from K. Abe et al. (T2K Collaboration), Phys. Rev. D, vol. 87, p. 012001 (2013)[72]. Copyright (2013) by the American Physical Society.

\subsection{Flux uncertainties}

When discussing systematic uncertainties for T2K analyses, covariance matrices are commonly employed as convenient descriptions of both the standard deviation of individual parameters affecting the analyses, and correlations among these parameters. For $n$ systematic parameters $y_{i}$, the associated covariance matrix $V$ is of dimension $\mathrm{n} \times \mathrm{n}$. The diagonal matrix elements, $\mathrm{V}_{\mathrm{ii}}=\sigma_{\mathrm{i}}^{2}$, describe the deviation $\sigma_{\mathrm{i}}$ of individual parameters $\mathrm{y}_{\mathrm{i}}$, whereas the off diagonal elements, $\mathrm{V}_{\mathrm{ij}}=\rho_{\mathrm{ij}} \sigma_{\mathrm{i}} \sigma_{\mathrm{j}}$, encode the strength of correlation $\rho_{\mathrm{ij}}$ among parameters $\mathrm{y}_{\mathrm{i}}$ and $\mathrm{y}_{\mathrm{j}}$. 


\subsubsection{Uncertainties from hadron interaction modelling}

The flux uncertainties due to the modelling of hadronic interactions are summarised in Figure 5.16 for the flux at Super-K, and in Figure 5.17 for the flux at ND280. These are the dominant type of uncertainties for the T2K flux calculation. The different sources of hadron interaction modelling uncertainties, and the techniques used for their estimation, will be discussed below.

\section{Multiplicity Uncertainty}

Variations in the thin target multiplicity measurements, commonly referred to as throws, are generated from the NA61 covariance matrix, which has been produced by $\mathrm{T} 2 \mathrm{~K}$, based on the evaluation of systematic and statistical errors on the NA61 measurement. This matrix is non-diagonal, reflecting existing correlations between $(p, \theta)$ analysis bins. Throws from such a non-diagonal covariance matrix are generated using the linear algebraic method of Cholesky decomposition[119]. Any well-defined physical covariance matrix $Q$, of dimension $n \times n$ is expected to be symmetric and positive-definite, and can thus be Cholesky decomposed into a product of a lower triangular matrix $\mathrm{L}$ and its transpose $\mathrm{L}^{\mathrm{T}}, \mathrm{Q}=\mathrm{LL}^{\mathrm{T}}$. Now, let us define the $\mathrm{n} \times 1$ column vector $\mathbf{y}$ to represent the $n$-dimensional random variable with covariance Q. Thus, the covariance matrix can be written as the expectation of the product of vector $\mathbf{y}$ and its transpose, $\mathrm{Q}=\mathbb{E}\left(\mathbf{y y}^{\mathrm{T}}\right)$. The covariance of a multi-dimensional variable $\mathbf{x}$, whose vector entries $\mathrm{x}_{\mathrm{i}}$ are fully uncorrelated random variables with zero mean and unit variance, $\mathbb{E}\left(\mathrm{x}_{\mathrm{i}} \mathrm{x}_{\mathrm{j}}\right)=\delta_{\mathrm{ij}}$, is simply the identity matrix: $\mathbb{E}\left(\mathbf{x x}^{\mathrm{T}}\right)=\mathbb{I}$. The covariance matrix of the random variable $L x$ is then given by:

$$
\mathbb{E}\left(\operatorname{Lx}(\mathrm{Lx})^{\mathrm{T}}\right)=\mathbb{E}\left(\mathrm{Lxx}^{\mathrm{T}} \mathrm{L}^{\mathrm{T}}\right)=\mathrm{LE}\left(\mathbf{x} \mathbf{x}^{\mathrm{T}}\right) \mathrm{L}^{\mathrm{T}}=\mathrm{LL}^{\mathrm{T}}=\mathrm{Q}
$$

i.e. the random variable $\mathrm{Lx}$ has the same correlation $\mathrm{Q}$ as the variable $\mathbf{y}$.

Following this recipe, multiplicities correlated according to the NA61 covariance are obtained by multiplying uncorrelated multiplicities thrown from standard normal distributions (with $\mu=0$ and $\sigma=1$ ) with the Cholesky decomposition of the 
NA61 covariance. Five hundred multiplicity throws have been made. Each of the multiplicity variations is treated as a toy data sample, and used to recalculate the tuned flux. Elements of the flux covariance matrix due to errors on NA61 multiplicity measurements, $\mathrm{V}_{\mathrm{i}, \mathrm{j}}^{\mathrm{NA} 61}$, are calculated from the resulting $\mathrm{N}=500$ tuned flux toys, with the following formula:

$$
\mathrm{V}_{\mathrm{i}, \mathrm{j}}^{\mathrm{NA} 61}=\frac{1}{\mathrm{~N}} \sum_{\mathrm{k}=1}^{\mathrm{N}}\left(\Phi_{\text {nom }}^{\mathrm{i}}-\Phi_{\text {toy k }}^{\mathrm{i}}\right)\left(\Phi_{\text {nom }}^{\mathrm{j}}-\Phi_{\text {toy k }}^{\mathrm{j}}\right)
$$

where $\Phi_{\text {nom }}^{\mathrm{i}}$ and $\Phi_{\text {toy k }}^{\mathrm{i}}$ are the flux contents in neutrino energy bin i of the nominal tuned flux and the toy $\mathrm{k}$ tuned flux, respectively. A similar approach has been used throughout this thesis, when calculating the effect on the flux error from the errors on various other datasets used in the tuning procedure.

The flux uncertainty due to the energy and target scaling procedures has also been estimated for all cases where such methods are used. Typically this involves the following procedures.

In the context of energy scaling, the BMPT parametrisation is used only when evaluating the flux uncertainty, whereas the nominal flux calculation relies purely on Feynman scaling. For the uncertainty estimate, the BMPT parametrisation is fitted to existing data at a range of beam energies. In regions where there is no data, extrapolations are made between the closest available datasets. This BMPT parametrisation is used to recalculate multiplicity weights at the true interaction energy. The difference in the nominal flux tuned with Feynman scaling, $\Phi_{\text {Feynman, }}$, and the flux tuned with the BMPT method, $\Phi_{\mathrm{BMPT}}$, is taken as the Feynman scaling uncertainty. Thus, the covariance error matrix due to Feynman scaling is estimated from

$$
\mathrm{V}_{\mathrm{i}, \mathrm{j}}^{\text {Feynman }}=\left(\Phi_{\text {Feynman }}^{\mathrm{i}}-\Phi_{\mathrm{BMPT}}^{\mathrm{i}}\right)\left(\Phi_{\text {Feynman }}^{\mathrm{j}}-\Phi_{\mathrm{BMPT}}^{\mathrm{j}}\right)
$$

where $\Phi_{\text {Feynman }}^{\mathrm{i}}$ and $\Phi_{\mathrm{BMPT}}^{\mathrm{i}}$ are the flux contents in neutrino energy bin $\mathrm{i}$ for the two tuning approaches.

The validity of the target scaling approximation is tested with the Eichten dataset, which was not used to derive the fit parameters in Table 5.7. The Eichten data on 
beryllium is scaled for aluminium and copper targets, and compared to real Eichten data at those targets. Additional multiplicity normalization and shape uncertainties are inferred from such comparisons, and propagated to flux uncertainties.

\section{Nucleon Uncertainty}

The uncertainty from the tuning of interactions of secondary baryons has been estimated. Analogously to the case of meson tuning, Cholesky decomposition is used to throw 100 variations on the measurements of baryon multiplicity from NA61 and Allaby. For every multiplicity variation, baryon tuning weights are recalculated and flux tuning reapplied, giving 100 tuned flux toys from which the covariance matrix associated with this error source has been estimated.

\section{Pion rescattering uncertainty}

The uncertainty due to pion rescattering has been estimated from the difference in the flux with and without applying pion rescattering multiplicity weights. Thus, the covariance error matrix due to pion rescattering is estimated from

$$
\mathrm{V}_{\mathrm{i}, \mathrm{j}}^{\pi \text { scat }}=\left(\Phi_{\text {without } \pi}^{\mathrm{i}}-\Phi_{\text {with } \pi}^{\mathrm{i}}\right)\left(\Phi_{\text {without } \pi}^{\mathrm{j}}-\Phi_{\text {with } \pi}^{\mathrm{j}}\right)
$$

where $\Phi_{\text {without } \pi}^{\mathrm{i}}$ and $\Phi_{\text {with } \pi}^{\mathrm{i}}$ are the flux contents in neutrino energy bin $\mathrm{i}$ of the tuned flux without and with pion rescattering weights applied, respectively. The uncertainty for rescattering on iron is accounted for by double the uncertainty from rescattering on aluminium. This uncertainty doubling is applied only for the wrong-sign flux component, where fake-data studies showed that equal numbers of interactions on both aluminium and iron is expected. For the right sign flux the number of out-of-target interactions is significantly reduced, and uncertainties in modelling of pion rescattering on iron have been neglected in this case.

\section{Hadron interaction length uncertainty}

There exists ambiguity in literature (see Fig. 5.12) on whether certain datasets (most notably Denisov et. al[120]) measure the production cross section or the total inelastic cross section (which also includes the quasi-elastic scattering of hadrons 
off from individual nucleons from the target nucleus). The uncertainty on the production cross section is thus taken to be equal to the quasi-elastic cross section at the relevant energy. Quasi-elastic cross sections are calculated based on the Bellettini et al. model[105] and using existing measurements of elastic scattering of pions, kaons and protons on individual nucleons:

$$
\begin{gathered}
\sigma_{\text {quasi-el }}(\mathrm{p}+\mathrm{N})=0.8\left(\sigma_{\mathrm{el}}(\mathrm{p}+\mathrm{p})+\sigma_{\mathrm{el}}(\mathrm{p}+\mathrm{n})\right) \mathrm{A}^{1 / 3} \\
\sigma_{\text {quasi-el }}(\pi+\mathrm{N})=0.8\left(\sigma_{\mathrm{el}}\left(\pi^{+}+\mathrm{p}\right)+\sigma_{\text {el }}\left(\pi^{-}+\mathrm{p}\right)\right) \mathrm{A}^{1 / 3} \\
\sigma_{\text {quasi-el }}\left(\mathrm{K}^{+}+\mathrm{N}\right)=1.6 \sigma_{\mathrm{el}}\left(\mathrm{K}^{+}+\mathrm{p}\right) \mathrm{A}^{1 / 3} \\
\sigma_{\text {quasi-el }}\left(\mathrm{K}^{-}+\mathrm{N}\right)=0.8\left(\sigma_{\mathrm{el}}\left(\mathrm{K}^{-}+\mathrm{p}\right)+\sigma_{\mathrm{el}}\left(\mathrm{K}^{-}+\mathrm{n}\right)\right) \mathrm{A}^{1 / 3}
\end{gathered}
$$

where A denotes the mass number of the target nucleus N. Using the above equations, the quasielastic cross sections have been calculated for different hadron species at a range of incident momenta relevant for $\nu$ production at T2K. For instance, the quasi-elastic cross section $\sigma_{\text {quasi-el }}(\mathrm{p}+\mathrm{C})$, derived from Equation 5.22, is plotted as a function of momentum in Figure 5.13.

The hadron interaction length uncertainty is propagated directly from the uncertainty on the measured hadron production cross section $\sigma_{\text {data }}$. As already mentioned, this uncertainty is taken to be of the same magnitude as the quasielastic cross section $\sigma_{\text {quasi-el }}$. Hadrons contributing dominantly to the T2K flux are split into 7 kinematic ranges as shown in Tab. 5.9, which act as dials for variations on the production cross section $\sigma_{\text {data }}$. These ranges have been introduced to facilitate for the correlated variation in the simulated production cross section of particles with certain properties. For instance, according to Tab. 5.9 (see dial 2), the production cross section of protons with momenta $p \in\{4,12\} \mathrm{GeV} / \mathrm{c}$ and pions with momenta $p \in\{2,6\} \mathrm{GeV} / \mathrm{c}$ is to be correlated. The reader will notice from Figure 5.14 that within this momentum range, most of the available production cross section measurements for both protons and pions come from the same dataset, namely that of Bobchenko et al.[123]. Thus, regions in momentum space populated 

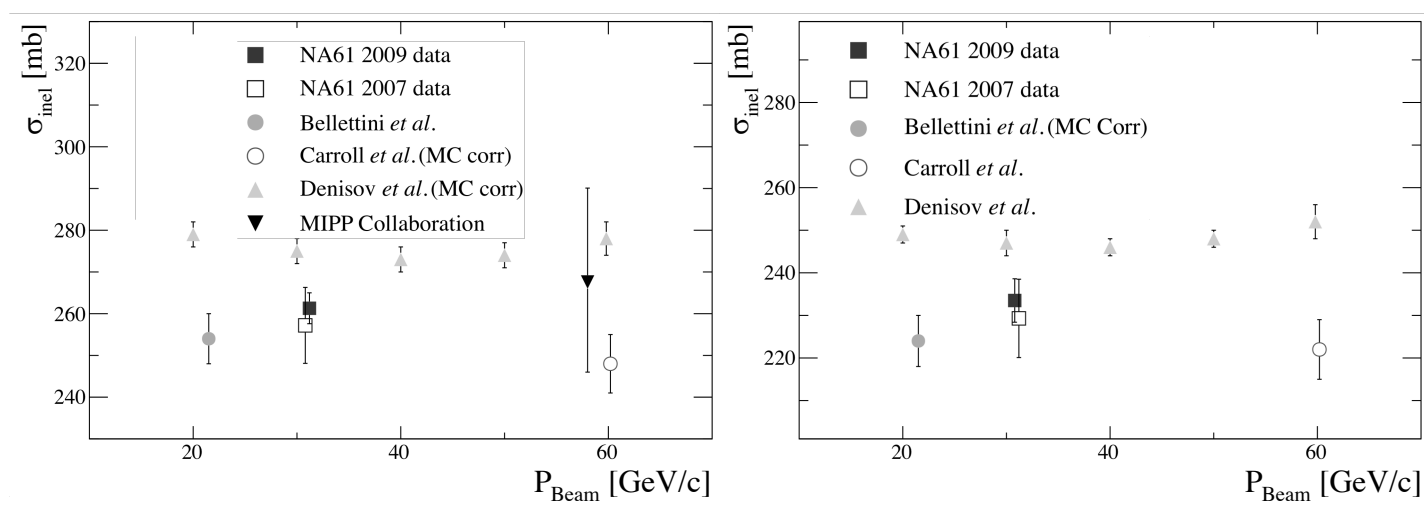

Figure 5.12: A review of measurements of inelastic (left) and production (right) cross sections of protons at different incident momenta. The following datasets have been considered: Bellettini et al. (full circles) [105], Carroll et al. (empty circles) [121], MIPP (black triangles) [122] and Denisov et al. measurements (grey triangles) [120]. Bellettini et. al, Carroll et. al and MIPP measured the inelastic cross section, while values from Denisov et al. correspond to production cross section as stated in their article, but the quantity is somewhat ambiguous. NA61 measurements are also shown (full and empty squares). To facilitate comparisons between datasets, the quasi-elastic cross section component, estimated with GEANT4, has been added to Carroll et al. and Denisov et al. measurements shown in the left plot, and subtracted from Bellettini et al. measurement shown in the right plot (labelled as MC corrected). Figure reprinted from [85]. Copyright by $\mathrm{T} 2 \mathrm{~K}$ Collaboration.

by production cross section measurements from the same dataset or groups of datasets are assigned to the same kinematic range in Table 5.9, guided by the fact that Monte Carlo generators are to a large degree tuned to existing data. The different dials are treated as independent, and each thrown from the standard normal distribution. The production cross sections are varied through variations of those dials via the following formula:

$$
\Delta \sigma_{\text {data }}(p)=\text { dial } \times \sigma_{\text {quasi-el }}(p)
$$

500 sets of dials have been thrown and used to generate 500 variations on the production cross sections. Each of the systematic cross section variations is treated as a toy data sample, and used to recalculate the tuned flux. The hadronic interaction length covariance matrix is calculated from the resulting 500 tuned flux toys. The hadron interaction length uncertainty on the T2K flux is shown in Figures 5.16 and 5.17, at Super-K and ND280, respectively. 


\begin{tabular}{c|c|c|c|c} 
dial & $\mathrm{p}$ & $\pi^{ \pm}$ & $\mathrm{K}^{+}$ & $\mathrm{K}^{-}$ \\
\hline 1 & $p<4$ & - & - & - \\
\hline 2 & $p \in\{4,12\}$ & $p \in\{2,6\}$ & - & - \\
\hline 3 & $p>12$ & - & - & - \\
\hline 4 & - & $p<2$ & - & - \\
\hline 5 & - & - & $p<5$ & $p<5$ \\
\hline 6 & - & $p>6$ & $p>5$ & $p \in\{5,17\}$ \\
\hline 7 & - & - & - & $p>17$
\end{tabular}

Table 5.9: Dials used for independently applying systematic variations on the production cross sections of pions, kaons and protons with different incident momenta (given in $\mathrm{GeV} / \mathrm{c}$ ), which contribute to the T2K neutrino flux. Once the value of the dial has been specified for a certain hadron, the variation on the production cross section is calculated from Eq. 5.26.

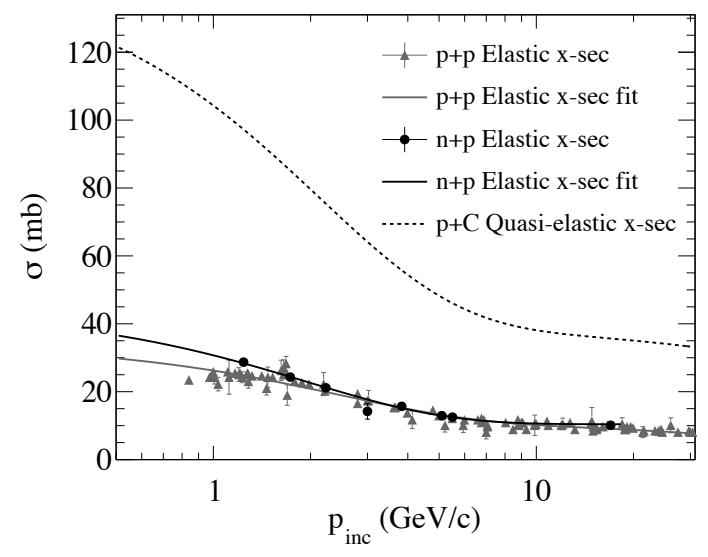

Figure 5.13: The quasi-elastic cross section $\sigma_{\text {quasi-el }}(\mathrm{p}+\mathrm{C})$ for protons interacting on carbon, derived from Equation 5.22, plotted as a function of incident proton momentum. The elastic scattering cross sections of nucleons, $\sigma_{\mathrm{el}}(\mathrm{p}+\mathrm{p})$ and $\sigma_{\mathrm{el}}(\mathrm{p}+\mathrm{n})$, are obtained from fits to all existing data, summarised in PDG 2018[54]. Reprinted figure with permission from K. Abe et al. (T2K Collaboration), Phys. Rev. D, vol. 87, p. 012001 (2013)[72]. Copyright (2013) by the American Physical Society.

A significant $(\sim 50 \%)$ reduction of the hadron interaction length uncertainty, for the right-sign flux at energies below $2 \mathrm{GeV}$, is achieved with the inclusion of the 2009 replica target dataset. This will be discussed in Chapter 6 .

\subsubsection{Other sources of T2K flux uncertainty}

Various flux systematics unrelated to hadron interaction modelling have also been considered. The flux uncertainties due to these error sources will be summarised 

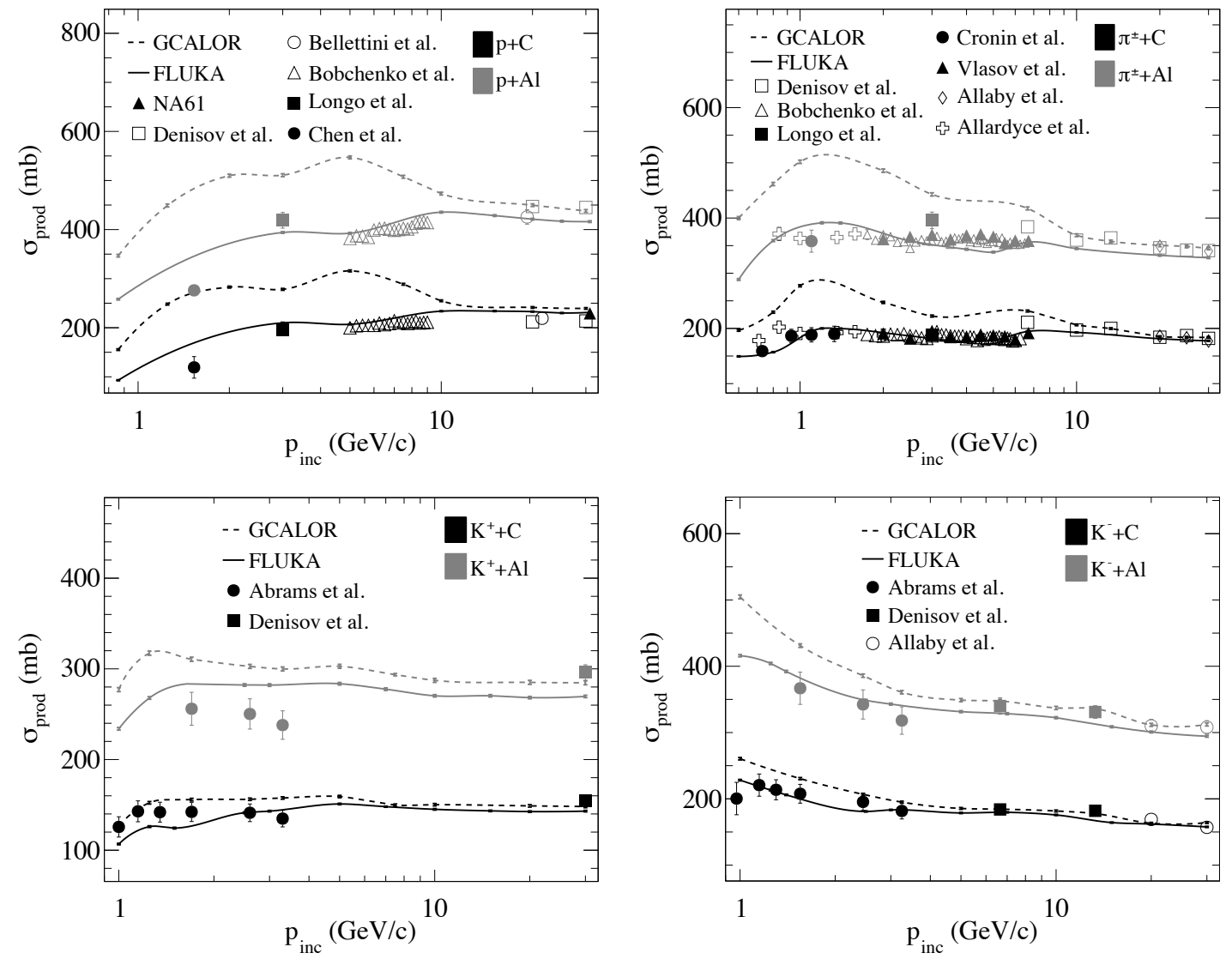

Figure 5.14: Measurements of proton (top left), $\pi^{+}$(top right), $K^{+}$(bottom left) and $K^{-}$(bottom right) production cross sections on carbon, alongside predictions from different simulations. For detailed references to all datasets shown in the Figure, the reader is referred to [72]. Reprinted figure with permission from K. Abe et al. (T2K Collaboration), Phys. Rev. D, vol. 87, p. 012001 (2013)[72]. Copyright (2013) by the American Physical Society.

in Figure 5.18 for the flux at Super-K, and in Figure 5.19 for the flux at ND280. The techniques used for the estimation of these flux uncertainties are discussed in detail in this chapter. For the sake of completeness, the total thin-tuned flux uncertainties, obtained by combining hadronic and non-hadronic error sources, will be given in Figure 5.20 (at SK) and Figure 5.21 (at ND280).

When estimating most of non-hadronic flux uncertainties, $\pm 1 \sigma$ variations in the position of different beamline components in the simulated geometry have been considered, and the associated covariance matrix calculated by examining 
the change in flux under those variations:

$$
\mathrm{V}_{\mathrm{i}, \mathrm{j}}^{\mathrm{syst}}=\frac{1}{2} \sum_{\mathrm{k}=+1 \sigma,-1 \sigma}\left(\Phi_{\mathrm{nom}}^{\mathrm{i}}-\Phi_{\mathrm{k}}^{\mathrm{i}}\right)\left(\Phi_{\mathrm{nom}}^{\mathrm{j}}-\Phi_{\mathrm{k}}^{\mathrm{j}}\right)
$$

where $\Phi_{\text {nom }}^{\mathrm{i}}$ is the nominal flux, and $\Phi_{ \pm 1 \sigma}^{\mathrm{i}}$ is the flux after applying the $\pm 1 \sigma$ variation to one of the parameters, in neutrino energy bin $i$.

Beam profile uncertainties are crucial inputs for modelling the flux. The importance of these parameters can best be understood by considering their impact on hadronic interactions. Variations in beam position, divergence and width directly affect the average distance travelled by beam protons through the target. For example, if the beam centre is moved sideways, parts of the beam might miss the target. Alternatively, if the beam has a large divergence, the fraction of noninteracting beam protons which exit from the sides of the target rather than through the downstream target face increases. Also, since ND280 and SK detectors are displaced from the beam axis mostly along the vertical direction, variations in ( $y$, $\left.\theta_{y}\right)$ affect the off-axis angle, and thus energy, of the T2K beam. For estimating these effects, variations in beam profile parameters are separately thrown from within their uncertainties, accounting for correlations between parameters such as position and divergence. The only non-negligible effect on the flux comes from uncertainties in $\left(y, \theta_{y}\right)$.

The direction of the T2K neutrino beam is measured for each data-taking period by the INGRID detector. Errors in beam direction affect the off-axis angle at the positions of ND280 and Super-K. Variations of $\pm 1 \sigma$ in the beam direction are applied in the simulation to estimate the effect of this uncertainty. Off-axis angle uncertainties are separately evaluated for neutrino and anti-neutrino modes of operation, and treated as uncorrelated.

The simulated horn current in all three horns was varied in a correlated manner for a conservative estimate of the horn current uncertainty. Current variations of $\sigma= \pm 5 \mathrm{kA}(2 \%)$, derived from horn CT measurements, have been applied. Additionally, from magnetic field measurements with the spare horn 1, an anomalous 
on-axis field component was observed[92], whereas strictly speaking the only nonzero field is expected between the walls of the inner and outer horn conductors. The measured field can be modelled as originating from an asymmetric vector current $\mathbf{I}$, whose magnitude is $2 \%$ of the total horn current $I_{0}$, and which is given by:

$$
\mathbf{I}=\frac{\alpha I_{0}}{2 \pi r} \sin \phi \mathbf{z}
$$

where $\alpha=2 \%, \phi$ is the azimuthal angle and $\mathbf{z}$ is the unit vector pointing along the horn axis (longitudinal direction). A schematic representation of this asymmetric field and the associated scalar field potential is shown in Figure 5.15. The effect on the flux uncertainty was estimated by observing the flux modelled in JNUBEAM with and without this asymmetric field component.

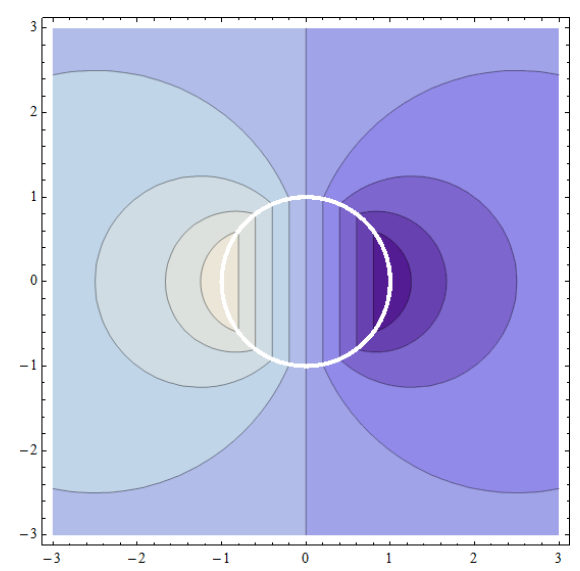

(a) Scalar Potential

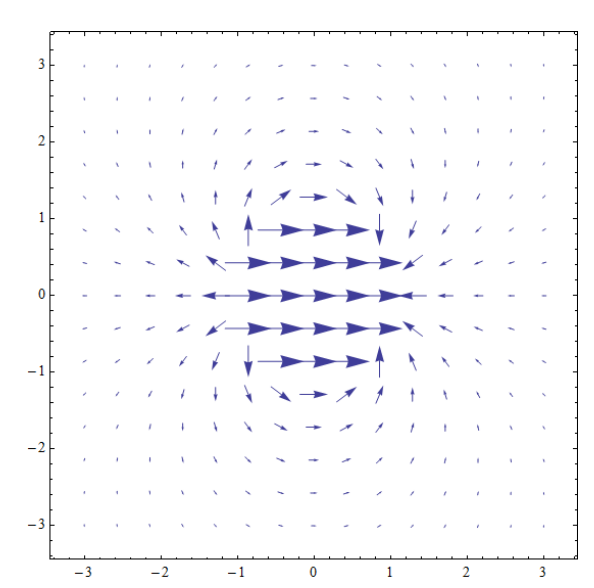

(b) Magnetic Field

Figure 5.15: The asymmetric horn field component modelled in JNUBEAM. On the left is the magnetic scalar potential, and on the right is the associated field. Figure reprinted from [124]. Copyright by T2K Collaboration.

The horn positions have been surveyed after instalment. From such surveys, positional alignment uncertainties of $\sigma=1 \mathrm{~mm}$ have been estimated for horns 2 and 3. The effect on the flux was estimated by changing the horn 2 and 3 positions in the simulation. The horn 1 position uncertainty is included in the beam profile uncertainty. Angular alignment uncertainties of horns 2 and 3 were shown to have a negligible effect on the flux. The angular alignment of horn 1 has a sizable effect on the flux as it results in the rotation of the whole T2K target. 
Horn 1 was rotated by $\pm 0.2 \mathrm{mrad}$ around the horizontal and vertical directions to estimate this component of the flux error.

The T2K target position after instalment was surveyed relative to the axis of the first horn. The upstream target face was measured to be centred on the horn axis, whereas the downstream target face was displaced from the horn axis. This resulted in a rotation by $1.3(0.1) \mathrm{mrad}$ in the horizontal (vertical) plane, of the downstream with respect to the upstream target face. The change in the predicted neutrino flux due to the measured target misalignment with respect to the first horn has been estimated by rotating the target position encoded in the Monte Carlo simulation, and comparing the new fluxes to the nominal.

Many of the secondary beamline components are continuously cooled during beam operation. Especially significant for neutrino production might be the horn cooling system of the first horn, and any accompanying water accumulation. To estimate this effect, an additional $1 \mathrm{~mm}$ thick water layer is simulated around the horn 1 inner conductor. The error is calculated from the difference in flux obtained with and without this additional water layer.

A fully correlated $2.6 \%$ uncertainty in POT number has been assigned for all neutrino energy bins, based on the absolute calibration and analysis of CT05 monitor current readings on a spill-by-spill basis. This uncertainty cancels out when taking the ratio of fluxes in ND280 and Super-K, and thus does not affect the T2K oscillation analysis. 
SK: Neutrino Mode, $v_{\mu}$

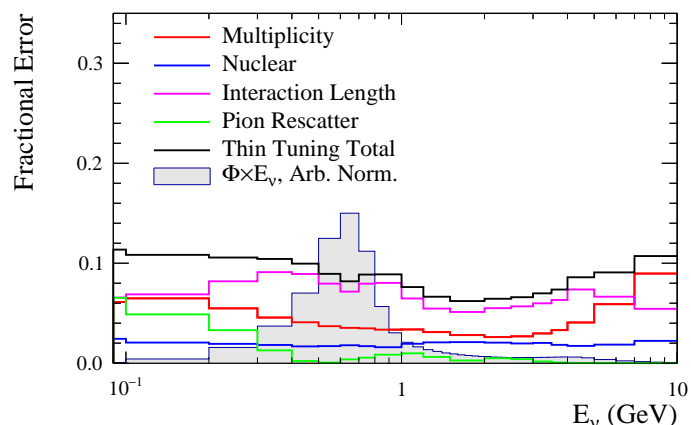

SK: Neutrino Mode, $v_{\mathrm{e}}$

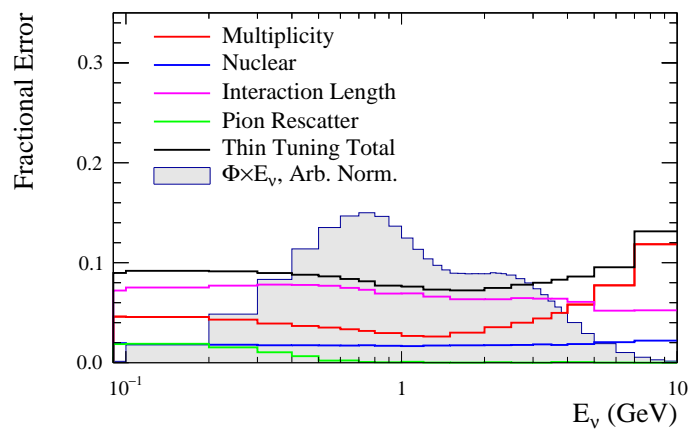

SK: Antineutrino Mode, $v_{\mu}$

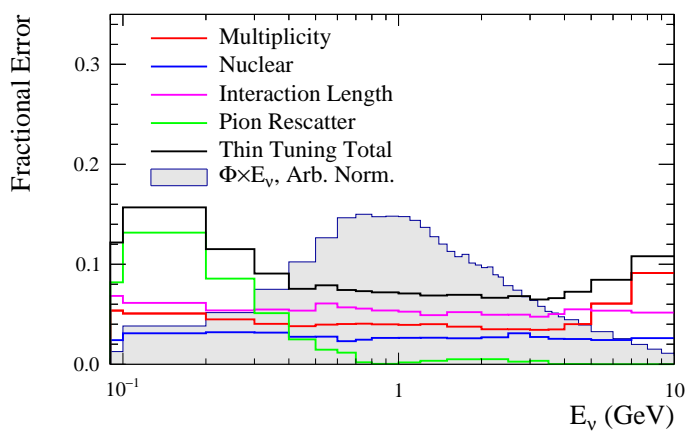

SK: Antineutrino Mode, $v_{\mathrm{e}}$

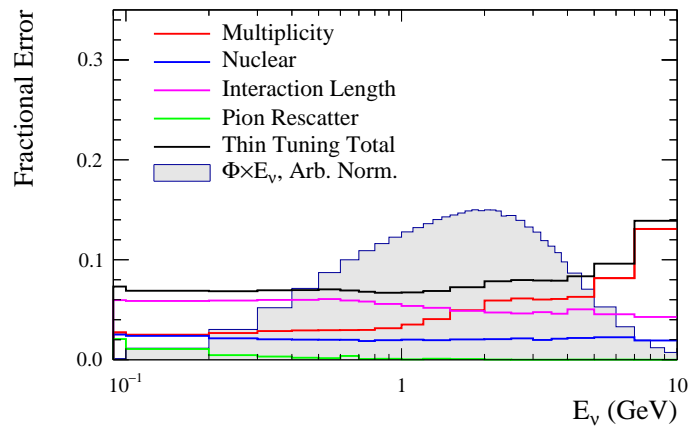

SK: Neutrino Mode, $\bar{v}_{\mu}$

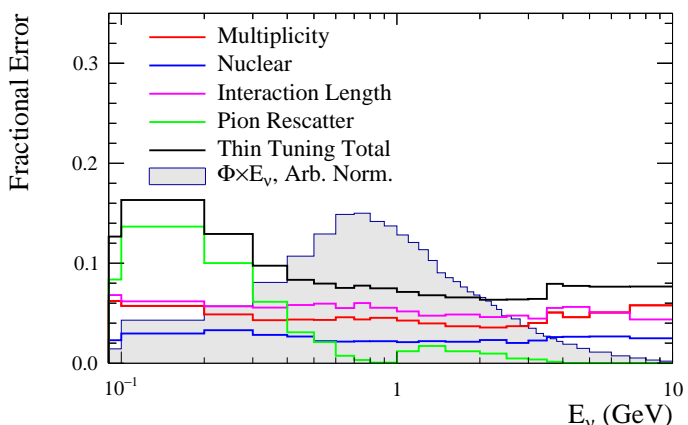

SK: Neutrino Mode, $\bar{v}_{\mathrm{e}}$

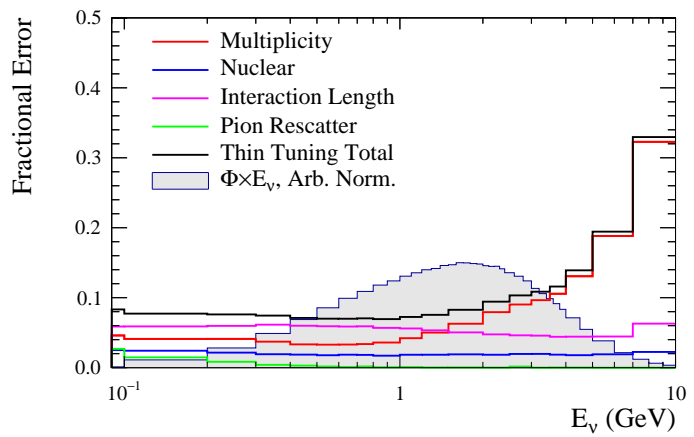

SK: Antineutrino Mode, $\bar{\nabla}_{\mu}$

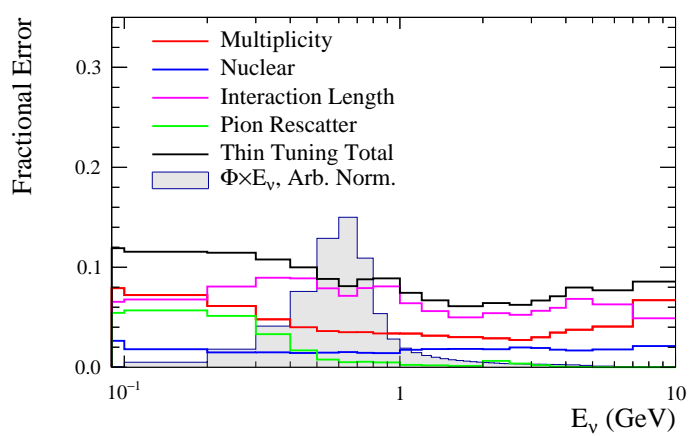

SK: Antineutrino Mode, $\overline{\mathrm{v}}_{\mathrm{e}}$

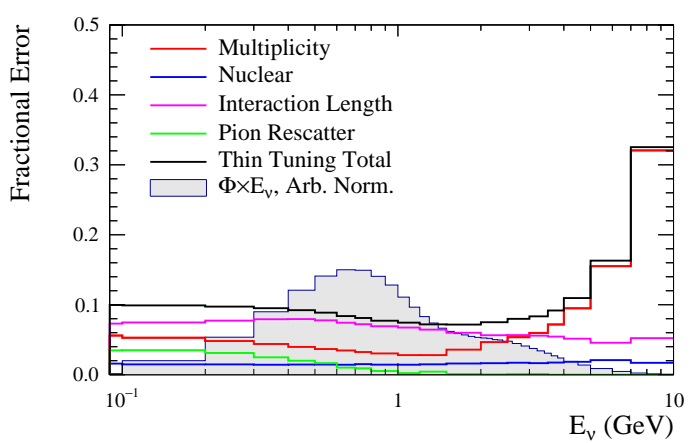

Figure 5.16: The hadronic interaction modelling portion of flux uncertainties at Super$\mathrm{K}$, evaluated when using only 2009 thin target data. The uncertainties have been estimated in neutrino mode (upper four plots) and anti-neutrino mode (lower four plots), for the $\nu_{\mu}$, $\bar{\nu}_{\mu}, \nu_{e}$ and $\bar{\nu}_{e}$ flux components. Copyright by T2K Collaboration. 

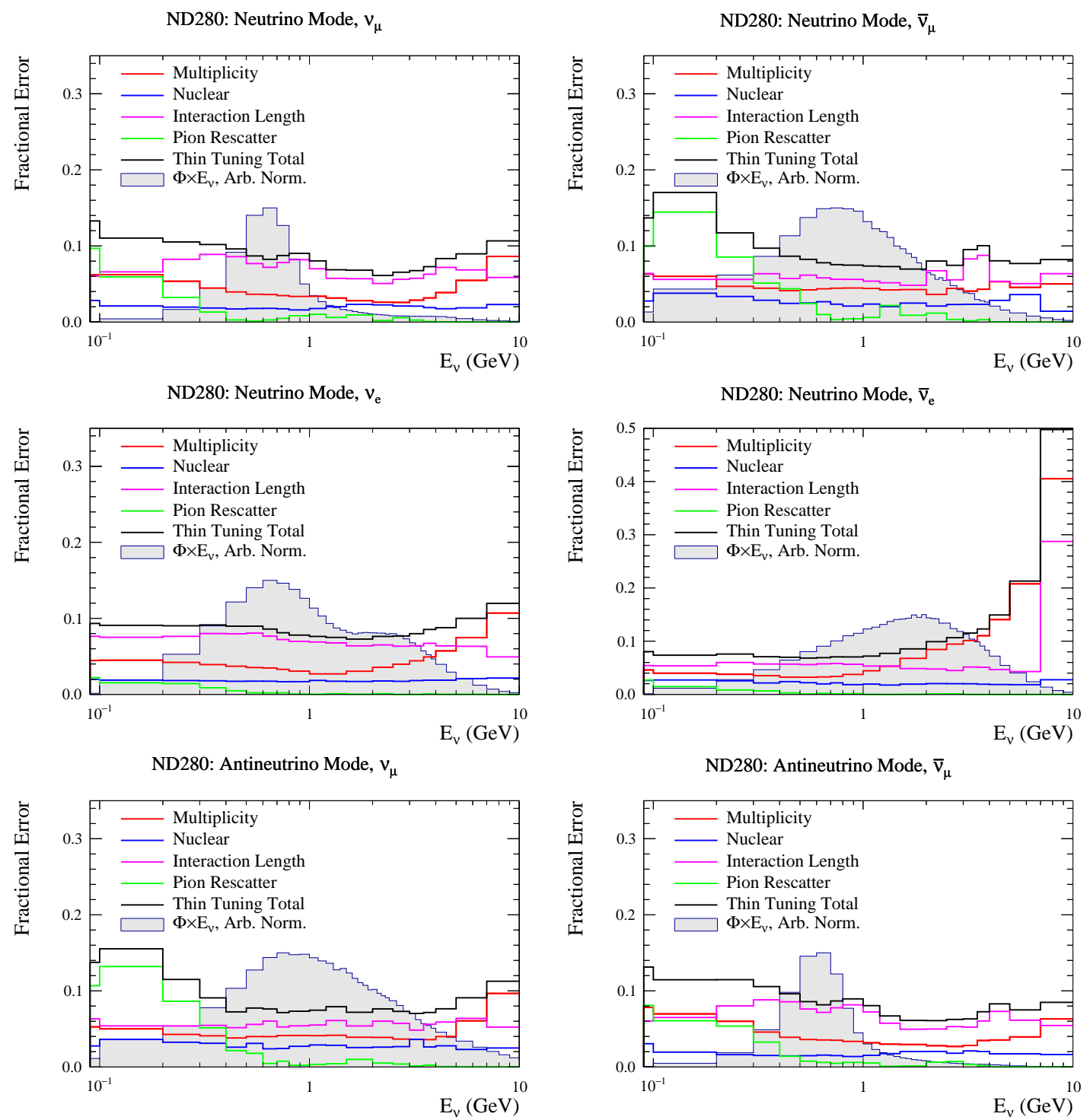

ND280: Antineutrino Mode, $v_{\mathrm{e}}$
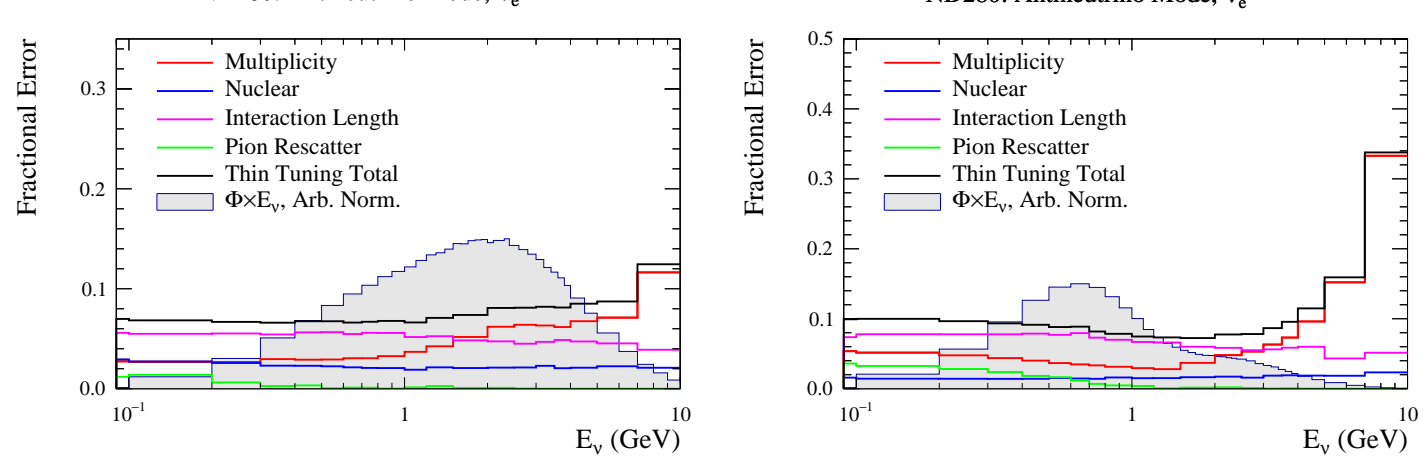

Figure 5.17: The hadronic interaction modelling portion of flux uncertainties at ND280, evaluated when using only 2009 thin target data. The uncertainties have been estimated in neutrino mode (upper four plots) and anti-neutrino mode (lower four plots), for the $\nu_{\mu}$, $\bar{\nu}_{\mu}, \nu_{e}$ and $\bar{\nu}_{e}$ flux components. Copyright by T2K Collaboration. 
SK: Neutrino Mode, $v_{\mu}$

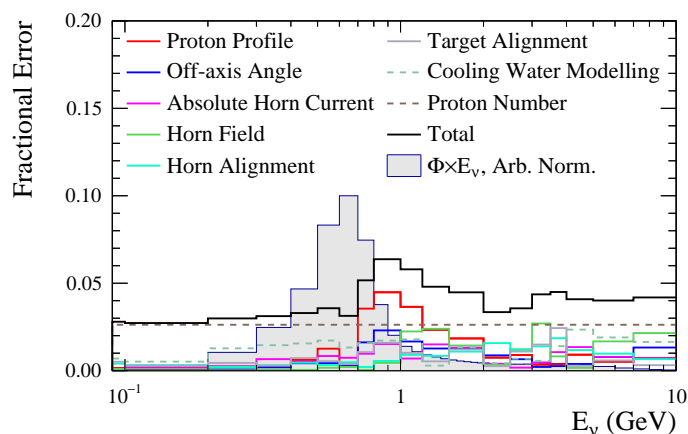

SK: Neutrino Mode, $v_{\mathrm{e}}$

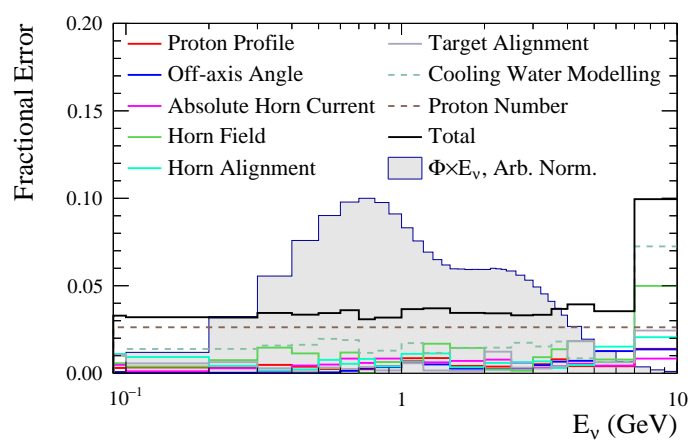

SK: Antineutrino Mode, $v_{\mu}$

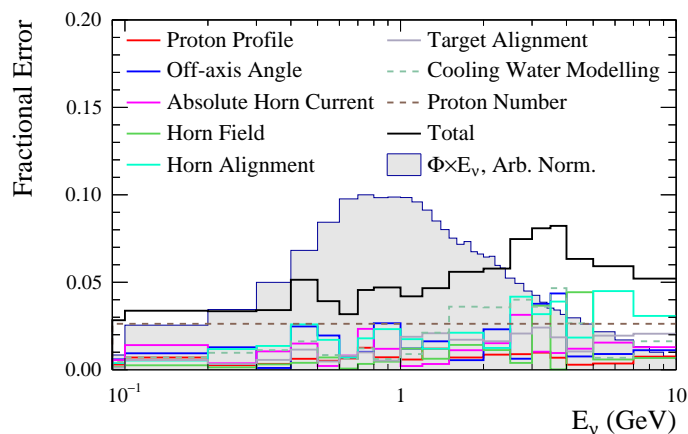

SK: Antineutrino Mode, $v_{\mathrm{e}}$

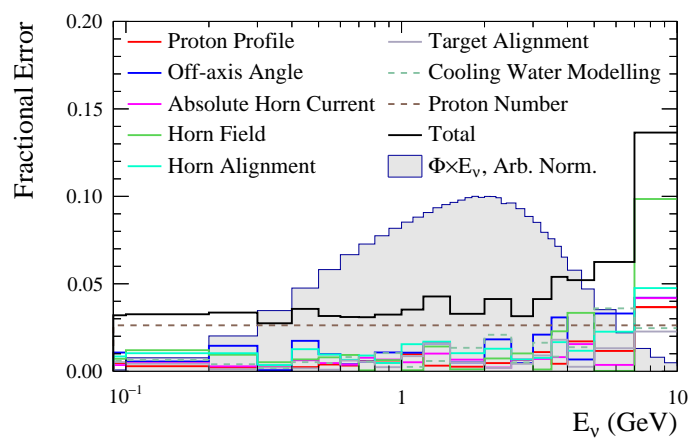

SK: Neutrino Mode, $\overline{\mathrm{v}}_{\mu}$

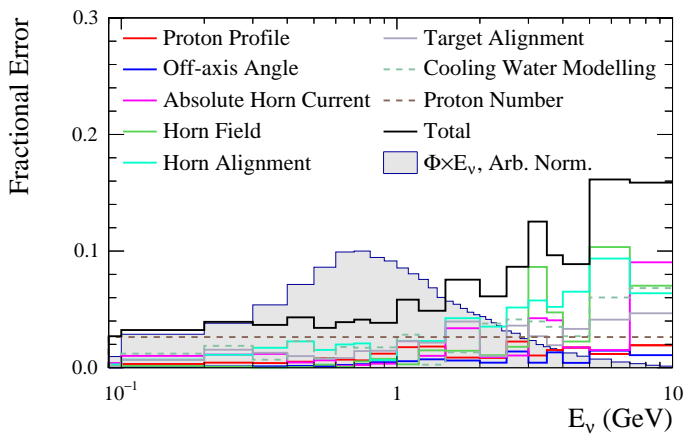

SK: Neutrino Mode, $\bar{v}_{\mathrm{e}}$

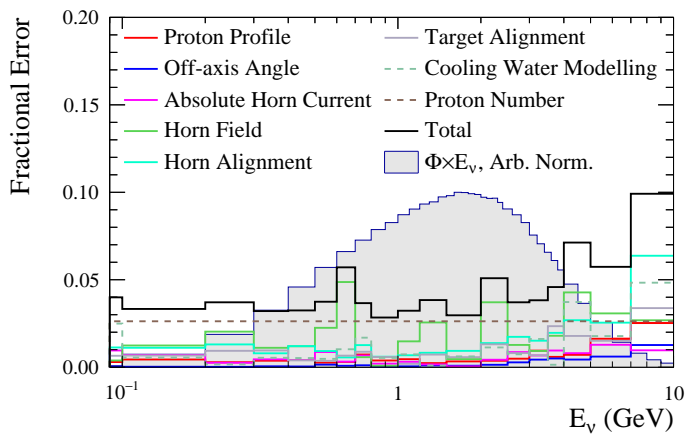

SK: Antineutrino Mode, $\nabla_{\mu}$

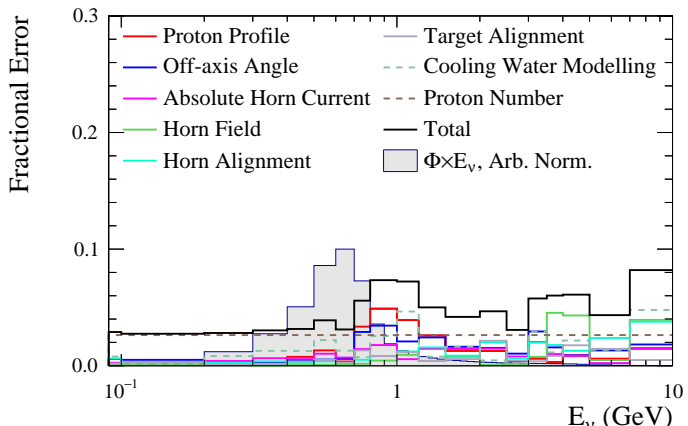

SK: Antineutrino Mode, $\overline{\mathrm{v}}_{\mathrm{e}}$

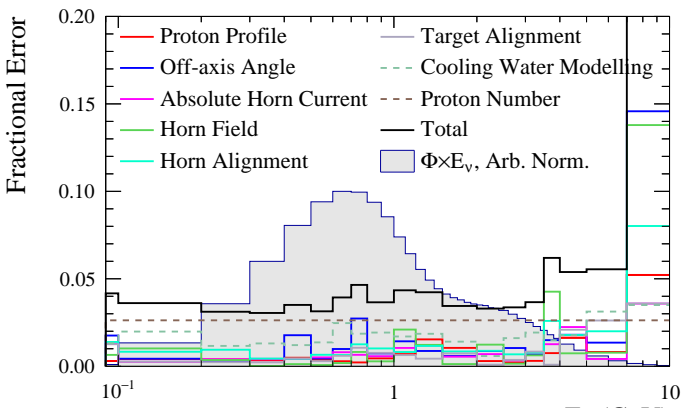

$\mathrm{E}_{\mathrm{v}}(\mathrm{GeV})$

Figure 5.18: Other sources of flux uncertainties at Super-K. The uncertainties have been estimated in neutrino mode (upper four plots) and anti-neutrino mode (lower four plots), for the $\nu_{\mu}, \bar{\nu}_{\mu}, \nu_{e}$ and $\bar{\nu}_{e}$ flux components. Copyright by T2K Collaboration. 
ND280: Neutrino Mode, $v_{\mu}$

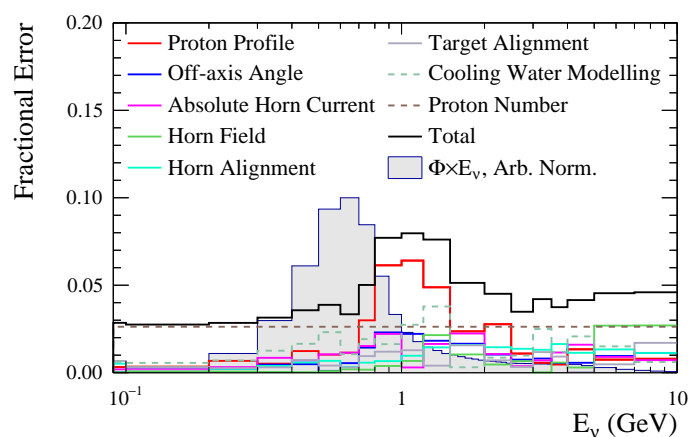

ND280: Neutrino Mode, $v_{\mathrm{e}}$

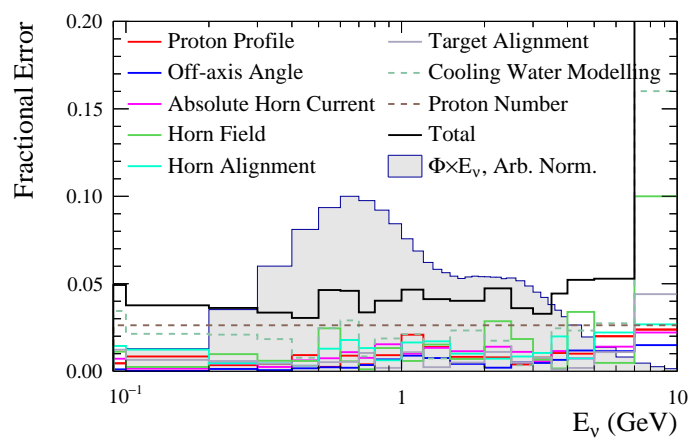

ND280: Antineutrino Mode, $v_{\mu}$

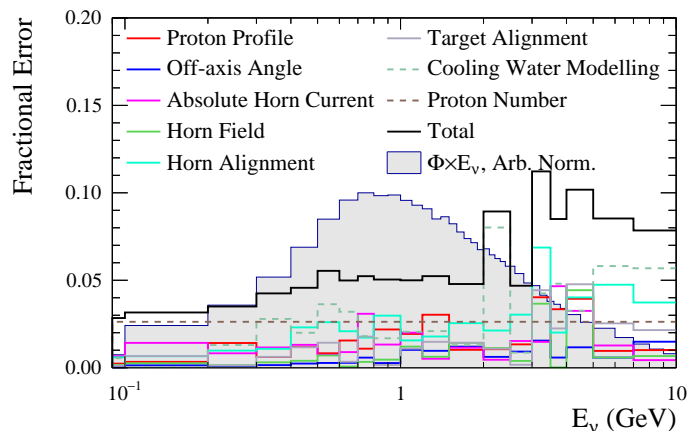

ND280: Antineutrino Mode, $v_{\mathrm{e}}$

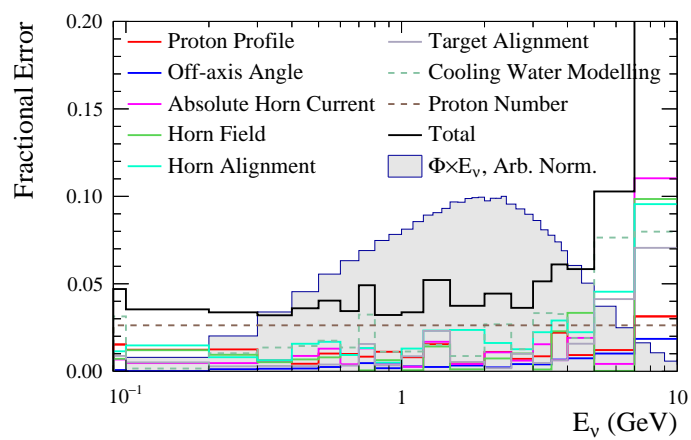

ND280: Neutrino Mode, $\overline{\mathrm{v}}_{\mu}$

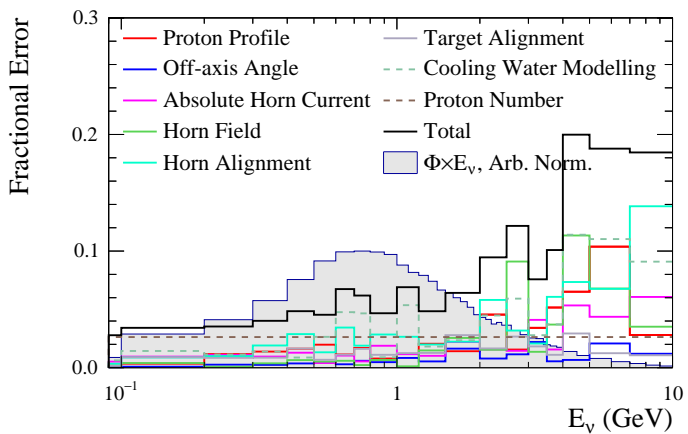

ND280: Neutrino Mode, $\overline{\mathrm{v}}_{\mathrm{e}}$

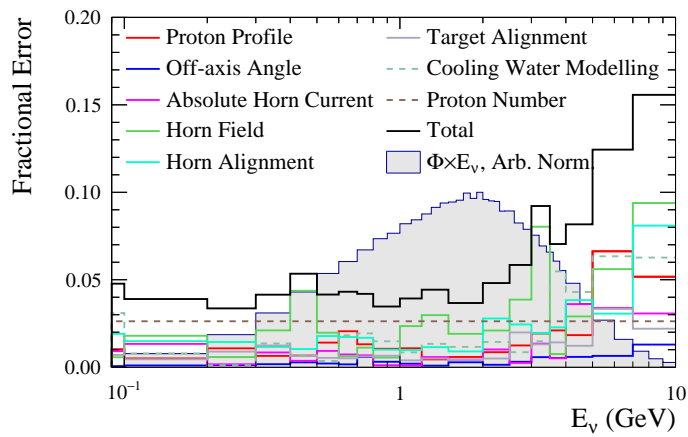

ND280: Antineutrino Mode, $\nabla_{\mu}$

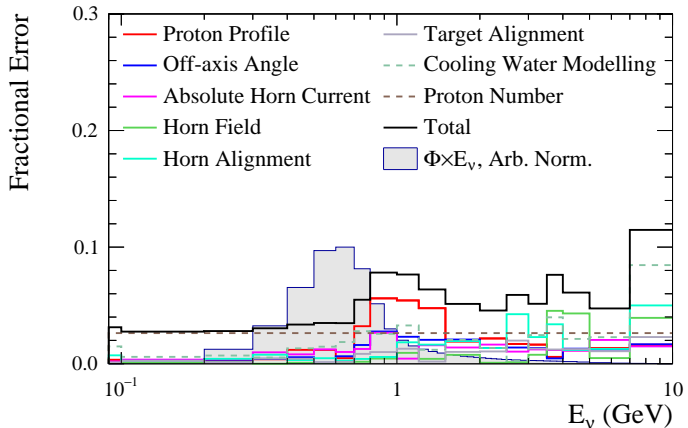

ND280: Antineutrino Mode, $\overline{\mathrm{v}}_{\mathrm{e}}$

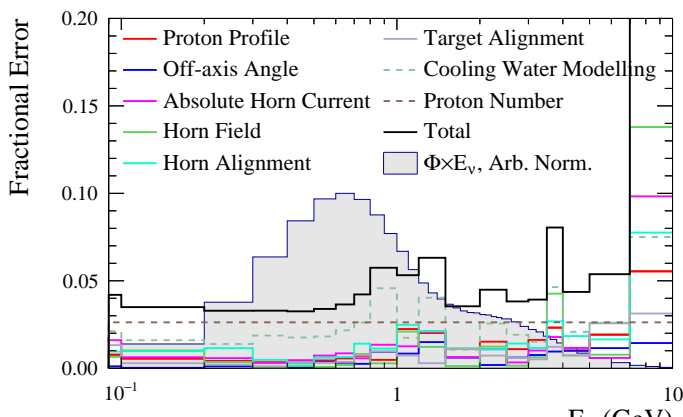

$\mathrm{E}_{\mathrm{v}}(\mathrm{GeV})$

Figure 5.19: Other sources of flux uncertainties at ND280. The uncertainties have been estimated in neutrino mode (upper four plots) and anti-neutrino mode (lower four plots), for the $\nu_{\mu}, \bar{\nu}_{\mu}, \nu_{e}$ and $\bar{\nu}_{e}$ flux components. Copyright by T2K Collaboration. 
SK: Neutrino Mode, $v_{\mu}$

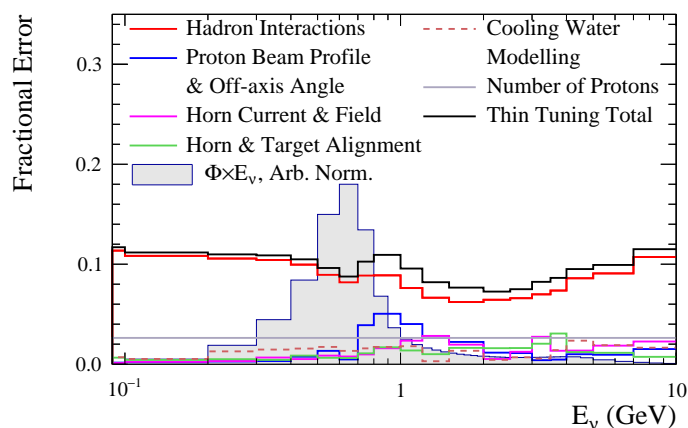

SK: Neutrino Mode, $v_{\mathrm{e}}$

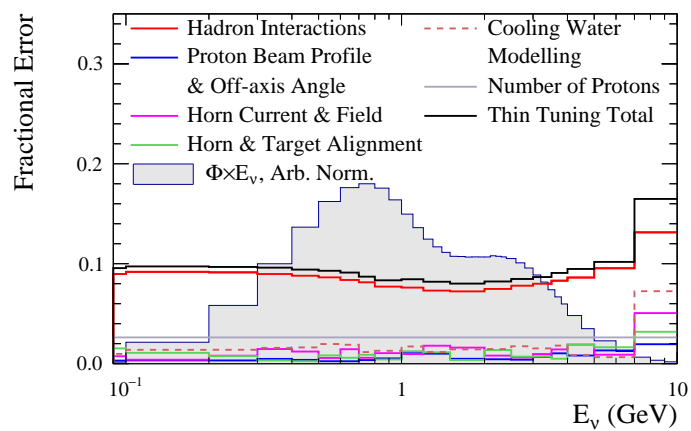

SK: Antineutrino Mode, $v_{\mu}$

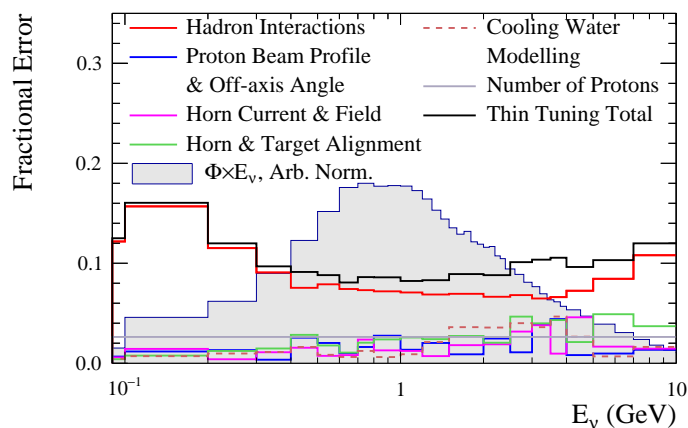

SK: Antineutrino Mode, $v_{\mathrm{e}}$

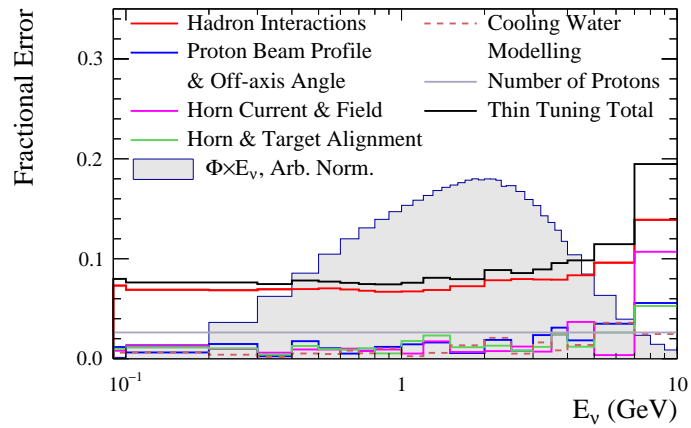

SK: Neutrino Mode, $\overline{\mathrm{v}}_{\mu}$

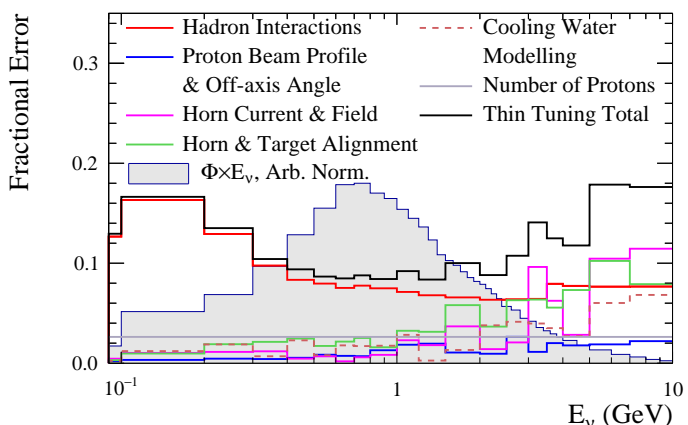

SK: Neutrino Mode, $\bar{v}_{\mathrm{e}}$

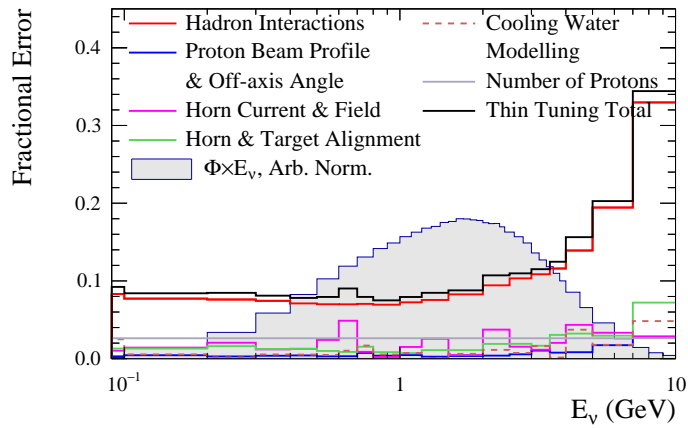

SK: Antineutrino Mode, $\bar{\nabla}_{\mu}$

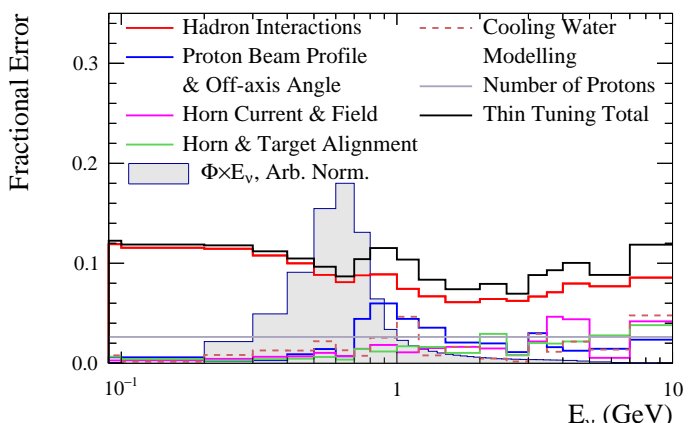

SK: Antineutrino Mode, $\bar{v}_{\mathrm{e}}$

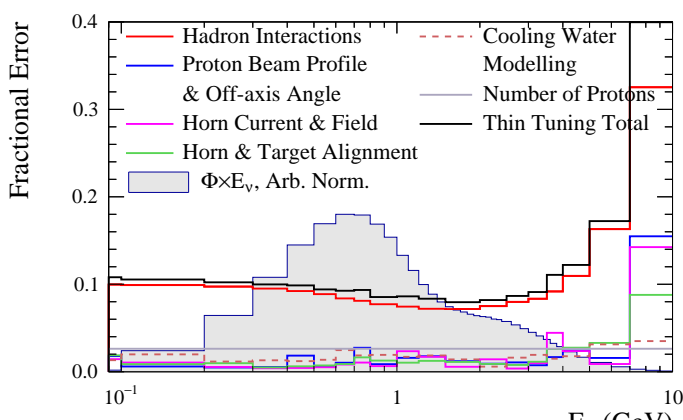

Figure 5.20: Total flux uncertainties at Super-K, evaluated when using only 2009 thin target data. The uncertainties have been estimated in neutrino mode (upper four plots) and anti-neutrino mode (lower four plots), for the $\nu_{\mu}, \bar{\nu}_{\mu}, \nu_{e}$ and $\bar{\nu}_{e}$ flux components. Copyright by T2K Collaboration. 
ND280: Neutrino Mode, $v_{\mu}$

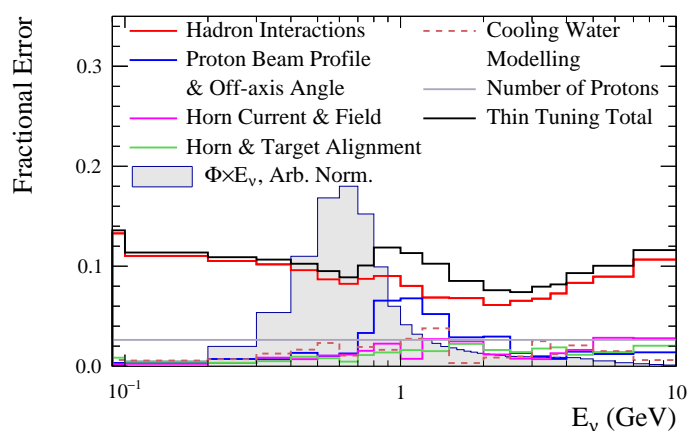

ND280: Neutrino Mode, $v_{\mathrm{e}}$

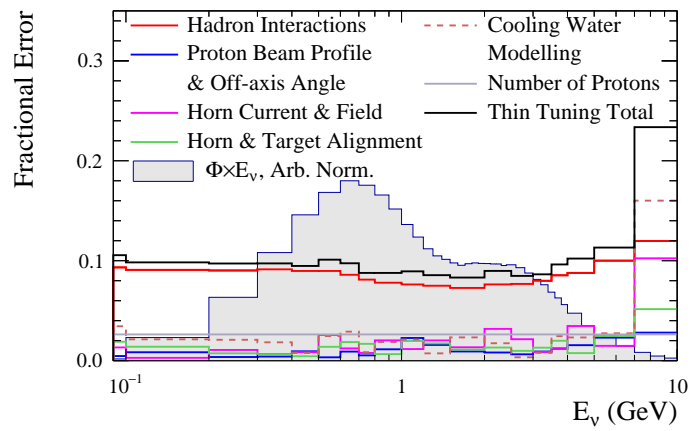

ND280: Antineutrino Mode, $v_{\mu}$

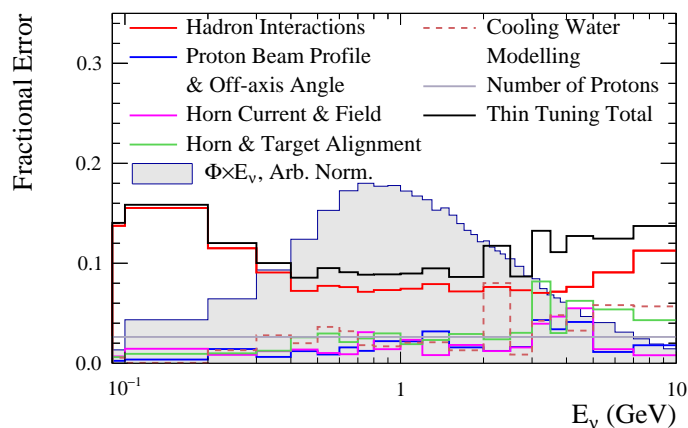

ND280: Antineutrino Mode, $v_{\mathrm{e}}$

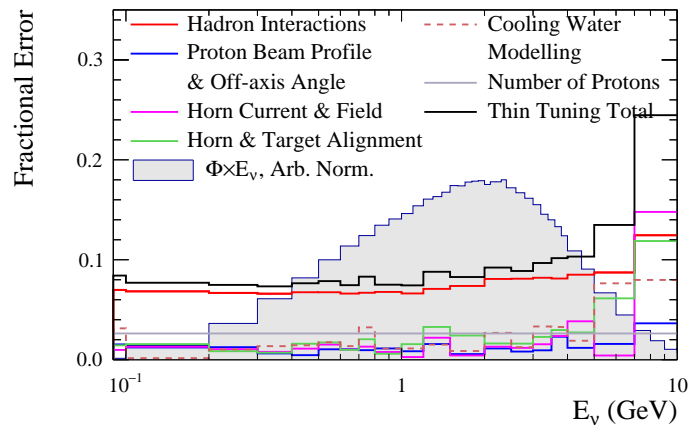

ND280: Neutrino Mode, $\overline{\mathrm{v}}_{\mu}$

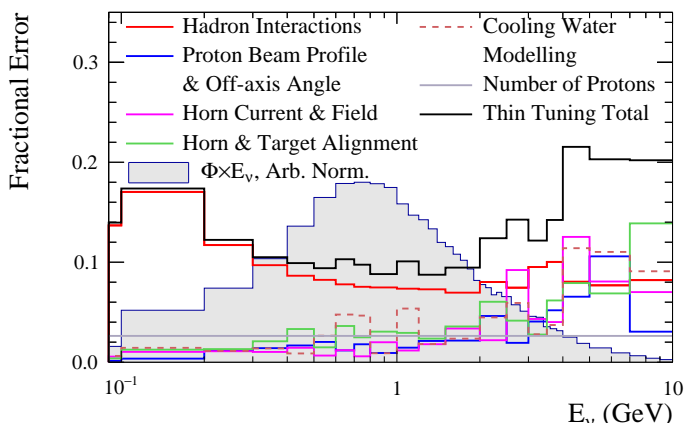

ND280: Neutrino Mode, $\overline{\mathrm{v}}_{\mathrm{e}}$

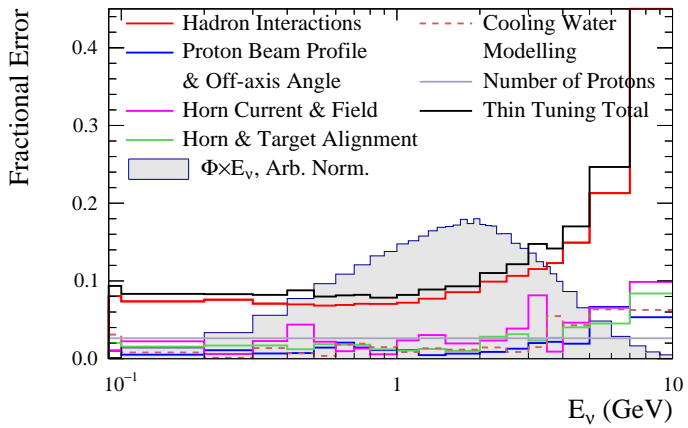

ND280: Antineutrino Mode, $\bar{v}_{\mu}$

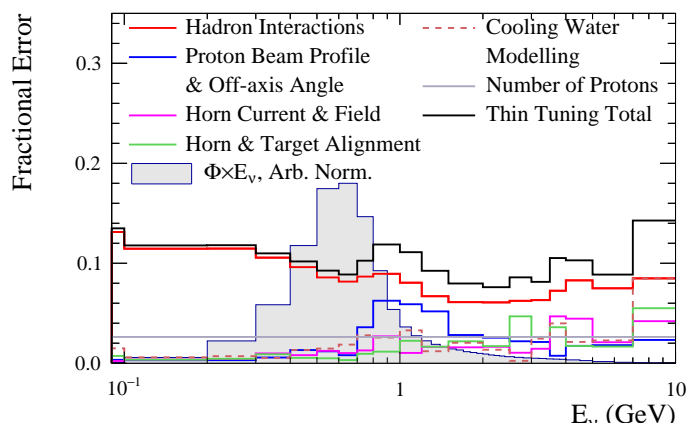

ND280: Antineutrino Mode, $\overline{\mathrm{v}}_{\mathrm{e}}$

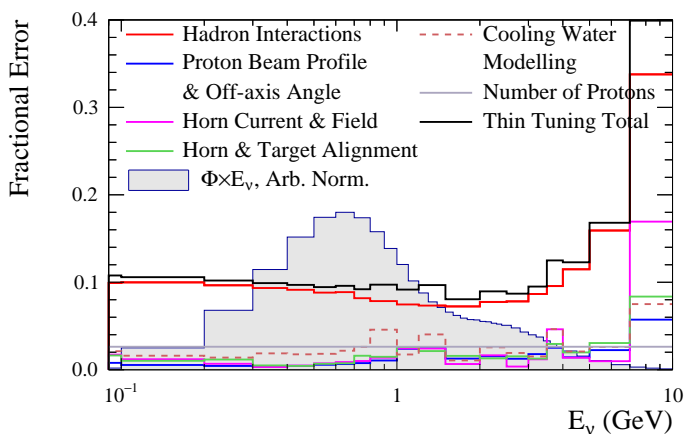

Figure 5.21: Total flux uncertainties at ND280, evaluated when using only 2009 thin target data. The uncertainties have been estimated in neutrino mode (upper four plots) and anti-neutrino mode (lower four plots), for the $\nu_{\mu}, \bar{\nu}_{\mu}, \nu_{e}$ and $\bar{\nu}_{e}$ flux components. Copyright by T2K Collaboration. 


\section{6} The T2K flux calculation tuned to replica-target data

This chapter presents an extension to the T2K flux calculation framework, accommodating for flux tuning to NA61 measurements with the replica-target. The "replica-tuning" technique is described in Chapter 6.1. The new flux prediction and uncertainty have been presented in Chapters 6.2 and 6.3, respectively. The flux uncertainty has been reduced by $\sim 50 \%$ with the inclusion of the 2009 replica-target dataset. Various new systematic uncertainties had been considered in Chapters 6.4, 6.5, 6.6 and 6.7, and shown to have a negligible effect on the flux calculation, with the only exception being the systematic uncertainty due to unconstrained interactions. Chapter 6.7 also highlights future hadron production measurements that could be beneficial for further improving the T2K flux calculation.

\subsection{Replica-tuning approach}

For NA61 measurements collected in 2009, with protons incident on a long target (see Fig. 4.4), the yield of pions exiting from the target $\mathrm{Y}_{\text {replica }}^{\alpha}$ is binned in the $(p, \theta, z)$ phase space, where $p$ is the pion momentum, $\theta$ is the pion angle with respect to the beam direction, $z$ is the longitudinal coordinate of the exiting position along the target length, and $\alpha \in\left\{\pi^{+}, \pi^{-}\right\}$is the exiting pion species. The reader is referred back to Chapter 4.2.2 for a detailed summary of the format of the NA61 2009 replica-target dataset. The multiplicity weight is again defined as the ratio of the measured and simulated (modelled) yields:

$$
\begin{aligned}
& \mathrm{W}_{\text {replica }}^{\alpha}(p, \theta, z)=\left[\mathrm{Y}_{\text {replica }}^{\alpha}(p, \theta, z)\right]_{\text {NA61 }} /\left[\mathrm{Y}_{\text {replica }}^{\alpha}(p, \theta, z)\right]_{\text {FLUKA }}= \\
& =\left[\frac{d \mathrm{n}_{\text {replica }}^{\alpha}}{d p}\right]_{\text {NA61 }} /\left[\frac{d \mathrm{n}_{\text {replica }}^{\alpha}}{d p}\right]_{\text {FLUKA }}
\end{aligned}
$$


Here, the FLUKA package is used for modelling in-target interactions. This weight is used to tune the pion yield from the T2K target. Thus, rather than applying tuning weights for individual in-target interactions, it is now sufficient to apply a single multiplicity weight at the exiting position of the resulting pion. A schematic diagram illustrating the difference between these two tuning approaches is given in Figure 6.1.
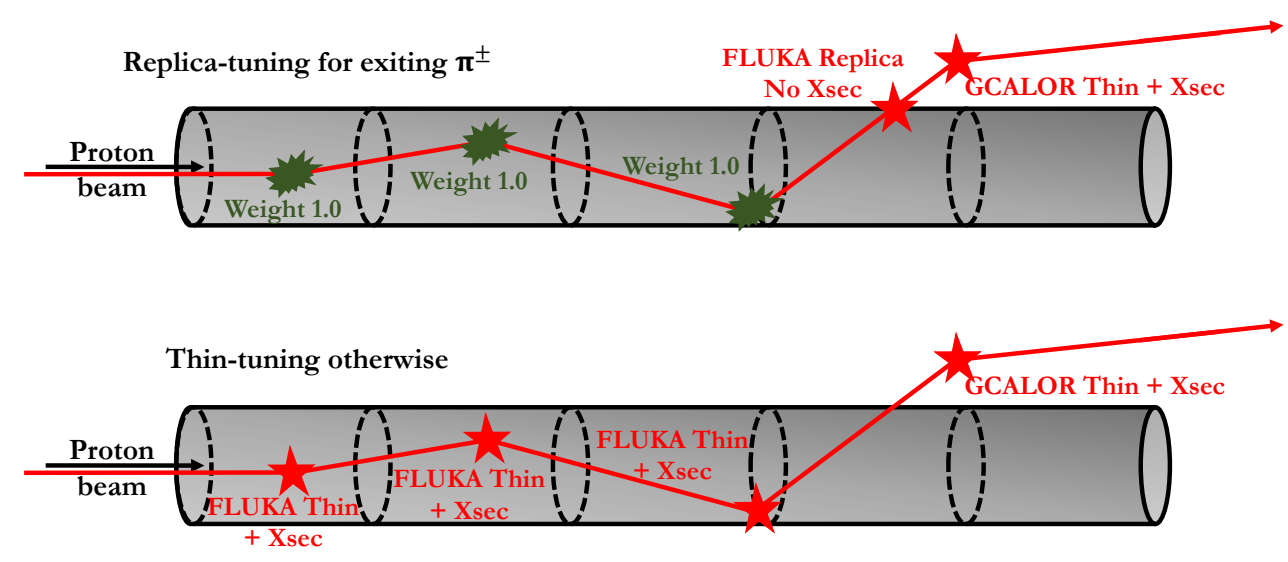

Figure 6.1: The implementation of pion yields from the replica-target into the flux calculation framework.

The replica-target multiplicity weights $\mathrm{W}_{\text {replica }}^{\pi^{ \pm}}(p, \theta, z)$ from Equation 6.1 are shown in Figures 6.3 and 6.4, for positive and negative pions, respectively. Because of the limited statistics of the 2009 dataset, only these pion yields were measured. Thus, thin-target data is still essential for constraining parts of the neutrino flux originating from other exiting particle species, and from out-of-target interactions. In these cases, thin-target weights from Equation 5.2 are used. Also, the flux from pions whose exiting $(p, \theta, z)$ coordinate lies outside the coverage of the 2009 replica-target dataset is tuned with thin-target data.

The aim of this thesis was to incorporate the 2009 NA61 replica-target dataset into the flux calculation framework, and use it whenever possible. Thus, in the remainder of this note, when referring to the replica-tuned flux, a combi- 
nation of replica and thin target data is assumed, with preference always given to replica-target data.

The fractions of the T2K neutrino flux at Super-K originating from different ancestor hadrons exiting from the target are shown in Figure 6.2. The majority of $\mathrm{T} 2 \mathrm{~K}$ neutrinos, especially around the $600 \mathrm{MeV}$ beam peak, have exiting pion ancestors, and are thus tunable with the 2009 dataset. There exists an additional dataset from 2010, collected with enough statistics to extract exiting $\pi^{ \pm}$, proton and $K^{ \pm}$yields, and its implementation in the T2K flux framework is currently ongoing, but won't be discussed in this thesis. The expected implications of tuning exiting proton and kaon yields would be a tighter constraint on the high energy tail of the T2K flux. These high energy neutrinos mostly come from kaons emitted from the target. 
SK: Neutrino Mode, $v_{\mu}$ flux by exiting hadron

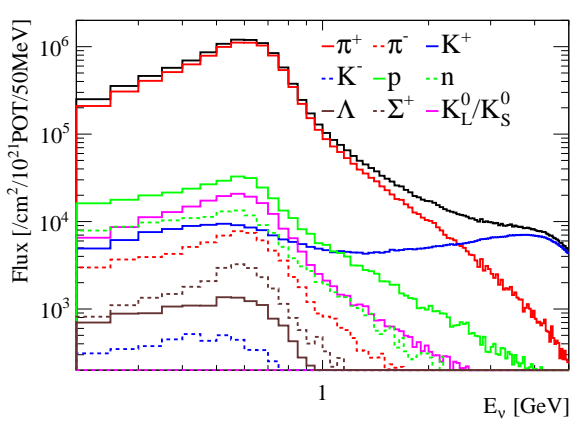

SK: Neutrino Mode, $v_{\mathrm{e}}$ flux by exiting hadron

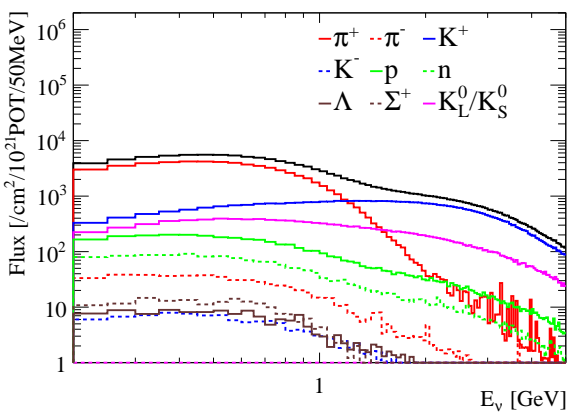

SK: Anti-Neutrino Mode, $v_{\mu}$ flux by exiting hadron

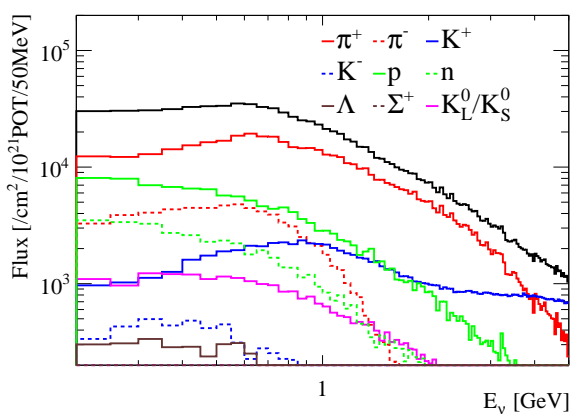

SK: Anti-Neutrino Mode, $v_{\mathrm{e}}$ flux by exiting hadron

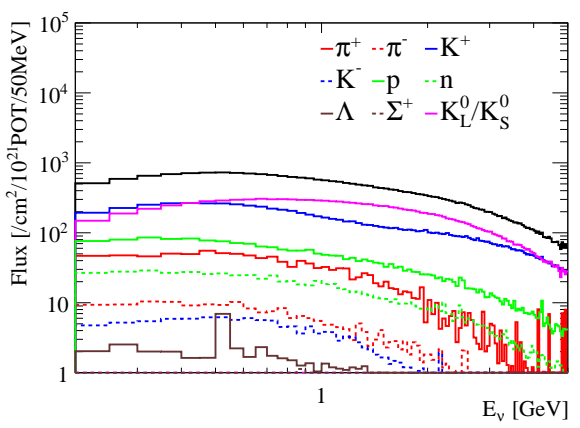

SK: Neutrino Mode, $\bar{v}_{\mu}$ flux by exiting hadron

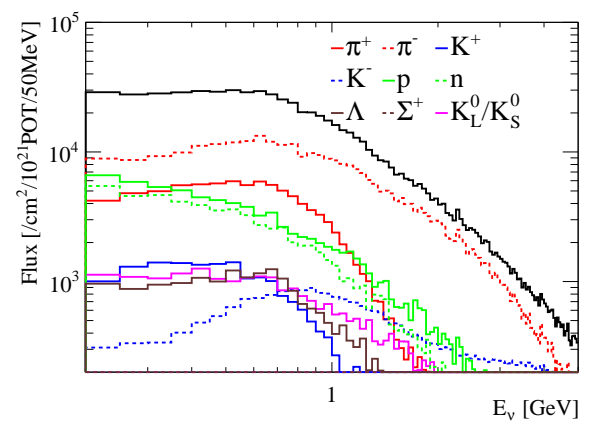

SK: Neutrino Mode, $\overline{\mathrm{v}}_{\mathrm{e}}$ flux by exiting hadron

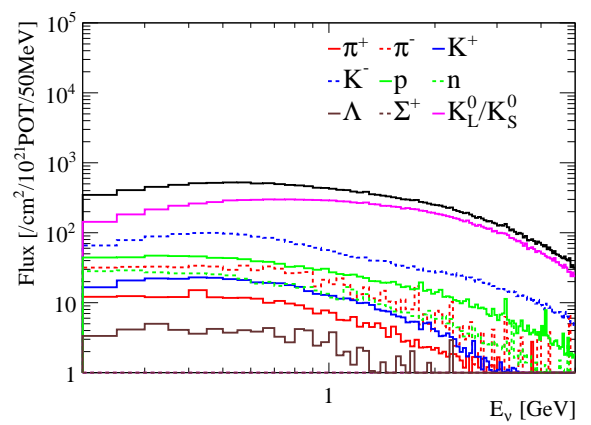

SK: Anti-Neutirno Mode, $\bar{v}_{\mu}$ flux by exiting hadron

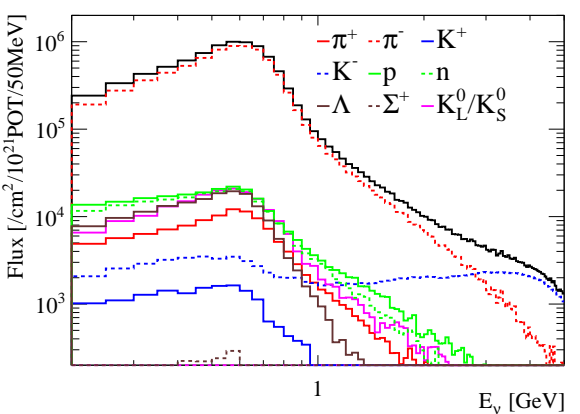

SK: Anti-Neutrino Mode, $\bar{v}_{\mathrm{e}}$ flux by exiting hadron

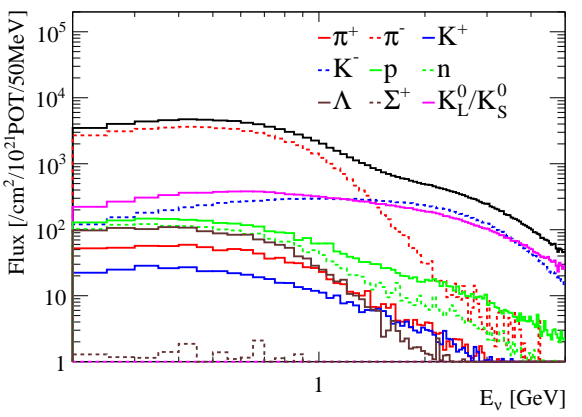

Figure 6.2: Contributions to the neutrino flux at Super-K from various hadrons exiting from the T2K target. Both the neutrino (upper four figures) and anti-neutrino (lower four figures) modes of operation have been considered, for the $\nu_{\mu}, \bar{\nu}_{\mu}, \nu_{e}$ and $\bar{\nu}_{e}$ flux components. 

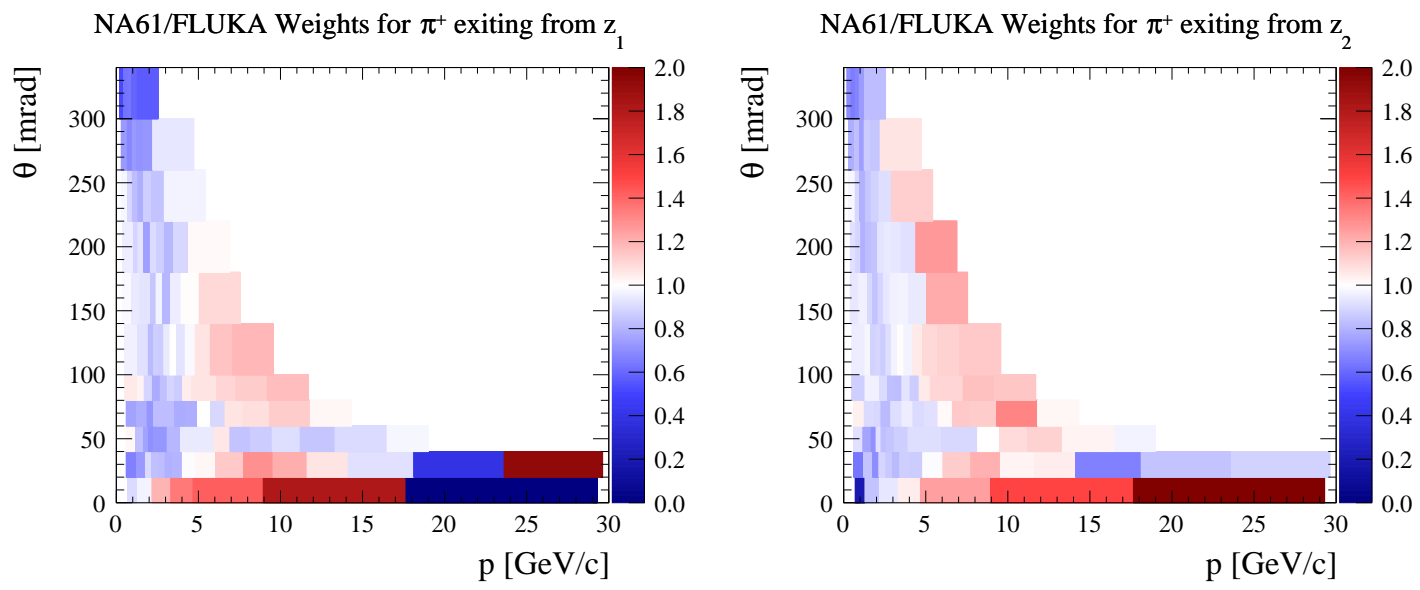

NA61/FLUKA Weights for $\pi^{+}$exiting from $z_{3}$

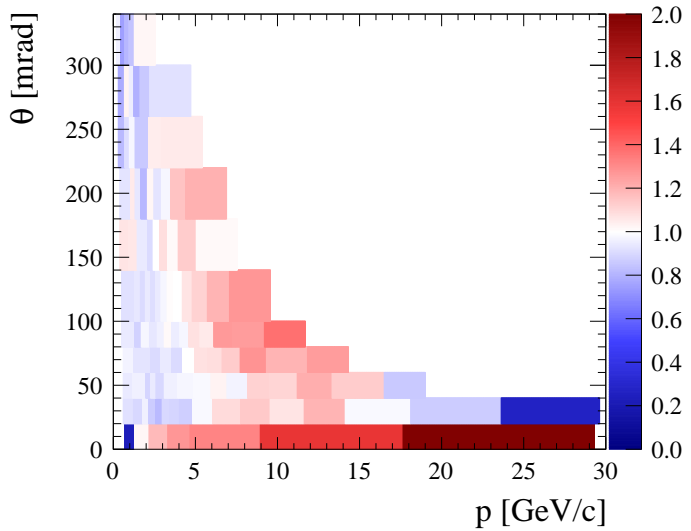

NA61/FLUKA Weights for $\pi^{+}$exiting from $\mathrm{z}_{4}$

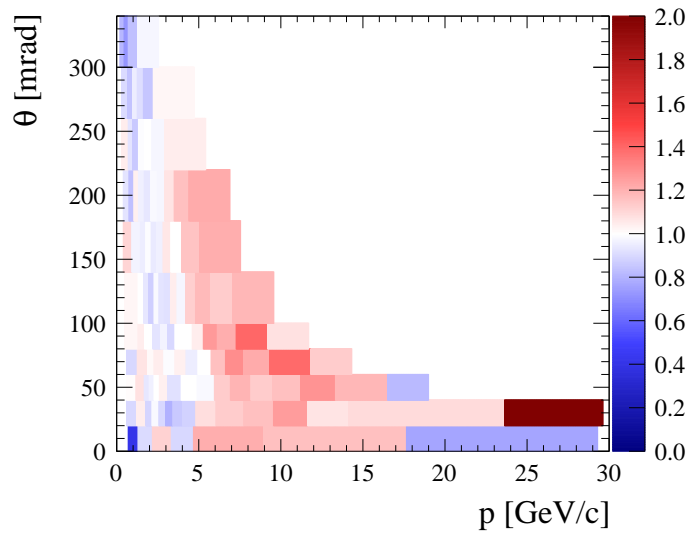

NA61/FLUKA Weights for $\pi^{+}$exiting from $z_{5}$ NA61/FLUKA Weights for $\pi^{+}$exiting from $\mathrm{z}_{6}$
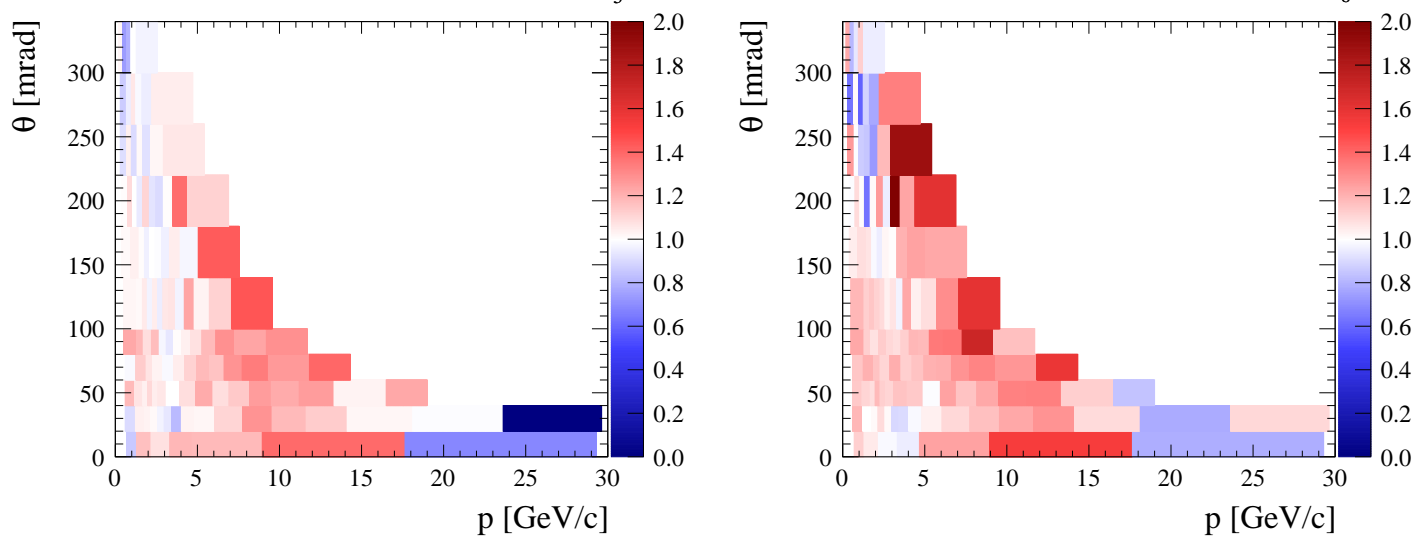

Figure 6.3: Replica-target $\pi^{+}$multiplicity weights used for constraining the unoscillated T2K neutrino flux prediction. Copyright by T2K Collaboration. 

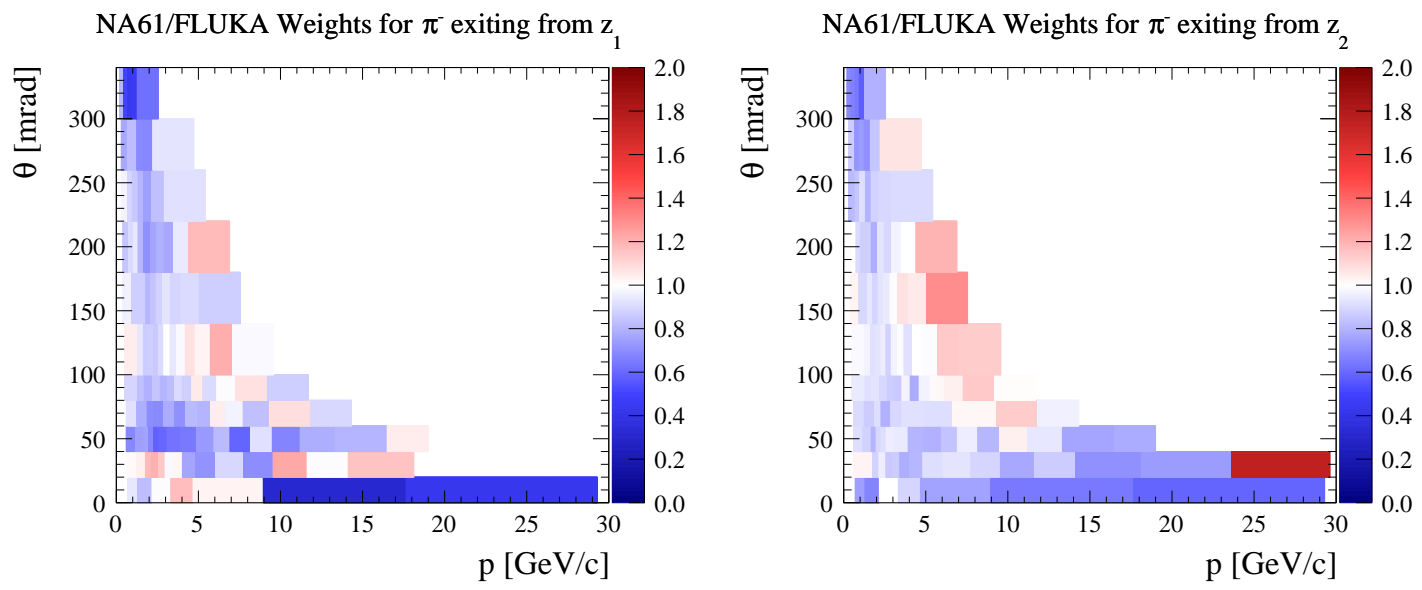

NA61/FLUKA Weights for $\pi^{-}$exiting from $z_{3}$

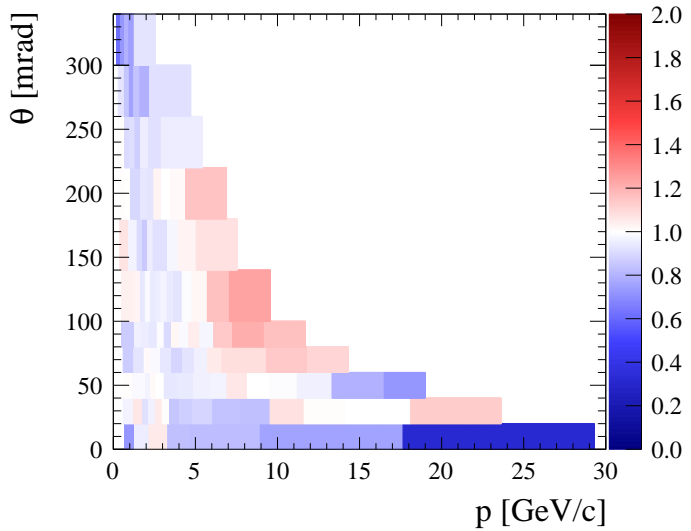

NA61/FLUKA Weights for $\pi^{-}$exiting from $\mathrm{z}_{4}$

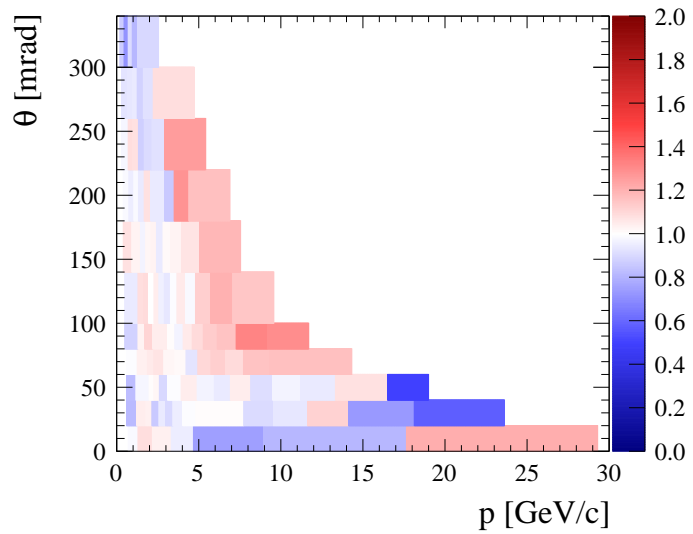

NA61/FLUKA Weights for $\pi$ exiting from $\mathrm{z}_{5}$

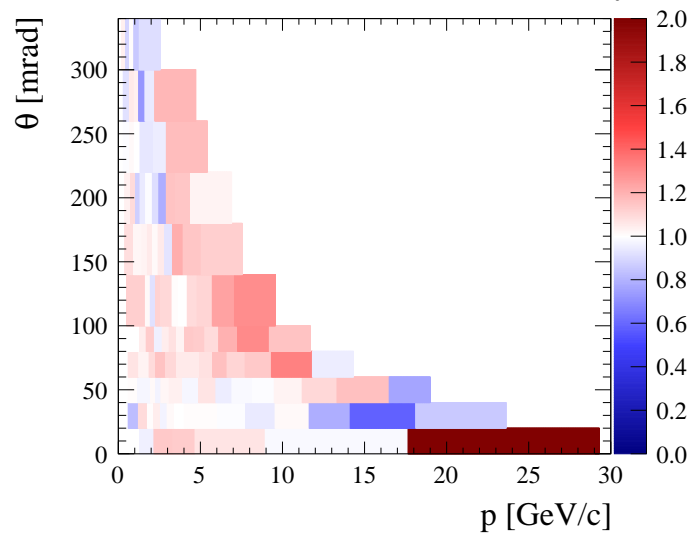

NA61/FLUKA Weights for $\pi^{-}$exiting from $\mathrm{z}_{6}$

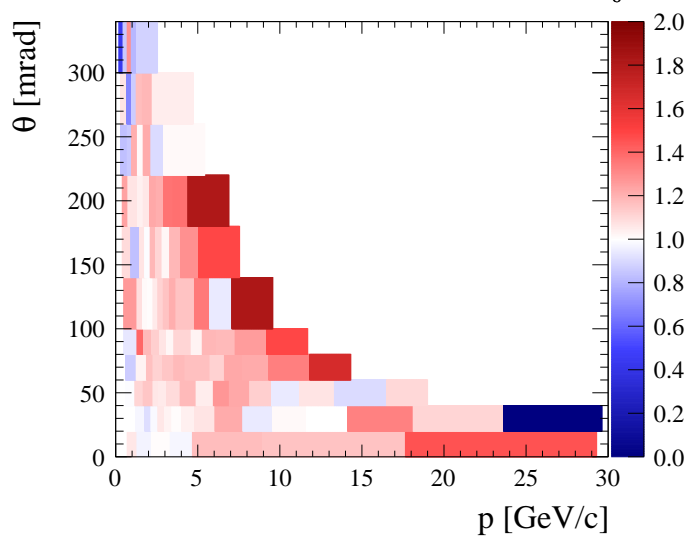

Figure 6.4: Replica-target $\pi^{-}$multiplicity weights used for constraining the unoscillated T2K neutrino flux prediction. Copyright by T2K Collaboration. 


\subsection{Replica-tuned flux prediction}

The replica-tuned flux for every T2K run is calculated by applying tuning weights to the raw prediction straight from the flux simulation tool. It is necessary to produce separate flux predictions for each run due to variations in beamline parameters, such as the primary proton beam profile and average focusing horn currents. Combined predictions of the flux across all T2K data-taking periods have also been derived. For every flux energy bin, the average POT weighted flux value is taken, as has been explained in Equation 5.1. For the combined replica-tuned flux for all T2K runs, in both neutrino and anti-neutrino mode, the reader is referred back to Figure 5.2. The Monte Carlo production for oscillation measurements uses the nominal flux calculation produced with typical beam conditions and no tuning. This production is then tuned as part of the oscillation analysis, using ratios of the T2K run specific tuned flux calculations to the nominal predictions. Such ratios of replica-tuned and nominal flux are shown in Figure 6.5, for the flux at ND280 and Super-K, in both neutrino and anti-neutrino mode. In all plots, the four species of neutrinos are presented, and no uncertainties are shown. These weights account for the effect of flux tuning, but also for any differences in beam profile parameters and horn current inputs between the nominal run and the real T2K physics run. One can see the weights at high energy are changed, mainly due to the inclusion of the NA61 kaon data $\left(\mathrm{K}^{+}, \mathrm{K}^{-}\right.$and $\left.\mathrm{K}_{\mathrm{S}}^{0}\right)$ in the re-weighting machinery. The addition of replica-target data affects the weights at energies around the T2K flux peak $(600 \mathrm{MeV})$.

The differences in thin-tuned and replica-tuned flux measurements, with the same beam profile parameter and horn current inputs, have also been examined. In every bin of the replica-tuned flux, some neutrino events will originate from pions exiting from the target, and thus get assigned replica-target weights, whereas other events in the same bin might use thin-target weights. Evidently, there is a degree of correlation between the current replica-tuned and thin-tuned flux predictions, reflecting the fact that both predictions use the same datasets to a certain extent. For instance, at the high energy flux tail, both tuning methods rely on kaon yields 

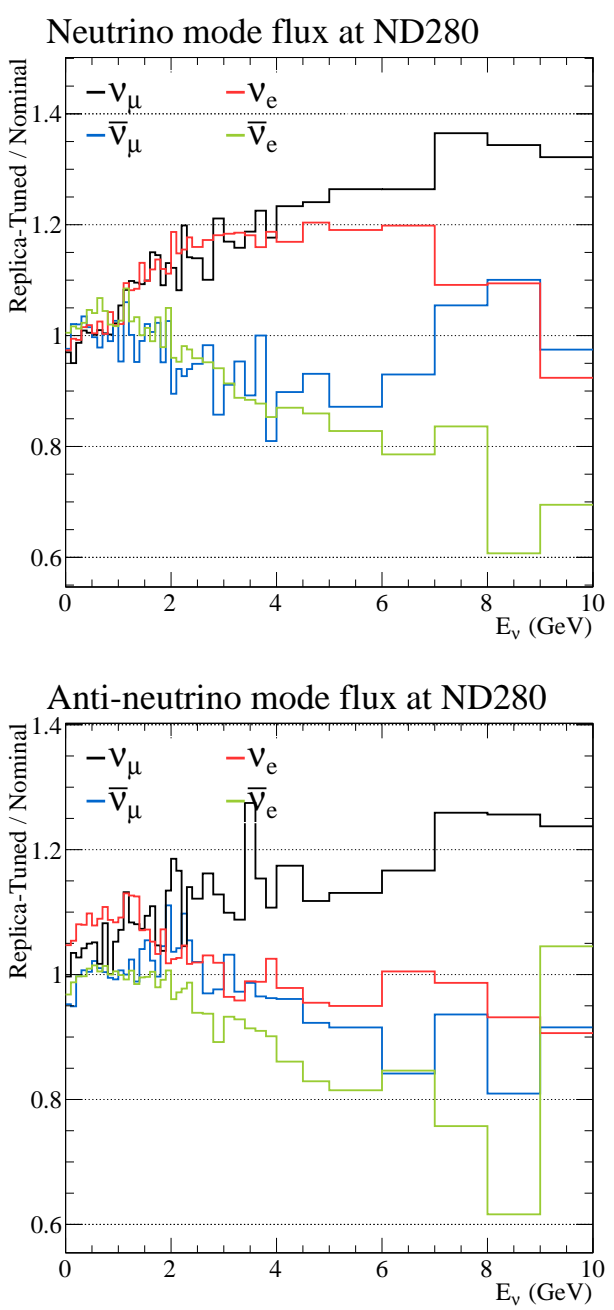

Neutrino mode flux at Super-K

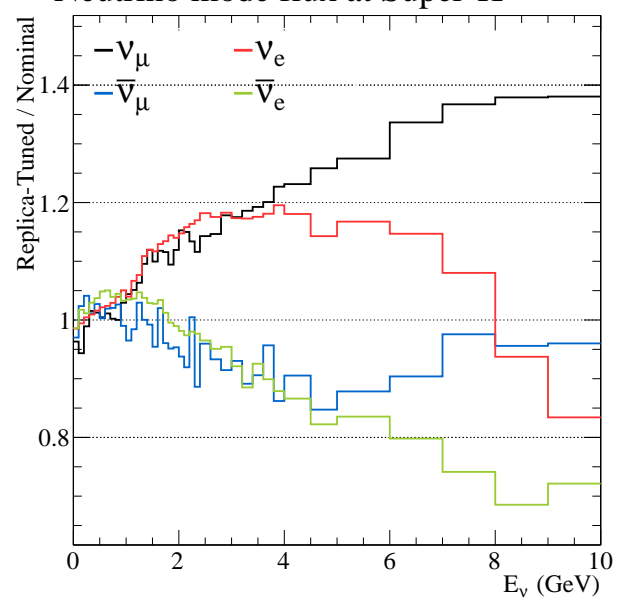

Anti-neutrino mode flux at Super-K

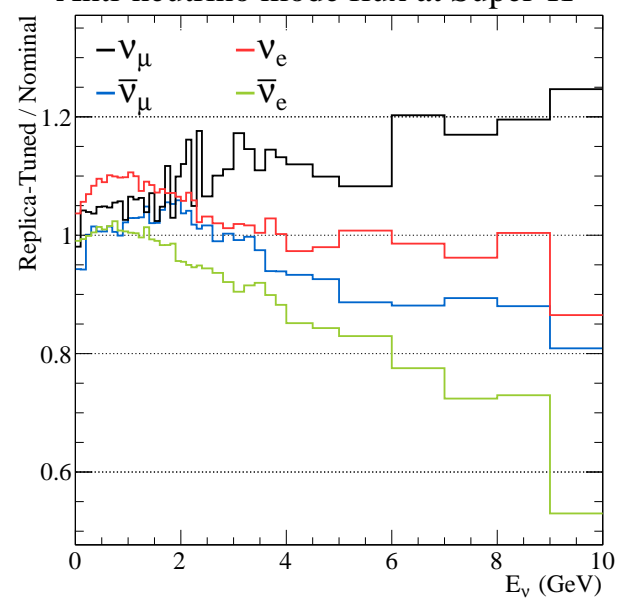

Figure 6.5: Ratios of the tuned flux for combined runs 1-9a to the 13a nominal flux, at ND280 (left) and at Super-Kamiokande (right). All species of neutrinos are shown. Errors are not presented. Copyright by T2K Collaboration.

from the NA61 2009 thin-target dataset. The ratio of the replica-tuned to the thintuned flux calculation at Super-K and ND280 is given in Figure 6.6 and Figure 6.7, respectively. The only difference between the two calculations is the inclusion of a new hadron production dataset. Consequently, flux errors due to effects unrelated to hadron production fully cancel in the ratio. The errors on the ratio due to multiplicity tuning and hadron interaction length tuning have been considered. First, multiplicities have been varied within their respective uncertainties, encoded in the NA61 thin-target and replica-target covariance matrices. Multiplicities have been varied in a correlated manner, using Cholesky decomposition, as described 
in Chapter 5.5.1. For every set of the multiplicity variations, the replica-tuned and thin-tuned flux was recalculated, and their ratio taken. This procedure was repeated 500 times, resulting in 500 toy samples of the flux ratio. The standard deviation on the ratio has been calculated from variations of these toys from their nominal value. When calculating the error on the ratio due to hadron interaction length weights, a similar procedure has been used, this time applying variations in hadron production cross sections, as described Chapter 5.5.1. The uncertainty on the flux ratio due to systematic variation in production cross section is depicted with the rectangular gray error bands in Fig. 6.6 and Fig. 6.7. The total uncertainty on the flux ratio, including both production cross section and multiplicity variations is shown with the vertical error bars. As expected, cancellation of errors is observed at high energies, where both flux versions rely almost exclusively on the kaon yields from NA61 thin-target measurements.

It is worth noting that the replica-tuned flux measurement predicts an approximately $5 \%$ lower $\nu_{\mu}\left(\bar{\nu}_{\mu}\right)$ flux around the $600 \mathrm{MeV}$ T2K beam peak in (anti)neutrino mode of operation. This likely reflects the preference of the replica-target dataset for a lower proton production cross section value, and consequently lower neutrino production. In fact, the measurements of exiting pion yields from the replica-target were compared to predictions using the FLUKA model. The FLUKA prediction overestimated the pion yield from the upstream bin $z_{1}$, and underestimate the yield in the most downstream bin $z_{6}$. When lowering the $30 \mathrm{GeV}$ proton production cross section to $\sigma_{\text {prod }}=200 \mathrm{mb}$ (compare this with the $\sigma_{\text {prod }}=230.7 \mathrm{mb}$ NA61 thin-target measurement introduced in Chapter 4.2.3), better agreement with data was observed. In any case, precise modelling of hadronic reinteractions inside a thick target is extremely challenging and highly sensitive to various underlying model parameters. The replica-tuned flux calculation offers an alternative, more robust estimate of the neutrino production at $\mathrm{T} 2 \mathrm{~K}$. The fact that the thin-tuning and replica-tuning methods produce predictions which agree within $5 \%$ is itself a remarkable testament of the hard and rigorous work of the T2K Beam Group over the last decade. 
SK: Neutrino Mode, $v_{\mu}$

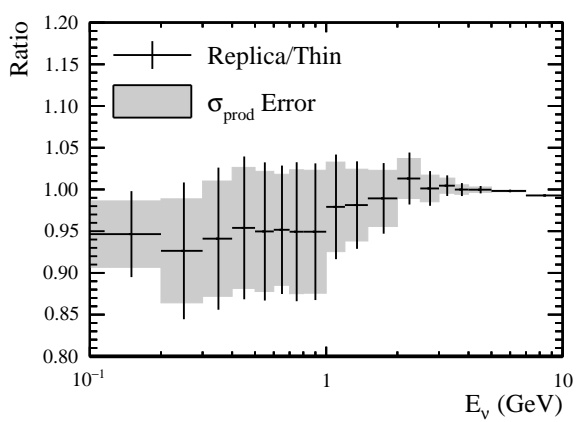

SK: Neutrino Mode, $v_{\mathrm{e}}$

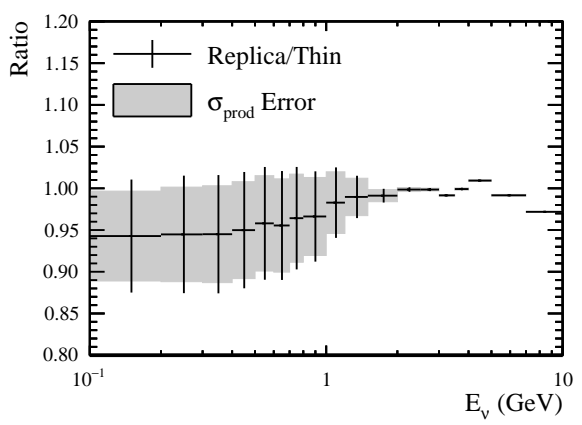

SK: Antineutrino Mode, $v_{\mu}$

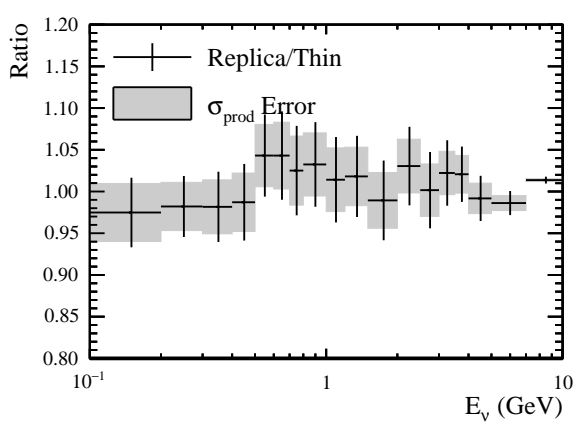

SK: Antineutrino Mode, $v_{\mathrm{e}}$

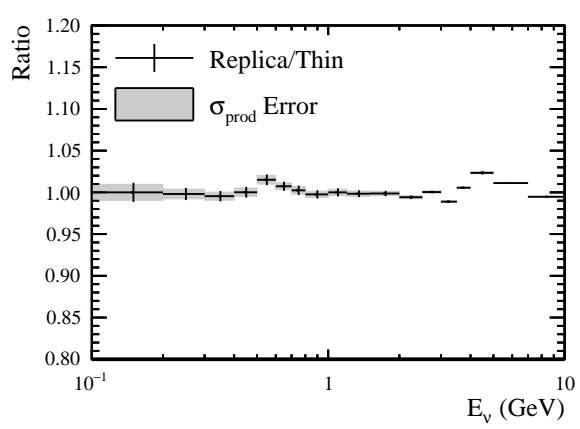

SK: Neutrino Mode, $\nabla_{\mu}$

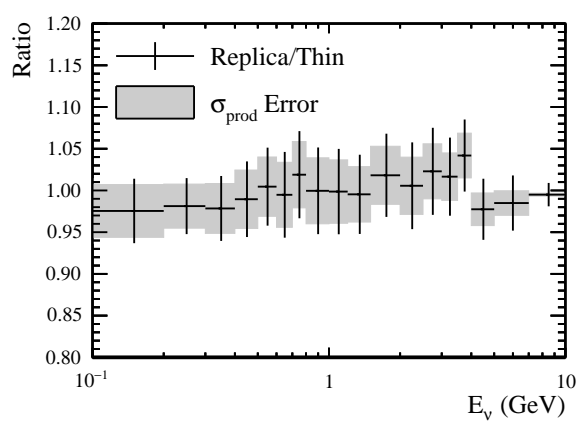

SK: Neutrino Mode, $\boldsymbol{\nabla}_{\mathrm{e}}$

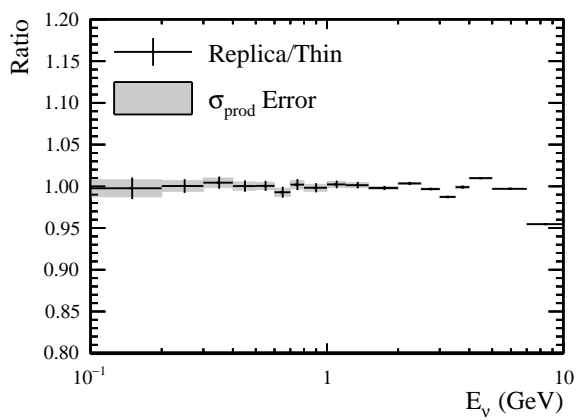

SK: Antineutrino Mode, $\nabla_{\mu}$

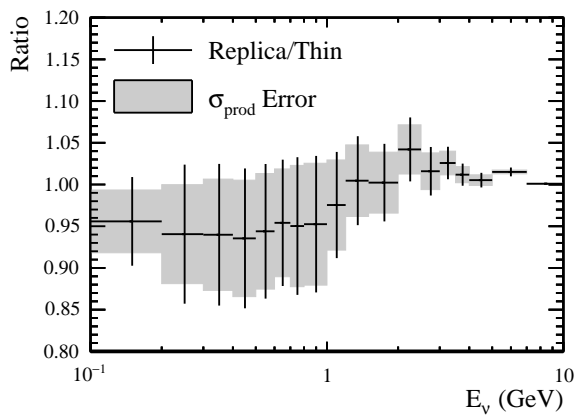

SK: Antineutrino Mode, $\nabla_{\mathrm{e}}$

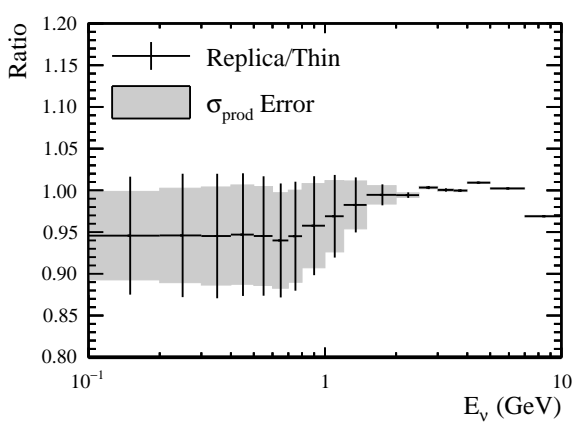

Figure 6.6: Ratio of the replica-tuned (13av4.0) flux to the thin-tuned (13av3.0) flux at Super-Kamiokande. Here tuning has been applied on the same set of nominal T2K flux files instead of the combined fluxes for all T2K runs. The total uncertainty on the ratio (vertical error bars) has been calculated by combining both multiplicity and interaction length ( $\sigma_{\text {prod }}$, grey error bands) uncertainties. All other flux uncertainties on the ratio cancel out because of full correlations between the two tuning procedures. Copyright by T2K Collaboration. 


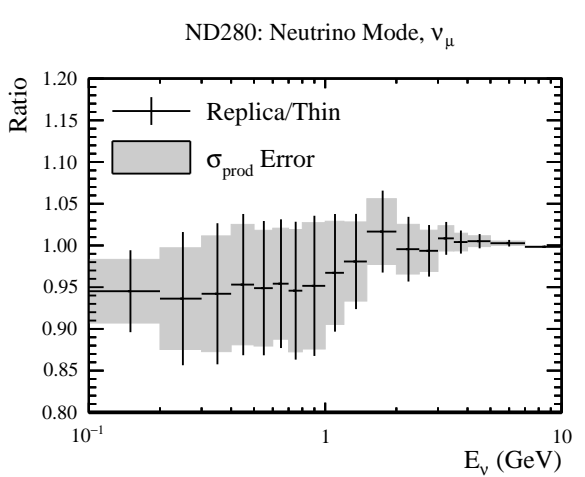

ND280: Neutrino Mode, $v_{\mathrm{e}}$

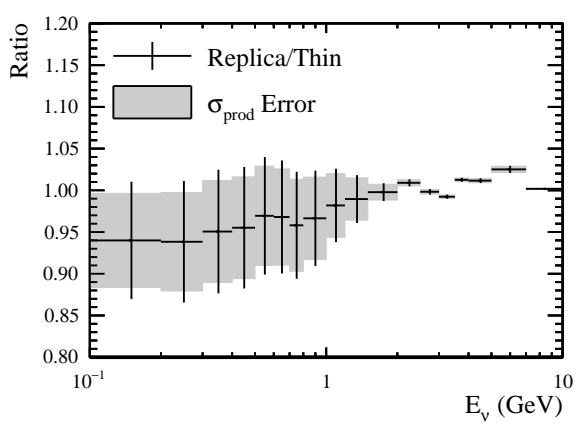

ND280: Antineutrino Mode, $v_{\mu}$

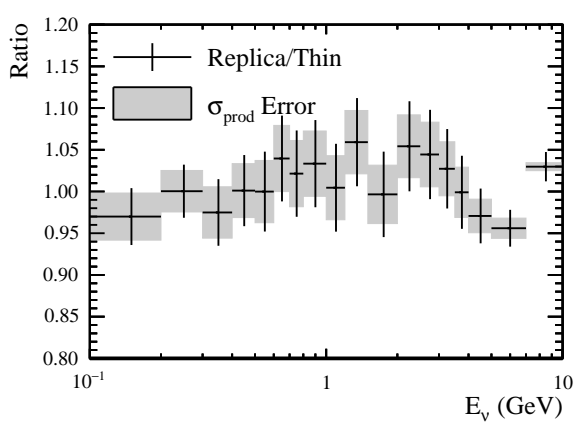

ND280: Antineutrino Mode, $v_{\mathrm{e}}$

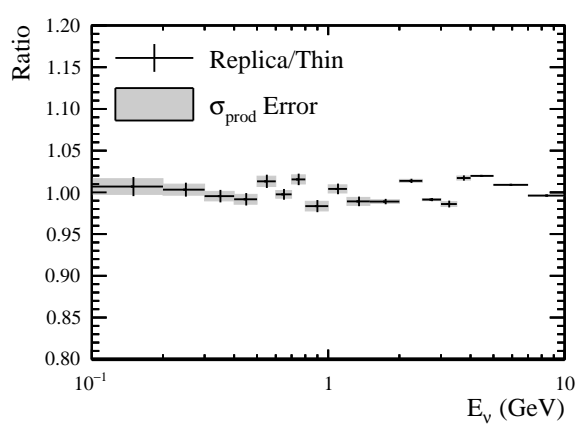

ND280: Neutrino Mode, $\nabla_{\mu}$

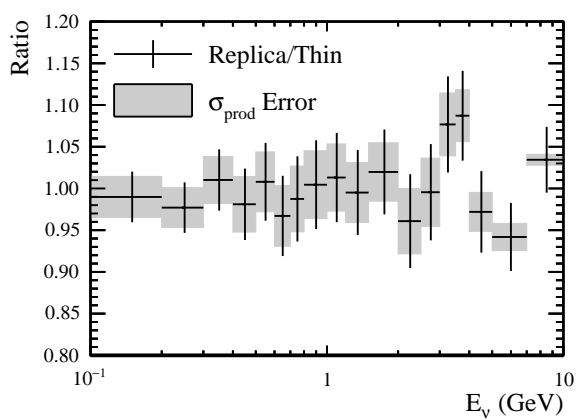

ND280: Neutrino Mode, $\boldsymbol{\nabla}_{\mathrm{e}}$

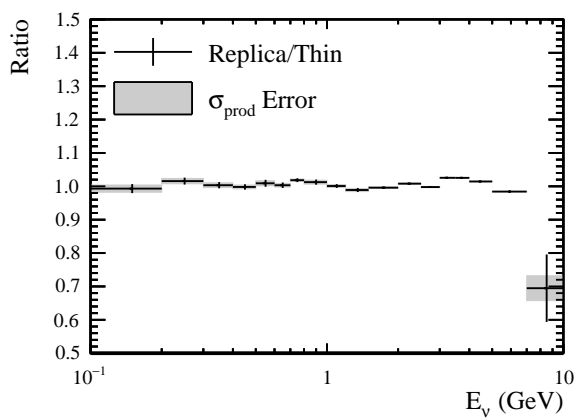

ND280: Antineutrino Mode, $\nabla_{\mu}$

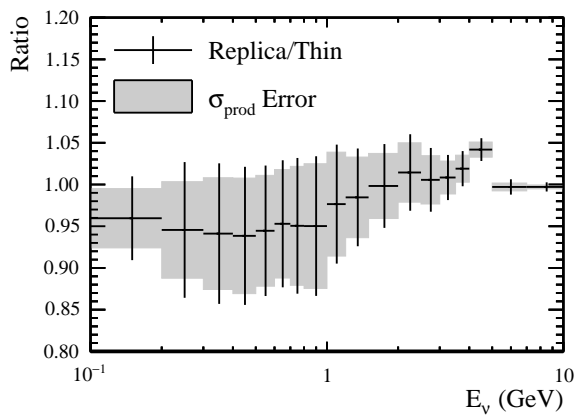

ND280: Antineutrino Mode, $\nabla_{\mathrm{e}}$

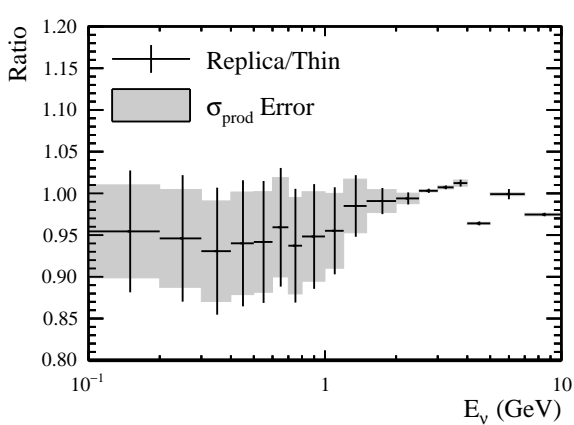

Figure 6.7: Ratio of the replica-tuned (13av4.0) flux to the thin-tuned (13av3.0) flux at ND280. Here tuning has been applied on the same set of nominal T2K flux files instead of the combined fluxes for all $\mathrm{T} 2 \mathrm{~K}$ runs. The total uncertainty on the ratio (vertical error bars) has been calculated by combining both multiplicity and interaction length ( $\sigma_{\text {prod }}$, grey error bands) uncertainties. All other flux uncertainties on the ratio cancel out because of full correlations between the two tuning procedures. Copyright by T2K Collaboration. 


\subsection{Replica-tuned flux uncertainty}

The uncertainty with replica-tuning has been estimated using techniques that have been introduced in Chapter 5.5. The flux covariance matrix combines flux bins at ND280 and Super-K, with forward and reverse horn current focusing, for the $\nu_{\mu}, \bar{\nu}_{\mu}, \nu_{e}$ and $\bar{\nu}_{e}$ components. The resulting correlation matrix is provided in Figure 6.9. The bins are defined with respect to the true neutrino energy, with finer binning used around the T2K flux peak.

The replica-tuned flux uncertainties due to hadronic interaction modelling are provided in Fig. 6.10 for Super-K and Figure 6.11 for ND280. The inclusion of replica-target measurements in the flux prediction dramatically reduces the hadronic interaction length uncertainty at lower neutrino energies. This uncertainty is due to the uncertainty on the modelled hadron production cross section, since it directly affects the distance travelled by hadrons before interacting. The pion rescattering uncertainty and multiplicity uncertainty are also reduced compared to the previous flux release. A new source of flux uncertainty due to unconstrained yields of daughter mesons in certain hadronic interactions has been included in the flux release. The uncertainty due to effects unrelated to interaction modelling remains unchanged, and the reader is referred back to Figures 5.18 and 5.19. The combined total flux uncertainty for the flux measurement with replica target data is given in Figure 6.12 for Super-K, and in Figure 6.13 for ND280. The total flux uncertainty at the T2K beam peak for the signal flux, $\nu_{\mu}$ in $\nu$-mode and $\bar{\nu}_{\mu}$ in $\bar{\nu}$-mode, has been reduced from roughly $10 \%$ to $5 \%$. The errors on the wrong-flavour intrinsic flux components, $\nu_{e}$ in $\nu$-mode and $\bar{\nu}_{e}$ in $\bar{\nu}$-mode, are also reduced in the low energy flux tail. The parents of the wrong-sign neutrinos, $\bar{\nu}_{\mu}$ in $\nu$-mode and $\nu_{\mu}$ in $\bar{\nu}$-mode, are mostly produced from out-of-target interactions, and consequently no significant reduction in flux uncertainty is observed. In certain regions the total uncertainty is increased compared to the thin-tuning method. This is due to the inclusion of the uncertainty from unconstrained interactions, which has been neglected in previous analyses. 


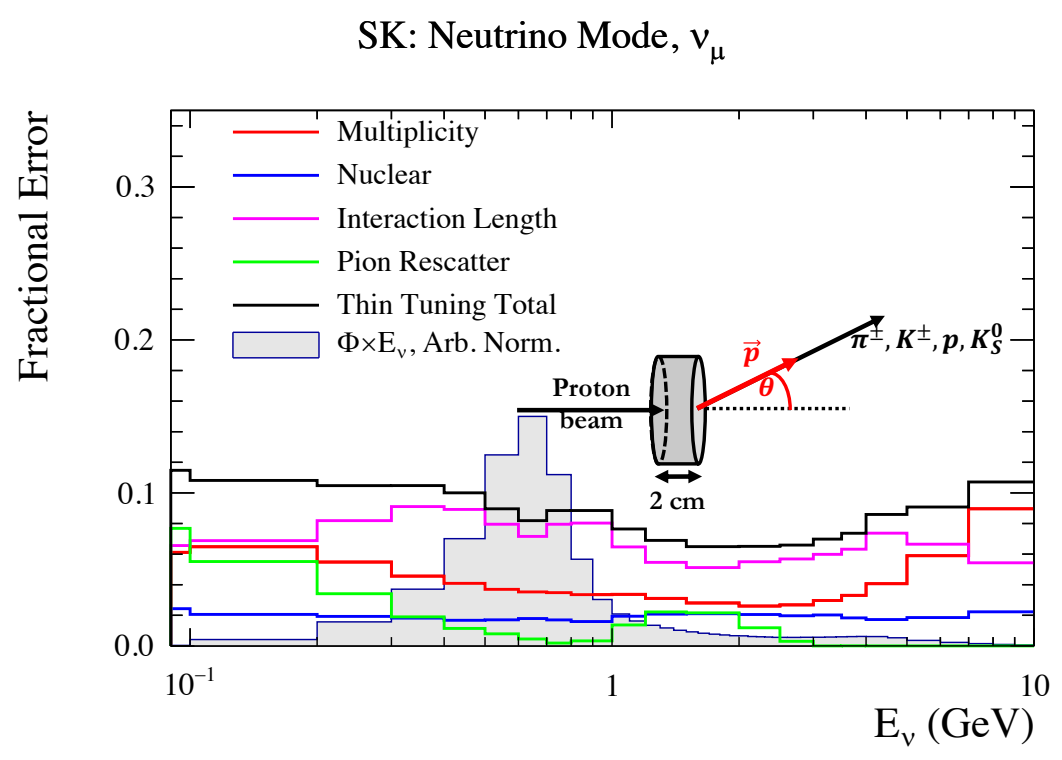

SK: Neutrino Mode, $v_{\mu}$

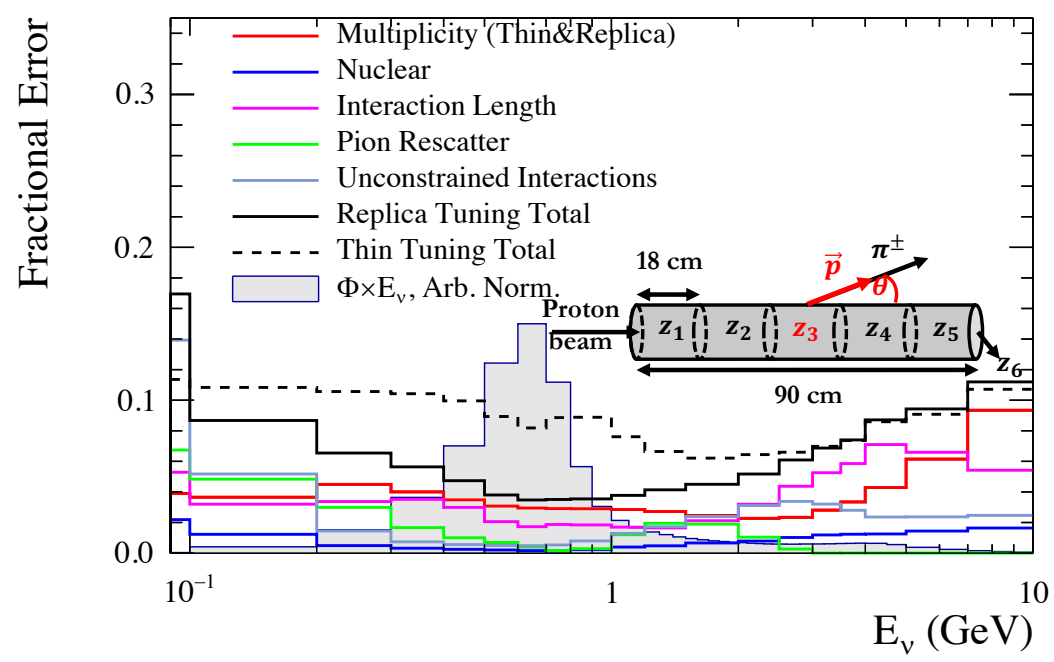

Figure 6.8: A direct comparison of the hadronic interaction modelling portion of flux uncertainties at Super-K with thin-tuning (upper figure) and replica-tuning (lower figure). The solid and dashed black lines in the lower figure correspond to the replica-tuned and the thin-tuned systematic flux uncertainties due to hadron interaction modelling, respectively. The reduction in flux uncertainty at the T2K flux peak $(600 \mathrm{MeV})$, achieved through the implementation of replica-tuning, is clearly visible in the lower figure. Copyright by T2K Collaboration.

The effect of the substantial flux error reduction and the slight shift in the absolute flux prediction with replica-tuning for the T2K oscillation analysis will be examined in the following chapters. 


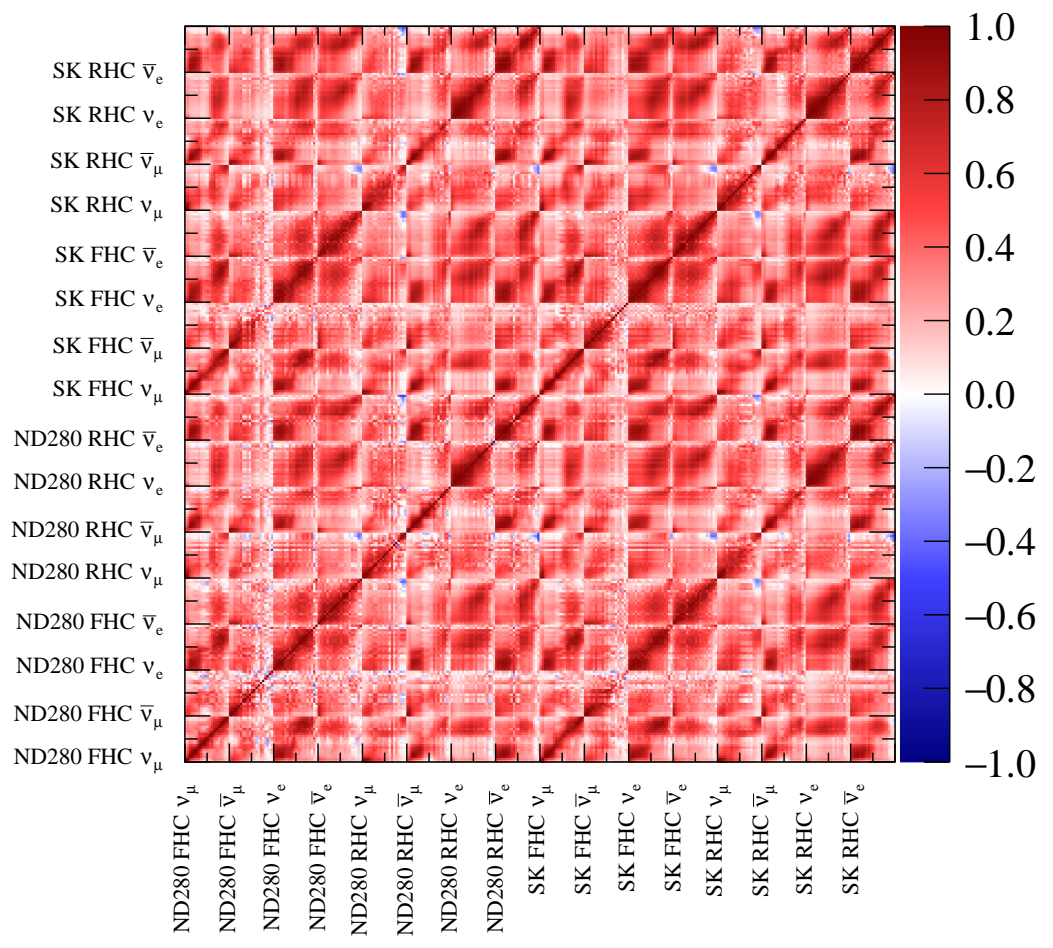

Figure 6.9: The correlation matrix for the replica-tuned flux prediction. Flux is binned in neutrino energy. The starting bins corresponding to flux at both ND280 and Super-K, with different modes of operation (FHC and RHC), and for each neutrino flavour, have been labelled. Copyright by T2K Collaboration. 


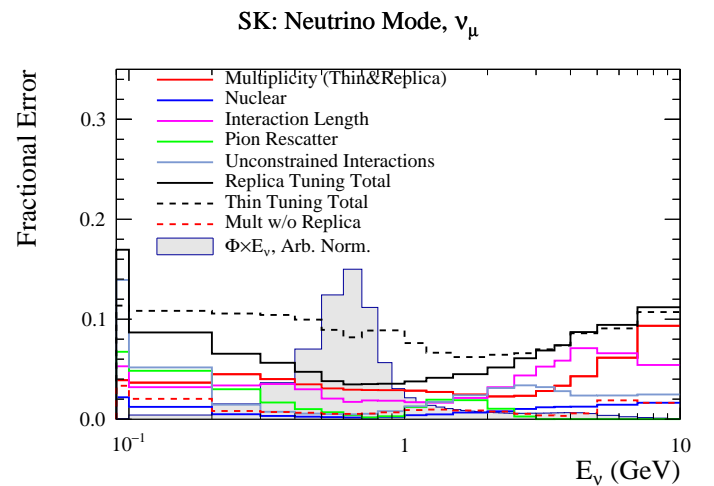

SK: Neutrino Mode, $v_{\mathrm{e}}$

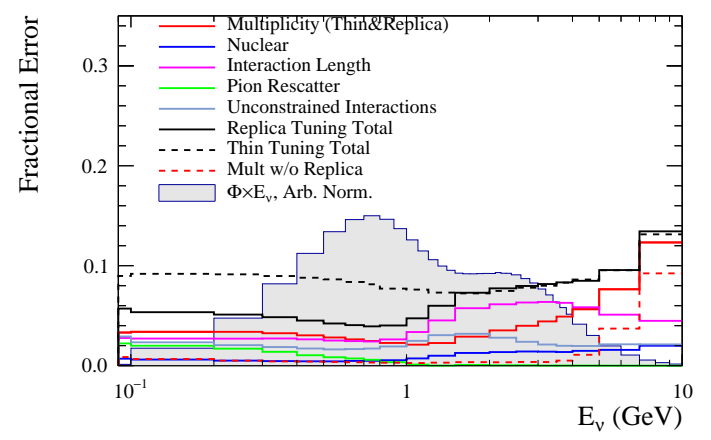

SK: Antineutrino Mode, $v_{\mu}$

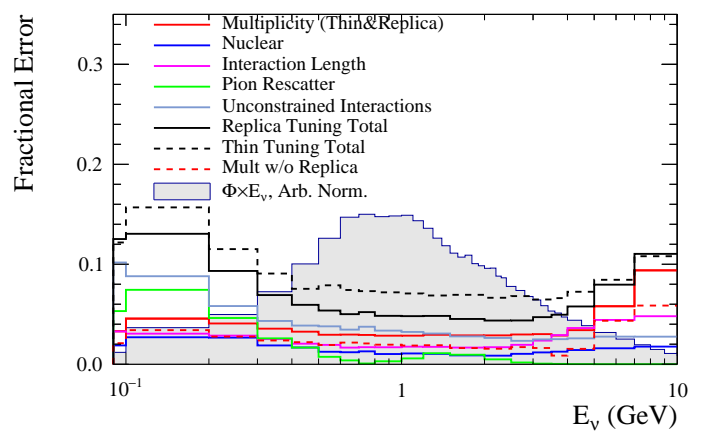

SK: Antineutrino Mode, $v_{\mathrm{e}}$

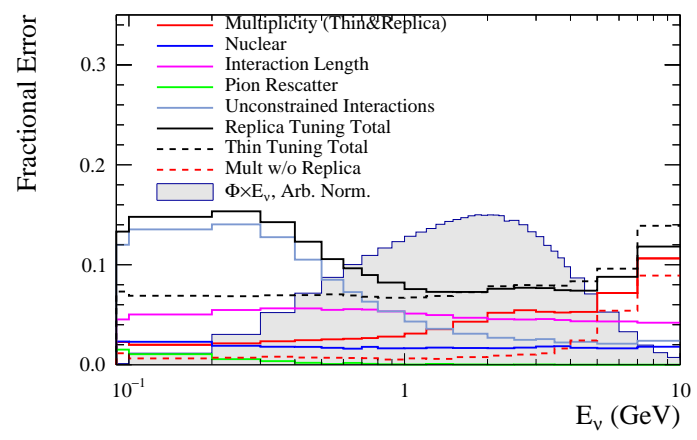

SK: Neutrino Mode, $\bar{v}_{\mu}$

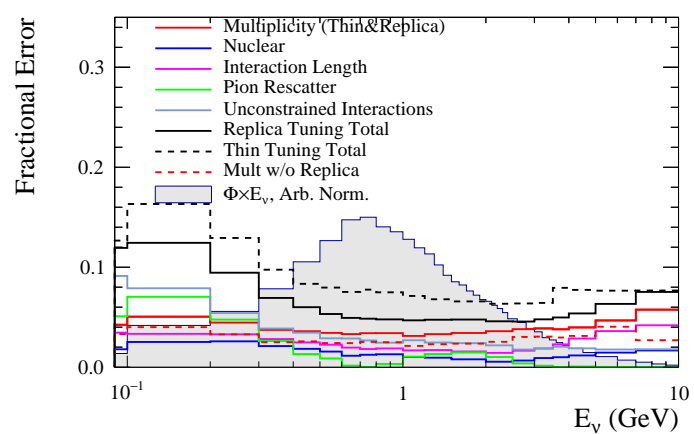

SK: Neutrino Mode, $\bar{v}_{\mathrm{e}}$

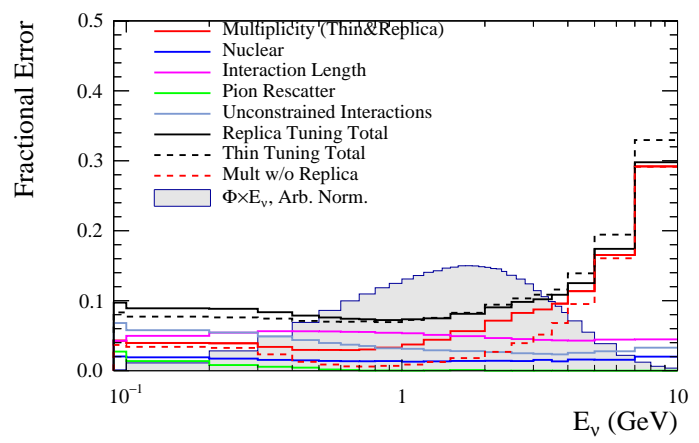

SK: Antineutrino Mode, $\overline{\mathrm{v}}_{\mu}$

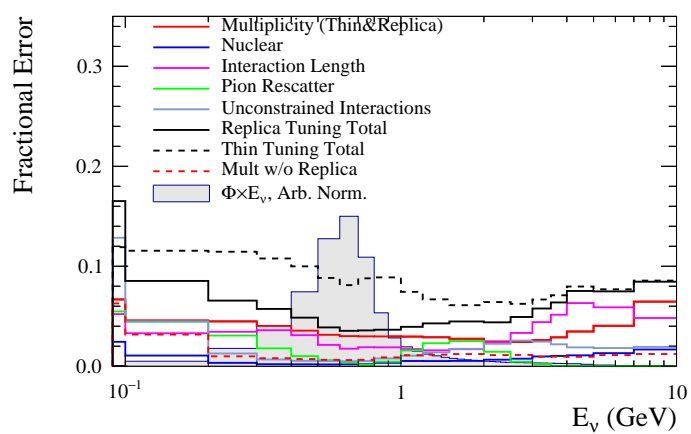

SK: Antineutrino Mode, $\bar{v}_{\mathrm{e}}$

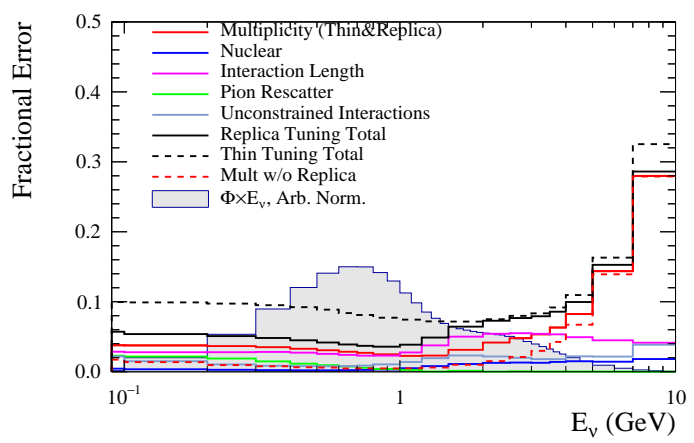

Figure 6.10: The hadronic interaction modelling portion of flux uncertainties at Super$\mathrm{K}$, evaluated with the addition of 2009 replica-target data. The uncertainties have been estimated in neutrino mode (upper four plots) and anti-neutrino mode (lower four plots), for the $\nu_{\mu}, \bar{\nu}_{\mu}, \nu_{e}$ and $\bar{\nu}_{e}$ flux components. Copyright by T2K Collaboration. 

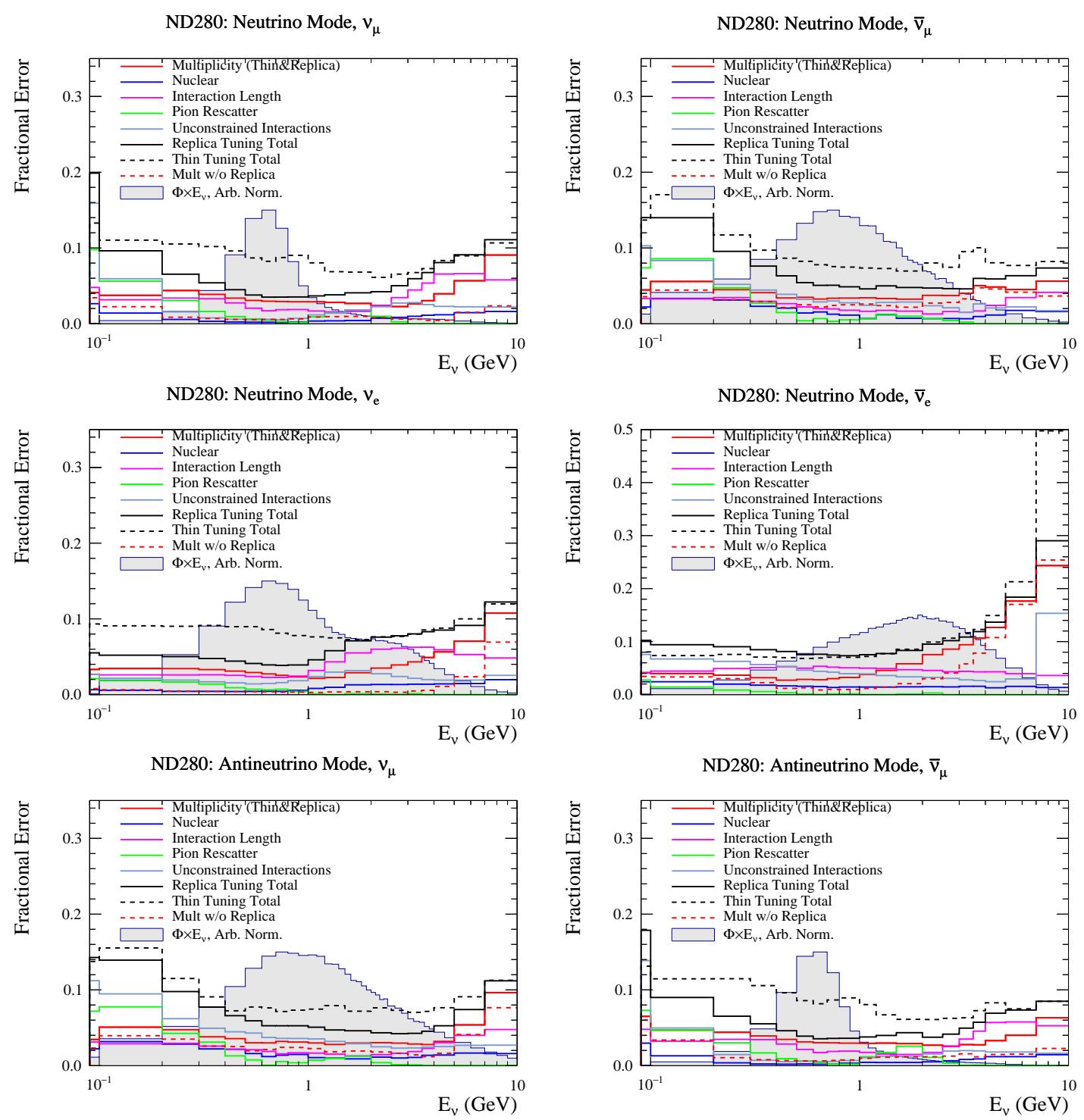

ND280: Antineutrino Mode, $v_{\mathrm{e}}$
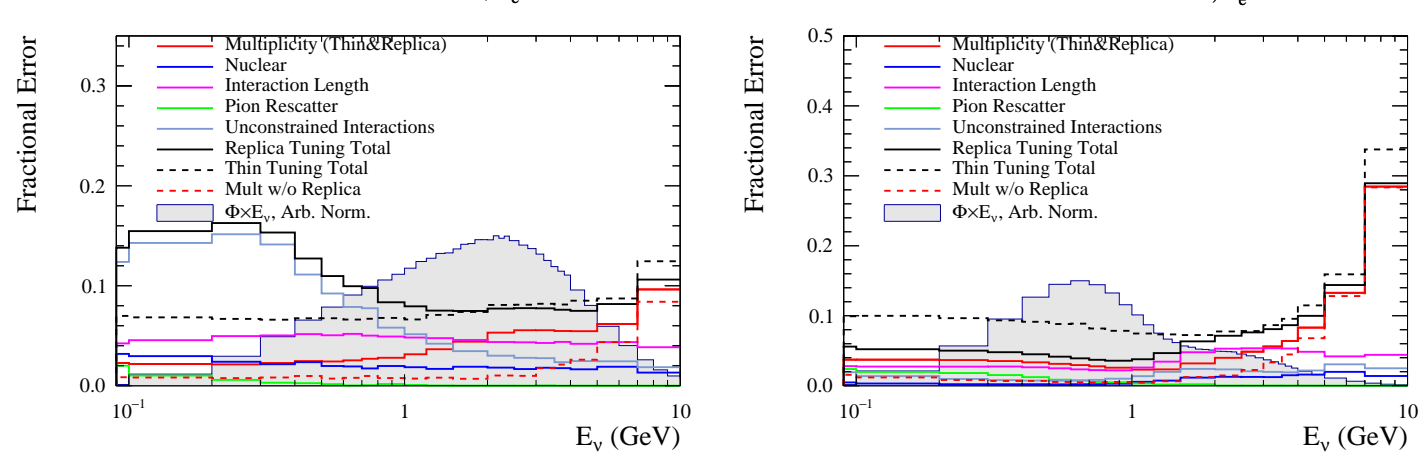

Figure 6.11: The hadronic interaction modelling portion of flux uncertainties at ND280, evaluated with the addition of 2009 replica-target data. The uncertainties have been estimated in neutrino mode (upper four plots) and anti-neutrino mode (lower four plots), for the $\nu_{\mu}, \bar{\nu}_{\mu}, \nu_{e}$ and $\bar{\nu}_{e}$ flux components. Copyright by T2K Collaboration. 
SK: Neutrino Mode, $v_{\mu}$

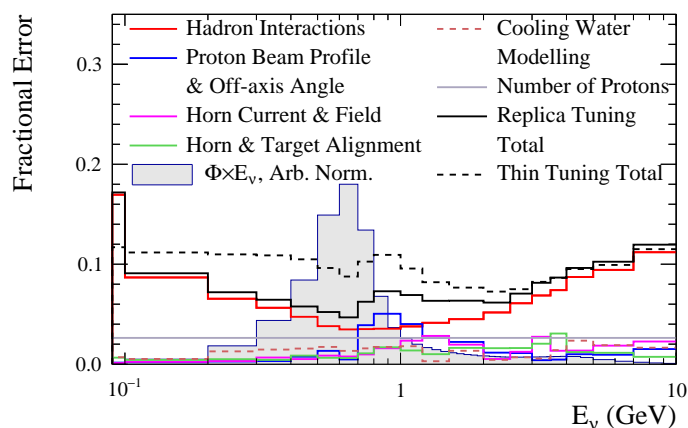

SK: Neutrino Mode, $v_{\mathrm{e}}$

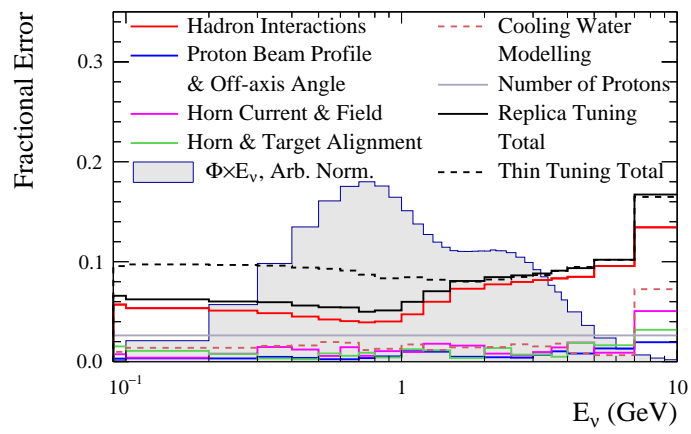

SK: Antineutrino Mode, $v_{\mu}$

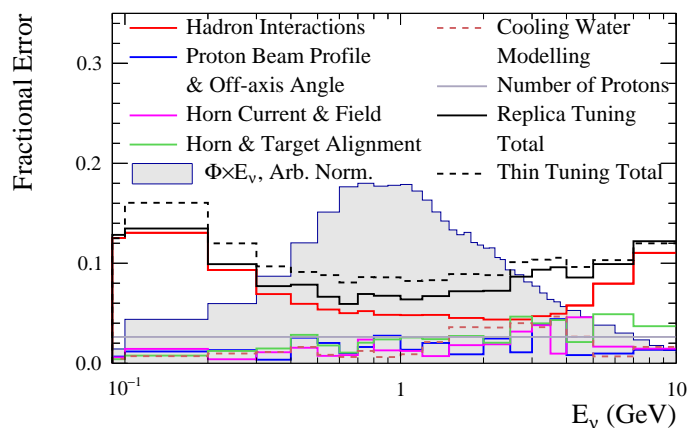

SK: Antineutrino Mode, $v_{\mathrm{e}}$

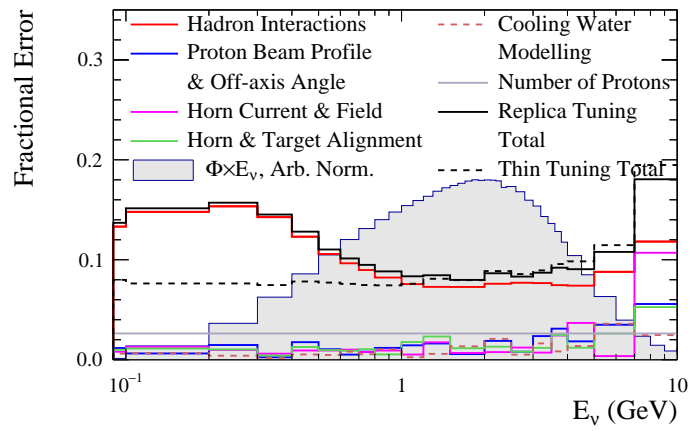

SK: Neutrino Mode, $\bar{v}_{\mu}$

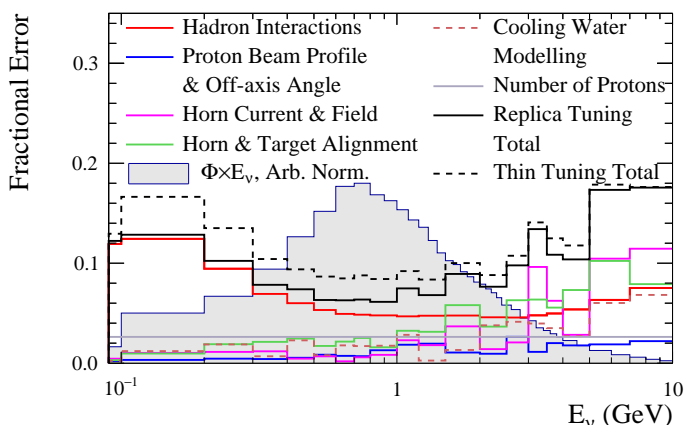

SK: Neutrino Mode, $\bar{v}_{\mathrm{e}}$

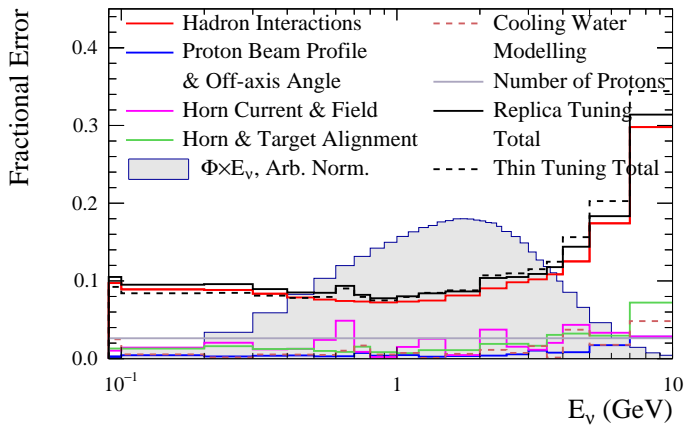

SK: Antineutrino Mode, $\bar{\nabla}_{\mu}$

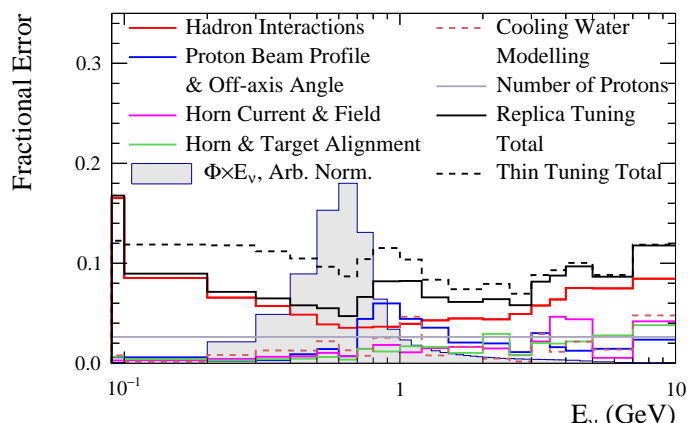

SK: Antineutrino Mode, $\bar{v}_{\mathrm{e}}$

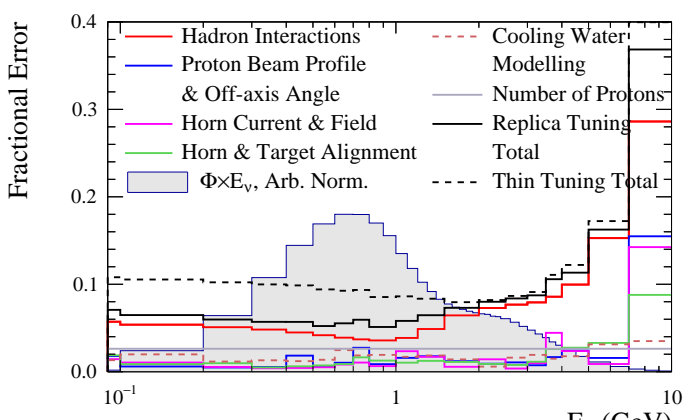

$\mathrm{E}_{\mathrm{v}}(\mathrm{GeV})$

Figure 6.12: Total flux uncertainties at Super-K, evaluated with the addition of 2009 replica-target data. The uncertainties have been estimated in neutrino mode (upper four plots) and anti-neutrino mode (lower four plots), for the $\nu_{\mu}, \bar{\nu}_{\mu}, \nu_{e}$ and $\bar{\nu}_{e}$ flux components. Copyright by T2K Collaboration. 
ND280: Neutrino Mode, $v_{\mu}$

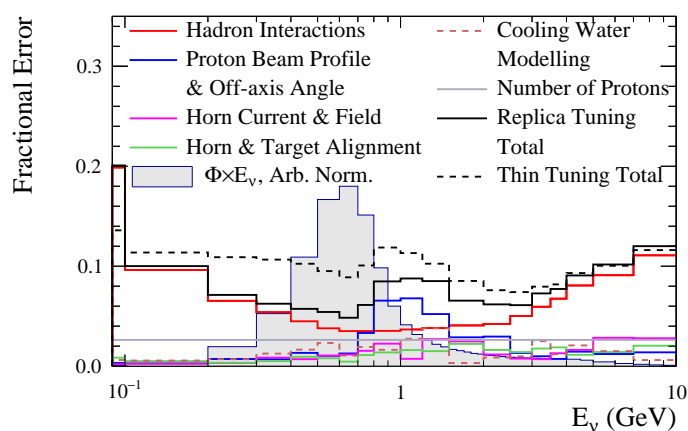

ND280: Neutrino Mode, $v_{\mathrm{e}}$

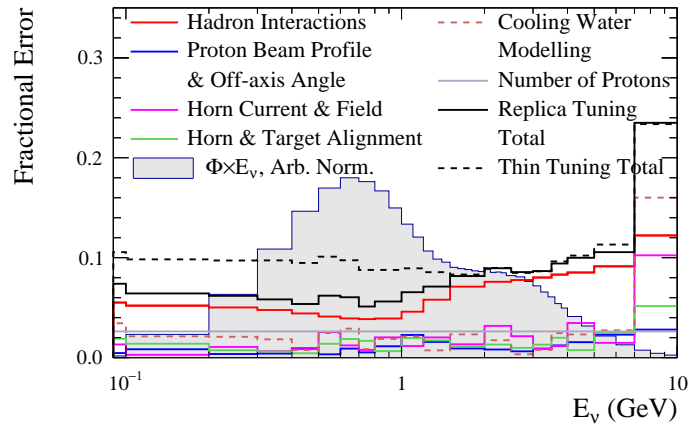

ND280: Antineutrino Mode, $v_{\mu}$

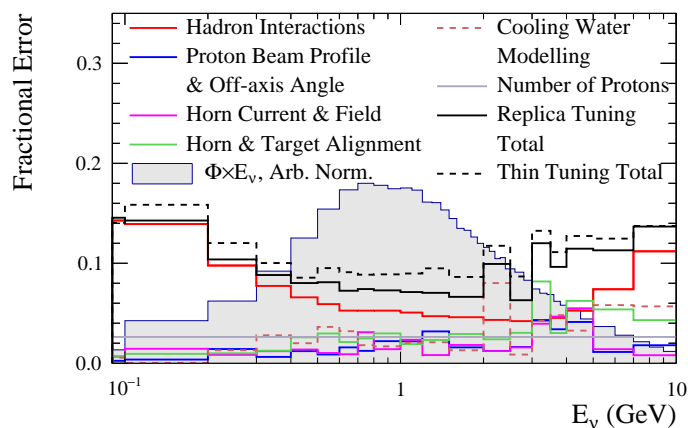

ND280: Antineutrino Mode, $v_{\mathrm{e}}$

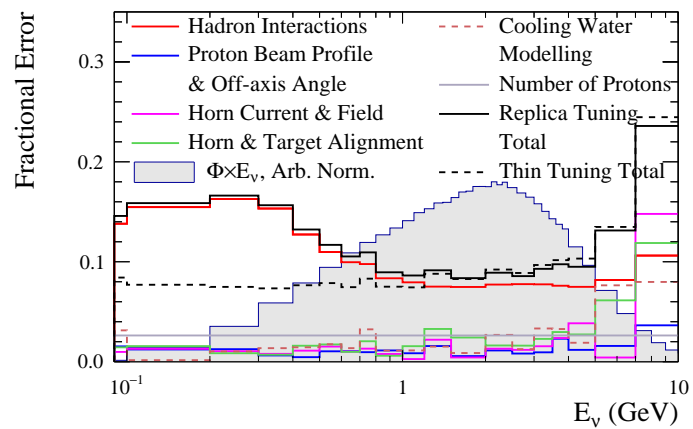

ND280: Neutrino Mode, $\bar{\nabla}_{\mu}$

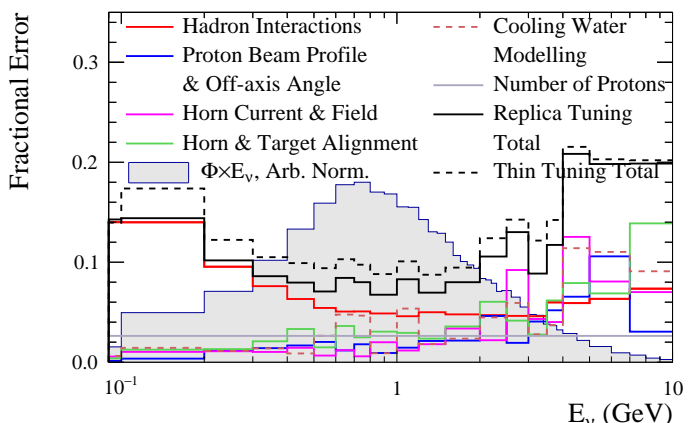

ND280: Neutrino Mode, $\overline{\mathrm{v}}_{\mathrm{e}}$

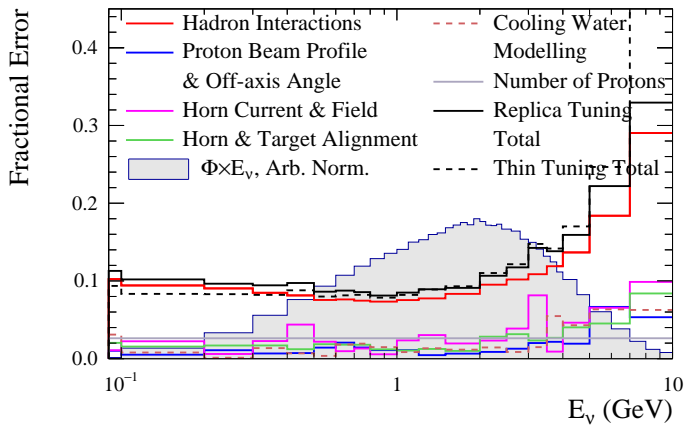

ND280: Antineutrino Mode, $\bar{v}_{\mu}$

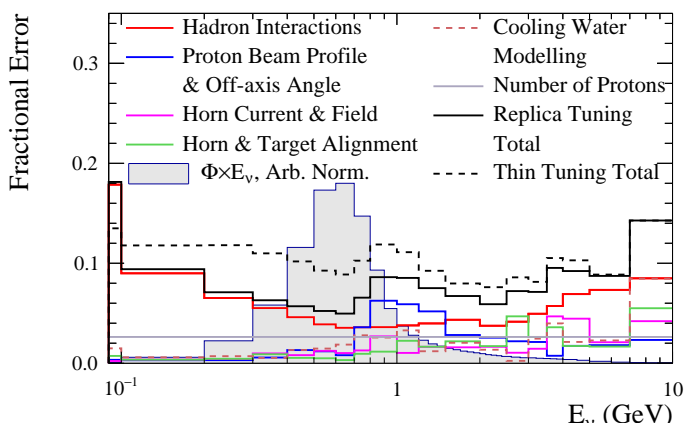

ND280: Antineutrino Mode, $\bar{v}_{\mathrm{e}}$

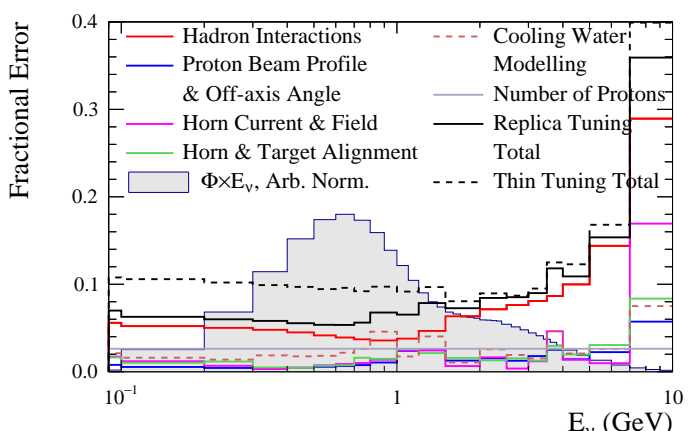

Figure 6.13: Total flux uncertainties at ND280, evaluated with the addition of 2009 replica-target data. The uncertainties have been estimated in neutrino mode (upper four plots) and anti-neutrino mode (lower four plots), for the $\nu_{\mu}, \bar{\nu}_{\mu}, \nu_{e}$ and $\bar{\nu}_{e}$ flux components. Copyright by T2K Collaboration. 


\subsection{Model dependence of NA61 measurement un- folding}

The NA61 replica-target $\pi^{ \pm}$exiting multiplicity has been released in $762(p, \theta, z)$ analysis bins, collectively denoted by $\vec{t}=\left(t_{1}, t_{2}, \ldots, t_{762}\right)$, where $t_{i}$ denotes the differential multiplicity in the $\mathrm{i}^{\text {th }}$ analysis bin $(p, \theta, z)$. The replica-target data that was passed on to T2K has already been unfolded. The unfolding method adopted by the NA61 collaboration calculates the "true" multiplicities $\overrightarrow{\mathrm{t}}$ by applying bin by bin corrections to the "reconstructed" (measured) multiplicities $\vec{r}$, as shown in the following equation:

$$
\begin{aligned}
& \left.\mathrm{t}_{\mathrm{i}}\right|_{\text {data }}=\left.\mathrm{r}_{\mathrm{i}}\right|_{\text {data }} \times \text { Correction }_{\mathrm{i}} \\
& =\left.\mathrm{r}_{\mathrm{i}}\right|_{\text {data }} \times\left.\left(\frac{\mathrm{t}_{\mathrm{i}}}{\mathrm{r}_{\mathrm{i}}(\overrightarrow{\mathrm{t}})}\right)\right|_{\text {FLUKA }}
\end{aligned}
$$

where the correction factors are calculated from simulations with FLUKA. The outlined unfolding technique is evidently model dependent. The study presented in this chapter estimates the degree of this model dependence. Following the NA61 approach, in this study correction factors were derived from the FLUKA model. Multiplicity predictions from GEANT4 are used as simulated data. These predictions have been generated from different physics lists, QGSP_BERT, FTF_BIC, FTFP_BERT and NuBeam, incorporating a wide range of underlying hadron interaction models. The validity of the correction factors is tested against these different models, thus ensuring a fairly conservative estimate of the NA61 unfolding uncertainty.

Naturally, it is impossible for a single modelling algorithm to capture the behaviour of hadronic interactions across many orders of energy scale. Typically, each of the GEANT4 physics lists incorporates different models overlapping across boundary regions. For example, QGSP_BERT is the physics list most commonly used in the high energy physics community, by collaborations such as ATLAS. It uses the quark-gluon string model[125] for interactions of hadrons with energies above $12 \mathrm{GeV}$. The Bertini style[126] intra-nuclear cascade model[127] (BERT) is implemented for energies below $9.9 \mathrm{GeV}$. The low energy parametrised (LEP) model[128] is used in the intermediate energy range, between $9.5 \mathrm{GeV}$ and $25 \mathrm{GeV}$. 
The FTF_BIC and FTFP_BERT lists both use Fritiof-like models[129] for high energy hadronic interactions. However, their treatment of lower energy interactions differs, with FTF_BIC using the binary and FTFP_BERT the Bertini cascade model. Finally, NuBeam is a GEANT4 physics list developed for the needs of the neutrino physics community. Here, special emphasis is placed on the primary beams and target materials commonly employed in neutrino beamlines.

The migration matrices necessary for transforming between the simulated (true) and the reconstructed pion multiplicities have been obtained from NA61 analysers[93], and are shown in Fig. 6.14. The reconstructed multiplicities $\overrightarrow{\mathrm{r}}$ can be obtained through simple multiplication of the true multiplicities $\vec{t}$ with the migration matrix M:

$$
\mathrm{r}_{\mathrm{i}}=\sum_{\mathrm{j}=1}^{762} \mathrm{M}_{\mathrm{ij}} \times \mathrm{t}_{\mathrm{j}}
$$

where $\mathrm{r}_{\mathrm{i}}$ is the reconstructed multiplicity in analysis bin i specified by the $(p, \theta, z)$ coordinate. Effectively, multiplying the nominal pion multiplicities from different Monte Carlo generators by the migration matrix accounts for both detector efficiency and resolution smearing.

For testing the model dependence of the unfolding procedure, true FLUKA multiplicity predictions have been smeared with the migration matrices, and bin by bin weights have been calculated for transforming from the smeared back to the unsmeared (true) values. These correction weights correspond to the multiplicative factor $\left.\left(\frac{\mathrm{t}_{\mathrm{i}}}{\mathrm{r}_{\mathrm{i}}(\overrightarrow{\mathrm{t}})}\right)\right|_{\text {FLUKA }}$ in Eq. 6.2. The correction weights for $\pi^{ \pm}$exiting multiplicities are provided in Fig. 6.15.

Next, GEANT4 is used to generate fake multiplicity datasets, which are then smeared according to the NA61 migration matrix. The smeared multiplicities are then unfolded through multiplication with the FLUKA correction factors, and compared to the starting GEANT4 multiplicities. In other words, $\left.t_{i}\right|_{\text {GEANT4 }}$ is compared to $\left.\left(\sum_{j=1}^{762} \mathrm{M}_{\mathrm{ij}} \times\left.\mathrm{t}_{\mathrm{j}}\right|_{\text {GEANT4 }}\right)\left(\frac{\mathrm{t}_{\mathrm{i}}}{\mathrm{r}_{\mathrm{i}}(\overrightarrow{\mathrm{t}})}\right)\right|_{\text {FLUKA }}$. Any difference between the 

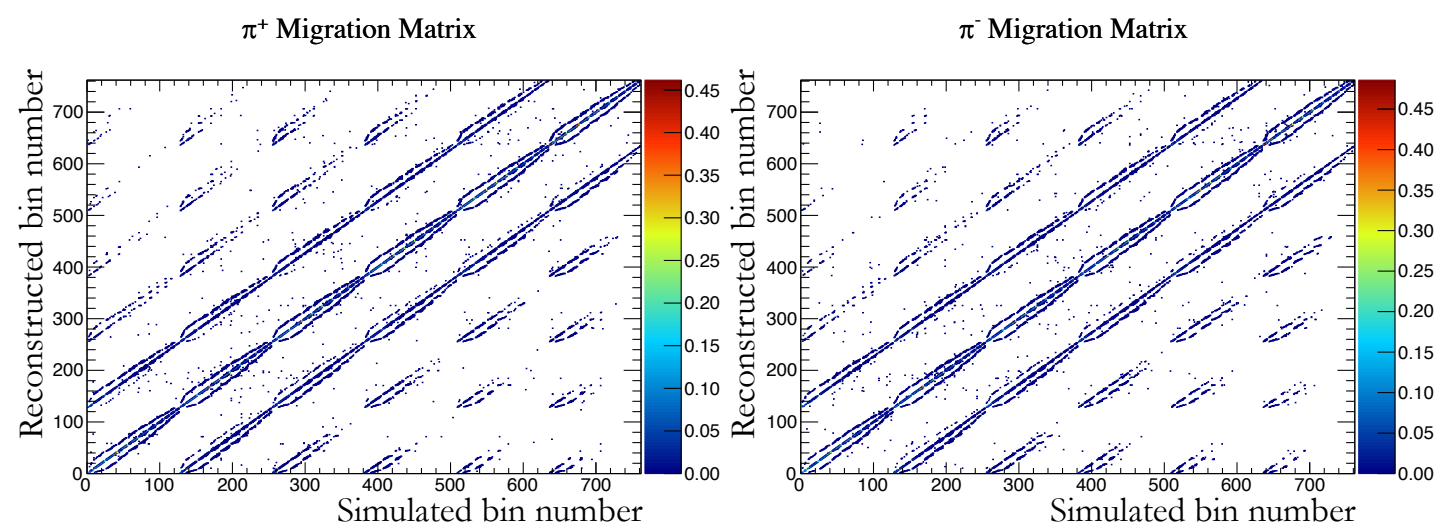

Figure 6.14: Migration matrices $\mathrm{M}_{\pi^{ \pm}}$describing how to transform from simulated pion multiplicities $\overrightarrow{\mathrm{t}}$ to what would actually be measured by the NA61 spectrometer $\overrightarrow{\mathrm{r}}$ : $\mathrm{r}_{\mathrm{i}}=\sum_{\mathrm{j}=1}^{762} \mathrm{M}_{\mathrm{ij}} \times \mathrm{t}_{\mathrm{j}}$. The vertical and horizontal axes list correspond to the 762 reconstructed and simulated bins of the NA61 dataset, respectively. The bin numbering scheme iterates across exiting position $z$ (upstream to downstream), angle $\theta$ (lowest to highest) and momentum $p$ (lowest to highest). The matrix element $\mathrm{M}_{\mathrm{ij}}<1$ gives the likelihood for pions exiting from bin $\mathrm{j}$ to be reconstructed as exiting from bin $\mathrm{i}$, given the finite resolution and efficiency of detector components.

$\pi^{+}$Unfolding Weight

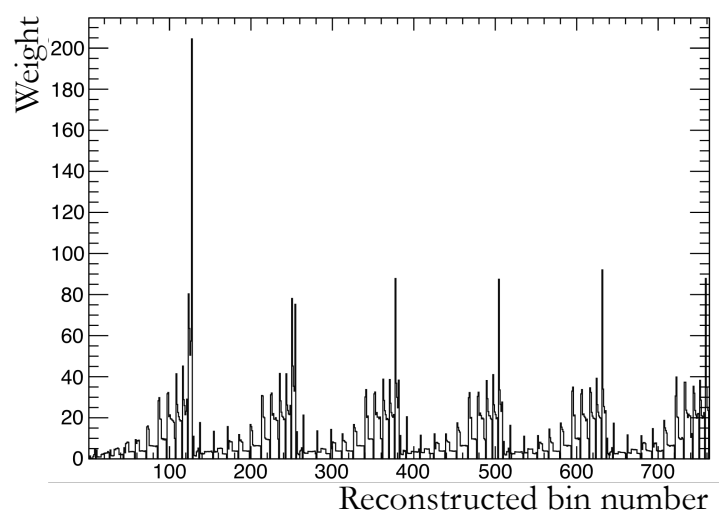

$\pi^{-}$Unfolding Weight

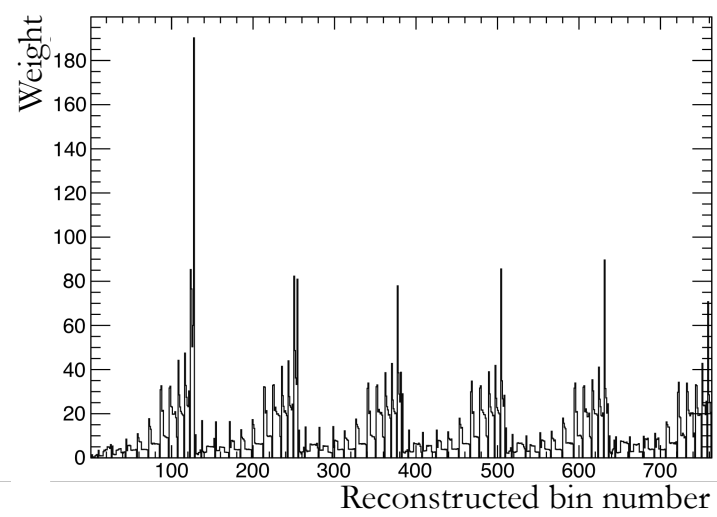

Figure 6.15: Weights to be applied on measured multiplicities to remove effects of geometric acceptance, detector inefficiencies and bin migrations (resolution), and calculate the "true" pion multiplicities. Efficiencies of various detector components have been absorbed into the unfolding weights, hence weights are generally greater than unity.

nominal multiplicity and the smeared plus unfolded multiplicity comes from the underlying difference between the FLUKA and GEANT4 Monte Carlo generators, since all input parameters such as the primary proton beam profile and replica-target geometry were kept constant throughout this analysis.

The greatest difference between the true and the smeared plus unfolded pion 
yields have been observed when using the QGSP_BERT generator, and are given in Fig. 6.16 and Fig. 6.17 for $\pi^{+}$and $\pi^{-}$yields, respectively. Comparisons of pion yields for other GEANT4 physics lists exhibit similar trends, but have not been included in this thesis due to space constraints. Even for this most extreme case where the multiplicity ratio differs from unity by as much as $60 \%$ for certain analysis bins, for the vast majority of $(p, \theta, z)$ bins the model dependent unfolding technique introduces multiplicity variations on the order of $1-2 \%$.

If NA61 replica-target data agreed better with one of the GEANT4 physics lists than with FLUKA, then the inverse of the ratios given in Fig. 6.16 and Fig. 6.17 could be used as a correction factor to the standard $\frac{\text { NA61 }\left.\right|_{\text {unfolded(FLUKA) }}}{\text { FLUKA }}$ multiplicity weights. The corrected multiplicity weights can then be used as new inputs for flux tuning, in order to study the sensitivity of the tuned flux on the Monte Carlo generator that was used to unfold the NA61 measurements. In other words, when different GEANT4 physics lists are used for NA61 replica-target data unfolding, the FLUKA unfolded multiplicities are corrected accordingly, and the flux tuned with these corrected weights is compared to the standard flux tuned to NA61 $\left.\right|_{\text {unfolded(FLUKA) }}$. The results are shown in Fig. 6.18 and Fig. 6.19, for tuned flux ratios at ND280 and the far detector, respectively. The right-sign flux changes by less than $0.5 \%$ when adjusting the unfolding procedure to different GEANT4 models. The wrong-sign flux typically changes by less than $1 \%$.

In conclusion, the unfolding technique adopted by NA61 could contribute at most a less than $1 \%$ variation on the tuned flux. Since very different Monte Carlo generators have been studied, this should be a fairly robust estimate. The magnitude of the fractional difference between fluxes tuned to NA61 data unfolded with FLUKA and QGSP_BERT is taken as the NA61 unfolding uncertainty. This uncertainty is negligible compared to other flux systematics, and has thus been neglected. 
$\pi^{+}$mult. ratios, bin z1, QGSP_BERT, smeared + unfolded / original

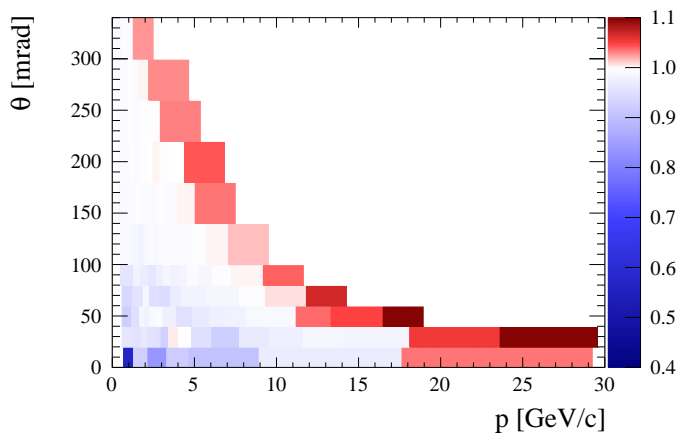

$\pi^{+}$mult. ratios, bin z3, QGSP_BERT, smeared + unfolded / original

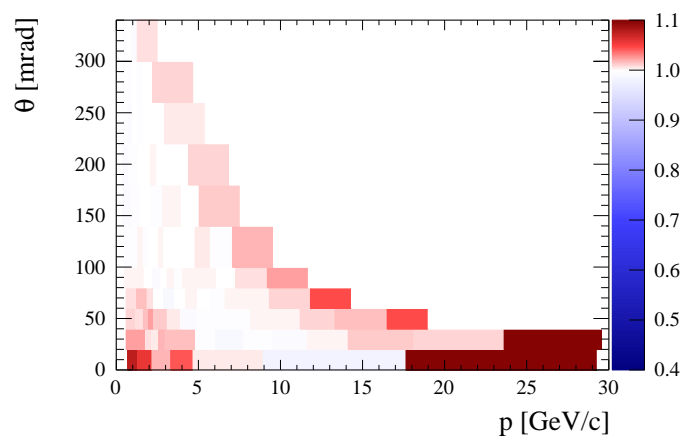

$\pi^{+}$mult. ratios, bin z5, QGSP_BERT, smeared + unfolded / original

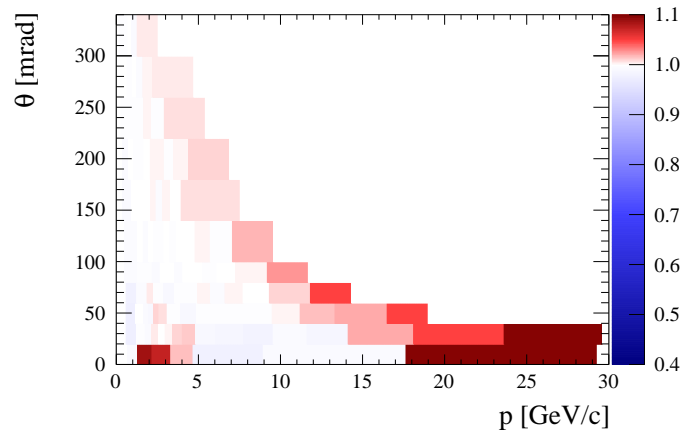

$\pi^{+}$mult. ratios, bin z2, QGSP_BERT, smeared + unfolded / original

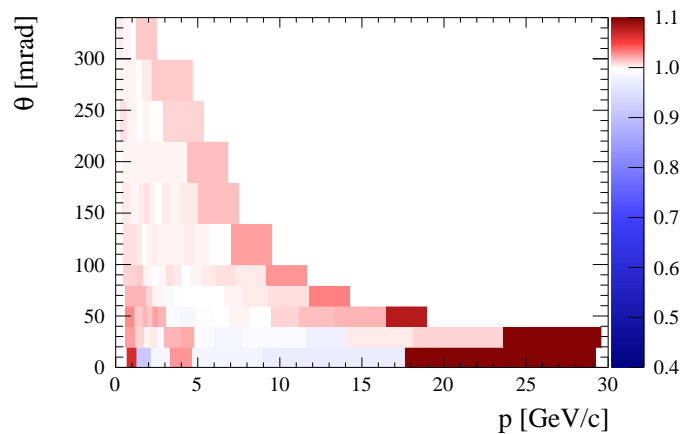

$\pi^{+}$mult. ratios, bin z4, QGSP_BERT, smeared + unfolded / original

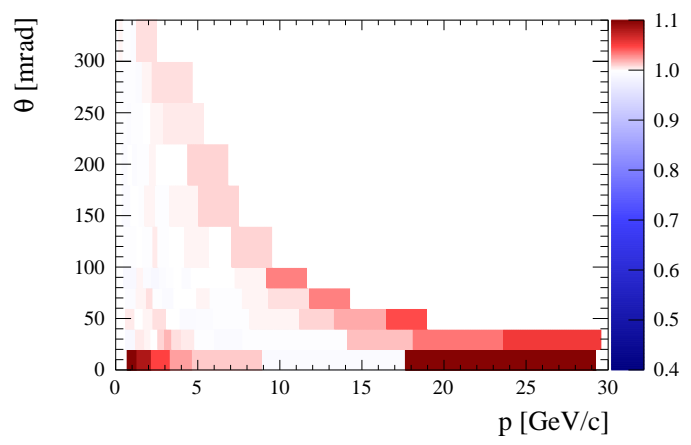

$\pi^{+}$mult. ratios, bin z6, QGSP_BERT, smeared + unfolded / original

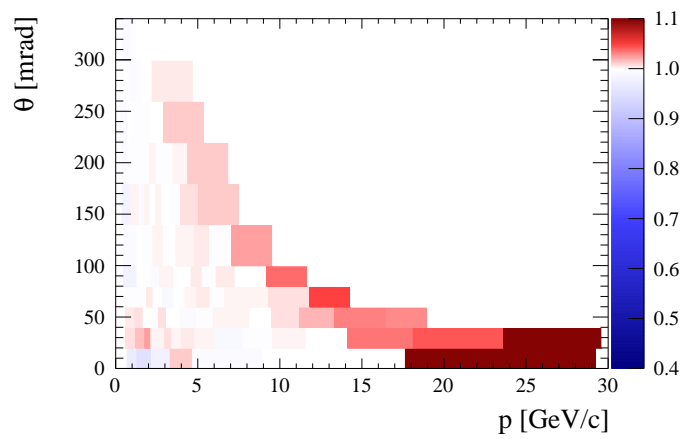

Figure 6.16: Ratio of the smeared plus unfolded and the nominal GEANT4 QGSP_BERT generated $\pi^{+}$multiplicities, where the bin by bin unfolding weights were derived from FLUKA. 
$\pi$ mult. ratios, bin z1, QGSP_BERT, smeared + unfolded / original

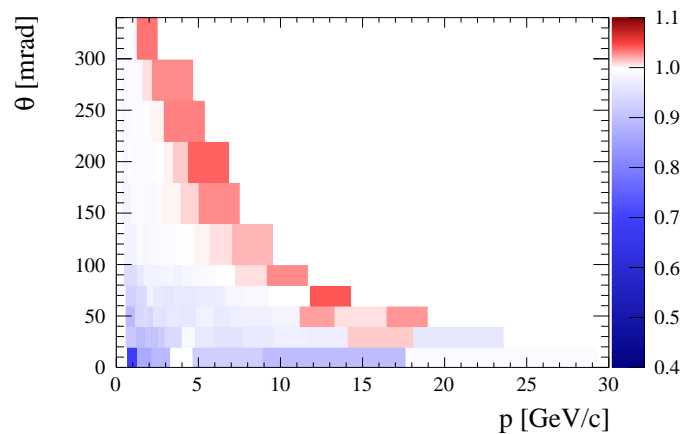

$\pi$ mult. ratios, bin z3, QGSP_BERT, smeared + unfolded / original

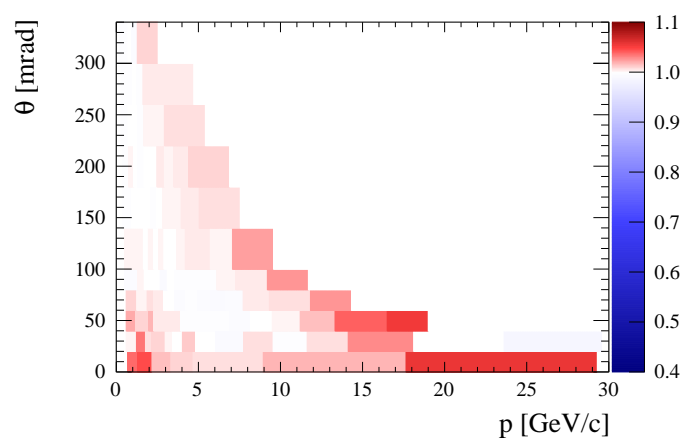

$\pi$ mult. ratios, bin z5, QGSP_BERT, smeared + unfolded / original

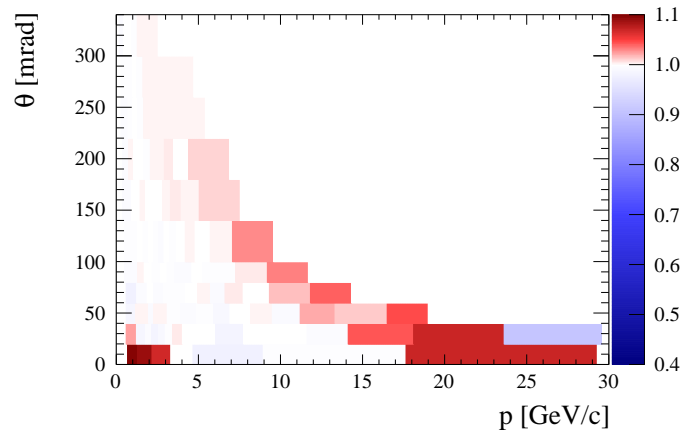

$\pi$ mult. ratios, bin z2, QGSP_BERT, smeared + unfolded / original

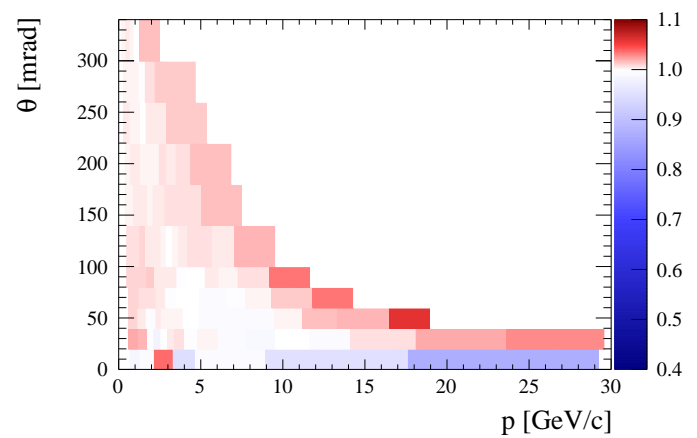

$\pi$ mult. ratios, bin z4, QGSP_BERT, smeared + unfolded / original

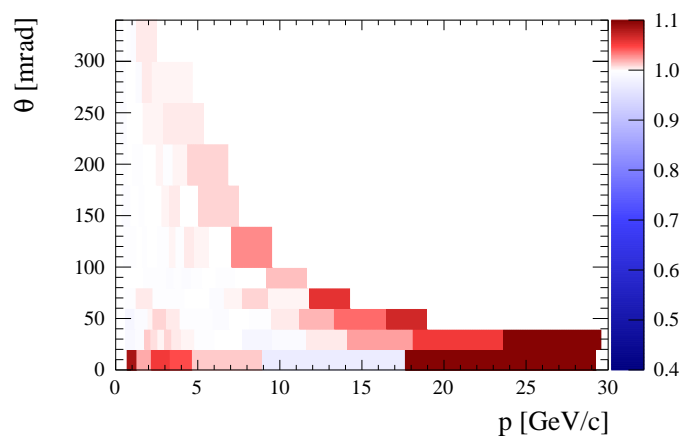

$\pi$ mult. ratios, bin z6, QGSP_BERT, smeared + unfolded / original

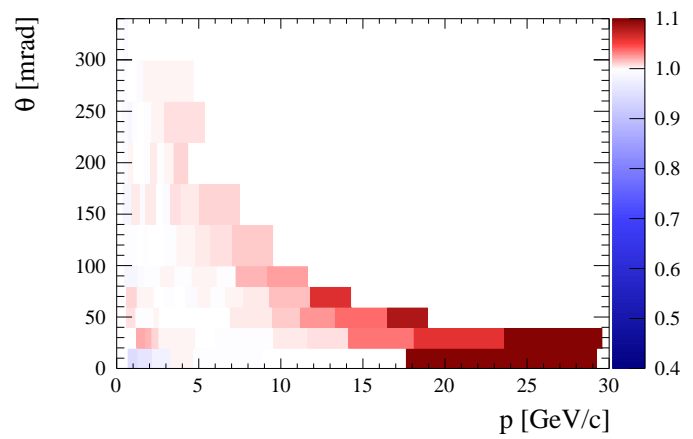

Figure 6.17: Ratio of the smeared plus unfolded and the nominal GEANT4 QGSP_BERT generated $\pi^{-}$multiplicities, where the bin by bin unfolding weights were derived from FLUKA. 
ND280: Neutrino Mode, $v_{\mu}$

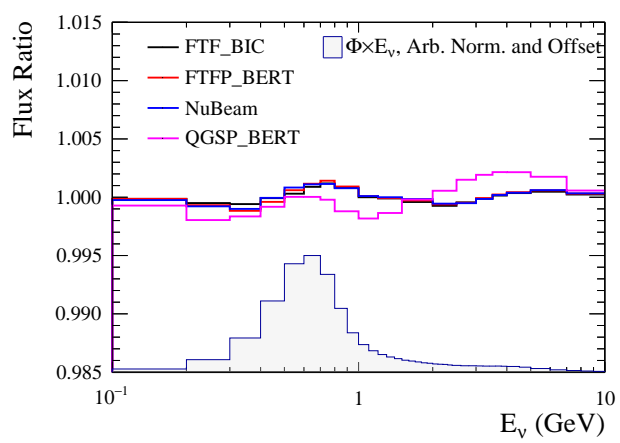

ND280: Neutrino Mode, $v_{\mathrm{e}}$

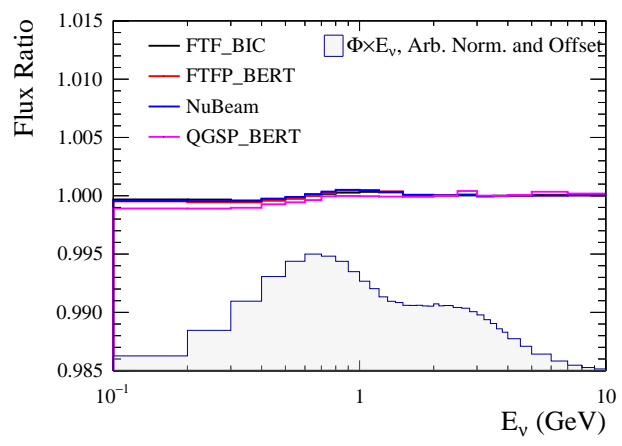

ND280: Anti-neutrino Mode, $v_{\mu}$

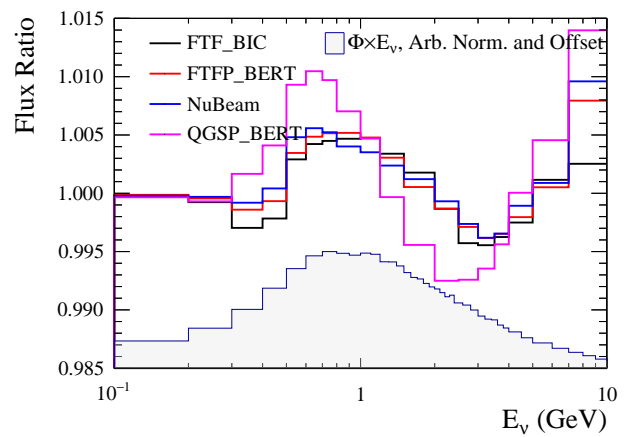

ND280: Anti-neutrino Mode, $v_{\mathrm{e}}$

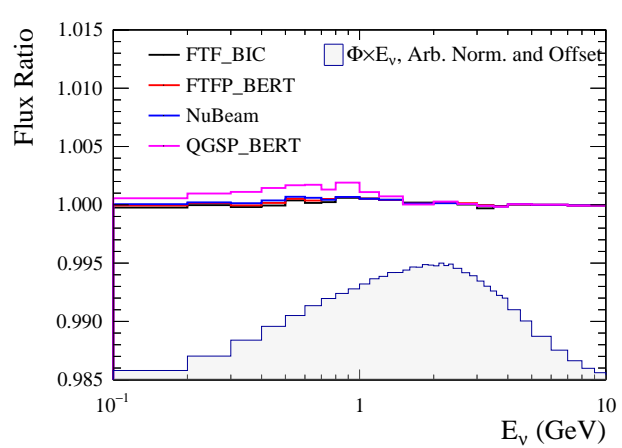

ND280: Neutrino Mode, $\bar{v}_{\mu}$

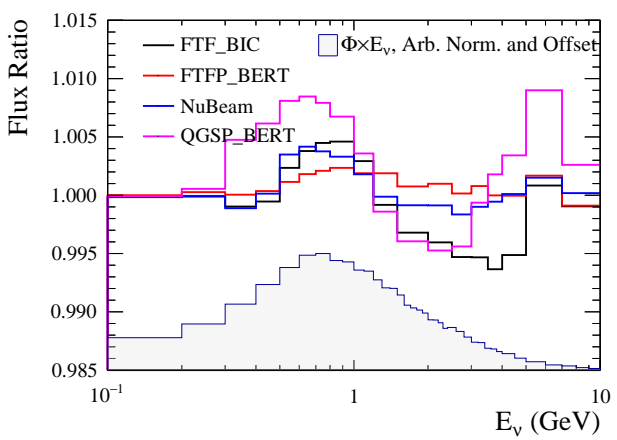

ND280: Neutrino Mode, $\bar{v}_{\mathrm{e}}$

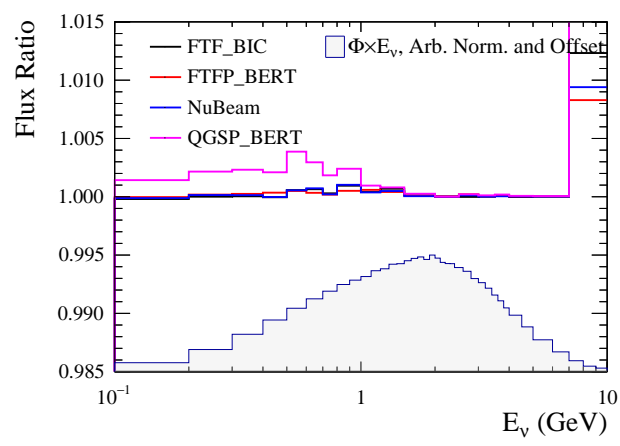

ND280: Anti-neutrino Mode, $\bar{\nabla}_{\mu}$

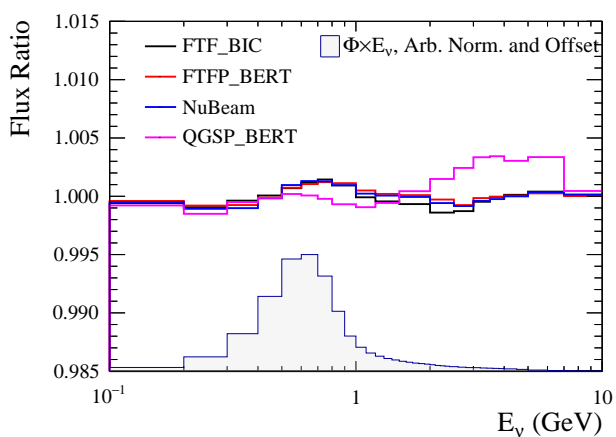

ND280: Anti-neutrino Mode, $\bar{v}_{\mathrm{e}}$

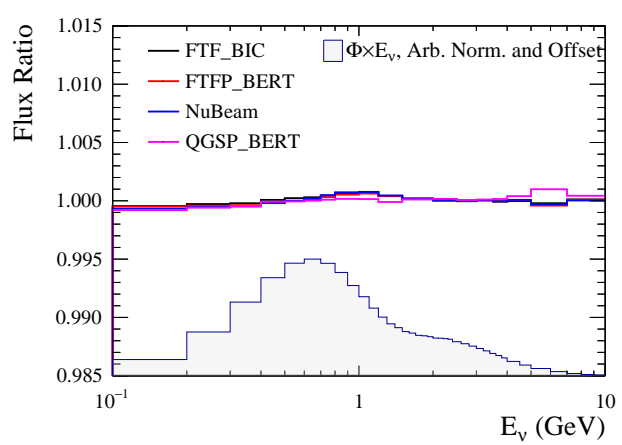

Figure 6.18: Difference in replica-tuned flux prediction at ND280, depending on the Monte Carlo model used for the unfolding. Different GEANT4 physics lists are compared to the FLUKA model chosen by NA61. The model dependence introduced by the unfolding has a sub-percent effect on the flux. 
SK: Neutrino Mode, $v_{\mu}$

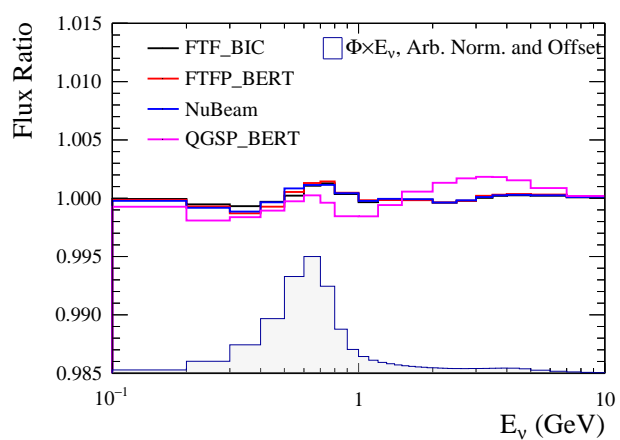

SK: Neutrino Mode, $v_{\mathrm{e}}$

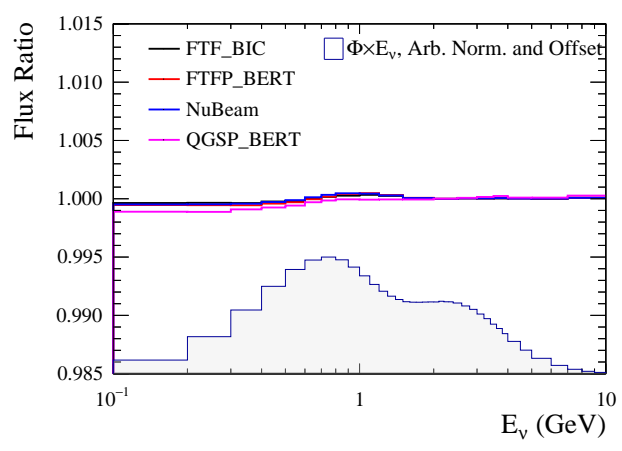

SK: Anti-neutrino Mode, $v_{\mu}$

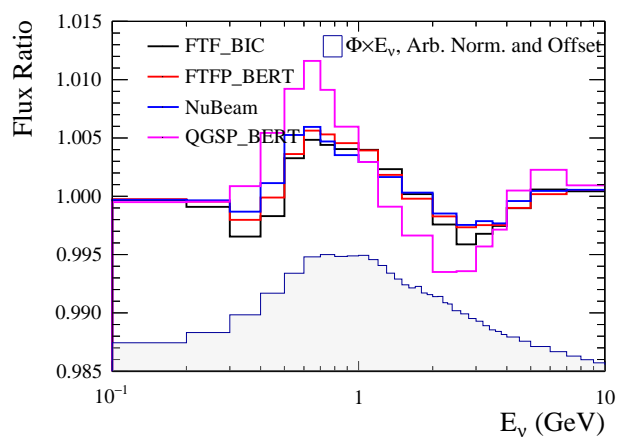

SK: Anti-neutrino Mode, $v_{\mathrm{e}}$

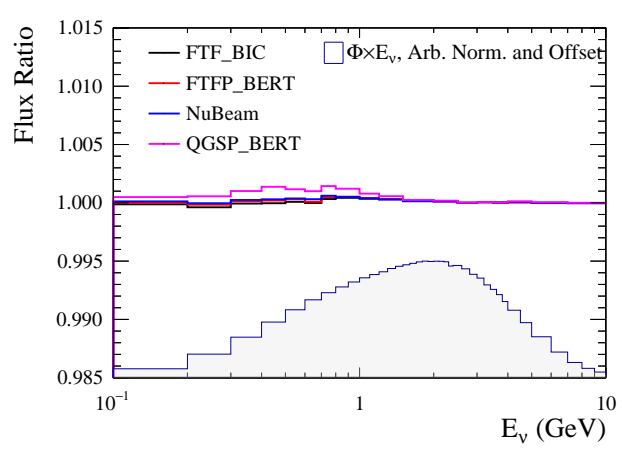

SK: Neutrino Mode, $\bar{v}_{\mu}$

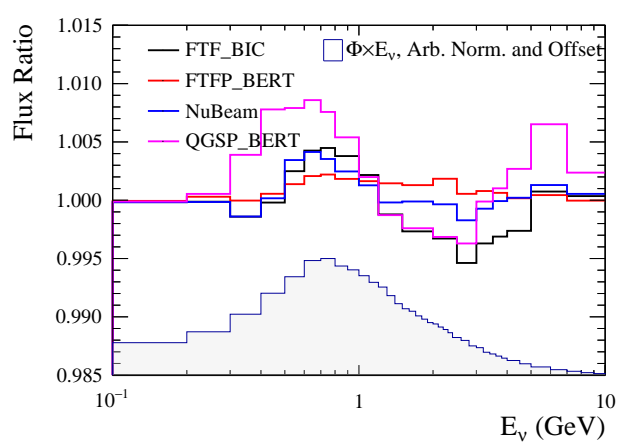

SK: Neutrino Mode, $\bar{v}_{\mathrm{e}}$

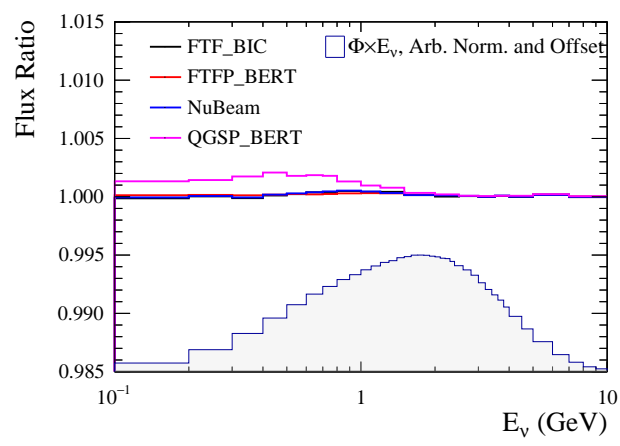

SK: Anti-neutrino Mode, $\bar{v}_{\mu}$

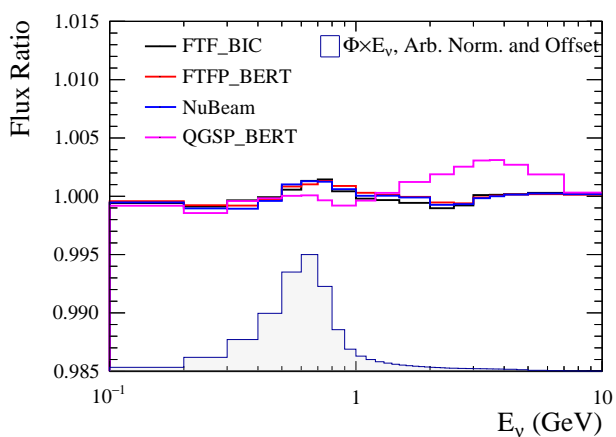

SK: Anti-neutrino Mode, $\bar{v}_{\mathrm{e}}$

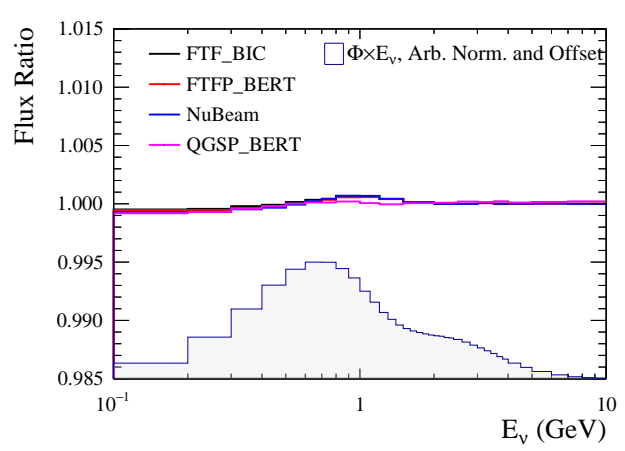

Figure 6.19: Difference in replica-tuned flux prediction at the far detector, depending on the Monte Carlo model used for the unfolding. Different GEANT4 physics lists are compared to the FLUKA model chosen by NA61. The model dependence introduced by the unfolding has a sub-percent effect on the flux. 


\subsection{Target density effects}

Both the T2K and NA61 long targets were manufactured by the Toyo-Tanso company, and are made out of isotropic graphite of grade IG43. Although the NA61 target was produced as a replica of the T2K target, the target density after machining is known to be different. The measured target densities, $\rho_{\text {meas }}$, are given in Table 6.1, alongside conservative estimates of uncertainties associated with the measurement procedure $(0.8 \%$ volume uncertainty and $1 \%$ mass uncertainty, resulting in $\sim 1.3 \%$ density uncertainty). The notation used in this chapter assumes that if the measured target density is $\rho_{\text {meas }} \pm 1 \sigma$, there is a $68.2 \%$ probability that the real density $\rho_{\text {real }}$ is within $\pm 1 \sigma$ of the measured value. The yield of pions exiting from the target varies with density, and this effect becomes important when using replica-target multiplicity measurements to estimate the exiting pion yields from the T2K target. The effects that have been studied are the uncertainty on the replica-target density, and the uncertainty on the T2K target density. The measured T2K and replica-target densities agree within the assigned uncertainty bands, so this effect has been neglected.

\begin{tabular}{c|c|c}
\hline \hline Target & $\begin{array}{c}\text { Measured Density } \\
\rho_{\text {meas }}\left[\frac{\mathrm{g}}{\mathrm{cm}^{3}}\right]\end{array}$ & $\begin{array}{c}\text { Uncertainty } \\
{[\%]}\end{array}$ \\
\hline T2K & 1.804 & 1.3 \\
\hline Replica & 1.831 & 1.3 \\
\hline \hline
\end{tabular}

Table 6.1: T2K and NA61 graphite target densities.

The multiplicity weights applied on the nominal pion yields exiting from the T2K target are:

$$
\mathrm{W}_{\rho_{\text {meas }}^{\text {NA61 }}}=\left[\frac{d \mathrm{n}_{\mathrm{NA61}}(p, \theta, z)}{d p}\right]_{\rho=\rho_{\text {real }}^{\mathrm{NA} 61}} /\left[\frac{d \mathrm{n}_{\text {FLUKA }}(p, \theta, z)}{d p}\right]_{\rho=\rho_{\text {meas }}^{\mathrm{NA} 61}}
$$

where $\frac{d \mathrm{n}(p, \theta, z)}{d p}$ is the differential multiplicity of the corresponding charged pion exiting from longitudinal bin $z$ along the replica-target. Additional multiplicity weights have been computed where the simulated replica-target density was shifted 
from the measured density by $\pm 1 \sigma$ :

$$
\begin{aligned}
& \mathrm{W}_{\rho_{\text {meas }}^{\mathrm{NA} 61}+1 \sigma}=\left[\frac{d \mathrm{n}_{\mathrm{NA61}}(p, \theta, z)}{d p}\right]_{\rho=\rho_{\text {real }}^{\mathrm{NA61}}} /\left[\frac{d \mathrm{n}_{\mathrm{FLUKA}}(p, \theta, z)}{d p}\right]_{\rho=\rho_{\text {meas }}^{\mathrm{NA61}}+1 \sigma} \\
& \mathrm{W}_{\rho_{\text {meas }}^{\mathrm{NA} 61}-1 \sigma}=\left[\frac{d \mathrm{n}_{\mathrm{NA} 61}(p, \theta, z)}{d p}\right]_{\rho=\rho_{\text {real }}^{\mathrm{NA61}}} /\left[\frac{d \mathrm{n}_{\mathrm{FLUKA}}(p, \theta, z)}{d p}\right]_{\rho=\rho_{\text {meas }}^{\mathrm{NA61}}-1 \sigma}
\end{aligned}
$$

Varying the density of the replica-target simulated by FLUKA by $\pm 1 \sigma$ changes the NA61 multiplicity weights, as shown in Eq. 6.5 and Eq. 6.6 compared to Equation 6.4. This is clearly shown when looking at the multiplicity ratios, presented in Figure 6.20 and Figure 6.21 for positive pion yields. With a higher density long target, the probability for primary protons to interact in the upstream region increases, thus decreasing the number of surviving protons that can interact in the downstream target portion. Consequently, for higher density targets the pion yield is increased from the upstream and decreased from the downstream longitudinal bins (see Figure 6.20). Decreasing the target density has the opposite effect, as shown in Figure 6.21.

The comparison of $\mathrm{T} 2 \mathrm{~K}$ neutrino flux predictions constrained with $\mathrm{W}_{\rho_{\text {meas }}^{\mathrm{NA}} \pm 1 \sigma}$ to those constrained with the nominal set of multiplicity weights $\mathrm{W}_{\rho_{\text {meas }}^{\mathrm{NA61}}}$ are provided in Figure 6.22 and Figure 6.23. The presented ratio shows the maximum change in the flux prediction introduced by variations of the replica-target density. This effect is small $(<0.5 \%)$. The flux covariance matrix due to the replica-target density uncertainty was built from the two variations (throws) of the tuned flux generated using $\mathrm{W}_{\rho_{\text {meas }}^{\mathrm{NA61}} \pm 1 \sigma}$ multiplicity weights. Compared to other flux systematics, the effect of differences in T2K and NA61 target densities could safely be neglected.

The T2K target density also enters into the flux calculation. Since the uncertainty on the $\mathrm{T} 2 \mathrm{~K}$ target and on the replica-target is the same, it is reasonable to expect that they will have the same effect on the flux. However, changing the T2K target density requires generating different Monte Carlo samples, so there is no cancellation of statistical errors in the flux ratio like in Figure 6.22 and Figure 6.23. The T2K Monte Carlo generator (FLUKA+GEANT3) had first to be run with the T2K target 

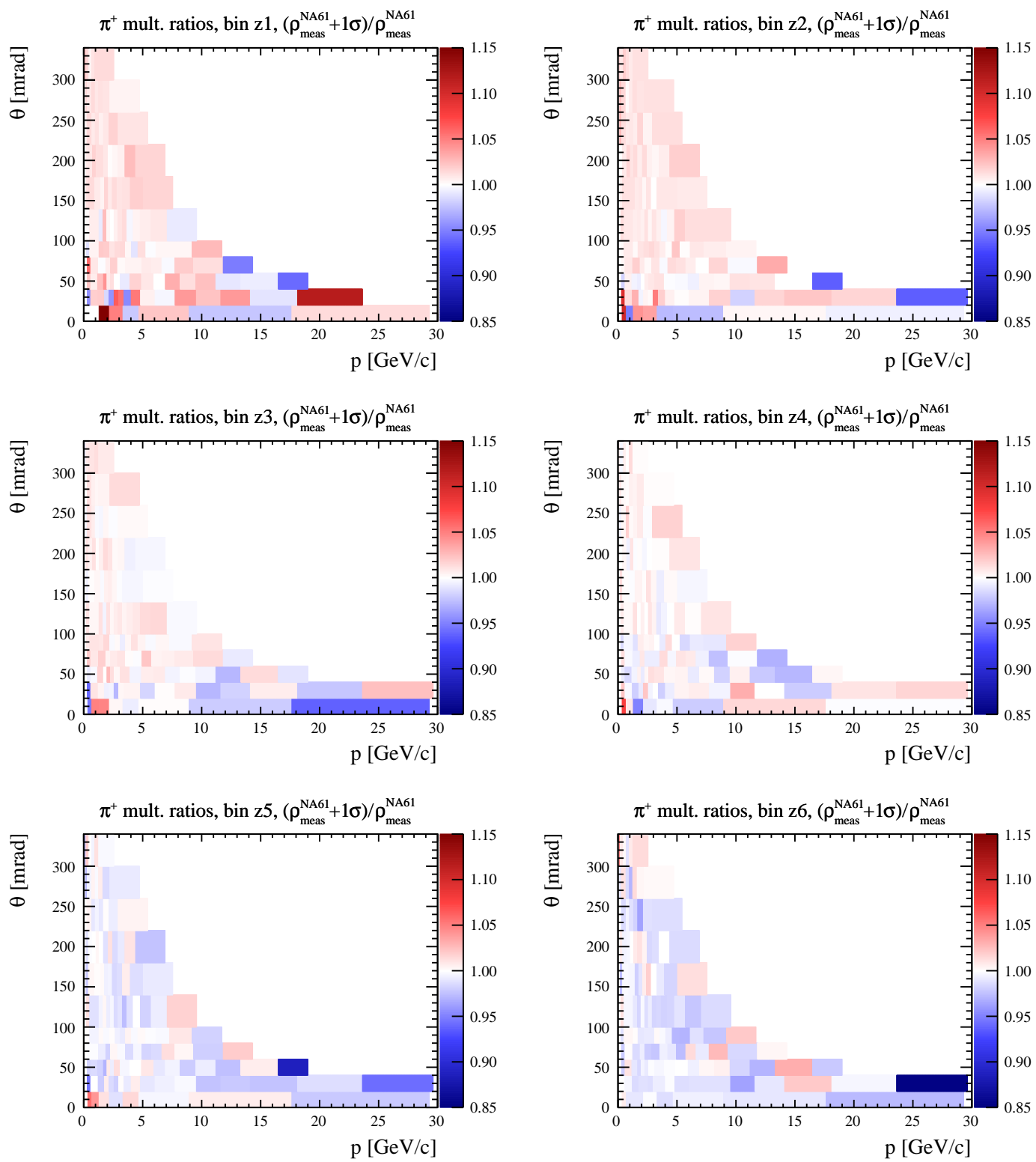

Figure 6.20: Variation in predicted positive pion multiplicities exiting from the NA61 target when increasing the measured replica-target density by $1 \sigma$.

density set to the measured value $\rho^{\mathrm{T} 2 \mathrm{~K}}=\rho_{\text {meas }}^{\mathrm{T} 2 \mathrm{~K}}$. The measured target density had then been shifted by the size of the measurement uncertainty $\rho^{\mathrm{T} 2 \mathrm{~K}}=\rho_{\text {meas }}^{\mathrm{T} 2 \mathrm{~K}} \pm 1 \sigma$, and the MC generator was rerun. A change in the target density affects both the nominal and the tuned T2K flux. The comparison of the tuned fluxes for different T2K target densities have been provided in Figure 6.24 and Figure 6.25, for fluxes at ND280 and Super-K, respectively. The statistical fluctuations increase at higher neutrino energies where there are fewer simulated events in the MC samples. Generating 

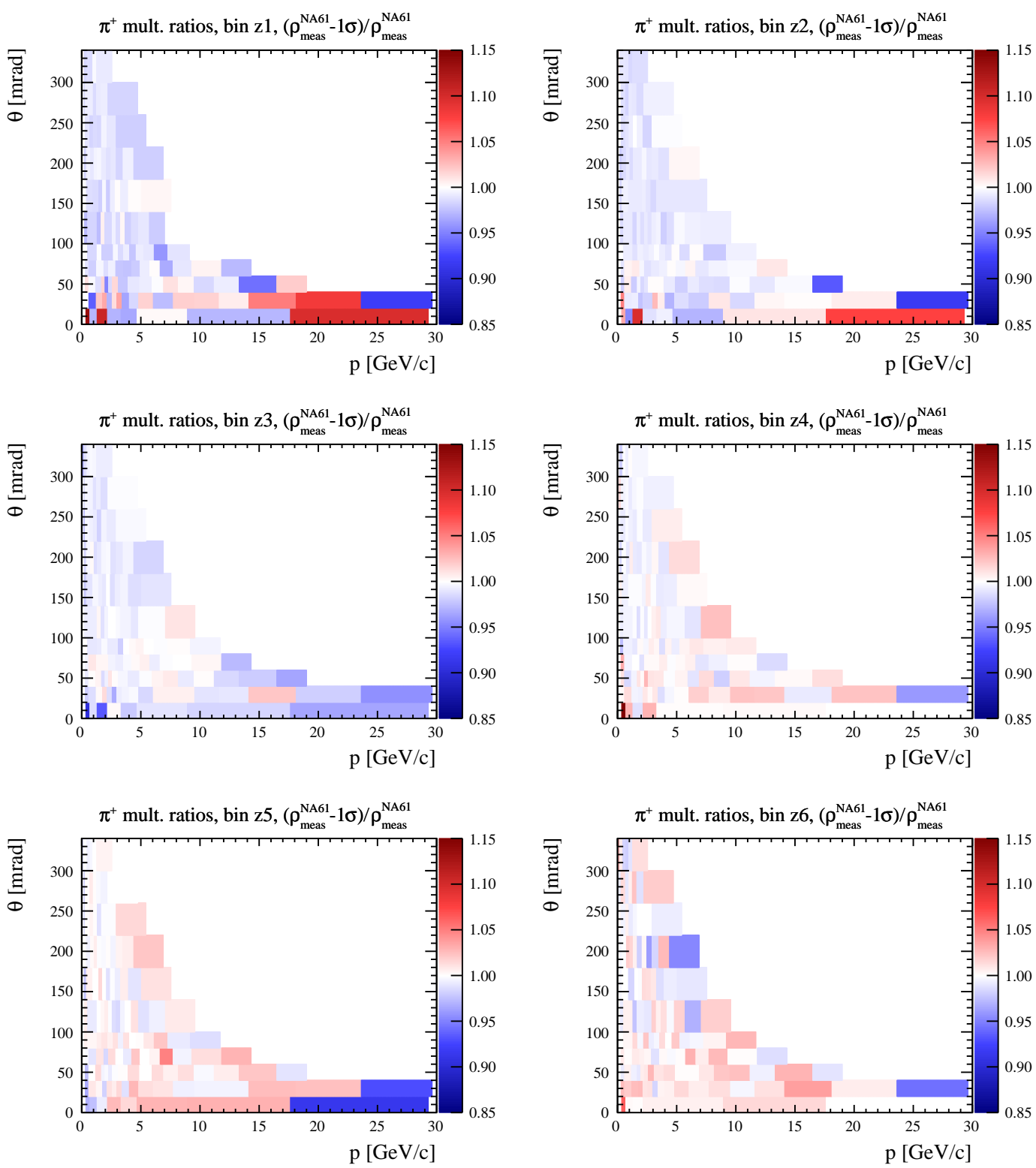

Figure 6.21: Variation in predicted positive pion multiplicities exiting from the NA61 target when decreasing the measured replica-target density by $1 \sigma$.

sufficient numbers of events at these high energies would be computationally very demanding. Variations in the T2K target density change the right-sign neutrino flux by less than $1 \%\left(\nu_{\mu}\right.$ component in neutrino-mode and $\bar{\nu}_{\mu}$ component in anti-neutrino mode). The right-sign flux component mostly comes from decays of strongly focused pions, produced through interactions of primary protons inside the T2K target. Decreasing the T2K target density reduces the number of potential target nuclei for the incident protons, thus reducing the flux around the T2K signal peak (and 
vice versa when increasing the $\mathrm{T} 2 \mathrm{~K}$ target density). For other (anti-)neutrino flavours, the change in flux with density variation is still less than $\sim 1.5 \%$, apart from a few high energy bins $(>3 \mathrm{GeV})$ in the $\bar{\nu}_{e}\left(\nu_{e}\right)$ component in (anti-)neutrino mode, where the pull on the nominal flux approaches $4 \%$. The flux covariance matrix due to the $\mathrm{T} 2 \mathrm{~K}$ target density uncertainty has been calculated from the two variations (throws) of the tuned flux with shifted density values. Uncertainties in the thin-target density have not been considered in this study, since hadrons incident on thin-targets very rarely undergo multiple interactions, and they do not travel appreciable distances through such targets for their survival probability to be affected by density uncertainties. 


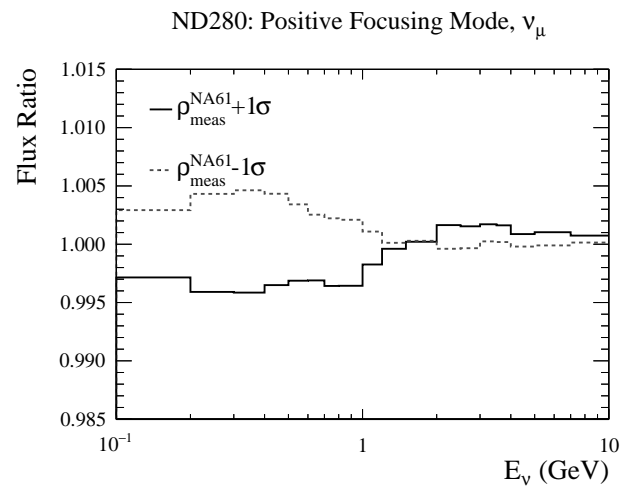

ND280: Positive Focusing Mode, $v_{\mathrm{e}}$

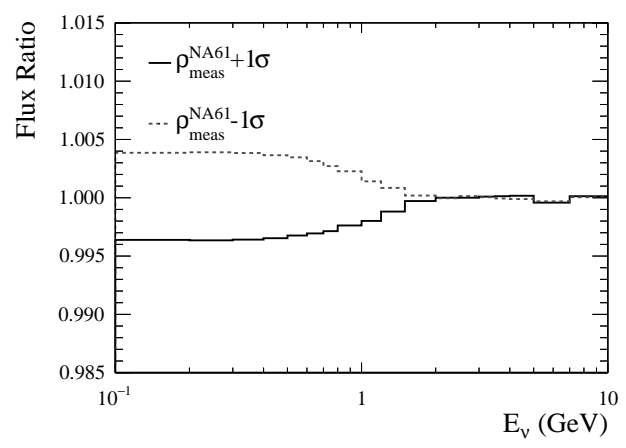

ND280: Negative Focusing Mode, $v_{\mu}$

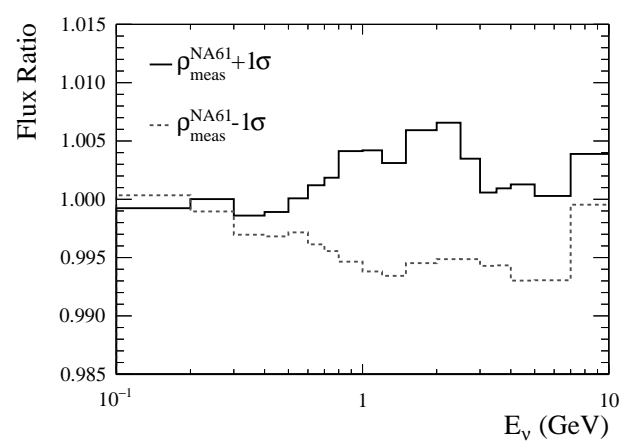

ND280: Negative Focusing Mode, $v_{\mathrm{e}}$

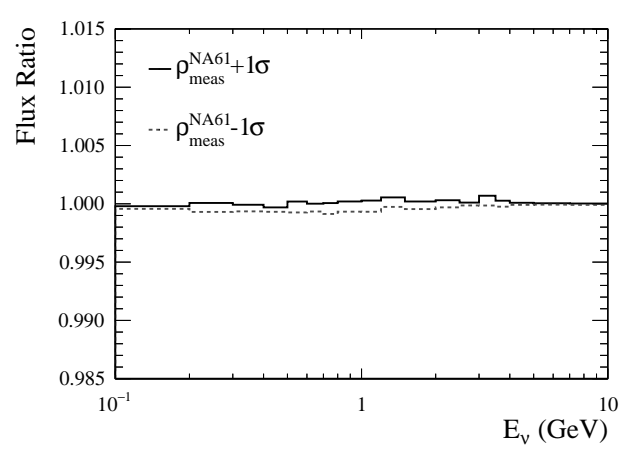

ND280: Positive Focusing Mode, $\bar{v}_{\mu}$

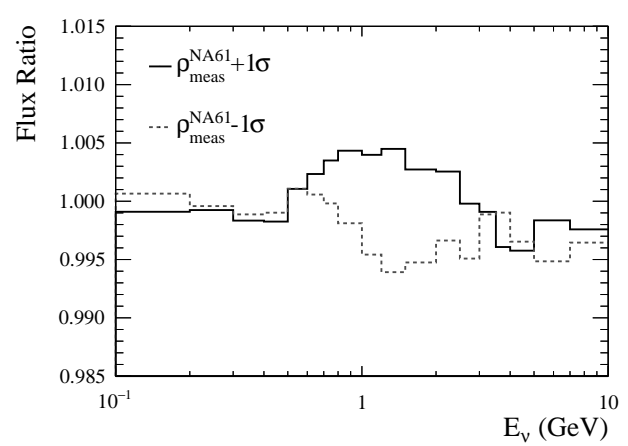

ND280: Positive Focusing Mode, $\bar{v}_{\mathrm{e}}$

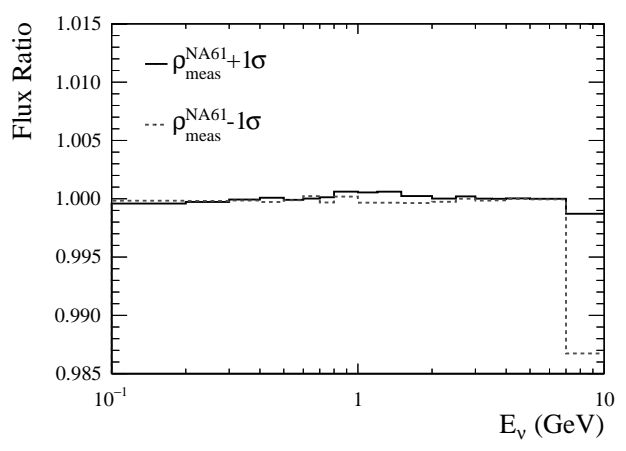

ND280: Negative Focusing Mode, $\bar{v}_{\mu}$

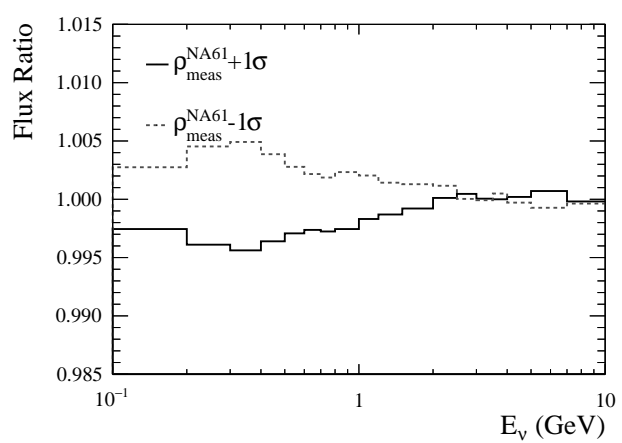

ND280: Negative Focusing Mode, $\bar{v}_{\mathrm{e}}$

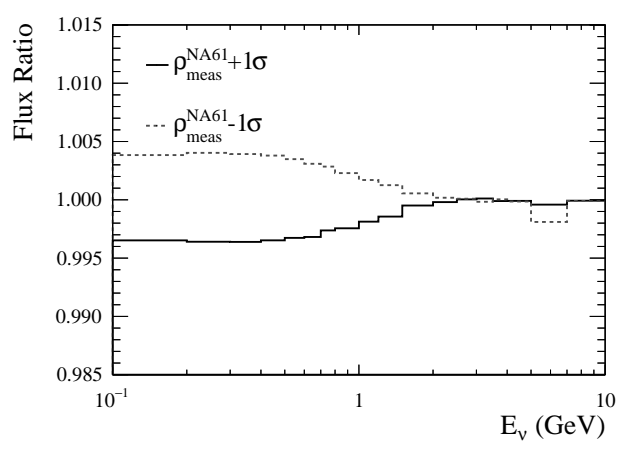

Figure 6.22: Comparing the neutrino flux at ND280 constrained with different sets of replica-target weights, where the modelled NA61 target's density was shifted by $\pm 1 \sigma$, to the flux constrained with the standard set of replica-target weights, based on the NA61 target's measured density. 
SK: Positive Focusing Mode, $v_{\mu}$

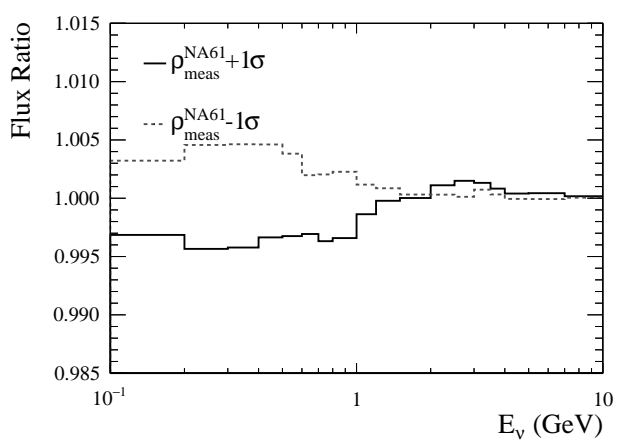

SK: Positive Focusing Mode, $v_{\mathrm{e}}$

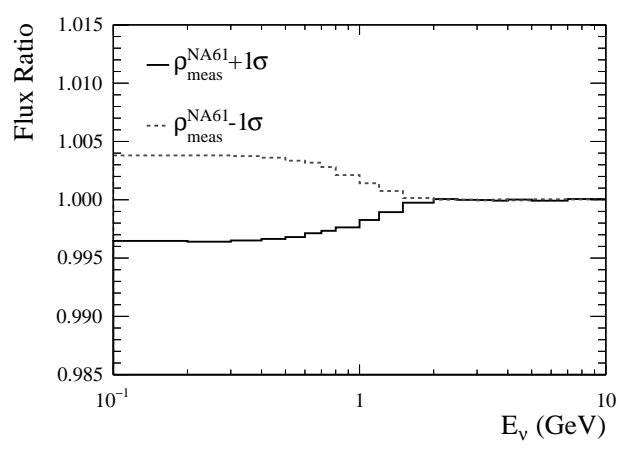

SK: Negative Focusing Mode, $v_{\mu}$

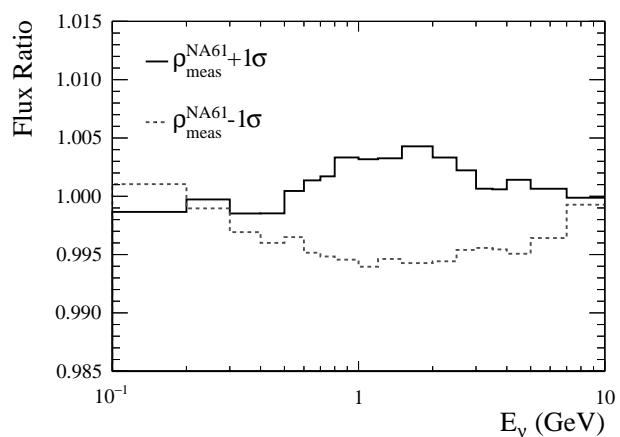

SK: Negative Focusing Mode, $v_{\mathrm{e}}$

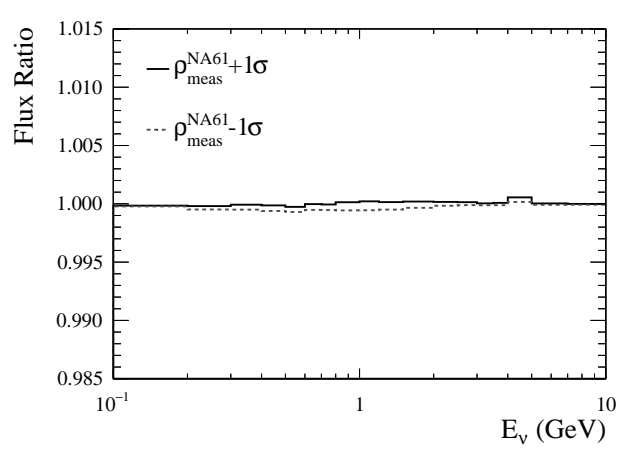

SK: Positive Focusing Mode, $\bar{v}_{\mu}$

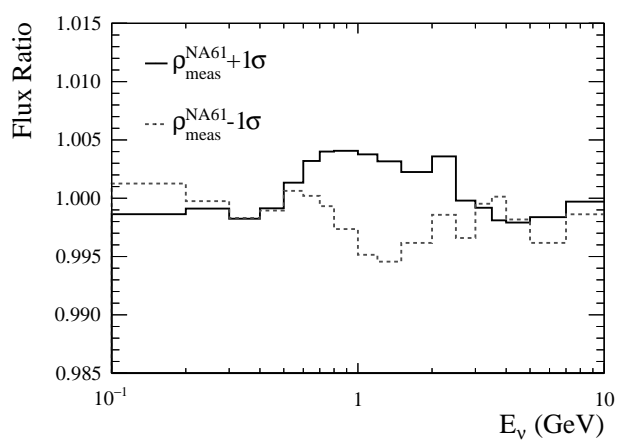

SK: Positive Focusing Mode, $\bar{v}_{\mathrm{e}}$

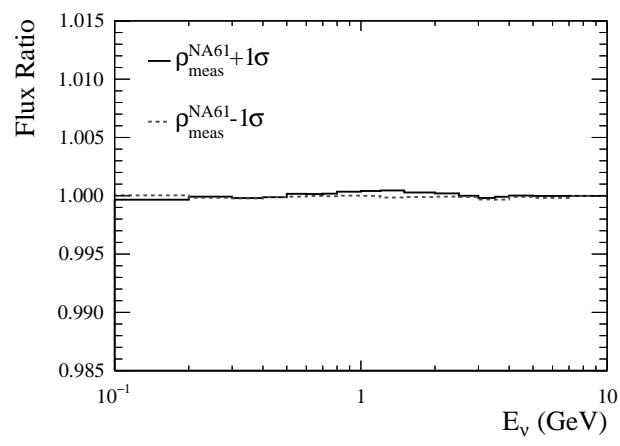

SK: Negative Focusing Mode, $\bar{\nabla}_{\mu}$

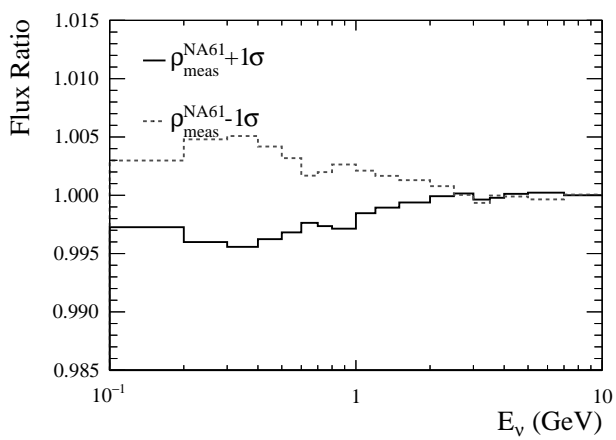

SK: Negative Focusing Mode, $\bar{v}_{\mathrm{e}}$

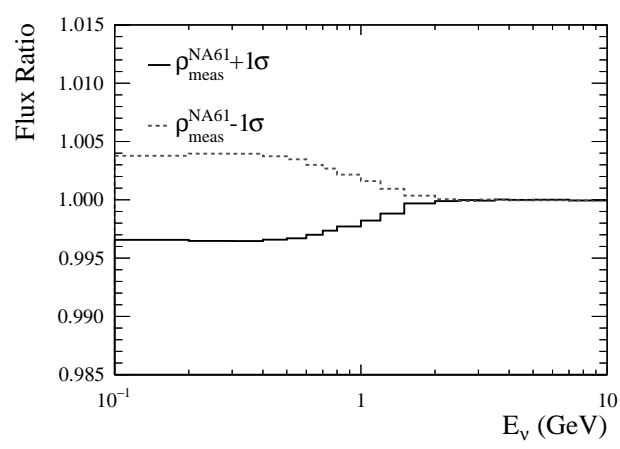

Figure 6.23: Comparing the neutrino flux at the far detector constrained with different sets of replica-target weights, where the modelled NA61 target's density was shifted by $\pm 1 \sigma$, to the flux constrained with the standard set of replica-target weights, based on the NA61 target's measured density. 


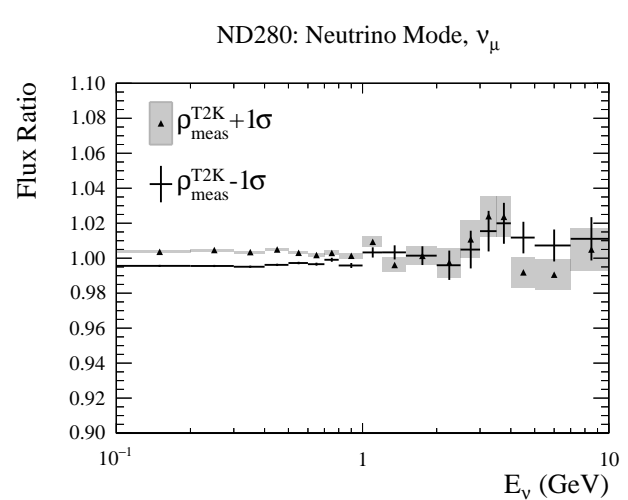

ND280: Neutrino Mode, $v_{\mathrm{e}}$

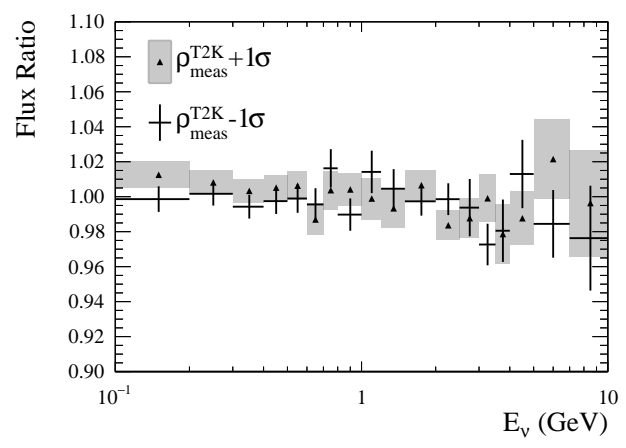

ND280: Anti-Neutrino Mode, $v_{\mu}$

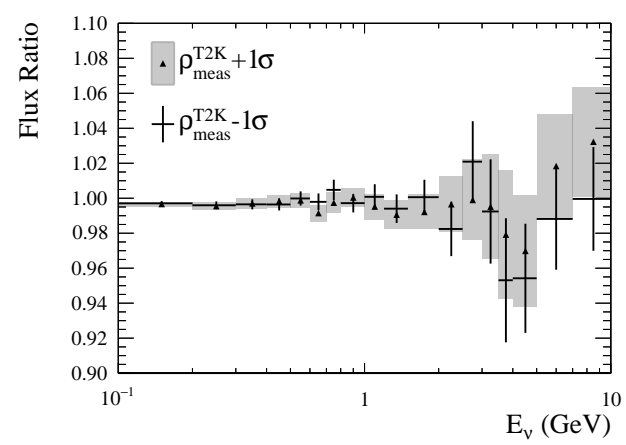

ND280: Anti-Neutrino Mode, $v_{\mathrm{e}}$

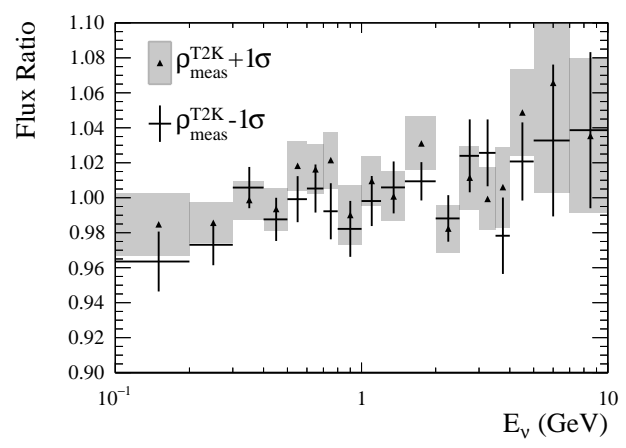

ND280: Neutrino Mode, $\bar{v}_{\mu}$

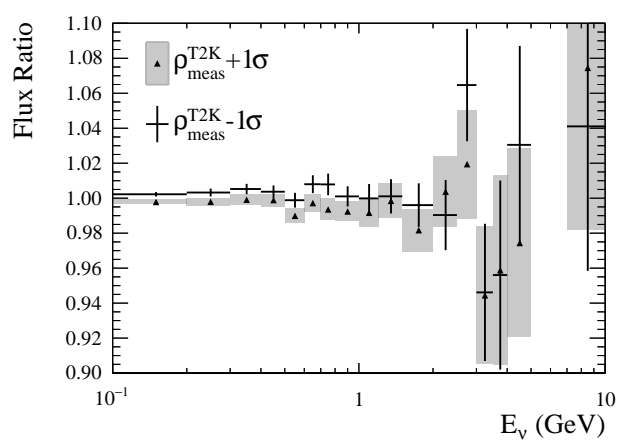

ND280: Neutrino Mode, $\bar{v}_{\mathrm{e}}$

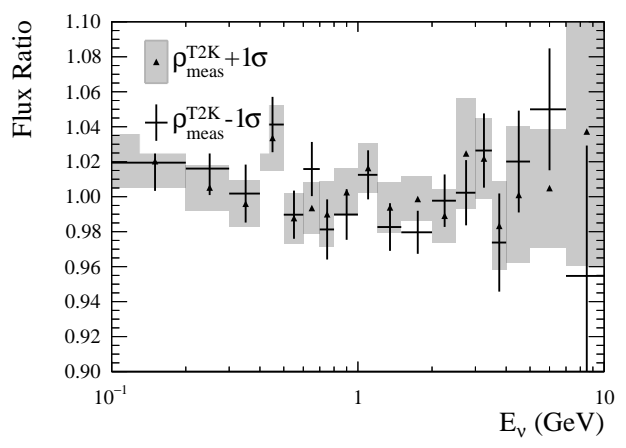

ND280: Anti-Neutirno Mode, $\bar{v}_{\mu}$

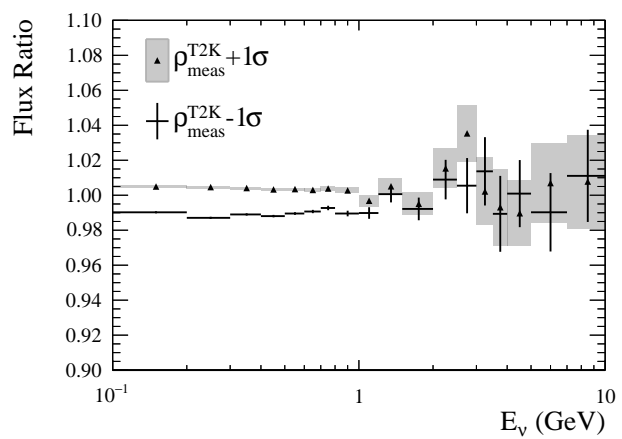

ND280: Anti-Neutrino Mode, $\bar{v}_{\mathrm{e}}$

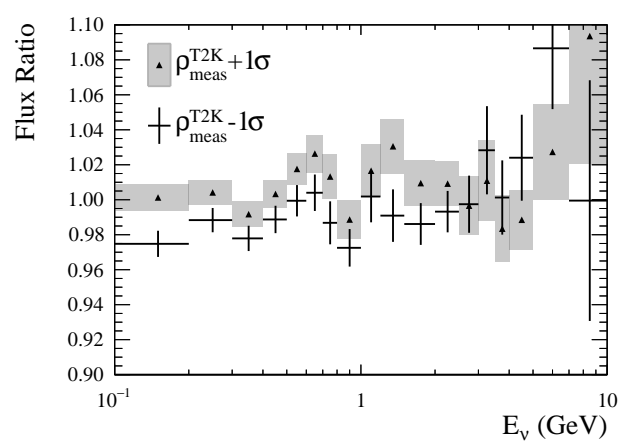

Figure 6.24: Comparison of tuned neutrino fluxes at ND280 modelled with the T2K target density set to $\rho_{\text {meas }}^{\mathrm{T} 2 \mathrm{~K}} \pm 1 \sigma$, and the tuned flux modelled with the measured T2K density $\rho_{\text {meas }}^{\mathrm{T} 2 \mathrm{~K}}$. Fluxes had to be calculated from different Monte Carlo samples, accounting for large statistical fluctuations on the ratio at higher neutrino energies with fewer events. The T2K target density was varied for each Monte Carlo sample, and every sample consists of $\sim 1000$ JNUBEAM files, each with 200,000 simulated POT. 
SK: Neutrino Mode, $v_{\mu}$

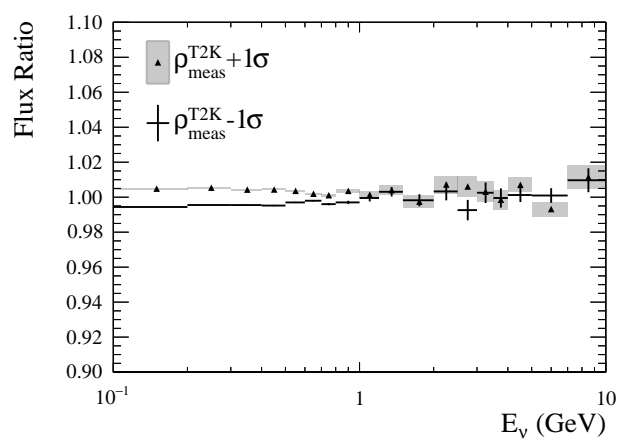

SK: Neutrino Mode, $v_{\mathrm{e}}$

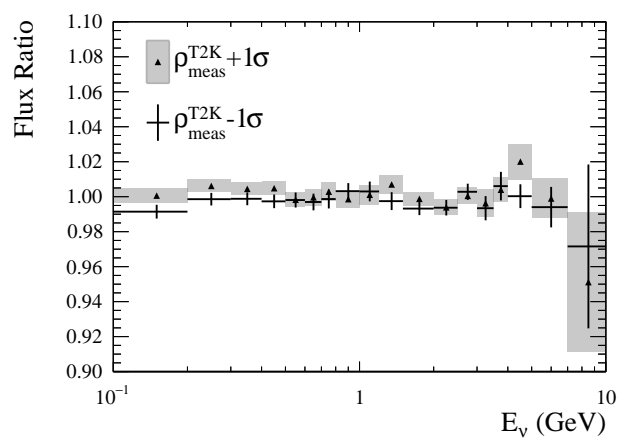

SK: Anti-Neutrino Mode, $v_{\mu}$

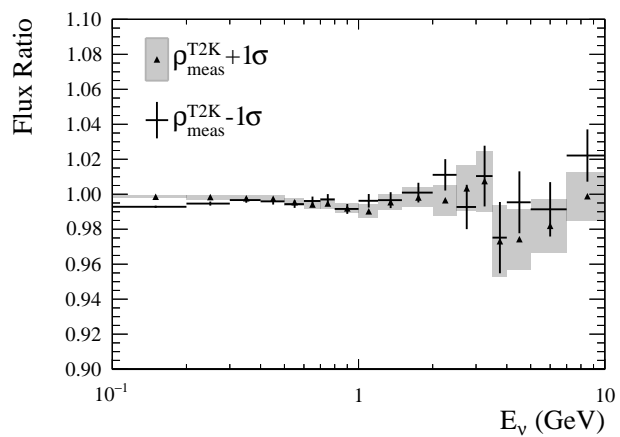

SK: Anti-Neutrino Mode, $v_{\mathrm{c}}$

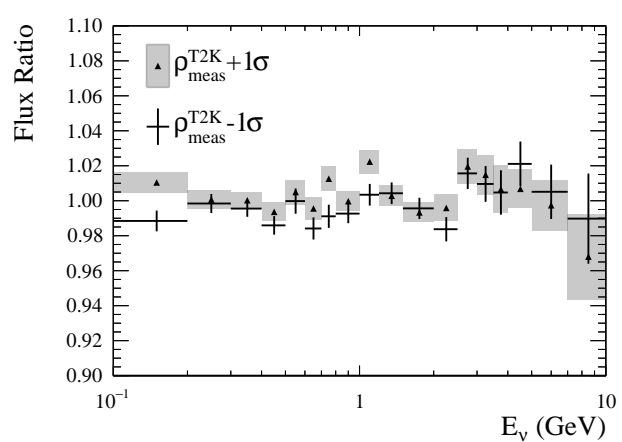

SK: Neutrino Mode, $\bar{v}_{\mu}$

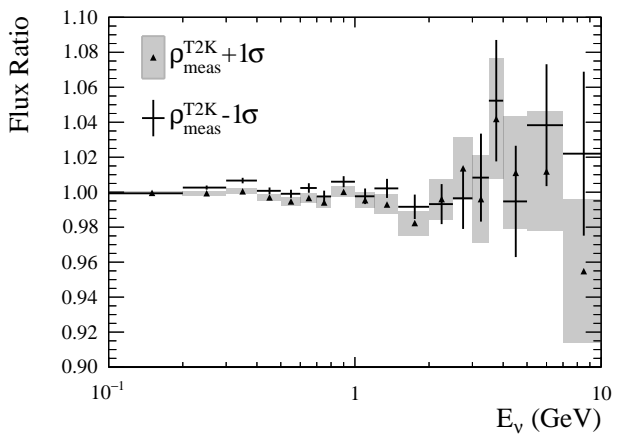

SK: Neutrino Mode, $\bar{v}_{\mathrm{e}}$

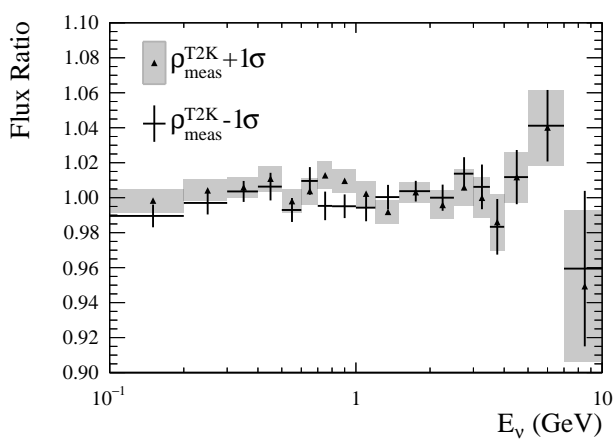

SK: Anti-Neutirno Mode, $\overline{\mathrm{v}}_{\mu}$

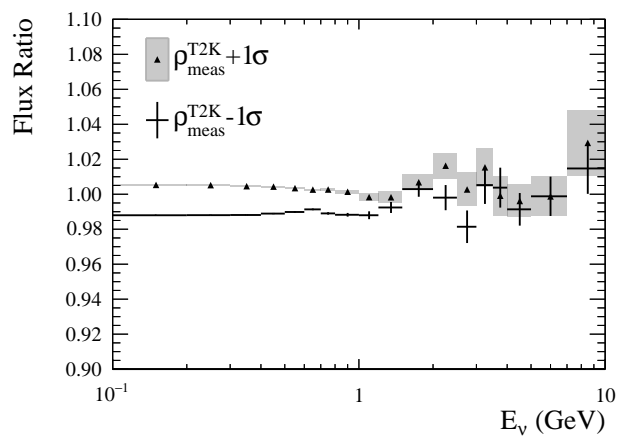

SK: Anti-Neutrino Mode, $\bar{v}_{\mathrm{e}}$

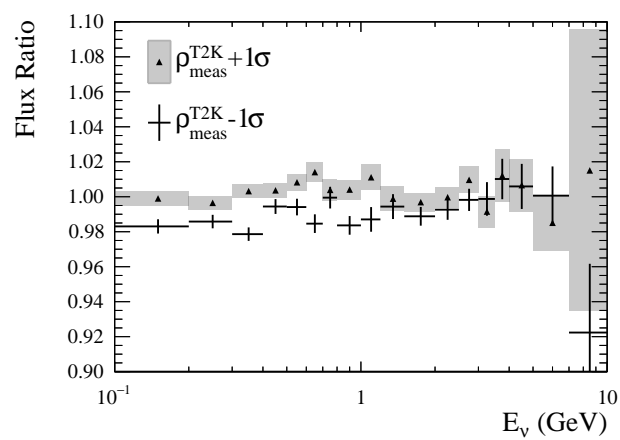

Figure 6.25: Comparison of tuned neutrino fluxes at the far detector modelled with the T2K target density set to $\rho_{\text {meas }}^{\mathrm{T} 2 \mathrm{~K}} \pm 1 \sigma$, and the tuned flux modelled with the measured T2K density $\rho_{\text {meas }}^{\mathrm{T} 2 \mathrm{~K}}$. Fluxes had to be calculated from different Monte Carlo samples, accounting for large statistical fluctuations on the ratio at higher neutrino energies with fewer events. The T2K target density was varied for each Monte Carlo sample, and every sample consists of $\sim 1000$ JNUBEAM files, each with 200,000 simulated POT. 


\subsection{Proton beam profile effects}

Systematic uncertainties related to the NA61 proton beam profile will be presented in this chapter. Also, differences between the NA61 and T2K beam profiles will be addressed, and quantitative estimates provided for their effect on the flux calculation. For the evaluation of flux systematics from uncertainties in the T2K proton beam profile the reader if referred back to Chapter 5.5.2.

The NA61 proton beam profile, used during the 2009 replica-target data-taking, and projected in the horizontal, $\left(x, \theta_{x}\right)$, and vertical, $\left(y, \theta_{y}\right)$, position-divergence phase space is shown in Figure 6.26. This profile was sampled with the BPD-3 beam profile detector, positioned $12.4 \mathrm{~cm}$ upstream from the replica target, as shown in Figure 4.1. The distributions of beam position and divergence in the transverse plane evolve as the beam spill propagates along the longitudinal direction, as explained in Chapter 2.3. The NA61 data acquisitioning trigger keeps only incident tracks which pass the proton particle identification cut and hit the upstream target face. Consequently, if the beam profile distributions from Figure 6.26 were to be propagated $12.4 \mathrm{~cm}$ forwards, to the z-position of the upstream face of the replica target, the $(x, y)$ beam distribution would neatly fall within a circle with target radius of $13 \mathrm{~mm}$. From these distributions, the average NA61 beam centre position and divergence at BPD-3 have been measured, as shown in Table 6.2. Proton beam tracks are fitted to combined cluster positions in BPD-1, BPD-2 and BPD-3 detectors, and the resulting uncertainty on the beam position from this procedure is $\sim 0.1 \mathrm{~mm}$ in both the horizontal and vertical direction. Next, it will be demonstrated that the impact of the $0.1 \mathrm{~mm}$ NA61 beam position error on replica-tuning can be neglected. This is to be expected, considering that the position uncertainty for the NA61 beam is typically significantly smaller than for the T2K beam (see Table 3.2). Studies of the effect of beam divergence, width, emittance and Twiss parameter uncertainties proceed in a similar manner, with the resulting conclusion being that they contribute negligibly to the T2K flux calculation. These will not be presented in this thesis due to space limitations. 
NA61 (x,x') Beam Profile for the 2009 Replica-Target Dataset

NA61 (y,y') Beam Profile for the 2009 Replica-Target Dataset
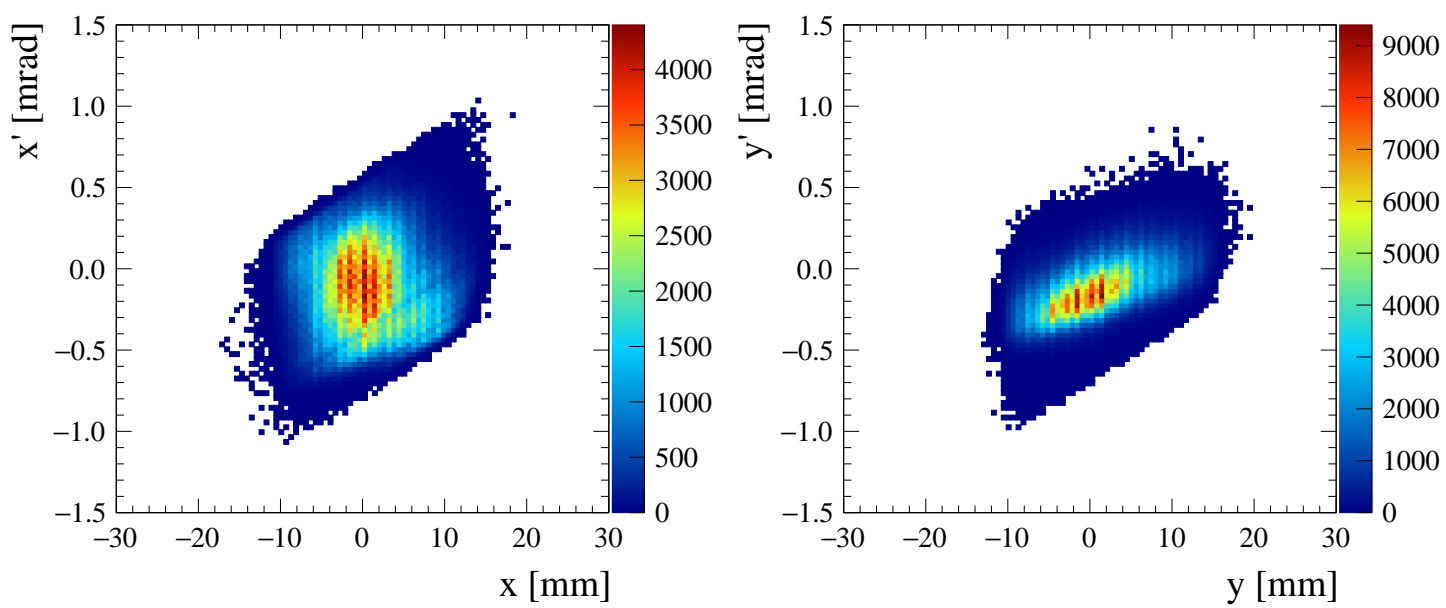

Figure 6.26: The average BPD-3 NA61 proton beam profile used for collecting the 2009 replica-target measurements.

\begin{tabular}{c|c|c|c|c} 
Direction & $\begin{array}{c}\text { Mean Position } \\
{[\mathrm{mm}]}\end{array}$ & $\begin{array}{c}\text { Position RMS } \\
{[\mathrm{mm}]}\end{array}$ & $\begin{array}{c}\text { Mean Direction } \\
{[\mathrm{mrad}]}\end{array}$ & $\begin{array}{c}\text { Direction RMS } \\
{[\mathrm{mrad}]}\end{array}$ \\
\hline Horizontal & 0.7 & 4.7 & -0.10 & 0.25 \\
\hline Vertical & 1.0 & 5.3 & -0.11 & 0.15
\end{tabular}

Table 6.2: The average NA61 proton beam profile at BPD3 (12.4 $\mathrm{cm}$ upstream from the replica-target). At NA61, every beam track hits the target. Beam profile detectors measure the relative target position at different longitudinal locations along the NA61 beamline.

The same notation was adopted as in Sec. 6.5, i.e. the probability for the measured beam positions $\mathrm{x}_{\text {meas }}^{\mathrm{NA} 61}$ and $\mathrm{y}_{\text {meas }}^{\mathrm{NA61}}$ to be within $\pm 1 \sigma$ of the real values $\mathrm{x}_{\text {real }}^{\mathrm{NA61}}$ and $\mathrm{y}_{\text {real }}^{\mathrm{NA}} \mathrm{in}$ is $68.2 \%$. The uncertainties on the measurement of the proton beam profile centre can be propagated to a variation in the tuned flux prediction. For the purposes of this study, the following sets of multiplicity weights are constructed: $\mathrm{W}_{\mathrm{x}_{\text {meas }}^{\mathrm{NA}} \pm 1 \sigma}$ and $\mathrm{W}_{\mathrm{y}_{\text {meas }}^{\mathrm{NA}} \pm 1 \sigma}$, where:

$$
\begin{aligned}
& \mathrm{W}_{\mathrm{x}_{\text {meas }}^{\mathrm{NA61}} \pm 1 \sigma}=\left[\frac{d n_{\mathrm{NA61}}(p, \theta, z)}{d p}\right]_{\mathrm{x}=\mathrm{x}_{\text {real }}^{\mathrm{NA61}}} /\left[\frac{d n_{\mathrm{FLUKA}}(p, \theta, z)}{d p}\right]_{\mathrm{x}=\mathrm{x}_{\text {meas }}^{\mathrm{NA61}} \pm 1 \sigma} \\
& \mathrm{W}_{\mathrm{y}_{\text {meas }}^{\mathrm{NA61}} \pm 1 \sigma}=\left[\frac{d n_{\mathrm{NA} 61}(p, \theta, z)}{d p}\right]_{\mathrm{y}=\mathrm{y}_{\text {real }}^{\mathrm{NA61}}} /\left[\frac{d n_{\mathrm{FLUKA}}(p, \theta, z)}{d p}\right]_{\mathrm{y}=\mathrm{y}_{\text {meas }}^{\mathrm{NA61}} \pm 1 \sigma} .
\end{aligned}
$$

The comparison between weights $\mathrm{W}_{\mathrm{x}_{\text {meas }}^{\mathrm{NA} 61} \pm 1 \sigma}$ and $\mathrm{W}_{\mathrm{y}_{\text {meas }}^{\mathrm{NA61}} \pm 1 \sigma}$, where the beam profile position has been shifted, and the nominal weights $\mathrm{W}_{\mathrm{x}_{\text {meas }}^{\mathrm{NA}}, \mathrm{y}_{\text {meas }}^{\mathrm{NA}} \mathrm{k1}}$, constructed 
using the measured NA61 proton beam profile parameters, has been made. The corresponding ratios of weights calculated when varying the horizontal beam position are given in Figure 6.27 for $+1 \sigma$ shifts, and Figure 6.28 for $-1 \sigma$ shifts, for positive pions. Changes in multiplicity weights under variations in the vertical beam position have also been considered, but will not be shown here due to space limitations. The main conclusion to take away is that the pion multiplicities typically change by small amounts $(<2.5 \%)$ for the vast majority of NA61 analysis bins.

The comparison of the neutrino flux prediction constrained using the modifed weights $\mathrm{W}_{\mathrm{x}_{\text {meas }}^{\mathrm{NA61}} \pm 1 \sigma}$ to the flux prediction with nominal weights $\mathrm{W}_{\mathrm{x}_{\text {meas }}^{\mathrm{NA61}}, \mathrm{y}_{\text {meas }}^{\mathrm{NA61}}}$ is provided in Figure 6.29 at ND280, and Figure 6.30 at Super-K. Variations in the NA61 beam profile centre produce sub-percent variations on the T2K tuned flux, and this systematic has been neglected for now, considering the overall flux uncertainty level of the order of a few percent.

Because of differences in the primary proton beamlines leading up to the target, the T2K and NA61 proton beam profiles are not identical. Maybe even more importantly, the T2K beam profile shows significant variations between different T2K physics runs (see Table 3.1). The multiplicity weights that are applied on the T2K flux are calculated from ratios of measurements to simulations with the NA61 beam profile, when ideally, they should be derived based on the T2K beam profile.

To study the impact of differences in the T2K and NA61 beam profiles on the tuned flux, the following toy multiplicity weights have been constructed, derived with both the NA61 beam profile $\mathrm{W}_{\text {NA61-beam }}$ and with the T2K beam profile $\mathrm{W}_{\text {T2K-beam, }}$ where:

$$
\begin{aligned}
& \mathrm{W}_{\text {NA61-beam }}=\left[\frac{d n_{\text {GEANT4 }}(p, \theta, z)}{d p}\right]_{\text {NA61-beam }} /\left[\frac{d n_{\mathrm{FLUKA}}(p, \theta, z)}{d p}\right]_{\text {NA61-beam }} \\
& \mathrm{W}_{\mathrm{T} 2 \mathrm{~K} \text {-beam }}=\left[\frac{d n_{\mathrm{GEANT} 4}(p, \theta, z)}{d p}\right]_{\mathrm{T} 2 \mathrm{~K} \text {-beam }} /\left[\frac{d n_{\mathrm{FLUKA}}(p, \theta, z)}{d p}\right]_{\mathrm{T} 2 \mathrm{~K} \text {-beam }} \cdot
\end{aligned}
$$

In equation 6.9, the average NA61 beam profile was used. In equation 6.10, the $\mathrm{T} 2 \mathrm{~K}$ beam parameters were separately varied across a range of values, spanning the 
scope of variations observed between T2K physics runs. Correlations among T2K beam parameter were deemed to be small enough to allow for parameters to be varied independently from each other. The difference between the T2K flux tuned with multiplicity weights derived using the NA61 beam profile and using the T2K beam profile was examined. The average deviation across all neutrino energy bins was quantified for variations in one of the T2K beam profile parameters at a time. This study was conducted by Lukas Berns from Tokyo Institute of Technology ${ }^{1}$, and is briefly mentioned in this thesis for the sake of completeness. For instance, for scans in the $\mathrm{T} 2 \mathrm{~K}$ beam position in the horizontal direction, the difference in the flux tuned with NA61 and T2K beam profile toy weights is shown in Figure 6.31. The takeaway from such beam parameter scans is that differences between the T2K and the NA61 beam profiles have a negligible effect on the T2K flux calculation.

\footnotetext{
${ }^{1}$ This study was presented at the 73 rd Annual JPS meeting[130].
} 

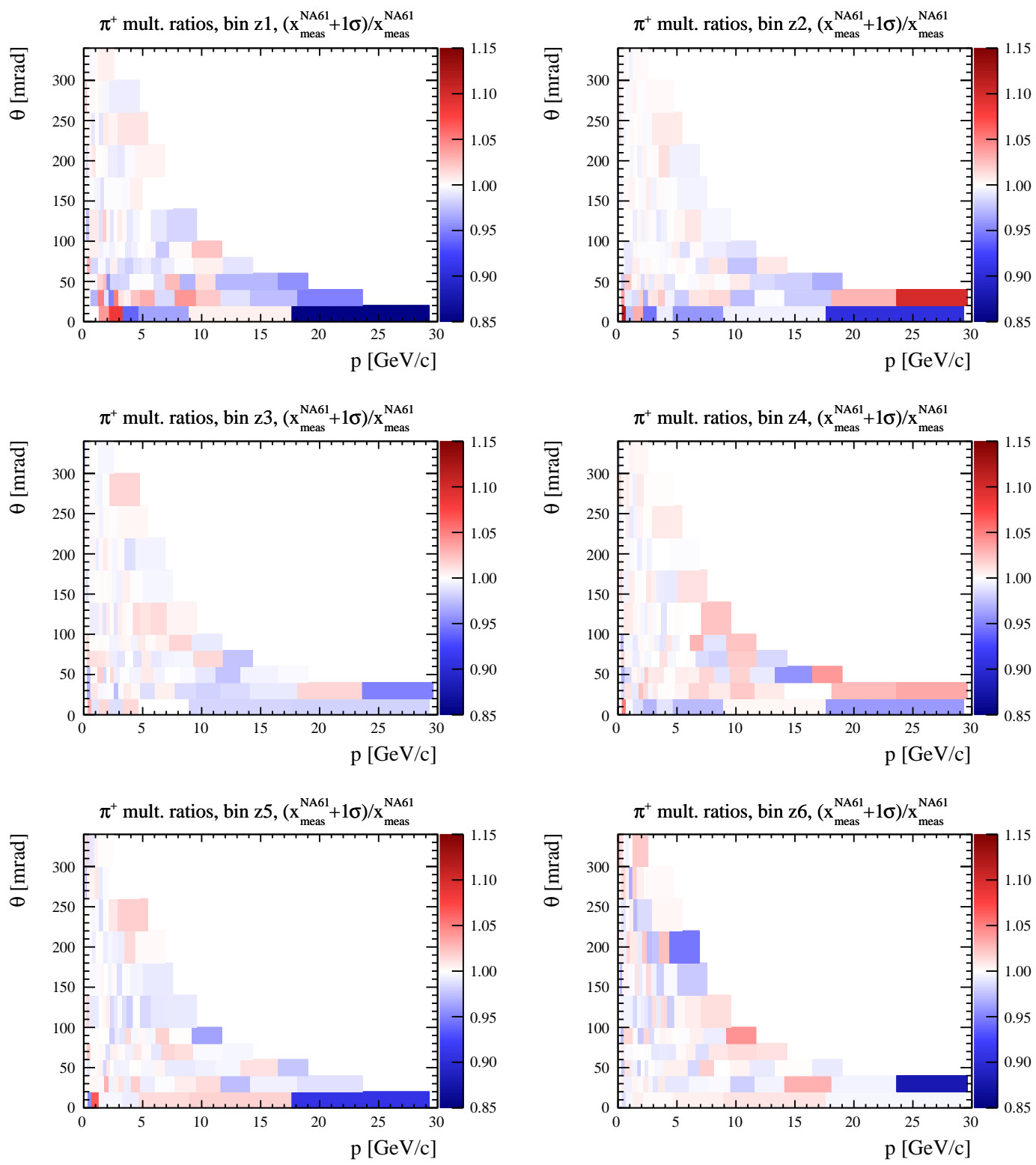

Figure 6.27: Variation in predicted positive pion multiplicities exiting from the NA61 target when increasing the measured NA61 proton beam profile horizontal position by $1 \sigma$. 

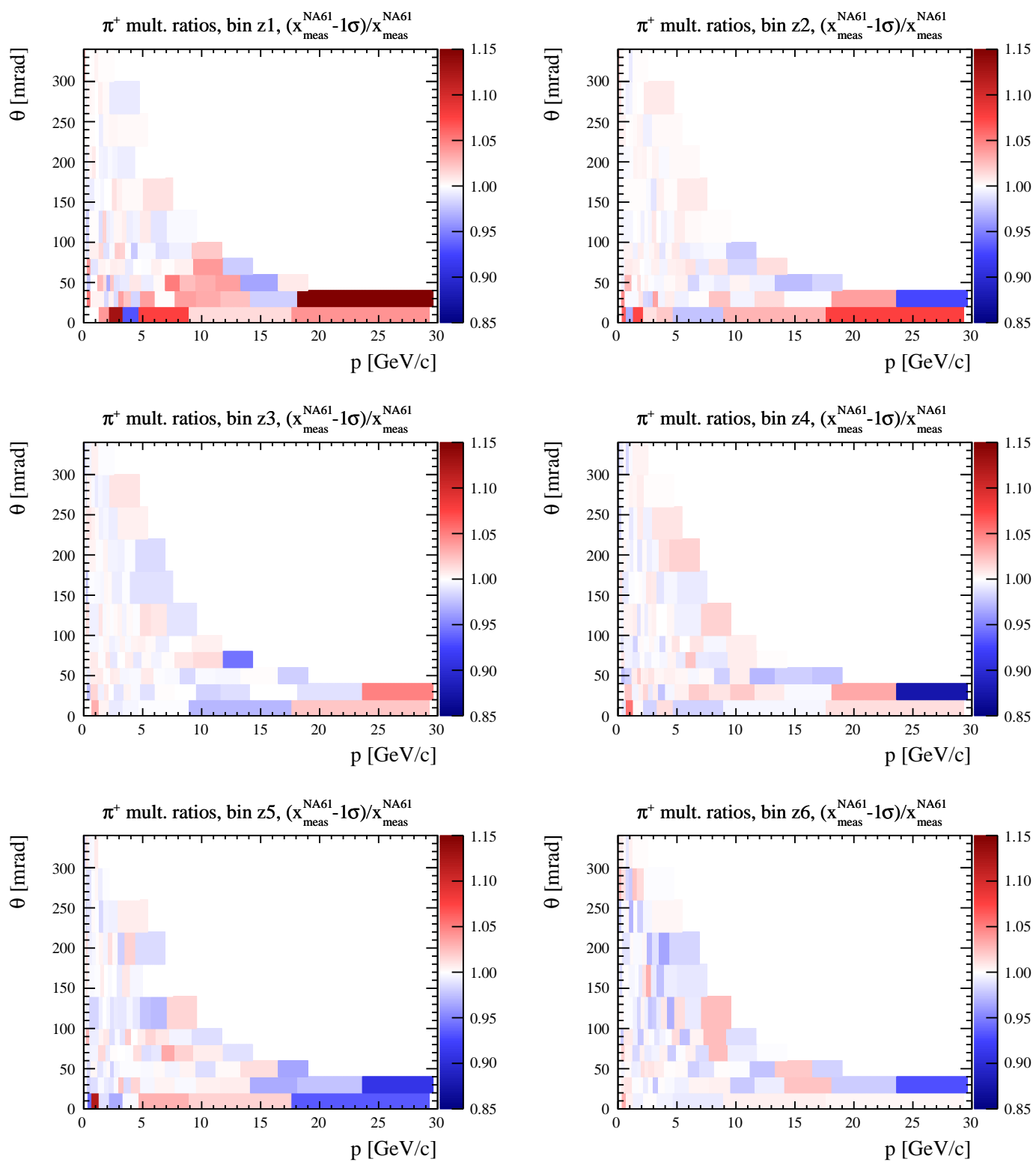

Figure 6.28: Variation in predicted positive pion multiplicities exiting from the NA61 target when decreasing the measured NA61 proton beam profile horizontal position by $1 \sigma$. 


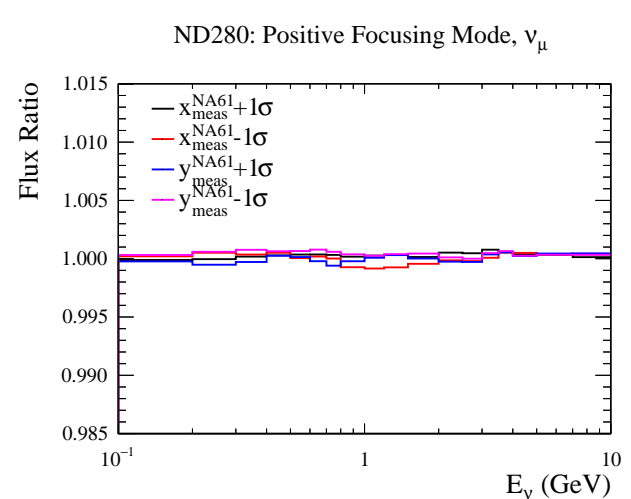

ND280: Positive Focusing Mode, $v_{\mathrm{e}}$

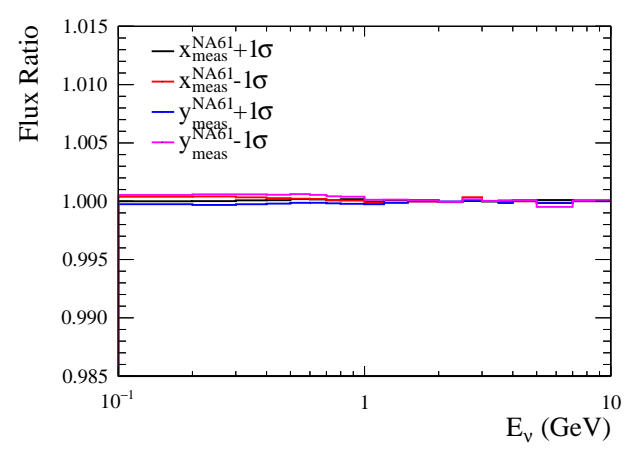

ND280: Negative Focusing Mode, $v_{\mu}$

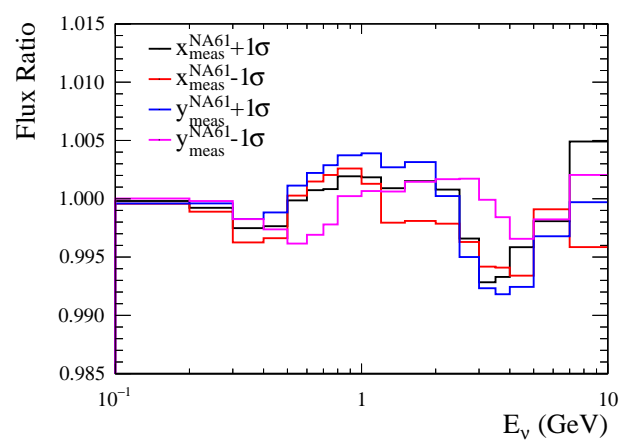

ND280: Negative Focusing Mode, $v_{\mathrm{e}}$

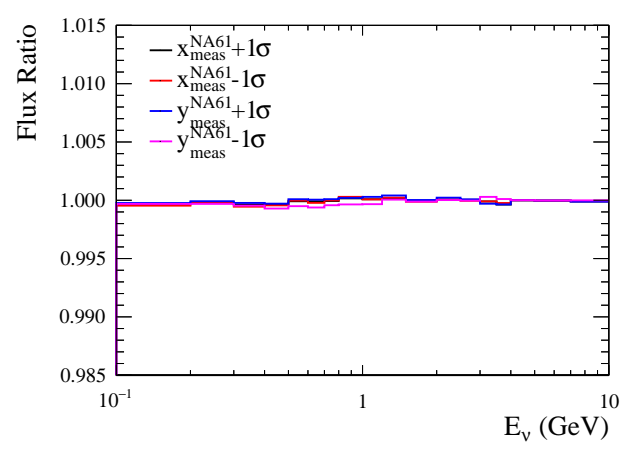

ND280: Positive Focusing Mode, $\bar{v}_{\mu}$

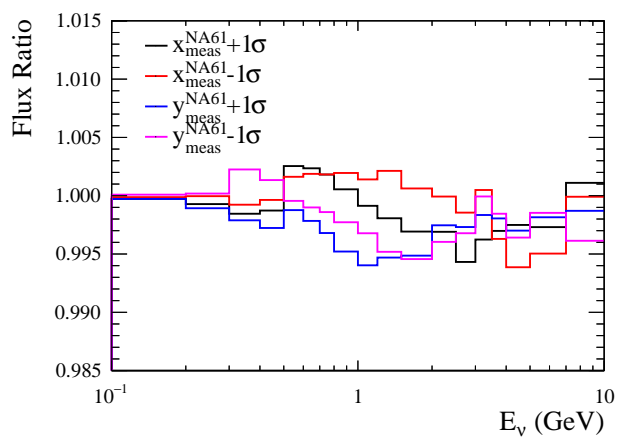

ND280: Positive Focusing Mode, $\bar{v}_{\mathrm{e}}$

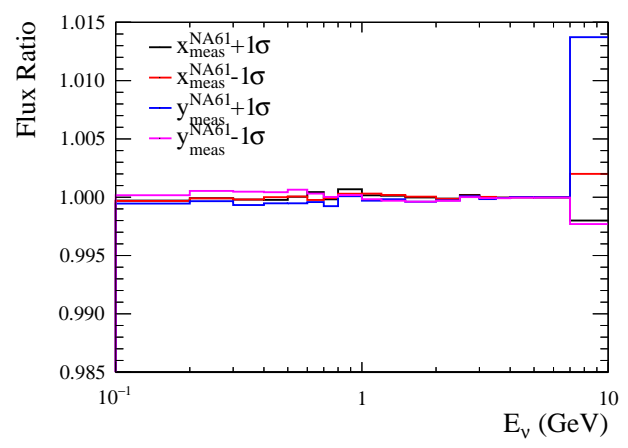

ND280: Negative Focusing Mode, $\bar{v}_{\mu}$

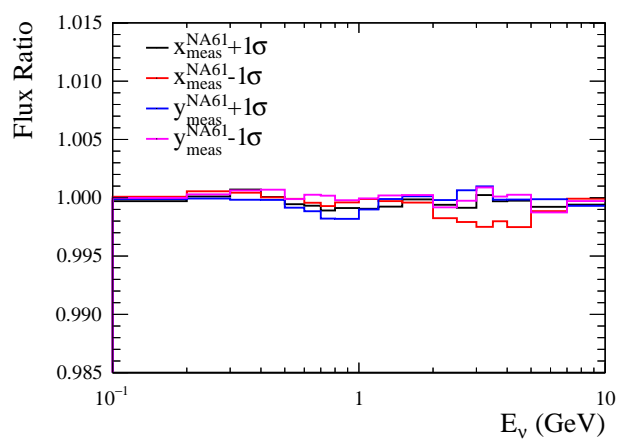

ND280: Negative Focusing Mode, $\bar{v}_{\mathrm{e}}$

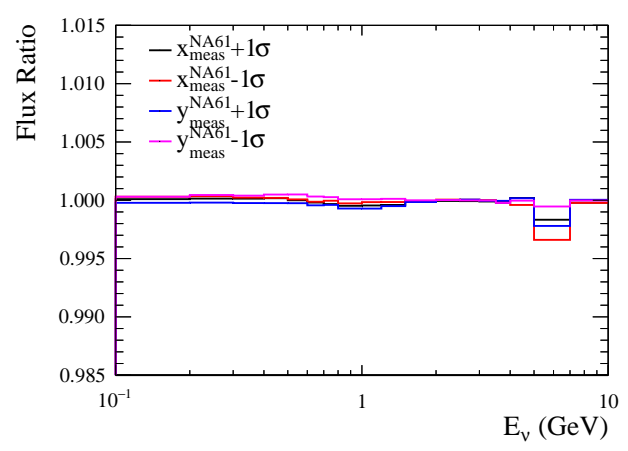

Figure 6.29: Comparing the neutrino flux at ND280 constrained with different sets of replica-target weights, where the modelled NA61 proton beam profile centre was shifted by $\pm 1 \sigma$ along either the horizontal or the vertical direction, to the flux constrained with the standard set of replica-target weights, based on the measured NA61 beam profile centre position. 
SK: Positive Focusing Mode, $v_{\mu}$

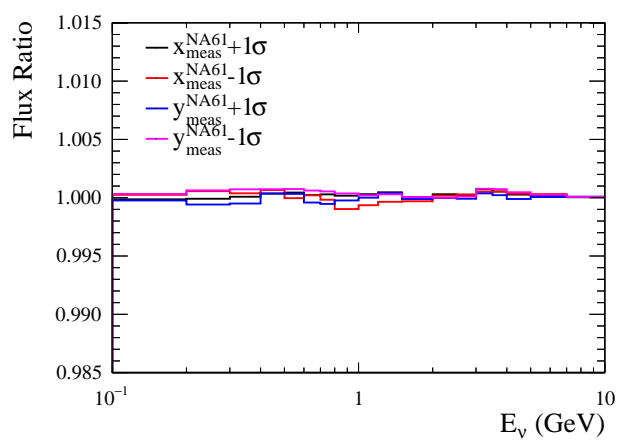

SK: Positive Focusing Mode, $v_{\mathrm{e}}$

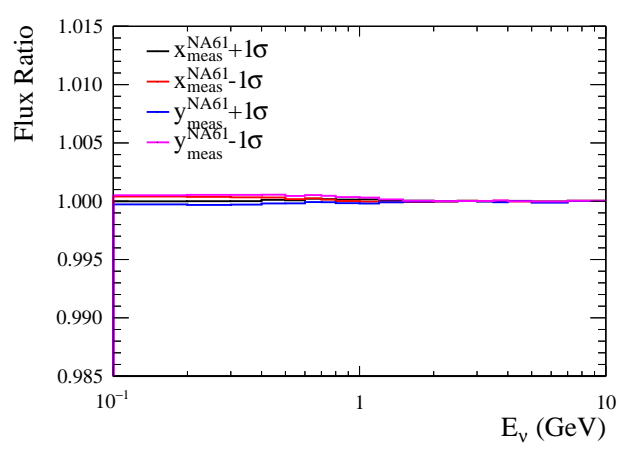

SK: Negative Focusing Mode, $v_{\mu}$

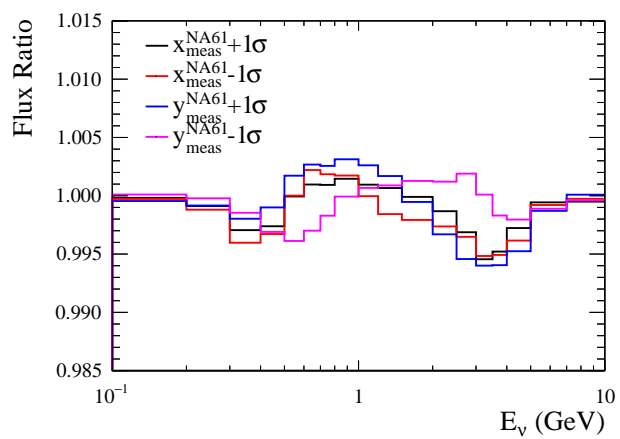

SK: Negative Focusing Mode, $v_{\mathrm{e}}$

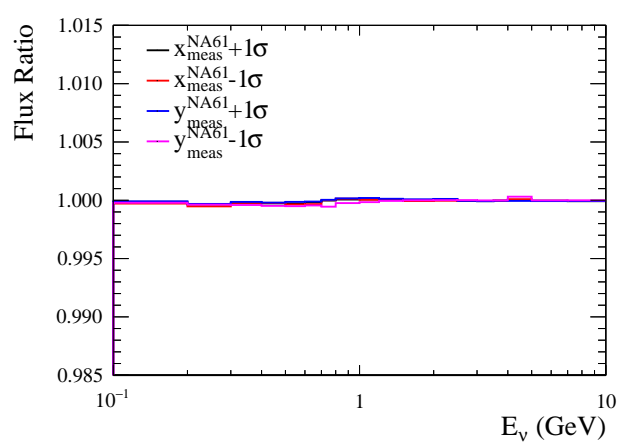

SK: Positive Focusing Mode, $\bar{\nabla}_{\mu}$

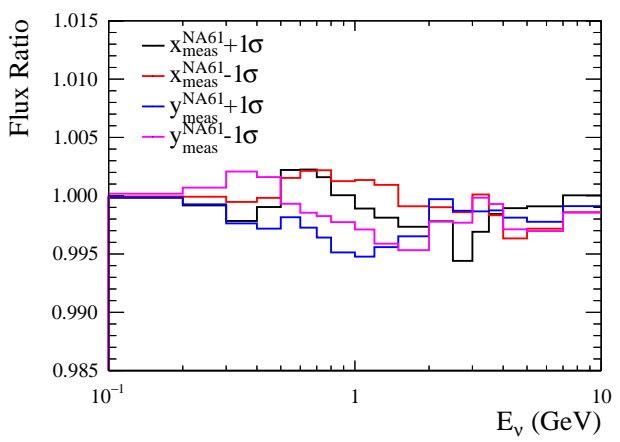

SK: Positive Focusing Mode, $\bar{v}_{\mathrm{e}}$

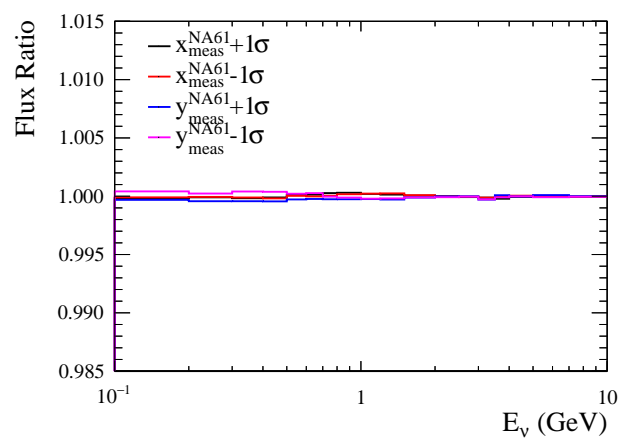

SK: Negative Focusing Mode, $\bar{v}_{\mu}$

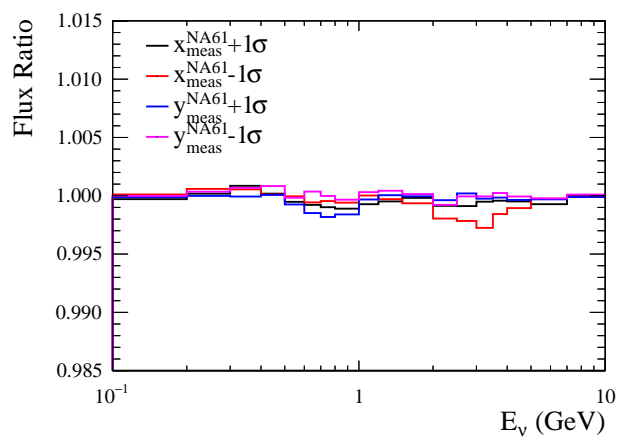

SK: Negative Focusing Mode, $\bar{v}_{\mathrm{e}}$

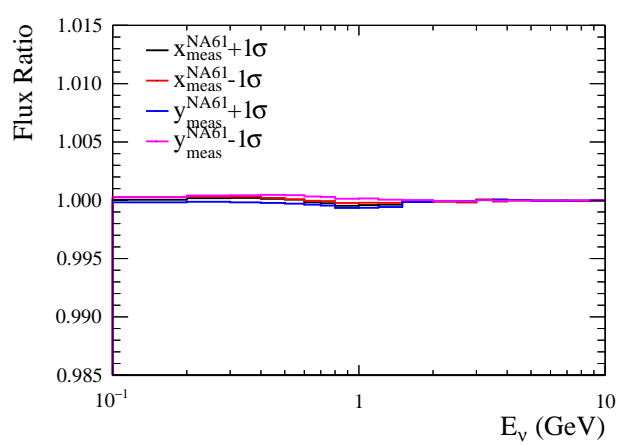

Figure 6.30: Comparing the neutrino flux at the far detector constrained with different sets of replica-target weights, where the modelled NA61 proton beam profile centre was shifted by $\pm 1 \sigma$ along either the horizontal or the vertical direction, to the flux constrained with the standard set of replica-target weights, based on the measured NA61 beam profile centre position. 

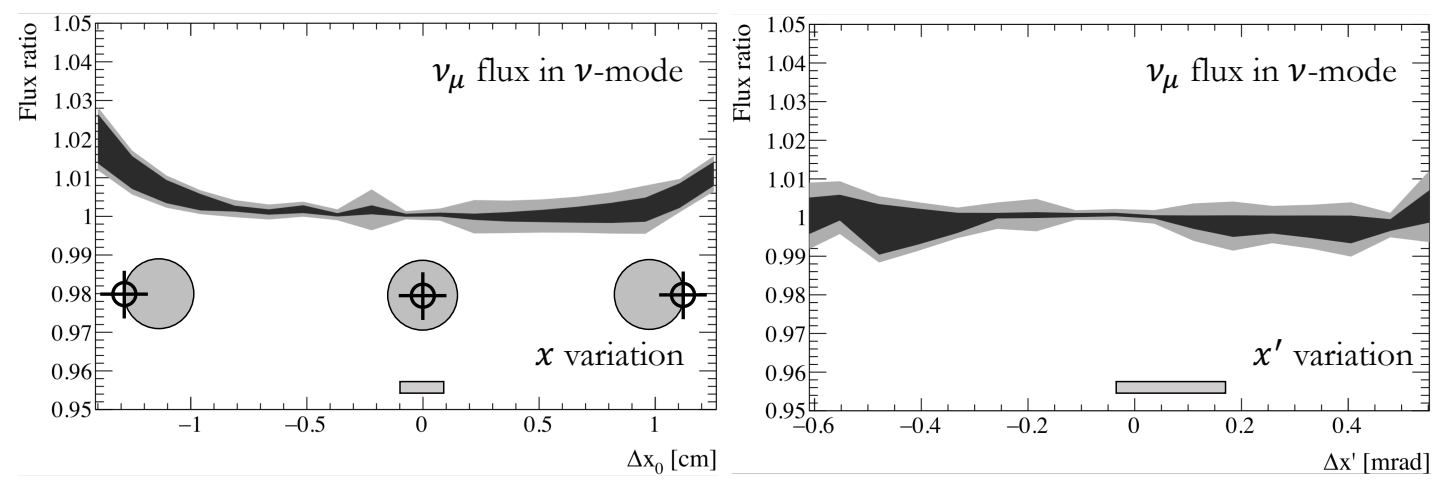

Figure 6.31: The vertical axis shows the ratio of the flux tuned with NA61 beam profile weights (kept fixed) with respect to the flux tuned with T2K beam profile weights (varied). The horizontal axis shows the variations in horizontal position (left) and divergence (right) of the T2K beam parameters with respect to the fixed NA61 value. The grey rectangle denotes the scope of variation observed in T2K runs 1-9. Thus, every parameter is varied across a wider range of values than the current maximum difference between the NA61 beam and the T2K beam for any physics run. Extreme displacements of the horizontal beam position of the T2K beam correspond to scenarios where half of the beam misses the target, resulting in a smaller neutrino flux. Reprinted with permission from L. Berns, "Systematic uncertainties for T2K neutrino flux tuning with 2010 NA61/SHINE data", 73rd Annual Meeting of the Japanese Physical Society (2018)[130]. 


\subsection{Unconstrained hadronic interactions}

Hadronic interactions in the neutrino ancestry chain where the exiting meson multiplicity could not be constrained with any of the available hadron production measurements should be accounted for when estimating the flux uncertainty. In other words, these are interactions for which no replica target (Eq. 6.1) and no thin target (Eq. 5.2) multiplicity weights can be assigned, simply because no data exists for these interactions.

The multiplicity weights used in this study have been extrapolated to cover the widest possible spectrum of hadronic interactions relevant for T2K neutrino productions. The weights were scaled to a range of incoming proton momenta and target nuclei species with energy and material scaling (refer to Chapter 5.3.1 for more details). The coverage of the outgoing hadron phase space was extended with parametrised BMPT fits to data (refer to Chapter 5.3.1). If possible, simulated interactions were tuned to HARP measurements of pion rescattering [118], which have been introduced in Chapter 5.3.3.

Finally, certain neutron interactions have been constrained with NA61 thin target data by invoking isospin arguments. The concept of isospin will be briefly reviewed in this paragraph, with a particular focus on its relevance for constraining interactions with neutrons incident on graphite, before proceeding to classify interactions which cannot be tuned to existing data in subsequent paragraphs. Isospin refers to an approximate flavour symmetry of the up and down quarks, from the point of view of strong interactions. The symmetry is an approximate symmetry of strong interactions, broken by the difference in the quark masses. It does not hold for

electromagnetic processes due to the $+\frac{2}{3}$ charge of the up quark and the $-\frac{1}{3}$ charge of the down quark. If a basis where the up and down quarks can be expressed as mutually orthogonal unit vectors is chosen,

$$
|u\rangle=\left(\begin{array}{l}
1 \\
0
\end{array}\right) \text { and }|d\rangle=\left(\begin{array}{l}
0 \\
1
\end{array}\right)
$$

then the symmetry under $u \leftrightarrow d$ transformations is equivalent to rotations $\mathbb{U}$ in the abstract isospin space, where $\mathbb{U}$ the complex unitary rotation matrix obeying 
$\mathbb{U} \mathbb{U}^{\dagger}=\mathbb{I}$. All the unitary matrices $\mathbb{U}$ comprise the unitary $U(2)$ group. The basis of this group consists of four independent matrices. One of these matrices corresponds to simple multiplication by a phase space factor

$$
\mathbb{U}=\left(\begin{array}{ll}
1 & 0 \\
0 & 1
\end{array}\right) e^{i \phi}
$$

and the other three form the special unitary SU(2) group. For infinitesimal transformations $\epsilon$, these matrices can be expressed in terms of Hermitian generators $\mathbb{G}$ via

$$
\mathbb{U}=1+i \epsilon \mathbb{G}
$$

A convenient choice for the Hermitian generators $\mathbb{G}$ of these remaining three matrices are the linearly independent Pauli matrices:

$$
\sigma_{1}=\left(\begin{array}{ll}
0 & 1 \\
1 & 0
\end{array}\right) \quad \sigma_{2}=\left(\begin{array}{cc}
0 & -i \\
i & 0
\end{array}\right) \quad \sigma_{3}=\left(\begin{array}{cc}
1 & 0 \\
0 & -1
\end{array}\right)
$$

Thus, it follows that isospin has exactly the same transformation properties as spin, and expressions for pion states follow from analogy with combinations of two spin- $\frac{1}{2}$ particles:

$$
\begin{aligned}
\left|\pi^{+}\right\rangle & =|u \bar{d}\rangle \\
\left|\pi^{0}\right\rangle & =\frac{1}{\sqrt{2}}(|u \bar{u}\rangle+|d \bar{d}\rangle) \\
\left|\pi^{-}\right\rangle & =|\bar{u} d\rangle
\end{aligned}
$$

Under isospin transformations, positive pions transform into negative pions and vice versa $\pi^{+} \leftrightarrow \pi^{-}$, and neutral pions remain unchanged. Similarly, protons which are made from two up quarks and one down quark transform to neutrons, $\mathrm{p} \leftrightarrow \mathrm{n}$, and vice versa. Keeping in mind that NA61 thin target measurements were collected using an isoscalar carbon target, with the same number of protons and neutrons in the nucleus, the following symmetries can be utilised:

$$
\begin{gathered}
\mathrm{n}+{ }^{12} \mathrm{C} \rightarrow \pi^{ \pm}+\ldots \quad \leftrightarrow \quad \mathrm{p}+{ }^{12} \mathrm{C} \rightarrow \pi^{\mp}+\ldots \\
\mathrm{n}+{ }^{12} \mathrm{C} \rightarrow \mathrm{n}+\ldots \leftrightarrow \mathrm{p}+{ }^{12} \mathrm{C} \rightarrow \mathrm{p}+\ldots
\end{gathered}
$$

This allows for neutron interactions with neutrons or pions in the final state to be tuned with multiplicity weights in Figure 5.7. Especially attractive is the feature of 
tuning interactions with neutrons in the final state, which are notoriously difficult to detect using conventional hadron production detectors.

A significant number of simulated interactions at T2K cannot be constrained with data even when using all of the above-mentioned techniques for extending the coverage of existing hadron production datasets. Out of the total number of interactions contributing to $\mathrm{T} 2 \mathrm{~K}$ neutrino production, $4.6 \%$ of interactions in neutrino mode, and $5.2 \%$ of interactions in anti-neutrino mode, could not be tuned to data. Interactions constrained through isospin arguments, such as inclusive interactions of neutrons with neutrons or pions in the final state, are considered as being covered by data, and are thus excluded from this study. Different datasets have been sequentially added, and the increase in the number of constrained interactions has been examined. These numbers have also been split by the neutrino flavour component, and the T2K mode of operation. The fraction of unconstrained interactions at ND280 is shown in Table 6.3, and at Super-K in Table 6.4.

\begin{tabular}{|c|c|c|c|c|c|c|c|c|}
\hline \multirow{3}{*}{ Dataset } & \multicolumn{8}{|c|}{ Tuned Hadronic Interactions in Neutrino Ancestry } \\
\hline & \multicolumn{4}{|c|}{ ND280 $\nu$-mode } & \multicolumn{4}{|c|}{ ND280 $\bar{\nu}$-mode } \\
\hline & $\nu_{\mu}$ & $\bar{\nu}_{\mu}$ & $\nu_{e}$ & $\bar{\nu}_{e}$ & $\nu_{\mu}$ & $\bar{\nu}_{\mu}$ & $\nu_{e}$ & $\bar{\nu}_{e}$ \\
\hline Thin & $86.0 \%$ & $80.1 \%$ & $84.0 \%$ & $75.8 \%$ & $80.4 \%$ & $85.5 \%$ & $76.6 \%$ & $83.5 \%$ \\
\hline +Replica & $94.0 \%$ & $83.4 \%$ & $89.7 \%$ & $76.2 \%$ & $83.7 \%$ & $93.7 \%$ & $76.9 \%$ & $90.2 \%$ \\
\hline+ HARP & $96.5 \%$ & $87.3 \%$ & $91.2 \%$ & $76.7 \%$ & $87.2 \%$ & $96.3 \%$ & $77.3 \%$ & $91.8 \%$ \\
\hline
\end{tabular}

Table 6.3: $\quad$ Fraction of hadronic interactions leading up to neutrinos at ND280, tuned with combinations of available multiplicity measurements.

\begin{tabular}{c|c|c|c|c|c|c|c|c}
\hline \multirow{2}{*}{ Dataset } & \multicolumn{6}{|c}{ Tuned Hadronic Interactions in Neutrino Ancestry } \\
\cline { 2 - 9 } & \multicolumn{4}{|c|}{ SK $\nu$-mode } & \multicolumn{4}{c}{ SK $\bar{\nu}$-mode } \\
\cline { 2 - 9 } & $\nu_{\mu}$ & $\bar{\nu}_{\mu}$ & $\nu_{e}$ & $\bar{\nu}_{e}$ & $\nu_{\mu}$ & $\bar{\nu}_{\mu}$ & $\nu_{e}$ & $\bar{\nu}_{e}$ \\
\hline Thin & $85.8 \%$ & $80.0 \%$ & $83.8 \%$ & $76.9 \%$ & $80.9 \%$ & $85.3 \%$ & $77.6 \%$ & $83.2 \%$ \\
\hline +Replica & $94.0 \%$ & $83.6 \%$ & $89.2 \%$ & $77.3 \%$ & $84.4 \%$ & $93.6 \%$ & $77.9 \%$ & $89.5 \%$ \\
\hline +HARP & $96.5 \%$ & $87.6 \%$ & $90.5 \%$ & $77.8 \%$ & $87.8 \%$ & $96.2 \%$ & $78.3 \%$ & $91.1 \%$ \\
\hline \hline
\end{tabular}

Table 6.4: Fraction of hadronic interactions leading up to neutrinos at Super-K, tuned with combinations of available multiplicity measurements. 
Plots showing the hadronic interactions which remain untuned, specified in terms of the incoming and outgoing hadron species, are shown in Figure 6.32, for the total flux at ND280 in both $\nu$-mode and $\bar{\nu}$-mode. These are inclusive interactions specified in terms of the outgoing hadron which ultimately contributes to neutrino production.
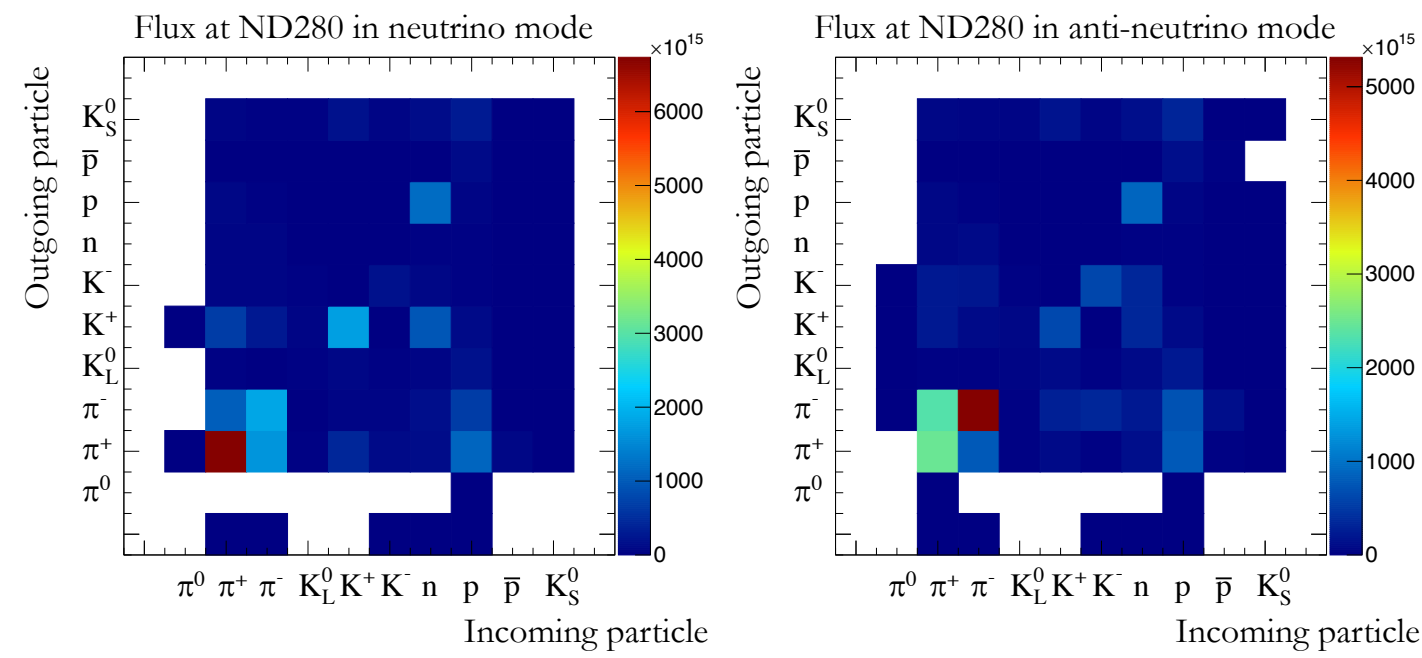

Figure 6.32: Incoming and outgoing particle types for unconstrained hadronic interactions of neutrino ancestors, in neutrino mode (left) and anti-neutrino mode (right). The colour scale in the figures corresponds to the relative number density of the corresponding interactions.

The material where the unconstrained interactions occur has also been examined. The cooling water around the inner horn conductor is not simulated in this study. In (anti)neutrino mode, the majority of untuned interactions are from $\pi^{+(-)}+$ $X \rightarrow \pi^{+(-)}+Y$ interactions, where the nuclear target $X$, in decreasing order of importance, is aluminium (horns), iron (decay volume walls), carbon (target and dump) and titanium (horn cooling structure). The limiting factor in constraining pion rescattering comes from the phase-space coverage of the HARP dataset. HARP measurements do not contain yields from pions emitted at low $(\theta>50 \mathrm{mrad})$ or high $(\theta>250 \mathrm{mrad})$ scattering angles.

Unconstrained interactions can be visualised in the phase space of the $\left(x_{\mathrm{F}}, p_{\mathrm{T}}\right)$ Feynman variables. The fraction of unconstrained interactions occupying different phase space regions for some of the main unconstrained interactions are given in Fig. 6.33 and Fig. 6.34, for the positive and negative horn focusing modes, 
respectively. For every unconstrained interaction, the $\left(x_{\mathrm{F}}, p_{\mathrm{T}}\right)$ phase space has been divided into 6 bins, the boundaries of which have been indicated with horizontal and vertical red lines. The production in each bin will be varied to evaluate the impact on the calculated flux.

In the absence of hadron production data that could cover the full phase space of hadrons contributing to the T2K neutrino production, a fake data study was performed. Toy data sets for exiting meson yields have been generated with both FLUKA and GEANT4 (FTF_BIC and FTFP_BERT physics lists). This study assumed a narrow beam profile incident on a thin target $(26 \mathrm{~mm}$ target diameter, and 20 $\mathrm{mm}$ target length along the beam direction). The beam profile was identical for all three hadron production generators. Different combinations of the beam hadron species $\left(\pi^{ \pm}, \mathrm{K}^{ \pm}\right)$, beam momentum $(3,5,8,12 \mathrm{GeV} / \mathrm{c})$ and target material (Al, C) were simulated in the Monte Carlo generators. Inclusive yields of various outgoing hadronic species $\left(\pi^{ \pm}, \mathrm{K}^{ \pm}\right)$were calculated from the fake data sets, and compared across the different generators. Comparisons of various meson yields across different models for interactions on graphite are summarised in Fig. 6.35, Fig. 6.36, Fig. 6.37, Fig. 6.38, Fig. 6.39 and Fig. 6.40. Similar levels of disagreement have been observed among the different Monte Carlo generator predictions irrespective of the target material, carbon or aluminium, thus justifying the assumption that unconstrained in-target and out-of-target interactions should be accounted for in the same manner. Exiting meson yields have been compared when plotted in the phase space of the $\left(x_{\mathrm{F}}, p_{\mathrm{T}}\right)$ Feynman variables. The scale of variation in the predicted yields among different generators was studied. For $3 \mathrm{GeV} / \mathrm{c}$ pion interactions, FLUKA and FTFP_BERT models exhibit good agreement, whereas the outliers are pion yields generated from FTF_BIC, which are generally lower than yields from both FLUKA and FTFP_BERT. For $3 \mathrm{GeV} / \mathrm{c}$ kaon interactions, the situation is reversed, and kaon yields generated from FTFP_BERT and FTF_BIC agree well with each other, but both differ from FLUKA predictions. It is interesting to note that the distribution of ratios of meson yields from different models does not follow a simple Gaussian distribution centred on unity: namely, the distribution tail on one side of unity is 
typically significantly steeper than on the other. Significant differences between various generators are observed even for phase space regions populated with HARP pion production measurements. It is unclear if any of the studied generators (FLUKA, FTFP_BERT and FTF_BIC) have been tuned to HARP measurements.

For every unconstrained interaction type, the simulated multiplicities can be systematically varied with 6 uncorrelated weights in $\left(x_{\mathrm{F}}, p_{\mathrm{T}}\right)$, and one overall normalisation weight. 100 sets of throws of these 7 weights are made for every interaction, specified by the incoming and outgoing hadron species. Each of these 7 weights is thrown from a Gaussian distribution centred at 1.0 and with standard deviation of 0.5 . The assignment of the $50 \%$ standard deviation for unconstrained meson yields in $(\mathrm{xF}, \mathrm{pT})$ bins contains a certain degree of freedom. The fake data studies presented in this chapter are meant to demonstrate an overall lack of agreement between different interaction generators, highlighting the intrinsic difficulty associated with assigning simulation uncertainties in areas of phase space not covered by data. The fractional variation in multiplicity $\mathrm{N}\left(x_{\mathrm{F}}, p_{\mathrm{T}}\right)$ is calculated via the following formula:

$$
\frac{\Delta \mathrm{N}}{\mathrm{N}}\left(x_{\mathrm{F}}, p_{\mathrm{T}}\right)=\operatorname{norm} \times \operatorname{weight}\left(x_{\mathrm{F}}, p_{\mathrm{T}}\right)-1
$$

The systematic variations on the outgoing meson rates are treated as toy data sets, and propagated to 100 toy variations in the tuned flux for each unconstrained interaction. The covariance matrix due to unconstrained interactions is calculated from all the tuned flux toys. Systematic variations are applied exclusively to outgoing meson multiplicities $\left(\pi^{ \pm}, \mathrm{K}^{ \pm}, \mathrm{K}^{0}\right)$, because baryon multiplicities are already tightly constrained by baryon number conservation. The contributions to the flux uncertainty from these interactions are given in Fig. 6.41, for the far detector flux predictions with positive and negative horn focusing, respectively. Flux uncertainties at ND280 due to unconstrained interactions are given in Fig. 6.42. The contributions to the flux uncertainty coming from unconstrained interactions are mostly of the order of a few percent. Greater unconstrained interaction uncertainties are generally at flux energy bins which are far from the T2K flux peak, e.g. for the $\nu_{\mu}$-flux in 
forward horn current focusing (top left plot in Fig. 6.41), this is pion scattering (black line) at low energies, and kaon scattering (orange line) and kaon production from incident neutrons (yellow-green line) at high energies. The exception is the $\nu_{e}$ untuned interaction error in reverse horn current focusing (bottom left plot in Fig. 6.42), where kaon scattering (cyan line) is the dominant flux uncertainty which now exceeds the total uncertainty assigned to this flux component in previous flux releases $\left(\nu_{e}\right.$ contamination in $\bar{\nu}_{\mu}$ beam is $\left.<0.1 \%\right)$.

The total flux uncertainty due to unconstrained interactions has been added to other systematic uncertainties caused by hadronic interaction modelling. The reader is reminded that this is a new systematic uncertainty, which has been estimated for a more robust treatment of flux parameters. The total hadron interaction component of the flux uncertainty is provided in Fig. 6.10 for the Super-K flux with both positive and negative horn focusing. The hadronic interaction component of flux uncertainty at ND280 is given in Fig. 6.11. Unconstrained interactions are the dominant uncertainty on the wrong sign flux at lower energies, with the most dramatic effect coming from $\mathrm{K}^{+}+X \rightarrow \mathrm{K}^{+}+Y$ interactions in reverse horn focusing mode. The hadronic component of the flux uncertainty was underestimated in the previous flux prediction using thin target measurements, most evidently for the $\nu_{e}$ flux in anti-neutrino mode.

For further constraining the T2K flux prediction, future hadron production experiments should populate the $\pi^{ \pm}+X \rightarrow \pi^{ \pm}+Y$ outgoing multiplicity measurements which are missing from the coverage of the low beam energy $(\sim 3 \mathrm{GeV})$ HARP dataset. Measuring exiting meson yields with a beam of kaons incident on graphite (and aluminium) would also be interesting, but would be very challenging due to the high proton and pion contamination in such a low energy kaon beam. 
$\pi^{+}+\mathrm{X} \rightarrow \pi^{+}+\ldots$

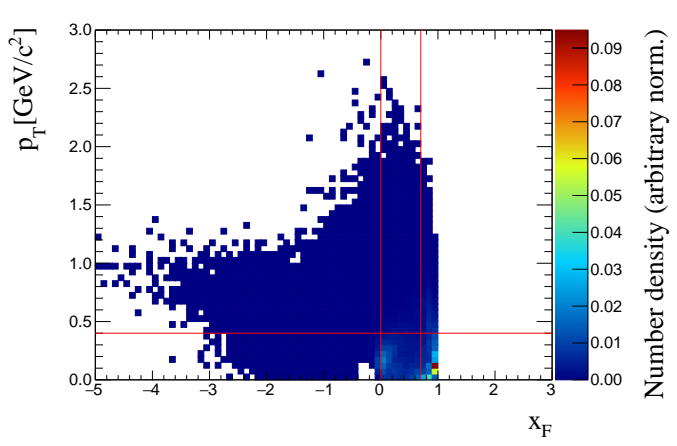

$\pi^{-}+\mathrm{X} \rightarrow \pi^{+}+\ldots$

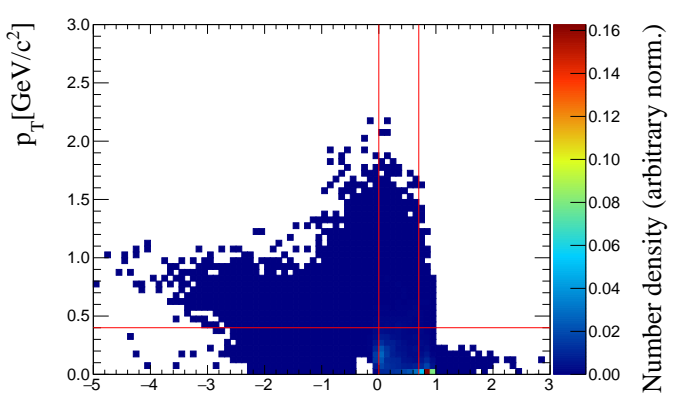

$\mathrm{X}_{\mathrm{F}}$

$\mathrm{K}^{+}+\mathrm{X} \rightarrow \mathrm{K}^{+}+\ldots$

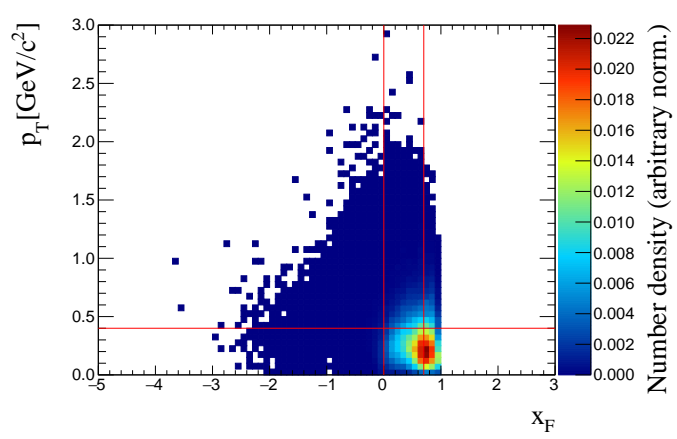

$\pi^{+}+\mathrm{X} \rightarrow \pi^{-}+\ldots$

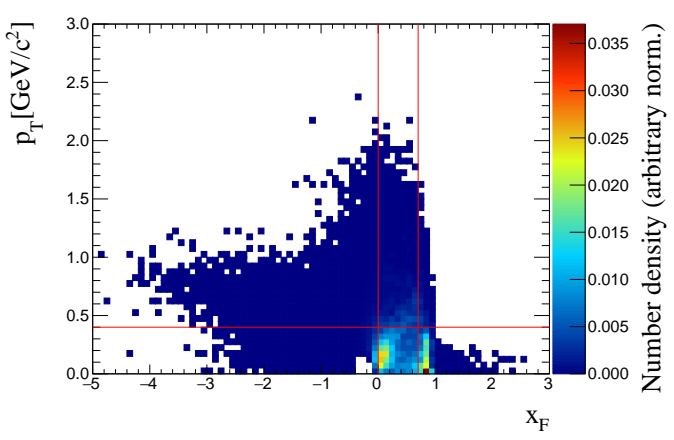

$\pi^{-}+\mathrm{X} \rightarrow \pi^{-}+\ldots$

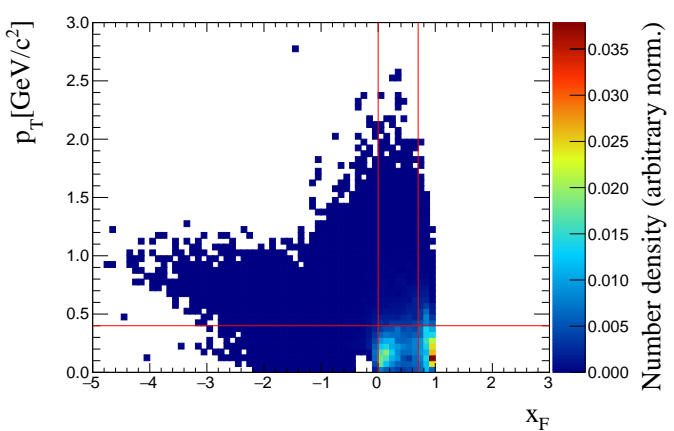

$\mathrm{K}^{-}+\mathrm{X} \rightarrow \mathrm{K}^{-}+\ldots$

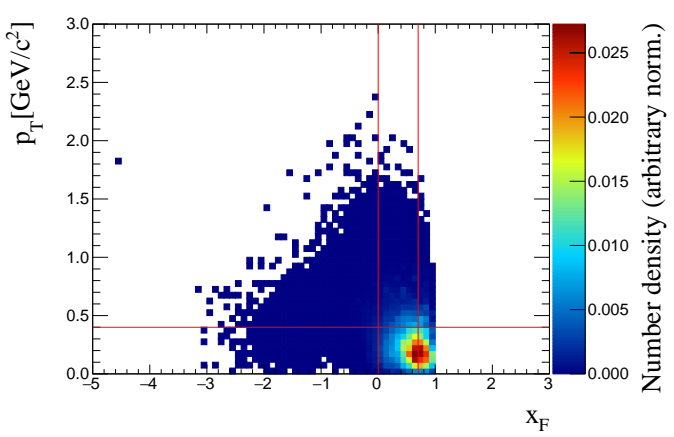

Figure 6.33: Depiction of the dominant untuned hadronic interactions at the far detector in forward horn current focusing mode. Untuned interactions have been visualised in the phase space of the $\left(x_{F}, p_{T}\right)$ Feynman variables of the daughter hadron, and the colour scale signifies the number density, in arbitrary units, of daughters in each $\left(x_{F}, p_{T}\right)$ bin. The red boundaries specify $6\left(x_{F}, p_{T}\right)$ bins used for estimating the effect of unconstrained interactions on the flux uncertainty. 

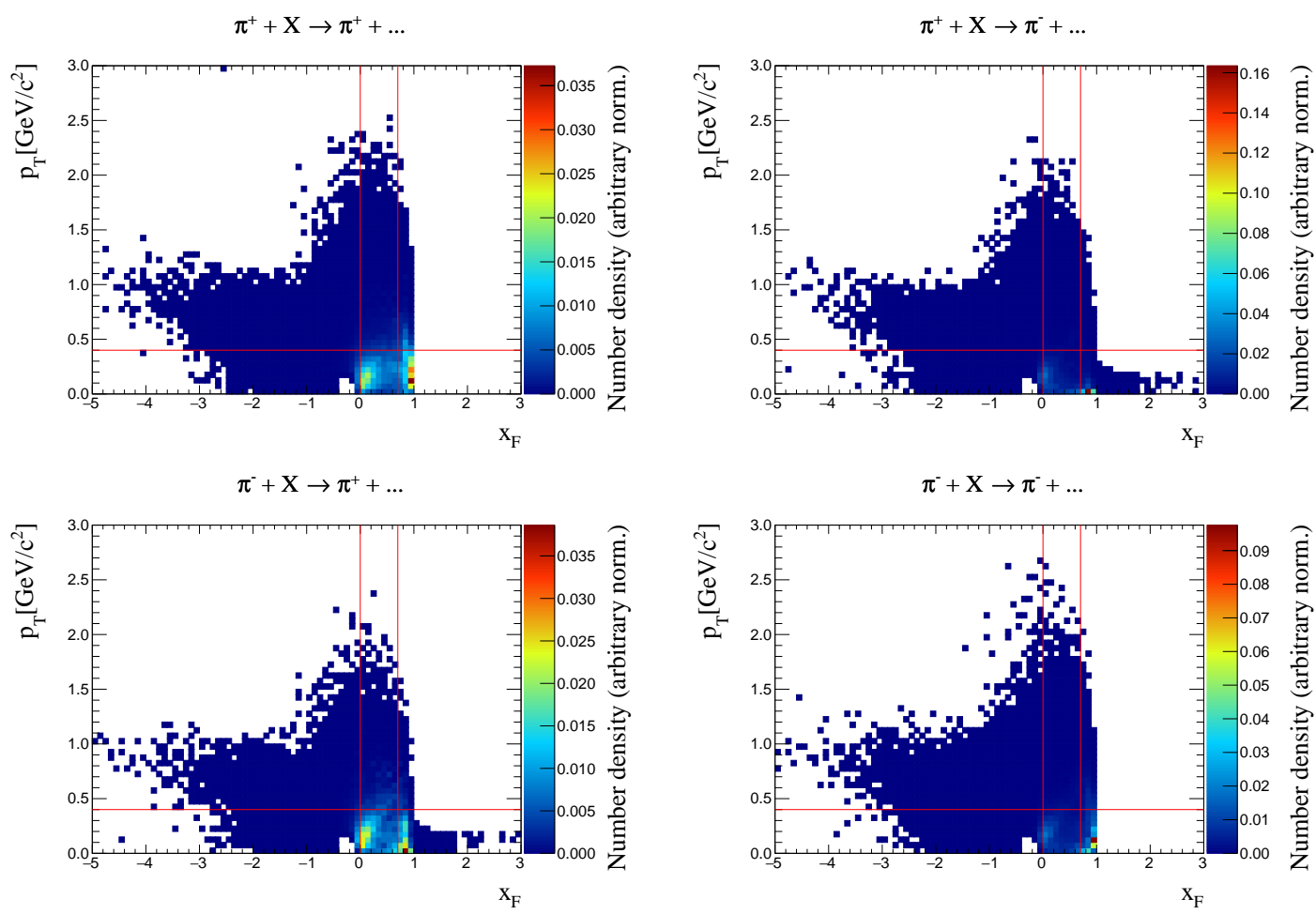

$\mathrm{K}^{+}+\mathrm{X} \rightarrow \mathrm{K}^{+}+\ldots$

$\mathrm{K}^{-}+\mathrm{X} \rightarrow \mathrm{K}^{-}+\ldots$
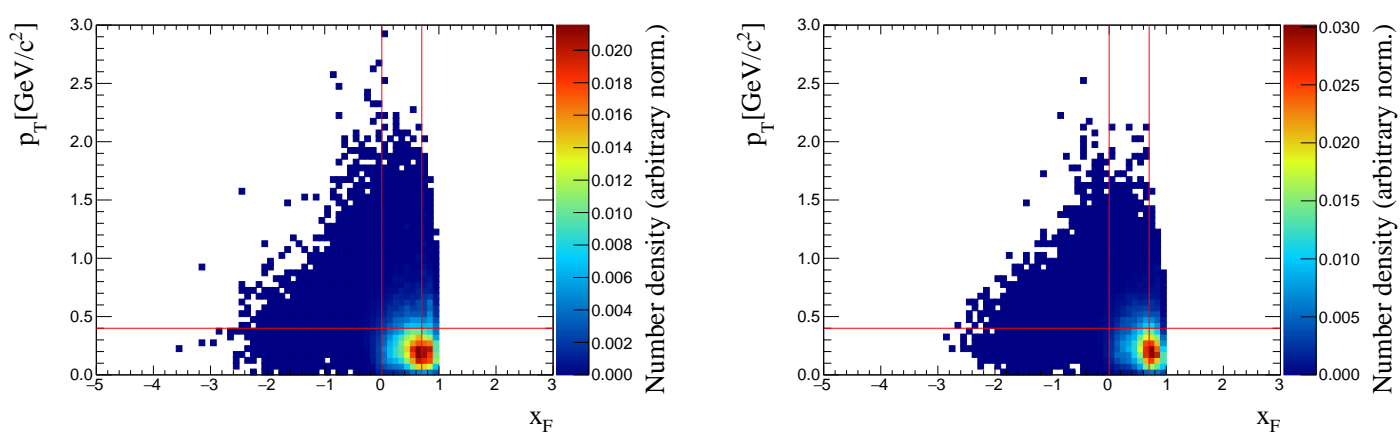

Figure 6.34: Depiction of the dominant untuned hadronic interactions at the far detector in reverse horn current focusing mode. Untuned interactions have been visualised in the phase space of the $\left(x_{F}, p_{T}\right)$ Feynman variables of the daughter hadron, and the colour scale signifies the number density, in arbitrary units, of daughters in each $\left(x_{F}, p_{T}\right)$ bin. The red boundaries specify $6\left(x_{F}, p_{T}\right)$ bins used for estimating the effect of unconstrained interactions on the flux uncertainty. 

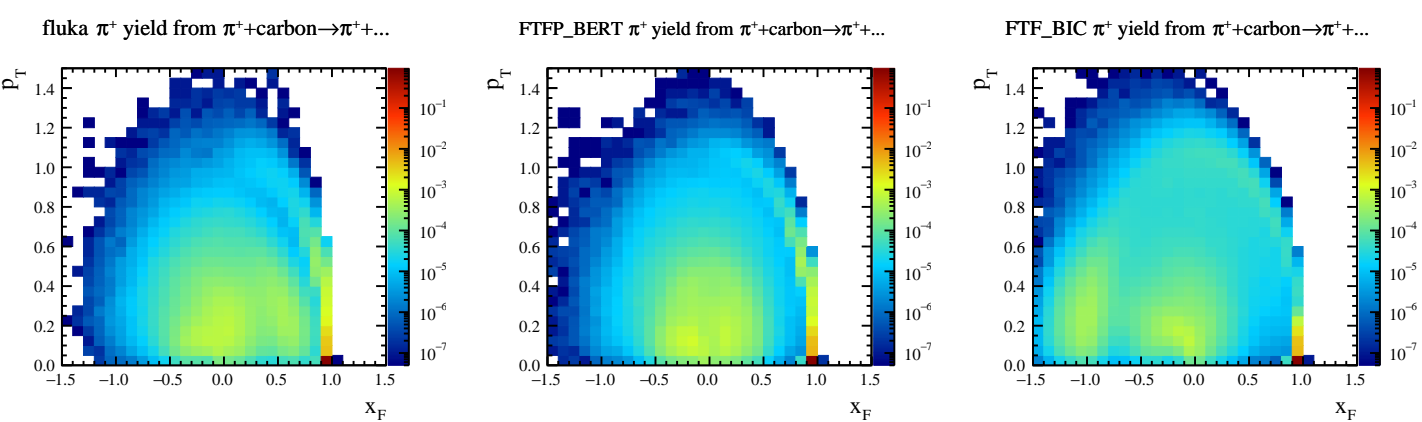

2D ratio of FTFP_BERT/FTF_BIC $\pi^{+}$yields

2D ratio of FTFP_BERT/fluka $\pi^{+}$yields

2D ratio of FTF_BIC/fluka $\pi^{+}$yields
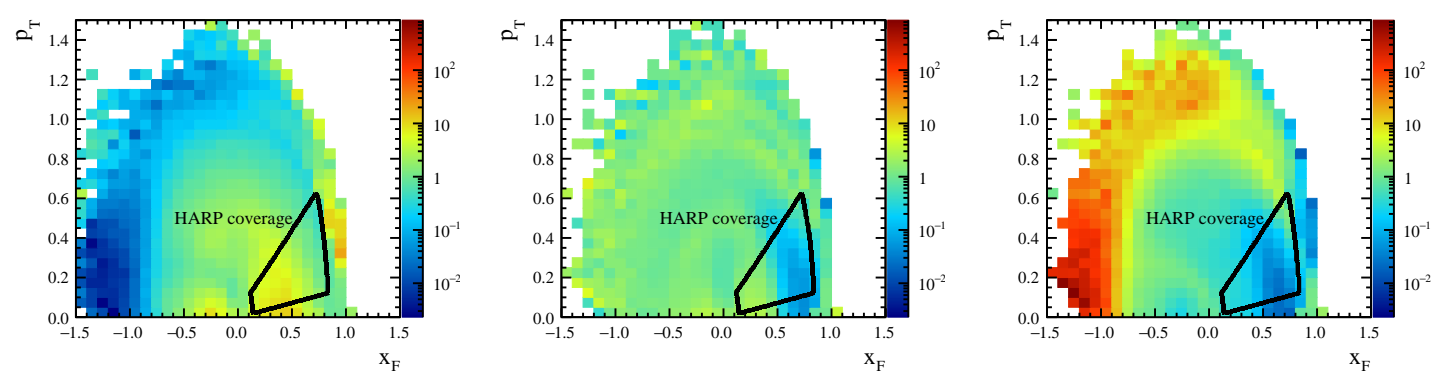

FTFP_BERT/FTF_BIC ratio distribution

FTFP_BERT/fluka ratio distribution
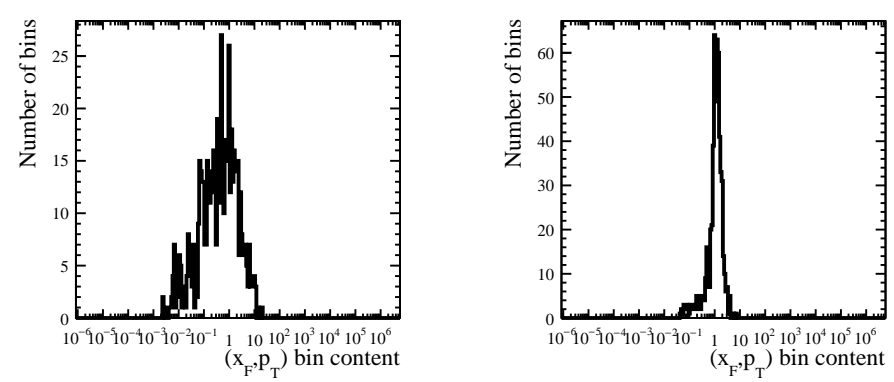

FTF_BIC/fluka ratio distribution

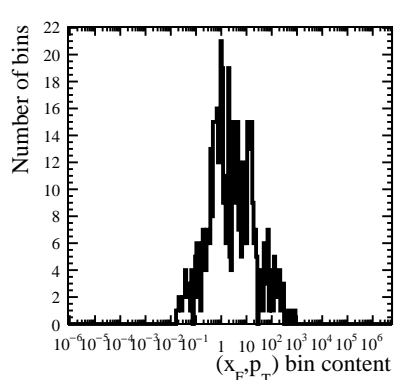

Figure 6.35: Study of differences in exiting $\pi^{+}$yields predicted by different Monte Carlo models for interactions of $3 \mathrm{GeV}$ positive pions on graphite $\pi^{+}+C \rightarrow \pi^{+}+X$. Plotted are the exiting pion yields in the $\left(x_{F}, p_{T}\right)$ phase space, predicted by FLUKA (top left), FTFP_BERT (top middle) and FTF_BIC (top right). Ratios of pion yields predicted by different models are shown: FTFP_BERT compared to FTF_BIC (middle left), FTFP_BERT compared to FLUKA (middle middle) and FTF_BIC compared to FLUKA (middle right). Finally, the frequency of different values for the yield ratio is examined in the bottom row of figures. 

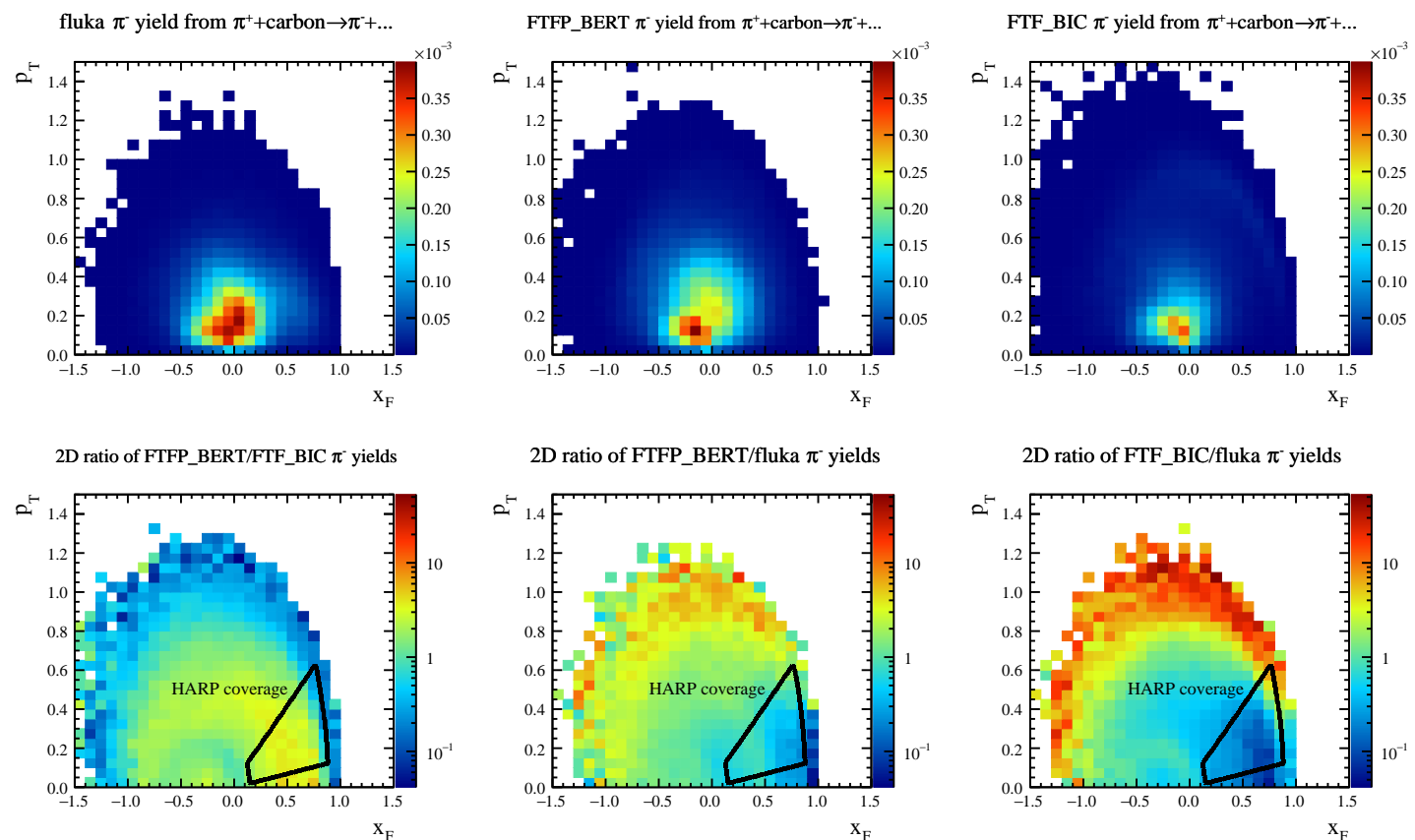

2D ratio of FTFP_BERT/fluka $\pi$ - yields

2D ratio of FTF_BIC/fluka $\pi$ yields
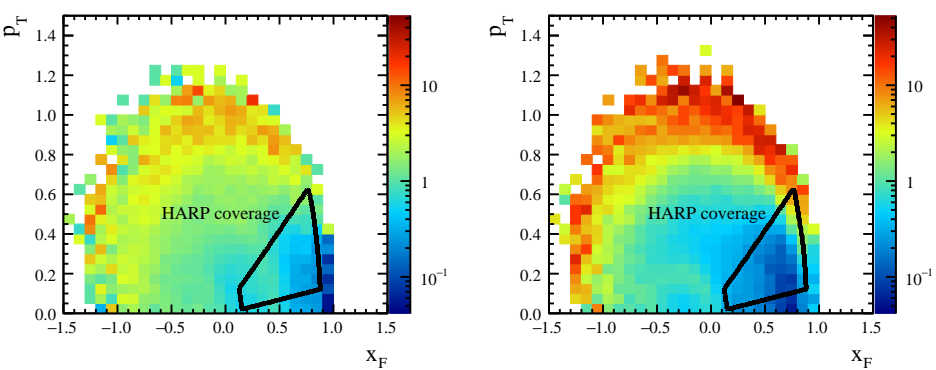

FTFP_BERT/FTF_BIC ratio distribution

FTFP_BERT/fluka ratio distribution
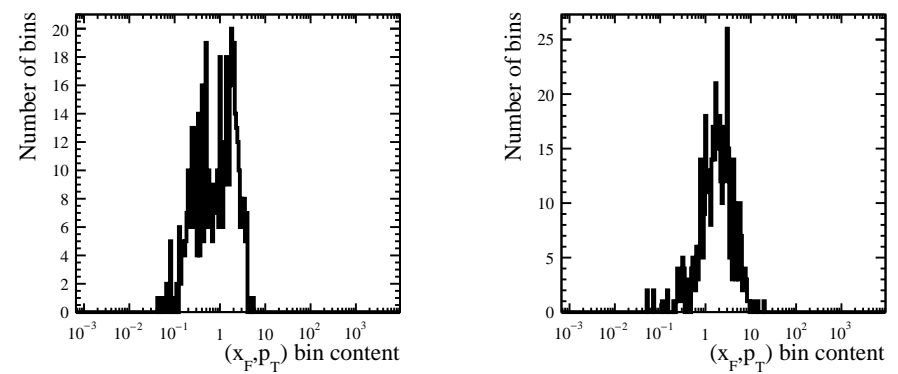

FTF_BIC/fluka ratio distribution

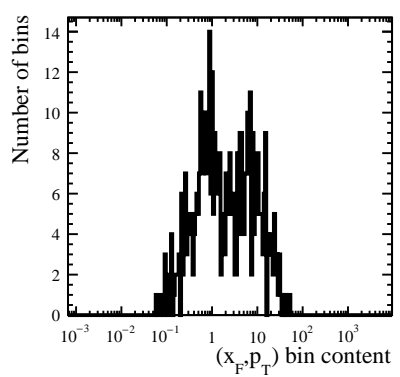

Figure 6.36: Study of differences in exiting $\pi^{-}$yields predicted by different Monte Carlo models for interactions of $3 \mathrm{GeV}$ positive pions on graphite $\pi^{+}+C \rightarrow \pi^{-}+X$. Plotted are the exiting pion yields in the $\left(x_{F}, p_{T}\right)$ phase space, predicted by FLUKA (top left), FTFP_BERT (top middle) and FTF_BIC (top right). Ratios of pion yields predicted by different models are shown: FTFP_BERT compared to FTF_BIC (middle left), FTFP_BERT compared to FLUKA (middle middle) and FTF_BIC compared to FLUKA (middle right). Finally, the frequency of different values for the yield ratio is examined in the bottom row of figures. 

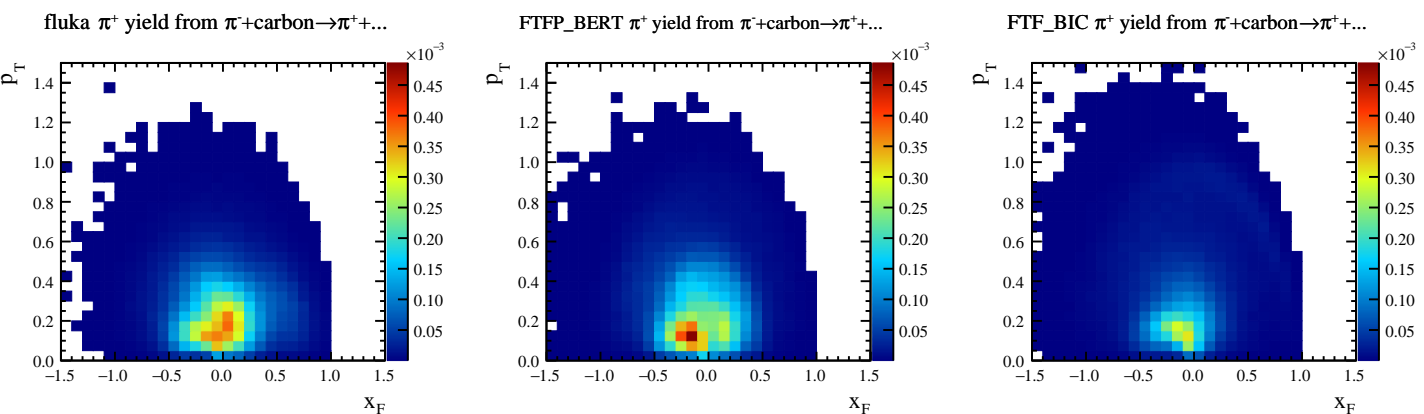

2D ratio of FTFP_BERT/FTF_BIC $\pi^{+}$yields

2D ratio of FTFP_BERT/fluka $\pi^{+}$yields

2D ratio of FTF_BIC/fluka $\pi^{+}$yields
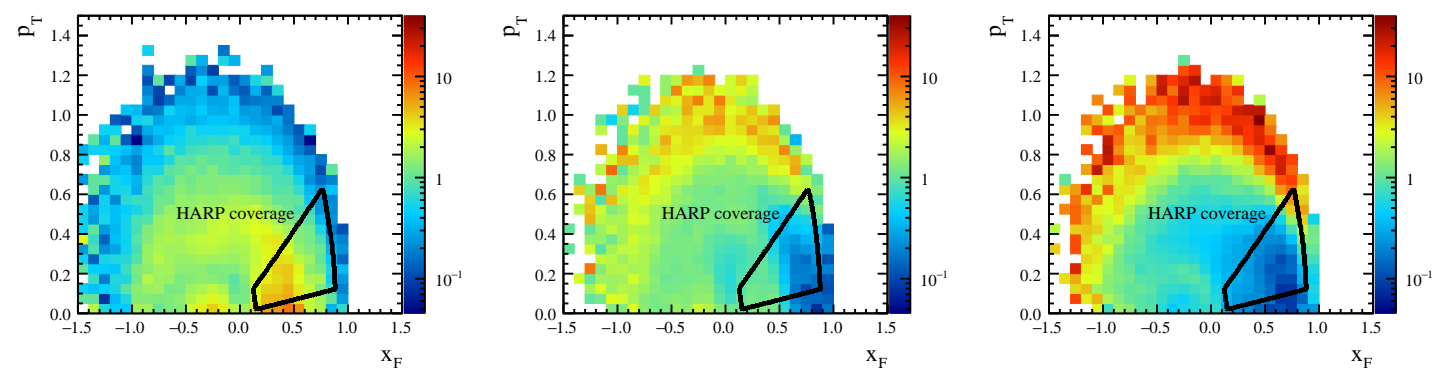

FTFP_BERT/FTF_BIC ratio distribution

FTFP_BERT/fluka ratio distribution
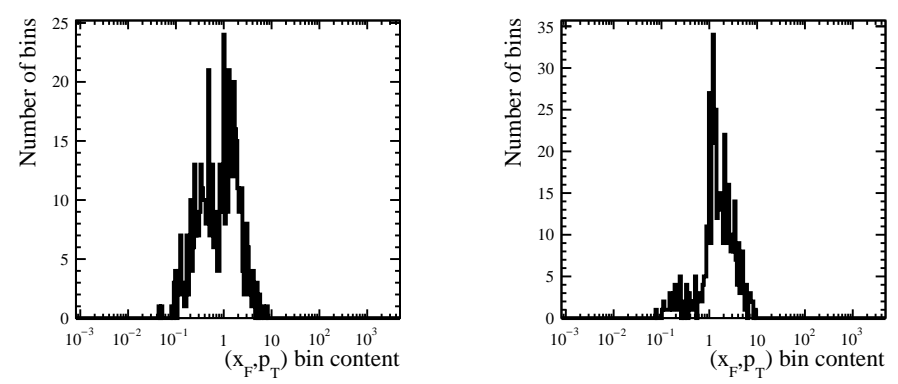

FTF_BIC/fluka ratio distribution

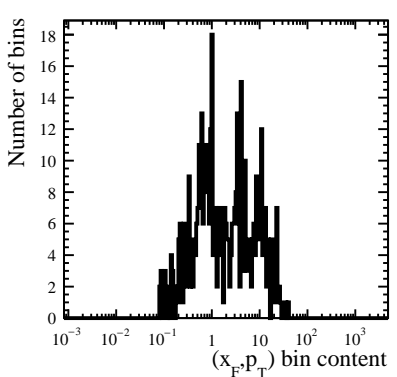

Figure 6.37: Study of differences in exiting $\pi^{+}$yields predicted by different Monte Carlo models for interactions of $3 \mathrm{GeV}$ negative pions on graphite $\pi^{-}+C \rightarrow \pi^{+}+X$. Plotted are the exiting pion yields in the $\left(x_{F}, p_{T}\right)$ phase space, predicted by FLUKA (top left), FTFP_BERT (top middle) and FTF_BIC (top right). Ratios of pion yields predicted by different models are shown: FTFP_BERT compared to FTF_BIC (middle left), FTFP_BERT compared to FLUKA (middle middle) and FTF_BIC compared to FLUKA (middle right). Finally, the frequency of different values for the yield ratio is examined in the bottom row of figures. 

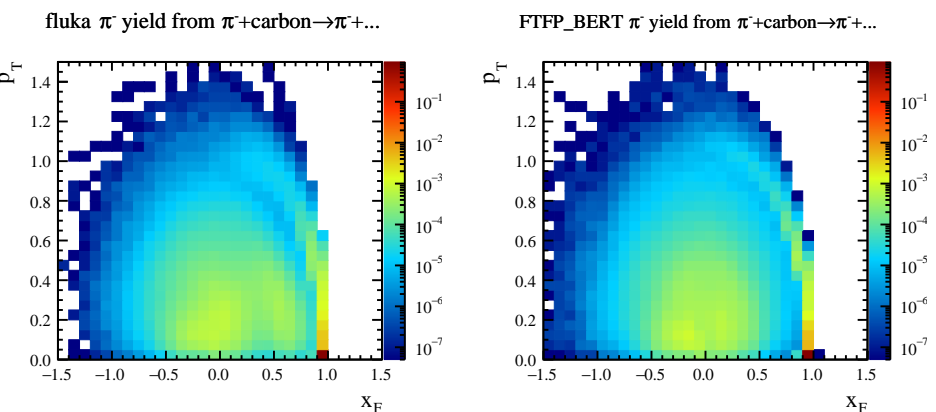

FTF_BIC $\pi$ yield from $\pi^{-}+$carbon $\rightarrow \pi^{-}+\ldots$

2D ratio of FTFP_BERT/FTF_BIC $\pi$ yields

2D ratio of FTFP_BERT/fluka $\pi$ yields
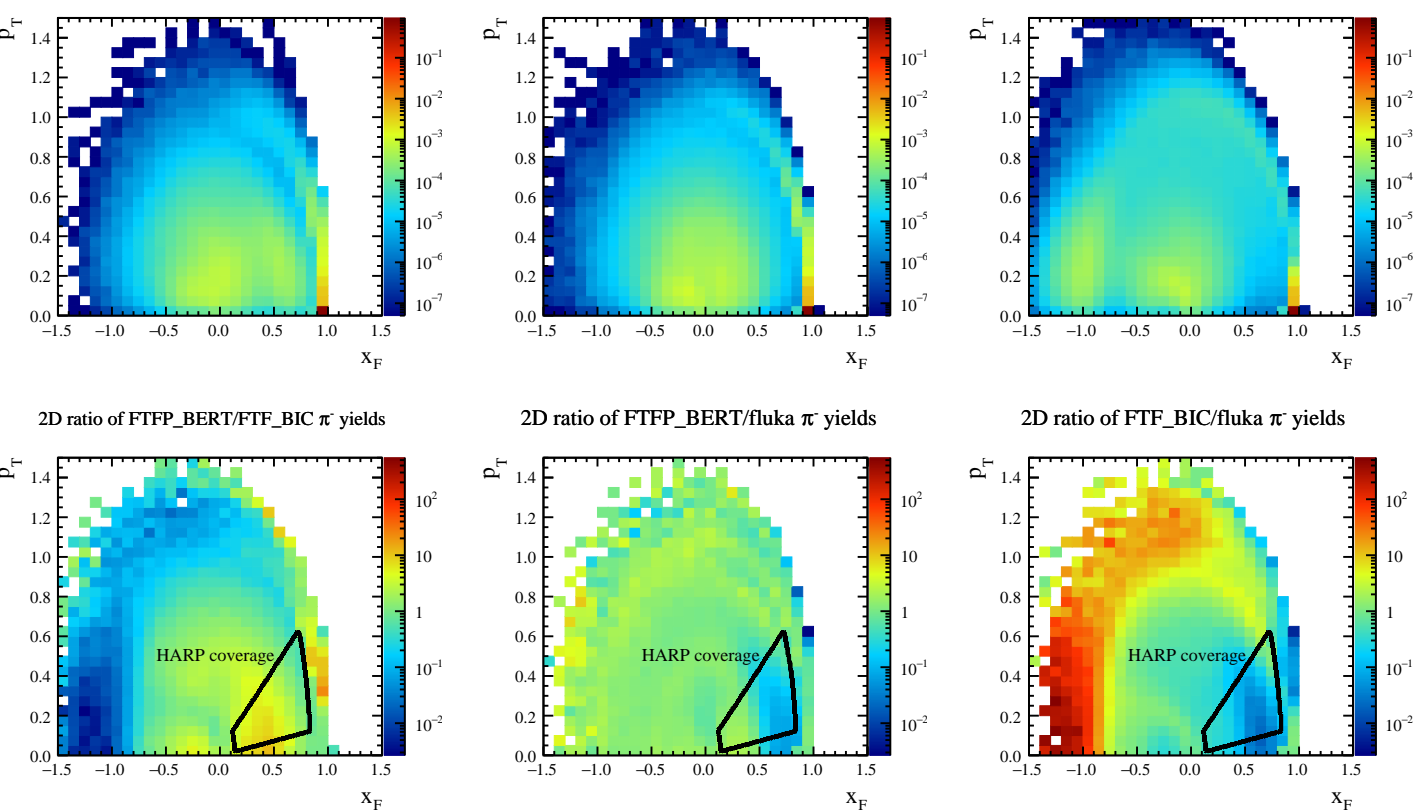

2D ratio of FTF_BIC/fluka $\pi$ yields

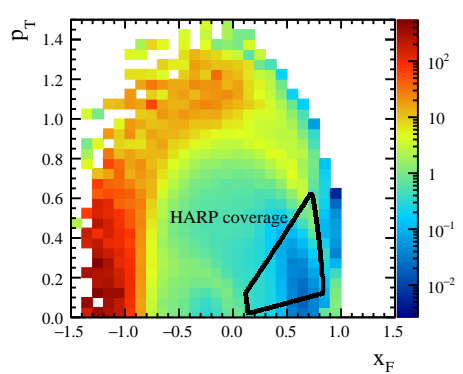

FTFP_BERT/FTF_BIC ratio distribution

FTFP_BERT/fluka ratio distribution
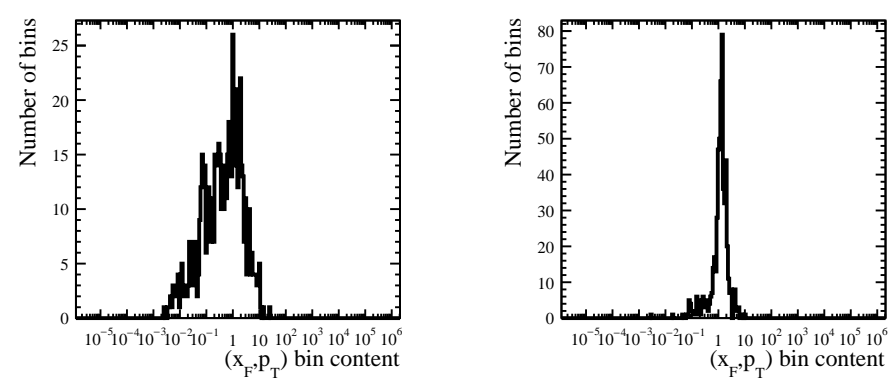

FTF BIC/fluka ratio distribution

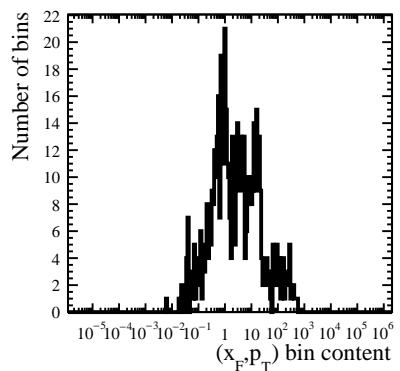

Figure 6.38: Study of differences in exiting $\pi^{-}$yields predicted by different Monte Carlo models for interactions of $3 \mathrm{GeV}$ negative pions on graphite $\pi^{-}+C \rightarrow \pi^{-}+X$. Plotted are the exiting pion yields in the $\left(x_{F}, p_{T}\right)$ phase space, predicted by FLUKA (top left), FTFP_BERT (top middle) and FTF_BIC (top right). Ratios of pion yields predicted by different models are shown: FTFP_BERT compared to FTF_BIC (middle left), FTFP_BERT compared to FLUKA (middle middle) and FTF_BIC compared to FLUKA (middle right). Finally, the frequency of different values for the yield ratio is examined in the bottom row of figures. 

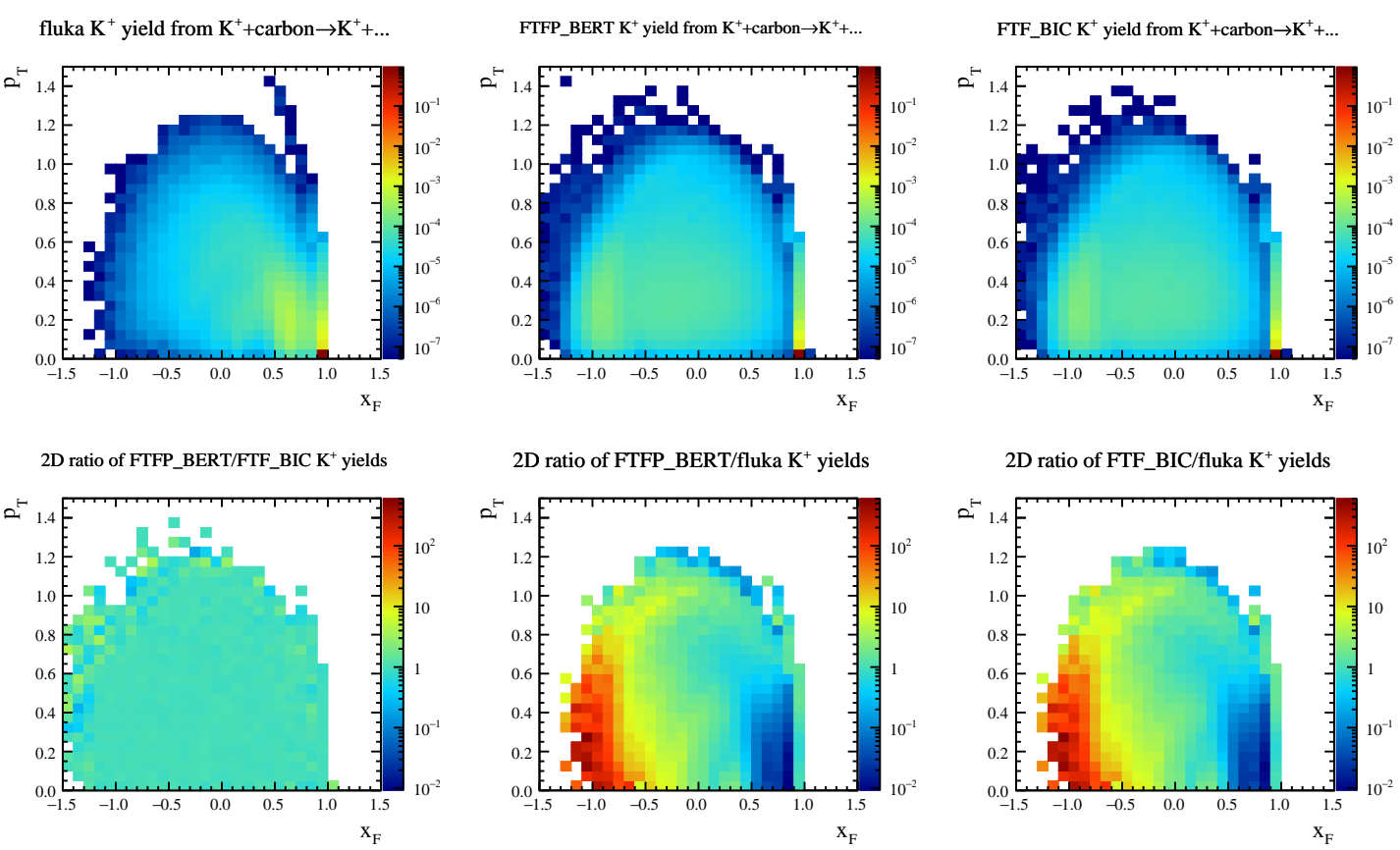

2D ratio of FTF_BIC/fluka $\mathrm{K}^{+}$yields

FTFP_BERT/FTF_BIC ratio distribution

FTFP_BERT/fluka ratio distribution
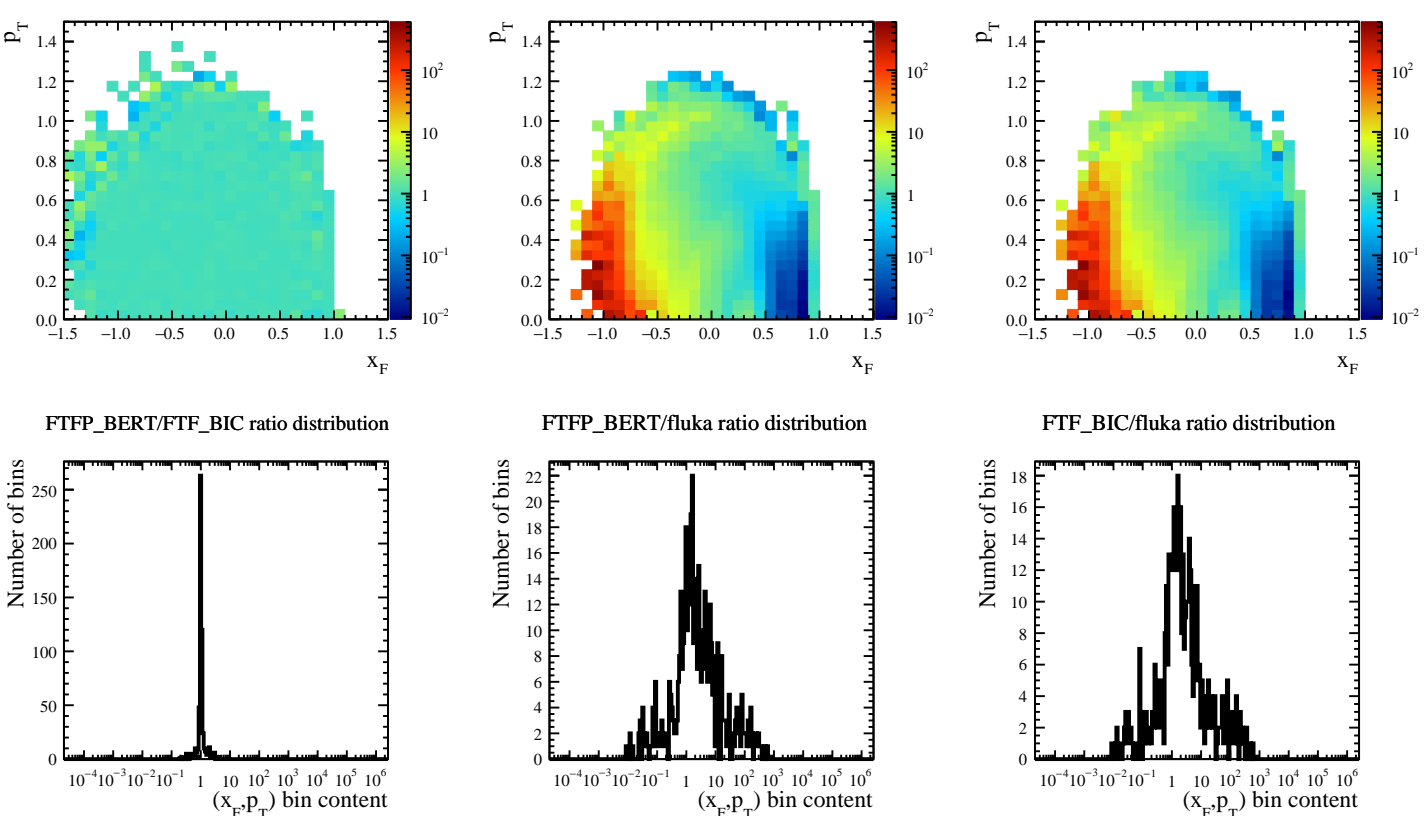

FTF_BIC/fluka ratio distribution

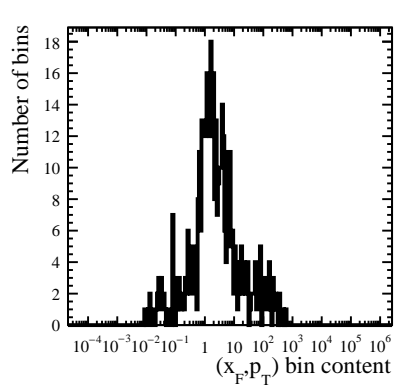

Figure 6.39: Study of differences in exiting $K^{+}$yields predicted by different Monte Carlo models for interactions of $3 \mathrm{GeV}$ positive kaons on graphite $K^{+}+C \rightarrow K^{+}+X$. Plotted are the exiting kaon yields in the $\left(x_{F}, p_{T}\right)$ phase space, predicted by FLUKA (top left), FTFP_BERT (top middle) and FTF_BIC (top right). Ratios of pion yields predicted by different models are shown: FTFP_BERT compared to FTF_BIC (middle left), FTFP_BERT compared to FLUKA (middle middle) and FTF_BIC compared to FLUKA (middle right). Finally, the frequency of different values for the yield ratio is examined in the bottom row of figures. 

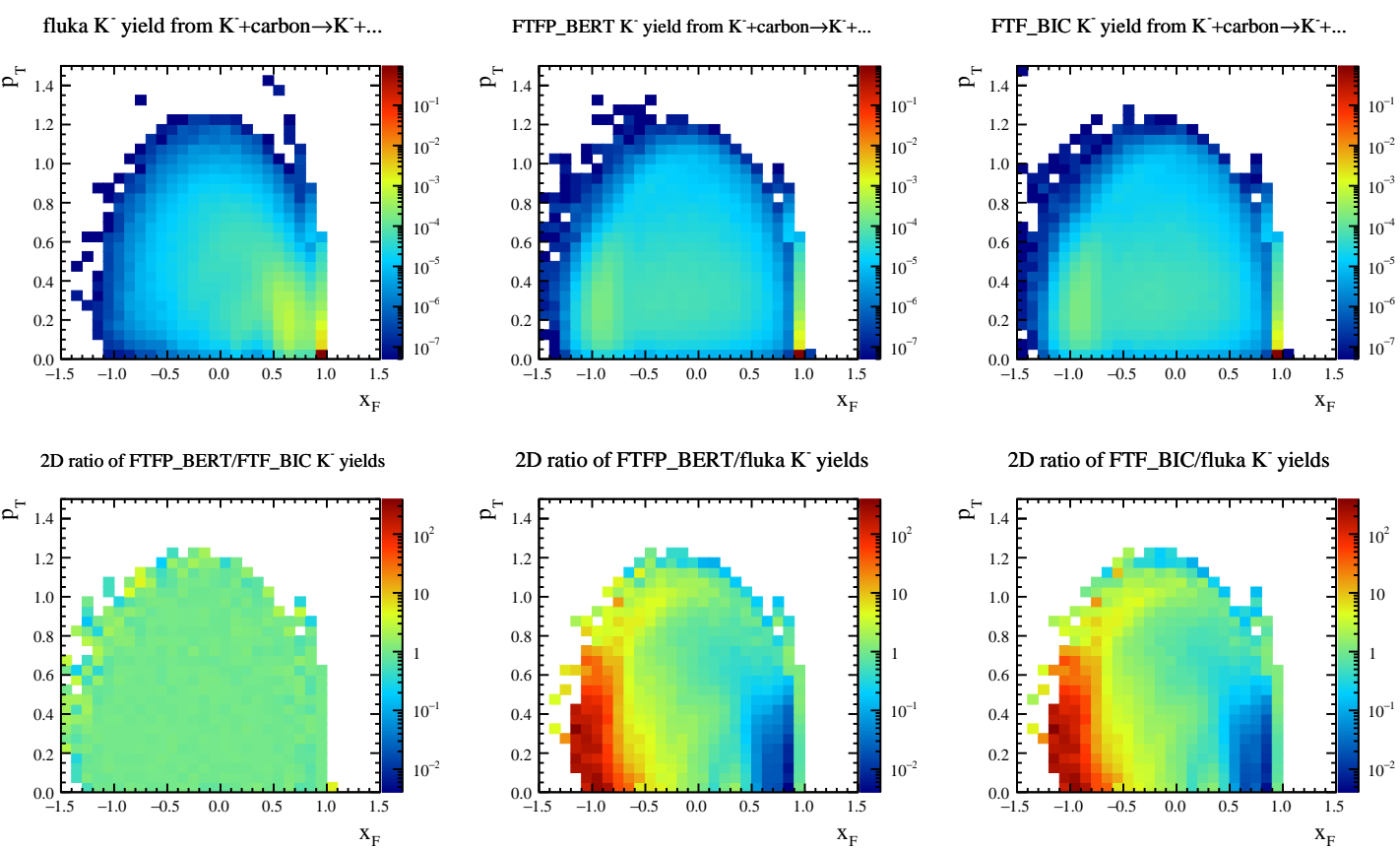

2D ratio of FTFP_BERT/fluka $\mathrm{K}^{-}$yields

2D ratio of FTF_BIC/fluka $\mathrm{K}^{-}$yields
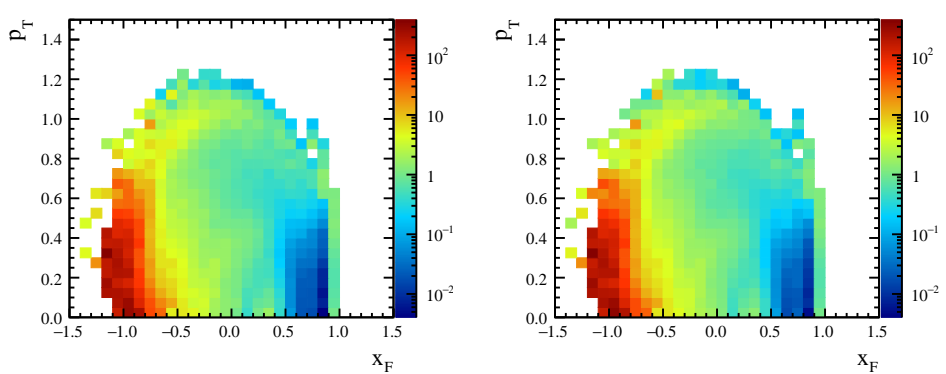

FTFP_BERT/FTF_BIC ratio distribution

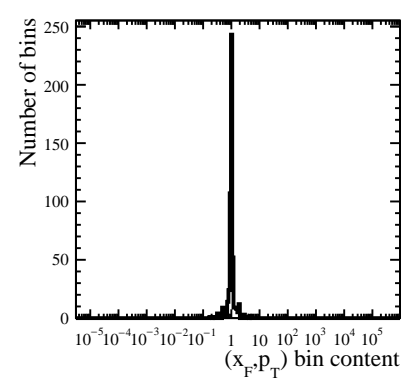

FTFP_BERT/fluka ratio distribution

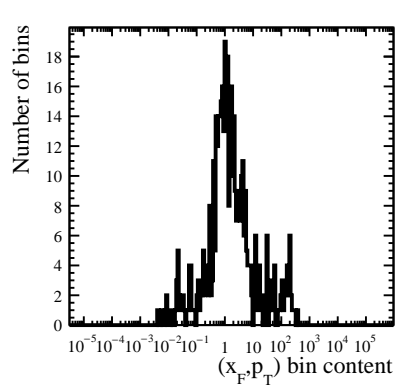

FTF_BIC/fluka ratio distribution

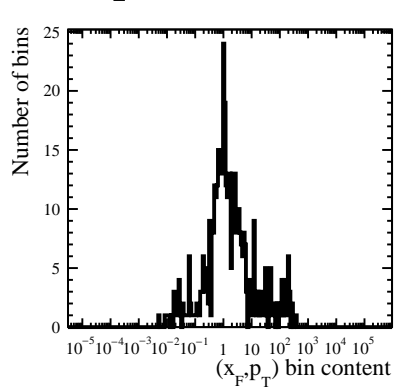

Figure 6.40: Study of differences in exiting $K^{-}$yields predicted by different Monte Carlo models for interactions of $3 \mathrm{GeV}$ negative kaons on graphite $K^{-}+C \rightarrow K^{-}+X$. Plotted are the exiting kaon yields in the $\left(x_{F}, p_{T}\right)$ phase space, predicted by FLUKA (top left), FTFP_BERT (top middle) and FTF_BIC (top right). Ratios of pion yields predicted by different models are shown: FTFP_BERT compared to FTF_BIC (middle left), FTFP_BERT compared to FLUKA (middle middle) and FTF_BIC compared to FLUKA (middle right). Finally, the frequency of different values for the yield ratio is examined in the bottom row of figures. 
SK: Positive Focusing Mode, $v_{\mu}$

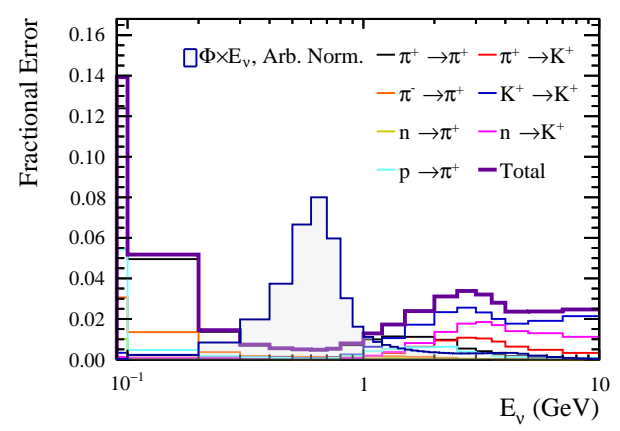

SK: Positive Focusing Mode, $v_{\mathrm{e}}$

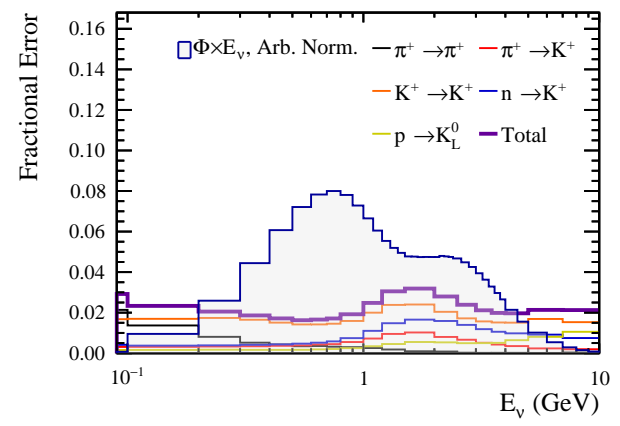

SK: Negative Focusing Mode, $v_{\mu}$

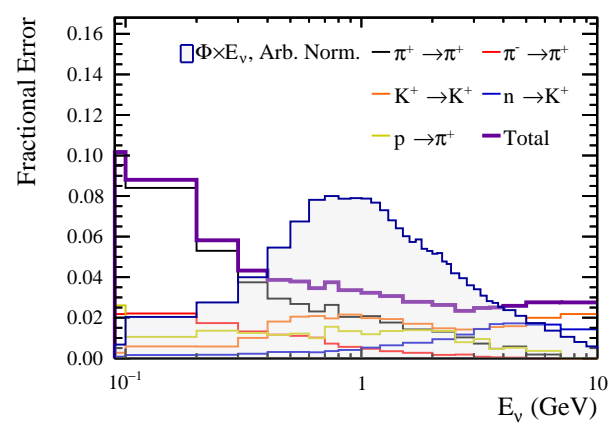

SK: Negative Focusing Mode, $v_{\mathrm{e}}$

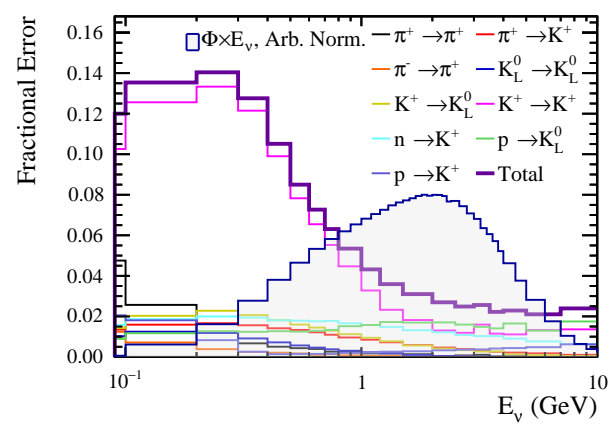

SK: Positive Focusing Mode, $\bar{\nabla}_{\mu}$

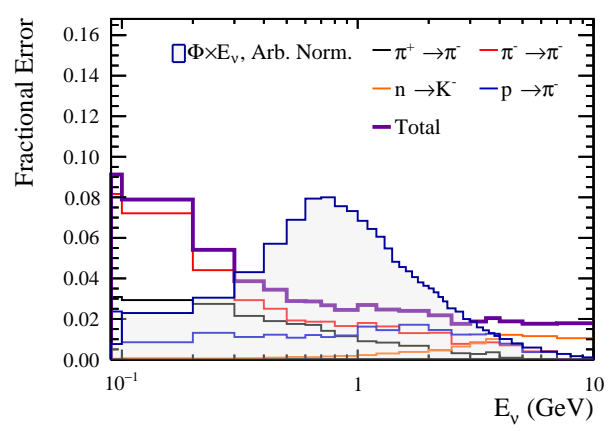

SK: Positive Focusing Mode, $\boldsymbol{\nabla}$

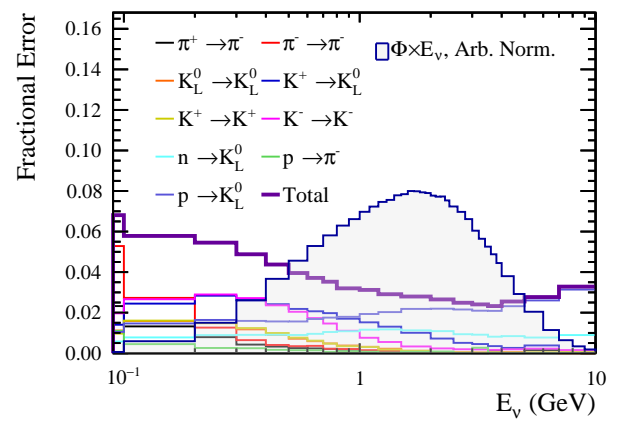

SK: Negative Focusing Mode, $\boldsymbol{\nabla}_{\mu}$

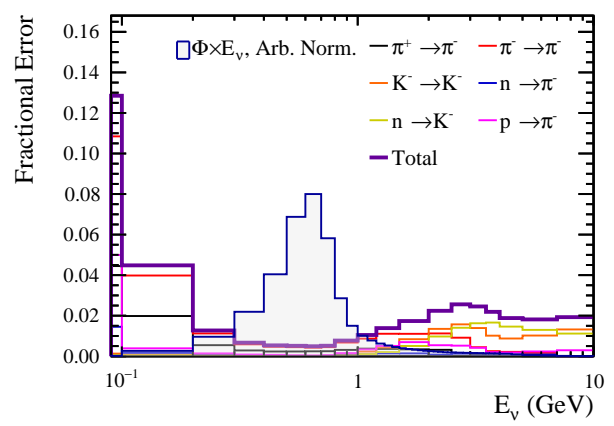

SK: Negative Focusing Mode, $\boldsymbol{\nabla}_{\mathrm{e}}$

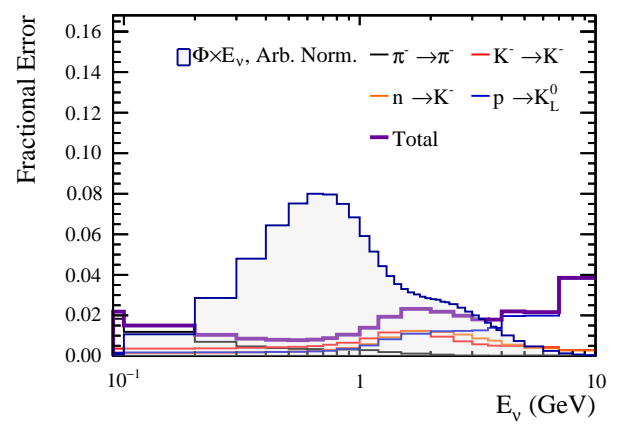

Figure 6.41: Contributions from unconstrained meson multiplicities to the flux uncertainty at the far detector in forward (top four plots) and reverse (bottom four plots) horn focusing mode. Only uncertainties exceeding $1 \%$ flux error in one of the energy bins have been plotted. Systematic flux uncertainties for neutrinos are shown on the left, and for anti-neutrinos on the right. 
ND280: Positive Focusing Mode, $v_{\mu}$

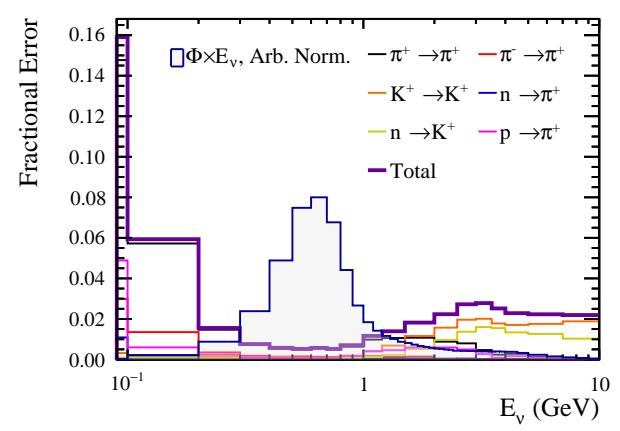

ND280: Positive Focusing Mode, $v_{\mathrm{e}}$

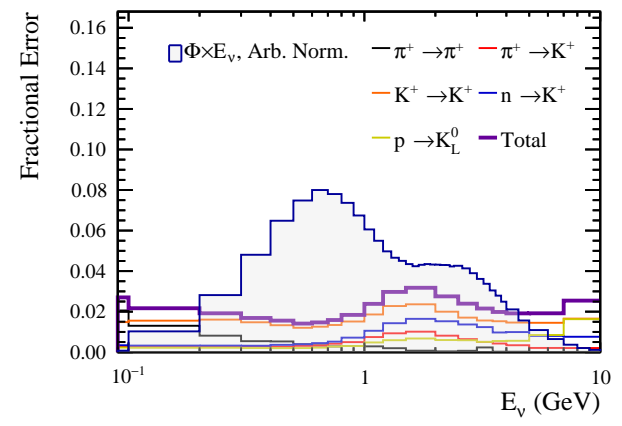

ND280: Negative Focusing Mode, $v_{\mu}$

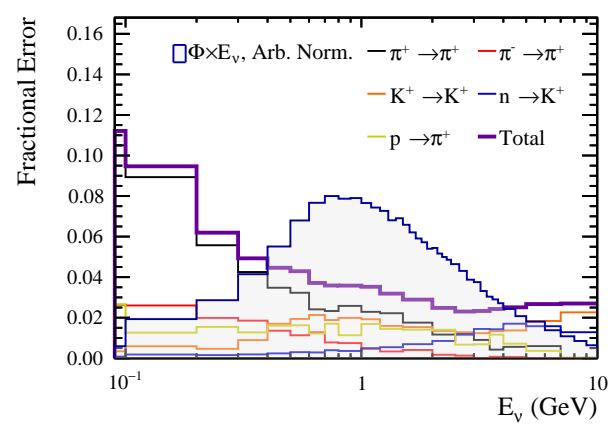

ND280: Negative Focusing Mode, $v_{\mathrm{e}}$

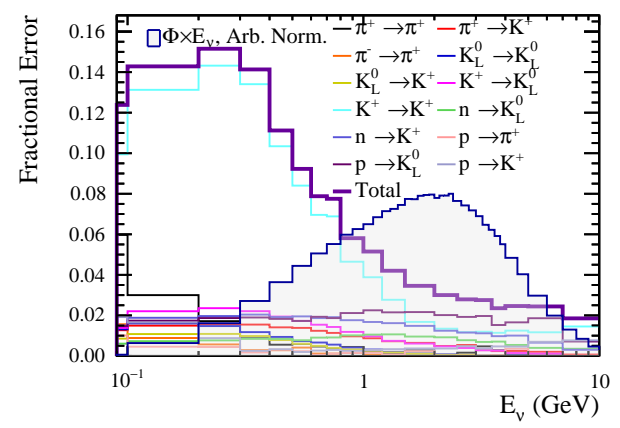

ND280: Positive Focusing Mode, $\bar{\nabla}_{\mu}$

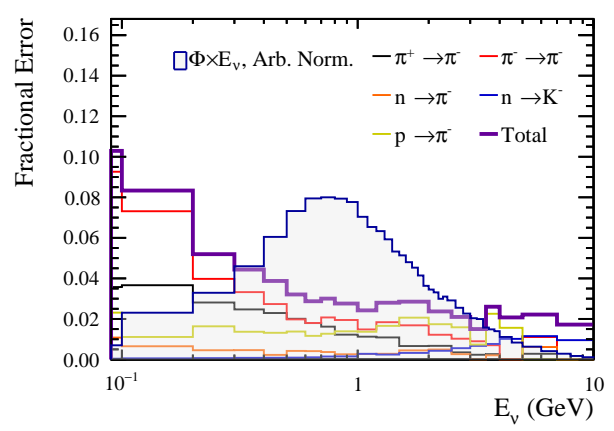

ND280: Positive Focusing Mode, $\boldsymbol{\nabla}_{\mathrm{e}}$

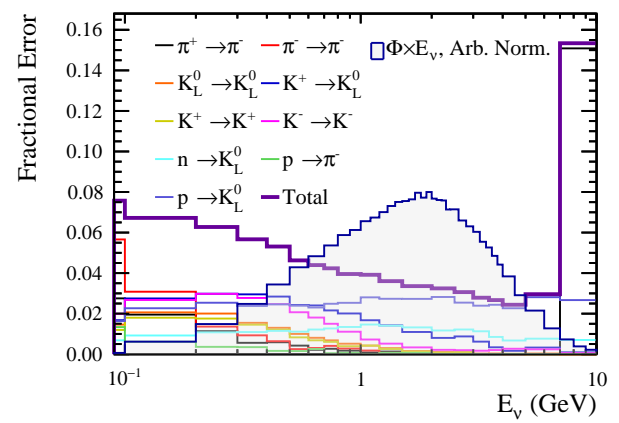

ND280: Negative Focusing Mode, $\boldsymbol{\nabla}_{\mu}$

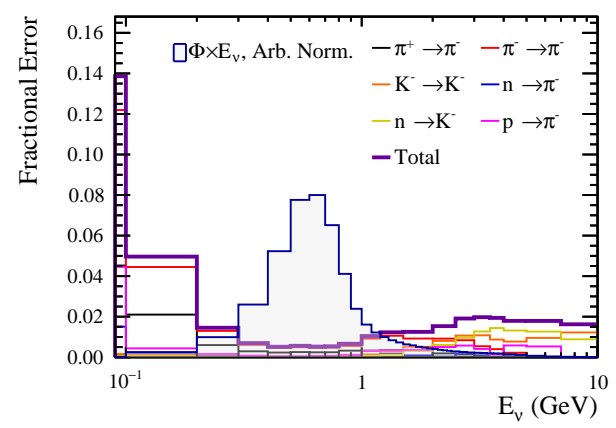

ND280: Negative Focusing Mode, $\boldsymbol{\nabla}_{\mathrm{e}}$

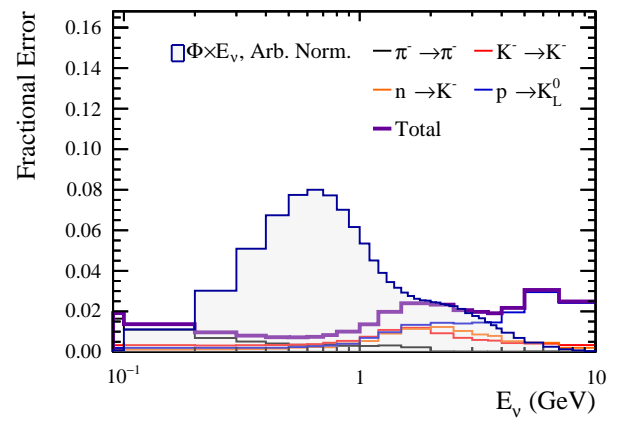

Figure 6.42: Contributions from unconstrained meson multiplicities to the flux uncertainty at ND280 in forward (top four plots) and reverse (bottom four plots) horn focusing mode. Only uncertainties exceeding $1 \%$ flux error in one of the energy bins have been plotted. Systematic flux uncertainties for neutrinos are shown on the left, and for anti-neutrinos on the right. 


\section{7}

\section{Bayesian statistics and the Markov Chain Monte Carlo technique for neutrino oscillation analyses}

The oscillation results presented in this thesis are to be interpreted in the Bayesian framework. This chapter starts with a brief discussion on Bayesian inference and Monte Carlo methods for sampling from multivariate posterior probability distributions. The probability distribution for oscillation parameters to take on certain values, given the collected $\mathrm{T} 2 \mathrm{~K}$ neutrino and anti-neutrino data, is also discussed. Finally, a summary of flux, cross section and detector inputs for the oscillation analysis has been presented.

\subsection{Bayesian Inference}

The outcome of an experiment can be summarised with the vector of collected data $\vec{d}$. For T2K, $\vec{d}$ would consist of numbers of neutrino events observed in each analysis bin. Naturally, the experiment outcome depends on a range of parameters $\vec{\theta}$. The goal of the experiment might be to estimate some of these parameters from the collected data. In the Bayesian framework, the joint probability for data and parameters is given by:

$$
P(\vec{d}, \vec{\theta})=P(\vec{d} \mid \vec{\theta}) \times P(\vec{\theta})
$$

where $P(\vec{d} \mid \vec{\theta})$ is the conditional probability of data given the parameter values, and $P(\vec{\theta})$ describes our prior knowledge of parameters. In Bayesian statistics, data and model parameters are on exactly the same footing, and the joint probability 
7. Bayesian statistics and the Markov Chain Monte Carlo technique for neutrino oscillation analyses

could just as well be written in the following form:

$$
P(\vec{d}, \vec{\theta})=P(\vec{\theta} \mid \vec{d}) \times P(\vec{d})
$$

Thus, the conditional probability for parameters to take up certain values, given the observed data, can be written as:

$$
P(\vec{\theta} \mid \vec{d})=\frac{P(\vec{d} \mid \vec{\theta}) \times P(\vec{\theta})}{P(\vec{d})}=\frac{P(\vec{d} \mid \vec{\theta}) \times P(\vec{\theta})}{\int P\left(\vec{d} \mid \overrightarrow{\theta^{\prime}}\right) \times P\left(\overrightarrow{\theta^{\prime}}\right) \mathrm{d} \overrightarrow{\theta^{\prime}}}
$$

Here, the probability $P(\vec{d})$ in the denominator is simply a normalization constant ${ }^{1}$, obtained by integrating the joint probability distribution $P(\vec{d}, \vec{\theta})$ over the parameter values. The value of experiment parameters $\vec{\theta}$ quoted as the experiment result is the value which maximises the posterior probability distribution $P(\vec{\theta} \mid \vec{d})$. For these considerations, the normalization constant can safely be neglected from the posterior likelihood expression:

$$
\mathcal{L}(\vec{\theta}, \vec{d})=P(\vec{\theta} \mid \vec{d}) \propto P(\vec{d} \mid \vec{\theta}) \times P(\vec{\theta})
$$

In T2K, the expected number of neutrino events is evaluated from the Monte Carlo. When evaluating $P(\vec{d} \mid \vec{\theta})$, the observed number of events, $\mathrm{N}^{\text {data }}(\vec{d})$, is assumed to be Poisson distributed around the expected event number $\mathrm{N}^{\mathrm{MC}}(\vec{\theta})$ :

$$
P(\vec{d} \mid \vec{\theta})=\prod_{\text {samples i }} \frac{\left(\mathrm{N}_{\mathrm{i}}^{\mathrm{MC}}\right)^{\mathrm{N}_{\mathrm{i}}^{\text {data }}} e^{-\mathrm{N}_{\mathrm{i}}^{\mathrm{MC}}}}{\left(\mathrm{N}_{\mathrm{i}}^{\text {data }}\right) !}
$$

The experiment parameters are usually taken to be Gaussian distributed around their nominal values:

$$
P(\vec{\theta}) \propto \prod_{\text {parameter groups } x} e^{-\frac{1}{2}\left(\overrightarrow{\theta_{x}}-\overrightarrow{\theta_{x}^{\text {nom }}}\right)^{\mathrm{T}} \mathbb{V}_{x}^{-1}\left(\overrightarrow{\theta_{x}}-\overrightarrow{\theta_{x}^{\text {nom }}}\right)}
$$

where the experiment parameters $\vec{\theta}$ are broken down in subgroups $\overrightarrow{\theta_{x}}$ of parameters with certain degrees of correlation, encoded by covariance matrices $\mathbb{V}_{x}$. For some parameters, there either isn't sufficient prior information to assign a Gaussian probability distribution, or the available information from different datasets might

\footnotetext{
${ }^{1}$ Strictly speaking, the probability for data is unity, since it has already happened.
} 
7. Bayesian statistics and the Markov Chain Monte Carlo technique for neutrino oscillation analyses

be conflicting. In such cases, uniform (flat) probability distributions are assigned across the parameter's range of validity.

The analysis presented in this thesis incorporates 579 ND280 parameters, 45 Super-K parameters, 100 flux parameters and 31 cross section parameters. In addition, the number of events observed at Super-K depends on 6 oscillation parameters. This gives a total of 761 parameters in the posterior likelihood. Naturally, when reporting the T2K result in publications, only the oscillation parameter values $\vec{o}$ are reported. The distinction is made between these parameters of interest, and the remaining nuisance parameters $\vec{\nu}$. The marginal likelihood is constructed from the total posterior likelihood in equation 7.4 , by integrating over the nuisance parameters:

$$
\mathcal{L}_{\text {marg }}(\vec{o}, \vec{d})=\int \mathcal{L}(\vec{o}, \vec{\nu}, \vec{d}) \mathrm{d} \vec{\nu}
$$

In the case of $\mathrm{T} 2 \mathrm{~K}$, the posterior likelihood $\mathcal{L}(\vec{o}, \vec{\nu}, \vec{d})$ has to be integrated over several hundreds of nuisance parameters. The resulting integral has no closed analytical form, and Markov Chain Monte Carlo techniques are used for its evaluation.

When presenting oscillation results, it is often convenient to depict regions $\zeta$ in oscillation parameter space $\vec{o}$, across which the marginal likelihood integrates to fraction $n$ :

$$
\int_{\text {region } \zeta} \mathcal{L}_{\text {marg }}\left(\overrightarrow{o^{\prime}}, \vec{d}\right) \mathrm{d} \overrightarrow{o^{\prime}}=n
$$

For example, in cases where $n=0.9$, these are usually referred to as $90 \%$ credible intervals. The oscillation probability at Super-K really depends on the values of just four oscillation parameters: $\sin ^{2} \theta_{13}, \sin ^{2} \theta_{23}, \delta_{\mathrm{CP}}$ and $\Delta m_{32}^{2}$. The remaining two oscillation parameters, $\sin ^{2} \theta_{12}$ and $\Delta m_{21}^{2}$, are treated as nuisance parameters and integrated over. The values of oscillation parameters of interest, which maximise the four-dimensional marginal likelihood $\mathcal{L}_{\text {marg }}\left(\sin ^{2} \theta_{13}, \sin ^{2} \theta_{23}, \delta_{\mathrm{CP}}, \Delta m_{32}^{2}\right)$, are collectively referred to as the " $4 \mathrm{D}$ best fit" point. Often, the total posterior likelihood is marginalised across all but two oscillation parameters, for easy representation of credible intervals as contours in two dimensional parameter space. Credible 
7. Bayesian statistics and the Markov Chain Monte Carlo technique for neutrino oscillation analyses

intervals in T2K are usually quoted in $\sin ^{2} \theta_{13}-\delta_{\mathrm{CP}}, \sin ^{2} \theta_{23}-\delta_{\mathrm{CP}}$ and $\sin ^{2} \theta_{23}-\Delta m_{32}^{2}$ 2D parameter space. The values of oscillation parameters which maximise such two-dimensional marginal likelihoods are referred as "2D best fit" points. Often, subtle differences exist between the best fit points evaluated in two-dimensional and four-dimensional parameter space, which are labelled as marginalisation effects in the T2K jargon. For more details on these effects, the reader is referred to [131].

\subsection{Markov Chain Monte Carlo method}

Monte Carlo methods are powerful tools for sampling random variables from multivariate likelihood functions, or alternatively when numerically evaluating complex multivariate integrals with no closed form. A concise review of different Monte Carlo techniques commonly used in the particle physics community is given in [132]. The Markov Chain Monte Carlo (MCMC) method is one such technique, which samples a chain of consecutive parameter states $\overrightarrow{\theta_{0}}, \overrightarrow{\theta_{1}}, \overrightarrow{\theta_{2}}, \ldots, \overrightarrow{\theta_{n}}$. The defining property of the Markov chain is that the current state of the system, $\overrightarrow{\theta_{n}}$, depends purely on the previous state, $\overrightarrow{\theta_{n-1}}$, and not on the full history of the chain:

$$
P\left(\overrightarrow{\theta_{n}} \mid \overrightarrow{\theta_{0}}, \overrightarrow{\theta_{1}}, \ldots, \overrightarrow{\theta_{n-1}}\right)=P\left(\overrightarrow{\theta_{n}} \mid \overrightarrow{\theta_{n-1}}\right)
$$

In other words, the history of the system affects the current state $\overrightarrow{\theta_{n}}$ only through its adjacent past state $\overrightarrow{\theta_{n-1}}$. Certain Markov chains eventually reach a stage where the conditional probability $P\left(\overrightarrow{\theta_{n}} \mid \overrightarrow{\theta_{0}}\right)$, for the system to be in state $\overrightarrow{\theta_{n}}$, given the initial system state $\overrightarrow{\theta_{0}}$, converges to a stationary distribution independent of the state number $n$ or the starting point $\overrightarrow{\theta_{0}}$ :

$$
P\left(\overrightarrow{\theta_{n}} \mid \overrightarrow{\theta_{0}}\right) \rightarrow f(\vec{\theta})
$$

For the T2K experiment, it is desirable for this stationary distribution function of parameters $\vec{\theta}$ to be the posterior likelihood of experimental parameters given the collected data: $f(\vec{\theta}) \equiv P(\vec{\theta} \mid \vec{d})$.

However, not all Markov chains have a stationary distribution, and even the ones that do sometimes reach it after exceedingly large times, thus making the 
7. Bayesian statistics and the Markov Chain Monte Carlo technique for neutrino oscillation analyses

oscillation analysis computationally prohibitive. The T2K experiment uses the Metropolis Hastings MCMC algorithm[133, 134] to ensure the existence of a stationary distribution for its Markov chains. The following recipe is used:

1. Start with the initial parameter state $\overrightarrow{\theta_{0}}$.

2. Generate the proposal state $\vec{\phi}$ from the current system state $\overrightarrow{\theta_{n}}$ by sampling from the proposal function $\varkappa\left(\vec{\phi} \mid \overrightarrow{\theta_{n}}\right)$. Here, the proposal function is symmetric in $\vec{\phi}$ and $\overrightarrow{\theta_{n}}$.

3. Determine whether the proposed step gets accepted by evaluating

$$
\alpha=\min \left[1, \frac{P(\vec{\phi} \mid \vec{d}) \varkappa\left(\overrightarrow{\theta_{n}} \mid \vec{\phi}\right)}{P\left(\overrightarrow{\theta_{n}} \mid \vec{d}\right) \varkappa\left(\vec{\phi} \mid \overrightarrow{\theta_{n}}\right)}\right],
$$

where the function $P$ is the desired stationary distribution of the chain. A random value $k$ is sampled with uniform probability from the $[0,1]$ interval. The proposed step gets accepted if $\alpha \geq k$. Otherwise, the proposed step is rejected. If the proposal is successful, the chain grows in size, and the current step $\overrightarrow{\theta_{n+1}}$ is set to the proposed step: $\overrightarrow{\theta_{n+1}}=\vec{\phi}$. In this way, steps for which the posterior probability is increased are always accepted, and steps where the posterior probability gets reduced are accepted with probability $\frac{P(\vec{\phi} \mid \vec{d})}{P\left(\overrightarrow{\theta_{n}} \mid \vec{d}\right)}<1$.

4. An attempt is made to build the chain further by going back to step 2 .

Depending on the choice of the initial parameter state $\overrightarrow{\theta_{0}}$, and the proposal function $\varkappa\left(\vec{\theta} \mid \overrightarrow{\theta_{0}}\right)$, it might take different amounts of time for the constructed chain to converge on its stationary distribution $P(\vec{\theta} \mid \vec{d})$. The choices of the starting parameter and proposal function have been optimised specifically for the purposes of the T2K oscillation analysis, and have been merely reused for the analysis presented in this thesis. The number of steps that has to be discarded at the start of the Markov chain, because the sampled parameter density does not yet converge on its stationary distribution, is denoted as the burn-in value. For fits to ND280 data presented in this thesis, a burn-in of 200,000 steps has been applied at the start of each Markov chain. For joint fits to ND280 and Super-K data, a conservative burn-in of 250,000 steps was applied. 
7. Bayesian statistics and the Markov Chain Monte Carlo technique for neutrino oscillation analyses

\subsection{Posterior likelihood at T2K}

The number of neutrino-induced events measured at ND280 depends on the complex interplay between hundreds of near detector $(\vec{n})$, flux $(\vec{f})$ and cross section $(\vec{x})$ parameters. Instead of dealing with the posterior likelihood $\mathcal{L}$, introduced in Equation 7.4, the natural logarithm of the likelihood, $\ln (\mathcal{L})$, is taken instead. This substantially simplifies the expression for the probability distribution, without changing the nature of the problem. The expressions $\ln (\mathcal{L})$ and $\mathcal{L}$ are maximised by the same set of parameter values $\vec{\theta}=(\vec{n}, \vec{f}, \vec{x})$. Thus, the posterior log-likelihood, $\ln \left(\mathcal{L}_{\text {posterior }}^{\text {ND280 }}\right)$, for these parameters $\vec{\theta}$ to take on certain values, given the collected ND280 data, is given by the following expression:

$$
\begin{aligned}
& -\ln \left(\mathcal{L}_{\text {posterior }}^{\mathrm{ND} 280}\right)= \\
& =\sum_{\mathrm{i}}^{\mathrm{ND} 280 \text { samples }} \mathrm{N}_{\mathrm{i}}^{\mathrm{ND}_{\mathrm{MC}}}-\mathrm{N}_{\mathrm{i}}^{\mathrm{ND}_{\text {data }}}+\mathrm{N}_{\mathrm{i}}^{\mathrm{ND}_{\text {data }}} \times \ln \left[\mathrm{N}_{\mathrm{i}}^{\mathrm{ND}_{\text {data }}} / \mathrm{N}_{\mathrm{i}}^{\mathrm{ND}_{\mathrm{MC}}}\right] \\
& +\frac{1}{2}\left(\vec{n}-\vec{n}_{\text {nom }}\right)^{\mathrm{T}} \mathbb{V}_{\vec{n}}^{-1}\left(\vec{n}-\vec{n}_{\text {nom }}\right) \\
& +\frac{1}{2}\left(\vec{f}-\vec{f}_{\text {nom }}\right)^{\mathrm{T}} \mathbb{V}_{\vec{f}}^{-1}\left(\vec{f}-\vec{f}_{\text {nom }}\right) \\
& +\frac{1}{2}\left(\vec{x}-\vec{x}_{\text {nom }}\right)^{\mathrm{T}} \mathbb{V}_{\vec{x}}^{-1}\left(\vec{x}-\vec{x}_{\text {nom }}\right)
\end{aligned}
$$

where $\mathrm{N}^{\mathrm{ND}_{\text {data }}}$ and $\mathrm{N}^{\mathrm{ND}_{\mathrm{MC}}}$ refer to the collected and predicted number of events at ND280, obtained from data and Monte Carlo (MC), respectively. In the above expression, $\mathbb{V}_{\vec{n}}^{-1}, \mathbb{V}_{\vec{f}}^{-1}$ and $\mathbb{V}_{\vec{x}}^{-1}$ are the inverses of the covariance matrices for the corresponding parameters. Effectively, expressions such as $(\Delta \vec{n})^{\mathrm{T}} \mathbb{V}_{\vec{n}}^{-1} \Delta \vec{n}$ act as penalty terms in the posterior probability distribution, in this case making it increasingly unlikely for ND280 parameters $\vec{n}$ to be displaced far from their nominal values $\vec{n}_{\text {nom }}$. Similar penalty terms have been included for the flux and cross section parameters. No prior correlations are assumed between these different classes of systematic parameters.

The posterior likelihood for fits to ND280 data can be adequately evaluated using equation 7.12. At the T2K beam energy, the chances for neutrinos to have oscillated 280 meters from their production point is vanishingly small. Thus, the predicted number of events at ND280 depends purely on the detector, flux and 
7. Bayesian statistics and the Markov Chain Monte Carlo technique for neutrino oscillation analyses

cross section parameters, $\mathrm{N}^{\mathrm{ND} 280_{\mathrm{MC}}} \equiv \mathrm{N}^{\mathrm{ND} 280_{\mathrm{MC}}}(\vec{n}, \vec{f}, \vec{x})$. The predicted number of events at the far detector depends crucially on the oscillation parameters, in addition to all other systematic parameters, $\mathrm{N}^{\mathrm{SK}_{\mathrm{MC}}} \equiv \mathrm{N}^{\mathrm{SK}_{\mathrm{MC}}}(\vec{n}, \vec{s}, \vec{f}, \vec{x}, \vec{o})$. Here, $\vec{s}$ and $\vec{o}$ are compact notations for the Super-K detector parameters and the oscillation parameters. When performing joint fits to ND280 and Super-K data, the posterior log-likelihood takes on a more complicated expression:

$$
\begin{aligned}
& -\ln \left(\mathcal{L}_{\text {posterior }}^{\text {joint }}\right)= \\
& -\ln \left(\mathcal{L}_{\text {posterior }}^{\text {ND280 }}\right) \\
& +\sum_{\mathrm{j}}^{\mathrm{SK} \text { samples }} \mathrm{N}_{\mathrm{j}}^{\mathrm{SK}_{\mathrm{MC}}}-\mathrm{N}_{\mathrm{j}}^{\mathrm{SK}_{\text {data }}}+\mathrm{N}_{\mathrm{j}}^{\mathrm{SK}_{\text {data }}} \times \ln \left[\mathrm{N}_{\mathrm{j}}^{\left.\mathrm{SK}_{\text {data }} / \mathrm{N}_{\mathrm{j}} \mathrm{SK}_{\mathrm{MC}}\right]}\right. \\
& +\frac{1}{2}\left(\vec{s}-\vec{s}_{\text {nom }}\right)^{\mathrm{T}} \mathbb{V}_{\vec{s}}^{-1}\left(\vec{s}-\vec{s}_{\text {nom }}\right) \\
& +\frac{1}{2}\left(\vec{o}-\vec{o}_{\text {nom }}\right)^{\mathrm{T}} \mathbb{V}_{\vec{o}}^{-1}\left(\vec{o}-\vec{o}_{\text {nom }}\right)
\end{aligned}
$$

Detector, flux and cross section systematic parameters are mostly treated as Gaussian distributed around their nominal values. These inputs are produced by dedicated T2K working groups and distributed to analysers prior to each round of oscillation analysis. These inputs will be discussed in more detail in the following chapter.

The T2K experiment has limited sensitivity to oscillation parameters $\sin ^{2} \theta_{12}$ and $\Delta m_{21}^{2}$, which are thus modelled as Gaussian distributed, with standard deviations and mean values taken from the Particle Data Group[54]. The fits presented in this thesis are performed with an external constraint on $\sin ^{2} \theta_{13}$ from reactor experiments, and are thus referred to as fits with reactor constraint (wRC). The $\sin ^{2} \theta_{23}, \Delta m_{32}^{2}$ and $\delta_{\mathrm{CP}}$ parameters are to be measured from T2K data. In this case, flat priors are assumed, making it equally likely for these parameters of interest to take on any value from their physically allowed range. Oscillation parameters are assumed to be mutually uncorrelated.

The numbers of neutrino events in ND280 and Super-K samples are modelled as Poisson distributed. The underlying assumption of there being a fixed probability of observing an event within a certain time interval, independent of the time passed 
7. Bayesian statistics and the Markov Chain Monte Carlo technique for neutrino oscillation analyses

since the last event, is logically sound. The goal of T2K is to measure the $\sin ^{2} \theta_{23}$, $\Delta m_{32}^{2}, \sin ^{2} \theta_{13}$ and $\delta_{\mathrm{CP}}$ oscillation parameters. For the purposes of this analysis, all other parameters are referred to as nuisance parameters.

\subsection{Systematic parameter inputs}

\subsubsection{Flux parameter inputs}

The Beam Group provides a fractional flux covariance matrix, with a total of 100 flux parameters. I have been responsible for producing such covariance matrices for T2K oscillation analyses. All flux inputs for oscillation measurements in this thesis are my own work. Each parameter denotes the expected flux within a certain neutrino energy range. The flux is binned more finely around the T2K beam peak, as well as for the right-sign flux components, as shown in Figure 7.1. For example, in neutrino-mode (FHC), the binning is fine for $\nu_{\mu}$ and $\nu_{e}$, and relatively coarse for $\bar{\nu}_{\mu}$ and $\bar{\nu}_{e}$. The number of flux parameters was carefully optimised, so that sufficient information of flux shape is passed on for oscillation analysis, without contributing with too many nuisance parameters. 
7. Bayesian statistics and the Markov Chain Monte Carlo technique for neutrino oscillation analyses

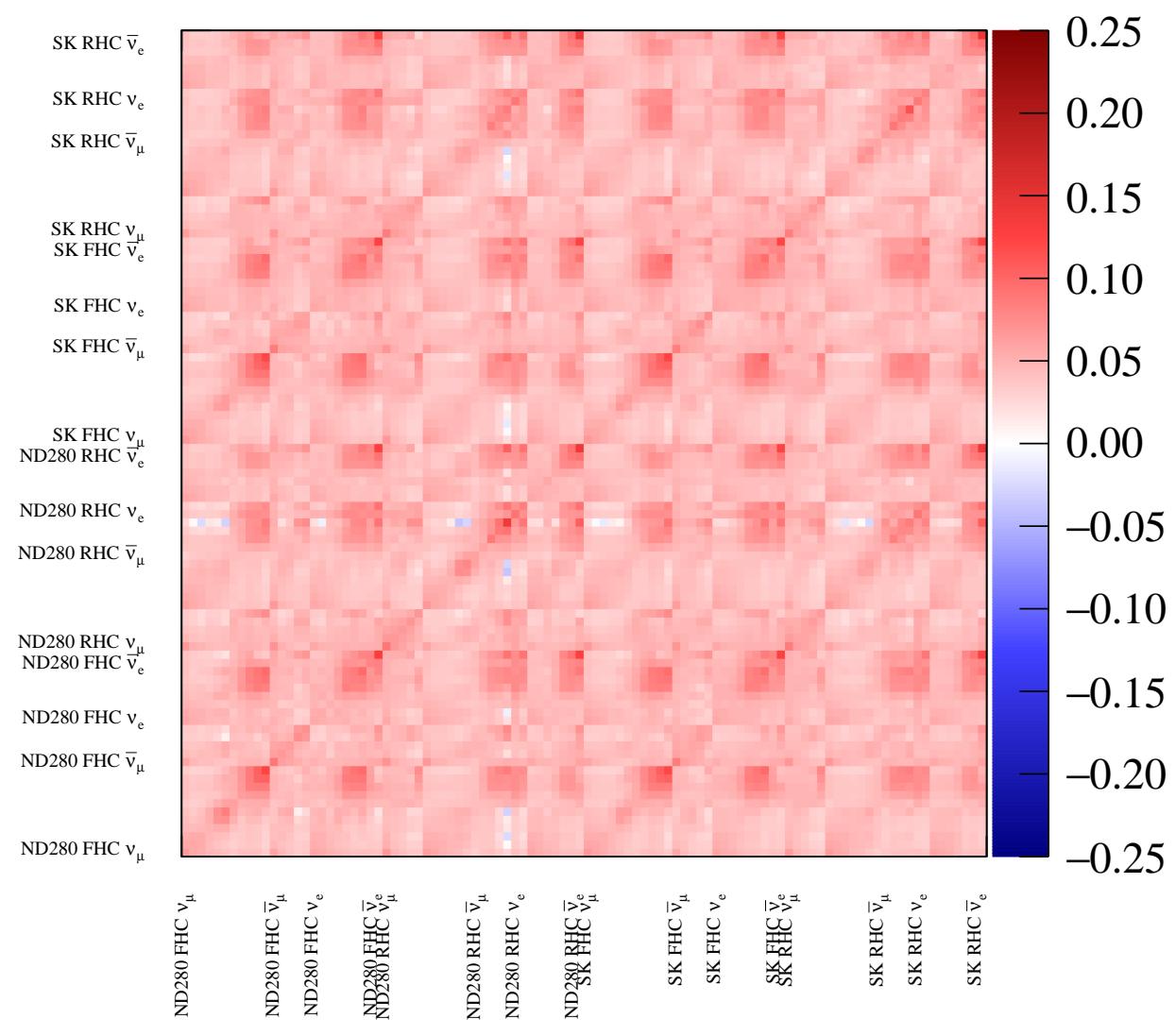

Figure 7.1: Fractional flux covariance matrix: $\operatorname{sign}\left(V_{i j}\right) \times \sqrt{\left|V_{i j}\right|}$. Here, replica-tuning has been used. 
7. Bayesian statistics and the Markov Chain Monte Carlo technique for neutrino oscillation analyses

\subsubsection{Cross section parameter inputs}

Interactions of T2K beam neutrinos at ND280 and Super-K are modelled with the NEUT event generator[135], which has been customized for the specific needs of T2K analyses. Neutrino interactions are an area of active research, with frequent updates to the leading theoretical models based on new measurements. Especially valuable in constraining nominal event predictions at $\mathrm{T} 2 \mathrm{~K}$ are neutrino cross section measurements from the MiniBooNE[136] and MINER $\nu \mathrm{A}[137]$ experiments. Target nuclei that neutrinos could be interacting with are provided by the components of the ND280 and Super-K detectors. The detector geometry is encoded in the GEANT4 framework.

Considerable thought has been put in devising parametrisations of neutrino interactions with a manageable number of free cross section parameters. The phase space of each one of these parameters is explored as the MCMC code samples from the T2K posterior distribution. Some of these parameters come with tight Gaussian shaped prior constraints inferred from existing measurements. Other cross section parameters come with limited or no prior information. For these parameters, a posterior constraint is imposed by $\mathrm{T} 2 \mathrm{~K}$ data. A distinction is made between two types of cross section parameters. Normalisation parameters affect all events from a certain class in the same way. For instance, one such parameter is the $2 \mathrm{p} 2 \mathrm{~h} \nu$ on ${ }^{12} \mathrm{C}$ normalisation. If the value of this parameter is shifted from the nominal 1.0 to 1.2 , every $2 \mathrm{p} 2 \mathrm{~h} \mathrm{CC} 0 \pi$ neutrino scattering event in the Monte Carlo prediction gets $20 \%$ more likely. In the other group are the so called shape parameters, such as the $\mathrm{M}_{\mathrm{A}}^{\mathrm{QE}}$ or BeRPA parameters. These are effective parametrisations for the modelled differential cross section as a function of event kinematics, for example the four momentum $Q^{2}=\left(P_{\nu}-P_{\mu}\right)^{2}$ transferred from the incident neutrino to hadronic products. These shape parameters are shifted from their nominal value $x \rightarrow x^{\prime}=x+N \sigma$, where $N \in\{ \pm 1, \pm 2, \ldots\}$, and the associated change in differential cross section $w\left(x^{\prime}\right)=\frac{\mathrm{d}^{n} \sigma\left(x^{\prime}\right)}{\mathrm{d} y^{n}} / \frac{\mathrm{d}^{n} \sigma(x)}{\mathrm{d} y^{n}}$ is calculated. For each event, a cubic polynomial (spline) is fitted to the $w\left(x^{\prime}\right)$ weights evaluated at several discrete $x^{\prime}$ points, and stored for future reference. Using these premade splines for shape parameters, in 
7. Bayesian statistics and the Markov Chain Monte Carlo technique for neutrino oscillation analyses

addition to normalisation parameters which are by construction trivial to apply, the Monte Carlo prediction can quickly be tuned for any shift in cross section parameters, by simply applying appropriate weights on an event by event basis. For instance, when proposing new steps in the Markov chain, parameters are shifted from their nominal value. When deciding whether to accept the proposed step, the Monte Carlo prediction with new parameter values needs to be recalculated. Instead of rerunning the whole Monte Carlo, simple multiplicative reweighting factors are applied. These reweighting factors are either the parameter itself for normalisation parameters, or the value from the stored spline for shape parameters.

Individual cross section parameters will now be reviewed in more detail. A summary of the nominal cross section parameter values and uncertainties recommended by the T2K Neutrino Interactions Working Group[138] is given in Table 7.1.

- $\mathrm{M}_{\mathrm{A}}^{\mathrm{QE}}$ (shape effect): the mass parameter in the axial dipole form factor describing CCQE interactions of neutrinos on single nucleons. The value of this parameter was constrained from fits to neutrino-deuterium scattering data in early bubble chamber experiments[139].

- $\mathrm{p}_{\mathrm{F}}^{\mathrm{C}}$ and $\mathrm{p}_{\mathrm{F}}^{\mathrm{O}}$ (shape effect): Momentum at the highest Fermi level occupied by nucleons in ${ }^{12} \mathrm{C}$ and ${ }^{16} \mathrm{O}$ nuclei, respectively, extracted from electron scattering data[140].

- $2 \mathrm{p} 2 \mathrm{~h}$ parameters (shape and normalisation effects): related to the short range correlations between pairs of nucleons, and implemented in the Nieves model[141]. Such correlations produce fluctuations in momentum of these nucleon pairs, above the expected Fermi momentum distribution. Neutrino scattering off from correlated nucleon pairs lead to two nucleons in the final state, which are then susceptible to further interactions in the nuclear medium. Separate $2 \mathrm{p} 2 \mathrm{~h}$ normalisation parameters have been introduced for interactions of $\nu$ and $\bar{\nu}$, as well as for nuclear scaling from ${ }^{12} \mathrm{C}$ to ${ }^{16} \mathrm{O}$. Shape effects have also been accounted through dedicated systematics for $\nu$ - and $\bar{\nu}$-induced events. 
7. Bayesian statistics and the Markov Chain Monte Carlo technique for neutrino oscillation analyses

- BeRPA parameters (shape effects): related to the treatment of collective nuclear effects in heavy targets. The non-perturbative Random Phase Approximation (RPA) is utilised, introduced in [141], and parametrised with third order Bernstein polynomials. Effectively, the presence of the nuclear medium modifies the neutrino interaction cross section, introducing a dependence on $Q^{2}$, the square of the four-momentum transfer to the hadronic system.

- $\mathrm{A}_{5}^{\mathrm{A}}, \mathrm{M}_{\mathrm{A}}^{\mathrm{RES}}$ and non-resonant $\mathrm{I}_{\frac{1}{2}}$ (shape effects): the axial normalisation, axial mass and isospin background parameters describing resonant scattering of neutrinos on nuclei, with $\pi^{ \pm}$production, implemented in the Rein-Sehgal $\operatorname{model}[142]$.

- $\nu_{e} / \nu_{\mu}$ and $\bar{\nu}_{e} / \bar{\nu}_{\mu}$ (shape effects): parameters describing any differences between $\mathrm{CC}$ neutrino interactions depending on their flavour.

- CC DIS (shape effect): a single parameter related to a class of topologically similar neutrino events. These include deep inelastic scattering, but also multi pion production and resonant scattering producing particles other than pions. The assigned systematic uncertainty is based on data from the MINOS experiment[143].

- $\mathrm{CC}$ and NC coherent parameters (normalisation effects): systematic parameters related to coherent neutrino-nucleus scattering, which results in single $\pi^{ \pm}$production without excitations of the target nucleus. Reweighting factors have been computed by comparing the Rein-Sehgal coherent model[142], used in T2K, with recent data from the MINERvA experiment[144]. These weights depend on the energy of the produced pions, and are applied to nominal NEUT predictions before constructing the posterior probability. Separate NC coherent parameters have been introduced, thus accounting for the non-zero lepton mass in the $\mathrm{CC}$ case.

- NC $1 \gamma$ and NC other (normalisation effects): The study in [145] showed that the nominal NEUT prediction underestimates the number of neutral 
7. Bayesian statistics and the Markov Chain Monte Carlo technique for neutrino oscillation analyses

current events accompanied by single photon production, when compared to predictions from the model in [146]. NEUT predicts $50 \%$ fewer such $\mathrm{NC} 1 \gamma$ events, which constitute and important background for $\nu_{e}$ appearance at Super-K. Consequently, the number of $\mathrm{NC} 1 \gamma$ events in the nominal NEUT prediction is doubled before attempting to construct the posterior probability distribution. A separate normalisation parameter for all other NC events is introduced.

- FSI parameters (shape effects): These parameters control interactions of hadrons before they leave the nuclear medium. At T2K neutrino energies, especially significant are final state interactions of pions, but nucleon, kaon and eta meson FSI are also implemented in NEUT. Dedicated cross section dials are introduced for pion absorption, production and quasi-elastic scattering at low and high energies. In addition, two dials control the pion charge exchange $^{2}$ at high and low energies, respectively. For a detailed description of FSI parametrisations in NEUT, the reader is referred to [147].

\footnotetext{
${ }^{2}$ processes where the charge of the pion changes through scattering
} 
7. Bayesian statistics and the Markov Chain Monte Carlo technique for neutrino oscillation analyses

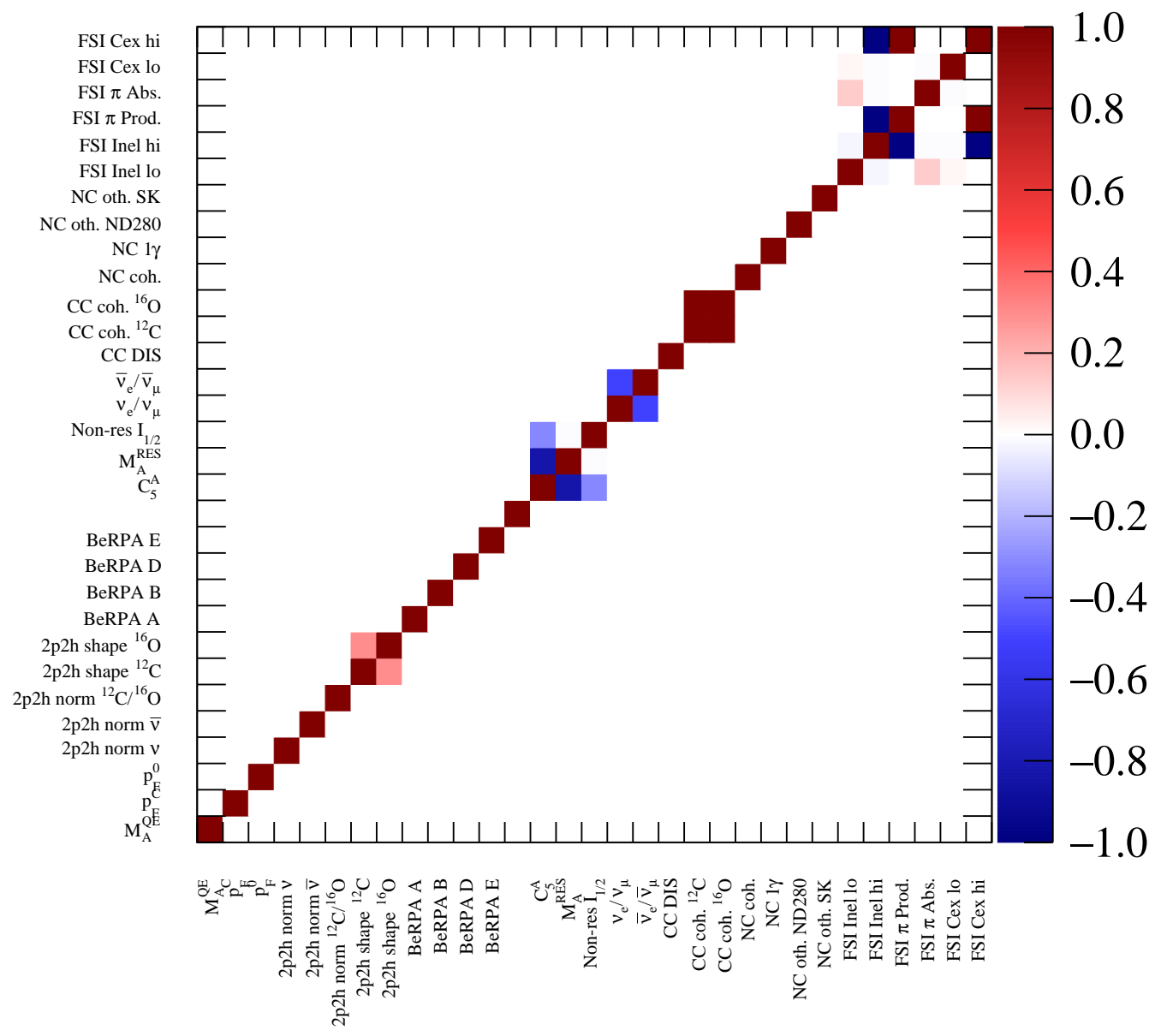

Figure 7.2: Correlations among cross section parameter inputs for oscillation analyses. Copyright by T2K Collaboration. 
7. Bayesian statistics and the Markov Chain Monte Carlo technique for neutrino oscillation analyses

\begin{tabular}{|c|c|c|c|c|}
\hline Parameter & Prior $\mu$ & Prior $\sigma$ & Prior shape & Type \\
\hline $\mathrm{M}_{\mathrm{A}}^{\mathrm{QE}}\left(\mathrm{GeV} / \mathrm{c}^{2}\right)$ & 1.21 & flat & flat & shape \\
\hline $\mathrm{p}_{\mathrm{F}}^{\mathrm{C}}(\mathrm{GeV} / \mathrm{c})$ & 217 & flat & flat & shape \\
\hline $\mathrm{p}_{\mathrm{F}}^{\mathrm{O}}(\mathrm{GeV} / \mathrm{c})$ & 225 & flat & flat & shape \\
\hline $2 \mathrm{p} 2 \mathrm{~h} \nu+{ }^{12} \mathrm{C}$ norm. & 1.0 & flat & flat & norm. \\
\hline $2 \mathrm{p} 2 \mathrm{~h} \bar{\nu}+{ }^{12} \mathrm{C}$ norm. & 1.0 & flat & flat & norm. \\
\hline $2 \mathrm{p} 2 \mathrm{~h}{ }^{16} \mathrm{O} /{ }^{12} \mathrm{C}$ & 1.0 & flat & flat & norm. \\
\hline $2 \mathrm{p} 2 \mathrm{~h} \nu+{ }^{12} \mathrm{C}$ shape & 0.0 & flat & flat & shape \\
\hline $2 \mathrm{p} 2 \mathrm{~h} \bar{\nu}+{ }^{12} \mathrm{C}$ shape & 0.0 & flat & flat & shape \\
\hline BeRPA A & 0.59 & 0.118 & Gauss & shape \\
\hline BeRPA B & 1.05 & 0.21 & Gauss & shape \\
\hline BeRPA D & 1.13 & 0.17 & Gauss & shape \\
\hline BeRPA E & 0.88 & 0.352 & Gauss & shape \\
\hline BeRPA U & 1.20 & - & - & - \\
\hline $\mathrm{M}_{\mathrm{A}}^{\mathrm{RES}}\left(\mathrm{GeV} / \mathrm{c}^{2}\right)$ & $1.07(0.95)$ & flat & flat & shape \\
\hline $\mathrm{C}_{5}^{\mathrm{A}}$ & $0.96(1.01)$ & 0.15 & Gauss & shape \\
\hline Non-res. $I_{\frac{1}{2}}$ & $0.96(1.30)$ & 0.4 & Gauss & shape \\
\hline $\mathrm{CC} \nu_{e} / \nu_{\mu}$ & 1.0 & $\sqrt{2} \times 0.02$ & Gauss & norm. \\
\hline $\mathrm{CC} \bar{\nu}_{e} / \bar{\nu}_{\mu}$ & 1.0 & $\sqrt{2} \times 0.02$ & Gauss & norm. \\
\hline CC DIS & 0.0 & 0.4 & Gauss & shape \\
\hline $\mathrm{CC}$ coh. ${ }^{12} \mathrm{C}$ & $\operatorname{weight}\left(\mathrm{E}_{\pi}\right)$ & 0.3 & Gauss & norm. \\
\hline $\mathrm{CC}$ coh. ${ }^{16} \mathrm{O}$ & weight $\left(\mathrm{E}_{\pi}\right)$ & 0.3 & Gauss & norm. \\
\hline NC coh. & 1.0 & 0.3 & Gauss & norm. \\
\hline $\mathrm{NC} 1 \gamma$ & $2.0(1.0)$ & 1.0 & Gauss & norm. \\
\hline NC oth. ND280 & 1.0 & 0.3 & Gauss & norm. \\
\hline NC oth. SK & 1.0 & 0.3 & Gauss & norm. \\
\hline FSI inel. low & 1.0 & 0.41 & Gauss & shape \\
\hline FSI inel. high & $1.8(1.0)$ & 0.34 & Gauss & shape \\
\hline FSI $\pi$ prod. & 1.0 & 0.5 & Gauss & shape \\
\hline FSI $\pi$ abs. & $1.1(1.0)$ & 0.41 & Gauss & shape \\
\hline FSI charge exch. high & 1.0 & 0.28 & Gauss & shape \\
\hline FSI charge exch. low & $1.8(1.0)$ & 0.57 & Gauss & shape \\
\hline
\end{tabular}

Table 7.1: Summary of cross section inputs for the oscillation analysis presented in this thesis. The parameter value $\mu$ and uncertainty $\sigma$ (for Gaussian priors) is given. The NEUT nominal value, if different from the parameter prior, is given in brackets. The parameter type is also specified: normalisation parameters affect all events from a certain event class in the same way, whereas shape parameters depend on specific event kinematics. Adapted from [138] 
7. Bayesian statistics and the Markov Chain Monte Carlo technique for neutrino oscillation analyses

\subsubsection{ND280 and Super-K parameter inputs}

These are nuisance parameters for the analysis presented in this thesis and thus won't be discussed in great detail. For more details on these, the reader should refer to [91].

Near detector parameters describe inefficiencies in selection (e.g. track matching efficiency between tracks in FGD and TPC), reconstruction (e.g. momentum resolution of the TPCs) and normalisation (e.g. uncertainty in FGD target mass) of the events selected at ND280. Events are binned by the detected muon's momentum $p_{\mu}$ and the cosine of the angle between the muon direction and the beam direction $\cos \theta_{\mu}$. ND280 parameters are applied as normalisations to the number of events in such $\left(p_{\mu}, \cos \theta_{\mu}\right)$ analysis bins of each sample. The fractional covariance matrix for ND280 parameters is shown in Figure 7.3, and the starting bin for every sample category has been labelled. For every momentum range, bin numbers iterate across all $\cos \theta_{\mu}$ bins. There is a total of 556 normalisation parameters at ND280.

Super-K parameters account for both detector effects (e.g. vertex resolution in the tank or random flasher events in the PMTs) and cross section modelling, such as interactions of hadrons from neutrino interactions both inside and outside of the nuclear medium. Photonuclear effects leading to photon absorption in Super-K have also been studied. For the estimation of some of these uncertainties, control samples have been selected, which are not part of the data samples used in the analysis. These could be for example cosmic muon events collected outside of T2K beam operation, or atmospheric neutrino events at Super-K. Data samples at Super-K are binned by the neutrino energy $\mathrm{E}_{\nu}$, and normalisation parameters control the number of events in every such analysis bin. The fractional covariance matrix for Super-K parameters is shown in Figure 7.4, and the starting bin for every sample category has been labelled. There is a total of 45 normalisation parameters at Super-K. Of course, these parameter inputs are still crucial during the construction of the Markov chain. Let us denote by $\overrightarrow{\theta^{\prime}}=\left(\overrightarrow{n^{\prime}}, \overrightarrow{s^{\prime}}, \overrightarrow{f^{\prime}}, \overrightarrow{x^{\prime}}\right)$ the proposed parameter values for a certain step in the Markov chain. The nominal Monte Carlo prediction 
7. Bayesian statistics and the Markov Chain Monte Carlo technique for neutrino oscillation analyses

is then reweighted on an event by event basis. Modelled events at ND280 and Super-K get assigned the following weights:

$$
\begin{aligned}
& w_{\mathrm{MC}}^{\mathrm{ND280}}=w_{\mathrm{POT}} \times w\left(n^{\prime}\right) \times w\left(f^{\prime}\right) \times w\left(x^{\prime}\right) \\
& w_{\mathrm{MC}}^{\mathrm{SK}}=w_{\mathrm{POT}} \times w\left(s^{\prime}\right) \times w\left(f^{\prime}\right) \times w\left(x^{\prime}\right)
\end{aligned}
$$

where $w_{\mathrm{POT}}$ is simply the reweighting factor for going from the simulated number of proton triggers to the total protons on target data exposure. Cross section parameters could be both normalisation and shape parameters. Flux and detector parameters are always applied as normalisations to the number of neutrino events.

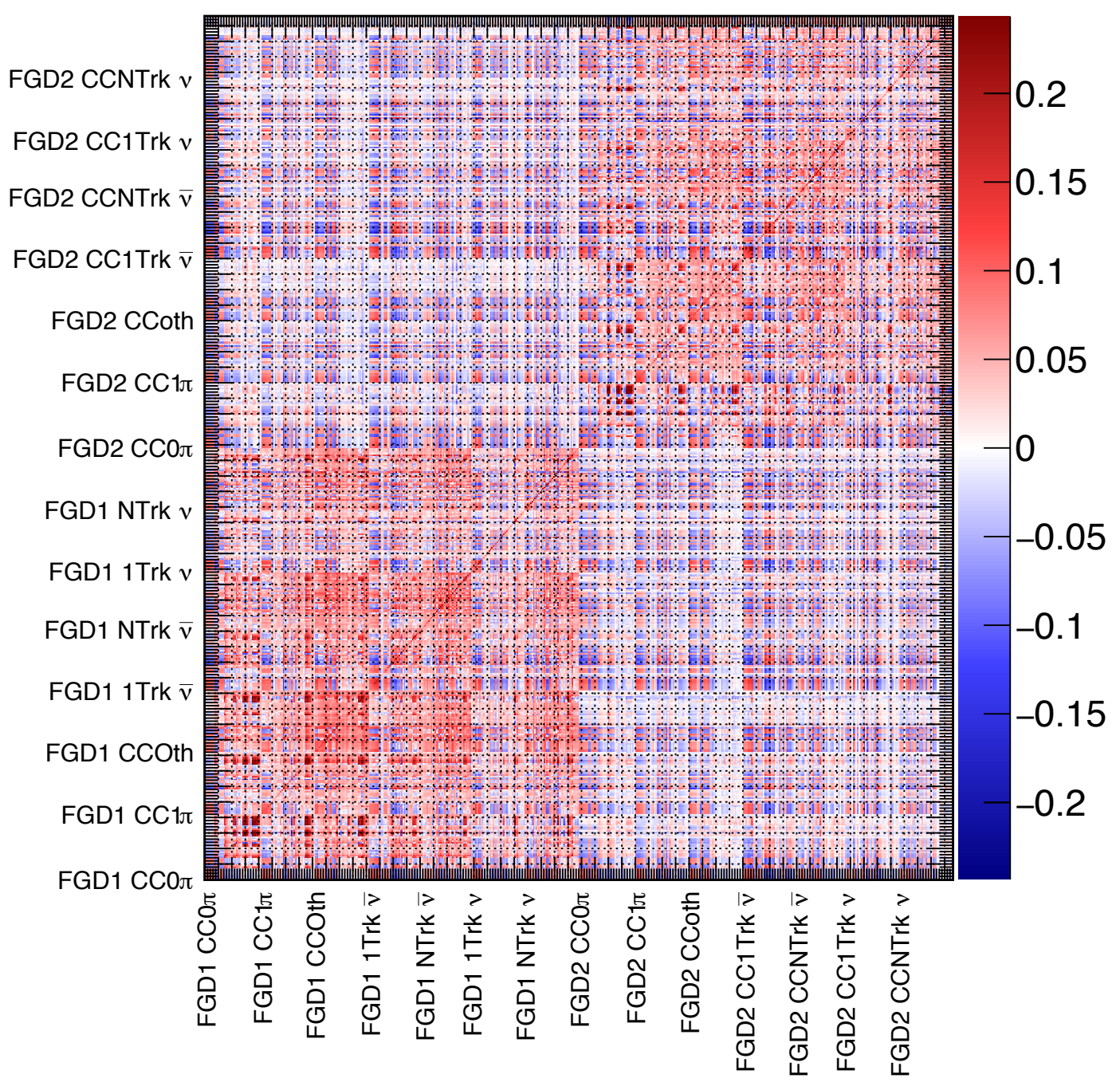

Figure 7.3: Fractional ND280 covariance matrix: $\operatorname{sign}\left(\mathrm{V}_{\mathrm{ij}}\right) \times \sqrt{\left|\mathrm{V}_{\mathrm{ij}}\right|}$. The starting bins for different ND280 samples have been labelled. Here, bins correspond to muon momentum and angle. Copyright by T2K Collaboration. 
7. Bayesian statistics and the Markov Chain Monte Carlo technique for neutrino oscillation analyses

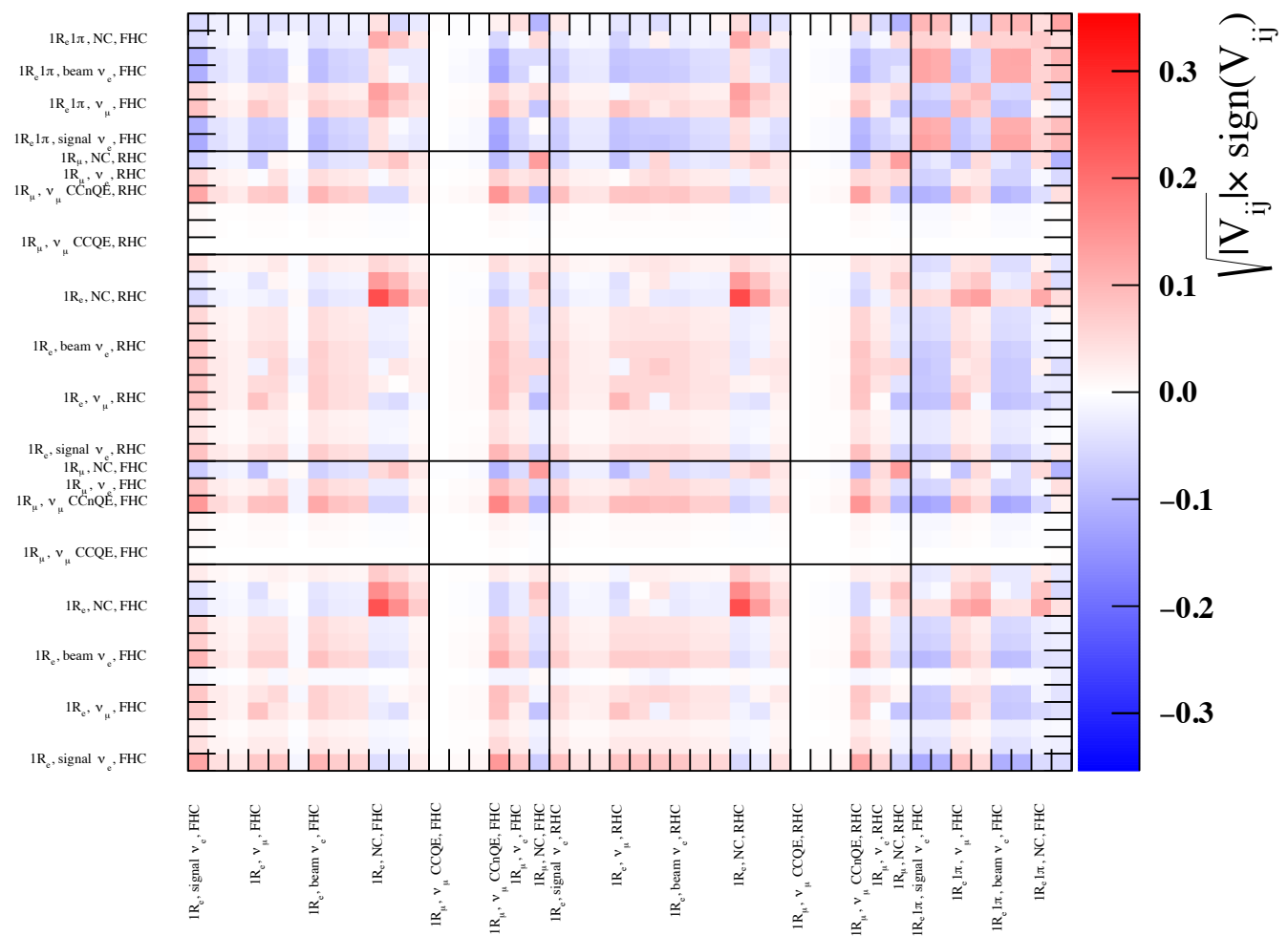

Figure 7.4: Fractional Super-K covariance matrix. The starting bins for different Super$\mathrm{K}$ samples have been labelled. Here, bins correspond to neutrino energy. Copyright by T2K Collaboration. 


\section{8}

\section{Joint $\nu+\bar{\nu} \mathrm{T} 2 \mathrm{~K}$ oscillation analysis with the reactor constraint and replica-tuned flux inputs}

The oscillation results presented in this chapter are the work of the author, and should not be interpreted as the official result of the T2K collaboration. For an up-todate official oscillation result by the T2K collaboration, the reader is referred to [8].

\subsection{Fits to ND280 data}

\subsubsection{ND280 data}

Fourteen data samples have been selected at ND280. Seven samples have been selected for interactions on each of the FGD1 and FGD2 detectors. In $\nu$-mode, interactions of $\nu_{\mu}$ are selected. For each detector, the event topology is split by the number of pions in final state: $\mathrm{CC}-0 \pi, \mathrm{CC}-1 \pi$ and $\mathrm{CC}$-Other, as depicted in Figure 8.1. The collected ND280 data for interactions on FGD1 are shown in Figure 8.4. In $\bar{\nu}$-mode, there is fewer statistics and event topologies are split by track number: CC-1Track and CC-NTrack. Both interactions of the signal $\bar{\nu}_{\mu}$ and the background $\nu_{\mu}$ are selected in $\bar{\nu}$-mode. Examples of event topologies selected in the RHC samples at ND280 are shown in Figure 8.2. Events are binned in terms of the detected muon kinematics $\left(p_{\mu}, \cos \theta_{\mu}\right)$. The collected ND280 data for interactions on FGD1 during runs 1-6 are shown in Figure 8.4. The predicted number of events in each of the samples is summarised in Table 8.1. The CC- $0 \pi$ interaction sample is the most abundant sample in $\nu$-mode, and consists of mostly CCQE and 2p2h $\nu_{\mu}$ interactions. A detailed MC based breakdown of interactions targeted by the CC- $0 \pi$ sample selection in FGD2 is given in Figure 8.3. The CC- $1 \pi$ 
8. Joint $\nu+\bar{\nu}$ T2K oscillation analysis with the reactor constraint and replica-tuned flux inputs

and CC-Other event samples select mostly CC-Res and CC-DIS interaction modes, respectively. In $\bar{\nu}$-mode, the most abundant is the CC-1Track sample, which is dominated by CCQE and 2p2h scattering.
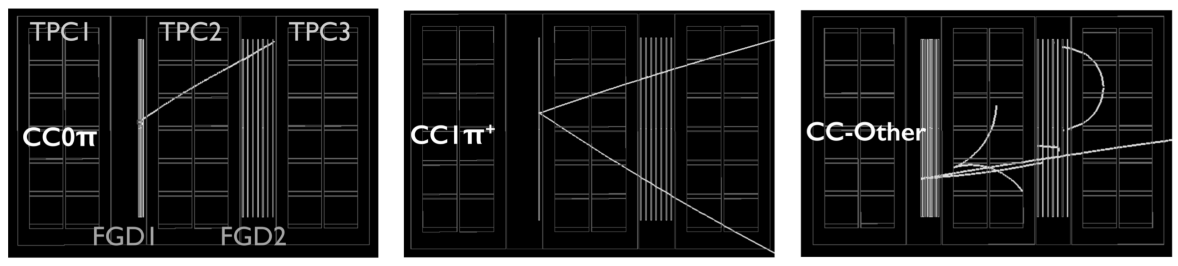

Figure 8.1: Examples of ND280 event displays for $\nu_{\mu} \mathrm{CC} 0 \pi, \nu_{\mu} \mathrm{CC} 1 \pi$ and $\nu_{\mu}$ CCOther FGD1 samples in neutrino mode. Figures from [148]. Copyright by speaker, Kirsty Duffy, on behalf of the T2K Collaboration. The proceedings for this conference talk are available at [149].
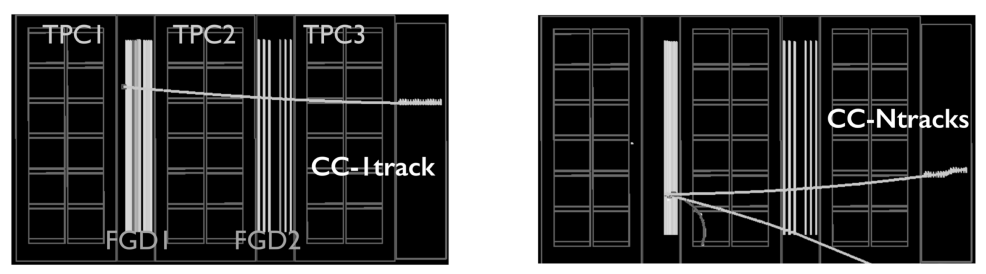

Figure 8.2: Examples of ND280 event displays for CC 1-Track and CC N-Track FGD1 samples in anti-neutrino mode. Figures from [148]. Copyright by speaker, Kirsty Duffy, on behalf of the T2K Collaboration. The proceedings for this conference talk are available at [149].

Events are selected by applying the following selection cuts. Only data collected during normal beam operation and coincident with the beam spill times is selected. At least one track with at least 18 hit clusters in the TPCs is required for every selected event. The interaction vertex (first hit position) of the highest momentum track must be contained within the fiducial volume of either FGD1 or FGD2, for efficient background removal. Here, backgrounds constitute interactions of T2K beam neutrinos on other parts of ND280, such as PØD and the ND280 magnet, and on external targets such as the sand surrounding the ND280 pit. When selecting $\nu_{\mu}$ $\left(\bar{\nu}_{\mu}\right)$ interactions, the curvature of the highest momentum track must be consistent with negative (positive) charge. Additional background removal cuts are imposed as follows. Events at FGD2 are rejected if there are reconstructed tracks present at FGD1. If the second highest momentum track starts more than $150 \mathrm{~mm}$ upstream 
8. Joint $\nu+\bar{\nu}$ T2K oscillation analysis with the reactor constraint and replica-tuned flux inputs

from the muon track, events are also rejected. These cuts remove backwardgoing events, and events where muons produced upstream from the FGDs undergo scattering before leaving track signatures in the FGD fiducial volume. In some cases, a single muon tracks is split into two shorter tracks during the reconstruction procedure, potentially resulting in misplacement of the interaction vertex. Events with at least one fully contained FGD track are required to have the separation between the muon candidate track and the upstream FGD face of less than $425 \mathrm{~mm}$.

In addition to these cuts for the selection of all CC-inclusive interactions of $\nu_{\mu}$ and $\bar{\nu}_{\mu}$, dedicated cuts are applied for individual ND280 sample selection. These cuts mostly rely on TPC energy loss measurements for particle identification. In CC- $0 \pi$ events, the highest momentum track candidate, given the TPC data, must be consistent with the $\mu^{-}$hypothesis (in other words, $e^{-}$and $\pi^{-}$PID hypotheses must be rejected). Additionally, for $\mathrm{CC}-1 \pi^{+}$events an additional track consistent with $\pi^{+}$or delayed Michel $e^{+}$is required. All events which pass the CC-inclusive cuts but are consistent with neither $\mathrm{CC}-0 \pi$ nor $\mathrm{CC}-1 \pi$ topologies are grouped in the CC-Other category. In $\bar{\nu}$-mode, CC-inclusive events are simply split based on the number of tracks. The highest momentum track in $\bar{\nu}_{\mu}$ (background $\nu_{\mu}$ ) interaction samples in $\bar{\nu}$-mode must be consistent with the $\mu^{+}\left(\mu^{-}\right)$PID hypothesis. 
8. Joint $\nu+\bar{\nu}$ T2K oscillation analysis with the reactor constraint and replica-tuned flux inputs

\begin{tabular}{|c|c|c|c|c|c|c|}
\hline \multirow[t]{2}{*}{ Sample } & \multirow[t]{2}{*}{ Target } & \multirow[t]{2}{*}{ Data } & \multicolumn{2}{|c|}{ Thin flux } & \multicolumn{2}{|c|}{ Replica flux } \\
\hline & & & Pre-fit & Post-fit & Pre-fit & Post-fit \\
\hline$\nu_{\mu} \mathrm{CC}-0 \pi$ & & 17136 & 17096.9 & 17121.2 & 16520.4 & 17120.2 \\
\hline$\nu_{\mu} \mathrm{CC}-1 \pi$ & & 3954 & 4479.27 & 4054.21 & 4403.03 & 4052.41 \\
\hline$\nu_{\mu}$ CC-Other & & 4149 & 4034.27 & 4106.71 & 4008.34 & 4108.37 \\
\hline $\bar{\nu}_{\mu}$ CC-1Track & FGD1 & 3527 & 3642.69 & 3508.73 & 3535.88 & 3504.53 \\
\hline $\bar{\nu}_{\mu}$ CC-NTrack & & 1054 & 1072.18 & 1061.59 & 1077.8 & 1061.46 \\
\hline$\nu_{\mu}$ CC-1Track & & 1363 & 1287.2 & 1349.05 & 1303.49 & 1358.85 \\
\hline$\nu_{\mu}$ CC-NTrack & & 1370 & 1373.56 & 1355.89 & 1387.74 & 1356.81 \\
\hline$\nu_{\mu} \mathrm{CC}-0 \pi$ & & 17443 & 17337.7 & 17501.5 & 16746.2 & 17488.4 \\
\hline$\nu_{\mu} \mathrm{CC}-1 \pi$ & & 3366 & 3643.83 & 3416.49 & 3583.38 & 3410.05 \\
\hline$\nu_{\mu}$ CC-Other & & 4075 & 3652.76 & 3915.71 & 3627.87 & 3914.65 \\
\hline $\bar{\nu}_{\mu}$ CC-1Track & FGD2 & 3732 & 3674.2 & 3680.07 & 3567.64 & 3681.74 \\
\hline $\bar{\nu}_{\mu}$ CC-NTrack & & 1026 & 1082.51 & 1106.22 & 1089.31 & 1105.48 \\
\hline$\nu_{\mu}$ CC-1Track & & 1320 & 1277.62 & 1324.93 & 1292.49 & 1336.2 \\
\hline$\nu_{\mu}$ CC-NTrack & & 1253 & 1261.53 & 1264.74 & 1275.45 & 1266.02 \\
\hline
\end{tabular}

Table 8.1: Summary of the number of events in ND280 samples for data and Monte Carlo. Both prefit (nominal) and postfit MC predictions are given, for thin-tuned and replica-tuned flux inputs.

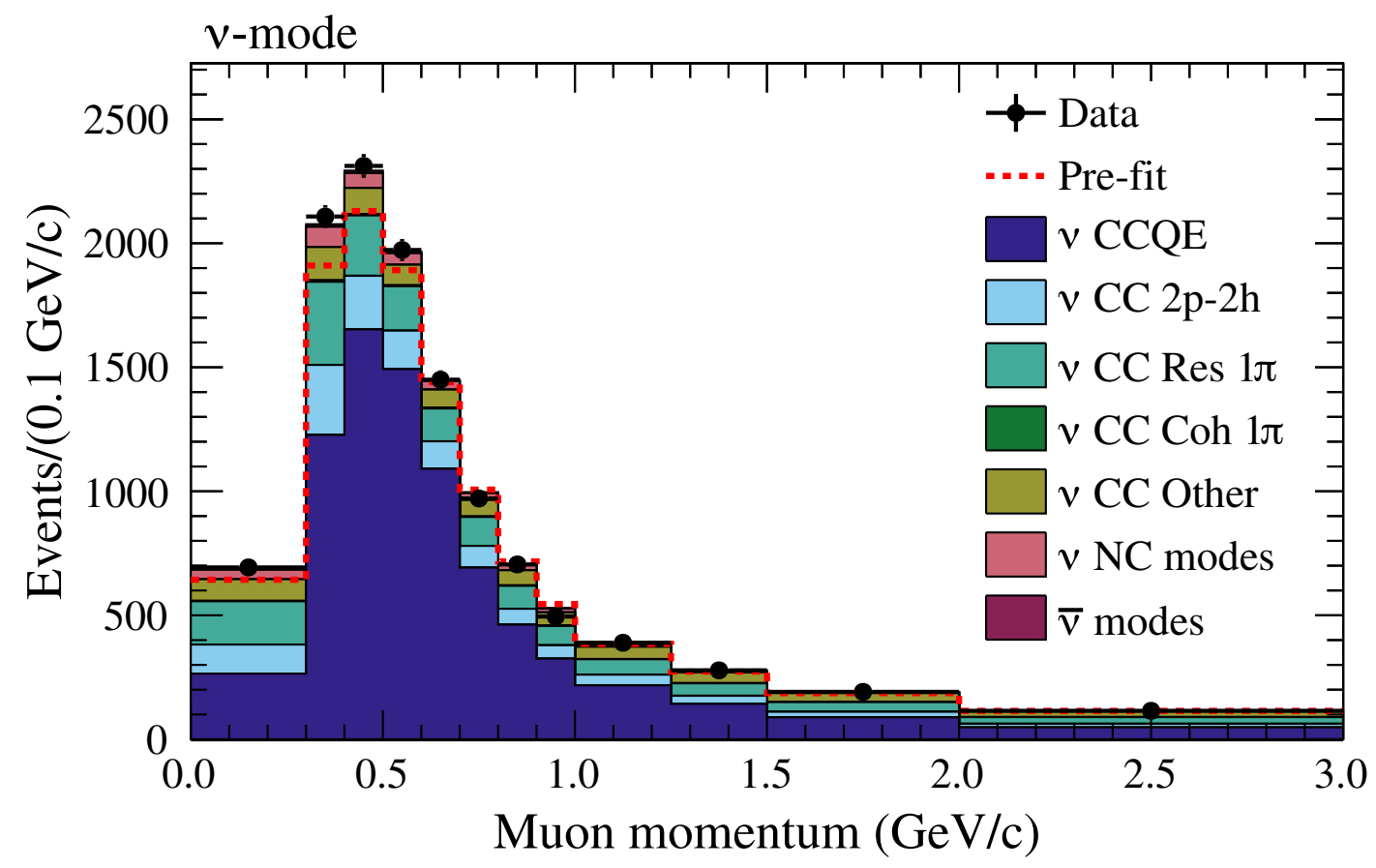

Figure 8.3: MC pre-fit prediction for types of interactions in the ND280 FGD2 CC-0 $\pi$ sample. The observed data from T2K Runs 1-6 is also given. The pre-fit MC prediction shown here assumes the thin-tuned neutrino flux. Figure reprinted from Physical Review Letters, Volume 121, K. Abe et al. (T2K Collaboration), "Search for $C P$ Violation in Neutrino and Antineutrino Oscillations by the T2K Experiment with $2.2 \times 10^{21}$ Protons on Target"[9], under the CC BY copyright licence. 
8. Joint $\nu+\bar{\nu}$ T2K oscillation analysis with the reactor constraint and replica-tuned flux inputs

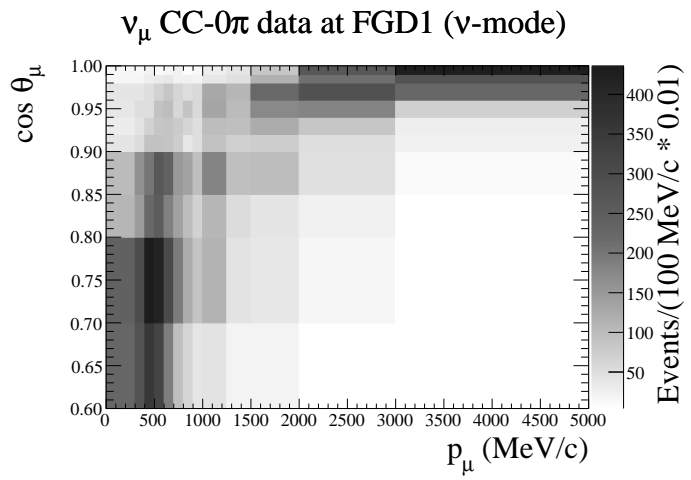

(a) $\mathrm{CC}-0 \pi, \nu$-mode $v_{\mu}$ CC- $1 \pi$ data at FGD1 ( $v$-mode)

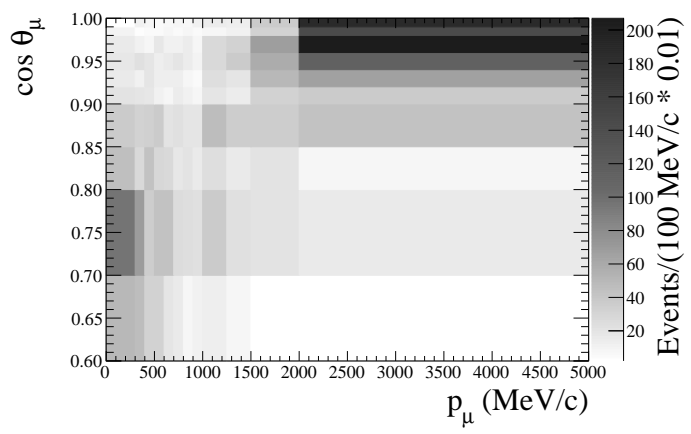

(c) $\mathrm{CC}-1 \pi, \nu$-mode

$v_{\mu}$ CC-Other data at FGD1 (v-mode)

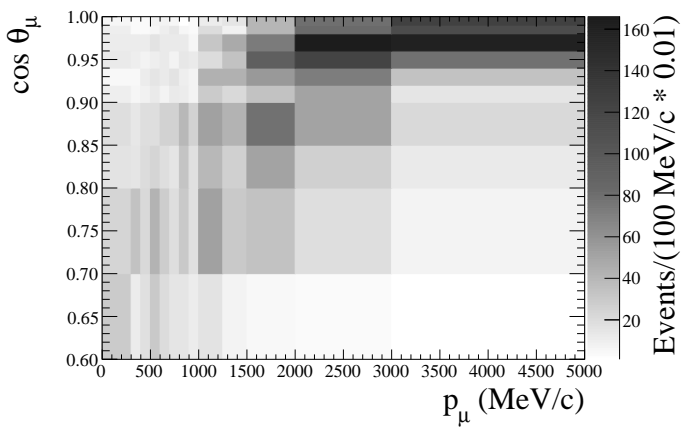

(e) CC-Other, $\nu$-mode

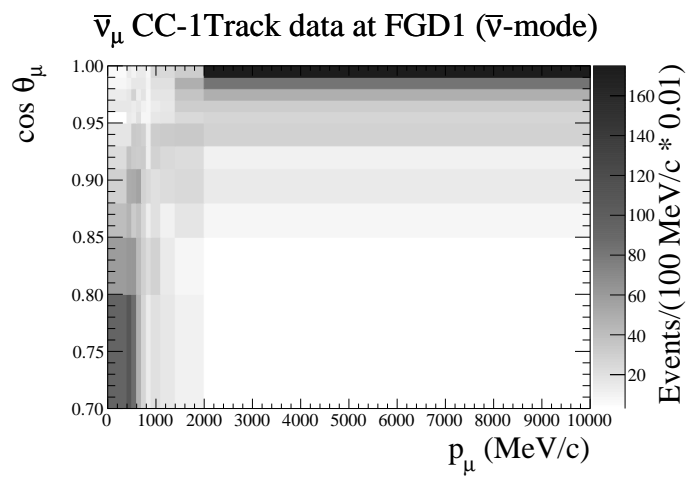

(b) CC-1Track, $\bar{\nu}$-mode $\bar{v}_{\mu}$ CC-NTrack data at FGD1 ( $\bar{v}$-mode)

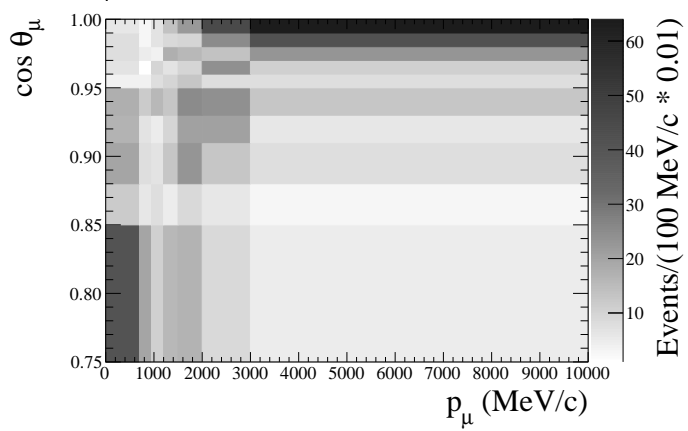

(d) CC-NTrack, $\bar{\nu}$-mode $v_{\mu}$ CC-1Track data at FGD1 ( $\bar{v}$-mode)

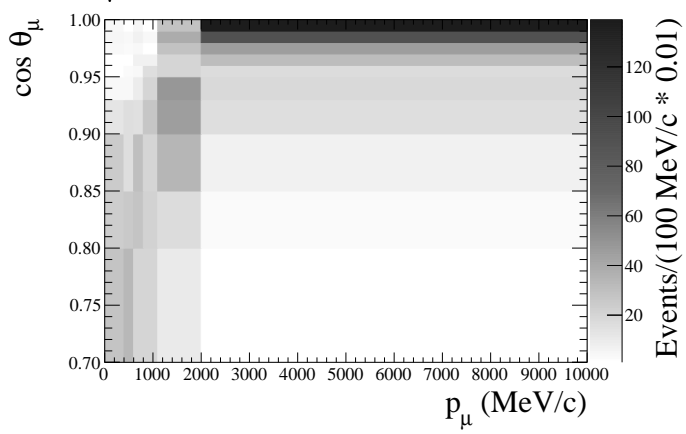

(f) CC-1Track background, $\bar{\nu}$-mode $v_{\mu}$ CC-NTrack data at FGD1 ( $\bar{v}$-mode)

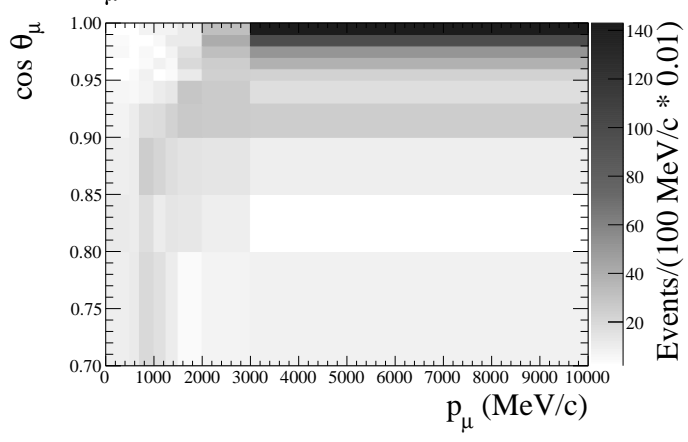

(g) CC-NTrack background, $\bar{\nu}$-mode

Figure 8.4: ND280 FGD1 data samples in $\nu$-mode (left) and $\bar{\nu}$-mode (right). 
8. Joint $\nu+\bar{\nu}$ T2K oscillation analysis with the reactor constraint and replica-tuned flux inputs

\subsubsection{Fit results}

This section presents the results of fits to ND280 data, in both neutrino and antineutrino mode, using the new replica-tuned flux systematic parameters. MCMC are constructed from the ND280 posterior log-likelihood. Firstly, the Monte Carlo event prediction after the ND280 data constraint (post-fit MC) is examined. The post-fit MC event spectrum is commonly referred to as the posterior predictive distribution. Finding the maximum of the posterior log-likelihood in the full 761 parameter space would be unfeasible from the standpoint of available computational resources. Instead, parameter points are sampled from Markov chains. For this study, 15,000 steps from the Markov chain, after burn-in removal, are chosen at random. By construction, the parameters for which the log-likelihood is maximised are more likely to be selected. For every selected set of parameters, the nominal MC prediction is reweighted, the ND280 event spectrum re-calculated. The best fit spectrum is taken as the mean of the 15,000 MC predictions from randomly chosen MCMC steps. The general trend will be discussed on the example of CC- $0 \pi$ events in FGD1, for which a comparison of both nominal (pre-fit) and post-fit MC prediction and collected data is shown in Figure 8.5, as a function of muon momentum. Here, both the thin-tuned and replica-tuned flux inputs have been considered. The replica-tuned flux calculation typically predicts a lower $\nu_{\mu}$ flux at T2K beam energies, and consequently fewer events in the $\mathrm{CC}-0 \pi$ sample (see ratio of pre-fit MC to data). The ND280 data constraint is remarkably powerful in tuning the MC event spectrum. Despite differences in nominal predictions, the post-fit MC event spectra for the thin-tuned and replica-tuned flux inputs show remarkable levels of agreement. This is demonstrated even more convincingly in Figure 8.6, where post-fit ND280 MC spectra with thin and replica tuning are compared in the two-dimensional $\left(p_{\mu}, \cos \theta_{\mu}\right)$ phase space. For most analysis bins, the agreement is better than 5\%. As expected, post-fit MC spectra also agree better with data (see Figure 8.5b).

The posterior value of flux and cross section parameters after fits to ND280 data has been examined. When calculating the parameter posterior, the distribution of 
8. Joint $\nu+\bar{\nu}$ T2K oscillation analysis with the reactor constraint and replica-tuned flux inputs

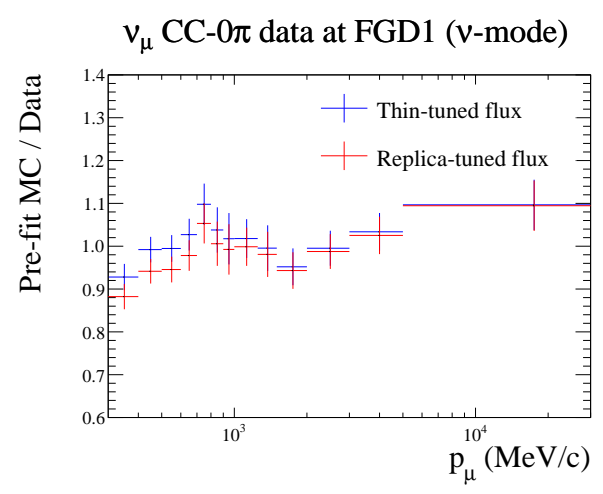

(a) Pre-fit MC

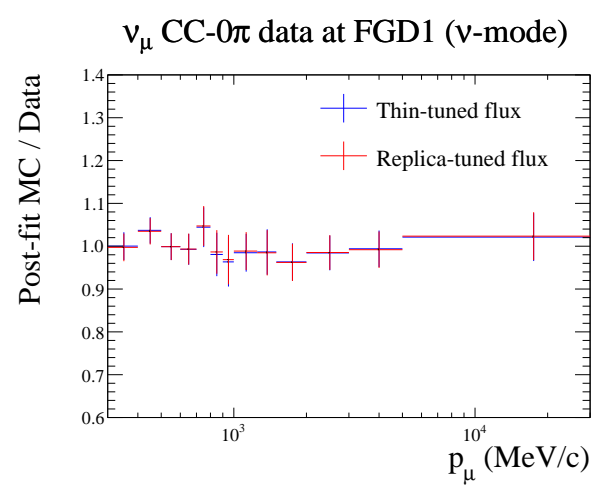

(b) Post-fit MC

Figure 8.5: Comparison of pre-fit (left) and post-fit (right) MC predictions to data, plotted as a function of muon momentum for the $\nu_{\mu}$ CC- $0 \pi$ sample in FGD1.

parameter values across all MCMC steps is taken. Such a distribution for beam parameter $b_{15}$ for example is given in Figure 8.7. Three methods for extracting the posterior value are used. The arithmetic method takes the arithmetic mean and standard deviation of the parameter distribution. Alternatively, the parameters of a fitted Gaussian are used to estimate the posterior. Finally, the posterior is also estimated to be at the highest posterior density of the parameter distribution, with asymmetrical errors calculated so that $\sim 34.1 \%$ of possible parameter values are contained on either side of the posterior. The ND280 posterior flux parameters are given in Figure 8.9 and Figure 8.10 for $\nu$-mode and $\bar{\nu}$-mode, respectively. The postfit flux predictions always lie within $\pm 1 \sigma$ of their assigned priors, indicating good underlying understanding of the T2K flux. In $\nu$-mode, the error on the right sign $\left(\nu_{\mu}\right.$ and $\left.\nu_{e}\right)$ flux components is reduced by more than $50 \%$. There is a tendency for ND280 data in $\nu$-mode to prefer a lower $\nu_{\mu}$ flux compared to the nominal prediction, for reconstructed neutrino energies $\mathrm{E}_{\nu} \in[0.6,2] \mathrm{MeV}$ and $\mathrm{E}_{\nu}>5 \mathrm{MeV}$. A similar, albeit less pronounced trend is observed in $\bar{\nu}$-mode. In $\bar{\nu}$-mode, a significant reduction in posterior $\nu_{\mu}$ (wrong-sign) flux uncertainty is observed, coming from the constraint imposed by the CC $\nu_{\mu}$ 1-Track and N-Track data samples.

The posterior cross section parameters are given in Figure 8.8, for fits with both thin-tuned and replica-tuned flux inputs. No significant change in cross section parameters has been observed based on different flux pre-fit values. Notably, ND280 
8. Joint $\nu+\bar{\nu}$ T2K oscillation analysis with the reactor constraint and replica-tuned flux inputs

data puts stringent constraints on many of the cross section parameters with flat priors, such as $2 \mathrm{p} 2 \mathrm{~h}$ normalisation and shape parameters. A substantial reduction in FSI parameter uncertainties is also achieved with fits to data.

A comparison between pre-fit and post-fit flux covariance and correlations is given in Figure 8.11. As expected, the flux uncertainties shrink dramatically after the data fit. Similarly, cross section parameter covariance and correlation before and after the fit are examined in Figure 8.12. The correlations among cross section parameters are significantly modified after fits to data. For example, $\mathrm{M}_{\mathrm{A}}^{\mathrm{QE}}$ and $\mathrm{p}_{\mathrm{F}}$ parameters get correlated to the BeRPA parameters, as they all contribute to the predicted number of events in the $\mathrm{CC}-0 \pi$ sample.

The goodness of fits to T2K data is estimated with the $\chi^{2}$ statistical hypothesis test. Here, the $\chi^{2}$ statistic is defined as the logarithm of the ratio of posterior probabilities:

$$
\chi^{2}=-2 \ln \left[\frac{P(\vec{d} \mid \vec{p})}{P(\vec{d} \mid \vec{d})}\right]=2 \times \sum_{\text {bins } \mathrm{i}}\left[d_{\mathrm{i}} \ln \left(\frac{d_{\mathrm{i}}}{p_{\mathrm{i}}}\right)+p_{\mathrm{i}}-d_{\mathrm{i}}\right]
$$

where $d_{\mathrm{i}}$ and $p_{\mathrm{i}}$ denote the observed and predicted (from MC) number of candidate events in the $\mathrm{i}^{\text {th }}$ analysis bin, respectively. This study reuses the 15,000 reweighted MC predictions from the posterior predictive spectra for each sample. $\chi_{\text {Data, Posterior }}^{2}$ is calculated by comparing these posterior spectra to the observed data. $\chi_{\text {Fluct. Posterior, Posterior }}^{2}$ is calculated by comparing posterior spectra to fake data, where the fake data is generated by taking random Poisson draws from each bin of the posterior spectra. Finally, the distribution of $\chi_{\text {Data, }}^{2}$ Posterior versus

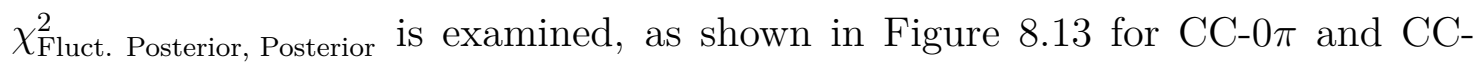
1Track samples in FGD1. The $\mathrm{p}$ value is defined as the fraction of points for which

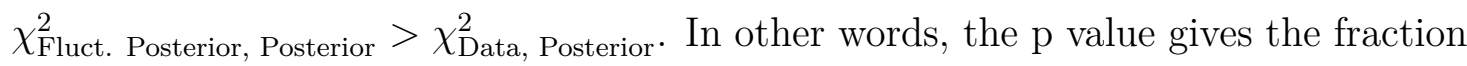
of points where the agreement between measured data and the fit result (posterior spectrum) is better than the agreement between the fit result and the fluctuated fit result. Prior to performing these fits, it has been agreed that fits with $\mathrm{p}>\alpha$, where $\alpha=0.05$, will be deemed acceptable. Typically, the significance level $\alpha$ is chosen anywhere between 0.05 and 0.10 . For $\mathrm{CC}-0 \pi$, the most abundant sample 
8. Joint $\nu+\bar{\nu}$ T2K oscillation analysis with the reactor constraint and replica-tuned flux inputs

in $\nu$-mode, the MCMC fit to data is successful, with a p value of 0.07 . Even more convincingly, for the CC-1Track sample in $\bar{\nu}$-mode, $\mathrm{p}=0.49$. The only sample where the fit fails to capture the collected data is for the CC-Other sample in FGD1. Marginal differences in $\mathrm{p}$ value are observed across all samples with the implementation of the replica-tuned flux priors in the fitting framework, as shown in Table 8.2. Ultimately, the pre-fit flux inputs are less important, since the post-fit predictions are tightly constrained with the collected ND280 data.

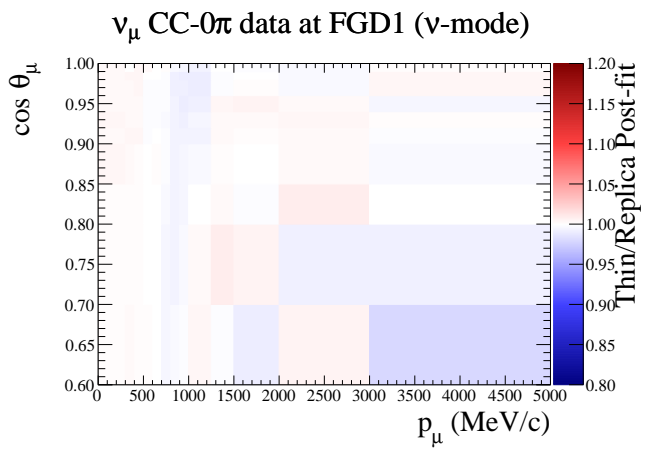

(a) CC- $0 \pi, \nu$-mode

$v_{\mu} \mathrm{CC}-1 \pi$ data at FGD1 (v-mode)

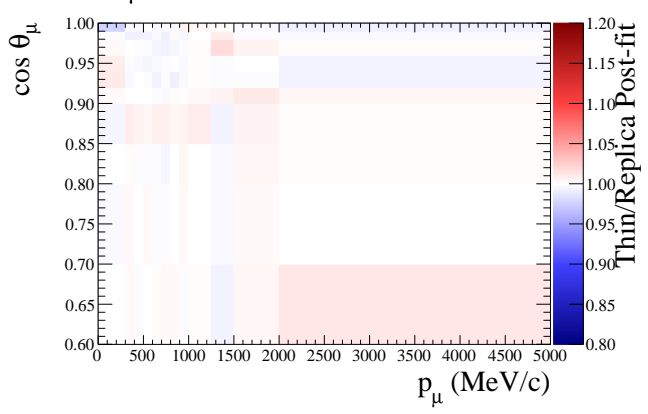

(c) $\mathrm{CC}-1 \pi, \nu$-mode

$v_{\mu}$ CC-Other data at FGD1 ( $v$-mode)

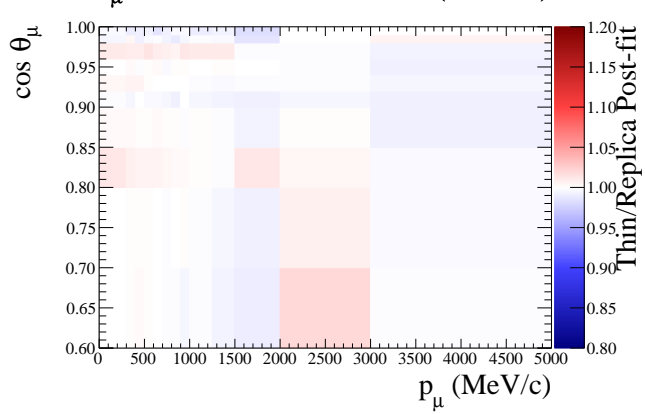

(e) CC-Other, $\nu$-mode

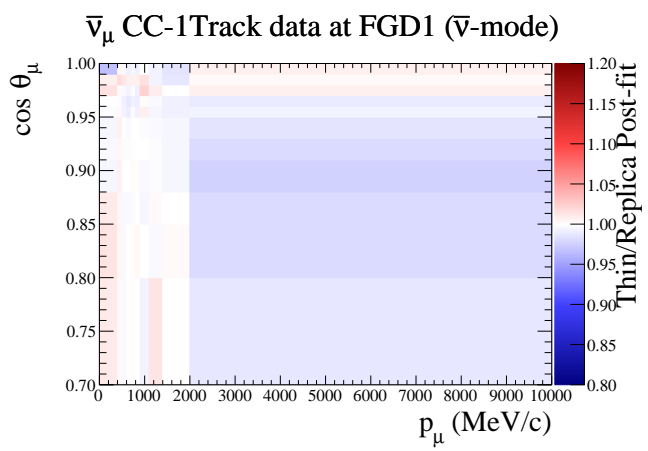

(b) CC-1Track, $\bar{\nu}$-mode $\overline{\mathrm{v}}_{\mu}$ CC-NTrack data at FGD1 ( $\overline{\mathrm{v}}$-mode)

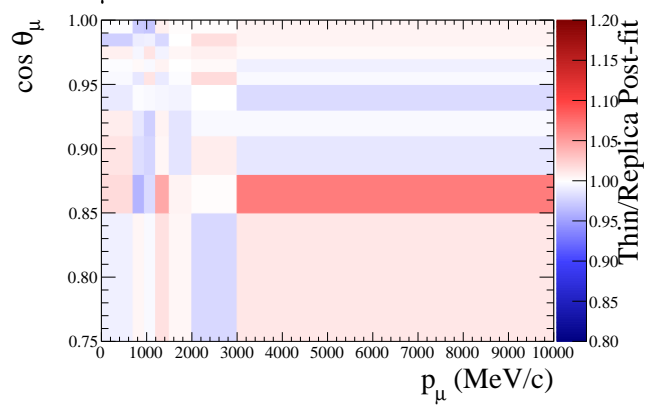

(d) CC-NTrack, $\bar{\nu}$-mode

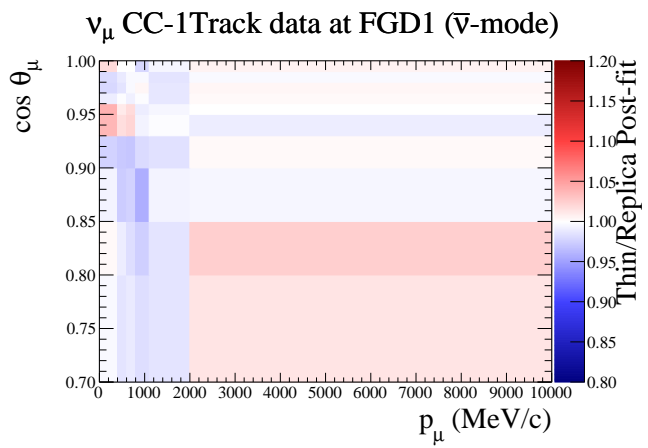

(f) CC-1Track background, $\bar{\nu}$-mode

Figure 8.6: Post-fit ratio of ND280 FGD1 MC samples in $\nu$-mode (left) and $\bar{\nu}$-mode (right), in $\left(p_{\mu}, \cos \theta_{\mu}\right)$ phase space. The posterior predictions of the two fits show good agreement despite different nominal neutrino flux inputs. 
8. Joint $\nu+\bar{\nu}$ T2K oscillation analysis with the reactor constraint and replica-tuned flux inputs

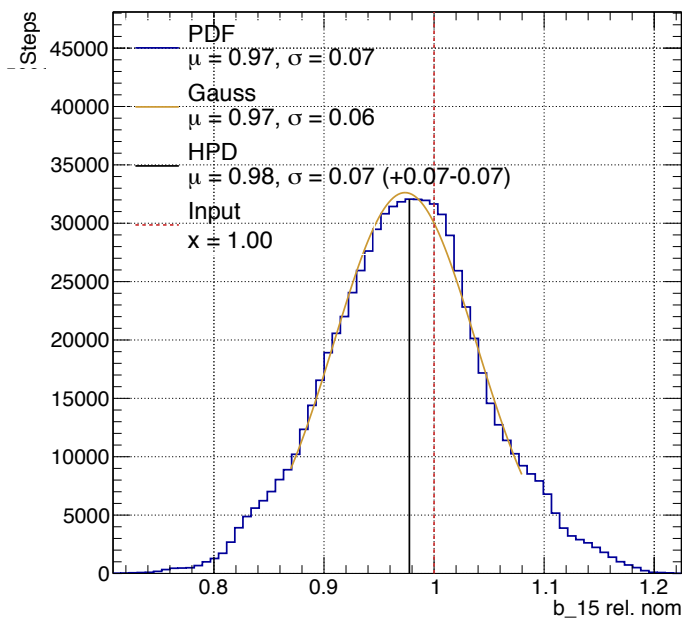

Figure 8.7: Distribution of values for flux parameter $\mathrm{b}_{15}$, for ND280 $\bar{\nu}_{\mu}$ flux with $\mathrm{E}_{\nu} \in[1.5,2.5] \mathrm{GeV} / \mathrm{c}$ in $\nu$-mode, across $750,000 \mathrm{MCMC}$ steps. The parameter posterior is calculated for arithmetic (PDF), Gaussian and highest posterior density (HPD) methods of extraction. For every method, the uncertainty on the mean is also given. The horizontal axis shows the parameter value normalised with respect to the thin-tuned nominal.

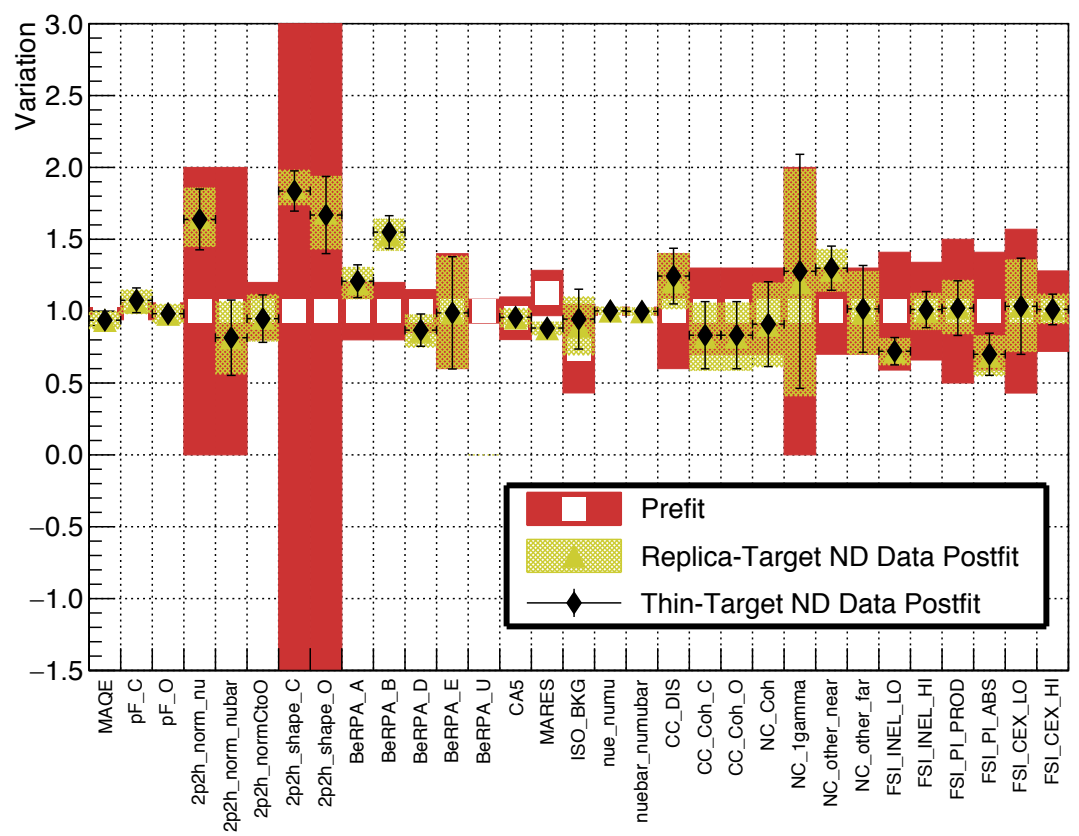

Figure 8.8: Posterior cross section parameters. Parameter values are normalised with respect to the nominal. The prior uncertainties are shown as red error bands. 
8. Joint $\nu+\bar{\nu}$ T2K oscillation analysis with the reactor constraint and replica-tuned flux inputs
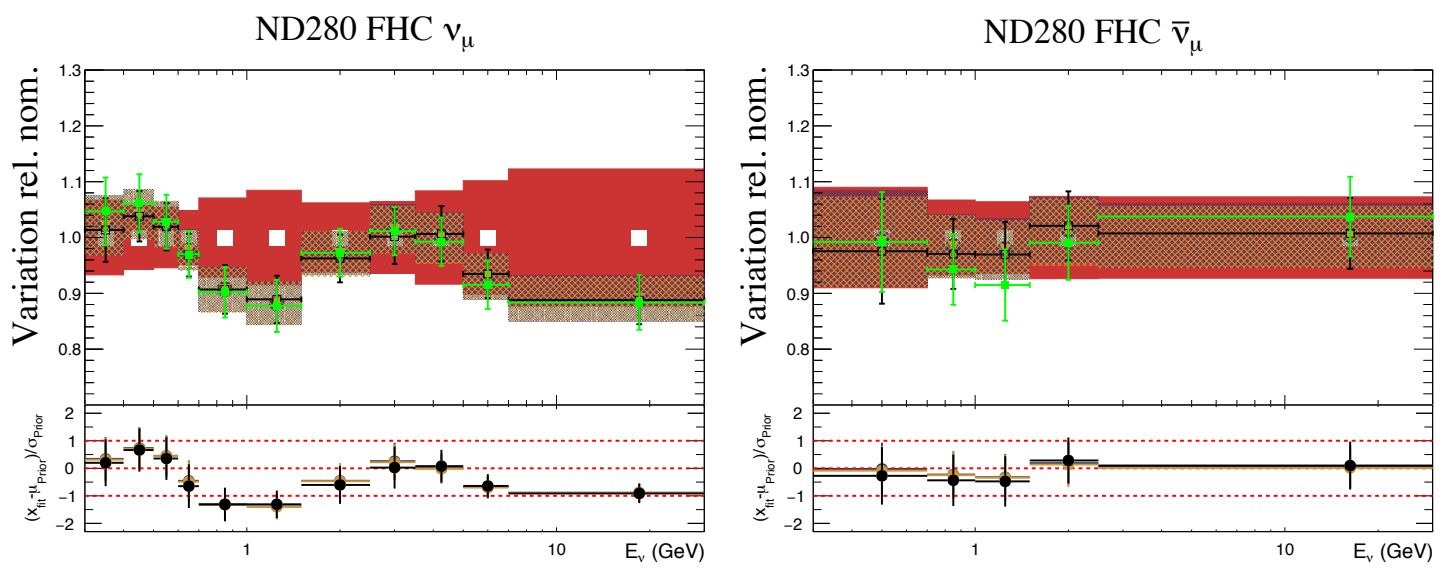

ND280 FHC $v_{\mathrm{e}}$

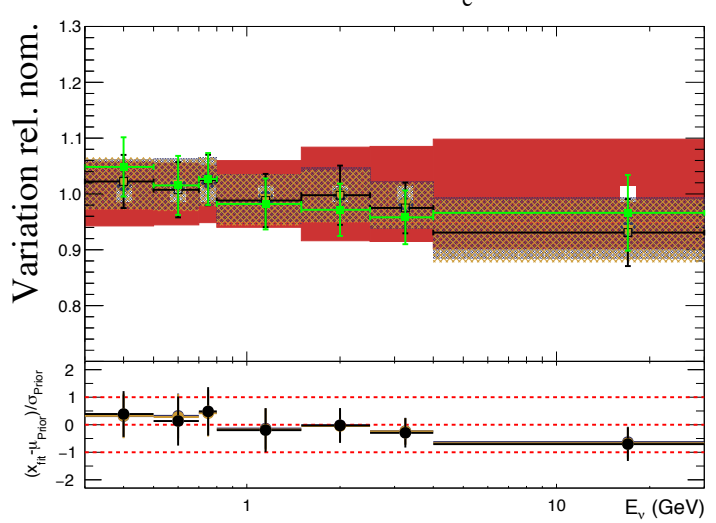

ND280 FHC $\bar{v}_{\mathrm{e}}$

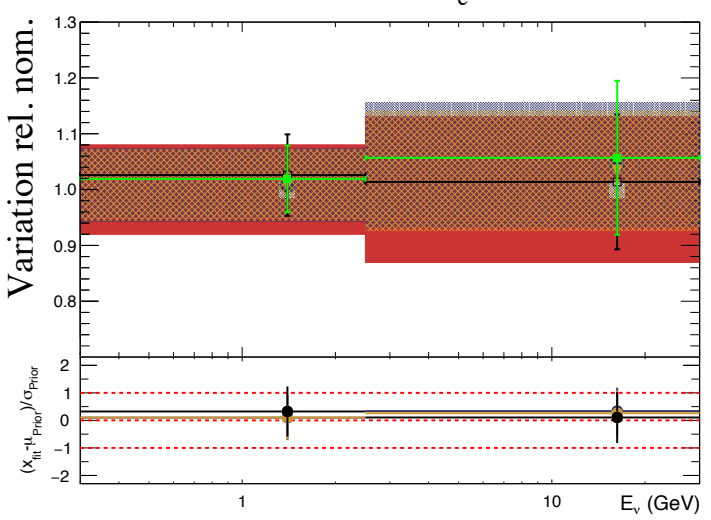

- Replica Prior

Arithmetic Replica Posterior

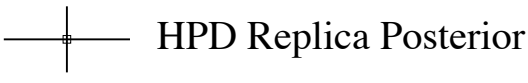

HPD Thin Posterior

\section{Gauss Replica Posterior}

Figure 8.9: Posterior flux parameters in neutrino mode, evaluated with the arithmetic, Gaussian and highest posterior density methods. All posterior values are normalised with respect to the replica-tuned flux priors. The replica-tuned prior uncertainties are shown as red bands. 
8. Joint $\nu+\bar{\nu}$ T2K oscillation analysis with the reactor constraint and replica-tuned flux inputs
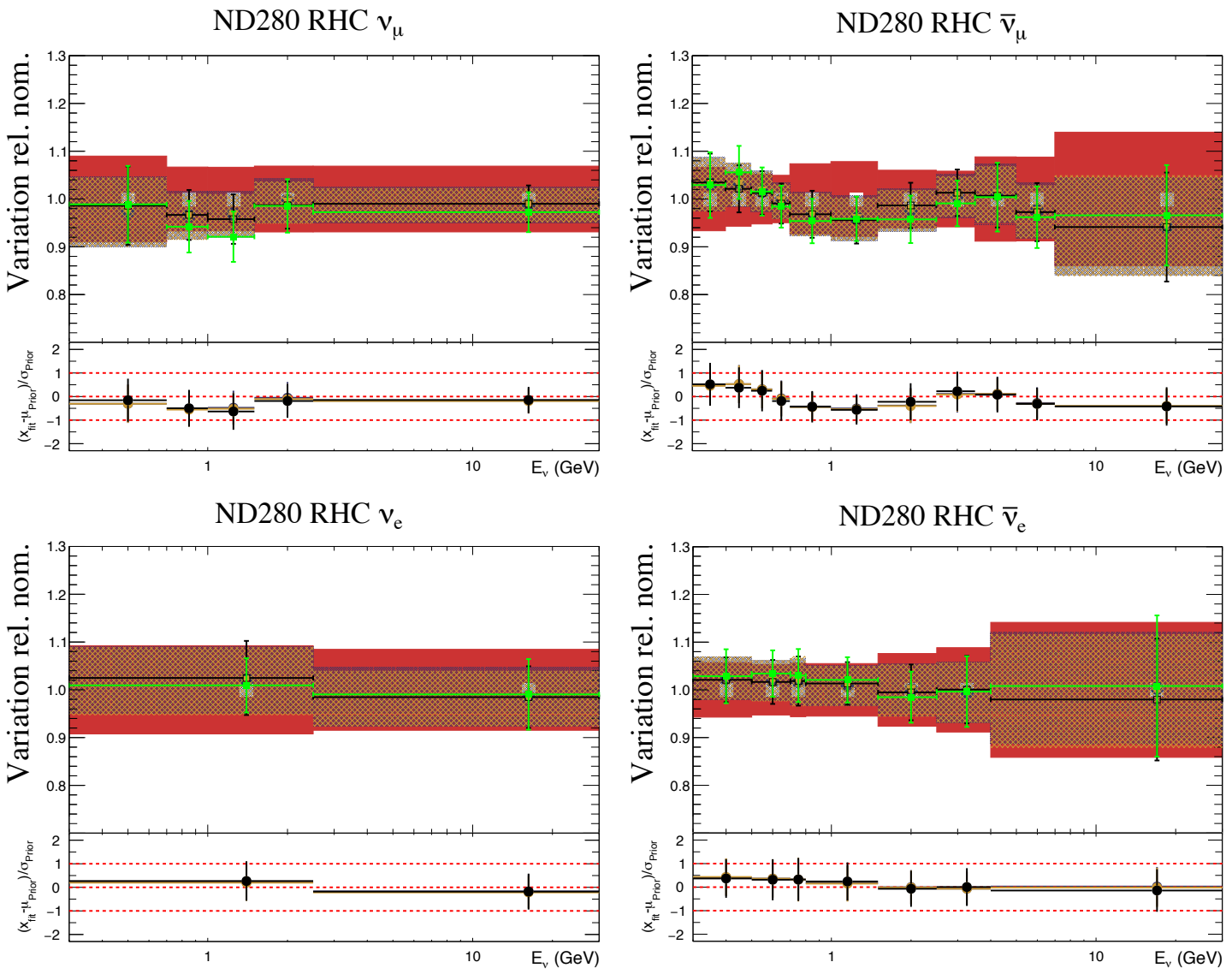

- Replica Prior

Arithmetic Replica Posterior

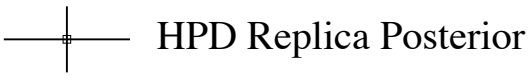

\section{HPD Thin Posterior}

\section{Gauss Replica Posterior}

Figure 8.10: Posterior flux parameters in anti-neutrino mode, evaluated with the arithmetic, Gaussian and highest posterior density methods. All posterior values are normalised with respect to the replica-tuned flux priors. The replica-tuned prior uncertainties are shown as red bands. 
8. Joint $\nu+\bar{\nu}$ T2K oscillation analysis with the reactor constraint and replica-tuned flux inputs
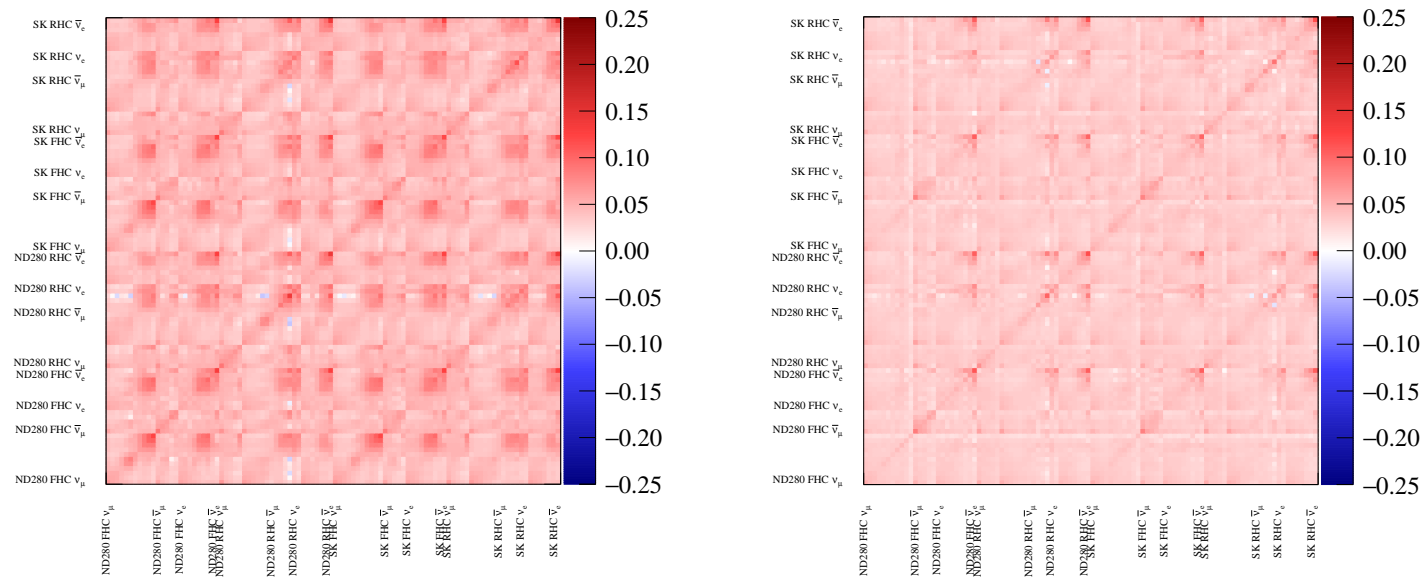

(a) Prefit covariance: $\operatorname{sign}\left(\mathrm{V}_{\mathrm{ij}}\right) \times \sqrt{\left|\mathrm{V}_{\mathrm{ij}}\right|}$

(b) Postfit covariance: $\operatorname{sign}\left(V_{\mathrm{ij}}\right) \times \sqrt{\left|\mathrm{V}_{\mathrm{ij}}\right|}$

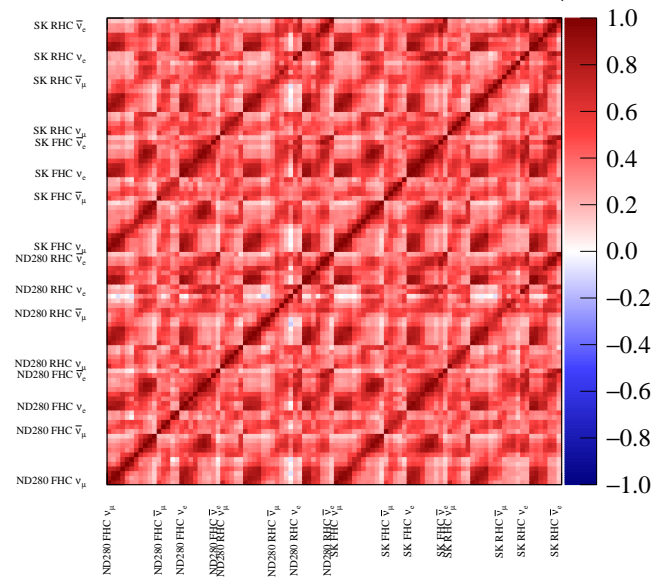

(c) Prefit correlation

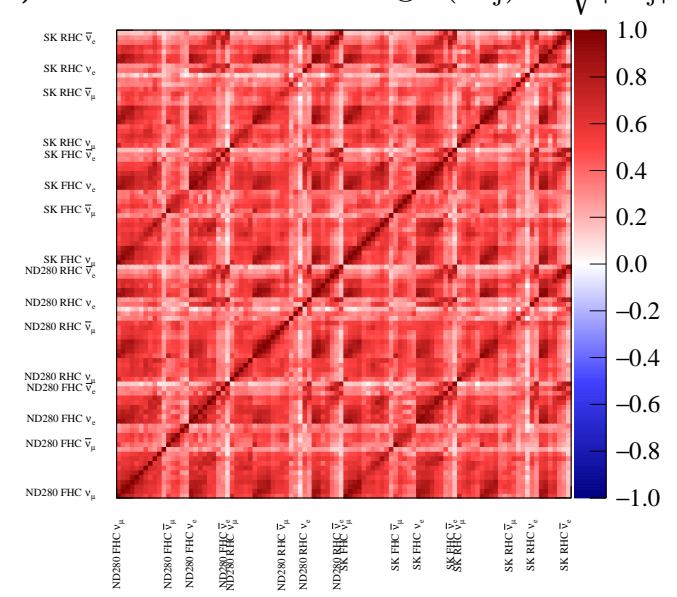

(d) Postfit correlation

Figure 8.11: Fractional covariance matrix and correlation matrix for replica-tuned flux parameters before (left) and after (right) the fit to ND280 data. 
8. Joint $\nu+\bar{\nu}$ T2K oscillation analysis with the reactor constraint and replica-tuned flux inputs
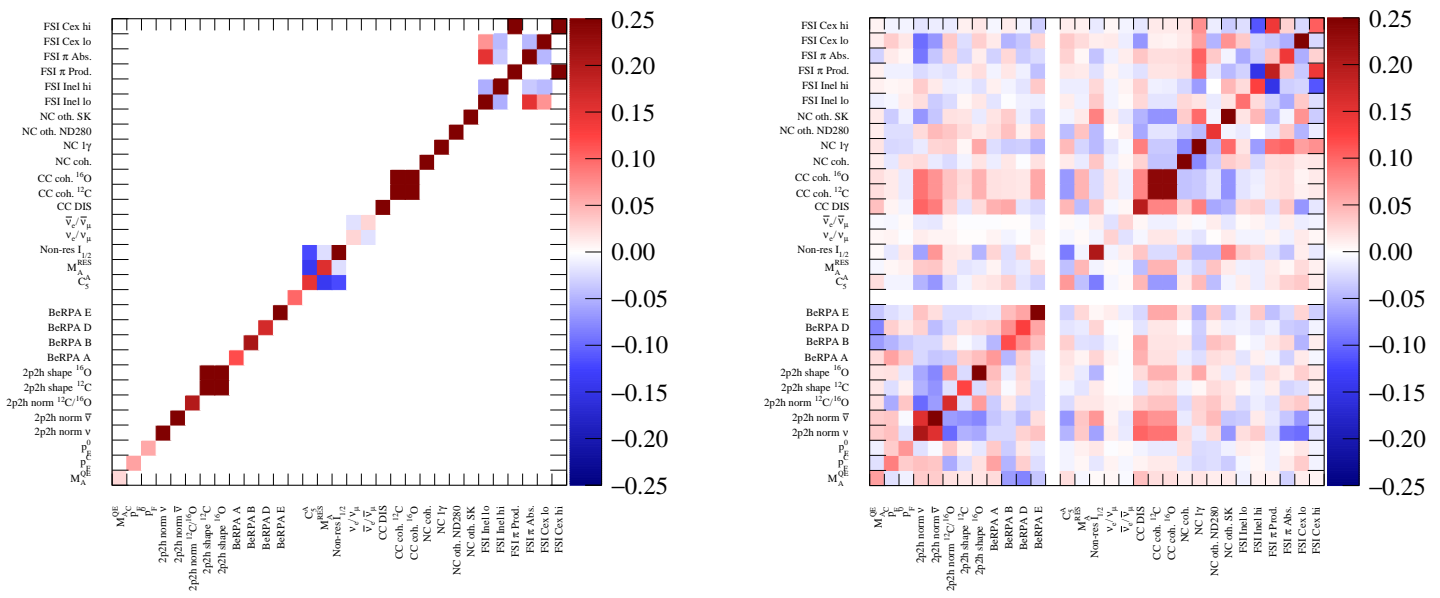

(a) Prefit covariance: $\operatorname{sign}\left(\mathrm{V}_{\mathrm{ij}}\right) \times \sqrt{\left|\mathrm{V}_{\mathrm{ij}}\right|}$

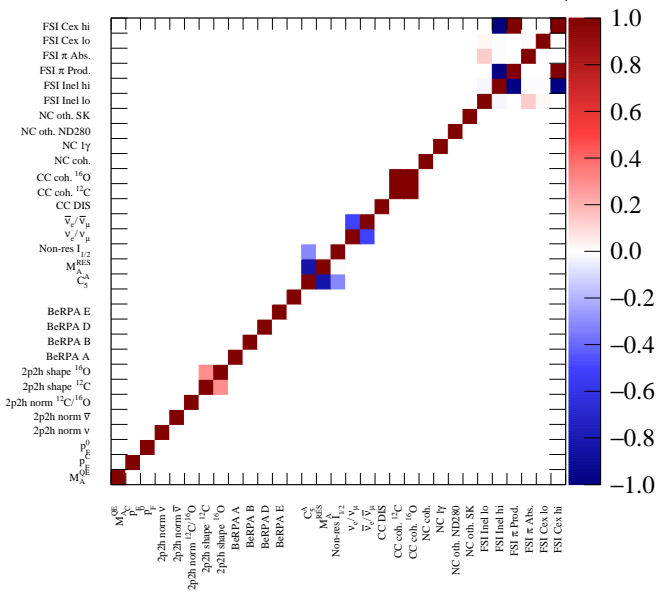

(c) Prefit correlation (b) Postfit covariance: $\operatorname{sign}\left(\mathrm{V}_{\mathrm{ij}}\right) \times \sqrt{\left|\mathrm{V}_{\mathrm{ij}}\right|}$

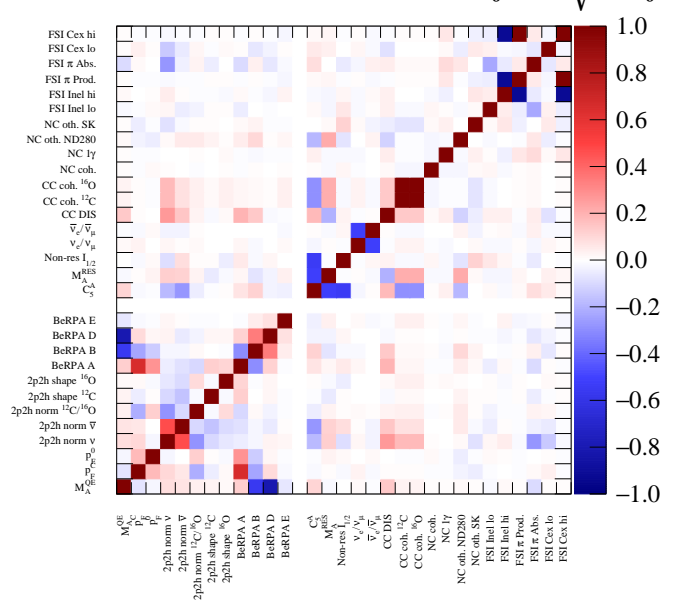

(d) Postfit correlation

Figure 8.12: Covariance and correlation among cross section parameters before (left) and after (right) the fit to ND280 data. Replica-tuned flux priors are inputs for this fit. 
8. Joint $\nu+\bar{\nu}$ T2K oscillation analysis with the reactor constraint and replica-tuned flux inputs
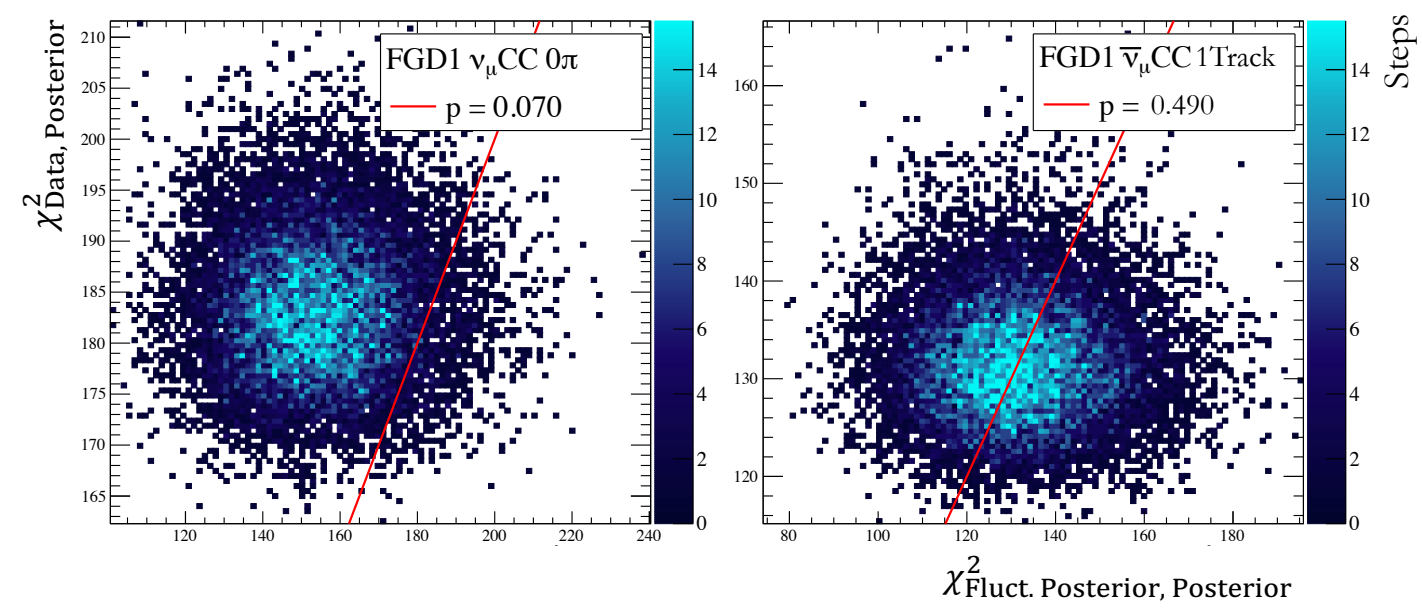

Figure 8.13: Testing the goodness of MCMC fits to ND280 CC- $0 \pi$ (left) and CC1Track (right) data samples at FGD1. The red line corresponds to $\chi_{\text {Data, }}^{2}$ Posterior $=$

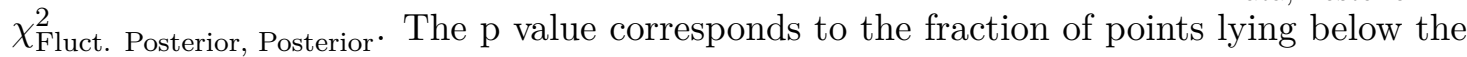
red line, and has been calculated for each sample. Fits with $\mathrm{p}>0.05$ are considered successful.

\begin{tabular}{c|c|c|c}
\hline \hline Sample & Target & $\begin{array}{c}\text { Thin fit } \\
\text { p-value }\end{array}$ & $\begin{array}{c}\text { Replica fit } \\
\text { p-value }\end{array}$ \\
\hline$\nu_{\mu}$ CC-0 $\pi$ & & 0.069 & 0.070 \\
$\nu_{\mu}$ CC-1 $\pi$ & & 0.082 & 0.083 \\
$\nu_{\mu}$ CC-Other & & 0.000 & 0.000 \\
$\bar{\nu}_{\mu}$ CC-1Track & FGD1 & 0.523 & 0.490 \\
$\bar{\nu}_{\mu}$ CC-NTrack & & 0.272 & 0.308 \\
$\nu_{\mu}$ CC-1Track & & 0.271 & 0.291 \\
$\nu_{\mu}$ CC-NTrack & & 0.857 & 0.848 \\
\hline$\nu_{\mu}$ CC-0 $\pi$ & & 0.110 & 0.099 \\
$\nu_{\mu}$ CC-1 $\pi$ & & 0.085 & 0.084 \\
$\nu_{\mu}$ CC-Other & & 0.095 & 0.099 \\
$\bar{\nu}_{\mu}$ CC-1Track & FGD2 & 0.275 & 0.300 \\
$\bar{\nu}_{\mu}$ CC-NTrack & & 0.222 & 0.224 \\
$\nu_{\mu}$ CC-1Track & & 0.327 & 0.315 \\
$\nu_{\mu}$ CC-NTrack & & 0.539 & 0.574 \\
\hline \hline
\end{tabular}

Table 8.2: Summary of p-values for MCMC fits to ND280 data, with different nominal flux inputs. Marginal differences are observed based on the method for the flux calculation. Fits to all data samples are successful $(\mathrm{p}>0.05)$ apart from CC-Other in FGD1. 
8. Joint $\nu+\bar{\nu}$ T2K oscillation analysis with the reactor constraint and replica-tuned flux inputs

\subsection{Joint fits to ND280 and Super-K data}

This section presents the results of joint fits to ND280 and Super-K data, in both neutrino and anti-neutrino mode, using the new replica-tuned flux inputs. Five SK data samples from T2K Runs 1-9 have been used, three in $\nu$-mode, FHC $1 \mathrm{R}_{\mu}, \mathrm{FHC}$ $1 \mathrm{R}_{e}$ and $\mathrm{FHC} 1 \mathrm{R}_{e} \mathrm{CC} 1 \pi^{+}$, and two in $\bar{\nu}$-mode, $\mathrm{RHC} 1 \mathrm{R}_{\mu}$ and $\mathrm{RHC} 1 \mathrm{R}_{e}$. For a reminder of data selection at Super-K, the reader is referred back to Chapter 3.5. The number of observed events in each of the Super-K samples is summarised in Table 8.4. The most abundant are $1 \mathrm{R}_{\mu}$ events, which are dominated by CCQE and 2p2h interactions of $\nu_{\mu}$ and $\bar{\nu}_{\mu}$. The ND280 Run 1-6 T2K data that is used in joint fits has already been discussed in Chapter 8.1.1. Predictions for different types of events in the single electron ring appearance samples, in both FHC and RHC modes of operation, are shown in Figure 8.14, alongside collected data. The biggest background in these samples are intrinsic $\nu_{e} / \bar{\nu}_{e}$ neutrinos in the beam produced at J-PARC, as well as $\mathrm{NC}$ events which pass the $1 \mathrm{R}_{e}$ selection cuts.

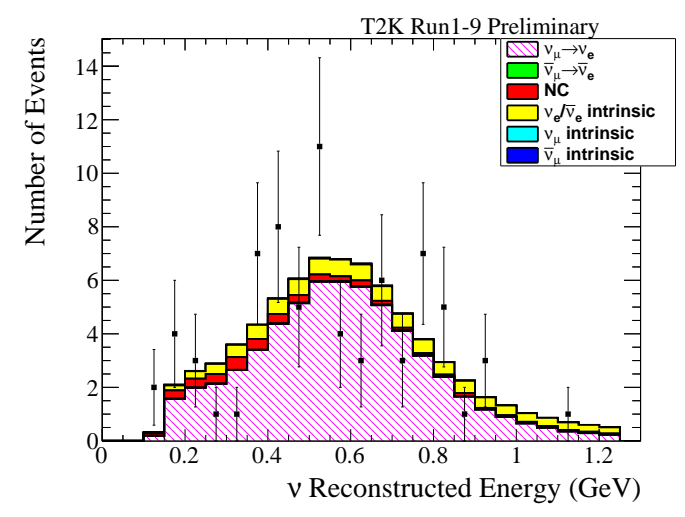

(a) $\mathrm{FHC} 1 \mathrm{R}_{e}$

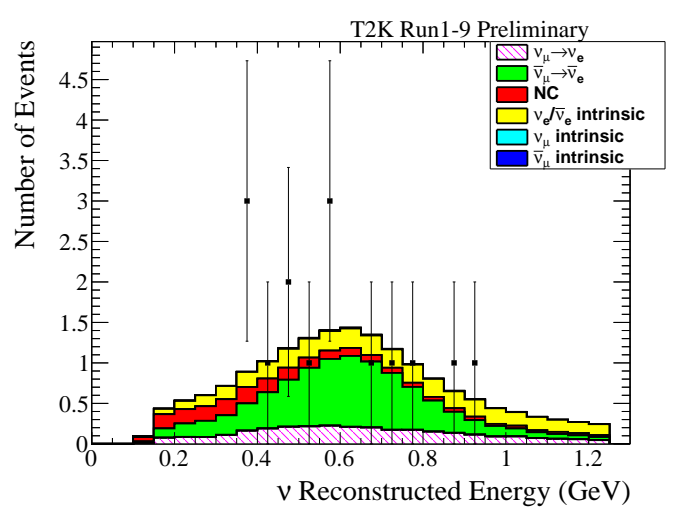

(b) $\mathrm{RHC} 1 \mathrm{R}_{e}$

Figure 8.14: Monte Carlo predictions for different types of events comprising the $1 \mathrm{R}_{e}$ sample at Super-K, in FHC (left) and RHC (right) modes of operation, plotted alongside Run 1-9 data. Copyright by T2K Collaboration.

\subsubsection{Asimov fits}

In T2K terminology, Asimov fits are fits to specific Monte Carlo simulated datasets, whose parameter values at every analysis bin are taken to be precisely at the MonteCarlo-predicted values for that parameter, with no statistical uncertainty. Real data 
8. Joint $\nu+\bar{\nu}$ T2K oscillation analysis with the reactor constraint and replica-tuned flux inputs

is always subject to statistical fluctuations. In fact, neutrino oscillation analyses usually suffer from limiting statistics due to the very nature of neutrinos as weakly interacting particles. The posterior parameter values calculated from Asimov fits are free from such data induced biases. Thus, Asimov fits are powerful tools for studying the performance of fitting algorithms with different data models, and are typically employed at T2K for consistency checks before proceeding with fits to real data.

The posterior flux, cross section and detector parameters from fits to ND280 data have been passed on as inputs for joint Asimov fits. Effectively, Asimov fits presented here are fits to real ND280 data and fake Super-K data. Different posterior flux inputs (thin-tuned and replica-tuned) have been used in these joint fits. In this study, the input oscillation parameters are taken to be:

$$
\begin{aligned}
& \sin ^{2} \theta_{12}=0.304 \\
& \sin ^{2} \theta_{23}=0.528 \\
& \sin ^{2} \theta_{13}=0.0219 \\
& \Delta m_{21}^{2}=7.53 \times 10^{-5} \mathrm{eV}^{2} \\
& \Delta m_{32}^{2}=2.509 \times 10^{-3} \mathrm{eV}^{2} \\
& \delta_{\mathrm{CP}}=-1.601
\end{aligned}
$$

The posterior predictive spectra at Super-K are given in Figure 8.15. These spectra have been calculated from 20,000 steps sampled at random from the joint Asimov MCMC. As expected, posterior spectra at Super-K with different flux inputs agree with each other within the assigned uncertainty, across all event samples and neutrino energies. This is because posterior flux and cross section parameters after fits to ND280 data, which are inputs for these Asimov fits, already show little variation among each other, irrespective of their pre-fit nominal values.

Finally, the $68 \%$ and $90 \%$ credible intervals and 2D best fit points for the measured oscillation parameters have been estimated. Confidence regions in $\sin ^{2} \theta_{13^{-}}$ $\delta_{\mathrm{CP}}$ and $\sin ^{2} \theta_{23}-\delta_{\mathrm{CP}}$ parameter space are given in Figure 8.16 and Figure 8.17, respectively. Measurement sensitivity to $\sin ^{2} \theta_{23}-\Delta m_{32}^{2}$ is examined in Figure 8.18. For each combination of parameter measurements, separate credible intervals have 
8. Joint $\nu+\bar{\nu}$ T2K oscillation analysis with the reactor constraint and replica-tuned flux inputs

been evaluated with normal $\left(\Delta m_{32}^{2}>0\right)$ and inverted $\left(\Delta m_{32}^{2}<0\right)$ mass hierarchy hypotheses, in addition to the case where no prior constraint is put on mass ordering (both hierarchies allowed). Overall, oscillation measurements prove remarkably robust under different flux inputs. The joint Asimov fits with replica-tuned flux have marginally lower $\delta_{\mathrm{CP}}$ and slightly higher $\sin ^{2} \theta_{23}$ sensitivity compared to fits using thin-tuned flux. Best fit values for $\delta_{\mathrm{CP}}, \sin ^{2} \theta_{13}$ and $\Delta m_{32}^{2}$ exhibit good agreement between the different fits. A slight variation in the $\sin ^{2} \theta_{23}$ best fit point is observed, most likely an artefact of marginalisation of posterior likelihood in 2D parameter space. The joint Asimov fit with replica-tuned flux has been estimated from 15 million MCMC steps before burn-in (11.25 million steps after burn-in). In Table 8.3, the preference of Asimov data for neutrino mass ordering and the $\sin ^{2} \theta_{23}$ octant has been calculated. Both versions of Asimov fits prefer the normal mass hierarchy and upper $\sin ^{2} \theta_{23}$ octant $\left(\sin ^{2} \theta_{23}>0.5\right)$. The normal ordering likelihood, given the Asimov data, is decreased by $2.8 \%$ when using replica-tuned compared to thin-tuned flux priors. Similarly, the probability for the upper octant gets reduced by $3.3 \%$. As expected, both Asimov fits contain sufficiently many Markov chain steps to successfully describe their respective Asimov datasets, with Bayesian p values in both cases above 40\%. Having observed good performance of joint Asimov fits with the new replica-tuned flux inputs, the new flux calculation will now be implemented in joint fits to real T2K Run 1-9 data in the next chapter.

\begin{tabular}{c|c|c|c} 
& $\sin ^{2} \theta_{23}<0.5$ & $\sin ^{2} \theta_{23}>0.5$ & Sum \\
\hline Normal hierarchy & $0.210(0.197)$ & $0.528(0.575)$ & $0.739(0.772)$ \\
Inverted hierarchy & $0.063(0.048)$ & $0.199(0.180)$ & $0.261(0.228)$ \\
\hline Sum & $0.273(0.245)$ & $0.727(0.755)$ & 1
\end{tabular}

Table 8.3: Summary of probabilities for the upper and lower octant, and normal and inverted mass hierarchy, for the posterior of joint Asimov fits with the reactor constraint. This fit uses replica-tuned pre-fit flux inputs. The probabilities from fits with thin-tuned pre-fit flux inputs are given in the brackets. 
8. Joint $\nu+\bar{\nu}$ T2K oscillation analysis with the reactor constraint and replica-tuned flux inputs

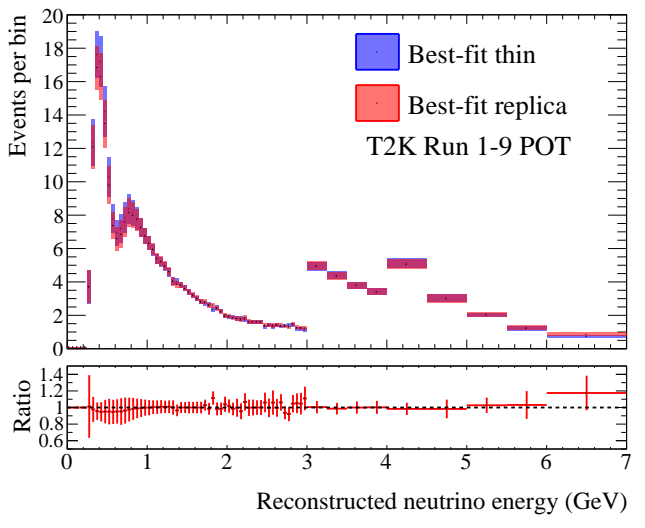

(a) $\mathrm{FHC} 1 \mathrm{R}_{\mu}$

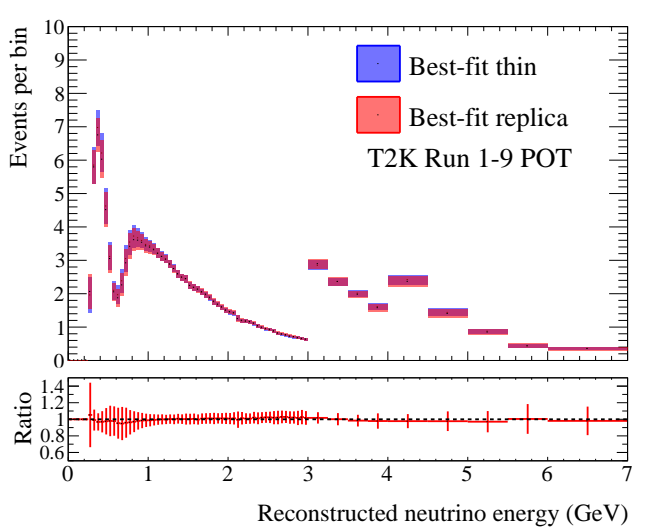

(c) $\mathrm{RHC} 1 \mathrm{R}_{\mu}$

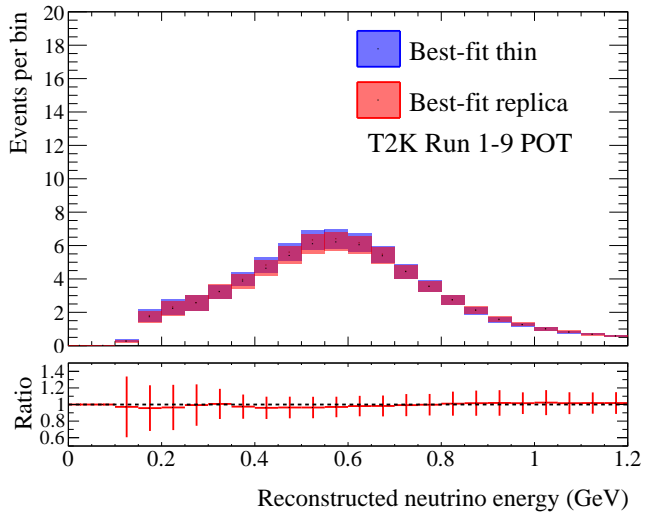

(b) $\mathrm{FHC} 1 \mathrm{R}_{e}$

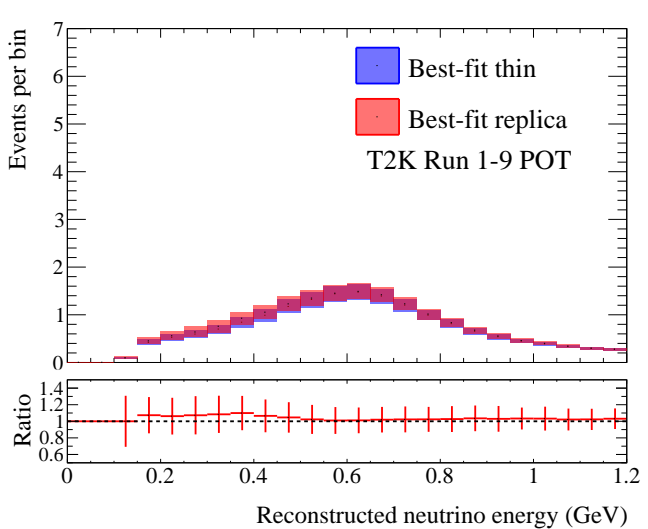

(d) $\mathrm{RHC} 1 \mathrm{R}_{e}$

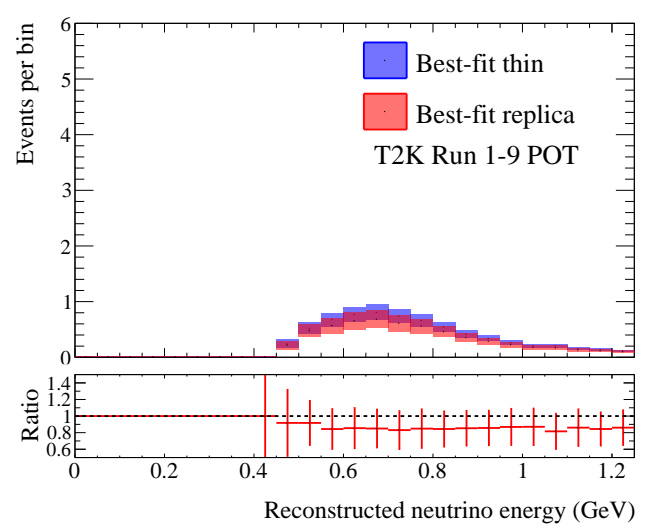

(e) $\mathrm{FHC} 1 \mathrm{R}_{e} \mathrm{CC} 1 \pi^{+}$

Figure 8.15: Posterior predictive spectra of joint Asimov fits with the reactor constraint. 
8. Joint $\nu+\bar{\nu}$ T2K oscillation analysis with the reactor constraint and replica-tuned flux inputs

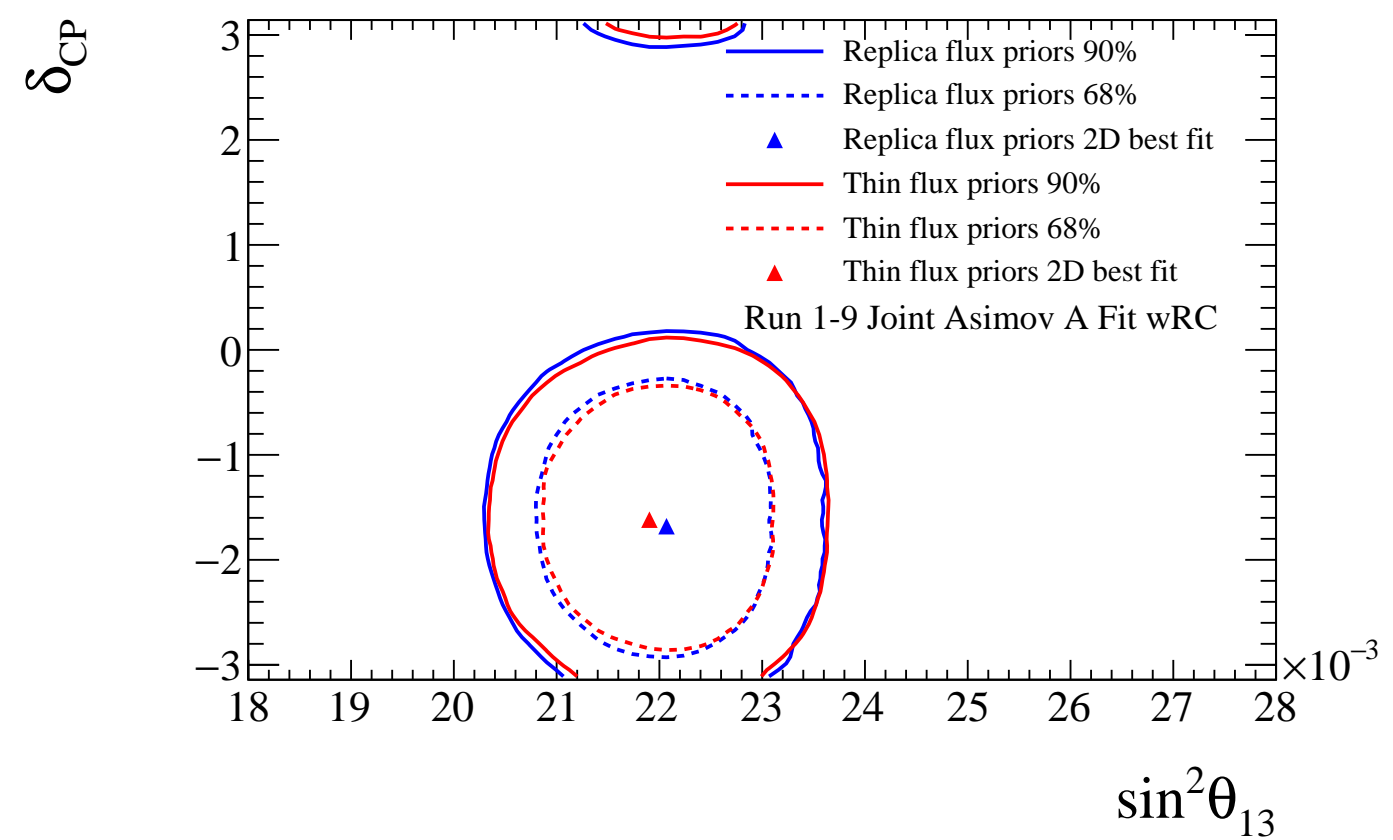

(a) Both mass hierarchies

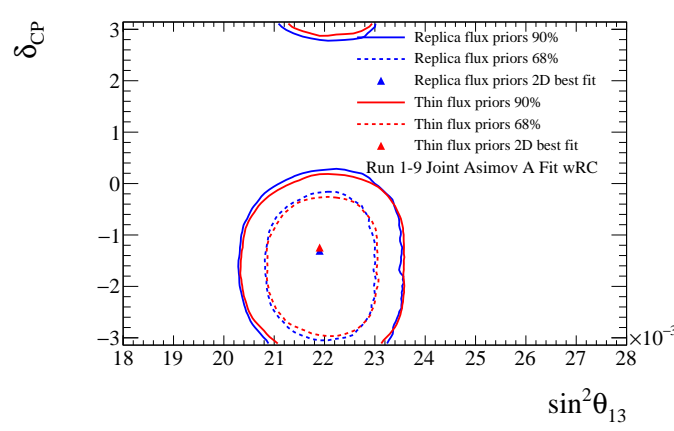

(b) Normal mass hierarchy

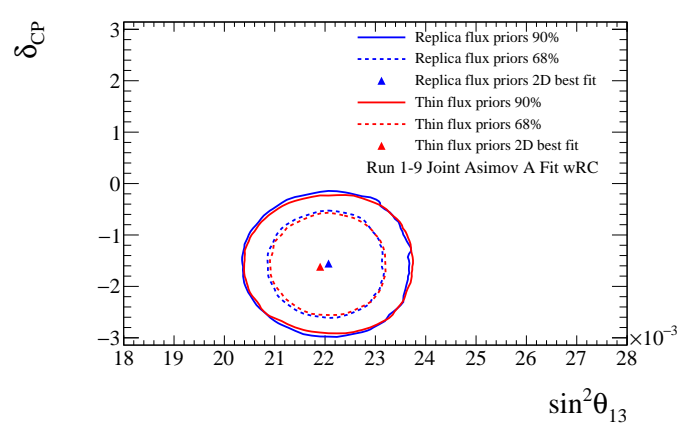

(c) Inverted mass hierarchy

Figure 8.16: Credible intervals in $\sin ^{2} \theta_{13}-\delta_{\mathrm{CP}}$, from joint Asimov A fits with the $\sin ^{2} \theta_{13}=(2.19 \pm 0.08) \times 10^{-2}$ reactor constraint. The best fit point in the two-dimensional $\sin ^{2} \theta_{13}-\delta_{\mathrm{CP}}$ posterior mode has been labelled. Fits with replica-tuned flux priors are shown in blue. Fits with thin-tuned flux priors are shown in red. 
8. Joint $\nu+\bar{\nu}$ T2K oscillation analysis with the reactor constraint and replica-tuned flux inputs

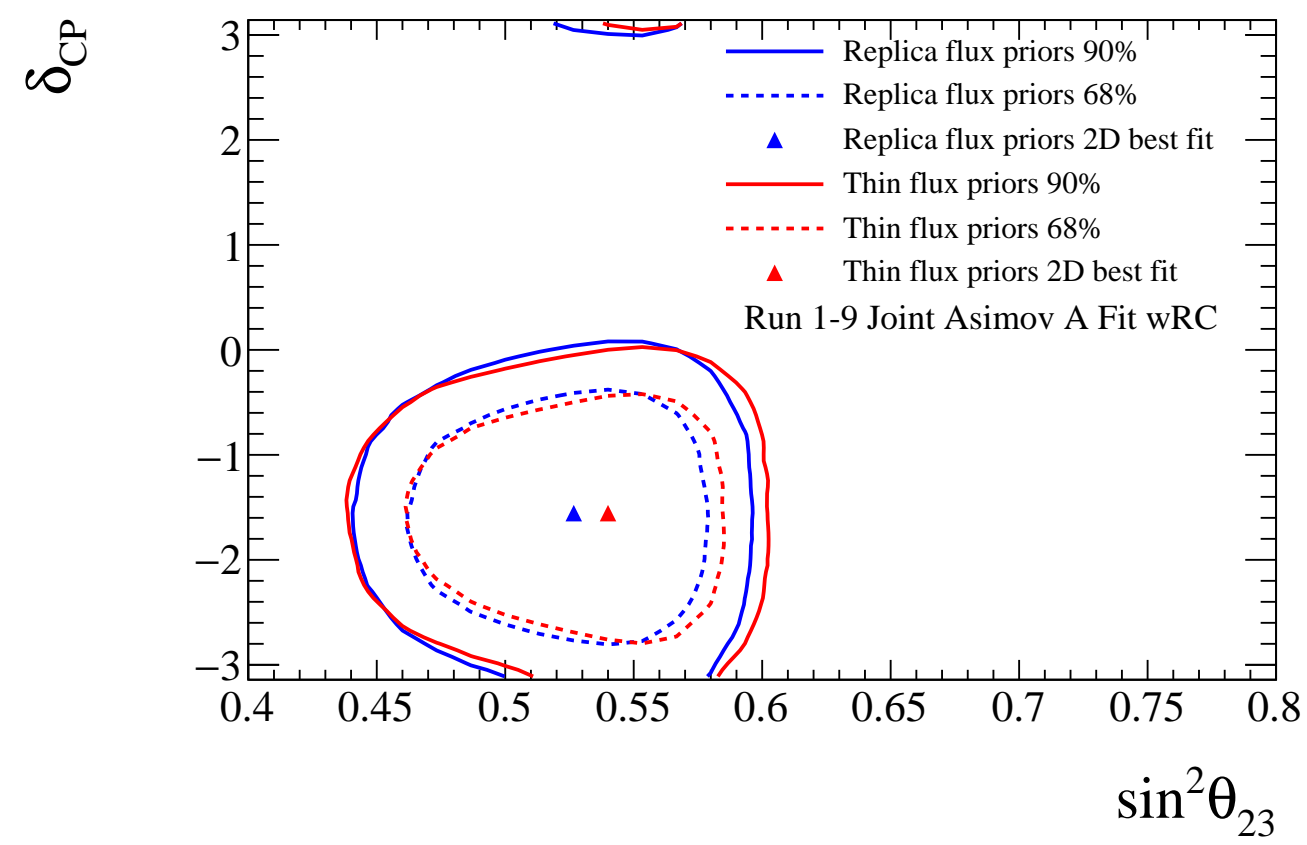

(a) Both mass hierarchies

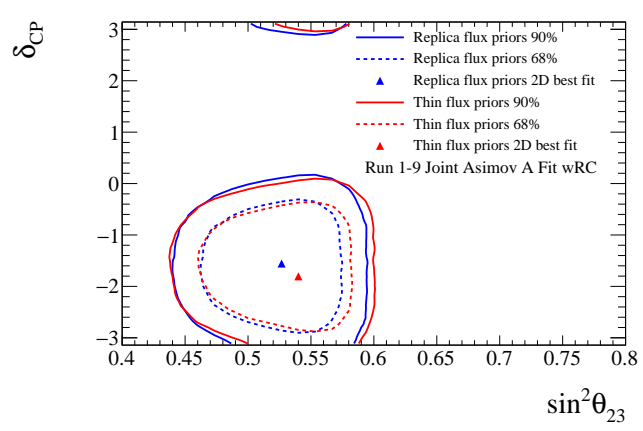

(b) Normal mass hierarchy

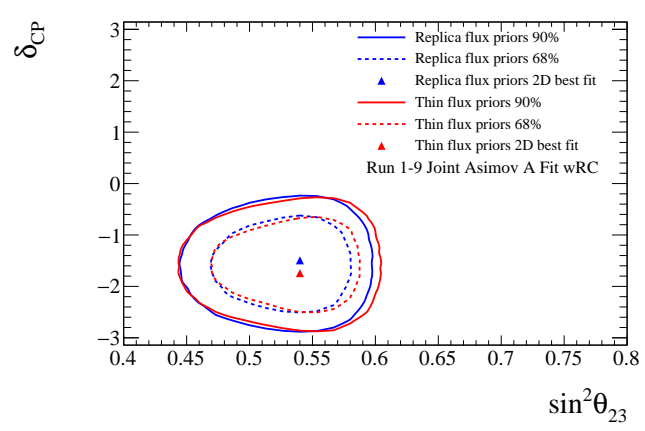

(c) Inverted mass hierarchy

Figure 8.17: Credible intervals in $\sin ^{2} \theta_{23}-\delta_{\mathrm{CP}}$, from joint Asimov A fits with the $\sin ^{2} \theta_{13}=(2.19 \pm 0.08) \times 10^{-2}$ reactor constraint. The best fit point in the two-dimensional $\sin ^{2} \theta_{23}-\delta_{\mathrm{CP}}$ posterior mode has been labelled. Fits with replica-tuned flux priors are shown in blue. Fits with thin-tuned flux priors are shown in red. 
8. Joint $\nu+\bar{\nu}$ T2K oscillation analysis with the reactor constraint and replica-tuned flux inputs

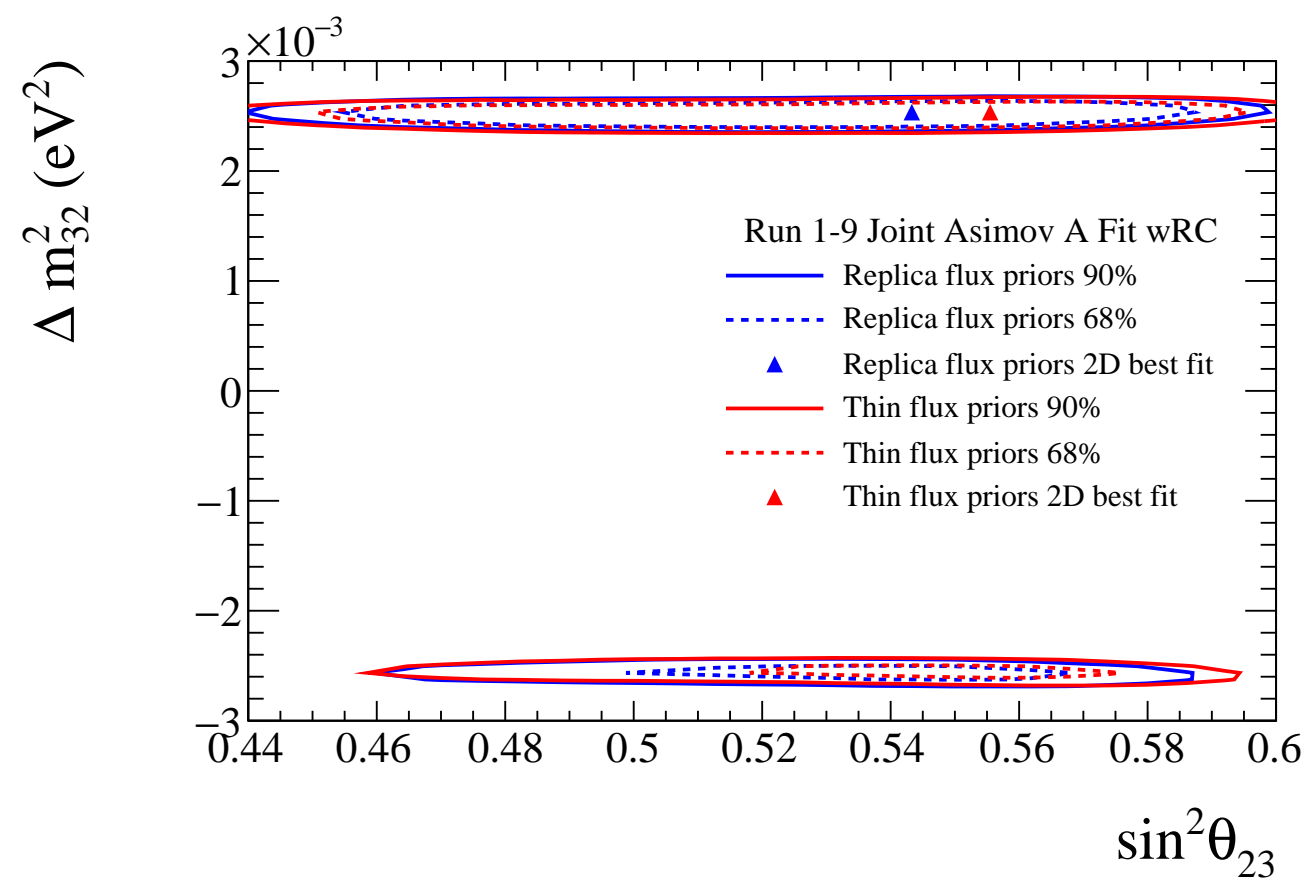

(a) Both mass hierarchies

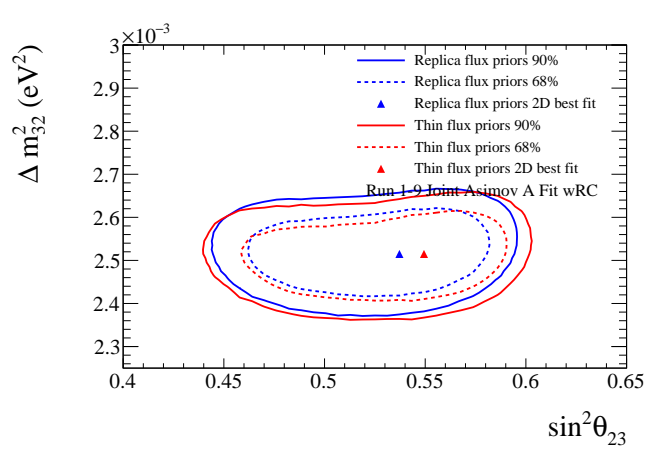

(b) Normal mass hierarchy

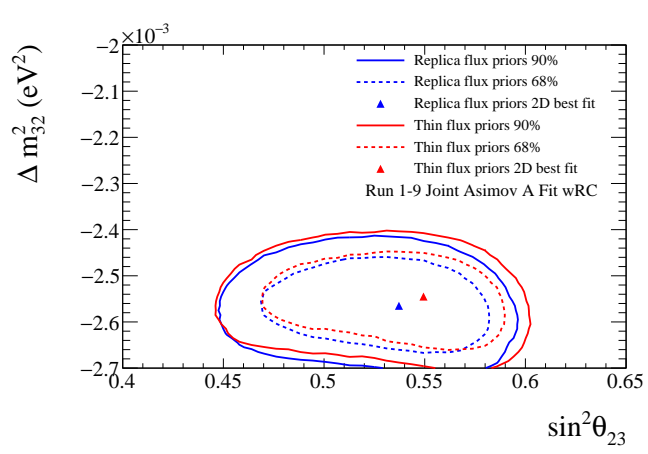

(c) Inverted mass hierarchy

Figure 8.18: Credible intervals in $\sin ^{2} \theta_{23}-\Delta m_{32}^{2}$, from joint Asimov $\mathrm{A}$ fits with the $\sin ^{2} \theta_{13}=(2.19 \pm 0.08) \times 10^{-2}$ reactor constraint. The best fit point in the two-dimensional $\sin ^{2} \theta_{23}-\Delta m_{32}^{2}$ posterior mode has been labelled. Fits with replica-tuned flux priors are shown in blue. Fits with thin-tuned flux priors are shown in red. 
8. Joint $\nu+\bar{\nu}$ T2K oscillation analysis with the reactor constraint and replica-tuned flux inputs

\subsubsection{Fits to combined $\nu+\bar{\nu}$ data}

Here, the effect of the replica-tuned flux calculation on the oscillation analysis with T2K Run 1-9 data has been examined. The posterior predictive number of events in each of the Super-K samples is given in Table 8.4, for fits with both thin-tuned and replica-tuned flux. The reader should not be surprised by the seemingly large discrepancy between the 15 observed and 6.9 expected (prior prediction with thintuned flux) events in the $\mathrm{FHC} 1 \mathrm{R}_{e} \mathrm{CC} 1 \pi^{+}$sample. In [9], it was calculated that there is a $12 \%$ chance to observe a fluctuation of such a significance in one of the five Super-K samples. Posterior predictive spectra as function of the reconstructed neutrino energy are given in Figure 8.19, alongside the measured T2K Run 1-9 spectra. Typically, the posterior spectra describe the data within the measurement uncertainty. Notably, joint fits with replica-tuned flux, compared to fits with thintuned flux, predict slightly fewer $1 \mathrm{R}_{e} \mathrm{CC} 1 \pi^{+}$, and marginally fewer events in the low and high energy tails of the $\mathrm{RHC} 1 \mathrm{R}_{e}$ distribution. The sensitivity of the $\mathrm{T} 2 \mathrm{~K}$ experiment to measurements of $\delta_{\mathrm{CP}}$ can be best appreciated from Table 8.5, showing the Monte Carlo predictions for the number of events at Super-K. As $\delta_{\mathrm{CP}}$ is varied, the expected number of events in the $\mathrm{FHC} 1 \mathrm{R}_{e}$ sample changes by $\sim 30 \%$, whereas the systematic uncertainty for the expected number of events corresponds to $\sim 9 \%$. The credible intervals and $2 \mathrm{D}$ best fit points are given in Figure 8.20 for $\sin ^{2} \theta_{13^{-}}$ $\delta_{\mathrm{CP}}$, Figure 8.21 for $\sin ^{2} \theta_{23}-\delta_{\mathrm{CP}}$ and Figure 8.22 for $\sin ^{2} \theta_{23}-\Delta m_{32}^{2}$. No significant difference between best-fit points has been observed with different versions of flux inputs. The constraint placed on neutrino mass hierarchy and $\sin ^{2} \theta_{23}$ octant from T2K Run 1-9 data is summarised in Table 8.6, with no clear difference observed between the fits. It is customary for $\Delta m_{32}^{2}$ and $\sin ^{2} \theta_{23}$ to be presented in form of two-dimensional credible intervals, because of high correlations between these oscillation parameters. The joint data fit with replica-tuned flux has been calculated from 21 million MCMC steps before burn-in (15 million steps after burn-in). The goodness-of-fit has been estimated using the same approach as for near detector fits. Again, the percentage of samples for which the observed data fits the MC prediction better than fake data is calculated. Fake data is obtained by making 20,000 Poisson 
8. Joint $\nu+\bar{\nu}$ T2K oscillation analysis with the reactor constraint and replica-tuned flux inputs

throws given the expected $\mathrm{MC}$ event number, and then randomly sampling these events from the MC spectrum. For joint data fits with replica-tuned flux, T2K data is better described by the MC prediction than by fake data for $68.8 \%$ of samples, as shown in Figure 8.23. Thus, there is no indication of disagreement between data and the best-fit MC prediction. For completeness, individual p-values for each of the Super-K samples, for both versions of nominal flux inputs, are given in Table 8.7.

\begin{tabular}{c|c|c|c|c|c} 
Sample & ${\mathrm{FHC} 1 \mathrm{R}_{\mu}}$ & $\mathrm{FHC} 1 \mathrm{R}_{e}$ & $\mathrm{FHC} 1 \mathrm{R}_{e} \mathrm{CC} 1 \pi^{+}$ & $\mathrm{RHC} 1 \mathrm{R}_{\mu}$ & $\mathrm{RHC} 1 \mathrm{R}_{e}$ \\
\hline Observed & 243 & 75 & 15 & 140 & 15 \\
Post-fit thin & 268.9 & 70.2 & 9.4 & 134.2 & 17.7 \\
Post-fit replica & 266.7 & 68.4 & 8.0 & 134.1 & 16.9
\end{tabular}

Table 8.4: Observed and posterior predicted number of events in each Super-K sample. The posterior predictions are from joint T2K Run 1-9 data fits with the reactor constraint, using either thin-tuned or replica-tuned fluxes as MCMC inputs. The accompanying posterior predictive event distributions at Super-K, as function of the reconstructed neutrino energy, are given in Figure 8.19.

\begin{tabular}{c|c|c|c|c|c} 
Sample & $\delta_{\mathrm{CP}}=-\pi / 2$ & $\delta_{\mathrm{CP}}=0$ & $\delta_{\mathrm{CP}}=\pi / 2$ & $\delta_{\mathrm{CP}}=\pi$ & Observed \\
\hline${\mathrm{FHC} 1 \mathrm{R}_{\mu}}_{\mathrm{FHC} 1 \mathrm{R}_{e}}$ & 272.4 & 272.0 & 272.4 & 272.8 & 243 \\
${\mathrm{FHC} 1 \mathrm{R}_{e} \mathrm{CC} 1 \pi^{+}}_{\mathrm{RHC} 1 \mathrm{R}_{\mu}}^{74.4}$ & 62.2 & 50.6 & 62.7 & 75 \\
$\mathrm{RHC} 1 \mathrm{R}_{e}$ & 139.5 & 6.1 & 4.9 & 5.9 & 15 \\
& 17.1 & 139.2 & 139.5 & 139.9 & 140 \\
& & 19.4 & 21.7 & 19.3 & 15
\end{tabular}

Table 8.5: Predicted numbers of events in different Super-K samples, after the datadriven ND280 constraint. The Monte Carlo method used for obtaining the predictions assumed normal mass ordering, with $\Delta m_{32}^{2}=2.51 \times 10^{-3}, \sin ^{2} \theta_{23}=0.528$ and $\sin ^{2} \theta_{13}=$ 0.0212 .

\begin{tabular}{c|c|c|c} 
& $\sin ^{2} \theta_{23}<0.5$ & $\sin ^{2} \theta_{23}>0.5$ & Sum \\
\hline Normal hierarchy & $0.180(0.184)$ & $0.708(0.705)$ & $0.888(0.889)$ \\
Inverted hierarchy & $0.0203(0.021)$ & $0.0915(0.090)$ & $0.112(0.111)$ \\
\hline Sum & $0.2(0.205)$ & $0.8(0.795)$ & 1
\end{tabular}

Table 8.6: Summary of probabilities for the upper and lower octant, and normal and inverted mass hierarchy, for the posterior of joint Run 1-9 T2K data fits with the reactor constraint. This fit uses replica-tuned pre-fit flux inputs. The probabilities from fits with thin-tuned pre-fit flux inputs are given in the brackets. 
8. Joint $\nu+\bar{\nu}$ T2K oscillation analysis with the reactor constraint and replica-tuned flux inputs

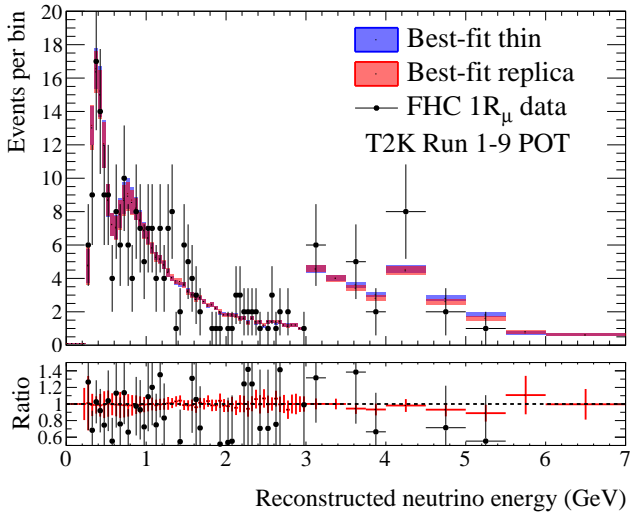

(a) $\mathrm{FHC} 1 \mathrm{R}_{\mu}$

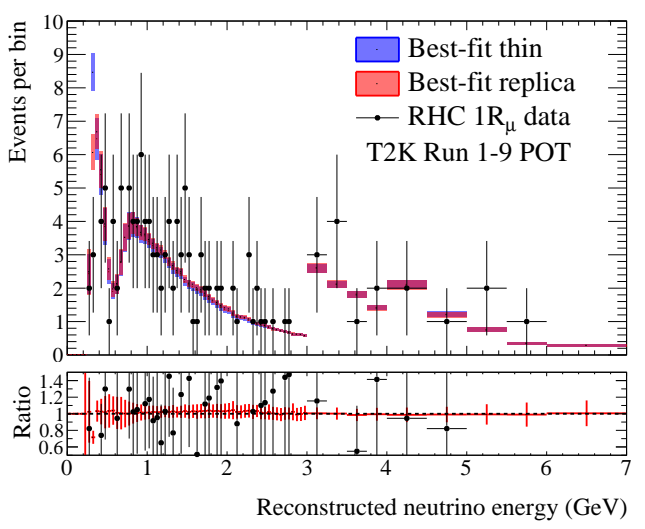

(c) $\mathrm{RHC} 1 \mathrm{R}_{\mu}$
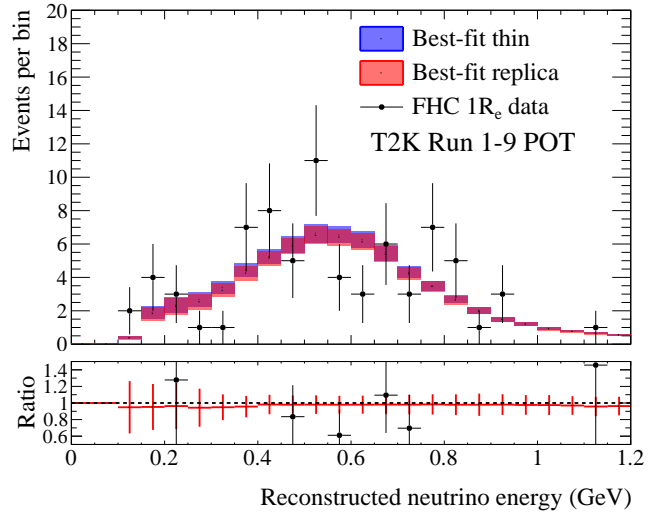

(b) $\mathrm{FHC} 1 \mathrm{R}_{e}$

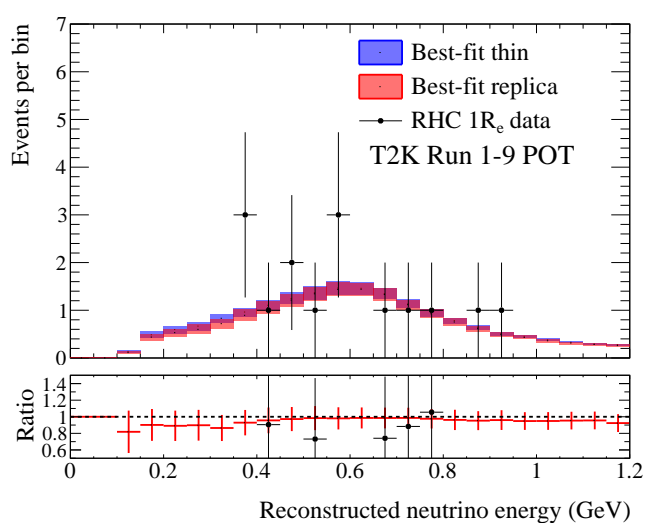

(d) $\mathrm{RHC} 1 \mathrm{R}_{e}$

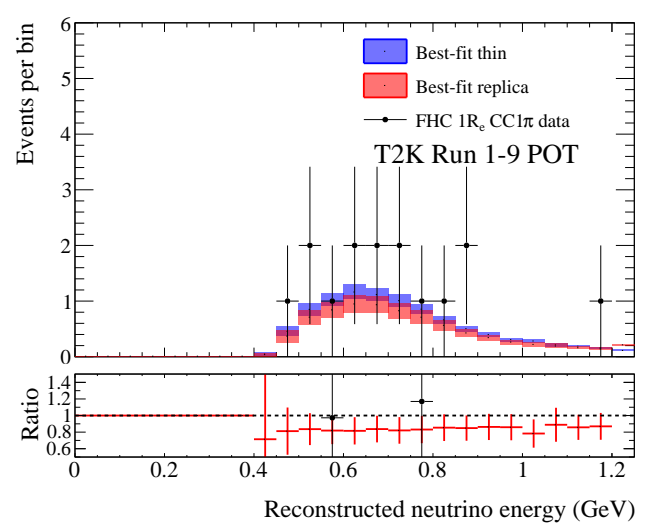

(e) $\mathrm{FHC} 1 \mathrm{R}_{e} \mathrm{CC} 1 \pi^{+}$

Figure 8.19: Posterior predictive spectra of joint Run 1-9 T2K data fits with the reactor constraint. 
8. Joint $\nu+\bar{\nu}$ T2K oscillation analysis with the reactor constraint and replica-tuned flux inputs

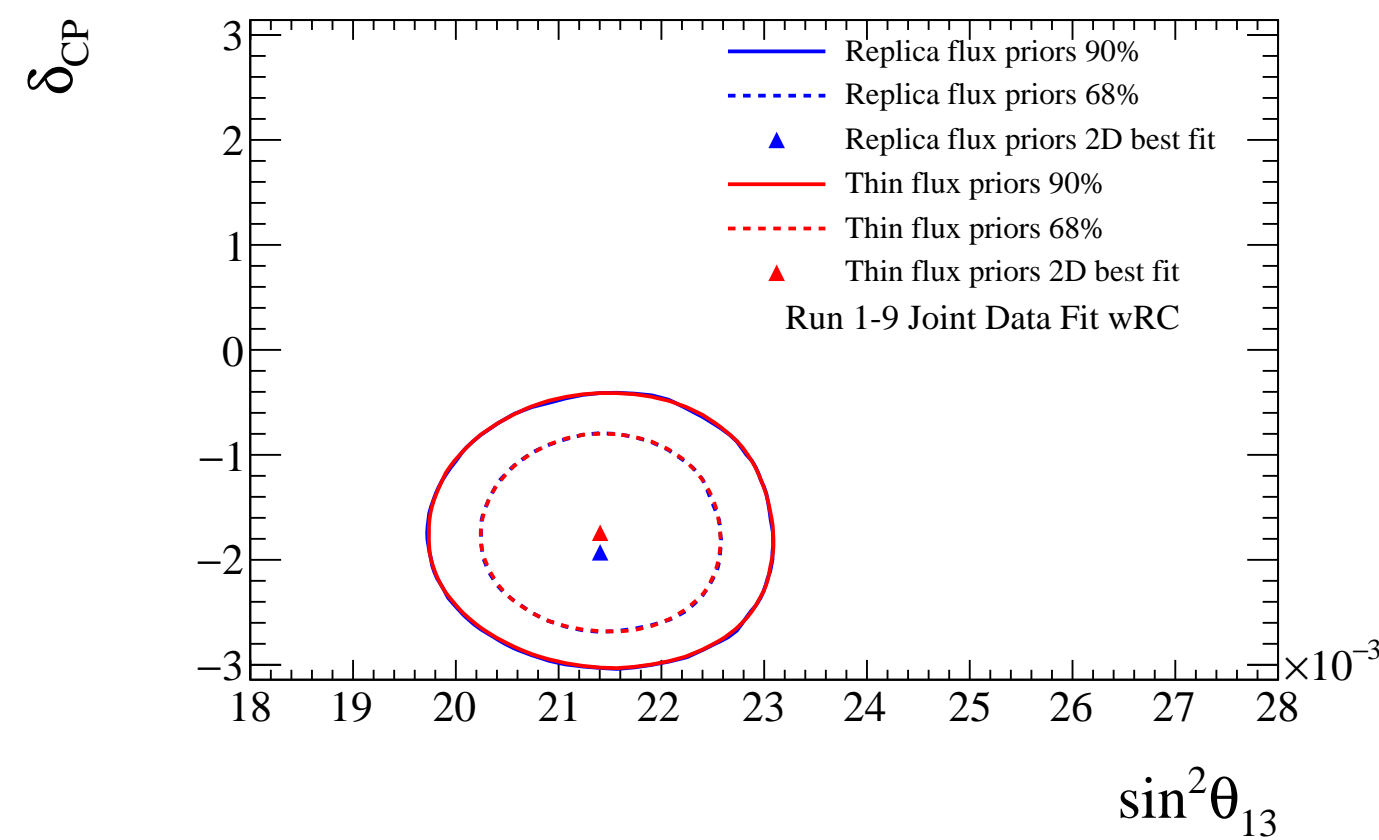

(a) Both mass hierarchies

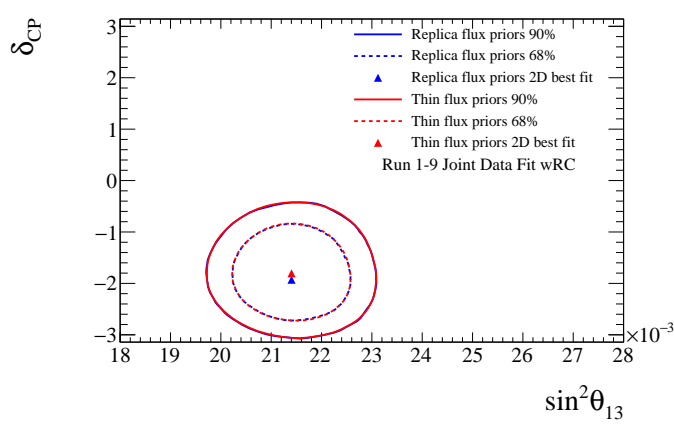

(b) Normal mass hierarchy

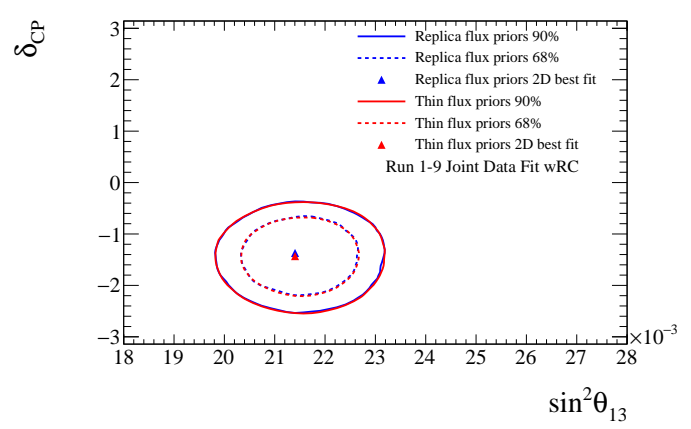

(c) Inverted mass hierarchy

Figure 8.20: Credible intervals in $\sin ^{2} \theta_{13}-\delta_{\mathrm{CP}}$, from joint data fits with the $\sin ^{2} \theta_{13}=$ $(2.12 \pm 0.08) \times 10^{-2}$ reactor constraint. The best fit point in the two-dimensional $\sin ^{2} \theta_{13^{-}}$ $\delta_{\mathrm{CP}}$ posterior mode has been labelled. Fits with replica-tuned flux priors are shown in blue. Fits with thin-tuned flux priors are shown in red. 
8. Joint $\nu+\bar{\nu}$ T2K oscillation analysis with the reactor constraint and replica-tuned flux inputs

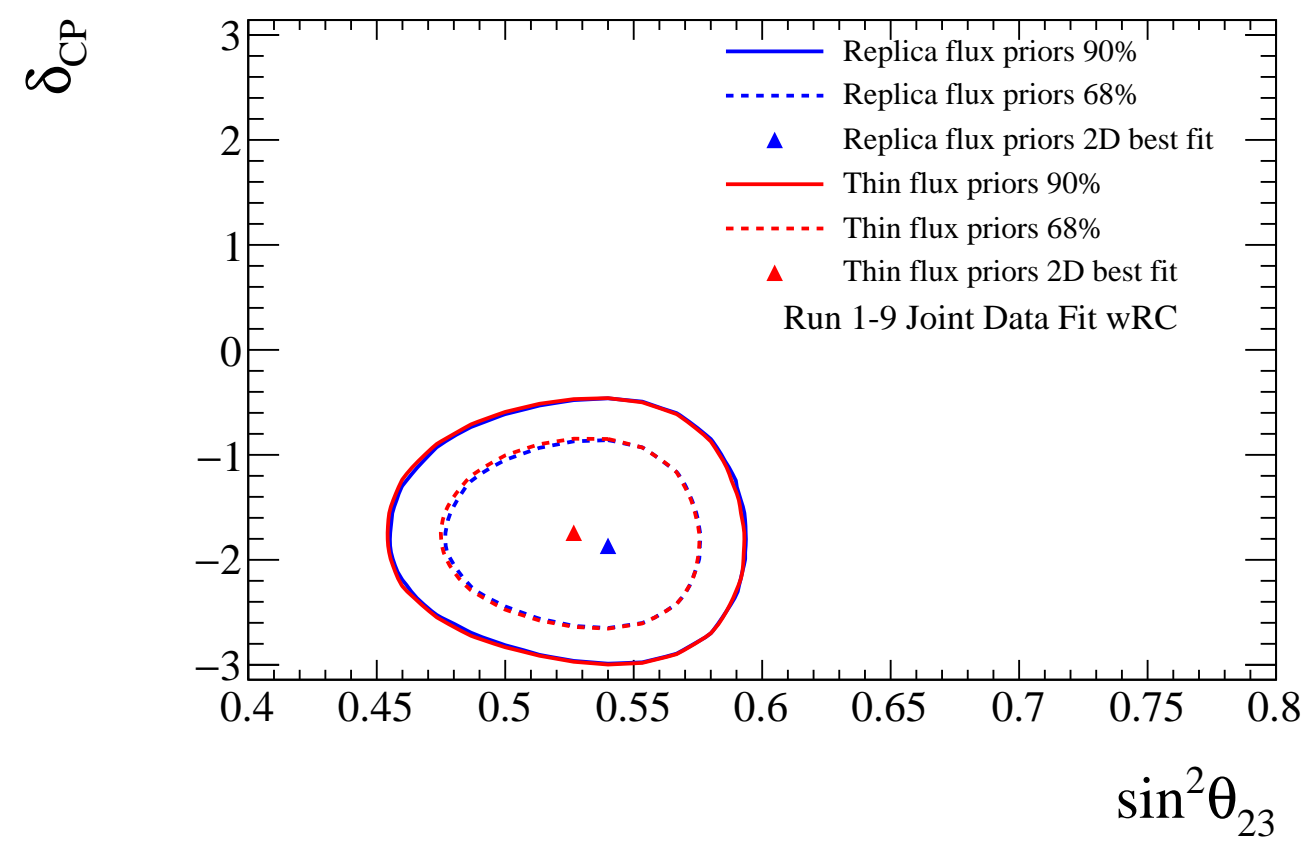

(a) Both mass hierarchies

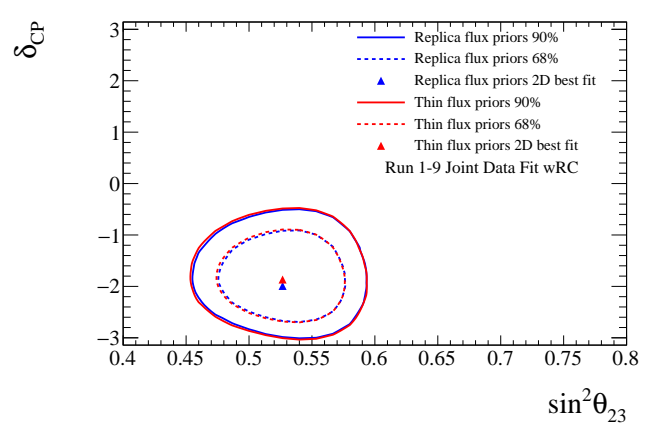

(b) Normal mass hierarchy

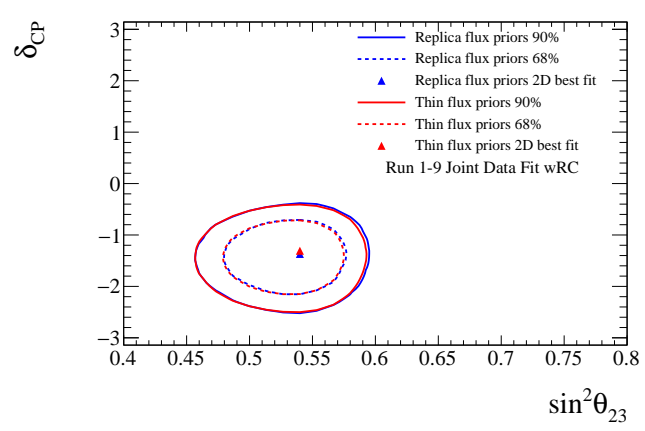

(c) Inverted mass hierarchy

Figure 8.21: Credible intervals in $\sin ^{2} \theta_{23}-\delta_{\mathrm{CP}}$, from joint data fits with the $\sin ^{2} \theta_{13}=$ $(2.12 \pm 0.08) \times 10^{-2}$ reactor constraint. The best fit point in the two-dimensional $\sin ^{2} \theta_{23^{-}}$ $\delta_{\mathrm{CP}}$ posterior mode has been labelled. Fits with replica-tuned flux priors are shown in blue. Fits with thin-tuned flux priors are shown in red. 
8. Joint $\nu+\bar{\nu}$ T2K oscillation analysis with the reactor constraint and replica-tuned flux inputs

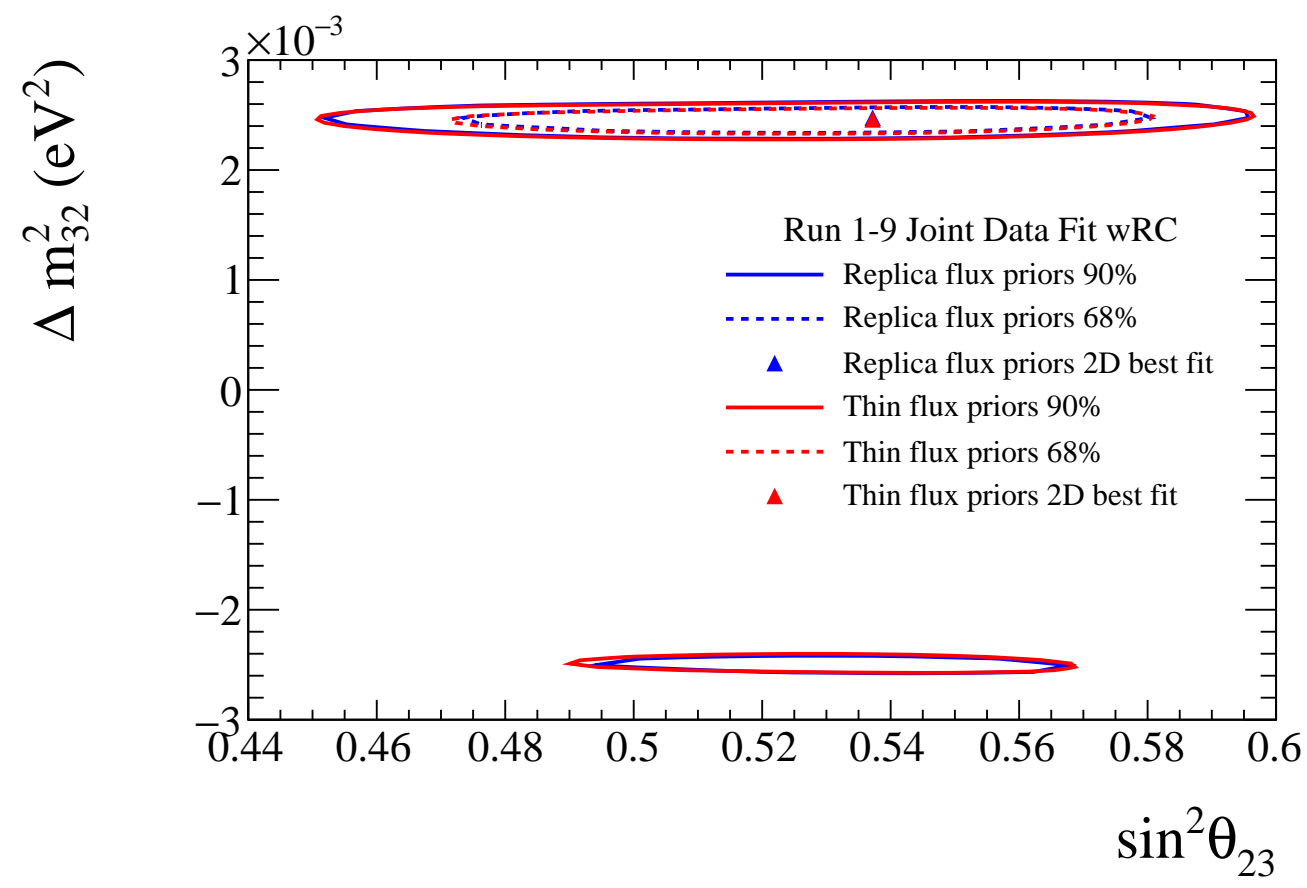

(a) Both mass hierarchies

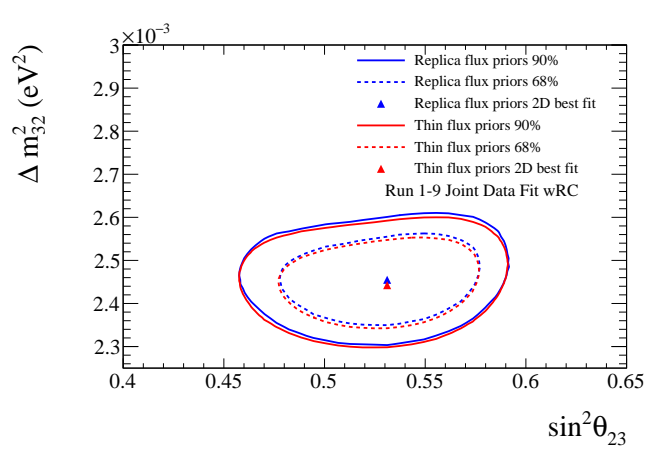

(b) Normal mass hierarchy

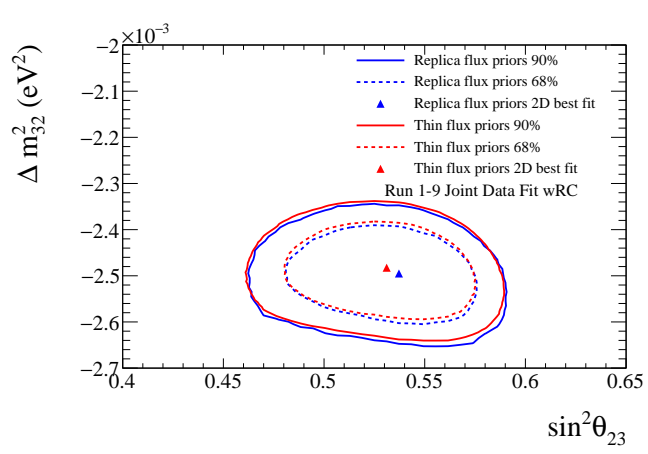

(c) Inverted mass hierarchy

Figure 8.22: Credible intervals in $\sin ^{2} \theta_{23}-\Delta m_{32}^{2}$, from joint data fits with the $\sin ^{2} \theta_{13}=$ $(2.12 \pm 0.08) \times 10^{-2}$ reactor constraint. The best fit point in the two-dimensional $\sin ^{2} \theta_{23^{-}}$ $\Delta m_{32}^{2}$ posterior mode has been labelled. Fits with replica-tuned flux priors are shown in blue. Fits with thin-tuned flux priors are shown in red. 
8. Joint $\nu+\bar{\nu}$ T2K oscillation analysis with the reactor constraint and replica-tuned flux inputs

\begin{tabular}{c|c|c|c|c|c} 
Sample & ${\mathrm{FHC} 1 \mathrm{R}_{\mu}}$ & $\mathrm{FHC} 1 \mathrm{R}_{e}$ & $\mathrm{FHC} 1 \mathrm{R}_{e} \mathrm{CC} 1 \pi^{+}$ & $\mathrm{RHC} 1 \mathrm{R}_{\mu}$ & $\mathrm{RHC} 1 \mathrm{R}_{e}$ \\
\hline Thin fit p-value & 0.43 & 0.13 & 0.84 & 0.87 & 0.82 \\
Replica fit p-value & 0.44 & 0.12 & 0.60 & 0.88 & 0.82
\end{tabular}

Table 8.7: Examining the goodness of joint fits, with different nominal flux inputs, to T2K Run 1-9 data samples. The quoted p-value gives the fraction of samples where measured data fits the posterior MC prediction better than fake data. The combined p-value for joint fits with replica-tuned nominal flux is 69\%, as shown in Figure 8.23

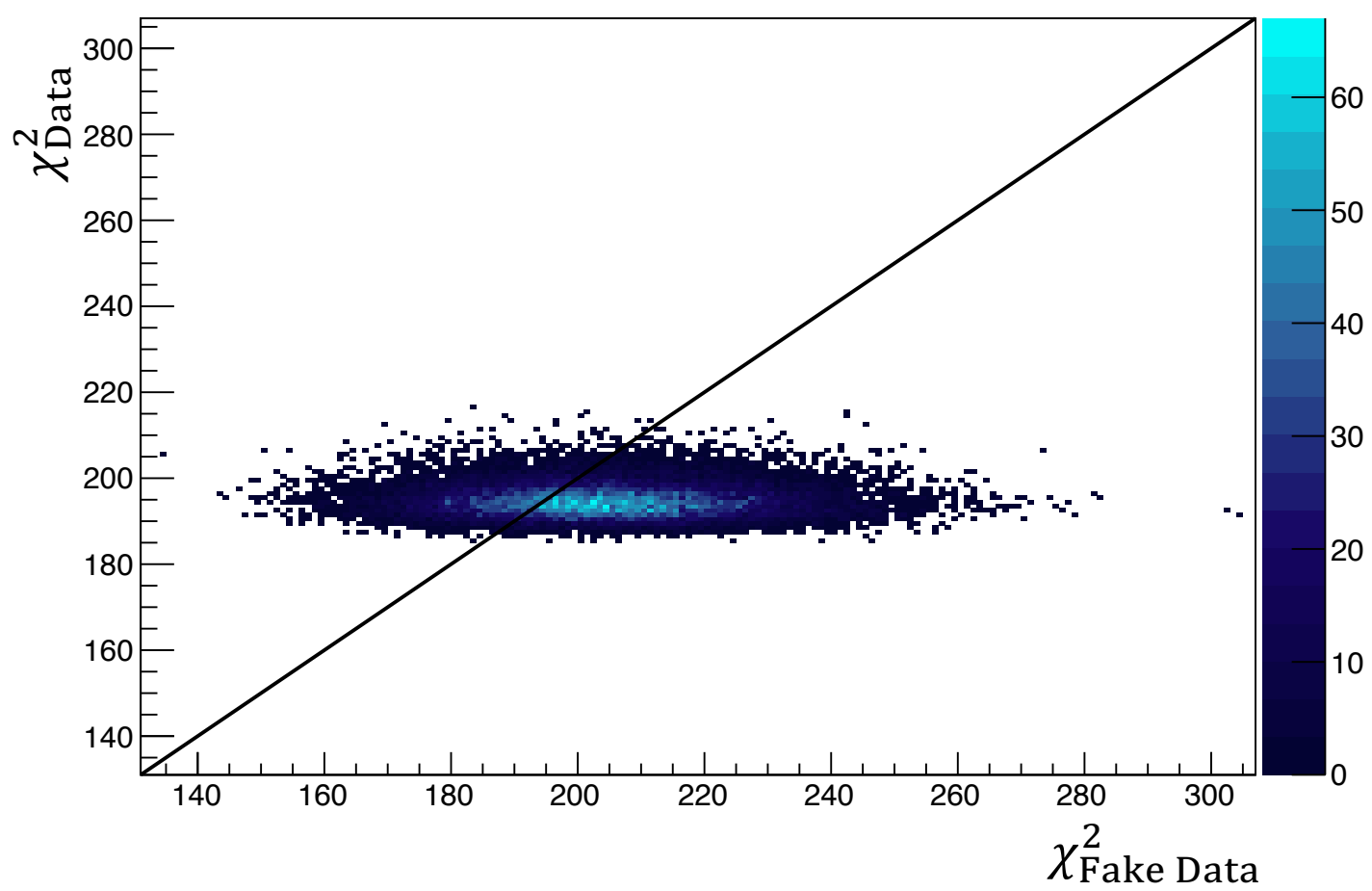

Figure 8.23: Testing the goodness of MCMC joint fits to T2K Run 1-9 data. The $\chi^{2}$ test statistic has been calculated for agreement between observed data and the MC prediction, denoted by $\chi_{\text {Data }}^{2}$, and for agreement between fake data and the MC prediction, $\chi_{\text {Fake Data }}^{2}$. For $69 \%$ of parameter sets sampled from the MCMC, the MC prediction is described by data better than fake data $\left(\chi_{\text {Data }}^{2}<\chi_{\text {Fake Data }}^{2}\right)$. 


\section{9 \\ Conclusions and future prospects}

Presented in this thesis is the first prediction of the unoscillated neutrino flux at T2K, using dedicated NA61 replica-target measurements from 2009. Previously, only external data collected with thin targets had been employed to tune the nominal, Monte Carlo based, T2K flux prediction. The flux calculation framework has been substantially modified to accommodate for the concept of tuning to thick target data. With the inclusion of this new dataset, the fractional systematic flux uncertainty has been reduced from $\sim 10 \%$ to $\sim 5 \%$.

This reduction of flux uncertainty has particularly significant implications for neutrino cross section modelling. For instance, the charged-current inclusive neutrino cross section on graphite measured at T2K has systematic errors ranging between $10 \%$ and $20 \%$ (see Figure 9.1), most of which come from flux uncertainties. Hence, with the improved flux model, it might be possible to put tighter constraints on the neutrino interaction model as well.

The effect of simply reducing the flux uncertainty does not impact the T2K oscillation analysis significantly, as has been demonstrated in Chapter 8. However, coupled with improvements in neutrino interaction modelling and expected reductions of other systematic uncertainties, it could still considerably improve the experiment's ability to draw conclusions about $\delta_{\mathrm{CP}}$, as shown in Figure 9.2. Assuming normal, but unknown, mass ordering, $\sin ^{2} \theta_{23}=0.5$ and maximally CP violating true $\delta_{\mathrm{CP}}=-\frac{\pi}{2}$, the ability to exclude $\delta_{\mathrm{CP}}=0, \pi$ at $3 \sigma$ confidence level is investigated as a function of accumulated POT[153]. With systematic improvements, including the $\sim 5 \%$ flux uncertainty achieved in this thesis, the sensitivity to exclude $\delta_{\mathrm{CP}}=0, \pi$ at $3 \sigma$ could be reached in $\sim 2024$, compared to $\sim 2026$ when assuming 


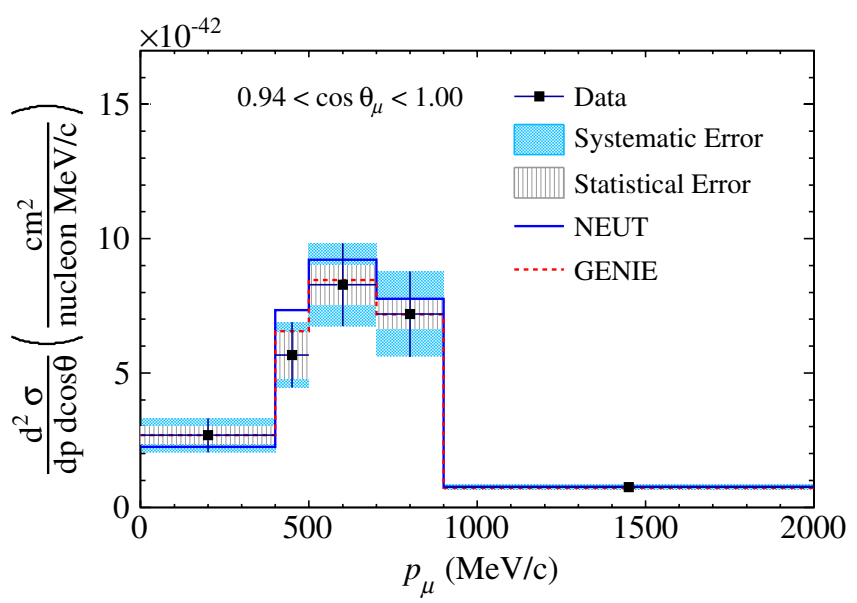

Figure 9.1: The CCQE inclusive differential cross section, for exiting muon angles $0.94<\cos \theta_{\mu}<1.00$, given as a function of muon momentum $p_{\mu}$. Predictions from NEUT[150] and GENIE[151] interaction generators are shown alongside data. Reprinted figure with permission from K. Abe et al. (T2K Collaboration), Physical Review D, Vol. 87, p. 092003 (2013)[152]. Copyright (2013) by the American Physical Society.

the same systematic errors as in the 2016 analysis ${ }^{1}$. Hence, reduction of systematic uncertainties is a significant factor in demonstrating the viability of the proposed extended T2K physics run, beyond $7.8 \times 10^{21}$ POT.

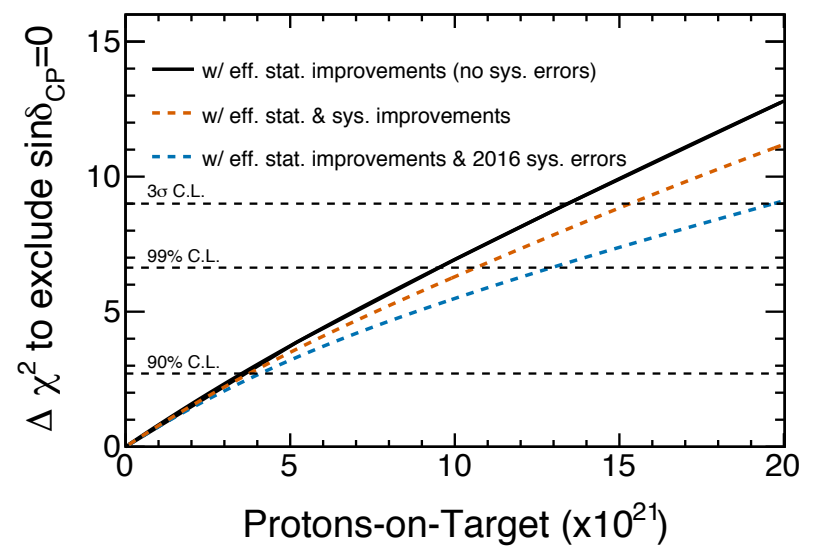

Figure 9.2: The potential of the $\mathrm{T} 2 \mathrm{~K}$ experiment to exclude $\sin \delta_{\mathrm{CP}}=0, \pi$ as a function of collected POT, and based on different assumptions regarding improvements in systematic parameters. The following has been calculated for the hypothesis that $\mathrm{CP}$ is maximally violated in the neutrino sector, $\sin \delta_{\mathrm{CP}}=\frac{\pi}{2}$, and assuming normal mass hierarchy and $\sin ^{2} \theta_{23}=0.5$. Figure reprinted from [153], with permission from K. Abe et al. (T2K Collaboration).

The study into additional hadron production measurements that could help

\footnotetext{
${ }^{1}$ Refer to [154] for details of the 2016 analysis systematics
} 
to further constrain the flux, described in Chapter 6.7, might be of interest when designing the target and beamline for the next generation of the T2K experiment, T2HK (Tokai-to-HyperK)[155]. Plans to further reduce the T2K flux uncertainty by measuring low angle pion and proton scattering on different materials using the EMPHATIC experiment[16, 156] are also in progress. Moreover, additional reductions of flux uncertainty at the high energy tail, above $\sim 1 \mathrm{GeV}$, are foreseen with the implementation of NA61 2010 replica-target dataset, which, in addition to pion multiplicities, includes also rates of kaons emitted from the replica-target. The NA61 collaboration is planning an ambitious programme of data taking after the long shutdown period at the Large Hadron Collider, one of the objectives of which will be to collect additional hadron production measurements with thin and extended targets. The NA61 DAQ system, readout electronics for the time projection chambers, beam trigger, vertex detector and time-of-flight walls are being upgraded[157]. These upgrades will facilitate a 10-fold increase of the data readout rate, and the overall reduction of systematic uncertainties of future NA61 measurements, with far reaching implications for high precision measurements of accelerator-based neutrino beams.

In summary, precise predictions of neutrino flux are inextricably linked to measurements of neutrino oscillations in both current and future neutrino experiments. The work presented in this thesis highlights a dramatic reduction in flux uncertainty achievable with dedicated ex-situ measurements of hadrons outgoing from replicatargets. I look forward to seeing the flux calculation with NA61 replica-target data used in the next published T2K oscillation result. 


\section{References}

[1] A. D. Sakharov. "Violation of CP Invariance, C Asymmetry, and Baryon Asymmetry of the Universe". In: Soviet Journal of Experimental and Theoretical Physics Letters 5 (Jan. 1967), p. 24.

[2] Antonio Riotto. Theories of Baryogenesis. 1998. arXiv: hep-ph/9807454 [hep-ph].

[3] A. Taroni. "Nobel Prize 2015: Kajita and McDonald". In: Nature Physics 11 (Oct. 2015), p. 891. URL: https://doi.org/10.1038/nphys3543.

[4] M. Fukugita and T. Yanagida. "Barygenesis without grand unification". In: Physics Letters B 174.1 (1986), pp. 45-47. URL: http://www.sciencedirect.com/science/article/pii/0370269386911263.

[5] Silvia Pascoli, S. Petcov, and Antonio Riotto. "Connecting Low Energy Leptonic CP-violation to Leptogenesis". In: Physical Review D 75 (Oct. 2006).

[6] C. Hagedorn et al. "CP violation in the lepton sector and implications for leptogenesis". In: International Journal of Modern Physics A 33.05n06 (2018), p. 1842006. URL: https://doi.org/10.1142/S0217751X1842006X.

[7] G. C. Branco, R. González Felipe, and F. R. Joaquim. "Leptonic CP violation". In: Rev. Mod. Phys. 84 (2 Apr. 2012), pp. 515-565. URL: https://link.aps.org/doi/10.1103/RevModPhys.84.515.

[8] K. Abe et al. (T2K Collaboration). "Constraint on the matter-antimatter symmetry-violating phase in neutrino oscillations". In: Nature 580 (7803 Apr. 2020), pp. 339-344. URL: https://doi.org/10.1038/s41586-020-2177-0.

[9] K. Abe et al. (T2K Collaboration). "Search for $C P$ Violation in Neutrino and Antineutrino Oscillations by the T2K Experiment with $2.2 \times 10^{21}$ Protons on Target". In: Phys. Rev. Lett. 121 (17 Oct. 2018), p. 171802. URL: https://link.aps.org/doi/10.1103/PhysRevLett.121.171802.

[10] Morgan Wascko. T2K Status, Results, and Plans. June 2018. URL: https://doi.org/10.5281/zenodo.1286752.

[11] Francis Bench. The T2K Experiment: Current Status and Results. 2019. URL: https://indico.cern.ch/event/773605/contributions/3498113/ attachments/1897012/3130048/NuFACT2019_T2K_plenary_FBench.pdf.

[12] Tomislav Vladisavljevic (On behalf of T2K Collaboration). "The T2K Neutrino Flux Predictions". In: Talk, 10th International Workshop on Neutrino Beams and Instrumentation (NBI2017): Tokai-mura, Japan, September 18-22, 2017. URL: https://conference-indico.kek.jp/indico/event/16/session/12/ contribution/15/material/slides/0.pdf.

[13] Tomislav Vladisavljevic (On behalf of T2K Collaboration). "Constraining the T2K Neutrino Flux Prediction with 2009 NA61/SHINE Replica-Target Data". In: Proceedings, Prospects in Neutrino Physics (NuPhys2017): London, UK, December 20-22, 2017. 2018, pp. 189-193. arXiv: 1804.00272 [physics.ins-det].

[14] Tomislav Vladisavljevic (On behalf of T2K Collaboration). "Constraining the T2K Neutrino Flux Prediction with 2009 NA61/SHINE Replica-Target Data". In: Poster, Neutrino2018 - XXVIII International Conference on Neutrino Physics and Astrophysics: Heidelberg, Germany, June 4-9, 2018. URL: https://doi.org/10.5281/zenodo. 1300546. 
[15] Tomislav Vladisavljevic (On behalf of T2K Collaboration). "The T2K Flux Prediction". In: Talk, 12th International Workshop on Neutrino-Nucleus Interactions in the Few-GeV Region (NuInt2018): Gran Sasso Science Institute, Italy, October 15-19, 2018. URL: https:

//indico.cern.ch/event/703880/contributions/3159044/attachments/ $1735667 / 2823451 /$ t2k_flux_calculations_nuint2018_vladisavljevic.pdf.

[16] Jonathan Paley. "EMPHATIC: A new hadron production experiment for improved neutrino flux predictions". In: Fermilab Physics Advisory Committee Meeting (2019). URL: https://indico.fnal.gov/event/19433/session/2/ contribution/7/material/slides/0.pdf.

[17] C. Sutton. "Spaceship Neutrino". In: Cambridge University Press, 1992.

[18] J Chadwick. "Intensitätsverteilung im magnetischen Spectrum der $\beta$-Strahlen von radium B + C". In: Verhandl. Dtsc. Phys. Ges. 16 (1914), p. 383. URL: https://cds.cern.ch/record/262756.

[19] Laurie M. Brown. "The idea of the neutrino". In: Physics Today 31.9 (Sept. 1978), pp. 23-28.

[20] J. Chadwick. "The Existence of a Neutron". In: Proceedings of the Royal Society of London Series A 136.830 (June 1932), pp. 692-708.

[21] C. L. Cowan, F. Reines, et al. "Detection of the Free Neutrino: a Confirmation". In: Science 124.3212 (1956), pp. 103-104. URL: https://science.sciencemag.org/content/124/3212/103.

[22] F. Reines and C. L. Cowan. "Free anti-neutrino absorption cross-section". In: Phys. Rev. 113 (1959), pp. 273-279.

[23] Martin Perl et al. "Evidence for anomalous lepton production in e + e annihilation". In: Physical Review Letters - PHYS REV LETT 35 (Jan. 1975), pp. 1489-1492.

[24] R. Brandelik et al. (DASP Collaboration). "Measurements of tau decay modes and a precise determination of the mass". In: Physics Letters B 73.1 (1978), pp. 109-114.

[25] ALEPH Collaboration et al. "Precision electroweak measurements on the Z resonance". In: Physics Reports 427.5 (2006), pp. 257-454. URL: http://www.sciencedirect.com/science/article/pii/S0370157305005119.

[26] Peter W. Higgs. "Broken Symmetries and the Masses of Gauge Bosons". In: Phys. Rev. Lett. 13 (16 Oct. 1964), pp. 508-509. URL: https://link.aps.org/doi/10.1103/PhysRevLett.13.508.

[27] F. Englert and R. Brout. "Broken Symmetry and the Mass of Gauge Vector Mesons". In: Phys. Rev. Lett. 13 (9 Aug. 1964), pp. 321-323. URL: https://link.aps.org/doi/10.1103/PhysRevLett.13.321.

[28] G. Aad et al. (ATLAS Collaboration). "Observation of a new particle in the search for the Standard Model Higgs boson with the ATLAS detector at the LHC". In: Physics Letters B 716.1 (2012), pp. 1-29. URL: http://www.sciencedirect.com/science/article/pii/S037026931200857X. 
[29] S. Chatrchyan et al. (CMS Collaboration). "Observation of a new boson at a mass of $125 \mathrm{GeV}$ with the CMS experiment at the LHC". In: Physics Letters B 716.1 (2012), pp. 30-61. URL:

http://www.sciencedirect.com/science/article/pii/S0370269312008581.

[30] K. Kodama et al. (DONUT Collaboration). "Observation of tau neutrino interactions". In: Physics Letters B 504.3 (2001), pp. 218-224. URL: http://www.sciencedirect.com/science/article/pii/S0370269301003070.

[31] Bruce T. Cleveland et al. "Measurement of the Solar Electron Neutrino Flux with the Homestake Chlorine Detector". In: The Astrophysical Journal 496.1 (Mar. 1998), pp. 505-526. URL: https://doi.org/10.1086/305343.

[32] John N. Bahcall and Raymond Davis. "Solar Neutrinos: A Scientific Puzzle". In: Science 191.4224 (1976), pp. 264-267. URL: https://science. sciencemag.org/content/191/4224/264.

[33] Q. R. Ahmad et al. (SNO Collaboration). "Measurement of the Rate of $\nu_{e}+d \rightarrow p+p+e^{-}$Interactions Produced by ${ }^{8} B$ Solar Neutrinos at the Sudbury Neutrino Observatory". In: Phys. Rev. Lett. 87 (7 July 2001), p. 071301. URL: https://link.aps.org/doi/10.1103/PhysRevLett.87.071301.

[34] Q. R. Ahmad et al. (SNO Collaboration). "Direct Evidence for Neutrino Flavor Transformation from Neutral-Current Interactions in the Sudbury Neutrino Observatory". In: Phys. Rev. Lett. 89 (1 June 2002), p. 011301. URL: https://link.aps.org/doi/10.1103/PhysRevLett.89.011301.

[35] J. Hosaka et al. (Super-Kamiokande Collaboration). "Solar neutrino measurements in Super-Kamiokande-I". In: Phys. Rev. D 73 (11 June 2006), p. 112001. URL: https://link.aps.org/doi/10.1103/PhysRevD.73.112001.

[36] John N. Bahcall, M. H. Pinsonneault, and Sarbani Basu. "Solar Models: Current Epoch and Time Dependences, Neutrinos, and Helioseismological Properties". In: The Astrophysical Journal 555.2 (July 2001), pp. 990-1012. URL: https://doi.org/10.1086/321493.

[37] Raymond Davis. "A review of the homestake solar neutrino experiment". In: Progress in Particle and Nuclear Physics 32 (1994), pp. 13-32. URL: http://www.sciencedirect.com/science/article/pii/0146641094900043.

[38] Y. Fukuda et al. "Atmospheric vuve ratio in the multi-GeV energy range". In: Physics Letters B 335.2 (1994), pp. 237-245. URL: http://www.sciencedirect.com/science/article/pii/0370269394914206.

[39] R. Becker-Szendy et al. "Electron- and muon-neutrino content of the atmospheric flux". In: Phys. Rev. D 46 (9 Nov. 1992), pp. 3720-3724. URL: https://link.aps.org/doi/10.1103/PhysRevD.46.3720.

[40] Y. Fukuda et al. (Super-Kamiokande Collaboration). "Evidence for Oscillation of Atmospheric Neutrinos". In: Physical Review Letters 81 (Aug. 1998).

[41] M. Drewes. "The Phenomenology of Right Handed Neutrinos". In: International Journal of Modern Physics E 22.08 (2013), p. 1330019. URL: https://doi.org/10.1142/S0218301313300191. 
[42] Ariel Goobar et al. "The neutrino mass bound from WMAP 3 year data, the baryon acoustic peak, the SNLS supernovae and the Lyman-alpha forest". In: Journal of Cosmology and Astroparticle Physics 2006.06 (2006). URL: https://doi.org/10.1088/1475-7516/2006/06/019.

[43] Joachim Wolf. "The KATRIN neutrino mass experiment". In: Nuclear Instruments and Methods in Physics Research Section A: Accelerators, Spectrometers, Detectors and Associated Equipment 623.1 (2010). 1st International Conference on Technology and Instrumentation in Particle Physics, pp. 442-444. URL: http://www.sciencedirect.com/science/article/pii/S0168900210005942.

[44] K. Hirata et al. "Observation of a neutrino burst from the supernova SN1987A". In: Phys. Rev. Lett. 58 (14 Apr. 1987), pp. 1490-1493. URL: https://link.aps.org/doi/10.1103/PhysRevLett.58.1490.

[45] R Bionta et al. "Observation of a neutrino burst in coincidence with supernova 1987A in the Large Magellanic Cloud". In: Phys. Rev. Lett. 58 (May 1987).

[46] Louis E Strigari et al. "The supernova relic neutrino backgrounds at KamLAND and Super-Kamiokande". In: Journal of Cosmology and Astroparticle Physics 2004.03 (Mar. 2004), pp. 007-007. URL: https://doi.org/10.1088/1475-7516/2004/03/007.

[47] IceCube Collaboration. "Evidence for High-Energy Extraterrestrial Neutrinos at the IceCube Detector". In: Science 342.6161 (2013). URL: https://science.sciencemag.org/content/342/6161/1242856.

[48] C. F. Turley et al. "A Coincidence Cosmic Neutrino and Gamma-Ray Emitting Sources Using IceCube and Fermi-LAT Public Data". In: The Astrophysical Journal 863.1 (Aug. 2018), p. 64. URL: https://doi .org/10.3847/1538-4357/aad195.

[49] L. Cardani. "Neutrinoless Double Beta Decay Overview". In: SciPost Phys. Proc. (1 2019), p. 24. URL: https://scipost.org/10.21468/SciPostPhysProc.1.024.

[50] M. A. Acero et al. (NOvA Collaboration). "First Measurement of Neutrino Oscillation Parameters using Neutrinos and Antineutrinos by NOvA". In: (2019). arXiv: 1906.04907 [hep-ex].

[51] J. H. Christenson et al. "Evidence for the $2 \pi$ Decay of the $K_{2}^{0}$ Meson". In: Phys. Rev. Lett. 13 (4 July 1964), pp. 138-140. URL: https://link . aps .org/doi/10.1103/PhysRevLett.13.138.

[52] B. Pontecorvo. "Inverse beta processes and nonconservation of lepton charge". In: Sov. Phys. JETP 7 (1958). [Zh. Eksp. Teor. Fiz.34,247(1957)], pp. 172-173.

[53] Ziro Maki, Masami Nakagawa, and Shoichi Sakata. "Remarks on the Unified Model of Elementary Particles". In: Progress of Theoretical Physics 28.5 (Nov. 1962), pp. 870-880. URL: https://doi.org/10.1143/PTP.28.870.

[54] M. Tanabashi et al. (Particle Data Group). "Review of Particle Physics". In: Phys. Rev. D 98 (3 Aug. 2018), p. 030001. URL: https://link.aps.org/doi/10.1103/PhysRevD.98.030001. 
[55] Norbert Schmitz. "Eigenschaften der Neutrinos". In: Neutrinophysik. Wiesbaden: Vieweg+Teubner Verlag, 1997, pp. 204-321. URL: https://doi.org/10.1007/978-3-322-80114-2_6.

[56] X. Qian and P. Vogel. "Neutrino mass hierarchy". In: Progress in Particle and Nuclear Physics 83 (2015), pp. 1-30. URL: http://www.sciencedirect.com/science/article/pii/S0146641015000307.

[57] C. Giganti, S. Lavignac, and M. Zito. "Neutrino oscillations: The rise of the PMNS paradigm". In: Progress in Particle and Nuclear Physics 98 (2018), pp. 1-54. URL: http://www. sciencedirect.com/science/article/pii/S014664101730087X.

[58] L. Wolfenstein. "Neutrino oscillations in matter". In: Phys. Rev. D 17 (9 May 1978), pp. 2369-2374. URL: https://link.aps.org/doi/10.1103/PhysRevD.17.2369.

[59] S. P. Mikheyev and A. Yu. Smirnov. "Resonant amplification of $\nu$ oscillations in matter and solar-neutrino spectroscopy". In: Il Nuovo Cimento C 9.1 (Jan. 1986), pp. 17-26. URL: https://doi.org/10.1007/BF02508049.

[60] A. Renshaw et al. (Super-Kamiokande Collaboration). "First Indication of Terrestrial Matter Effects on Solar Neutrino Oscillation". In: Phys. Rev. Lett. 112 (9 Mar. 2014), p. 091805. URL: https://link.aps.org/doi/10.1103/PhysRevLett.112.091805.

[61] E. Kh. Akhmedov and A. Yu. Smirnov. "Paradoxes of neutrino oscillations". In: Physics of Atomic Nuclei 72.8 (Aug. 2009), pp. 1363-1381. URL: https://doi.org/10.1134/S1063778809080122.

[62] Bernhard J. Holzer. "Beam optics and lattice design for particle accelerators". In: arXiv:1303.6514 (Mar. 2013), 171-206. 36 p. URL: https://cds.cern.ch/record/1533020.

[63] K. Abe et al. (T2K Collaboration). "The T2K experiment". In: Nuclear Instruments and Methods in Physics Research Section A: Accelerators, Spectrometers, Detectors and Associated Equipment 659.1 (2011), pp. 106-135. URL: http://www.sciencedirect.com/science/article/pii/S0168900211011910.

[64] K. Abe et al. (T2K Collaboration). "Observation of Electron Neutrino Appearance in a Muon Neutrino Beam". In: Phys. Rev. Lett. 112 (6 Feb. 2014), p. 061802. URL: https://link.aps.org/doi/10.1103/PhysRevLett.112.061802.

[65] K. Suzuki et al. "Measurement of the muon beam direction and muon flux for the T2K neutrino experiment". In: Progress of Theoretical and Experimental Physics 2015.5 (May 2015). 053C01. URL: https://doi.org/10.1093/ptep/ptv054.

[66] S. Bhadra et al. "Optical transition radiation monitor for the T2K experiment". In: Nuclear Instruments and Methods in Physics Research Section A: Accelerators, Spectrometers, Detectors and Associated Equipment 703 (2013), pp. 45-58. URL: http://www. sciencedirect.com/science/article/pii/S0168900212013812.

[67] T. Sekiguchi et al. "Development and operational experience of magnetic horn system for T2K experiment". In: Nuclear Instruments and Methods in Physics Research Section A: Accelerators, Spectrometers, Detectors and Associated Equipment 789 (2015), pp. 57-80. URL: http://www.sciencedirect.com/science/article/pii/S0168900215004672. 
[68] N. Abgrall et al. Neutrino Flux Prediction. www.t2k.org/docs/technotes/038/. 2011.

[69] T. Nakadaira. "The graphite target for J-PARC neutrino beam-line". In: Journal of Radioanalytical and Nuclear Chemistry 305.3 (Sept. 2015), pp. 777-782. URL: https://doi.org/10.1007/s10967-015-4120-7.

[70] C. Xiao et al. "An overview of integratable current sensor technologies". In: 38th IAS Annual Meeting on Conference Record of the Industry Applications Conference, 2003. Vol. 2. Oct. 2003, 1251-1258 vol.2.

[71] A.K. Ichikawa. "Design concept of the magnetic horn system for the T2K neutrino beam". In: Nuclear Instruments and Methods in Physics Research Section A: Accelerators, Spectrometers, Detectors and Associated Equipment 690 (2012), pp. 27-33. URL: http://www.sciencedirect.com/science/article/pii/S0168900212007115.

[72] K. Abe et al. (T2K Collaboration). "The T2K Neutrino Flux Prediction". In: Phys.Rev. D87 (2013), p. 012001. arXiv: 1211.0469 [hep-ex].

[73] K. Matsuoka et al. "Design and performance of the muon monitor for the T2K neutrino oscillation experiment". In: Nuclear Instruments and Methods in Physics Research Section A: Accelerators, Spectrometers, Detectors and Associated Equipment 624.3 (2010), pp. 591-600. URL: http://www.sciencedirect.com/science/article/pii/S016890021002098X.

[74] M. Barranco Luque et al. (UA1 Collaboration). "The construction of the central detector for an experiment at the CERN $\overline{\mathrm{p}}$-p collider". In: Nuclear Instruments and Methods 176.1 (1980), pp. 175-180. URL: http://www.sciencedirect.com/science/article/pii/0029554X80906990.

[75] J. Altegoer et al. (NOMAD Collaboration). "The NOMAD experiment at the CERN SPS". In: Nuclear Instruments and Methods in Physics Research Section A: Accelerators, Spectrometers, Detectors and Associated Equipment 404.1 (1998), pp. 96-128. URL: http://www.sciencedirect.com/science/article/pii/S0168900297010796.

[76] S. Assylbekov et al. "The T2K ND280 off-axis pi-zero detector". In: Nuclear Instruments and Methods in Physics Research Section A: Accelerators, Spectrometers, Detectors and Associated Equipment 686 (2012), pp. 48-63. URL: http://www.sciencedirect.com/science/article/pii/S0168900212005153.

[77] N. Abgrall et al. "Time projection chambers for the T2K near detectors". In: Nuclear Instruments and Methods in Physics Research Section A: Accelerators, Spectrometers, Detectors and Associated Equipment 637.1 (2011), pp. 25-46. URL: http://www.sciencedirect.com/science/article/pii/S0168900211003421.

[78] P.-A. Amaudruz et al. "The T2K fine-grained detectors". In: Nuclear Instruments and Methods in Physics Research Section A: Accelerators, Spectrometers, Detectors and Associated Equipment 696 (2012), pp. 1-31. URL: http://www.sciencedirect.com/science/article/pii/S0168900212008789. 
[79] G. Charpak et al. "Micromegas, a multipurpose gaseous detector". In: Nuclear Instruments and Methods in Physics Research Section A: Accelerators, Spectrometers, Detectors and Associated Equipment 478.1 (2002). Proceedings of the ninth Int. Conf. on Instrumentation, pp. 26-36. URL: http://www. sciencedirect.com/science/article/pii/S0168900201017132.

[80] D Allan et al. "The electromagnetic calorimeter for the T2K near detector ND280". In: Journal of Instrumentation 8.10 (Oct. 2013), P10019-P10019. URL: https://doi.org/10.1088/1748-0221/8/10/p10019.

[81] T. Yano et al. "The Side Muon Range Detector for the T2K experiment". In: Nuclear Physics B - Proceedings Supplements 229-232 (2012). Neutrino 2010, p. 454. URL: http://www.sciencedirect.com/science/article/pii/S0920563212003088.

[82] K. Abe et al. (T2K Collaboration). "Measurements of the T2K neutrino beam properties using the INGRID on-axis near detector". In: Nuclear Instruments and Methods in Physics Research Section A: Accelerators, Spectrometers, Detectors and Associated Equipment 694 (2012), pp. 211-223. URL: http://www.sciencedirect.com/science/article/pii/S0168900212002987.

[83] T. Kikawa et al. INGRID New Analysis Technical Note. www.t2k.org/docs/technotes/132. 2013.

[84] M. Otani et al. INGRID Analysis Technical Note. www.t2k.org/docs/technotes/041. 2012.

[85] A. Fiorentini et al. Flux Prediction and Uncertainty Updates with NA61 2009 Thin Target Data and Negative Focussing Mode Predictions. www.t2k.org/docs/technotes/217. 2016.

[86] S. Fukuda et al. (Super-Kamiokande Collaboration). "The Super-Kamiokande detector". In: Nuclear Instruments and Methods in Physics Research Section A: Accelerators, Spectrometers, Detectors and Associated Equipment 501.2 (2003), pp. 418-462. URL: http://www.sciencedirect.com/science/article/pii/S016890020300425X.

[87] E. V. Bugaev et al. "Atmospheric muon flux at sea level, underground, and underwater". In: Phys. Rev. D 58 (5 July 1998), p. 054001. URL: https://link.aps.org/doi/10.1103/PhysRevD.58.054001.

[88] D. Casper. "The nuance neutrino physics simulation, and the future". In: Nuclear Physics B - Proceedings Supplements 112.1 (2002), pp. 161-170. URL: http://www.sciencedirect.com/science/article/pii/S0920563202017565.

[89] J. A. Formaggio and G. P. Zeller. "From eV to EeV: Neutrino cross sections across energy scales". In: Rev. Mod. Phys. 84 (3 Sept. 2012), pp. 1307-1341. URL: https://link.aps.org/doi/10.1103/RevModPhys.84.1307.

[90] A. Himmel et al. Super-Kamiokande Events and Data Quality Studies for T2K Runs 5 and 6. www.t2k.org/docs/technotes/219. 2015.

[91] K. Abe et al. (T2K Collaboration). "Measurement of neutrino and antineutrino oscillations by the T2K experiment including a new additional sample of $\nu_{e}$ interactions at the far detector". In: Phys. Rev. D 96 (9 Nov. 2017), p. 092006. URL: https://link.aps.org/doi/10.1103/PhysRevD.96.092006. 
[92] Andrew David Missert. "Neutrino Oscillation Measurements Using a Maximum Likelihood Event Reconstruction Algorithm". PhD thesis. University of Colorado at Boulder, 2017.

[93] Alexis Hasler. "T2K Replica Target Hadron Production Measurements in NA61/SHINE and T2K Neutrino Flux Predictions". PhD thesis. University of Geneva, 2015. URL: https://cds.cern.ch/record/2039148.

[94] N. Abgrall et al. (NA61/SHINE Collaboration). "NA61/SHINE facility at the CERN SPS: beams and detector system". In: Journal of Instrumentation 9.06 (June 2014), P06005-P06005. URL: https://doi.org/10.1088/1748-0221/9/06/p06005.

[95] S Afanasiev et al. "The NA49 large acceptance hadron detector". In: Nuclear Instruments and Methods in Physics Research Section A: Accelerators, Spectrometers, Detectors and Associated Equipment 430 (July 1999), pp. 210-244.

[96] C. Bovet et al. "The Cedar (Cerenkov Differential Counters with Achromatic Ring Focus) Project". In: IEEE Transactions on Nuclear Science 25.1 (Feb. 1978), pp. $572-576$.

[97] N. Abgrall et al. (NA61/SHINE Collaboration). "Measurements of $\pi^{ \pm}, K^{ \pm}$and proton double differential yields from the surface of the T2K replica target for incoming $31 \mathrm{GeV} / \mathrm{c}$ protons with the NA61/SHINE spectrometer at the CERN SPS". In: The European Physical Journal C 79.2 (Jan. 2019). URL: https://doi.org/10.1140/epjc/s10052-019-6583-0.

[98] N. Abgrall et al. (NA61/SHINE Collaboration). "Measurements of $\pi^{ \pm}, K^{ \pm}, K_{S}^{0}$, $\Lambda$ and proton production in proton-carbon interactions at $31 \mathrm{GeV} / \mathrm{c}$ with the NA61/SHINE spectrometer at the CERN SPS". In: Eur. Phys. J. C76.2 (2016), p. 84. URL: https://doi.org/10.1140/epjc/s10052-016-3898-y.

[99] N. Abgrall et al. (NA61/SHINE Collaboration). "Measurements of $\pi^{ \pm}$differential yields from the surface of the T2K replica target for incoming $31 \mathrm{GeV} / \mathrm{c}$ protons with the NA61/SHINE spectrometer at the CERN SPS". In: Eur. Phys. J. C76.11 (2016), p. 617. URL: https://doi.org/10.1140/epjc/s10052-016-4440-y.

[100] N. Abgrall et al. (NA61/SHINE Collaboration). "Measurements of cross sections and charged pion spectra in proton-carbon interactions at $31 \mathrm{GeV} / c$ ". In: Phys. Rev. C 84 (3 Sept. 2011), p. 034604. URL: https://link.aps.org/doi/10.1103/PhysRevC.84.034604.

[101] N. Abgrall et al. (NA61/SHINE Collaboration). "Measurement of production properties of positively charged kaons in proton-carbon interactions at $31 \mathrm{GeV} / c$ ". In: Phys. Rev. C 85 (3 Mar. 2012), p. 035210. URL: https://link.aps.org/doi/10.1103/PhysRevC.85.035210.

[102] O. Buss et al. "Transport-theoretical description of nuclear reactions". In: Physics Reports 512.1 (2012). Transport-theoretical Description of Nuclear Reactions, pp. 1-124. URL: http://www.sciencedirect.com/science/article/pii/S0370157311003619.

[103] S. Agostinelli et al. (GEANT4 Collaboration). "GEANT4: A Simulation toolkit". In: Nucl. Instrum. Meth. A506 (2003), pp. 250-303. 
[104] L. Zheng and X. Zhang. "Chapter 8 - Numerical Methods". In: Modeling and Analysis of Modern Fluid Problems. Ed. by Liancun Zheng and Xinxin Zhang. Mathematics in Science and Engineering. Academic Press, 2017, pp. 361-455. URL: http:

//wWw.sciencedirect.com/science/article/pii/B9780128117538000086.

[105] G. Bellettini et al. "Proton-nuclei cross sections at $20 \mathrm{GeV}$ ". In: Nuclear Physics 79.3 (1966), pp. 609-624. URL:

http://wWw.sciencedirect.com/science/article/B73DR-46YSSCV7F/2/030e70b8f6a86f3a5cb1e16a3000ae4b.

[106] A. Schiz et al. "High-statistics study of +p, -p, and pp elastic scattering at 200 GeV/c". English (US). In: Physical review D: Particles and fields 24.1 (1981), pp. $26-45$.

[107] Alfredo Ferrari et al. "FLUKA: A multi-particle transport code (Program version 2005)". In: (2005).

[108] René Brun et al. "GEANT Detector Description and Simulation Tool". In: (1994).

[109] C. Zeitnitz and T.A. Gabriel. "The GEANT-CALOR interface and benchmark calculations of ZEUS test calorimeters". In: Nuclear Instruments and Methods in Physics Research Section A: Accelerators, Spectrometers, Detectors and Associated Equipment 349.1 (1994), pp. 106-111. URL:

http://www.sciencedirect.com/science/article/pii/0168900294906130.

[110] M. Bonesini et al. "On particle production for high energy neutrino beams". In: The European Physical Journal C - Particles and Fields 20.1 (Apr. 2001), pp. 13-27. URL: https://doi.org/10.1007/s100520100656.

[111] T. Eichten et al. "Particle production in proton interactions in nuclei at 24 GeV/c". In: Nuclear Physics B 44.2 (1972), pp. 333-343. URL: http://www.sciencedirect.com/science/article/pii/0550321372901204.

[112] J. V. Allaby et al. "High-energy particle spectra from proton interactions at 19.2 GeV/c". In: Technical Report 70-12, CERN (1970).

[113] Richard P. Feynman. "Very High-Energy Collisions of Hadrons". In: Phys. Rev. Lett. 23 (24 Dec. 1969), pp. 1415-1417. URL: https://link.aps.org/doi/10.1103/PhysRevLett.23.1415.

[114] D. S. Barton et al. "Experimental study of the $A$ dependence of inclusive hadron fragmentation". In: Phys. Rev. D 27 (11 June 1983), pp. 2580-2599. URL: https://link.aps.org/doi/10.1103/PhysRevD.27.2580.

[115] P. Skubic et al. "Neutral-strange-particle production by $300-\mathrm{GeV}$ protons". In: Phys. Rev. D 18 (9 Nov. 1978), pp. 3115-3144. URL: https://link.aps.org/doi/10.1103/PhysRevD.18.3115.

[116] T. Abbott et al. (E-802 Collaboration). "Measurement of particle production in proton induced reactions at 14.6-GeV/c". In: Phys.Rev. D45 (1992), pp. 3906-3920.

[117] M. Apollonio et al. (HARP Collaboration). "Forward production of charged pions with incident protons on nuclear targets at the CERN PS". In: Phys.Rev. C80 (2009), p. 035208. arXiv: 0907.3857 [hep-ex]. 
[118] M. Apollonio et al. (HARP Collaboration). "Forward production of charged pions with incident $\pi^{ \pm}$on nuclear targets measured at the CERN PS". In: Nuclear Physics A 821.1 (2009), pp. 118-192. URL: http://www.sciencedirect.com/science/article/pii/S0375947409000232.

[119] Michael McCool, Arch D. Robison, and James Reinders. "Chapter 15 - Cholesky Factorization". In: Structured Parallel Programming. Ed. by Michael McCool, Arch D. Robison, and James Reinders. Boston: Morgan Kaufmann, 2012, pp. 315-322. URL: http:

//www.sciencedirect.com/science/article/pii/B9780124159938000153.

[120] S.P. Denisov et al. "Absorption cross-sections for pions, kaons, protons and anti-protons on complex nuclei in the $6-\mathrm{GeV} / \mathrm{c}$ to $60-\mathrm{GeV} / \mathrm{c}$ momentum range". In: Nucl.Phys. B61 (1973), pp. 62-76.

[121] A. S. Carroll et al. "Absorption cross section of [pi],$\pm \mathrm{K} \pm$, p and on nuclei between 60 and $280 \mathrm{GeV} / \mathrm{c}$ ". In: Physics Letters B 80.3 (1979), pp. 319-322. URL: http://www.sciencedirect.com/science/article/B6TVN-46YKH5DWP/2/bb31d269058cc69e5d831a46acb9f345.

[122] Sonam Mahajan and Rajendran Raja. "Particle Production Measurements using the MIPP Detector at Fermilab". In: (2013). arXiv: 1311.2258 [hep-ex].

[123] B.M. Bobchenko et al. "Measurement of total inelastic cross-sections from proton interactions with nuclei in the momentum range from $5 \mathrm{GeV} / \mathrm{c}$ to $9 \mathrm{GeV} / \mathrm{c}$ and $\pi^{-}$mesons with nuclei in the momentum range from $1.75 \mathrm{GeV} / \mathrm{c}$ to $6.5 \mathrm{GeV} / \mathrm{c}$ ". In: Sov. J. Nucl. Phys. 30 (1979), p. 805.

[124] N. Abgrall et al. Flux Prediction and Uncertainties for the 2012a Oscillation Analysis. www.t2k.org/docs/technotes/099. 2013.

[125] Yu. M. Shabelski. "Multiple hadron production in the quark-gluon string model". In: Nuclear Physics B - Proceedings Supplements 52.3 (1997), pp. 116-119. URL: http://www.sciencedirect.com/science/article/pii/S0920563296008559.

[126] Hugo W. Bertini. "Intranuclear-Cascade Calculation of the Secondary Nucleon Spectra from Nucleon-Nucleus Interactions in the Energy Range 340 to $2900 \mathrm{MeV}$ and Comparisons with Experiment". In: Phys. Rev. 188 (4 Dec. 1969), pp. 1711-1730. URL: https://link.aps.org/doi/10.1103/PhysRev.188.1711.

[127] R. Serber. "The Production of High Energy Neutrons by Stripping". In: Phys. Rev. 72 (11 Dec. 1947), pp. 1008-1016. URL:

https://link.aps.org/doi/10.1103/PhysRev.72.1008.

[128] Dennis H. Wright et al. "Low and High Energy Modeling in Geant4". In: AIP Conference Proceedings 896.1 (2007), pp. 11-20. URL: https://aip.scitation.org/doi/abs/10.1063/1.2720453.

[129] B. Andersson, G. Gustafson, and B. Nilsson-Almqvist. "A model for low-pT hadronic reactions with generalizations to hadron-nucleus and nucleus-nucleus collisions". In: Nuclear Physics B 281.1 (1987), pp. 289-309. URL: http://www.sciencedirect.com/science/article/pii/0550321387902574.

[130] Lukas Berns. "Systematic uncertainties for T2K neutrino flux tuning with 2010 NA61/SHINE data". In: JPS 73rd Annual Meeting (2018). URL: https: //w4.gakkai-web.net/jps_search/2018spe/data/html/programsr.html. 
[131] Kirsty Duffy. "Measurement of the neutrino oscillation parameters $\sin ^{2} \theta_{23}, \Delta m_{32}^{2}$, $\sin ^{2} \theta_{13}$, and $\delta_{\mathrm{CP}}$ in neutrino and antineutrino oscillation at T2K". PhD thesis. University of Oxford, 2016. URL: https://ora.ox.ac.uk/objects/uuid: 4485185d-3f72-49d0-866c-af 2bab55d916.

[132] J. Beringer et al. (Particle Data Group). "Review of Particle Physics". In: Phys. Rev. D 86 (1 July 2012), p. 010001. URL: https://link.aps.org/doi/10.1103/PhysRevD.86.010001.

[133] Nicholas Metropolis et al. "Equation of State Calculations by Fast Computing Machines". In: The Journal of Chemical Physics 21.6 (1953), pp. 1087-1092. eprint: https://doi.org/10.1063/1.1699114. URL: https://doi.org/10.1063/1.1699114.

[134] W. K. Hastings. "Monte Carlo sampling methods using Markov chains and their applications". In: Biometrika 57.1 (Apr. 1970), pp. 97-109. eprint: http: //oup.prod.sis.lan/biomet/article-pdf/57/1/97/23940249/57-1-97.pdf. URL: https://doi.org/10.1093/biomet/57.1.97.

[135] Yoshinari Hayato. "A Neutrino Interaction Simulation Program Library NEUT". In: Acta Physica Polonica B - ACTA PHYS POL B 40 (Sept. 2009).

[136] A.A. Aguilar-Arevalo et al. "The MiniBooNE detector". In: Nuclear Instruments and Methods in Physics Research Section A: Accelerators, Spectrometers, Detectors and Associated Equipment 599.1 (2009), pp. 28-46. URL: http://www.sciencedirect.com/science/article/pii/S0168900208015404.

[137] L. Aliaga et al. "Design, calibration, and performance of the MINERvA detector". In: Nuclear Instruments and Methods in Physics Research Section A: Accele rators, Spectrometers, Detectors and Associated Equipment 743 (2014), pp. 130-159. URL: http://www.sciencedirect.com/science/article/pii/S0168900214000035.

[138] The Neutrino Interaction Working Group. NIWG model and uncertainties for 2017 oscillation analysis. www.t2k.org/docs/technotes/315. 2017.

[139] Véronique Bernard, Latifa Elouadrhiri, and Ulf-G Meißner. "Axial structure of the nucleon". In: Journal of Physics G: Nuclear and Particle Physics 28.1 (Nov. 2001), R1-R35. URL: https://doi.org/10.1088/0954-3899/28/1/201.

[140] E. J. Moniz et al. "Nuclear Fermi momenta from quasielastic electron scattering". In: Phys. Rev. Lett. 26 (1971), pp. 445-448.

[141] J. Nieves, I. Ruiz Simo, and M. J. Vicente Vacas. "Inclusive charged-current neutrino-nucleus reactions". In: Phys. Rev. C 83 (4 Apr. 2011), p. 045501. URL: https://link.aps.org/doi/10.1103/PhysRevC.83.045501.

[142] Dieter Rein and Lalit M Sehgal. "Neutrino-excitation of baryon resonances and single pion production". In: Annals of Physics 133.1 (1981), pp. 79-153. URL: http://www.sciencedirect.com/science/article/pii/0003491681902426.

[143] P. Adamson et al. (MINOS Collaboration). "Neutrino and antineutrino inclusive charged-current cross section measurements with the MINOS near detector". In: Phys. Rev. D 81 (7 Apr. 2010), p. 072002. URL:

https://link.aps.org/doi/10.1103/PhysRevD.81.072002. 
[144] A. Higuera et al. (MINERvA Collaboration). "Measurement of Coherent Production of $\pi^{ \pm}$in Neutrino and Antineutrino Beams on Carbon from $E_{\nu}$ of 1.5 to $20 \mathrm{GeV}$ ". In: Phys. Rev. Lett. 113 (26 Dec. 2014), p. 261802. URL:

https://link.aps.org/doi/10.1103/PhysRevLett.113.261802.

[145] E. Wang et al. "Photon emission in neutral current interactions at the T2K experiment". In: Phys. Rev. D 92 (5 Sept. 2015), p. 053005. URL: https://link.aps.org/doi/10.1103/PhysRevD.92.053005.

[146] E. Wang et al. "Photon emission in neutral-current interactions at intermediate energies". In: Phys. Rev. C 89 (1 Jan. 2014), p. 015503. URL: https://link.aps.org/doi/10.1103/PhysRevC.89.015503.

[147] Patrick de Perio. "NEUT Pion Final State Interactions". In: (2014). eprint: arXiv: 1405.3973.

[148] Kirsty Duffy. "Current Status and Future Plans of T2K". In: Conference Talk, Prospects in Neutrino Physics (NuPhys2016): London, UK, December 12-14, 2016. 2017. URL: https://indico.ph.qmul.ac.uk/indico/getFile.py/access? contribId=44\&res $I d=0 \& m a t e r i a l I d=s l i d e s \& \operatorname{conf} I d=112$.

[149] Kirsty Duffy. "Current Status and Future Plans of T2K". In: Proceedings, Prospects in Neutrino Physics (NuPhys2016): London, UK, December 12-14, 2016. 2017. arXiv: 1705.01764 [hep-ex].

[150] Yoshinari Hayato. "A neutrino interaction simulation program library NEUT". In: Acta Phys. Polon. B40 (2009), pp. 2477-2489.

[151] C. Andreopoulos et al. "The GENIE Neutrino Monte Carlo Generator". In: Nucl. Instrum. Meth. A614 (2010), pp. 87-104. arXiv: 0905.2517 [hep-ph].

[152] K. Abe et al. (T2K Collaboration). "Measurement of the inclusive $\nu_{\mu}$ charged current cross section on carbon in the near detector of the T2K experiment". In: Phys. Rev. D 87 (9 May 2013), p. 092003. URL: https://link.aps.org/doi/10.1103/PhysRevD.87.092003.

[153] K. Abe et al. (T2K Collaboration). "Proposal for an Extended Run of T2K to $20 \times 10^{21}$ POT". In: (2016). arXiv: 1609.04111 [hep-ex].

[154] K. Abe et al. (T2K Collaboration). "Combined Analysis of Neutrino and Antineutrino Oscillations at T2K". In: Phys. Rev. Lett. 118 (15 Apr. 2017), p. 151801. URL: https://link.aps.org/doi/10.1103/PhysRevLett.118.151801.

[155] K. Abe et al. (Hyper-Kamiokande Working Group). "A Long Baseline Neutrino Oscillation Experiment Using J-PARC Neutrino Beam and Hyper-Kamiokande". In: 2014. arXiv: 1412.4673 [physics.ins-det].

[156] Khalid Gameil. "Hadron production measurements of the EMPATHIC group". PhD thesis. University of British Columbia, 2018. URL: https: //open.library.ubc.ca/collections/ubctheses/24/items/1.0371134.

[157] D. Larsen (NA61/SHINE Collaboration). "Upgrade of the NA61/SHINE Facility beyond 2020 for an Expanded Physics Programme". In: Universe 5.1 (Jan. 2019), p. 24. URL: http://dx.doi.org/10.3390/universe5010024. 